\title{
Long period variables in NGC 5128
}

\section{Catalogue $e^{\star}, \star$}

\author{
M. Rejkuba ${ }^{1,2}$, D. Minniti ${ }^{2}$, and D. R. Silva ${ }^{1}$ \\ 1 European Southern Observatory, Karl-Schwartzschild-Strasse 2, 85748 Garching, Germany \\ e-mail:mrejkuba@eso.org,dsilva@eso.org \\ 2 Department of Astronomy, P. Universidad Católica, Casilla 306, Santiago 22, Chile \\ e-mail: dante@astro.puc.cl
}

Received 5 March 2003 / Accepted 6 May 2003

\begin{abstract}
The first variable star catalogue in a giant elliptical galaxy NGC 5128 (Centaurus A) is presented. Using multi-epoch observations with ISAAC at the VLT we have detected 1504 red variables in two halo fields covering 10.46 arcmin square. For the variables with at least 10 good measurements, periods and amplitudes were determined using Fourier analysis and non-linear sine-curve fitting algorithms. The final catalogue contains 1146 long period variables with well established light curve parameters. Periods, amplitudes, $J_{\mathrm{s}} H K_{\mathrm{s}}$ photometry as well as individual $K_{\mathrm{s}}$-band magnitudes are provided for all the variables. The distribution of amplitudes ranges from 0.3 to a few magnitudes in the $K_{\mathrm{s}}$-band, with a median value around 0.7 mag. The amplitudes, mean magnitudes and periods indicate that the majority of variables belong to the class of long period variables with semiregular and Mira variables. Exhaustive simulations were performed in order to assess the completeness of our catalogue and the accuracy of the derived periods.
\end{abstract}

Key words. galaxies: elliptical and lenticular, $\mathrm{cD}$ - galaxies: stellar content - stars: variables: general stars: AGB and post-AGB - galaxies: individual: NGC 5128

\section{Introduction}

In a stellar population older than few hundred Myr the near-IR light is dominated by red giants. Of these, first ascent giants are the brightest among the metal-poor stars older than 1-2 Gyr. In composite stellar populations, like those found in galaxies, the first ascent giants are mixed with asymptotic giant branch (AGB) stars. The red giants fainter than the tip of the first ascent giant branch are usually referred to as red giant branch (RGB) stars. In the following RGB is used to denote the first ascent giants, although it should be kept in mind that it is not possible to separate RGB and AGB stars that are fainter than the RGB tip.

In intermediate-age populations ( $1-5$ Gyr old) numerous bright asymptotic giant branch (AGB) stars are located above the tip of the RGB. However, among old populations like

Send offprint requests to: M. Rejkuba, e-mail: mrejkuba@eso.org

* Based on observations collected at the European Southern Observatory, Paranal, Chile, within the Observing Programmes 63.N-0229, 65.N-0164, 67.B-0503, 68.B-0129 and 69.B-0292, and at La Silla Observatory, Chile, within the Observing Programme 64.N-0176(B).

$\star \star$ Tables 3-8 and Figs. 7-14 together with the complete Sect. 6 are only available in electronic form at http://www. edpsciences.org
Galactic globular clusters with $[\mathrm{Fe} / \mathrm{H}] \gtrsim-1.0$ dex and in the Galactic bulge, bright stars have also been detected above the tip of the RGB (Frogel \& Elias 1988; Guarnieri et al. 1997), implying the presence of bright AGB stars in metal-rich and old populations. Essentially all of the bright giants above the RGB tip in globular clusters seem to be long period variables (LPVs; Frogel \& Elias 1988; Frogel \& Whitelock 1998). The frequency of LPVs in old metal-rich globular clusters of the MW and in the Bulge has been studied by Frogel \& Whitelock (1998). Old populations of lower metallicity are known not to have AGB stars brighter than the RGB tip. The presence or absence of these bright giants located above the tip of the first ascent giant branch has important implications for the magnitude of the surface brightness fluctuations specifically in the near-IR (e.g. Mei et al. 2001; Liu et al. 2002), a method used to determine distances to galaxies that are too distant to have their stellar content resolved, but that still present fluctuations due to the underlying light of giant stars (Tonry \& Schneider 1988).

The properties of LPVs have been reviewed by Habing (1996). They are thermally pulsing asymptotic giant branch (TP-AGB) stars with main sequence masses between 1 and $6 M_{\odot}$. They present variability with periods of 80 days or longer, and often the longest period variables show variable 
or multiple periods. Two main classes of LPVs are Mira variables (Miras) and semiregular variables (SRs). SRs usually have smaller amplitudes as well as shorter periods and more irregular light curves than Miras. SRs are sometimes subdivided into subclasses ( $\mathrm{SRa}, \mathrm{SRb}$ ) depending on the regularity and multiplicity of their periods and shape of their light curves. The separation between Miras and SRs is not always clear (e.g. Kerschbaum \& Hron 1992, 1994). The classical definition requires that Miras have $V$-band amplitudes larger than $2.5 \mathrm{mag}$ and regular periods in the range of 80-1000 days (GCVS Kholopov 1985). Mean $K$-band amplitudes of Miras are 0.6 mag (e.g. Feast et al. 1982; Wood et al. 1983).

In the gE NGC 5128 Soria et al. (1996), based on VI HST CMD, and Marleau et al. (2000), based on JH NICMOS data, suggested a presence of up to $10 \%$ bright AGB stars belonging to an intermediate-age population. Harris et al. (1999, 2000) on the contrary did not find bright AGB stars in their VI CMDs of two halo fields in NGC 5128. However, $V$ and $I$ bands are not very sensitive indicators of these cool giants and thus some of them might have been confused with the RGB tip or foreground stars and few stellar blends or stars with larger photometric errors.

Our group has obtained VLT images with FORS1 and ISAAC in the halo of NGC 5128 in order to study the bright giants in the halo of the galaxy (Rejkuba et al. 2001). We have observed two halo fields in the $V$ and $K_{\mathrm{s}}$-bands finding a large number of bright AGB stars, extending up to bolometric magnitude of -5 . Field 1 coincides with the prominent north-eastern diffuse stellar shell (Malin et al. 1983; Rejkuba et al. 2001). Crossing it in diagonal there is a chain of young stars (Mould et al. 2000; Fassett \& Graham 2000; Rejkuba et al. 2002). Field 2 is located away from the known stellar shells and dust bands, $\sim 9^{\prime}$ south from the galactic nucleus. It coincides with the field observed with HST in $V$ and $I$-bands with WFPC2 by Soria et al. (1996) and in $J$ and $H$-bands with NICMOS by Marleau et al. (2000). Part of the VLT ISAAC $K_{\mathrm{s}}$-band data analyzed here were already described in Rejkuba et al. (2001). Already with the first few epochs of $K_{\mathrm{s}}$-band imaging it was obvious that variable stars were present among the bright red giants. The preliminary results of the multi-epoch imaging in $K_{\mathrm{s}}$-band were presented by Rejkuba (2002).

We present here the full near-IR data-set containing multiepoch $K_{\mathrm{s}}$-band imaging and single-epoch data in the $J_{\mathrm{s}^{-}}$and $H$-bands obtained over the period of 3 years with ISAAC at the VLT. In this paper the data reduction, the photometry, and the catalogue of the variable stars are presented. In the following papers these data are used to investigate the variability characteristics of the population of stars found above the RGB tip, and to derive the distance to NGC 5128 using the Mira periodluminosity relation in the $K$-band. Multicolor information will further be used to investigate the chemical composition of these variables, and to put constraints to their ages. In the next section we briefly present the data and the reduction procedures. Section 3 contains the photometry. The technique used to detect the variable stars is described next. The light curves and periods of the long period variables are derived in Sect. 5. A detailed description of the completeness simulations is given only in the electronic version of the article where also the complete catalogue of all the variable stars can be found. Section 6 presents the main results of our simulations, while the last section summarizes the results.

\section{The data}

\subsection{The observations}

We have obtained a total of 20 epochs of $K_{\mathrm{s}}$-band photometry in Field 1 and 24 epochs in Field 2 between April 1999 and July 2002. Most of our data were obtained using the ISAAC near-IR imaging spectrometer at the ESO Paranal UT1 Antu $8.2 \mathrm{~m}$ telescope. These data were obtained in Service Mode. One Field 2 epoch comes from data obtained in Visitor Mode at the ESO La Silla NTT $3.5 \mathrm{~m}$ telescope equipped with SOFI near-IR imaging spectrometer.

The instrument setup for the observations was the following: all but 2 epochs for each field were observed with the short wavelength arm of ISAAC, which is equipped with a $1024 \times 1024$ pixel Hawaii Rockwell array with a pixel scale of 0 "'148. The last two epochs of the $K_{\mathrm{s}}$-band series for both fields were observed with the long wavelength arm of ISAAC with a $1024 \times 1024 \mathrm{InSb}$ Aladdin array from Santa Barbara Research Center. The pixel size of the Aladdin array with $0 . ' 1478$ is almost identical to that of Hawaii detector.

During the service observations of 3 epochs of Field 2, the integration was interrupted before the end of the sequence due to changing weather conditions. Additional observations for these epochs were taken a few days after the original sequences. Thus we reduced them as separate epochs, yielding 24 data points for Field 2, including the epoch observed with SOFI at NTT, and 20 epochs for Field 1 . Additional $J_{\mathrm{s}^{-}}$and $\mathrm{H}$-band single epoch observations were obtained in April and May 2002 with ISAAC, also in service mode. $J_{\mathrm{s}}$ and $H$-band observations were secured with a short wavelength arm of ISAAC. The $J_{\mathrm{s}}$ filter was preferred over the $J$ filter, due to the presence of red leaks in the $K$-band of the latter. From here on " $J$-band" is used to denote observations taken with $J_{\mathrm{s}}$ filter and " $K$-band" for $K_{\mathrm{s}}$ observations. The observation taken with $K_{\mathrm{s}}$ filter with SOFI at NTT is described in Rejkuba (2001).

The summary of the observations is given in Table 1, where on the left we describe the Field 1 observations and on the right the Field 2 observations. For each field, the first column is the Julian date of the observation in the form MJD = JD - 2400000 , in the second column the exposure time in minutes is given, the next is airmass, then seeing in arcseconds, and in the last field the filter and the sequential number of the $K$-band epoch are presented.

\subsection{Data reduction}

Data reduction followed the procedure described in Rejkuba et al. (2001). We first correct for the electronic ghost using the ESO supplied routine in the ECLIPSE package. Then we subtract the dark and divide with the sky flats provided from the service observing standard calibrations. The DIMSUM package (Stanford et al. 1995) in IRAF was used to subtract the sky in a double sky-subtraction run, the second time masking the 
Table 1. Near-IR multi-epoch photometry observing log. On the left Field 1 observations, and on the right Field 2 observations are described. MJD is JD-2400000, Exp is the exposure time in minutes and $X$ is the mean Airmass during the observing sequence.

\begin{tabular}{ccccc|ccccc}
\hline \hline MJD & $\begin{array}{c}\text { Exp } \\
\text { min }\end{array}$ & $\begin{array}{c}\text { Seeing } \\
\prime\end{array}$ & $\begin{array}{c}\text { filter } \\
\text { epoch }\end{array}$ & MJD & $\begin{array}{c}\text { Exp } \\
\text { min }\end{array}$ & $\begin{array}{c}\text { Seeing } \\
\prime\end{array}$ & $\begin{array}{c}\text { filter } \\
\text { epoch }\end{array}$ \\
\hline 51277.1 & 60 & 1.15 & 0.41 & $1 \mathrm{Ks} 01$ & 51277.2 & 60 & 1.05 & 0.37 & $2 \mathrm{Ks} 01$ \\
51306.1 & 60 & 1.05 & 0.40 & $1 \mathrm{Ks} 02$ & 51306.1 & 60 & 1.10 & 0.38 & $2 \mathrm{Ks} 02$ \\
51327.1 & 60 & 1.14 & 0.52 & $1 \mathrm{Ks} 03$ & 51328.0 & 60 & 1.06 & 0.44 & $2 \mathrm{Ks} 03$ \\
51650.1 & 65 & 1.09 & 0.36 & $1 \mathrm{Ks} 04$ & 51594.4 & 45 & 1.04 & 0.55 & $2 \mathrm{Ks} 04^{1}$ \\
51675.2 & 65 & 1.22 & 0.43 & $1 \mathrm{Ks} 05$ & 51650.3 & 65 & 1.09 & 0.40 & $2 \mathrm{Ks} 05$ \\
51702.9 & 65 & 1.13 & 0.61 & $1 \mathrm{Ks} 06$ & 51677.2 & 51 & 1.22 & 0.52 & $2 \mathrm{Ks} 06$ \\
51734.0 & 65 & 1.15 & 0.31 & $1 \mathrm{Ks} 07$ & 51684.2 & 20 & 1.22 & 0.44 & $2 \mathrm{Ks} 07$ \\
52037.1 & 60 & 1.05 & 0.64 & $1 \mathrm{Ks} 08$ & 51703.0 & 41 & 1.13 & 0.58 & $2 \mathrm{Ks} 08$ \\
52060.1 & 60 & 1.08 & 0.46 & $1 \mathrm{Ks} 09$ & 51705.0 & 30 & 1.13 & 0.41 & $2 \mathrm{Ks} 09$ \\
52096.0 & 60 & 1.05 & 0.33 & $1 \mathrm{Ks} 10$ & 51734.1 & 65 & 1.15 & 0.40 & $2 \mathrm{Ks} 10$ \\
52299.3 & 56 & 1.44 & 0.55 & $1 \mathrm{Ks} 11$ & 52037.2 & 60 & 1.10 & 0.59 & $2 \mathrm{Ks} 11$ \\
52309.3 & 56 & 1.27 & 0.49 & $1 \mathrm{Ks} 12$ & 52060.1 & 60 & 1.06 & 0.50 & $2 \mathrm{Ks} 12$ \\
52322.2 & 56 & 1.38 & 0.47 & $1 \mathrm{Ks} 13$ & 52096.1 & 60 & 1.12 & 0.38 & $2 \mathrm{Ks} 13$ \\
52348.3 & 56 & 1.06 & 0.47 & $1 \mathrm{Ks} 14$ & 52300.3 & 56 & 1.30 & 0.55 & $2 \mathrm{Ks} 14$ \\
52360.1 & 56 & 1.17 & 0.41 & $1 \mathrm{Ks} 15$ & 52315.3 & 56 & 1.10 & 0.46 & $2 \mathrm{Ks} 15$ \\
52379.2 & 56 & 1.06 & 0.50 & $1 \mathrm{Ks} 16$ & 52332.3 & 56 & 1.13 & 0.36 & $2 \mathrm{Ks} 16$ \\
52403.2 & 56 & 1.27 & 0.38 & $1 \mathrm{Ks} 17$ & 52348.3 & 54 & 1.10 & 0.37 & $2 \mathrm{Ks} 17$ \\
52422.0 & 56 & 1.10 & 0.43 & $1 \mathrm{Ks} 18$ & 52360.2 & 56 & 1.06 & 0.36 & $2 \mathrm{Ks} 18$ \\
52444.0 & 56 & 1.11 & 0.53 & $1 \mathrm{Ks} 19^{2}$ & 52379.1 & 56 & 1.08 & 0.50 & $2 \mathrm{Ks} 19$ \\
52474.0 & 56 & 1.07 & 0.49 & $1 \mathrm{Ks} 20^{2}$ & 52398.1 & 35 & 1.06 & 0.38 & $2 \mathrm{Ks} 20$ \\
52385.1 & 70 & 1.08 & 0.38 & $1 \mathrm{Js}$ & 52405.0 & 56 & 1.30 & 0.58 & $2 \mathrm{Ks} 21$ \\
52411.1 & 56 & 1.09 & 0.47 & $1 \mathrm{H}$ & 52422.1 & 56 & 1.06 & 0.49 & $2 \mathrm{Ks} 22$ \\
& & & & & 52444.0 & 56 & 1.06 & 0.62 & $2 \mathrm{Ks} 23^{2}$ \\
& & & & & 52474.0 & 56 & 1.16 & 0.59 & $2 \mathrm{Ks} 24^{2}$ \\
& & & & & 52385.2 & 70 & 1.06 & 0.48 & $2 \mathrm{Js}$ \\
\hline
\end{tabular}

(1) Observed with SOFI@NTT.

(2) Observed with LW arm of ISAAC@VLT.

objects detected in the registered and combined frames after the first sky subtraction. The double sky subtraction procedure is particularly important for these crowded images. We noticed that in a single sky subtraction pass, bright regions were often over-subtracted producing dark halos around the bright stars. Finally the sky-subtracted images were aligned with IMALIGN and all the images taken in a single jittered sequence were combined with IMCOMBINE task in IRAF.

\section{The photometry}

The PSF fitting photometry was done for each single epoch image individually. First we used DAOPHOT II and ALLSTAR (Stetson 1987) to create a PSF for each frame and to define the coordinate transformations between the frames. The complete star list was created from the median combined image that had only the best seeing epochs (i.e. those with FWHM stellar profiles measured $<3$ pix). These included epochs $1 \mathrm{Ks} 01$, 1Ks02, 1Ks04, 1Ks05, 1Ks07, $1 \mathrm{Ks} 10,1 \mathrm{Ks} 15$, 1Ks17, $1 \mathrm{Ks} 18$ and $1 \mathrm{Js}$ for Field 1, and epochs $2 \mathrm{Ks} 01$, 2Ks02, 2Ks05, 2Ks09, 2Ks10, 2Ks13, 2Ks16, 2Ks17 and 2Ks18 for Field 2. The total exposure time of these median combined images are 9.4 and 8.4 hours. We found that better results were obtained in this way instead of combining all the images. PSF fitting photometry using this star list and coordinate transformations was performed simultaneously on all images of a single field with the ALLFRAME programme (Stetson 1994). The final photometric catalogue contains 13111 stars in Field 1 and 16435 stars in Field 2, that have been detected on at least $3 \mathrm{~K}$-band frames and in $J$ - and $H$-band images. The areas covered with at least three pointings in $K$-band are $2.28 \times 2.30$ and $2.25 \times 2 ! 31$, and the total exposure times in $K$-band are 19.67 and 21.17 hours, for Fields 1 and 2, respectively. These are the deepest near-IR images obtained so far in an external galaxy halo.

\subsection{The photometric calibration}

Not all the epochs were observed in photometric conditions. The $K$-band photometry was brought to the system of one of the photometric nights that had excellent seeing: epochs $1 \mathrm{Ks} 07$ and $2 \mathrm{Ks} 10$ for Fields 1 and 2, respectively, both observed on July 8, 2000. The zero point of $24.257 \pm 0.042$ for that night has been derived from the observations of Persson et al. (1998) standards supplied by ESO service observing (Rejkuba et al. 2001). $J$ - and $H$-band photometric zero points were derived using the observations of Persson standard stars obtained 

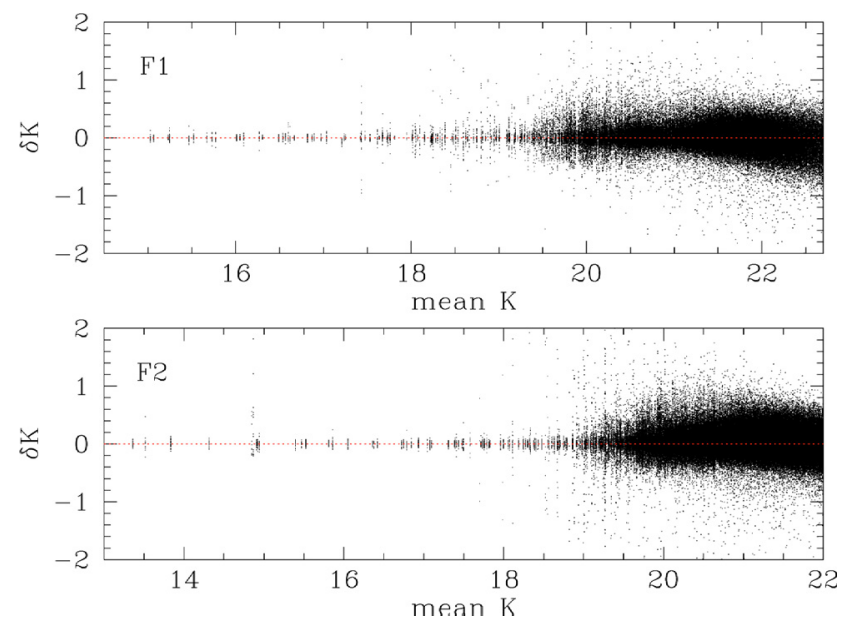

Fig. 1. Photometric accuracy for the $K$-band data. Plotted are differences of individual epoch measurements with respect to their mean magnitudes. Only individual measurements with ALLFRAME $\sigma$ error smaller than 0.2 are plotted. At the bright end the limiting accuracy of $\pm 0.05 \mathrm{mag}$ can be seen. The variables are located where an excessive scatter with respect to the mean is present with both positive and negative excursions.

during the same night as the NGC 5128 observations in the corresponding filter (see Table 1) and their values are $24.737 \pm$ 0.039 and $24.626 \pm 0.033$. For all three filters extinction coefficients measured by ESO observatory staff and reported on ISAAC web pages were assumed ( 0.06 for $J_{\mathrm{s}}$ and $H$-band and 0.07 for $K_{\mathrm{s}}$-band).

The quality of the photometry can be assessed from Fig. 1 where we plot differences of individual epoch measurements vs. mean magnitudes. The mean magnitudes were calculated weighting the individual measurements with their photometric errors calculated by ALLFRAME:

$\bar{K}=\frac{\sum_{i=1}^{n} \frac{K_{i}}{\sigma_{i}^{2}}}{\sum_{i=1}^{n} \frac{1}{\sigma_{i}^{2}}}$.

Only the stars with individual ALLFRAME $\sigma$ uncertainty measurements smaller than 0.2 mag are plotted. The limiting accuracy of our photometry at the bright end is \pm 0.05 mag, getting worse at faintest magnitudes. The region around $K=20$ has more scattered measurements than the mean. This is where most variable stars are found. The mean magnitude calculated with the above equation will, however, be overestimated (too bright) for variable stars with large amplitudes because brighter phases will typically get higher weights.

\section{Search for variable stars}

Variable stars were identified using a procedure similar to the one described by Welch \& Stetson (1993) and Stetson (1996). First, we selected all the stars with mean of all photometric errors given by ALLFRAME, as measured on each individual epoch frame, smaller than $0.2 \mathrm{mag}$. We then required for each star to be detected on more than 5 frames and constructed
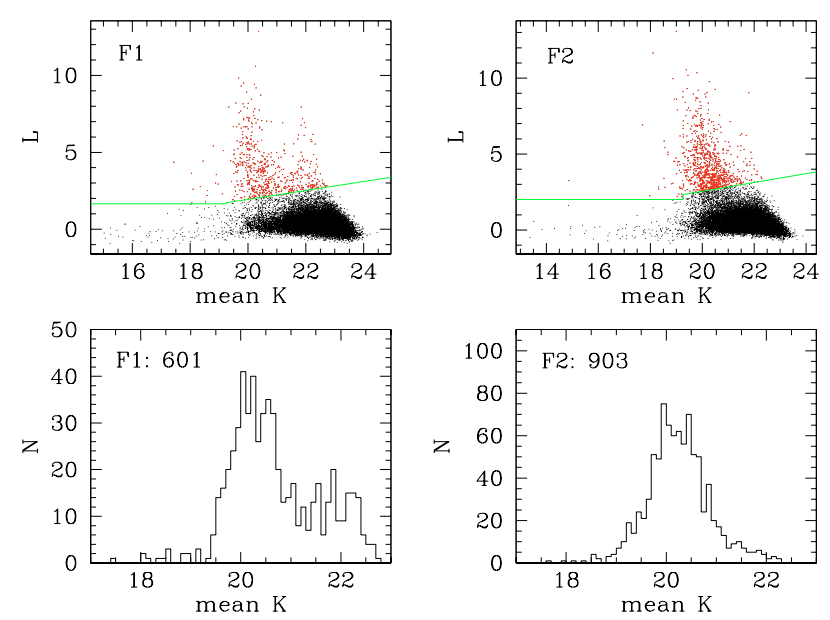

Fig. 2. Variability index $L$ vs. magnitude is plotted in the upper two panels for fields 1 (left) and 2 (right). Variable stars have higher index $L$ values than non-variables and are found above the solid line. In the lower panels the magnitude distribution of variable stars is shown. The number of variables with at least 6 data points is also given.

variability indexes $J, K$ and $L$, following the prescriptions by Stetson (1996, Eqs. (1-3)).

In particular the index $J$ is used for single observations assuming $P_{i}=\delta_{i}^{2}-1$, where $\delta_{i}$ is a normalized residual of the measurement from the mean magnitude. The mean magnitude is calculated using a weighting with inverse square of the measurement error (weight $w_{i}$ ) according to Eq. (1).

The index $J$ is a robust measure of the external repeatability relative to the internal precision. For single epoch observations containing only random noise its value tends to zero. For a physical variable it is a positive number. The index $K$ is a robust measure of the kurtosis of the magnitude histogram and its value is fixed by the shape of the light curve, with $K=0.9$ for a pure sinusoid and $K=0.798$ for a Gaussian magnitude distribution, a limit approached when random measurement errors dominate over physical variation. Including the information about the variability and the shape of the light curve, Stetson defined the final variability index $L$ as:

$L=\left(\frac{J K}{0.798}\right)\left(\frac{\sum w_{i}}{w_{\text {all }}}\right)$.

The second term in this expression assigns an additional weight to stars with the highest number of measurements.

Figure 2 is a plot of the variability index $L$ for all the stars in Field 1 and 2 in the left and right panels, respectively. The stars above the solid line (upper panels), plotted with slightly larger symbols, were searched for periodic variations. The limits that separate variable from non-variable sources were determined in the following way: in completeness simulations stars with constant magnitude light curves were added to the frames and their photometry and variability indexes were measured in identical way as for the programme stars. A similar plot of the variability index vs. magnitude was used to decide on the separation line between the variable and non-variable stars (Fig. 3). With the adopted variable star detection limits (solid lines in the upper panels) less than $2 \%$ of sources from these simulations are found above the lines. Note however, that at least some of these 

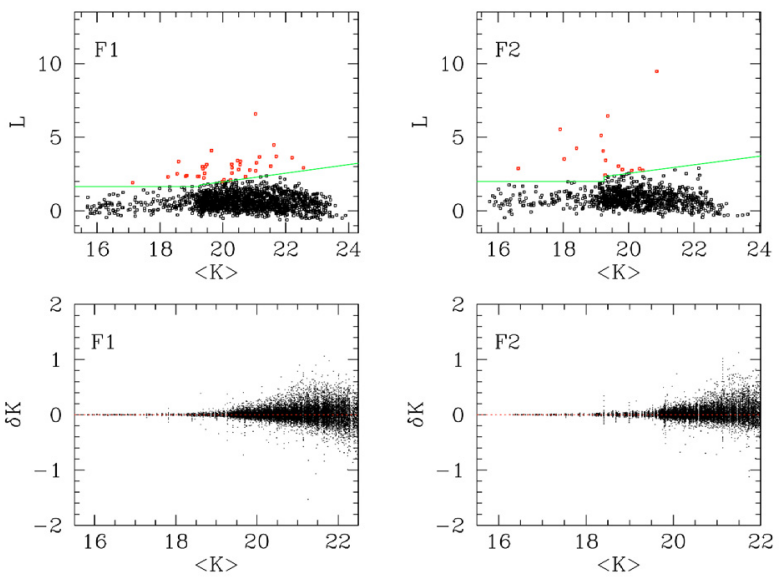

Fig. 3. Variability index $L$ vs. magnitude for simulated non-variable stars, i.e. those with light curve constant in time, is plotted in the upper two panels for fields 1 (left) and 2 (right). This diagram is used to decide the detection limit for the variable stars. In the lower panels scatter of the individual magnitude measurements around the mean magnitude is shown.

may have their light curves contaminated by a neighbouring variable star. Simulations necessary to evaluate the completeness of the variable star search are described below.

With such selection criteria 601 stars in Field 1 and 903 stars in Field 2 are found to be variable. Of these 536 and 878 had at least 10 measurements with individual errors smaller than $0.5 \mathrm{mag}$, and light curves were constructed for them.

\section{Light curves and periods of LPVs}

Fourier analysis of the $K$-band light curves was used to search for the periodic signal in the data in the range of $30<P<$ 1700 days. Only individual measurements with ALLFRAME uncertainty smaller than 0.5 mag were used. First, an initial guess of the period was obtained using a routine that calculates spectral power as a function of angular frequency $(\omega=2 \pi f$; Lomb 1976):

$$
\begin{aligned}
P_{N}(\omega)= & \frac{\left[\sum_{j}\left(K_{j}-\bar{K}\right) \cos \omega\left(t_{j}-\tau\right)\right]^{2}}{2 \sigma^{2} \sum_{j} \cos ^{2} \omega\left(t_{j}-\tau\right)} \\
& +\frac{\left[\sum_{j}\left(K_{j}-\bar{K}\right) \sin \omega\left(t_{j}-\tau\right)\right]^{2}}{2 \sigma^{2} \sum_{j} \sin ^{2} \omega\left(t_{j}-\tau\right)}
\end{aligned}
$$

where $K_{j}$ and $\bar{K}$ are the individual and mean magnitudes, $\sigma$ is the variance, $t_{j}$ is the Julian Date (JD) of the measurement, and $\tau$ is defined as:

$\tan (2 \omega \tau)=\frac{\sum_{j} \sin 2 \omega t_{j}}{\sum_{j} \cos 2 \omega t_{j}}$.

The period obtained from the frequency with largest power corresponds to the most probable sinusoidal component. It was further improved with a non-linear least-square fitting of the function

$K(t)=A \cos \left(2 \pi \frac{\left(t-t_{0}\right)}{P}\right)+B \sin \left(2 \pi \frac{\left(t-t_{0}\right)}{P}\right)+K_{0}$.
From this, the best fitting period $(P)$, amplitude $\left(2 \times \sqrt{A^{2}+B^{2}}\right)$, mean magnitude $\left(K_{0}\right)$, and phase $\left(t_{0}\right)$ were obtained.

In optical passbands Miras often have asymmetric light curves, usually steeply rising to the maximum and with a shallower decline. In near-IR the variations are more regular and nearly sinusoidal (e.g. Whitelock et al. 2000). Hence a sinewave gives a reasonable approximation to most of the LPVs.

Our code that determines the best fitting period was tested on a subsample of data on Mira variables published by Whitelock et al. (2000). Their data are similar to ours, with 10-15 data points per star and photometric errors of the order of $0.05 \mathrm{mag}$ in $K$. In 8 out of 10 cases we obtain the same period as Whitelock et al. In two cases our periods were significantly different (in one case we derived 74 days period with respect to the published value of 185 days and in the second case our period is 422 days where Whitelock et al. got 327 days), but the light curves folded with these periods looked as good or even smoother than the published light curves. In some cases is possible to obtain more than one acceptable period when there are less than $\sim 15$ data points. It is not clear however, if these stars really pulsate with two different periods or the insufficient data allow to derive such different periods due to aliases in the sampling of the data.

In Fig. 4 we show two examples of a periodogram, the original light curve in time domain and the light curves folded with the period obtained from the highest power frequency from the periodogram and by a sine curve fitting. In a few cases there are evidences for the presence of the second period or a luminosity modulation in the data (e.g. star F2 \#194 in Fig. 4), but we have determined only the main period, because for most of the variables the data were not good enough for a more detailed analysis. Most of the times when a good period could be derived, the two periods were similar, but sometimes they differed by up to 20-30 days and both light curves were of acceptable quality. This illustrates that the accuracy of the derived periods and amplitudes varies considerably from one star to the next, depending on the number of observations, their individual errors and the stability of the light curve over the sampling interval.

It is interesting to note that some LPVs in the LMC with periods in excess of $\sim 420$ days, which appear to be rather bright for their periods when compared to other LPVs in the $K$-band or $M_{\text {bol }}$ vs. $\log P$ diagram, show a pronounced hump on the rising branch. These stars are tentatively identified as being in the Hot Bottom Burning stage of evolution on account of, for example, the strong Li apparent in some of their spectra (Glass \& Lloyd Evans 2003). Star F2 \#194 shows such a hump and it is lying 0.28 mag above the mean period-luminosity relation (Rejkuba 2003, in preparation). Reviewing the light curves of all the variables with $P>420$ days that are at least 0.3 mag brighter than the mean $K$-band magnitude at a given period, we found that approximately $1 / 3(\sim 15$ stars $)$ present a similar hump. For some of the other $2 / 3$ stars the light curve coverage is not sufficiently good to exclude the presence of a hump.

The comparison of the periods obtained through Fourier analysis and by a sine-curve fitting is shown in Fig. 6. Variables in Field 1 are shown with open squares and those in Field 2 with crosses. The upper left panel is a direct comparison of the periods determined with the two methods, while the other 

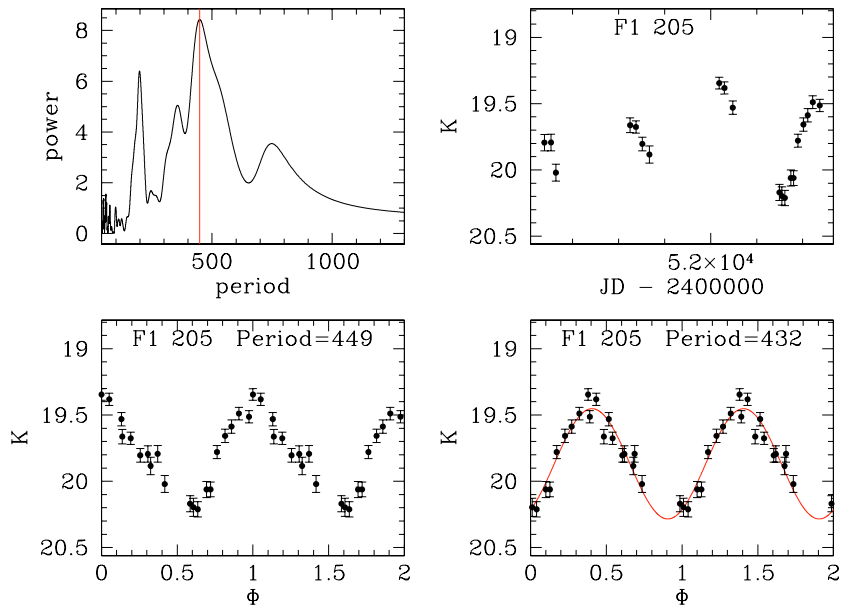
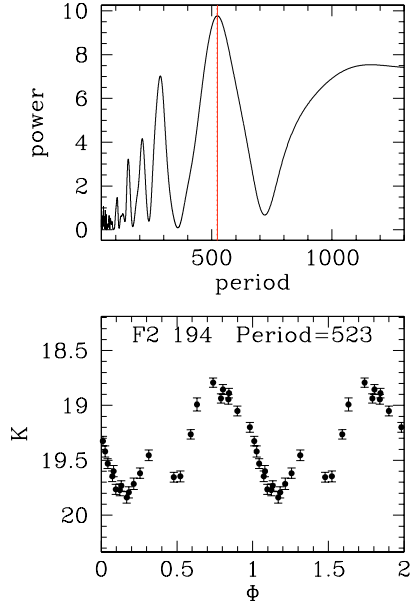

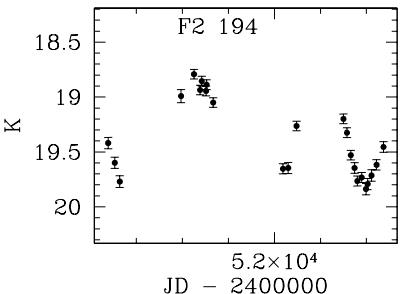

JD -2400000

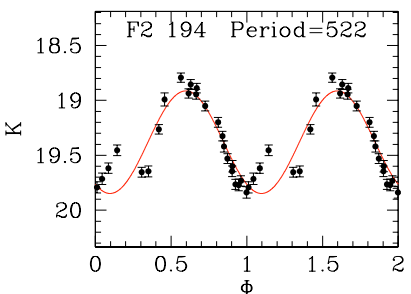

Fig. 4. From left to right, top: periodogram and the original light curve in time domain; bottom: the light curve folded with the period obtained from the highest power frequency from the periodogram and the same light curve folded by the best period obtained from sine curve fitting. Each point in the phased light curves is plotted twice to emphasize the periodicity. Over the last light curve the fitting function is over-plotted. In the left panels the four diagrams are plotted for star \#205 in Field 1 and in the right panels for star \#194 in Field 2.

panels show how the difference depends on period (upper right), on significance of the period obtained from Fourier analysis (lower left) and on reduced $\chi^{2}$ of the sine-curve fit. There is no significant difference between the two fields. The larger differences are more probable for variables with low $\chi^{2}$ of the sine-curve fit. This is an expected result due to the fact that not all the variables have strictly sinusoidal light curves. On the contrary, there is no strong dependence on the significance parameter which measures the strength of the peak in the periodogram (e.g. Fig. 4). Significance is a smaller number for stronger (more significant) period. We have visually inspected all the variables folded with both periods and in cases where one of the two gave clearly much smoother light curve that period was retained, otherwise the final period is a mean of the two. A more quantitative accuracy of the periods, as well as the completeness of the detection of variables with a given period was derived using Monte Carlo simulations (see next section).

For 99 variable stars in Field 1 and 169 in Field 2 no acceptable periods could be obtained because of the non-sinusoidal variations, large errors combined with small amplitudes, periodicity outside our probed range $(30<P \lesssim 1000$ day), presence of multiple periods, irregularity (cycle-to-cycle variations) in Miras or semiregulars, or absence of period (e.g. microlensing, background AGN or SN). More data are necessary to determine the nature of these variables. Among these are also LPVs that have periods longer than $\sim 1000$ days, for which our observations did not cover much more than 1 period and thus it was not possible to determine an accurate period.

The light curves for 437 variables in Field 1 and 709 in Field 2, for which we could determine periods, as well as the tables with the best fitting parameters for these stars, are presented only in the electronic version of the article. In Fig. 5 we show a sample of light curves folded with the periods that are indicated in each panel. In the example there are bright and faint variables from both fields. Table 2 lists for these stars: ID number, $x$ and $y$ position with respect to the reference frame (1Ks07 and $2 \mathrm{Ks} 10$ for Field 1 and Field 2, respectively), number of epochs with magnitude measurements with $\sigma<0.5$, periods, semi-amplitudes $\left(\sqrt{A^{2}+B^{2}}\right)$ as obtained from sine-curve fitting, reduced $\chi^{2}$ of the fits, significances, the $J$ - and $H$-band magnitudes, and the $K$-band magnitudes from the sine-curve fit. Negative numbers for $x$ and $y$ positions mean that the star was out of the limits of the reference frame. The initial positions $(x, y)=(1,1)$ correspond to $(\alpha, \delta)=\left(13^{\mathrm{h}} 26^{\mathrm{m}} 17^{\mathrm{s}} .5,-42^{\circ} 51^{\prime} 03^{\prime \prime} \cdot 3\right)$ for Field 1 and to $(\alpha, \delta)=\left(13^{\mathrm{h}} 25^{\mathrm{m}} 18.0,-43^{\circ} 09^{\prime} 03^{\prime \prime} \cdot 0\right)$ for Field 2. This table is a subset of Tables 3 and 4 presented in the electronic version. Additionally, in the electronic version of the article we list all the raw $K$-band measurements for 1504 variables in both fields (Tables 7 and 8). Magnitude 99.99 and the error 9.99 denote that no measurement for that epoch was obtained. Here we give only first three lines of these tables.

It should be noted that while the large majority of the variables with the determined periods are long period variables with periods in excess of 100 days, there are 27 stars in Field 1 and 13 stars in Field 2 for which most probable periods were shorter than 51 days. However, the significance of these periods is in all cases is very low, with significance parameter $>0.8$ for all but two stars in Field 1 for which signif $>0.65$. If the quoted periods are real, these stars could be Cepheids or some other kind of variables. Cepheids are expected to be found among younger populations in star forming regions like that present in our Field 1 (Rejkuba et al. 2001). Unfortunately our sampling is not good enough to determine reliably the variability type from the light curve shape.

\section{Completeness and contamination}

Two sets of crowding experiments were made in order to assess the completeness of the photometric catalogue as well as to estimate the completeness and contamination in the LPV catalogue. The full description of these experiments is given only in the electronic version of the article. Here we present a summary with the main results. 

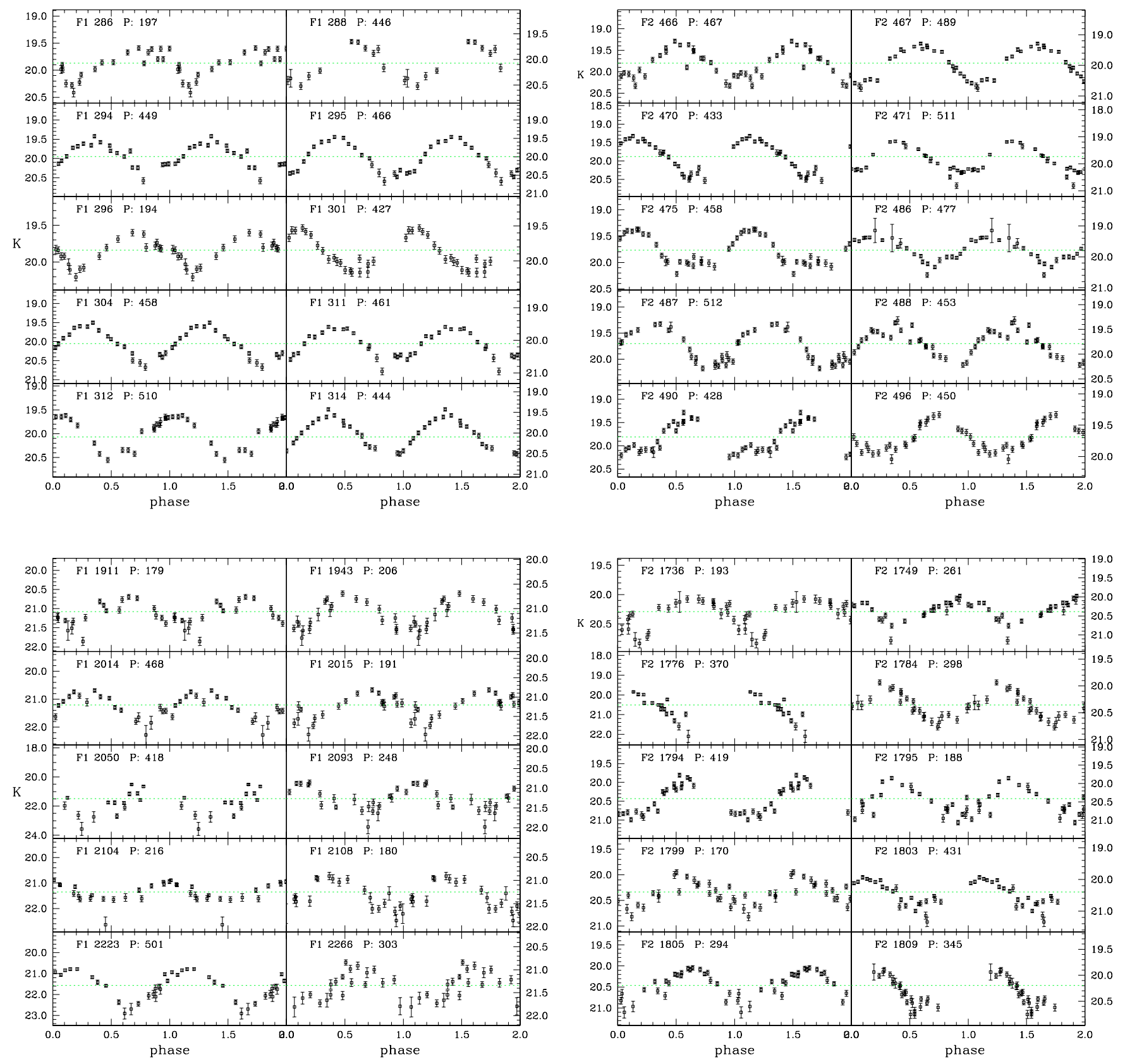

Fig. 5. A sample of phased light curves for variables in both fields and with a range of mean magnitudes. Each point is plotted twice to emphasize the variability.

In the first set of ten different crowding experiments nonvariable stars with magnitudes uniformly distributed between $15.7<K<25.2$ and $J-K, H-K$ mean red giant branch colors were added to the original images. A realistic, observed, amount of Poissonian noise was added as well. The photometry was then re-calculated in the same way as described above. Our photometry is complete for more than $50 \%$ in the $K$ and $H$-bands for stars brighter than 22.5 and 21.6 mag for fields 1 and 2, respectively. These numbers for the $J$-band are 23.25 and 22.5 mag. Apart from the completeness and photometric error estimations (see Figs. 7-10 in the electronic version), these simulations were also used to determine criteria for the detection of variable stars. The results are summarized in Fig. 3 and are described in Sect. 4.
Dependence of the detection probability of a variable star with a given period on the actual distribution of the observations can be estimated with numerical simulations similar to those by Saha \& Hoessel (1990) and Bersier \& Wood (2002). Given a period $\mathrm{P}$ and initial phase $\phi_{0}$ at the time $t_{0}$ of the first observation, the phase distribution can be calculated for our set of observations (see Fig. 11 in electronic version). However, it should be noted that the period detection probability will depend not only on the time sampling, but also on photometric incompleteness, magnitude, and amplitude distributions of variable stars.

We obtained a more realistic estimate of the dependence of the detection probability on magnitude, period and amplitude distribution of variable stars with our second set 
Table 2. Data for a sample of variables with light curves plotted in Fig. 5. The columns from left to right list: star ID, $x$ and $y$ position with respect to the reference epoch, the best fitting period, semi-amplitude, reduced $\chi^{2}$ of the sine-curve fit, significance of the period from Fourier analysis, single epoch $J$ and $H$-band magnitudes, and mean $K$-band magnitude from the sine-curve fit.

\begin{tabular}{|c|c|c|c|c|c|c|c|c|c|c|}
\hline ID & $x$ & $y$ & $N$ & $P$ & $A$ & $\chi^{2}$ & signif & $J$ & $H$ & $<K>$ \\
\hline F1 286 & 279.230 & 67.260 & 20 & 197 & 0.247 & 9.20 & 0.550 & 21.07 & 20.13 & 19.87 \\
\hline F1 288 & 14.460 & 549.217 & 11 & 446 & 0.408 & 1.70 & 0.690 & 21.98 & 20.87 & 20.07 \\
\hline F1 294 & 124.558 & 330.030 & 20 & 449 & 0.387 & 5.20 & 0.160 & 21.18 & 20.12 & 19.95 \\
\hline F1 295 & 445.246 & 622.988 & 20 & 466 & 0.527 & 2.20 & 0.060 & 21.56 & 20.49 & 20.00 \\
\hline F1 296 & 395.486 & 18.707 & 19 & 194 & 0.217 & 2.50 & 0.260 & 20.89 & 20.00 & 19.84 \\
\hline F1 301 & 364.732 & 570.560 & 20 & 427 & 0.283 & 1.30 & 0.080 & 20.97 & 20.24 & 19.84 \\
\hline F1 304 & 169.875 & 226.678 & 20 & 458 & 0.483 & 1.50 & 0.050 & 21.24 & 20.21 & 20.05 \\
\hline F1 311 & 51.930 & 187.867 & 20 & 461 & 0.508 & 3.40 & 0.090 & 22.13 & 20.67 & 20.10 \\
\hline F1 312 & 326.187 & 501.922 & 20 & 510 & 0.436 & 2.20 & 0.160 & 21.27 & 20.32 & 20.07 \\
\hline F1 314 & 108.418 & 332.330 & 20 & 444 & 0.521 & 1.40 & 0.050 & 21.24 & 20.35 & 20.10 \\
\hline F1 1911 & 865.938 & 292.732 & 19 & 179 & 0.315 & 5.10 & 0.690 & 22.38 & 21.50 & 21.08 \\
\hline F1 1943 & 652.101 & 471.075 & 20 & 206 & 0.366 & 2.10 & 0.310 & 21.72 & 21.20 & 21.06 \\
\hline F1 2014 & 862.100 & 33.721 & 19 & 468 & 0.496 & 2.80 & 0.300 & 23.10 & 21.63 & 21.21 \\
\hline F1 2015 & 644.197 & 327.874 & 20 & 191 & 0.320 & 4.40 & 0.550 & 21.84 & 21.08 & 21.21 \\
\hline F1 2050 & 54.552 & 767.989 & 15 & 418 & 0.821 & 26.00 & 0.960 & 23.15 & 99.99 & 21.48 \\
\hline F1 2093 & 719.435 & 768.779 & 20 & 248 & 0.359 & 2.60 & 0.410 & 22.09 & 21.33 & 21.26 \\
\hline F1 2104 & 728.300 & 354.414 & 19 & 216 & 0.376 & 2.90 & 0.680 & 24.72 & 23.11 & 21.35 \\
\hline F1 2108 & 722.411 & 633.752 & 20 & 180 & 0.053 & 12.90 & 0.380 & 22.70 & 22.00 & 21.25 \\
\hline F1 2223 & 760.724 & 516.453 & 20 & 501 & 0.823 & 3.10 & 0.070 & 26.89 & 23.28 & 21.57 \\
\hline F1 2266 & 324.743 & 541.049 & 20 & 303 & 0.311 & 6.50 & 0.760 & 22.09 & 21.40 & 21.34 \\
\hline F2 466 & 497.668 & 115.838 & 24 & 467 & 0.381 & 7.50 & 0.060 & 21.14 & 20.87 & 19.80 \\
\hline F2 467 & 219.420 & 287.679 & 24 & 489 & 0.615 & 3.20 & 0.010 & 22.38 & 21.42 & 19.93 \\
\hline F2 470 & 413.810 & 765.776 & 24 & 433 & 0.514 & 2.50 & 0.010 & 20.93 & 19.89 & 19.88 \\
\hline F2 471 & 654.271 & 480.820 & 24 & 511 & 0.583 & 3.70 & 0.060 & 22.62 & 21.25 & 19.74 \\
\hline F2 475 & 388.723 & 379.739 & 24 & 458 & 0.327 & 4.70 & 0.040 & 20.78 & 19.92 & 19.76 \\
\hline F2 486 & 12.504 & 328.661 & 23 & 477 & 0.448 & 3.60 & 0.030 & 20.75 & 19.97 & 19.78 \\
\hline F2 487 & 215.930 & 745.931 & 24 & 512 & 0.432 & 2.90 & 0.040 & 21.68 & 20.80 & 19.70 \\
\hline F2 488 & 409.287 & 716.276 & 24 & 453 & 0.235 & 9.10 & 0.070 & 20.86 & 20.12 & 19.78 \\
\hline F2 490 & 597.413 & 11.477 & 24 & 428 & 0.377 & 5.70 & 0.030 & 21.52 & 20.59 & 19.81 \\
\hline F2 496 & 442.349 & 576.357 & 24 & 450 & 0.169 & 6.40 & 0.030 & 21.42 & 20.35 & 19.69 \\
\hline F2 1736 & 40.346 & 627.771 & 24 & 193 & 0.247 & 5.20 & 0.240 & 22.55 & 21.26 & 20.29 \\
\hline F2 1749 & 832.748 & 178.959 & 24 & 261 & 0.319 & 3.70 & 0.210 & 21.17 & 20.41 & 20.39 \\
\hline F2 1776 & 337.907 & -7.156 & 16 & 370 & 0.607 & 11.10 & 0.660 & 24.74 & 22.21 & 20.51 \\
\hline F2 1784 & 711.917 & 559.903 & 24 & 298 & 0.330 & 2.00 & 0.030 & 21.95 & 20.99 & 20.36 \\
\hline F2 1794 & 491.605 & 75.748 & 23 & 419 & 0.488 & 4.00 & 0.020 & 22.87 & 21.76 & 20.43 \\
\hline F2 1795 & 405.954 & 409.103 & 24 & 188 & 0.394 & 10.60 & 0.410 & 22.01 & 20.87 & 20.41 \\
\hline F2 1799 & 575.492 & 194.880 & 24 & 170 & 0.231 & 5.80 & 0.240 & 22.14 & 20.99 & 20.33 \\
\hline F2 1803 & 786.644 & 606.898 & 24 & 431 & 0.405 & 5.30 & 0.080 & 21.49 & 20.58 & 20.40 \\
\hline F2 1805 & 359.442 & 396.001 & 24 & 294 & 0.356 & 4.30 & 0.090 & 21.35 & 20.46 & 20.46 \\
\hline F2 1809 & 849.471 & 740.460 & 24 & 345 & 0.386 & 3.30 & 0.060 & 21.67 & 20.85 & 20.19 \\
\hline
\end{tabular}

of crowding experiments in which we have added artificial variable stars with sinusoidal light curves with given periods, amplitudes and magnitudes. The magnitude range was chosen between $19.5<K<21.5$, similar to that of detected LPVs in NGC 5128 halo. Amplitudes ranged from 0.1 to 1.4 mag and periods from 50 to 1100 days. In particular variable stars with 182 and 365 day periods were also simulated. One series of completeness experiments tested our detection sensitivity to the amplitudes of variable stars, hence we fixed period and measured the detection sensitivity and measurement 
Table 3. LPVs in Field 1. The columns from left to right list: star ID, $x$ and $y$ position with respect to the reference epoch, the best fitting period, semi-amplitude, reduced $\chi^{2}$ of the sine-curve fit, significance of the period from Fourier analysis, single epoch $J$ and $H$-band magnitudes and mean $K$-band magnitude. Magnitude 99.99 denotes that no measurement for that star was obtained.

\begin{tabular}{cccccrrrrrr}
\hline \hline F1 ID & $x$ & $y$ & $N$ & $P$ & $A$ & $\chi^{2}$ & signif & $J$ & $H$ & $<K>$ \\
\hline \hline 31 & 460.926 & 401.982 & 20 & 432. & 0.513 & 21.10 & 0.650 & 19.11 & 18.05 & 17.37 \\
53 & 107.785 & 832.967 & 19 & 448. & 0.203 & 2.30 & 0.310 & 19.91 & 19.01 & 18.09 \\
64 & 610.320 & 803.826 & 20 & 430. & 0.202 & 18.50 & 1.000 & 18.03 & 18.45 & 18.36 \\
\hline
\end{tabular}

Table 4. LPVs in Field2. The columns from left to right list: star ID, $x$ and $y$ position with respect to the reference epoch, the best fitting period, semi-amplitude, reduced $\chi^{2}$ of the sine-curve fit, significance of the period from Fourier analysis, single epoch $J$ and $H$-band magnitudes and mean $K$-band magnitude.Magnitude 99.99 denotes that no measurement for that star was obtained.

\begin{tabular}{rccccrrrrrr}
\hline \hline F2 ID & $x$ & $y$ & $N$ & $P$ & $A$ & $\chi^{2}$ & signif & $J$ & $H$ & $\langle K>$ \\
\hline \hline 35 & 115.155 & -29.046 & 10 & 35. & 0.978 & 226.70 & 0.940 & 18.76 & 18.30 & 18.48 \\
49 & 41.708 & 172.990 & 10 & 988. & 1.463 & 2.20 & 0.980 & 99.99 & 99.99 & 17.87 \\
61 & 591.542 & 445.812 & 24 & 696. & 0.180 & 1.70 & 0.300 & 19.67 & 18.66 & 18.41 \\
\hline
\end{tabular}

Table 5. Additional variable stars in Field 1 for which periods could not be determined. The columns from left to right list: star ID, $x$ and $y$ position with respect to the reference epoch, single epoch $J$ and $H$-band magnitudes and mean $K$-band magnitude. Magnitude 99.99 denotes that no measurement for that star was obtained.

\begin{tabular}{ccccrrr}
\hline \hline F1 ID & $x$ & $y$ & $N$ & $J$ & $H$ & $<K>$ \\
\hline \hline 50 & 763.941 & 462.473 & 20 & 19.70 & 18.76 & 18.23 \\
51 & 219.227 & 79.584 & 20 & 18.48 & 18.02 & 17.97 \\
69 & 113.406 & 403.575 & 12 & 99.99 & 99.99 & 21.80 \\
\hline
\end{tabular}
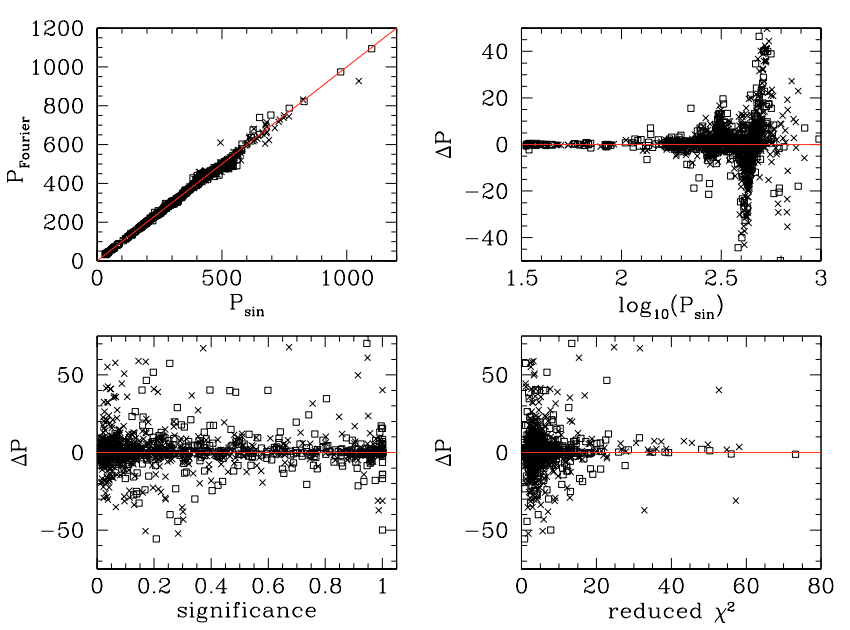

Fig. 6. Comparison of periods derived through Fourier analysis $\left(P_{\text {Fourier }}\right)$ and sine-curve fitting $\left(P_{\sin }\right)$ for variables in Field 1 (open squares) and Field 2 (crosses) and the dependence of the differences between the two methods on quality of the fit parameters: significance measures strength of the periodicity signal in Fourier analysis and reduced $\chi^{2}$ is a direct indicator of the quality of the sine-curve fit.

accuracy as a function of amplitude. In the second series of experiments, amplitude was fixed at a typical amplitude for LPVs ( $\Delta K=0.7 \mathrm{mag}$ ) in order to measure dependence on period.

The results of these simulations are summarized in Figs. 12-14 (to appear only in the electronic version). As illustrated in these figures, our periods seem to be accurate to \pm 25 days except in the range of 700-800 days. In that specific range, our periods appear to be systematically overestimated by as much as 50-100 days. An additional potential systematic error could come from period aliasing. Our observations do not span time continuously. Rather, they are grouped in three to six month windows with inter-window gaps of 200 to 320 days. Thus, it is possible that stars with actual periods in the range 175-200 days were assigned periods of order of one year, because only alternate maxima fell within our observing windows.

A systematic bias is present in our catalogue in the sense that we detect stars brighter than they are and with somewhat larger amplitudes. The magnitude of this bias is proportional to the amplitude of the variable. Partially this difference may be due to the way the mean output magnitude is calculated, but using different means still produces a slight bias. As expected the simulations have shown that most of the large amplitude variables $(\Delta K>0.3)$ will be detected for stars with shorter periods. Our variable star catalogue is highly incomplete for stars with amplitudes of 0.2 mag or smaller, irrespective of period.

\section{Conclusions}

We have presented the first catalogue of variable stars in a giant elliptical galaxy. 1504 red variables were detected in two halo fields of NGC 5128 (Centaurus A) covering 10.46 arcmin square. For 1146 variables with at least 10 good measurements we have determined periods, amplitudes and mean $K$-band magnitudes using Fourier analysis and non-linear sine-curve fitting algorithms. Periods, amplitudes and $J H K$ photometry are given for 1146 long period variables. Additionally, individual $K$-band measurements are given for all the red variables.

Our extensive completeness simulations show that periods determined for these long period variables are accurate to 
Table 6. Additional variable stars in Field 2 for which periods could not be determined. The columns from left to right list: star ID, $x$ and $y$ position with respect to the reference epoch, single epoch $J$ and $H$-band magnitudes and mean $K$-band magnitude. Magnitude 99.99 denotes that no measurement for that star was obtained.

\begin{tabular}{cccrrrr}
\hline \hline F2 ID & $x$ & $y$ & $N$ & $J$ & $H$ & $<K>$ \\
\hline \hline 5 & 110.132 & 451.042 & 17 & 99.99 & 18.68 & 15.51 \\
25 & 111.583 & -16.658 & 14 & 18.96 & 18.89 & 18.39 \\
63 & 133.142 & -27.505 & 9 & 19.13 & 18.49 & 18.96 \\
\hline
\end{tabular}

Table 7. Individual measurements of the $K$-band magnitudes for all the red variable stars detected in Field 1 . The columns from left to right list: star ID, $x$ and $y$ position with respect to the reference epoch, the reference epoch $K$-band magnitudes and errors and all the other individual $K$-band magnitudes and the errors of the measurements as given by ALLFRAME to which 0.04 mag calibration error have been added in quadrature. Magnitude 99.99 and the error 9.99 denote that no measurement for that star was obtained.

\begin{tabular}{cccccccccc}
\hline \hline F1 ID & $x$ & $y$ & $1 \mathrm{Ks} 07(\sigma)$ & $1 \mathrm{Ks} 01(\sigma)$ & $1 \mathrm{Ks} 02(\sigma)$ & $1 \mathrm{Ks} 03(\sigma)$ & $1 \mathrm{Ks} 04(\sigma)$ & $1 \mathrm{Ks} 05(\sigma)$ & $1 \mathrm{Ks} 06(\sigma)$ \\
& & & $1 \mathrm{Ks} 08(\sigma)$ & $1 \mathrm{Ks} 09(\sigma)$ & $1 \mathrm{Ks} 10(\sigma)$ & $1 \mathrm{Ks} 11(\sigma)$ & $1 \mathrm{Ks} 12(\sigma)$ & $1 \mathrm{Ks} 13(\sigma)$ & $1 \mathrm{Ks} 14(\sigma)$ \\
& & & $1 \mathrm{Ks} 15(\sigma)$ & $1 \mathrm{Ks} 16(\sigma)$ & $1 \mathrm{Ks} 17(\sigma)$ & $1 \mathrm{Ks} 18(\sigma)$ & $1 \mathrm{Ks} 19(\sigma)$ & $1 \mathrm{Ks} 20(\sigma)$ & \\
\hline \multirow{2}{*}{31} & 460.926 & 401.982 & $17.38(0.09)$ & $17.41(0.08)$ & $16.54(0.08)$ & $16.70(0.06)$ & $17.24(0.09)$ & $17.24(0.06)$ & $16.48(0.07)$ \\
& & & $17.37(0.05)$ & $17.50(0.06)$ & $17.54(0.06)$ & $17.49(0.05)$ & $17.26(0.05)$ & $17.85(0.05)$ & $18.04(0.08)$ \\
& & & $18.04(0.08)$ & $17.86(0.09)$ & $17.88(0.09)$ & $17.66(0.05)$ & $18.33(0.11)$ & $17.67(0.07)$ & \\
\multirow{2}{*}{50} & \multirow{2}{*}{763.941} & 462.473 & $18.00(0.08)$ & $18.12(0.05)$ & $17.98(0.05)$ & $17.94(0.04)$ & $17.92(0.07)$ & $18.00(0.05)$ & $17.80(0.05)$ \\
& & & $17.85(0.04)$ & $18.04(0.05)$ & $18.03(0.07)$ & $18.09(0.04)$ & $17.99(0.05)$ & $18.08(0.05)$ & $18.19(0.05)$ \\
& & & $18.18(0.05)$ & $18.18(0.04)$ & $18.17(0.06)$ & $18.14(0.05)$ & $18.21(0.04)$ & $18.14(0.05)$ & \\
\multirow{3}{*}{51} & 219.227 & \multirow{2}{*}{79.584} & $17.91(0.04)$ & $17.83(0.04)$ & $17.71(0.06)$ & $17.78(0.05)$ & $17.71(0.05)$ & $17.90(0.04)$ & $17.76(0.05)$ \\
& & & $18.12(0.04)$ & $18.06(0.04)$ & $18.07(0.04)$ & $18.23(0.04)$ & $18.18(0.04)$ & $18.24(0.04)$ & $18.29(0.04)$ \\
& & & $18.23(0.05)$ & $18.18(0.04)$ & $18.18(0.05)$ & $18.06(0.04)$ & $18.25(0.05)$ & $18.13(0.04)$ \\
\hline
\end{tabular}

Table 8. Individual measurements of the $K$-band magnitudes for all the red variable stars detected in Field 2 . The columns from left to right list: star ID, $x$ and $y$ position with respect to the reference epoch, the reference epoch $K$-band magnitudes and errors and all the other individual $K$-band magnitudes and the errors of the measurements as given by ALLFRAME to which 0.04 mag calibration error have been added in quadrature. Magnitude 99.99 and the error 9.99 denote that no measurement for that star was obtained.

\begin{tabular}{|c|c|c|c|c|c|c|c|c|}
\hline \multirow[t]{4}{*}{ F2 ID } & \multirow[t]{4}{*}{$x$} & \multirow[t]{4}{*}{$y$} & $2 \mathrm{Ks} 10(\sigma)$ & $2 \mathrm{Ks} 01(\sigma)$ & $2 \mathrm{Ks} 02(\sigma)$ & $2 \mathrm{Ks} 03(\sigma)$ & $2 \mathrm{Ks} 05(\sigma)$ & $2 \mathrm{Ks} 06(\sigma)$ \\
\hline & & & $2 \mathrm{Ks} 07(\sigma)$ & $2 \mathrm{Ks} 08(\sigma)$ & $2 \mathrm{Ks} 09(\sigma)$ & $2 \mathrm{Ks} 11(\sigma)$ & $2 \mathrm{Ks} 12(\sigma)$ & $2 \mathrm{Ks} 13(\sigma)$ \\
\hline & & & $2 \mathrm{Ks} 14(\sigma)$ & $2 \mathrm{Ks} 15(\sigma)$ & $2 \mathrm{Ks} 16(\sigma)$ & $2 \mathrm{Ks} 17(\sigma)$ & $2 \mathrm{Ks} 18(\sigma)$ & $2 \mathrm{Ks} 19(\sigma)$ \\
\hline & & & $2 \mathrm{Ks} 20(\sigma)$ & $2 \mathrm{Ks} 21(\sigma)$ & $2 \mathrm{Ks} 22(\sigma)$ & $2 \mathrm{Ks} 23(\sigma)$ & $2 \mathrm{Ks} 24(\sigma)$ & $2 \mathrm{Ks} 04(\sigma)$ \\
\hline \multirow[t]{4}{*}{5} & 110.132 & 451.042 & $14.76(0.04)$ & $15.33(0.14)$ & $15.04(0.15)$ & $15.00(0.12)$ & $14.66(0.05)$ & $14.69(0.04)$ \\
\hline & & & $14.68(0.04)$ & $14.68(0.04)$ & $14.69(0.04)$ & $15.37(0.06)$ & $15.41(0.07)$ & $15.50(0.13)$ \\
\hline & & & $16.69(0.13)$ & $17.02(0.16)$ & $15.97(0.28)$ & $16.08(0.18)$ & 99.99 (9.99) & 99.99 (9.99) \\
\hline & & & 99.99 (9.99) & 99.99 (9.99) & 99.99 (9.99) & 99.99 (9.99) & 99.99 (9.99) & $14.73(0.07)$ \\
\hline \multirow[t]{4}{*}{25} & 111.583 & -16.658 & 99.99 (9.99) & $22.55(0.25)$ & 99.99 (9.99) & 99.99 (9.99) & 99.99 (9.99) & 99.99 (9.99) \\
\hline & & & 99.99 (9.99) & 99.99 (9.99) & 99.99 (9.99) & $17.57(0.07)$ & $18.26(0.11)$ & $16.74(0.04)$ \\
\hline & & & $18.18(0.21)$ & $17.87(0.11)$ & 99.99 (9.99) & 99.99 (9.99) & $21.48(0.34)$ & $19.79(0.24)$ \\
\hline & & & $20.17(0.09)$ & $21.46(0.18)$ & $18.18(0.16)$ & $19.47(0.09)$ & $19.93(0.10)$ & $19.98(0.22)$ \\
\hline \multirow[t]{4}{*}{35} & 115.155 & -29.046 & 99.99 (9.99) & 99.99 (9.99) & 99.99 (9.99) & 99.99 (9.99) & 99.99 (9.99) & 99.99 (9.99) \\
\hline & & & 99.99 (9.99) & 99.99 (9.99) & 99.99 (9.99) & $17.02(0.06)$ & 99.99 (9.99) & $16.71(0.06)$ \\
\hline & & & $19.02(0.27)$ & $17.75(0.18)$ & $24.37(6.32)$ & 99.99 (9.99) & $22.20(0.49)$ & 99.99 (9.99) \\
\hline & & & $20.37(0.10)$ & $21.65(0.31)$ & $18.49(0.19)$ & 99.99 (9.99) & $21.58(0.34)$ & $19.99(0.23)$ \\
\hline
\end{tabular}

\pm 25 days except in the range of 700-800 days where the accuracy does not exceed \pm 50 to 100 days. Additionally there may be a few cases where an aliasing period may introduce a larger error due to less than optimal sampling.

Mean magnitudes of variable stars are measured to be brighter than simulated mean magnitudes by up to $\sim 0.3 \mathrm{mag}$ and this brightening depends on the amplitude of a variable stars. Typically for variables with amplitudes smaller than $\sim 1$ mag measured mean magnitudes are very similar to the input magnitudes. This brightening is partially due to the way the mean magnitude is calculated. For constant light curve stars in the same magnitude range as the variable stars 
$(19.5<K<21.5)$ there is no bias, i.e. the difference between measured and input magnitude is consistent with zero.

Our variable star catalogue is close to $90 \%$ complete for short period long amplitude variables. For variable stars with variability amplitudes of 0.2 mag or smaller detection probability is $50 \%$ or smaller. There is a strong dependence of the detection probability on the period of a variable. Our simulations show that most variable stars with periods in the range of 700800 days will have their periods strongly biased toward larger values, overestimating the period by as much as 50-100 days.

In the next paper the complete analysis of the period and amplitude distribution as well as their dependence on the magnitude and color of the variable stars will be presented. The comparison with long period variables in the Milky Way and the Magellanic Clouds will be made.

Acknowledgements. We are indebted to many ESO staff astronomers who took the data presented in this paper in service mode operations at Paranal Observatory. Useful input by Tim Bedding in the early stages of the project is gratefully acknowledged. We wish to thank the referee, Tom Lloyd Evans, for useful suggestions and Ian Glass for communicating their results in advance of the publication. MR thanks Mariarosa Cioni, Manuela Zoccali and Peter Stetson for useful discussions. DM is sponsored by FONDAP Center for Astrophysics 15010003. MR acknowledges the ESO studentship programme during which most of this work was done.

\section{References}

Bersier, D., \& Wood, P. R. 2002, AJ, 123, 840

Bertelli, G., Bressan, A., Chiosi, C., Fagotto, F., \& Nasi, E. 1994, A\&AS, 106, 275

Fassett, C. I., \& Graham, J. A. 2000, ApJ, 538, 594

Feast, M. W., Robertson, B. S. C., Catchpole, R. M., et al. 1982, MNRAS, 201, 439

Frogel, J. A., \& Whitford, A. E. 1987, ApJ, 320, 199

Frogel, J. A., \& Elias, J. H. 1988, ApJ, 324, 823
Frogel, J. A., \& Whitelock, P. A. 1998, AJ, 116, 754

Glass, I. S., \& Lloyd Evans, T. 2003, MNRAS, in press

Guarnieri, M. D., Renzini, A., \& Ortolani, S. 1997, ApJ, 477, L21

Habing, H. J. 1996, A\&ARv., 7, 97

Harris, G. L. H., \& Harris, W. E. 2000, AJ, 120, 2423

Harris, G. L. H., Harris, W. E., \& Poole, G. B. 1999, AJ, 117, 855

Kennicutt, R. C. 1998, ARA\&A, 36, 189

Kerschbaum, F., \& Hron, J. 1992, A\&A, 263, 97

Kerschbaum, F., \& Hron, J. 1994, A\&AS, 106, 397

Kholopov, P. N., et al. 1985, General Catalogue of Variable Stars, 4th edn. (Moscow: Nauka Publishing House), (GCVS)

Liu, M. C., Graham, J. R., \& Charlot, S. 2002, ApJ, 564, 216

Lomb, N. R. 1976, Ap\&SS, 39, 447

Malin, D. F., Quinn, P. J., \& Graham, J. A. 1983, ApJ, 272, L5

Marleau, F. R., Graham, J. R., Liu, M. C., \& Charlot, S. 2000, AJ, 120, 1779

Mei, S., Silva, D. R., \& Quinn, P. J. 2001, A\&A, 366, 54

Mould, J. R., Ridgewell, A., Gallagher, J. S. III, et al. 2000, ApJ, 536, 266

Persson, S. E., Murphy, D. C., Krzeminski, W., Roth, M., \& Rieke, M. J. 1998, AJ, 116, 2475

Rejkuba, M. 2001, A\&A, 369, 812

Rejkuba, M. 2002, Ph.D. Thesis, P. Universidad Católica de Chile

Rejkuba, M., Minniti, D., Bedding, T., \& Silva, D. R. 2001, A\&A, 379,781

Rejkuba, M., Minniti, D., Courbin, F., \& Silva, D. R. 2002, ApJ, 564, 688

Saha, A., \& Hoessel, J. G. 1990, AJ, 99, 97

Soria, R., Mould, J. R., Watson, A. M., et al. 1996, ApJ, 465, 79

Stanford, S. A., Eisenhardt, P. R. M., \& Dickinson, M. 1995, ApJ, 450, 512

Stetson, P. B. 1996, PASP, 108, 851

Stetson, P. B. 1994, PASP, 106, 250

Stetson, P. B. 1987, PASP, 99, 191

Tonry, J., \& Schneider, D. P. 1988, AJ, 96, 807

Tonry, J. L., \& Schechter, P. L. 1990, AJ, 100, 1794

Welch, D. L., \& Stetson, P. B. 1993, AJ, 105, 1813

Whitelock, P., Marang, F., \& Feast, M. 2000, MNRAS, 319, 728

Wood, P. R., Bessell, M. S., \& Fox, M. W. 1983, ApJ, 272, 99 


\section{Online Material}


M. Rejkuba et al.: LPV catalogue in Cen A, Online Material p 2

\section{Completeness and contamination}

The completeness of the photometric catalogue in the $J, H$ and $K$-bands was determined with crowding experiments. In ten different crowding experiments a set of stars with magnitudes uniformly distributed between $15.7<K<25.2$ was added to all the images after the appropriate re-scaling for the photometric zero point differences and shifting the stars so that they all fall at the same physical position in the sky coordinates. The $J-K$ and $H-K$ colors of the stars were chosen to follow a mean ridge line of the observed red giant branch (Fig. 7). A realistic, observed, amount of Poissonian noise was added as well. The photometry was then re-calculated in the same way as described above.

The results of these completeness experiments are shown in Figs. 8, 9 and 10. Our photometry is complete more than $50 \%$ in the $K$ and $H$-bands for stars brighter than 22.5 and 21.6 mag for Fields 1 and 2, respectively. These numbers for the $J$-band are 23.25 and $22.5 \mathrm{mag}$. Some bins have completeness larger than $100 \%$ due to false detections or migrations due to blends with original stars in the images. Note however, that we assume that the simulated star is detected only if its measured magnitude does not differ from the input value by more than 0.75 mag.

The left panels of Figs. 8 to 10 show differences between input (added) and recovered (measured) magnitudes which is indicative of photometric errors at a given magnitude. From them it is evident that the stars fainter than $50 \%$ completeness have very large photometric errors. Apart from the completeness and photometric error estimations, these simulations were also used to determine criteria for the detection of variable stars. The results are summarized in Fig. 3 and are described in Sect. 4.

Further simulations of variable stars were used to gain information on the completeness and contamination in our LPV catalogue. Since the detection probability of a variable
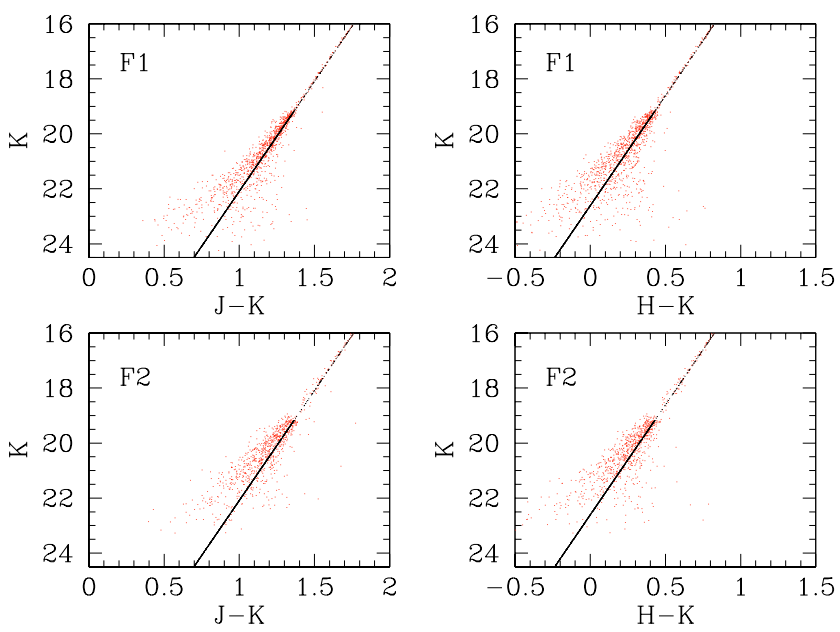

Fig. 7. Completeness simulations: black dots distributed in a line that represents a mean RGB ridge line were added to all frames. Red dots scattered in the vicinity of that line are measured color-magnitude diagrams for these added stars. Stars simulated in the Field 1 crowding experiments are shown in the top panels and those in Field 2 are in the bottom panels.
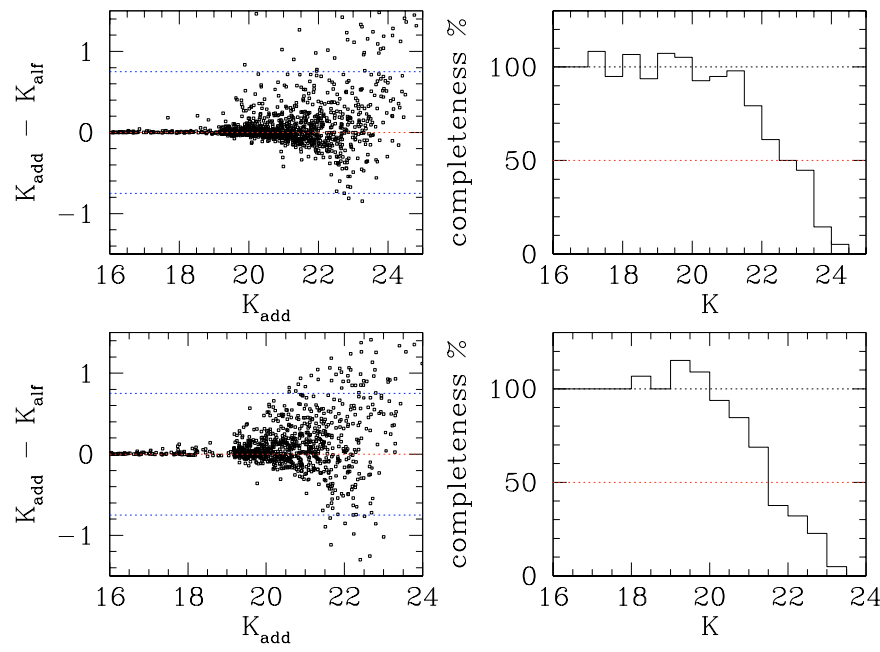

Fig. 8. $K$-band photometric errors (left) and completeness (right): The simulations for Field 1 are in the upper and for Field 2 in the lower diagrams. Only stars that were measured at the same coordinates as the added stars and having $J, H$ and $K$-band magnitudes within $-0.75<$ $\delta K<0.75$ (dotted lines on the left diagrams) are considered to be detected in the crowding experiments.
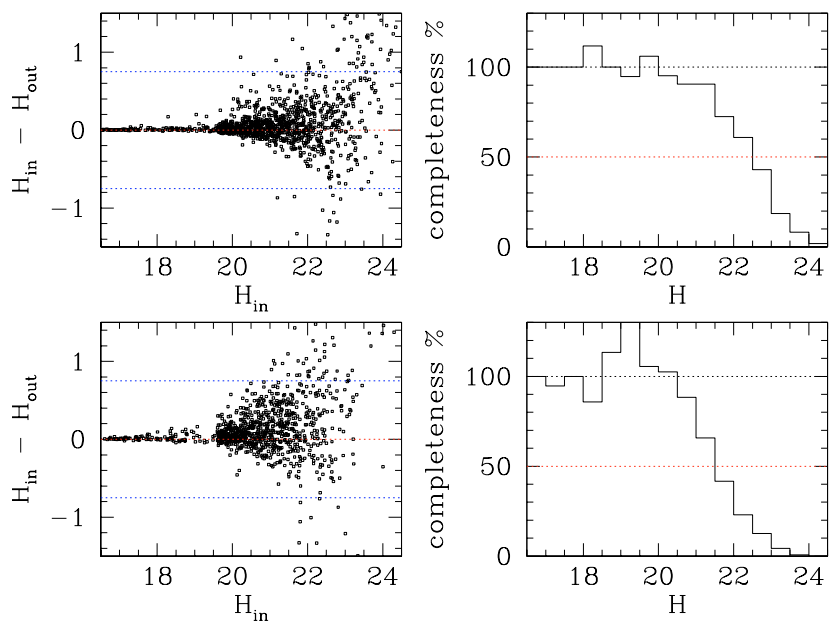

Fig. 9. Same as Fig. 8 but for the $H$-band.
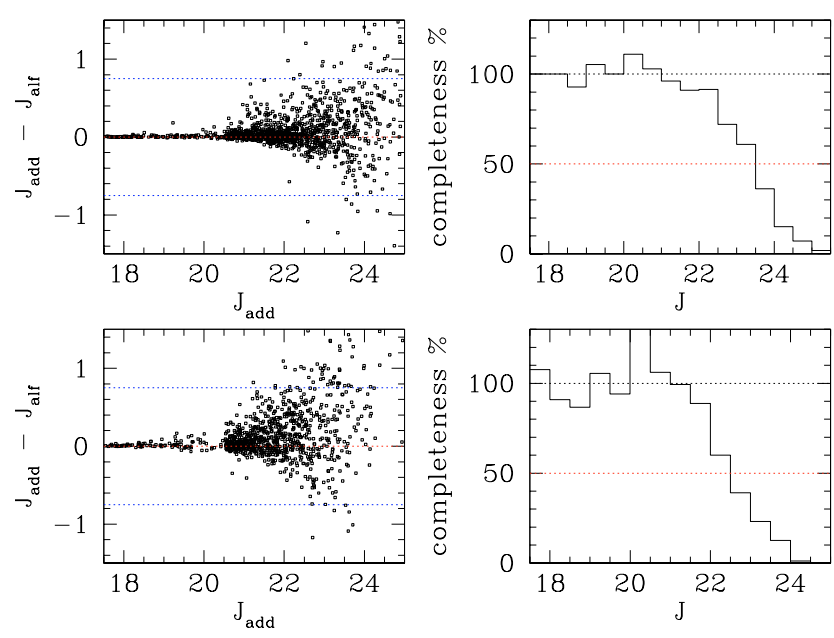

Fig. 10. Same as Fig. 8 but for the $J$-band. 
M. Rejkuba et al.: LPV catalogue in Cen A, Online Material p 3
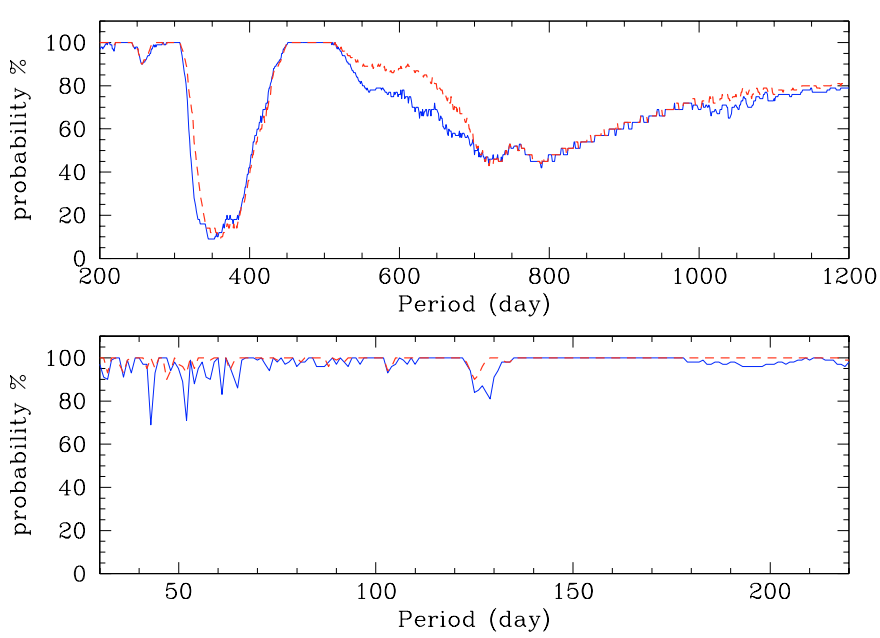

Fig. 11. Probability of detecting a variable star as a function of period assuming an even distribution of measurements in phase (see text). The solid line is for Field 1 and the dashed line for observations of Field 2.

depends not only on the mean magnitude of the star, but also on period and amplitude, as well as on sampling distribution, different sets of simulations were carried out.

Dependence of the probability of the detection of a variable star with a given period on the actual distribution of the observations can be estimated with numerical simulations similar to those by Saha \& Hoessel (1990) and Bersier \& Wood (2002). Given a period $\mathrm{P}$ and initial phase $\phi_{0}$ at the time $t_{0}$ of the first observation, the phase distribution can be calculated for our set of observations. Assuming that to detect a variable a uniform coverage in phase is required, with (1) at least two observations between phases 0 and 0.2 , (2) at least two detections with phases between 0.2 and 0.5 and (3) at least two measurements with phases between 0.5 and 0.8 , the resulting period detection probability is presented in Fig. 11. Note that for a more realistic estimate this curve should be multiplied by the incompleteness corresponding to the mean magnitude of the variable. An additional factor in the period detection probability is the amplitude of the variable.

A better estimate of the detection probability for variables with given magnitudes, amplitudes and periods, can be obtained through simulations similar to the crowding experiments. In a series of such experiments we have added artificial variable stars with sinusoidal light curves with given periods, amplitudes and magnitudes. The magnitude range was chosen between $19.5<K<21.5$, similar to that of detected LPVs in NGC 5128 halo.

One series of completeness experiments tested our detection sensitivity to the amplitudes of variable stars. Variable stars with amplitudes between 0.1 and 1.4 mag for two fixed periods, 100 and 450 days were added to the original frames in 18 different crowding experiments for each field. In each single experiment all the variables had the same amplitude and period, but a range of magnitudes as mentioned above. In Fig. 12 the percentage of detected variables, those with $-0.75 \leq \Delta$ mag $\leq 0.75$ and $-25<\Delta \mathrm{P}<25$, as a function of amplitude is given for variables brighter than $K=20.5$ (triangles) and those with

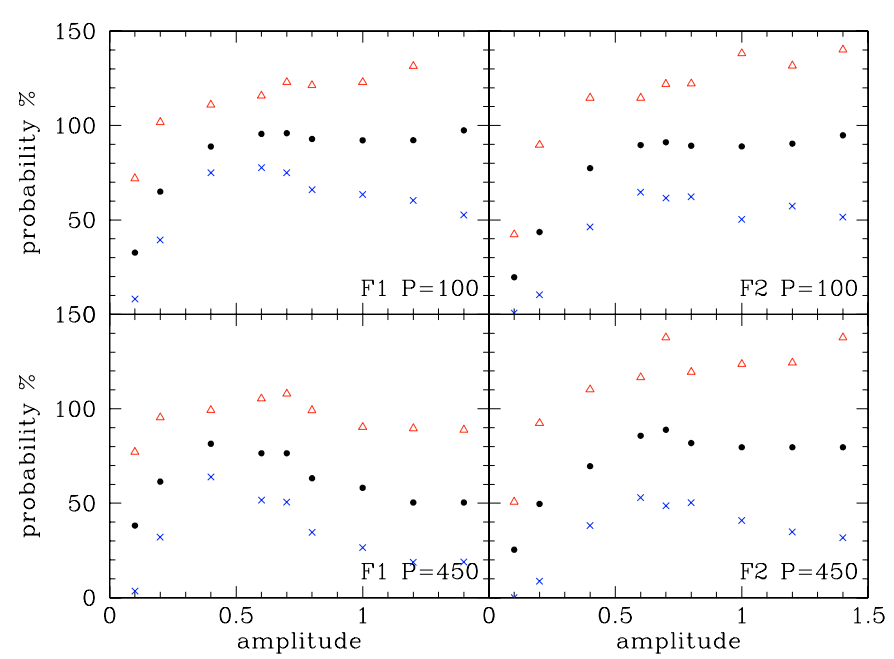

Fig. 12. Probability of detecting a variable star as a function of amplitude for simulated variable stars with periods of $P=100$ day (top panels) and $P=450$ days (bottom panels). A simulated variable is considered to be detected only if $|\Delta m a g|<0.75$ and $|\Delta P| \leq 25$. Simulations for Field 1 are on the left and those for Field 2 on the right. Different symbols are used for different magnitude bins: triangles for stars brighter than $K=20.5$, crosses for $K>20.5$ and filled dots for all the stars irrespective of their magnitude.

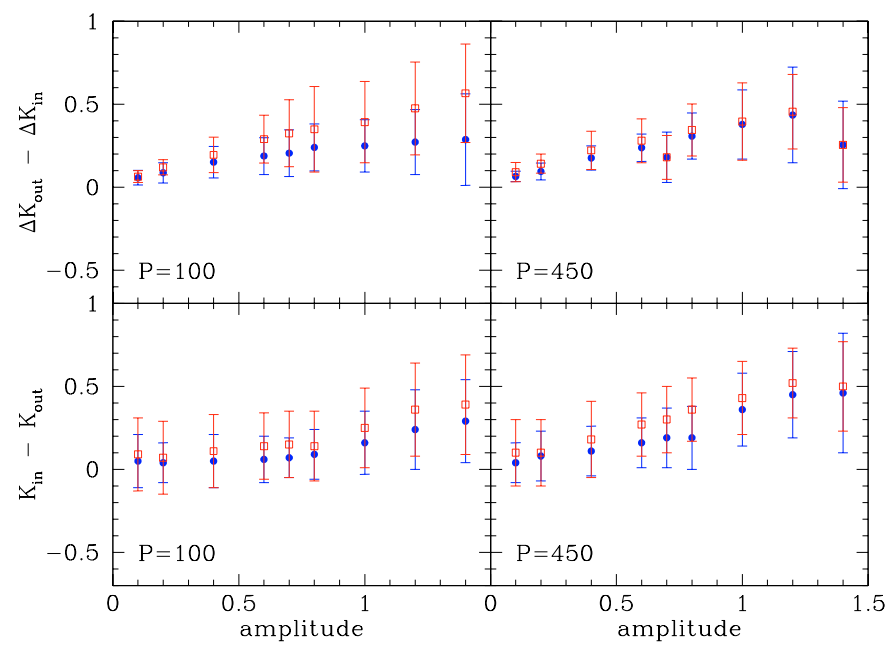

Fig. 13. The difference between the detected and input amplitude (top panels) and the difference between the detected and input magnitudes (bottom panels) for simulated variables with periods of $P=100$ days (left) and $P=450$ days as a function of input amplitude. Simulations of Field 1 variables are shown with filled circles and those for Field 2 with open squares.

$K>20.5$ (crosses). Filled dots denote detection probability (or completeness) as a function of amplitude of variables for all the stars irrespective of their magnitudes. It is obvious from the fact that the bright stars have completeness higher than $100 \%$ that there is some migration of faint stars into the brighter magnitude bin.

This effect can also be seen in Fig. 13 where we plot differences between detected and input magnitudes (bottom panels) and differences in detected and input amplitudes (top panels) as a function of input amplitude for all the variables. Simulations of Field 1 variables are plotted with filled dots and those of 


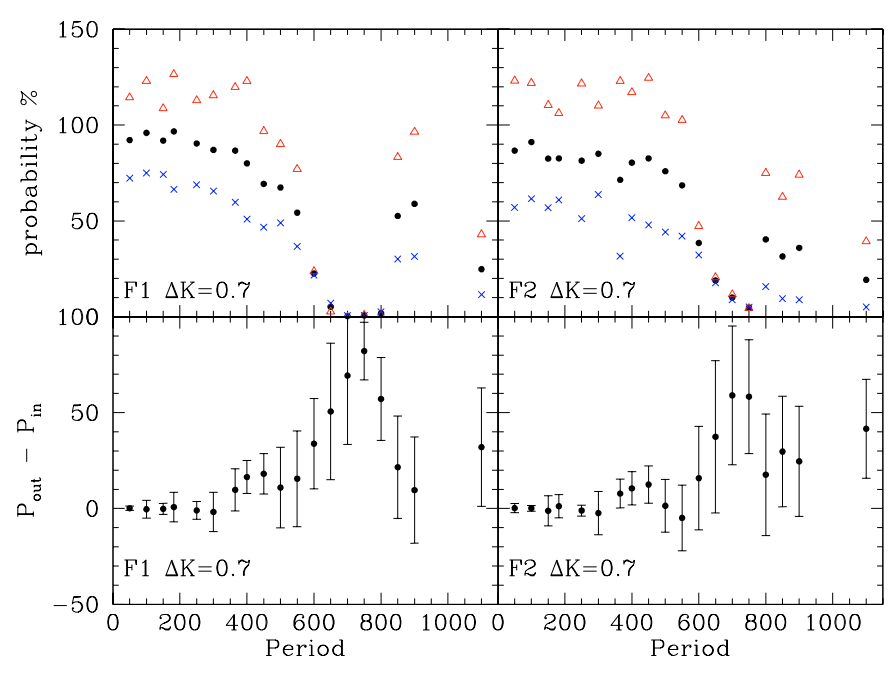

Fig. 14. Probability of detecting a variable star as a function of period for simulated variable stars with amplitudes of $\Delta K=0.7$ mag for Field 1 (top left) and Field 2 (top right panel). Here a simulated variable is considered to be detected only if its detected magnitude did not differ from the measured value by more than 0.75 mag and if its measured period did not differ from the input one by more than \pm 25 days. Bottom panels show difference in measured and input periods for variables as a function of period.

Field 2 with open squares. There is a systematic bias in the sense that we detect stars brighter than they are and with somewhat larger amplitudes. The magnitude of this bias is proportional to the amplitude of the variable. Partially this difference may be due to the way the mean output magnitude is calculated, but using different means still produces a slight bias. In order to have an estimate of bias independent of an a priori knowledge about the period and phase, because for some of the variables we could not determine periods and some stars in the field escaped detection as variable stars, we plot in Fig. 13 differences in mean magnitudes as they get calculated by DAOMASTER task in DAOPHOT.

From Fig. 12 it is obvious that most of the large amplitude variables $(\Delta K>0.3)$ will be detected for stars with shorter periods. Our variable star catalogue is highly incomplete for stars with amplitudes of 0.2 mag or smaller, irrespective of period. The absolute value of which percentage of variables with a given amplitude will be detected for longer period LPVs depends strongly on our choice of detection criterion. If we consider that a variable star is detected only if its measured period does not deviate from the input one by \pm 25 days, detection probability at $P=450$ days is between 50 and $80 \%$, but if we allow a larger uncertainty in period detection, our catalogue is more complete.

In order to see what is the completeness of our catalogue with respect to period of variable stars and how precise our period measurements are we performed a series of experiments in which we have added to our frames artificial variable stars with sinusoidal light curves with typical amplitudes for LPVs $(\Delta K=0.7 \mathrm{mag})$ and magnitudes in the range between $19.5<K<21.5$, similar to that of detected LPVs in NGC 5128 halo. Periods were chosen at 50 day interval, ranging from 50 to 1100 days. In particular 365 day and 182 day period vari- able stars were also added. Photometry of all the added stars was performed in the same way as before and the light curve parameters calculated as explained above. The measured detection probability as a function of period is presented in Fig. 14. Again we plot the detection probability for all the simulated stars with a given period with filled dots. Open triangles and crosses are used for bright $(K<20.5)$ and faint $(K>20.5)$ stars, respectively. In plotting the top panels of Fig. 14 we assumed a variable star to be detected only if (i) its mean magnitude did not differ from the input mean magnitude by more than $0.75 \mathrm{mag}$, (2) if it was detected in at least 10 frames, and (iii) if its measured period did not differ from the input period by more than \pm 25 days. Due to this last restriction there is an almost zero probability to detect variables with periods around 700-800 days. However, in our catalogue there are variables with these periods. In fact the bottom panels of Fig. 14 show a dependence of the difference between measured and input periods as a function of input period. From this it is obvious that for the variables with periods in the range between 700 and 800 days we systematically overestimate their periods by as much as 50-100 days. In the top panels these variables are considered non-detections. Periods for variables with periods longer than 800 days can be determined more accurately. An additional potential systematic error could come from period aliasing. Our observations do not span time continuously. Rather, they are grouped in three to six month windows with inter-window gaps of 200 to 320 days. Thus, it is possible that stars with actual periods in the range 175-200 days were assigned periods of order of one year, because only alternate maxima fell within our observing windows. 
M. Rejkuba et al.: LPV catalogue in Cen A, Online Material p 5

Table 3. LPVs in Field 1. The columns from left to right list: star ID, $x$ and y position with respect to the reference epoch, the best fitting period, semi-amplitude, reduced $\chi^{2}$ of the sine-curve fit, significance of the period from Fourier analysis, single epoch $J$ and $H$-band magnitudes and mean $K$-band magnitude. Magnitude 99.99 denotes that no measurement for that star was obtained.

\begin{tabular}{|c|c|c|c|c|c|c|c|c|c|c|}
\hline F1 ID & $\mathrm{x}$ & $\mathrm{y}$ & $\mathrm{N}$ & $\mathrm{P}$ & $\mathrm{A}$ & $\chi^{2}$ & signif & $\mathrm{J}$ & $\mathrm{H}$ & $<K>$ \\
\hline 31 & 460.926 & 401.982 & 20 & 432. & 0.513 & 21.10 & $\overline{0.650}$ & 19.11 & 18.05 & 17.37 \\
\hline 53 & 107.785 & 832.967 & 19 & 448. & 0.203 & 2.30 & 0.310 & 19.91 & 19.01 & 18.09 \\
\hline 64 & 610.320 & 803.826 & 20 & 430. & 0.202 & 18.50 & 1.000 & 19.03 & 18.45 & 18.36 \\
\hline 73 & 688.817 & 405.675 & 20 & 137. & 0.182 & 15.90 & 1.000 & 19.88 & 19.16 & 18.55 \\
\hline 77 & 859.402 & 627.796 & 19 & 411. & 0.353 & 19.00 & 1.000 & 18.93 & 18.41 & 18.72 \\
\hline 84 & 218.231 & 79.339 & 16 & 432. & 0.469 & 27.70 & 0.490 & 19.16 & 19.01 & 18.94 \\
\hline 91 & 860.939 & 628.112 & 19 & 440. & 0.235 & 13.10 & 0.960 & 19.64 & 19.28 & 18.88 \\
\hline 100 & 469.319 & 296.353 & 20 & 618. & 0.418 & 1.80 & 0.130 & 20.72 & 19.78 & 19.33 \\
\hline 104 & 860.140 & 629.895 & 19 & 232. & 0.181 & 4.90 & 0.940 & 19.91 & 19.28 & 19.13 \\
\hline 129 & 366.442 & 678.245 & 20 & 515. & 0.304 & 14.80 & 0.850 & 20.93 & 19.97 & 19.36 \\
\hline 133 & 16.431 & 426.376 & 12 & 486. & 0.322 & 1.30 & 0.550 & 21.43 & 20.39 & 19.44 \\
\hline 146 & 241.723 & 710.178 & 20 & 471. & 0.293 & 9.40 & 0.470 & 20.83 & 20.03 & 19.45 \\
\hline 148 & 251.540 & -35.913 & 11 & 248. & 0.489 & 48.10 & 1.000 & 99.99 & 19.57 & 19.57 \\
\hline 151 & 383.324 & 278.858 & 20 & 501. & 0.400 & 2.50 & 0.120 & 20.64 & 19.75 & 19.61 \\
\hline 155 & 822.695 & 291.891 & 19 & 558. & 0.685 & 4.10 & 0.090 & 21.26 & 19.94 & 19.69 \\
\hline 160 & 157.838 & 174.017 & 20 & 425. & 0.211 & 6.00 & 0.910 & 21.06 & 20.14 & 19.52 \\
\hline 161 & 1.411 & 593.494 & 20 & 501. & 0.328 & 6.00 & 0.600 & 21.03 & 20.23 & 19.45 \\
\hline 164 & 645.768 & 675.400 & 20 & 527. & 0.384 & 15.90 & 0.640 & 21.17 & 19.99 & 19.46 \\
\hline 166 & 476.846 & 747.158 & 20 & 214. & 0.254 & 4.30 & 0.340 & 20.95 & 19.82 & 19.46 \\
\hline 168 & 63.609 & 662.896 & 20 & 434. & 0.432 & 4.90 & 0.070 & 21.27 & 20.46 & 19.61 \\
\hline 170 & 280.584 & 257.958 & 20 & 499. & 0.276 & 2.60 & 0.120 & 22.35 & 20.94 & 19.55 \\
\hline 171 & 205.283 & 725.663 & 20 & 452. & 0.328 & 1.90 & 0.060 & 21.72 & 20.23 & 19.49 \\
\hline 172 & 651.896 & 747.256 & 20 & 446. & 0.281 & 1.10 & 0.080 & 20.72 & 19.87 & 19.61 \\
\hline 173 & 132.979 & 205.212 & 20 & 449. & 0.647 & 6.10 & 0.060 & 20.85 & 19.75 & 19.79 \\
\hline 174 & 876.368 & 141.793 & 18 & 474. & 0.283 & 3.50 & 0.220 & 20.94 & 19.86 & 19.58 \\
\hline 177 & 387.634 & 211.691 & 20 & 394. & 0.199 & 4.50 & 0.590 & 20.93 & 20.11 & 19.53 \\
\hline 178 & 281.918 & 543.030 & 20 & 538. & 0.398 & 1.70 & 0.410 & 21.43 & 20.27 & 19.48 \\
\hline 182 & 679.090 & -5.543 & 18 & 442. & 0.175 & 0.90 & 0.150 & 20.50 & 19.89 & 19.51 \\
\hline 183 & 110.136 & 846.650 & 11 & 452. & 0.446 & 2.10 & 0.800 & 22.02 & 99.99 & 19.71 \\
\hline 184 & 35 & 68.748 & 20 & 478. & 0.295 & 3.90 & 0.150 & 21.54 & 20.46 & 19.58 \\
\hline 185 & 144.836 & 180.467 & 20 & 511. & 0.360 & 7.10 & 0.260 & 21.20 & 20.23 & 19.64 \\
\hline 188 & 108.023 & 247.863 & 20 & 454. & 0.302 & 2.50 & 0.250 & 21.06 & 20.04 & 19.69 \\
\hline 190 & 489.946 & 400.930 & 20 & 429. & 0.405 & 4.50 & 0.180 & 20.83 & 20.08 & 19.66 \\
\hline 191 & 794.769 & 177.227 & 20 & 452. & 0.365 & 5.00 & 0.110 & 21.25 & 20.52 & 19.64 \\
\hline 193 & 143.056 & 171.690 & 20 & 195. & 0.213 & 2.30 & 0.140 & 20.77 & 19.85 & 19.62 \\
\hline 194 & 428.622 & 185.952 & 20 & 461. & 0.238 & 3.40 & 0.200 & 21.03 & 20.24 & 19.62 \\
\hline 195 & 373.988 & 172.810 & 20 & 467. & 0.385 & 2.00 & 0.060 & 20.83 & 20.11 & 19.73 \\
\hline 197 & 129.658 & 501.311 & 20 & 516. & 0.422 & 4.30 & 0.310 & 21.09 & 20.25 & 19.80 \\
\hline 199 & 52.553 & 723 & 20 & 432. & 0.289 & 3.00 & 0.540 & 21.52 & 20.34 & 19.62 \\
\hline 200 & 447.286 & 399.913 & 20 & 448. & 0.438 & 4.60 & 0.100 & 21.31 & 20.68 & 19.85 \\
\hline 201 & 344.898 & 770.497 & 20 & 189. & 0.296 & 10.90 & 0.390 & 20.52 & 19.70 & 19.67 \\
\hline 204 & 31.785 & 419.365 & 20 & 449. & 0.311 & 2.50 & 0.080 & 20.73 & 19.78 & 19.73 \\
\hline 205 & 740.307 & 514.147 & 20 & 440. & 0.416 & 2.70 & 0.100 & 21.18 & 20.06 & 19.87 \\
\hline 206 & 672.810 & 492.074 & 20 & 466. & 0.412 & 2.30 & 0.060 & 20.60 & 19.83 & 19.74 \\
\hline 207 & 349.012 & 58.330 & 20 & 314. & 0.278 & 8.80 & 0.470 & 20.74 & 19.79 & 19.63 \\
\hline 210 & 173.593 & 109.992 & 20 & 430. & 0.396 & 3.40 & 0.090 & 20.84 & 20.04 & 19.75 \\
\hline 213 & 113.806 & 521.844 & 20 & 526. & 0.592 & 17.20 & 0.320 & 21.70 & 20.73 & 19.98 \\
\hline 216 & 266.856 & 305.468 & 20 & 446. & 0.269 & 3.00 & 0.110 & 21.17 & 20.20 & 19.71 \\
\hline 218 & 506.923 & 159.390 & 20 & 496. & 0.364 & 1.60 & 0.130 & 20.85 & 20.01 & 19.87 \\
\hline 219 & 178.324 & 64.073 & 20 & 423. & 0.493 & 4.70 & 0.080 & 20.75 & 20.08 & 19.91 \\
\hline 224 & 314.828 & 367.392 & 20 & 562. & 0.546 & 1.90 & 0.490 & 21.45 & 20.25 & 19.97 \\
\hline 227 & 746.930 & 775.240 & 20 & 439. & 0.327 & 2.80 & 0.110 & 20.85 & 19.94 & 19.83 \\
\hline 229 & 623.448 & 577.709 & 20 & 201. & 0.201 & 4.80 & 0.920 & 20.81 & 19.92 & 19.67 \\
\hline 230 & 479.669 & 410.112 & 20 & 462. & 0.382 & 5.30 & 0.140 & 21.05 & 20.05 & 19.80 \\
\hline 232 & 263.283 & 697.783 & 20 & 463. & 0.313 & 2.90 & 0.090 & 20.95 & 19.92 & 19.80 \\
\hline 233 & 89.820 & 829.313 & 19 & 358. & 0.338 & 4.20 & 0.710 & 21.60 & 20.45 & 19.64 \\
\hline 234 & 871.714 & 142.695 & 19 & 510. & 0.494 & 2.90 & 0.090 & 20.81 & 19.87 & 19.79 \\
\hline 236 & 130.784 & 541.867 & 20 & 475. & 0.589 & 6.80 & 0.200 & 22.91 & 21.39 & 19.83 \\
\hline 237 & 57.420 & 528.362 & 20 & 444. & 0.295 & 7.70 & 0.860 & 21.17 & 20.00 & 19.88 \\
\hline 238 & 16.670 & 152.924 & 12 & 427. & 0.456 & 1.10 & 0.520 & 20.88 & 20.04 & 19.95 \\
\hline
\end{tabular}


Table 3. continuation.

\begin{tabular}{|c|c|c|c|c|c|c|c|c|c|c|}
\hline$\overline{\text { F1 ID }}$ & $\mathrm{x}$ & $\mathrm{y}$ & $\overline{\mathrm{N}}$ & $\overline{\mathrm{P}}$ & $\overline{\mathrm{A}}$ & $\chi^{2}$ & signif & $\overline{\mathrm{J}}$ & $\overline{\mathrm{H}}$ & $<K>$ \\
\hline 240 & 139.735 & 4474.178 & 20 & 499. & 0.223 & 2.50 & 0.230 & 21.85 & 20.32 & 19.72 \\
\hline 244 & 802.609 & 464.726 & 20 & 442. & 0.349 & 2.40 & 0.070 & 21.47 & 20.38 & 19.87 \\
\hline 245 & 189.929 & 129.833 & 20 & 441. & 0.228 & 4.50 & 0.310 & 20.92 & 19.98 & 19.82 \\
\hline 246 & 575.943 & 702.232 & 20 & 458. & 0.422 & 3.70 & 0.070 & 20.90 & 20.22 & 19.85 \\
\hline 248 & 182.270 & 335.708 & 20 & 437. & 0.369 & 3.90 & 0.140 & 21.08 & 20.39 & 19.73 \\
\hline 250 & 889.374 & 396.430 & 18 & 477. & 0.409 & 3.50 & 0.160 & 21.20 & 20.37 & 19.86 \\
\hline 252 & 377.710 & 736.657 & 20 & 421. & 0.260 & 4.10 & 0.140 & 21.21 & 20.42 & 19.80 \\
\hline 258 & 584.651 & 646.161 & 20 & 501. & 0.539 & 12.60 & 0.280 & 21.00 & 20.09 & 19.78 \\
\hline 259 & 564.125 & 580.467 & 20 & 473. & 0.100 & 21.40 & 0.130 & 21.03 & 20.37 & 19.87 \\
\hline 260 & 550.035 & 697.299 & 20 & 476. & 0.484 & 2.50 & 0.070 & 21.91 & 21.06 & 19.98 \\
\hline 261 & 366.244 & 211.569 & 20 & 544. & 0.550 & 5.70 & 0.090 & 21.21 & 20.24 & 19.87 \\
\hline 263 & 194.740 & 832.315 & 19 & 457. & 0.383 & 18.40 & 0.770 & 20.47 & 20.39 & 19.84 \\
\hline 264 & 125.830 & 18.280 & 20 & 436. & 0.269 & 11.00 & 0.680 & 21.37 & 20.37 & 19.81 \\
\hline 269 & 783.385 & 778.874 & 20 & 197. & 0.309 & 4.20 & 0.230 & 20.81 & 19.99 & 19.79 \\
\hline 272 & 449.464 & 717.144 & 20 & 455. & 0.489 & 4.10 & 0.070 & 21.38 & 20.74 & 19.87 \\
\hline 275 & 97.280 & 801.841 & 20 & 475. & 0.355 & 12.10 & 0.380 & 21.12 & 19.80 & 19.88 \\
\hline 283 & 347.964 & -3.955 & 19 & 185. & 0.178 & 3.80 & 0.660 & 20.85 & 20.08 & 19.83 \\
\hline 284 & 193.238 & 500.106 & 20 & 431. & 0.466 & 5.80 & 0.100 & 20.83 & 20.10 & 20.00 \\
\hline 286 & 279.230 & 67.260 & 20 & 197. & 0.247 & 9.20 & 0.550 & 21.07 & 20.13 & 19.87 \\
\hline 288 & 14.460 & 549.217 & 11 & 446. & 0.408 & 1.70 & 0.690 & 21.98 & 20.87 & 20.07 \\
\hline 294 & 124.558 & 330.030 & 20 & 449. & 0.387 & 5.20 & 0.160 & 21.18 & 20.12 & 19.95 \\
\hline 295 & 445.246 & 622.988 & 20 & 466. & 0.527 & 2.20 & 0.060 & 21.56 & 20.49 & 20.00 \\
\hline 296 & 395.486 & 18.707 & 19 & 194. & 0.217 & 2.50 & 0.260 & 20.89 & 20.00 & 19.84 \\
\hline 301 & 364.732 & 570.560 & 20 & 427. & 0.283 & 1.30 & 0.080 & 20.97 & 20.24 & 19.84 \\
\hline 304 & 169.875 & 226.678 & 20 & 458. & 0.483 & 1.50 & 0.050 & 21.24 & 20.21 & 20.05 \\
\hline 311 & 51.930 & 187.867 & 20 & 461. & 0.508 & 3.40 & 0.090 & 22.13 & 20.67 & 20.10 \\
\hline 312 & 326.187 & 501.922 & 20 & 510. & 0.436 & 2.20 & 0.160 & 21.27 & 20.32 & 20.07 \\
\hline 314 & 108.418 & 332.330 & 20 & 444. & 0.521 & 1.40 & 0.050 & 21.24 & 20.35 & 20.10 \\
\hline 315 & 483.406 & 293.818 & 20 & 466. & 0.331 & 7.00 & 0.270 & 21.63 & 20.47 & 19.93 \\
\hline 316 & 721.808 & 688.040 & 20 & 448. & 0.371 & 1.90 & 0.050 & 21.79 & 20.83 & 19.99 \\
\hline 317 & 400.985 & 356.026 & 20 & 404. & 0.480 & 0.90 & 0.280 & 20.94 & 20.07 & 20.13 \\
\hline 319 & 83.836 & 591.370 & 20 & 483. & 0.620 & 1.00 & 0.050 & 21.56 & 20.22 & 20.15 \\
\hline 322 & 574.036 & 475.742 & 20 & 426. & 0.498 & 4.40 & 0.060 & 21.78 & 20.86 & 20.08 \\
\hline 323 & 563.345 & 653.749 & 20 & 416. & 0.478 & 2.40 & 0.150 & 21.02 & 20.14 & 20.11 \\
\hline 325 & 589.059 & 492.799 & 20 & 379. & 0.453 & 1.80 & 0.370 & 21.45 & 20.70 & 19.76 \\
\hline 327 & 853.249 & 736.471 & 19 & 449. & 0.406 & 3.60 & 0.140 & 21.50 & 20.72 & 19.91 \\
\hline 330 & 636.715 & 523.009 & 20 & 511. & 1.014 & 22.50 & 0.220 & 22.70 & 21.44 & 20.52 \\
\hline 331 & 480.523 & 569.934 & 20 & 517. & 0.539 & 1.10 & 0.250 & 22.79 & 21.42 & 19.98 \\
\hline 334 & 86.817 & 788.080 & 20 & 380. & 0.342 & 5.20 & 0.260 & 20.99 & 20.30 & 20.04 \\
\hline 335 & 259.608 & 230.597 & 20 & 446. & 0.476 & 3.20 & 0.070 & 21.05 & 20.12 & 20.05 \\
\hline 338 & 87.810 & 792.326 & 20 & 1097. & 1.078 & 4.00 & 0.110 & 24.91 & 23.11 & 20.48 \\
\hline 339 & 886.358 & 801.067 & 18 & 370. & 0.340 & 4.40 & 0.380 & 21.39 & 20.65 & 19.85 \\
\hline 342 & 359.105 & 464.911 & 20 & 461. & 0.510 & 0.60 & 0.040 & 21.94 & 20.75 & 20.07 \\
\hline 343 & 87.019 & 36.775 & 20 & 386. & 0.447 & 9.10 & 0.540 & 21.01 & 20.29 & 20.14 \\
\hline 350 & 895.862 & 228.496 & 16 & 481. & 0.670 & 8.40 & 0.270 & 22.04 & 20.98 & 20.23 \\
\hline 352 & 249.665 & 451.350 & 20 & 432. & 0.309 & 8.10 & 0.330 & 21.25 & 20.34 & 20.01 \\
\hline 353 & 900.235 & 821.974 & 16 & 652. & 0.378 & 56.00 & 0.630 & 24.04 & 22.55 & 20.26 \\
\hline 355 & 66.638 & 549.981 & 20 & 441. & 0.414 & 4.20 & 0.130 & 21.13 & 20.16 & 20.09 \\
\hline 357 & 757.542 & -12.799 & 17 & 627. & 0.523 & 10.80 & 0.730 & 21.18 & 20.35 & 20.27 \\
\hline 360 & 641.885 & 372.593 & 20 & 361. & 0.218 & 2.90 & 0.250 & 21.28 & 20.30 & 19.99 \\
\hline 361 & 266.155 & 53.191 & 20 & 403. & 0.226 & 2.90 & 0.220 & 21.15 & 20.29 & 19.98 \\
\hline 362 & 267.730 & 522.355 & 20 & 190. & 0.198 & 5.40 & 0.940 & 21.10 & 20.36 & 19.96 \\
\hline 364 & 461.075 & 786.365 & 20 & 480. & 0.426 & 5.60 & 0.110 & 21.00 & 20.11 & 20.02 \\
\hline 368 & 158.315 & 592.526 & 20 & 401. & 0.585 & 4.50 & 0.260 & 21.29 & 20.39 & 20.37 \\
\hline 369 & 825.349 & 606.238 & 19 & 436. & 0.454 & 4.30 & 0.120 & 21.77 & 20.79 & 20.00 \\
\hline 373 & 857.805 & 68.719 & 19 & 463. & 0.382 & 3.10 & 0.100 & 21.88 & 20.94 & 19.97 \\
\hline 375 & 394.411 & 672.023 & 20 & 196. & 0.272 & 4.70 & 0.300 & 21.07 & 20.23 & 19.98 \\
\hline 377 & 506.196 & 698.151 & 20 & 532. & 0.616 & 4.20 & 0.200 & 22.52 & 20.71 & 20.02 \\
\hline 382 & 147.260 & 308.850 & 20 & 464. & 0.718 & 2.50 & 0.050 & 23.11 & 21.97 & 20.38 \\
\hline 383 & 549.156 & 223.944 & 20 & 432. & 0.508 & 3.20 & 0.080 & 21.14 & 20.23 & 20.17 \\
\hline
\end{tabular}


M. Rejkuba et al.: LPV catalogue in Cen A, Online Material $p 7$

Table 3. continuation.

\begin{tabular}{|c|c|c|c|c|c|c|c|c|c|c|}
\hline F1 ID & $\mathrm{x}$ & $\mathrm{y}$ & $\overline{\mathrm{N}}$ & $\mathrm{P}$ & $\mathrm{A}$ & $\chi^{2}$ & signif & $\bar{J}$ & $\overline{\mathrm{H}}$ & $<K>$ \\
\hline$\overline{\overline{386}}$ & $\overline{844.386}$ & $\overline{7709.244}$ & $\overline{19}$ & 450. & $\overline{0.601}$ & 2.60 & $\overline{0.110}$ & 21.28 & 20.33 & 20.25 \\
\hline 388 & 0.293 & 20.148 & 20 & 372. & .346 & 1.30 & .060 & 21.14 & 0.48 & 20.02 \\
\hline 392 & 70.626 & 170.136 & 20 & 463. & 0.448 & 2.50 & 0.060 & 21.22 & 20.32 & 20.10 \\
\hline 393 & 34.904 & 18.061 & 20 & 191. & 0.220 & 6.70 & 0.540 & 21.04 & 20.20 & 20.02 \\
\hline 396 & 52.867 & 628.918 & 19 & 234. & .453 & 13.20 & .850 & 21.23 & 20.30 & 20.04 \\
\hline 397 & 9.331 & 15.644 & 20 & 389. & 0.424 & 3.20 & 0.360 & 21.01 & 20.28 & 20.19 \\
\hline 408 & 15.215 & 176.524 & 19 & 457. & 0.448 & 1.90 & 0.090 & 22.56 & 21.04 & 20.00 \\
\hline 410 & 5.327 & 271.408 & 12 & 428. & 0.483 & 6.90 & 0.690 & 21.21 & 20.19 & 20.23 \\
\hline 419 & 248.910 & 805.041 & 20 & 450. & 0.076 & 21.10 & 0.500 & 21.37 & 20.71 & 19.98 \\
\hline 423 & 25.791 & -23.687 & 13 & 401. & 0.435 & 1.50 & 0.440 & 21.32 & 20.65 & 20.10 \\
\hline 424 & 99.815 & 4.421 & 20 & 459. & 0.467 & 4.00 & 0.080 & 21.55 & 20.38 & 20.15 \\
\hline 425 & 608.033 & 515.828 & 20 & 174. & 0.242 & 4.00 & 0.220 & 21.08 & 20.30 & 20.07 \\
\hline 429 & 624.594 & 728.730 & 20 & 442. & 0.479 & 9.40 & 0.140 & 22.43 & 21.46 & 20.13 \\
\hline 430 & 4.655 & 456.5 & 20 & 563. & 0.604 & 0.90 & .110 & 22.90 & 21.20 & 20.39 \\
\hline 431 & .194 & 565.0 & 20 & 183. & 0.226 & 3.70 & 0.440 & 21.12 & 20.31 & 20.10 \\
\hline 435 & 51.769 & 514.580 & 20 & 357. & 0.384 & 1.80 & 0.210 & 21.23 & 20.63 & 20.23 \\
\hline 438 & 624.755 & 854.3 & 11 & 186. & 0.264 & 1.40 & 0.750 & 21.27 & 99.99 & 20.06 \\
\hline 440 & .923 & 388.467 & 20 & 408. & 355 & 3.40 & 0.400 & 21.34 & 20.72 & 19.94 \\
\hline 441 & & 108. & 20 & 175. & 243 & 3.40 & 0.250 & 21.07 & 20.50 & 20.08 \\
\hline 444 & 21.751 & 657.1 & 20 & 479. & 0.574 & 4.20 & 0.160 & 22.74 & 20.96 & 20.05 \\
\hline 449 & 1.643 & 462.9 & 20 & 413. & 0.572 & & 140 & 22.30 & & 20.42 \\
\hline 451 & 6.261 & 525. & 20 & 409. & 0.297 & .30 & 0.350 & 21.67 & 20.70 & 20.08 \\
\hline 452 & & & 20 & 349. & & & 190 & 21.16 & 20.18 & 20.17 \\
\hline 456 & & 557. & 20 & 450. & 0.278 & 10 & .110 & 21.21 & 20.39 & 20.05 \\
\hline 459 & .563 & 541. & 20 & 390. & & 9.80 & 0.470 & 21.50 & 20.76 & 19.91 \\
\hline 462 & .738 & 56.930 & 11 & 371. & 0.3 & 3.90 & 0.910 & 21.46 & 99.99 & 20.21 \\
\hline 463 & & & 20 & 39 & & & & 21.56 & 20.82 & 20.08 \\
\hline 464 & & & 20 & 432. & 177 & 6.20 & 0.300 & 21.83 & 20.70 & 20.01 \\
\hline 465 & .571 & 247 & 20 & 301. & & & 0.500 & 21.41 & 20.61 & 20.15 \\
\hline 466 & 2.225 & 35 & 20 & 418. & 463 & & 0.120 & 21.58 & & 20.09 \\
\hline 468 & & & 20 & 277. & & & & 21.79 & 21.03 & 20.14 \\
\hline 469 & & & 20 & 408. & & 4 & & 22.07 & 20.65 & 20.28 \\
\hline 472 & 803 & 504. & 20 & 443. & 0.214 & 22.80 & 0.170 & 21.11 & 20.41 & 20.16 \\
\hline 474 & & 724. & 20 & 189. & & & & 21.28 & & 20.13 \\
\hline 476 & 36 & 69 & 20 & 650. & & & & 23.09 & 21.84 & 20.14 \\
\hline 477 & & & 20 & 426. & 331 & 1 & 0.090 & 21.16 & 20.49 & 20.08 \\
\hline 479 & .278 & 33 & 20 & 319. & 0.283 & 1.40 & 0.070 & 21.56 & 20.84 & 20.08 \\
\hline 483 & & & 20 & 329. & & & & 21.21 & & 20.12 \\
\hline 485 & & & 20 & 447. & & & & 21.31 & & 20.17 \\
\hline 494 & 38 & 65 & 20 & 403. & & & & 21.09 & 20.42 & 20.23 \\
\hline 497 & & & 20 & 403. & .312 & & 0.490 & 21.04 & 20.67 & 19.96 \\
\hline 503 & & 286.7 & 20 & 432. & & 4.20 & 0.140 & 21.79 & 20.87 & 20.17 \\
\hline 504 & 2 & & 20 & 369. & & & & 21.25 & & 20.22 \\
\hline 509 & & & 20 & 37 & & & & 21.32 & 20.60 & 20.12 \\
\hline 519 & .972 & & 20 & 397. & 0.411 & & 0.130 & 21.65 & 20.76 & 20.21 \\
\hline 522 & & 53 & 20 & 174. & 0.237 & .00 & 0.260 & 21.20 & 20.34 & 20.19 \\
\hline 524 & & 617.082 & 20 & 442. & & 7.20 & & 21.33 & 20.72 & 20.10 \\
\hline 527 & 378.870 & & 20 & 189. & & 3.20 & 0.650 & 21.43 & 20.55 & 20.15 \\
\hline & & & 20 & 324. & & & & 21.19 & 20.23 & 20.20 \\
\hline & 3.447 & 536.537 & 20 & 248. & 0.258 & 6.30 & 0.620 & 22.03 & 20.92 & 20.17 \\
\hline 533 & & & 20 & 376. & & 11.10 & & 21.54 & 20.75 & 20.22 \\
\hline 536 & 6.016 & 793.034 & 20 & 177. & & 4.30 & 0.280 & 21.18 & 20.62 & 20.17 \\
\hline & & 709. & 20 & 446. & & 2.7 & 0.090 & 21.35 & 20.35 & 20.28 \\
\hline & & 426.247 & 20 & 486. & & 00 & 0.980 & 21.61 & 20.55 & 20.17 \\
\hline 549 & 748.629 & 626.308 & 20 & 333. & 0.193 & 2.20 & 0.640 & 21.45 & 20.56 & 20.10 \\
\hline & 424.360 & 615.109 & 20 & 404. & 0.447 & 6.90 & 0.220 & 21.27 & 20.65 & 20.22 \\
\hline & & 39 & 20 & 428. & & 1. & 0.280 & 21.56 & 20.84 & 19.96 \\
\hline & & & 20 & 458. & & 2.0 & & 21.80 & 20.78 & 20.26 \\
\hline 560 & 175.575 & 324.498 & 20 & 368. & 0.372 & 3.80 & 0.350 & 21.74 & 21.12 & 20.04 \\
\hline 561 & 159.943 & 560.438 & 20 & 384. & 0.385 & 3.50 & 0.120 & 21.38 & 20.94 & 20.14 \\
\hline
\end{tabular}


Table 3. continuation.

\begin{tabular}{|c|c|c|c|c|c|c|c|c|c|c|}
\hline F1 ID & $\mathrm{x}$ & $\mathrm{y}$ & $\mathrm{N}$ & $\mathrm{P}$ & $\mathrm{A}$ & $\chi^{2}$ & signif & $\mathrm{J}$ & $\mathrm{H}$ & $<K>$ \\
\hline 564 & 70.924 & 715.081 & 20 & 417. & 0.582 & 2.20 & 0.050 & 21.23 & 20.51 & 20.40 \\
\hline 566 & 143.276 & 386.319 & 20 & 275. & 0.165 & 4.50 & 0.930 & 21.29 & 20.54 & 20.18 \\
\hline 567 & 796.243 & 303.054 & 20 & 393. & 0.396 & 1.70 & 0.080 & 21.29 & 20.70 & 20.23 \\
\hline 571 & 204.220 & 129.730 & 20 & 357. & 0.259 & 2.70 & 0.500 & 21.62 & 20.74 & 20.04 \\
\hline 572 & 831.839 & 266.819 & 19 & 439. & 0.587 & 5.90 & 0.130 & 21.70 & 20.71 & 20.49 \\
\hline 573 & 438.028 & 228.245 & 20 & 345. & 0.497 & 1.70 & 0.060 & 21.41 & 21.08 & 20.38 \\
\hline 575 & 214.401 & 198.591 & 20 & 362. & 0.393 & 6.20 & 0.420 & 21.22 & 20.74 & 20.34 \\
\hline 579 & 83.866 & 395.534 & 20 & 170. & 0.237 & 10.40 & 0.980 & 21.32 & 20.64 & 20.24 \\
\hline 581 & 822.800 & 526.661 & 19 & 215. & 0.293 & 8.20 & 0.590 & 21.80 & 21.04 & 20.29 \\
\hline 583 & 656.805 & 627.704 & 20 & 413. & 0.427 & 4.40 & 0.110 & 21.44 & 20.73 & 20.14 \\
\hline 584 & 459.402 & 172.627 & 20 & 268. & 0.246 & 6.20 & 0.580 & 21.56 & 20.71 & 20.19 \\
\hline 585 & 70.548 & 307.259 & 20 & 316. & 0.264 & 1.10 & 0.400 & 21.62 & 20.46 & 20.09 \\
\hline 587 & 90.366 & 126.397 & 20 & 356. & 0.345 & 2.10 & 0.170 & 21.45 & 20.43 & 20.12 \\
\hline 588 & 69.022 & 385.081 & 20 & 451. & 0.217 & 8.50 & 0.740 & 21.71 & 20.81 & 20.17 \\
\hline 590 & 147.305 & 746.683 & 20 & 322. & 0.238 & 1.90 & 0.150 & 21.65 & 20.69 & 20.23 \\
\hline 600 & 694.275 & 800.618 & 20 & 344. & 0.293 & 4.90 & 0.340 & 21.32 & 20.71 & 20.29 \\
\hline 603 & 122.595 & 494.816 & 20 & 371. & 0.228 & 1.50 & 0.140 & 21.71 & 20.86 & 20.21 \\
\hline 608 & 227.197 & 81.820 & 20 & 822. & 0.288 & 4.00 & 0.190 & 21.51 & 20.56 & 20.27 \\
\hline 610 & 875.921 & 243.440 & 18 & 431. & 0.141 & 4.60 & 0.980 & 21.54 & 20.77 & 20.23 \\
\hline 621 & 570.552 & 27.635 & 20 & 199. & 0.192 & 7.00 & 1.000 & 21.56 & 20.74 & 20.29 \\
\hline 623 & 445.251 & 779.298 & 20 & 447. & 0.035 & 31.50 & 0.240 & 21.74 & 21.00 & 20.21 \\
\hline 624 & 63.818 & 31.241 & 19 & 555. & 0.196 & 7.50 & 1.000 & 21.85 & 20.73 & 20.26 \\
\hline 628 & 372.952 & 316.404 & 20 & 380. & 0.278 & 5.80 & 0.970 & 21.80 & 21.32 & 20.12 \\
\hline 632 & 687.298 & 541.673 & 20 & 290. & 0.217 & 5.10 & 0.610 & 21.97 & 20.98 & 20.26 \\
\hline 633 & 765.892 & 33.885 & 20 & 448. & 0.086 & 28.60 & 0.210 & 22.47 & 21.42 & 20.25 \\
\hline 641 & 198.166 & 560.710 & 20 & 182. & 0.179 & 5.00 & 0.760 & 21.24 & 20.54 & 20.25 \\
\hline 643 & 856.354 & 845.953 & 14 & 355. & 0.358 & 3.00 & 0.830 & 21.56 & 99.99 & 20.34 \\
\hline 648 & 323.482 & 229.953 & 20 & 326. & 0.490 & 0.90 & 0.050 & 21.33 & 20.47 & 20.48 \\
\hline 652 & 522.784 & 730.552 & 20 & 540. & 0.145 & 3.80 & 1.000 & 21.68 & 20.91 & 20.26 \\
\hline 657 & 708.216 & 431.793 & 20 & 486. & 0.558 & 6.40 & 0.110 & 22.19 & 21.49 & 20.52 \\
\hline 661 & 572.238 & 20.186 & 20 & 349. & 0.378 & 5.00 & 0.840 & 21.42 & 20.85 & 20.47 \\
\hline 663 & 49.071 & 207.327 & 20 & 306. & 0.307 & 4.80 & 0.560 & 21.49 & 20.42 & 20.22 \\
\hline 668 & 761.629 & 384.585 & 20 & 177. & 0.228 & 7.60 & 0.880 & 22.07 & 20.95 & 20.27 \\
\hline 669 & 819.291 & 784.309 & 19 & 359. & 0.298 & 3.70 & 0.660 & 21.67 & 21.01 & 20.20 \\
\hline 670 & 437.063 & 810.658 & 20 & 173. & 0.193 & 4.60 & 0.730 & 21.61 & 20.78 & 20.26 \\
\hline 682 & 361.295 & 534.566 & 20 & 424. & 0.481 & 6.80 & 0.170 & 21.39 & 20.82 & 20.28 \\
\hline 683 & 682.074 & 17.049 & 20 & 411. & 0.475 & 2.50 & 0.080 & 22.41 & 21.52 & 20.35 \\
\hline 685 & 502.782 & 296.070 & 20 & 354. & 0.382 & 3.20 & 0.250 & 21.72 & 20.73 & 20.23 \\
\hline 688 & 215.140 & 349.738 & 20 & 279. & 0.355 & 1.80 & 0.060 & 21.72 & 21.00 & 20.40 \\
\hline 702 & 680.365 & 528.461 & 20 & 379. & 0.483 & 2.00 & 0.060 & 21.56 & 20.87 & 20.43 \\
\hline 703 & 801.073 & 764.353 & 20 & 391. & 0.351 & 3.70 & 0.910 & 21.89 & 21.14 & 20.26 \\
\hline 706 & 37.628 & 472.303 & 20 & 344. & 0.227 & 3.10 & 0.630 & 21.65 & 20.82 & 20.41 \\
\hline 707 & 511.431 & 752.320 & 20 & 374. & 0.458 & 2.50 & 0.360 & 21.52 & 20.85 & 20.51 \\
\hline 708 & 88.775 & 79.997 & 20 & 302. & 0.383 & 7.60 & 0.380 & 21.21 & 20.38 & 20.44 \\
\hline 733 & 705.344 & 239.892 & 20 & 315. & 0.513 & 2.10 & 0.090 & 21.92 & 21.54 & 20.61 \\
\hline 734 & 157.074 & 330.983 & 20 & 363. & 0.361 & 3.70 & 0.170 & 21.82 & 21.21 & 20.31 \\
\hline 735 & 139.861 & 121.752 & 20 & 311. & 0.351 & 2.40 & 0.360 & 21.32 & 20.52 & 20.53 \\
\hline 737 & 508.883 & 516.055 & 20 & 371. & 0.440 & 2.00 & 0.100 & 21.60 & 20.89 & 20.40 \\
\hline 741 & 128.502 & 494.060 & 20 & 418. & 0.423 & 4.50 & 0.200 & 21.49 & 20.89 & 20.36 \\
\hline 744 & 904.262 & 474.976 & 14 & 285. & 0.325 & 2.80 & 0.920 & 21.82 & 21.00 & 20.33 \\
\hline 745 & 338.569 & 126.905 & 20 & 299. & 0.260 & 4.00 & 0.510 & 21.40 & 20.58 & 20.44 \\
\hline 746 & 225.747 & -14.905 & 17 & 170. & 0.344 & 6.90 & 0.960 & 21.60 & 20.64 & 20.39 \\
\hline 751 & 105.709 & 309.710 & 20 & 298. & 0.370 & 4.60 & 0.220 & 21.54 & 20.90 & 20.52 \\
\hline 753 & 628.524 & 761.190 & 20 & 304. & 0.298 & 5.00 & 0.530 & 21.45 & 20.79 & 20.55 \\
\hline 757 & 193.930 & 833.109 & 16 & 454. & 0.571 & 23.20 & 0.760 & 21.28 & 20.21 & 20.79 \\
\hline 775 & 188.193 & 504.862 & 20 & 432. & 0.163 & 14.40 & 0.530 & 22.24 & 21.56 & 20.34 \\
\hline 787 & 31.338 & 245.559 & 20 & 436. & 0.594 & 12.40 & 0.480 & 22.36 & 21.26 & 20.62 \\
\hline 790 & 126.739 & 688.708 & 18 & 465. & 0.800 & 14.60 & 0.520 & 22.51 & 23.09 & 20.42 \\
\hline 793 & 205.793 & 127.571 & 20 & 455. & 0.077 & 50.30 & 0.090 & 22.55 & 21.42 & 20.56 \\
\hline 797 & 706.228 & 562.504 & 20 & 277. & 0.350 & 2.20 & 0.110 & 21.76 & 21.21 & 20.51 \\
\hline
\end{tabular}


M. Rejkuba et al.: LPV catalogue in Cen A, Online Material $p 9$

Table 3. continuation.

\begin{tabular}{|c|c|c|c|c|c|c|c|c|c|c|}
\hline F1 ID & $\mathrm{x}$ & $\mathrm{y}$ & $\overline{\mathrm{N}}$ & $\mathrm{P}$ & $\overline{\mathrm{A}}$ & $\overline{\chi^{2}}$ & signif & $\bar{J}$ & $\overline{\mathrm{H}}$ & $<K>$ \\
\hline$\overline{7799}$ & $\overline{398.332}$ & $\overline{38.844}$ & 20 & 626. & 0.238 & $\overline{7.80}$ & $\overline{1.000}$ & 21.45 & 20.62 & $\overline{20.45}$ \\
\hline 802 & 73.116 & 77.463 & 20 & 305. & 203 & 5.50 & 0.930 & 21.47 & 20.77 & 20.36 \\
\hline 809 & 04.597 & 582.775 & 20 & 328. & .257 & 2.90 & 0.400 & 21.65 & 20.59 & 20.35 \\
\hline 813 & 15.885 & 336.602 & 20 & 308. & 0.455 & 1.30 & 0.120 & 21.35 & 20.62 & 20.63 \\
\hline 816 & 9.942 & 37.022 & 20 & 172. & .220 & 5.60 & 0.650 & 22.16 & 21.26 & 20.44 \\
\hline 818 & 0.903 & 306.009 & 19 & 176. & 0.218 & 6.40 & 1.000 & 21.85 & 20.82 & 20.43 \\
\hline 821 & 1.008 & 814.429 & 19 & 311. & 0.388 & 14.10 & 0.970 & 21.51 & 20.49 & 20.65 \\
\hline 828 & 81.336 & 780.161 & 18 & 287. & 0.228 & 6.80 & 1.000 & 22.64 & 21.82 & 20.35 \\
\hline 831 & 66.563 & 225.020 & 20 & 290. & 0.409 & 2.30 & 0.130 & 21.37 & 20.92 & 20.57 \\
\hline 838 & 46.087 & 213.945 & 20 & 303. & 0.383 & 4.50 & 0.210 & 21.36 & 20.72 & 20.60 \\
\hline 839 & 63.239 & 20.287 & 20 & 311. & 0.375 & 3.30 & 0.100 & 21.46 & 20.50 & 20.55 \\
\hline 846 & 36.441 & 380.184 & 20 & 474. & 0.270 & 1.40 & 0.130 & 23.79 & 22.05 & 20.49 \\
\hline 847 & 563.904 & 47.281 & 20 & 591. & 0.225 & 3.60 & 0.420 & 21.79 & 21.06 & 20.46 \\
\hline 854 & 7.899 & 800.704 & 18 & 237. & 0.391 & 20.10 & 1.000 & 21.51 & 21.55 & 20.44 \\
\hline 870 & 38.892 & 828.252 & 18 & 285. & 326 & .50 & 1.000 & 20.82 & 20.54 & 20.55 \\
\hline 880 & 0.677 & -0.487 & 18 & 491. & 0.586 & 14.10 & 0.990 & 22.97 & 21.50 & 20.73 \\
\hline 890 & 809.620 & 316.646 & 19 & 282. & 0.306 & 5.20 & 0.810 & 21.83 & 21.02 & 20.55 \\
\hline 892 & 30.380 & 244.955 & 16 & 370. & 0.727 & 21.30 & 0.680 & 22.53 & 21.35 & 20.99 \\
\hline 902 & & 02 & 20 & 345. & $\$ 12$ & 3.30 & 170 & 22.01 & 21.47 & 20.38 \\
\hline 903 & 86 & 370.159 & 20 & 173. & 0.228 & 8.80 & 0.950 & 22.02 & 21.13 & 20.45 \\
\hline 904 & 4.146 & 292.289 & 20 & 286. & 0.272 & 6.20 & 0.810 & 21.88 & 21.10 & 20.59 \\
\hline 908 & 8.452 & 540.397 & 20 & 445. & 0.299 & 5.60 & 0.270 & 21.47 & 20.56 & 20.55 \\
\hline 910 & 26 & 21 & 19 & 366. & & 0.90 & & 21.79 & 21.14 & 20.40 \\
\hline 911 & & & 20 & 115. & 269 & 6.70 & 0.910 & 21.77 & 21.22 & 20.50 \\
\hline 924 & 25.735 & 704.143 & 20 & 345. & 0.408 & 3.00 & 0.090 & 21.78 & 21.23 & 20.53 \\
\hline 928 & 0.283 & 437.0 & 20 & 319. & 0.311 & 6.20 & 0.640 & 21.65 & 20.65 & 20.39 \\
\hline 930 & 99 & 9.322 & 18 & 433. & 0.391 & 14.60 & 0.890 & 22.09 & 21.36 & 20.43 \\
\hline 32 & & & 20 & 293. & & & 0.250 & 22.17 & 21.38 & 20.62 \\
\hline 942 & & 65 & 20 & 449. & 0.340 & 9.20 & 0.270 & 22.01 & 21.28 & 20.58 \\
\hline 945 & 63 & 11.692 & 20 & 461. & & 7.20 & 0.380 & 23.35 & 21.72 & 20.60 \\
\hline 966 & & 05 & 19 & 291. & & 5.00 & 210 & 22.20 & 21.57 & 20.67 \\
\hline 972 & & & 20 & 321. & 224 & 3.70 & 750 & 21.87 & 21.17 & 20.43 \\
\hline 983 & & 52 & 20 & 270. & 0.349 & 2.70 & 0.370 & 21.38 & 20.65 & 20.63 \\
\hline 985 & 99 & 2.349 & 20 & 306. & & 8.50 & 0.890 & 21.67 & 20.89 & 20.45 \\
\hline 987 & & 4.607 & 20 & 261. & & 4.20 & & 21.65 & & 20.56 \\
\hline & & 90 & 14 & 261. & & 3.70 & & 22.44 & 21.50 & 20.66 \\
\hline 95 & & & 20 & 331. & & 5.90 & 0.730 & 21.87 & 21.13 & 20.38 \\
\hline 996 & 1.072 & 2.105 & 20 & 299. & & 4.60 & 0.220 & 21.76 & 20.70 & 20.44 \\
\hline 1001 & & 759.7 & 20 & 184. & & & & 22.24 & 1.44 & 20.50 \\
\hline & & & 20 & 287. & & 4.50 & & 21.72 & 21.26 & 20.68 \\
\hline 1017 & & & 18 & 241. & 346 & 10.00 & 0.920 & 22.05 & 21.38 & 20.57 \\
\hline 1019 & & 64.701 & 18 & 294. & & 9.10 & 0.790 & 21.60 & 20.76 & 20.81 \\
\hline & & & 20 & 297. & & & & 21.51 & 20.61 & 20.58 \\
\hline & & & 19 & 274. & & & & 21.92 & 21.19 & 20.58 \\
\hline & 72 & & 20 & 28 & & & & 21.79 & 21.26 & 20.72 \\
\hline & & & 20 & 465. & & 4.90 & & 22.90 & 21.60 & 20.82 \\
\hline & & & 20 & & & & & 21.66 & & 20.65 \\
\hline & 30 & & 20 & 283. & & 3.60 & & 22.08 & 21.20 & 20.57 \\
\hline & & & 20 & 346. & & 4.90 & 0.310 & 22.29 & 21.45 & 20.55 \\
\hline 1071 & & 15.068 & 20 & 303. & 0.452 & 3.20 & 0.180 & 21.77 & 20.98 & 20.46 \\
\hline 1082 & & & 20 & 696. & & 0.80 & 0.210 & 21.93 & 20.95 & 20.66 \\
\hline & 175.552 & 62.948 & 20 & 321. & & 2.80 & 0.320 & 22.15 & 21.26 & 20.46 \\
\hline & & & 20 & 272. & & 1.30 & 0.140 & 22.09 & 21.09 & 20.64 \\
\hline & & & 20 & 160. & & 3.60 & 0.320 & 21.95 & 21.00 & 20.65 \\
\hline 1097 & 331.354 & 394.755 & 20 & 277. & 0.299 & 4.30 & 0.220 & 21.66 & 20.62 & 20.59 \\
\hline 1112 & & & 20 & 282. & & 4.20 & & 21.74 & 21.15 & 20.75 \\
\hline & 347.32 & 344.2 & 20 & 182. & & 4.90 & 0.860 & 22.07 & 21.30 & 20.56 \\
\hline & & & 20 & 504. & & 6.40 & 0.470 & 23.68 & 21.81 & 20.94 \\
\hline & & 376.012 & 20 & 307. & & 1.80 & 0.380 & 21.78 & 20.75 & 20.55 \\
\hline 1133 & 579.644 & 774.993 & 20 & 276. & 0.187 & 3.40 & 0.640 & 21.77 & 20.96 & 20.60 \\
\hline
\end{tabular}


M. Rejkuba et al.: LPV catalogue in Cen A, Online Material p 10

Table 3. continuation.

\begin{tabular}{|c|c|c|c|c|c|c|c|c|c|c|}
\hline$\overline{F 1}$ ID & $\mathrm{x}$ & $\mathrm{y}$ & $\mathrm{N}$ & $\overline{\mathrm{P}}$ & $\overline{\mathrm{A}}$ & $\chi^{2}$ & signif & $\bar{J}$ & $\overline{\mathrm{H}}$ & $<K>$ \\
\hline 1143 & 754.050 & 6599.413 & $\overline{20}$ & 238. & 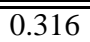 & 3.60 & 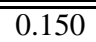 & 222.07 & 21.91 & 20.72 \\
\hline 1151 & 393.950 & 764.899 & 20 & 313. & 0.418 & 5.00 & 0.700 & 21.84 & 20.76 & 20.43 \\
\hline 1159 & 409.233 & 469.687 & 20 & 176. & 0.221 & 6.60 & 0.880 & 21.98 & 21.20 & 20.59 \\
\hline 1167 & 176.566 & 702.620 & 20 & 309. & 0.338 & 3.00 & 0.380 & 21.86 & 20.98 & 20.54 \\
\hline 1177 & 854.540 & 771.523 & 18 & 286. & 0.509 & 8.70 & 0.770 & 21.72 & 21.34 & 20.94 \\
\hline 1178 & 87.904 & 336.986 & 20 & 331. & 0.373 & 1.70 & 0.110 & 22.01 & 21.45 & 20.53 \\
\hline 1195 & 723.969 & 300.127 & 20 & 352. & 0.525 & 2.70 & 0.120 & 22.23 & 21.61 & 20.51 \\
\hline 1198 & 793.095 & 281.772 & 20 & 306. & 0.426 & 5.00 & 0.220 & 22.43 & 21.55 & 20.65 \\
\hline 1199 & 808.362 & 642.118 & 19 & 579. & 0.658 & 1.70 & 0.320 & 24.94 & 22.75 & 21.12 \\
\hline 1202 & 130.634 & 718.495 & 20 & 628. & 0.265 & 4.20 & 0.440 & 21.87 & 20.89 & 20.63 \\
\hline 1207 & 252.832 & 598.495 & 20 & 495. & 0.578 & 3.70 & 0.400 & 24.73 & 22.32 & 20.87 \\
\hline 1216 & 694.102 & 639.796 & 20 & 254. & 0.216 & 0.80 & 0.120 & 21.74 & 20.85 & 20.69 \\
\hline 1222 & 283.927 & 656.067 & 20 & 253. & 0.214 & 1.70 & 0.580 & 21.50 & 20.72 & 20.71 \\
\hline 1227 & 123.256 & 499.771 & 20 & 210. & 0.302 & 9.90 & 0.830 & 21.74 & 21.05 & 20.76 \\
\hline 1232 & 62.177 & 65.747 & 20 & 278. & 0.355 & 6.60 & 0.720 & 21.71 & 20.83 & 20.72 \\
\hline 1241 & 252.580 & 54.482 & 20 & 294. & 0.455 & 5.00 & 0.190 & 21.51 & 20.62 & 20.79 \\
\hline 1242 & 593.086 & 698.145 & 20 & 275. & 0.249 & 11.50 & 0.960 & 22.03 & 21.43 & 20.70 \\
\hline 1254 & 323.129 & 308.024 & 20 & 321. & 0.381 & 3.30 & 0.870 & 21.94 & 20.96 & 20.59 \\
\hline 1291 & 455.682 & 392.499 & 20 & 279. & 0.309 & 4.80 & 0.980 & 22.08 & 20.96 & 20.73 \\
\hline 1298 & 390.213 & -6.731 & 12 & 34. & 0.860 & 5.10 & 0.990 & 21.67 & 22.11 & 20.91 \\
\hline 1304 & 380.244 & 314.110 & 17 & 61. & 0.478 & 8.70 & 1.000 & 22.00 & 22.17 & 20.70 \\
\hline 1327 & 320.303 & 693.920 & 20 & 208. & 0.202 & 2.80 & 0.430 & 21.97 & 21.20 & 20.76 \\
\hline 1333 & 705.585 & 752.688 & 20 & 294. & 0.431 & 3.70 & 0.120 & 21.77 & 20.87 & 20.82 \\
\hline 1356 & 275.836 & 724.749 & 20 & 170. & 0.265 & 9.30 & 0.820 & 22.00 & 21.10 & 20.72 \\
\hline 1361 & 62.584 & 30.180 & 13 & 39. & 0.545 & 35.00 & 1.000 & 22.00 & 21.32 & 21.05 \\
\hline 1370 & 119.517 & 697.211 & 16 & 38. & 0.834 & 2.00 & 0.700 & 99.99 & 21.42 & 21.06 \\
\hline 1381 & 412.053 & 131.619 & 20 & 264. & 0.269 & 7.50 & 0.990 & 22.51 & 21.58 & 20.76 \\
\hline 1390 & 252.473 & 92.608 & 20 & 251. & 0.243 & 3.40 & 0.790 & 21.93 & 20.95 & 20.81 \\
\hline 1394 & 324.783 & 739.677 & 20 & 248. & 0.273 & 2.00 & 0.120 & 21.98 & 21.38 & 20.76 \\
\hline 1396 & 305.918 & 295.709 & 20 & 778. & 0.461 & 5.90 & 0.980 & 22.24 & 21.24 & 20.85 \\
\hline 1416 & 719.443 & 827.022 & 19 & 250. & 0.361 & 3.20 & 0.380 & 21.64 & 20.93 & 20.86 \\
\hline 1445 & 53.525 & 768.300 & 18 & 256. & 0.268 & 6.80 & 0.990 & 22.10 & 20.92 & 20.89 \\
\hline 1450 & 331.171 & 253.491 & 20 & 536. & 0.606 & 3.20 & 0.120 & 23.85 & 22.03 & 21.00 \\
\hline 1485 & 65.611 & 460.413 & 20 & 214. & 0.251 & 3.90 & 0.760 & 21.74 & 21.38 & 20.87 \\
\hline 1495 & 839.865 & 829.356 & 16 & 291. & 0.474 & 18.00 & 0.960 & 99.99 & 22.34 & 20.98 \\
\hline 1513 & 281.031 & 309.679 & 20 & 196. & 0.430 & 1.50 & 0.130 & 21.90 & 21.40 & 20.93 \\
\hline 1533 & 412.255 & -22.898 & 11 & 69. & 1.286 & 18.00 & 0.930 & 22.55 & 21.48 & 21.32 \\
\hline 1539 & 89.590 & 146.109 & 20 & 204. & 0.289 & 3.50 & 0.720 & 22.15 & 21.74 & 20.99 \\
\hline 1554 & 164.071 & 564.966 & 20 & 203. & 0.488 & 2.70 & 0.430 & 21.58 & 20.89 & 21.02 \\
\hline 1564 & 300.273 & 611.715 & 20 & 267. & 0.315 & 3.40 & 0.420 & 21.67 & 20.91 & 20.94 \\
\hline 1629 & 768.512 & 220.448 & 20 & 216. & 0.277 & 3.30 & 0.220 & 22.37 & 21.55 & 21.05 \\
\hline 1649 & 777.550 & 813.482 & 20 & 232. & 0.454 & 2.40 & 0.290 & 22.10 & 21.49 & 21.13 \\
\hline 1670 & 534.016 & 605.585 & 20 & 239. & 0.270 & 3.50 & 0.420 & 22.29 & 21.62 & 20.95 \\
\hline 1671 & 74.539 & 825.258 & 20 & 226. & 0.365 & 5.00 & 0.500 & 21.98 & 21.65 & 21.12 \\
\hline 1682 & 206.538 & 46.923 & 20 & 206. & 0.267 & 3.90 & 0.920 & 22.29 & 21.25 & 20.97 \\
\hline 1735 & 511.708 & -8.212 & 18 & 202. & 0.260 & 2.00 & 0.770 & 22.12 & 21.43 & 21.05 \\
\hline 1775 & 763.695 & 286.694 & 20 & 217. & 0.267 & 5.00 & 0.950 & 21.98 & 21.53 & 21.11 \\
\hline 1806 & 65.875 & 646.566 & 20 & 231. & 0.395 & 7.20 & 0.510 & 21.92 & 21.24 & 21.22 \\
\hline 1810 & 606.950 & -17.238 & 16 & 304. & 0.506 & 2.60 & 0.360 & 22.42 & 21.51 & 21.01 \\
\hline 1832 & 105.314 & 515.071 & 20 & 197. & 0.414 & 4.40 & 0.800 & 21.75 & 20.94 & 21.05 \\
\hline 1840 & 263.550 & 751.289 & 20 & 230. & 0.301 & 3.80 & 0.460 & 22.09 & 21.68 & 21.16 \\
\hline 1851 & 227.825 & 255.322 & 20 & 271. & 0.303 & 6.60 & 0.880 & 22.40 & 21.84 & 21.17 \\
\hline 1867 & 878.280 & 140.257 & 18 & 250. & 0.246 & 16.30 & 0.940 & 22.55 & 22.14 & 21.03 \\
\hline 1888 & 151.663 & 580.097 & 20 & 445. & 0.154 & 16.70 & 0.170 & 24.35 & 22.80 & 21.22 \\
\hline 1893 & 447.366 & 406.122 & 20 & 35. & 0.371 & 8.00 & 1.000 & 22.84 & 21.43 & 21.14 \\
\hline 1897 & 357.604 & 318.509 & 17 & 490. & 0.471 & 13.40 & 0.950 & 22.18 & 21.24 & 20.97 \\
\hline 1903 & 251.772 & 690.018 & 20 & 226. & 0.530 & 12.10 & 0.860 & 22.05 & 21.87 & 21.43 \\
\hline 1911 & 865.938 & 292.732 & 19 & 179. & 0.315 & 5.10 & 0.690 & 22.38 & 21.50 & 21.08 \\
\hline 1943 & 652.101 & 471.075 & 20 & 206. & 0.366 & 2.10 & 0.310 & 21.72 & 21.20 & 21.06 \\
\hline 2014 & 862.100 & 33.721 & 19 & 468. & 0.496 & 2.80 & 0.300 & 23.10 & 21.63 & 21.21 \\
\hline
\end{tabular}


Table 3. continuation.

\begin{tabular}{|c|c|c|c|c|c|c|c|c|c|c|}
\hline F1 ID & $\mathrm{x}$ & $\mathrm{y}$ & $\overline{\mathrm{N}}$ & $\mathrm{P}$ & $\mathrm{A}$ & $\chi^{2}$ & signif & $\bar{J}$ & $\overline{\mathrm{H}}$ & $<K>$ \\
\hline 2015 & 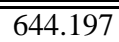 & 327.874 & $\overline{20}$ & 191. & $\overline{0.320}$ & 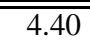 & 0.550 & 21.84 & 21.08 & 21.21 \\
\hline 2050 & & 67.989 & 15 & 418. & 0.821 & 26.00 & & 3.15 & 9.99 & 21.48 \\
\hline 2093 & 19.435 & 768.779 & 20 & 248. & 0.359 & 2.60 & 0.410 & 22.09 & 21.33 & 21.26 \\
\hline 2104 & 28.300 & 354.414 & 19 & 216. & 0.376 & 2.90 & 0.680 & 24.72 & 23.11 & 21.35 \\
\hline 2108 & 22.411 & 3.752 & 20 & 180. & 0.053 & 12.90 & .380 & 22.70 & 2.00 & 1.25 \\
\hline 2144 & 63.752 & 3.934 & 16 & 34. & 0.323 & 9.30 & 0.970 & 22.77 & 9.99 & 21.59 \\
\hline 2223 & 50.724 & 516.453 & 20 & 501. & 0.823 & 3.10 & 0.070 & 26.89 & 23.28 & 21.57 \\
\hline 2266 & 24.743 & 541.049 & 20 & 303. & 0.311 & .50 & 0.760 & 22.09 & 21.40 & 21.34 \\
\hline 2297 & 4.922 & 85.100 & 19 & 36. & 0.468 & 8.90 & 1.000 & 22.86 & 21.89 & 21.27 \\
\hline 2306 & 40.396 & 703.310 & 12 & 123. & 904 & 6.70 & .980 & 99.99 & 21.71 & 21.70 \\
\hline 2364 & 21.966 & 828.285 & 19 & 244. & 0.517 & 8.40 & 1.000 & 21.86 & 21.51 & 21.26 \\
\hline 2394 & 14.065 & 834.808 & 12 & 37. & 0.962 & 2.50 & 0.750 & 21.88 & 22.81 & 21.56 \\
\hline 2461 & 855.807 & 772.237 & 17 & 193. & 0.516 & 12.60 & 1.000 & 24.04 & 22.38 & 21.54 \\
\hline 2860 & 6.907 & 513.450 & 20 & 172. & & 3.20 & 640 & 22.30 & 21.85 & 1.57 \\
\hline 2872 & & 524.680 & 20 & 125. & 0.245 & 7.90 & .000 & 22.91 & 21.52 & 1.45 \\
\hline 2896 & 0.140 & 68.674 & 13 & 68. & 0.796 & 8.80 & 990 & 24.17 & 24.25 & 21.57 \\
\hline 2900 & 1.874 & 584.0 & 20 & 456. & & 5.80 & 1.000 & 22.57 & 21.62 & 21.48 \\
\hline 2927 & & 18 & 20 & 458. & 12 & 3.70 & 990 & 22.65 & 21.67 & 21.50 \\
\hline 3153 & & & 16 & 40. & & 10 & 990 & 23.91 & 22.88 & 2.06 \\
\hline 3212 & 2.689 & 680.5 & 17 & 36. & & 6.80 & 1.000 & 99.99 & 22.32 & 21.64 \\
\hline 3229 & & 707.4 & 13 & 62. & & 4.40 & & 99.99 & 22.11 & 21.62 \\
\hline 3239 & & 7 & 16 & 34. & & 8.80 & & 22.93 & 21.44 & 21.65 \\
\hline & & & 19 & 42. & & & & 22.70 & 21.54 & 1.66 \\
\hline 10 & & & 16 & 58. & & 7.00 & & 22.92 & 24.42 & 1.71 \\
\hline & & & 17 & 178. & & 4.50 & & 23.41 & 4.46 & 21.54 \\
\hline 45 & 3 & 0.910 & 12 & 149. & & 38.30 & & 23.60 & 99.99 & 22.07 \\
\hline & & & 18 & 308. & & & & 22.74 & 22.11 & 1.63 \\
\hline 3609 & & & 11 & 66. & & 50 & & 24.71 & 22.83 & 2.01 \\
\hline 4036 & & & 11 & 166. & & & & 24.56 & 4.10 & 21.98 \\
\hline 4052 & 7 & 8.905 & 14 & 144. & & & & 24.46 & & 22.12 \\
\hline 4098 & & & 16 & 89. & & & & 23.27 & 21 & 21.88 \\
\hline 4107 & & & 12 & 34. & & & & 25.37 & 23.45 & 21.84 \\
\hline 4211 & & & 18 & 574. & & 9.7 & 1.000 & 22.04 & 21.71 & 21.73 \\
\hline 4237 & & & 19 & 270. & & & & 23.23 & & 21.90 \\
\hline 4374 & & & 19 & 138. & & & & 22.84 & 91 & 21.75 \\
\hline 4586 & & & 10 & 35. & & & & 23.22 & 21.33 & 21.42 \\
\hline 4712 & & & 10 & 169. & & 3.20 & & 26.58 & 3.18 & 2.49 \\
\hline 4795 & & & 10 & 256. & & & & & & 21.72 \\
\hline 4809 & & & 12 & 66. & & & & & & 22.12 \\
\hline 4856 & & & 19 & 42. & & & & 23. & 22.84 & 21.96 \\
\hline 4895 & & & 15 & 975. & & & & 99.99 & 22.74 & 22.03 \\
\hline 4919 & & & 17 & 252. & & 11.10 & & 22.94 & 22.37 & 21.97 \\
\hline 4973 & & & 12 & 34. & & & & & & 21.99 \\
\hline 4980 & & & 19 & 85. & & & & 23.17 & 22.70 & 22.02 \\
\hline 5123 & & & 18 & 70. & & & & 23.64 & 23.08 & 22.10 \\
\hline 5225 & & 27 & 20 & 116. & & & & 22.50 & 22.27 & 21.96 \\
\hline & & & 18 & 133. & & 13.90 & & & & 21.97 \\
\hline & & 2 & 11 & 268. & & & & 24.30 & 22.83 & 22.59 \\
\hline & & & 17 & 152. & & & & 24.56 & 22.69 & 22.30 \\
\hline & & & 11 & 42. & & & & 24.06 & 23.72 & 22.47 \\
\hline & & & 15 & 207. & & 10.30 & & & 22.33 & 22.11 \\
\hline & & & 14 & 225. & & 7.4 & & 23.41 & 22.43 & 22.10 \\
\hline & & & 18 & 105. & & 9.4 & & 23.84 & 23.30 & 22.35 \\
\hline & & & 16 & 66. & & & & 24.30 & 22.89 & 22.24 \\
\hline & & & 16 & 34. & & & & 23.54 & 22.47 & 22.46 \\
\hline & & & 12 & 86. & & 18.40 & 1.000 & 23.09 & 23.58 & 22.53 \\
\hline & & & 12 & 567. & & & 1.0 & 23.51 & 23.23 & 21.99 \\
\hline & & & 1 & 42. & & & & & 23.18 & 22.24 \\
\hline 7098 & 567.725 & & 16 & 85. & & 0.40 & 0.960 & 23.69 & 23.11 & 22.46 \\
\hline 7165 & 97.672 & 421.263 & 18 & 36. & 0.623 & 5.10 & 1.000 & 25.34 & 22.74 & 22.42 \\
\hline
\end{tabular}


M. Rejkuba et al.: LPV catalogue in Cen A, Online Material p 12

Table 3. continuation.

\begin{tabular}{ccccccrcrrr}
\hline \hline F1 ID & $\mathrm{x}$ & $\mathrm{y}$ & $\mathrm{N}$ & $\mathrm{P}$ & $\mathrm{A}$ & $\chi^{2}$ & signif & $\mathrm{J}$ & $\mathrm{H}$ & $<K>$ \\
\hline \hline 7219 & 394.372 & 341.060 & 16 & 84. & 0.540 & 3.80 & 1.000 & 23.36 & 22.18 & 22.31 \\
7345 & 119.065 & 839.147 & 14 & 38. & 0.530 & 4.10 & 0.970 & 23.87 & 22.61 & 22.33 \\
7416 & 474.764 & 416.396 & 13 & 45. & 1.003 & 3.20 & 0.970 & 24.42 & 99.99 & 22.27 \\
7432 & 709.054 & 98.738 & 15 & 83. & 1.133 & 4.70 & 1.000 & 24.03 & 22.75 & 22.80 \\
7608 & 695.119 & 51.824 & 11 & 71. & 1.197 & 12.50 & 0.990 & 24.20 & 23.57 & 22.60 \\
7630 & 731.075 & 3.369 & 10 & 34. & 0.814 & 4.00 & 0.990 & 24.32 & 23.12 & 22.72 \\
7689 & 532.141 & 813.352 & 18 & 37. & 0.698 & 6.90 & 0.970 & 23.44 & 22.83 & 22.42 \\
7740 & 751.847 & 22.710 & 10 & 114. & 0.806 & 4.60 & 1.000 & 24.03 & 99.99 & 22.57 \\
7753 & 684.468 & 63.865 & 17 & 59. & 0.820 & 5.50 & 0.950 & 23.54 & 21.82 & 22.35 \\
7843 & 690.220 & 56.601 & 11 & 37. & 0.864 & 3.30 & 0.990 & 24.22 & 22.79 & 22.54 \\
8034 & 117.399 & 721.861 & 11 & 45. & 1.529 & 4.90 & 0.980 & 23.88 & 24.19 & 22.47 \\
8208 & 578.446 & 642.597 & 14 & 42. & 0.996 & 3.40 & 1.000 & 24.41 & 22.52 & 22.52 \\
8319 & 28.685 & 532.373 & 18 & 136. & 0.612 & 6.00 & 1.000 & 23.76 & 22.94 & 22.54 \\
8343 & 442.410 & 137.706 & 13 & 45. & 0.779 & 2.80 & 0.970 & 24.13 & 22.84 & 22.53 \\
8575 & 455.378 & 425.579 & 14 & 236. & 0.871 & 5.20 & 0.960 & 23.71 & 22.93 & 22.49 \\
9271 & 482.027 & 808.044 & 13 & 120. & 0.644 & 1.20 & 0.890 & 24.36 & 23.13 & 22.82 \\
10375 & 623.502 & 566.500 & 13 & 138. & 0.684 & 5.30 & 1.000 & 24.01 & 23.70 & 22.91 \\
\hline
\end{tabular}


Table 4. LPVs in Field2. The columns from left to right list: star ID, $x$ and y position with respect to the reference epoch, the best fitting period, semi-amplitude, reduced $\chi^{2}$ of the sine-curve fit, significance of the period from Fourier analysis, single epoch $J$ and $H$-band magnitudes and mean $K$-band magnitude.Magnitude 99.99 denotes that no measurement for that star was obtained.

\begin{tabular}{|c|c|c|c|c|c|c|c|c|c|c|}
\hline F2 ID & $\mathrm{x}$ & $\mathrm{y}$ & $\mathrm{N}$ & $\mathrm{P}$ & $\mathrm{A}$ & $\chi^{2}$ & signif & $\mathrm{J}$ & $\mathrm{H}$ & $<K>$ \\
\hline 35 & 115.155 & -29.046 & $\overline{10}$ & 35. & $\overline{0.978}$ & $\overline{226.70}$ & 0.940 & 18.76 & 18.30 & $\overline{18.48}$ \\
\hline 49 & 41.708 & 172.990 & 10 & 988. & 1.463 & 2.20 & 0.980 & 99.99 & 99.99 & 17.87 \\
\hline 61 & 591.542 & 445.812 & 24 & 696. & 0.180 & 1.70 & 0.300 & 19.67 & 18.66 & 18.41 \\
\hline 75 & 33.955 & 173.506 & 14 & 158. & 1.141 & 20.00 & 1.000 & 21.50 & 18.95 & 18.41 \\
\hline 94 & 143.469 & -15.349 & 10 & 43. & 2.160 & 10.00 & 0.960 & 18.74 & 18.55 & 19.96 \\
\hline 104 & 875.231 & 67.293 & 23 & 622. & 0.381 & 5.70 & 0.290 & 20.90 & 19.60 & 19.06 \\
\hline 105 & 119.195 & -22.547 & 11 & 79. & 1.246 & 15.40 & 0.990 & 99.99 & 99.99 & 18.99 \\
\hline 106 & 34.529 & 166.407 & 20 & 438. & 1.167 & 57.20 & 0.980 & 19.73 & 20.56 & 19.07 \\
\hline 111 & 586.548 & 279.567 & 24 & 486. & 0.367 & 3.20 & 0.140 & 20.52 & 19.62 & 18.94 \\
\hline 112 & 42.165 & 180.308 & 15 & 300 . & 1.128 & 34.10 & 0.970 & 21.18 & 99.99 & 19.25 \\
\hline 118 & 736.158 & 751.081 & 24 & 645. & 0.337 & 5.50 & 0.130 & 21.10 & 19.82 & 19.02 \\
\hline 123 & 255.404 & 276.950 & 12 & 61. & 0.871 & 5.10 & 0.940 & 20.22 & 99.99 & 19.09 \\
\hline 124 & 51.280 & 179.498 & 23 & 313. & 1.191 & 37.30 & 0.910 & 19.95 & 19.74 & 19.35 \\
\hline 137 & 802.342 & 660.341 & 24 & 430. & 0.186 & 1.00 & 0.040 & 20.33 & 19.38 & 19.08 \\
\hline 140 & 500.951 & 593.051 & 24 & 602. & 0.565 & 7.30 & 0.310 & 20.49 & 19.47 & 19.40 \\
\hline 141 & 789.272 & 224.574 & 24 & 569. & 0.249 & 5.20 & 0.100 & 20.45 & 19.65 & 19.12 \\
\hline 143 & 613.780 & 471.722 & 24 & 522. & 0.639 & 2.80 & 0.010 & 21.96 & 20.16 & 19.32 \\
\hline 148 & 850.071 & -34.921 & 12 & 494. & 0.526 & 4.00 & 0.710 & 21.20 & 99.99 & 19.46 \\
\hline 154 & 834.447 & 691.914 & 24 & 465. & 0.205 & 4.40 & 0.180 & 20.89 & 19.94 & 19.20 \\
\hline 156 & 679.925 & 778.012 & 24 & 496. & 0.455 & 2.50 & 0.010 & 20.25 & 19.46 & 19.34 \\
\hline 157 & 257.797 & -11.193 & 15 & 500. & 0.507 & 2.50 & 0.940 & 20.45 & 19.59 & 19.42 \\
\hline 163 & 45.964 & 179.276 & 17 & 42. & 0.938 & 6.90 & 0.820 & 99.99 & 20.86 & 19.20 \\
\hline 164 & 744.544 & 648.632 & 24 & 534. & 0.475 & 3.10 & 0.010 & 21.21 & 20.08 & 19.33 \\
\hline 166 & 538.063 & 121.359 & 24 & 466. & 0.301 & 3.00 & 0.020 & 20.30 & 19.34 & 19.24 \\
\hline 167 & 679.250 & 59.629 & 24 & 490. & 0.350 & 4.90 & 0.120 & 20.48 & 19.69 & 19.28 \\
\hline 169 & 298.943 & 642.226 & 24 & 554. & 0.327 & 9.80 & 0.130 & 21.08 & 20.10 & 19.22 \\
\hline 173 & 66.678 & 695.443 & 24 & 1229. & 0.511 & 10.60 & 0.240 & 20.97 & 20.00 & 19.31 \\
\hline 177 & 526.510 & 8.394 & 24 & 557. & 0.364 & 1.00 & 0.010 & 20.35 & 19.66 & 19.32 \\
\hline 178 & 687.346 & 575.031 & 24 & 689. & 0.846 & 12.90 & 0.110 & 22.28 & 20.89 & 19.22 \\
\hline 182 & 206.565 & 721.171 & 24 & 478. & 0.391 & 6.20 & 0.030 & 21.34 & 20.44 & 19.25 \\
\hline 183 & 118.156 & 302.625 & 24 & 655. & 0.405 & 1.80 & 0.280 & 20.76 & 19.66 & 19.52 \\
\hline 194 & 587.021 & 98.805 & 24 & 522. & 0.467 & 9.60 & 0.040 & 21.49 & 20.30 & 19.38 \\
\hline 197 & 100.756 & 704.3 & 24 & 461. & 0.366 & 2.50 & 0.020 & 20.35 & 19.45 & 19.35 \\
\hline 199 & 229.905 & 503.969 & 24 & 556. & 0.257 & 6.20 & 0.950 & 20.69 & 19.79 & 19.18 \\
\hline 204 & 832.484 & 675.812 & 24 & 489. & 0.332 & 2.50 & 0.030 & 21.22 & 20.09 & 19.40 \\
\hline 208 & 691.197 & 210.117 & 24 & 485. & 0.288 & 2.40 & 0.030 & 20.55 & 19.61 & 19.37 \\
\hline 210 & 789.583 & 543.906 & 24 & 493. & 0.329 & 3.30 & 0.030 & 21.14 & 20.16 & 19.37 \\
\hline 214 & 60.696 & 435.243 & 24 & 658. & 0.931 & 18.50 & 0.040 & 21.04 & 20.21 & 19.39 \\
\hline 219 & 329.618 & 569.642 & 24 & 541. & 0.361 & 3.60 & 0.110 & 21.45 & 20.08 & 19.48 \\
\hline 224 & 483.740 & 722.181 & 24 & 460. & 0.219 & 1.60 & 0.020 & 20.86 & 19.85 & 19.38 \\
\hline 226 & 89.463 & 42.892 & 24 & 698. & 0.353 & 7.70 & 0.350 & 21.53 & 20.22 & 19.29 \\
\hline 231 & 811.270 & 254.707 & 24 & 461. & & 4.20 & 0.010 & 21.39 & 20.40 & 19.53 \\
\hline 233 & 596.110 & 808.644 & 24 & 488. & 0.540 & 7.10 & 0.020 & 21.03 & 19.74 & 19.55 \\
\hline 237 & 693.643 & 383.014 & 24 & 492. & 0.394 & 7.70 & 0.200 & 20.88 & 19.99 & 19.34 \\
\hline 238 & 791.968 & 662.156 & 24 & 451. & 0.296 & 2.80 & 0.050 & 20.70 & 19.66 & 19.33 \\
\hline 240 & 563.219 & 365.034 & 24 & 500. & 0.265 & 8.90 & 0.270 & 21.00 & 20.07 & 19.40 \\
\hline 252 & 767.647 & 146.556 & 24 & 455. & 0.313 & 2.80 & 0.040 & 21.25 & 20.07 & 19.38 \\
\hline 255 & 78.276 & 117.655 & 24 & 458. & 0.356 & 2.90 & 0.030 & 20.98 & 20.00 & 19.44 \\
\hline 256 & 236.027 & 677.916 & 24 & 474. & 0.396 & 2.50 & 0.040 & 21.05 & 19.92 & 19.56 \\
\hline 257 & 614.979 & 263.849 & 24 & 477. & 0.462 & 2.60 & 0.010 & 21.51 & 20.26 & 19.50 \\
\hline 258 & 23.309 & 678.350 & 23 & 193. & 0.257 & 4.20 & 0.450 & 20.67 & 19.93 & 19.50 \\
\hline 259 & 891.108 & 292.001 & 19 & 463. & 0.368 & 2.80 & 0.110 & 20.45 & 19.52 & 19.50 \\
\hline 262 & 629.264 & 657.023 & 24 & 521. & 0.588 & 2.70 & 0.020 & 21.79 & 20.47 & 19.66 \\
\hline 268 & 617.266 & 11.399 & 24 & 502. & 0.330 & 4.60 & 0.070 & 21.03 & 20.12 & 19.38 \\
\hline 269 & 7.301 & 183.796 & 19 & 437. & 0.238 & 4.30 & 0.300 & 21.17 & 20.28 & 19.44 \\
\hline 272 & 725.302 & 96.781 & 24 & 687. & 0.369 & 4.60 & 0.220 & 20.54 & 19.73 & 19.57 \\
\hline 273 & 217.894 & 526.151 & 24 & 419. & 0.278 & 1.70 & 0.010 & 20.66 & 19.57 & 19.46 \\
\hline 274 & 829.010 & 545.445 & 24 & 212. & 0.281 & 4.60 & 0.090 & 20.90 & 20.24 & 19.45 \\
\hline 276 & 308.469 & 327.348 & 24 & 492. & 0.306 & 7.30 & 0.090 & 20.51 & 19.70 & 19.48 \\
\hline 282 & 433.087 & 63.769 & 24 & 464. & 0.373 & 4.50 & 0.030 & 20.97 & 19.93 & 19.47 \\
\hline
\end{tabular}


Table 4. continuation.

\begin{tabular}{|c|c|c|c|c|c|c|c|c|c|c|}
\hline F2 ID & $\mathrm{x}$ & $\mathrm{y}$ & $\overline{\mathrm{N}}$ & $\mathrm{P}$ & $\mathrm{A}$ & $\overline{\chi^{2}}$ & signif & $\mathrm{J}$ & $\overline{\mathrm{H}}$ & $\overline{<<K>}$ \\
\hline 285 & $\overline{5664.710}$ & $\overline{4888.882}$ & $\overline{24}$ & $\overline{506 .}$ & 0.386 & $\overline{9.90}$ & 0.160 & $\overline{21.22}$ & $\overline{20.38}$ & $\overline{19.46}$ \\
\hline 289 & 24.248 & 01.533 & 23 & 497. & .644 & 10.00 & 0.660 & 21.16 & 0.87 & 19.73 \\
\hline 296 & 43.817 & 426.917 & 24 & 557. & 0.554 & 5.20 & 0.020 & 22.52 & 20.89 & 19.69 \\
\hline 299 & 58.945 & 470.513 & 24 & 430. & 0.377 & 3.10 & 0.040 & 21.31 & 20.42 & 19.67 \\
\hline 302 & 8.003 & 141.525 & 24 & 444. & 353 & 2.60 & .030 & 20.88 & 9.79 & 19.49 \\
\hline 307 & 2.659 & 165.534 & 24 & 444. & 0.211 & 4.60 & 0.320 & 21.01 & 19.89 & 19.58 \\
\hline 309 & 02.863 & 824.379 & 24 & 483. & 0.348 & 2.70 & 0.050 & 21.43 & 20.40 & 19.46 \\
\hline 312 & 479.483 & 204.810 & 24 & 594. & 0.459 & 3.90 & 0.090 & 21.43 & 20.43 & 19.46 \\
\hline 313 & 5.465 & 671.823 & 24 & 468. & 340 & 2.30 & 0.080 & 21.02 & 20.21 & 19.55 \\
\hline 315 & 19.328 & 571.688 & 24 & 482. & 0.629 & 2.20 & 0.010 & 20.77 & 19.76 & 19.84 \\
\hline 319 & 41.857 & 410.714 & 24 & 446. & 0.371 & 2.30 & 0.030 & 20.72 & 20.03 & 19.65 \\
\hline 322 & 466.585 & 163.313 & 24 & 443. & 0.239 & 5.90 & 0.360 & 21.03 & 19.91 & 19.46 \\
\hline 324 & 891.658 & 765.781 & 17 & 487. & 0.316 & 2.50 & 0.440 & 20.98 & 20.51 & 19.56 \\
\hline 326 & 8.559 & 274.274 & 23 & 485. & .554 & 3.60 & 0.030 & 20.60 & 9.89 & 19.71 \\
\hline 338 & 74.833 & 518.023 & 24 & 501. & 0.369 & 3.70 & 0.040 & 20.80 & 19.87 & 19.68 \\
\hline 343 & 29.215 & 91.401 & 23 & 501. & 0.529 & 3.00 & 0.020 & 20.65 & 19.91 & 19.64 \\
\hline 346 & 608.595 & 215.0 & 21 & 549. & 0.6 & 15.40 & 0.950 & 21.60 & & 19.58 \\
\hline 348 & 54 & 305.110 & 22 & 511. & 350 & 3.70 & 0.080 & 20.58 & & 19.60 \\
\hline 351 & & & 24 & 488. & & 2.30 & 060 & 21.44 & .18 & 19.91 \\
\hline 352 & 26.321 & 178.747 & 19 & 268. & 0.634 & 45.50 & 1.000 & 19.84 & 19.06 & 19.72 \\
\hline 357 & 783.957 & 585.8 & 24 & 469. & & & & 20.88 & & 19.70 \\
\hline 362 & .414 & 166.625 & 20 & 432. & 1.0 & & 0.990 & 19.52 & 9.03 & 19.48 \\
\hline 363 & & & 24 & 422. & & & 40 & 20.86 & & 19.77 \\
\hline 372 & 53 & 598. & 24 & 483. & 462 & 00 & 0.020 & 22.06 & 20.99 & 19.78 \\
\hline 373 & 603.818 & 657.3 & 24 & 490. & 0.611 & 6.30 & 0.020 & 22.17 & & 19.80 \\
\hline 375 & 8.261 & 403. & 24 & 434. & 0.1 & 4.60 & 0.190 & 20.72 & & 19.58 \\
\hline 389 & 36 & & 24 & 447. & & & & 21.24 & & 19.72 \\
\hline 390 & & & 24 & 490. & & & & 21.05 & 20.39 & 19.60 \\
\hline 392 & 1.989 & 714.767 & 24 & 470. & & 1.20 & 0.010 & 20.72 & & 19.69 \\
\hline 393 & 2.743 & 659.931 & 24 & 503. & & & 0.040 & 21.29 & & 19.56 \\
\hline 395 & 7 & 375.8 & 24 & 445. & & & & 20.93 & & 19.76 \\
\hline 3 & & & 24 & 50 & & & & 21.75 & & 19.65 \\
\hline 398 & .470 & & 24 & 493. & & & 0.050 & 21.42 & 20.38 & 19.77 \\
\hline 406 & & & 15 & 451. & & & & & & 19.89 \\
\hline 408 & 9.302 & 607. & 24 & 468. & & 4. & 20 & 20.88 & & 19.71 \\
\hline 10 & .202 & & 24 & 462. & 370 & 2.4 & 0.020 & 21.22 & 20.38 & 19.73 \\
\hline 415 & 7.163 & 429.572 & 24 & 465. & 0.128 & 22.20 & 0.010 & 20.99 & 20.02 & 19.71 \\
\hline 416 & & & 24 & 561. & & & & 22.69 & & 19.80 \\
\hline & & & 24 & 428. & & & & & & 19.63 \\
\hline & 8.382 & & 24 & 492. & & & & 21.96 & & 19.74 \\
\hline 422 & & & 24 & 468. & & & 0.0 & 21.43 & 20.73 & 19.77 \\
\hline 425 & 11625 & 365.705 & 24 & 483. & & & & & & 19.72 \\
\hline 427 & & & 24 & 439. & & & & 20.97 & & 19.62 \\
\hline & & & 24 & 361. & & & & 20.95 & & 19.74 \\
\hline & .273 & 309. & 24 & 492. & & 3.8 & 0.05 & 21.94 & 21.03 & 19.64 \\
\hline 432 & 5.275 & 2.508 & 24 & 451. & & & 0.180 & 20.68 & 19.78 & 19.71 \\
\hline 436 & 0447 & & 24 & 470. & & & & 21.32 & & 19.74 \\
\hline & & 609.696 & 24 & 460. & & 2.8 & & 22.11 & & 19.69 \\
\hline & 94 & 170.8 & 24 & 529. & & 2.40 & & 21.49 & & 19.82 \\
\hline 441 & 1.100 & & 24 & 474. & 0.061 & 43.40 & 0.05 & 21.24 & 20.11 & 19.81 \\
\hline & & & 24 & 450. & & & & 21.46 & & 19.77 \\
\hline 445 & & 180.724 & 24 & 454. & 0.466 & 16.30 & 0.110 & 21.85 & 20 & 19.84 \\
\hline & & & 24 & 455. & & & 0.040 & 21.51 & 20.42 & 19.70 \\
\hline & & & 24 & 473. & & & 0.020 & 20.74 & 19.92 & 19.71 \\
\hline 449 & & & 24 & 513. & 0.521 & 2.60 & 0.020 & 21.01 & 20.03 & 19.88 \\
\hline 450 & 405.718 & 554.259 & 24 & 484. & 0.398 & 4.60 & 0.020 & 21.82 & 20.64 & 19.77 \\
\hline & 66.825 & & 24 & 449. & & 4.8 & 0.0 & 21.63 & 20.72 & 19.74 \\
\hline & & & 23 & 47 & & & & & & 19.69 \\
\hline 455 & 409.322 & 736.005 & 24 & 483. & 0.273 & 0.00 & 0.010 & 21.18 & 20.11 & 19.68 \\
\hline 458 & 376.220 & 655.209 & 24 & 416. & 0.282 & 3.20 & 0.030 & 21.30 & 20.53 & 19.70 \\
\hline
\end{tabular}


Table 4. continuation.

\begin{tabular}{|c|c|c|c|c|c|c|c|c|c|c|}
\hline F2 ID & $\mathrm{x}$ & $\mathrm{y}$ & $\overline{\mathrm{N}}$ & $\mathrm{P}$ & $\overline{\mathrm{A}}$ & $\overline{\chi^{2}}$ & signif & $\bar{J}$ & $\overline{\mathrm{H}}$ & $<K>$ \\
\hline$\overline{4463}$ & 217.575 & $\overline{433.394}$ & $\overline{24}$ & 458. & 0.367 & 2.80 & $\overline{0.030}$ & 20.89 & 20.17 & $\overline{19.79}$ \\
\hline 465 & 34.136 & 505.911 & 24 & 449. & 0.298 & 4.90 & 0.070 & 21.00 & 20.04 & 19.76 \\
\hline 466 & 97.668 & 115.838 & 24 & 467. & 0.381 & 7.50 & 0.060 & 21.14 & 20.87 & 19.80 \\
\hline 467 & 19.420 & 287.679 & 24 & 489. & 0.615 & 3.20 & 0.010 & 22.38 & 21.42 & 19.93 \\
\hline 470 & 3.810 & 765.776 & 24 & 433. & 0.514 & 2.50 & 0.010 & 20.93 & 19.89 & 19.88 \\
\hline 471 & 54.271 & 480.820 & 24 & 511. & 0.583 & 3.70 & 0.060 & 22.62 & 21.25 & 19.74 \\
\hline 475 & 88.723 & 379.739 & 24 & 458. & 0.327 & 4.70 & 0.040 & 20.78 & 19.92 & 19.76 \\
\hline 486 & 12.504 & 328.661 & 23 & 477. & 0.448 & 3.60 & 0.030 & 20.75 & 19.97 & 19.78 \\
\hline 487 & 215.930 & 745.931 & 24 & 512. & 0.432 & 2.90 & 0.040 & 21.68 & 20.80 & 19.70 \\
\hline 488 & 9.287 & 716.276 & 24 & 453. & 0.235 & 9.10 & 0.070 & 20.86 & 20.12 & 19.78 \\
\hline 490 & 9.413 & 11.477 & 24 & 428. & 0.377 & 5.70 & 0.030 & 21.52 & 20.59 & 19.81 \\
\hline 496 & 442.349 & 576.357 & 24 & 450. & 0.169 & 6.40 & 0.030 & 21.42 & 20.35 & 19.69 \\
\hline 503 & 91.055 & 118.358 & 24 & 439. & 0.264 & 3.10 & 0.070 & 21.28 & 20.39 & 19.69 \\
\hline 504 & .530 & 766.677 & 24 & 426. & 0.313 & 3.00 & 0.180 & 21.59 & 20.63 & 19.79 \\
\hline 505 & 24.158 & 628.283 & 24 & 431. & 550 & 3.40 & 0.020 & 21.93 & 21.07 & 19.99 \\
\hline 508 & 9.389 & -20.741 & 15 & 418. & 0.389 & 4.10 & 0.390 & 20.94 & 19.99 & 19.80 \\
\hline 510 & 53.145 & 557.418 & 24 & 558. & 0.567 & 6.30 & 0.020 & 21.19 & 20.29 & 19.88 \\
\hline 511 & 59.647 & 843.494 & 23 & 434. & 0.320 & 3.70 & 0.030 & 21.75 & 20.81 & 19.82 \\
\hline 514 & 1.504 & 5.202 & 24 & 456. & 336 & 2.70 & .200 & 21.09 & 20.32 & 19.75 \\
\hline 515 & 17.426 & 544.319 & 24 & 457. & 0.300 & 7.20 & 0.030 & 20.91 & 20.20 & 19.80 \\
\hline 517 & 1.276 & 414.502 & 24 & 466. & 0.463 & 3.20 & 0.010 & 21.82 & 21.00 & 19.87 \\
\hline 518 & 8.647 & 647.864 & 24 & 554. & 0.531 & 2.20 & 0.030 & 21.19 & 20.58 & 19.70 \\
\hline 19 & & 36 & 24 & 466. & 94 & 1.40 & 0.010 & 20.76 & 20.09 & 19.87 \\
\hline 525 & & & 24 & 454. & 507 & 2.20 & 0.010 & 20.86 & 19.94 & 19.92 \\
\hline 528 & 0.748 & 227.502 & 24 & 389. & 0.277 & 3.10 & 0.050 & 21.21 & 20.48 & 19.82 \\
\hline 529 & 6.288 & 331.541 & 24 & 750. & 0.498 & 6.90 & 0.170 & 21.18 & 20.28 & 19.72 \\
\hline 531 & 1.483 & 398.036 & 24 & 444. & 0.457 & 2.40 & 0.020 & 20.96 & 20.22 & 19.89 \\
\hline 532 & & & 24 & 452. & & 2.50 & 0.020 & 21.01 & 20.01 & 19.77 \\
\hline 542 & & 430. & 24 & 457. & 0.436 & 1.50 & 0.010 & 21.84 & 20.83 & 19.87 \\
\hline 543 & 94 & 197.5 & 24 & 409. & 0.315 & 8.00 & 0.180 & 21.21 & 20.36 & 19.76 \\
\hline 545 & 2 & 57 & 24 & 576. & 0. & 2.80 & 170 & 22.32 & 20.67 & 20.11 \\
\hline 46 & & & 24 & 45 & 71 & 3.70 & 40 & 21.59 & 20.42 & 19.71 \\
\hline 548 & & 37 & 14 & 529. & 1.418 & 18.40 & 0.950 & 22.52 & 99.99 & 20.48 \\
\hline 551 & 36 & 1.737 & 24 & 730. & 0.862 & 6.70 & 0.030 & 22.22 & 20.88 & 19.87 \\
\hline 554 & 0 & 581.529 & 24 & 469. & & 4.50 & & 20.81 & 20.02 & 19.89 \\
\hline 567 & & 263.4 & 24 & 477. & & 1.80 & & 20.85 & 20.05 & 19.86 \\
\hline 70 & & 213. & 24 & 437. & 35 & 1.20 & 0.020 & 20.86 & 20.01 & 19.93 \\
\hline 577 & & 1.845 & 20 & 479. & 0.556 & 52.80 & 1.000 & 21.38 & 20.29 & 19.61 \\
\hline & & 206.3 & 24 & 504. & & 1.90 & & 21.37 & & 19.89 \\
\hline & & 702.3 & 24 & 432. & .390 & 3.10 & & 20.76 & 19. & 19.87 \\
\hline 594 & & 544 & 24 & 197. & 0.248 & 3.80 & 0.080 & 20.78 & 20.03 & 19.74 \\
\hline 597 & & 476.8 & 24 & 622. & 0.272 & 31.60 & 0.370 & 22.64 & 21.46 & 19.76 \\
\hline & & & 24 & 446. & & 2.70 & & 21.23 & 20.50 & 19.95 \\
\hline 601 & 7.961 & 38 & 24 & 451. & 0.249 & 11.60 & 0.030 & 20.90 & 20.25 & 19.83 \\
\hline 02 & & 49 & 24 & 458. & 0.208 & 6.10 & 0.030 & 21.16 & 20.45 & 19.84 \\
\hline & & 10 & 24 & 412. & 0.292 & 4.00 & & 21.43 & 20.52 & 19.81 \\
\hline & & & 24 & 326. & & 2.40 & & 20.88 & 20.14 & 20.04 \\
\hline 607 & 0.220 & 119.771 & 24 & 436. & & 5.10 & & 21.38 & 20.39 & 19.77 \\
\hline & & 752.2 & 24 & 426. & 0.332 & 6.20 & 0.060 & 21.46 & 20.59 & 19.87 \\
\hline 616 & & 149.4 & 24 & 456. & 0.333 & 1.60 & 0.010 & 21.89 & 20.70 & 19.86 \\
\hline 620 & & & 24 & 462. & & 2.30 & 0.020 & 21.84 & 20.64 & 19.85 \\
\hline 625 & 16.687 & 846.436 & 23 & 461. & 0.309 & 4.70 & 0.080 & 21.76 & 20.71 & 19.85 \\
\hline 631 & & 551.661 & 23 & 547. & 0.542 & 1.10 & 0.090 & 22.83 & 21.21 & 19.82 \\
\hline & & 31.204 & 24 & 454. & 0.363 & 1.90 & 0.010 & 21.45 & & 19.87 \\
\hline 639 & 9.303 & 20.019 & 24 & 453. & 0.145 & 15.30 & 0.320 & 21.98 & 20.55 & 19.87 \\
\hline 640 & & 255.813 & 24 & 429. & & 2.60 & 0.050 & 21.07 & 20.04 & 19.83 \\
\hline & & 172.9 & 23 & 431. & 0.500 & 9.30 & 0.050 & 21.84 & 20.94 & 20.04 \\
\hline & & & 24 & 457. & & 1.80 & 0.040 & 21.27 & 20.59 & 19.92 \\
\hline 645 & 753.390 & 308.952 & 24 & 445. & 0.552 & 6.50 & 0.020 & 21.84 & 21.19 & 20.10 \\
\hline 647 & 442.445 & -0.474 & 20 & 636. & 0.815 & 5.10 & 0.170 & 22.50 & 21.03 & 20.41 \\
\hline
\end{tabular}


Table 4. continuation.

\begin{tabular}{|c|c|c|c|c|c|c|c|c|c|c|}
\hline F2 ID & $\mathrm{x}$ & $\mathrm{y}$ & $\mathrm{N}$ & $\mathrm{P}$ & $\mathrm{A}$ & $\chi^{2}$ & signif & $\bar{J}$ & $\overline{\mathrm{H}}$ & $<K>$ \\
\hline 651 & $\overline{501.926}$ & 11.122 & 24 & 447. & 0.537 & 2.20 & $\overline{0.010}$ & 22.11 & 21.27 & 20.09 \\
\hline 653 & 1.328 & 478.053 & 24 & 402. & 320 & 3.80 & 0.070 & 21.20 & 0.63 & 19.94 \\
\hline 654 & 75.946 & 540.901 & 24 & 375. & 0.281 & 3.30 & 0.060 & 21.06 & 20.07 & 19.74 \\
\hline 655 & 45.450 & 186.668 & 18 & 36. & 1.268 & 17.30 & 0.990 & 20.82 & 20.10 & 19.84 \\
\hline 656 & 607.630 & 715.505 & 24 & 459. & 426 & 2.50 & 020 & 21.40 & 0.79 & 19.90 \\
\hline 660 & 1.747 & 653.525 & 24 & 551. & 0.621 & 3.70 & 0.050 & 21.76 & 20.42 & 20.18 \\
\hline 663 & 97.577 & 237.269 & 24 & 198. & 0.220 & 1.80 & 0.040 & 20.97 & 20.19 & 19.80 \\
\hline 664 & 624.931 & 400.023 & 24 & 492. & 0.538 & 4.90 & 0.020 & 22.74 & 21.41 & 19.98 \\
\hline 665 & 12.844 & 108.361 & 24 & 397. & .510 & 3.30 & 0.010 & 22.41 & 21.18 & 20.02 \\
\hline 666 & 770.632 & 463.310 & 24 & 370. & 308 & 2.10 & .040 & 20.95 & 20.07 & 19.91 \\
\hline 676 & 265.506 & 126.520 & 24 & 451. & 0.322 & 3.20 & 0.080 & 21.38 & 20.47 & 19.93 \\
\hline 677 & 535.476 & 226.278 & 24 & 455. & 0.472 & 12.20 & 0.060 & 22.29 & 21.14 & 19.97 \\
\hline 679 & 672.370 & 757.677 & 24 & 465. & 0.339 & 2.80 & 0.030 & 21.95 & 20.86 & 19.91 \\
\hline 683 & 8.621 & 199.772 & 24 & 369. & & 5.40 & 0.130 & 21.21 & 20.59 & 19.84 \\
\hline 685 & 7.669 & 746.478 & 24 & 419. & 404 & 3.90 & .030 & 21.65 & 20.90 & 20.00 \\
\hline 691 & 1.947 & 593.063 & 24 & 473. & 0.482 & 3.20 & 0.020 & 21.30 & 20.72 & 19.95 \\
\hline 692 & 64.060 & 165.640 & 24 & 395. & 0.410 & 1.40 & 0.020 & 20.93 & 19.94 & 19.97 \\
\hline 700 & & 784.9 & 24 & 200. & 224 & 2.80 & 0.130 & 21.09 & 20.40 & 19.82 \\
\hline 701 & & & 24 & 424. & & 3.10 & .040 & 21.85 & 21.03 & 20.04 \\
\hline 715 & 6.303 & 157.950 & 24 & 436. & 0.361 & 2.40 & 0.100 & 21.29 & 20.30 & 20.01 \\
\hline 720 & 6 & 232.4 & 24 & 427. & & 3.20 & 0.010 & 21.27 & 20.17 & 20.09 \\
\hline 21 & 8.034 & 712.025 & 24 & 621. & .544 & 2.00 & 0.220 & 21.93 & 20.66 & 20.19 \\
\hline 22 & & & 24 & 431. & & 2.50 & & 21.16 & .15 & 9.98 \\
\hline 723 & & 350. & 24 & 426. & & 4.20 & 0.020 & 20.96 & 20.02 & 20.02 \\
\hline 724 & 1.042 & 384.463 & 24 & 370. & 0.8 & 13.40 & 0.160 & 22.86 & 21.94 & 20.56 \\
\hline 26 & 964 & 7.804 & 18 & 419. & & 7.30 & 0.390 & 21.10 & 20.08 & 19.99 \\
\hline 27 & & & 24 & 46 & 199 & 33.80 & 20 & 21.0 & 20.19 & 19.98 \\
\hline 730 & & 279. & 24 & 467. & & 5.60 & 0.080 & 20.96 & 20.13 & 19.85 \\
\hline 734 & & 269.139 & 23 & 431. & & 8.10 & & 22.42 & 21.32 & 20.12 \\
\hline 735 & & 2.846 & 24 & 456. & 0.4 & 2.30 & 0.010 & 21.06 & 20.24 & 20.02 \\
\hline 38 & & 33 & 24 & 337. & 8 & 2.10 & 0.040 & 21.17 & 20.27 & 19.88 \\
\hline 43 & & & 24 & 452. & & & & 21. & 20 & 19.98 \\
\hline 745 & & & 24 & 196. & & 6.90 & 0.240 & 21.57 & 20.84 & 20.00 \\
\hline 46 & & & 24 & 418. & & & & & & 20.06 \\
\hline 18 & & 3 & 24 & 420. & & 5.10 & & 21.83 & 21.33 & 20.24 \\
\hline 1 & & & 24 & 398. & 326 & 1.6 & 0.120 & 21.29 & 20.22 & 19.80 \\
\hline 754 & & 569. & 24 & 457. & 0.431 & 4.20 & 0.030 & 21.15 & 20.37 & 20.03 \\
\hline 762 & & & 23 & 391. & & & & & & 20.01 \\
\hline 66 & & & 24 & 487. & & & 20 & 22.87 & 21.19 & 20.14 \\
\hline & 067 & & 24 & 722. & & 5.1 & 0.270 & 21.49 & 20.36 & 20.03 \\
\hline 768 & & 4 & 24 & 471. & 8 & 3.00 & 10 & 21.23 & 20.23 & 20.03 \\
\hline 69 & & & 24 & 402. & & & & & 20.55 & 19.92 \\
\hline 7 & & 32 & 23 & 452. & & & & & & 20.03 \\
\hline & & & 23 & 397. & & 4.90 & & 21.24 & 20.65 & 20.00 \\
\hline 75 & 662 & .731 & 24 & 431. & 3 & 2.0 & 0.0 & 21.55 & 20.84 & 19.97 \\
\hline 777 & & & 24 & 194. & & & & 21.20 & 20.48 & 19.96 \\
\hline 778 & & & 24 & 476. & & 4.90 & & 21.66 & 20.83 & 19.93 \\
\hline 779 & .909 & 44.180 & 24 & 505. & & 1.80 & 0.010 & 20.82 & 20.27 & 19.94 \\
\hline & & & 23 & 428. & 0.495 & 1.6 & 0.020 & 21.18 & 20.15 & 20.02 \\
\hline & & & 24 & 500. & & 5.10 & $0.0^{\circ}$ & 21.84 & 20.92 & 19.93 \\
\hline & & & 24 & 483. & & & & 21.12 & 20.26 & 20.05 \\
\hline 797 & & 298.404 & 24 & 398. & & 1.60 & 0.0 & 21.40 & 20.82 & 20.15 \\
\hline & & & 23 & 480. & & 7.20 & 0.030 & 22.46 & 21.30 & 20.30 \\
\hline & & & 24 & 423. & & 6.30 & & 21.54 & 20.81 & 20.02 \\
\hline 808 & & 412.300 & 24 & 401. & 0.410 & 1.10 & 0.010 & 21.04 & 20.18 & 19.97 \\
\hline 809 & & 25.982 & 24 & 451. & 0.138 & 26.40 & 0.900 & 21.22 & 20.24 & 19.91 \\
\hline & & 201. & 24 & 35 & & 2.90 & & 21.33 & 20.63 & 19.93 \\
\hline & & & 2 & 438. & & & & & 21.02 & 20.01 \\
\hline 816 & 549.096 & 166.588 & 24 & 529. & 1.001 & 12.90 & 0.060 & 21.72 & 20.54 & 20.66 \\
\hline 817 & 346.288 & 464.295 & 24 & 177. & 0.279 & 5.50 & 0.210 & 21.03 & 20.30 & 20.00 \\
\hline
\end{tabular}


Table 4. continuation.

\begin{tabular}{|c|c|c|c|c|c|c|c|c|c|c|}
\hline F2 ID & $\mathrm{x}$ & $\mathrm{y}$ & $\overline{\mathrm{N}}$ & $\mathrm{P}$ & $\overline{\mathrm{A}}$ & $\overline{\chi^{2}}$ & signif & $\bar{J}$ & $\overline{\mathrm{H}}$ & $<K>$ \\
\hline 820 & 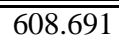 & 220.076 & 23 & $\overline{511 .}$. & 0.625 & $\overline{4.50}$ & $\overline{0.500}$ & 22.11 & $\overline{21.49}$ & 19.97 \\
\hline 828 & 28.831 & 805.376 & 24 & 498. & 433 & 7.00 & 0.070 & 21.76 & 20.50 & 19.96 \\
\hline 832 & 51.849 & 373.813 & 24 & 439. & 0.402 & 6.10 & 0.060 & 21.75 & 20.96 & 20.12 \\
\hline 839 & 28.852 & 173.045 & 24 & 442. & 0.467 & 3.80 & 0.030 & 21.05 & 20.22 & 20.12 \\
\hline 841 & 50.130 & 761.766 & 24 & 310. & 266 & 4.70 & .710 & 21.23 & 20.20 & 20.06 \\
\hline 843 & 386.502 & 661.370 & 24 & 446. & 0.237 & 16.90 & 0.330 & 21.73 & 20.71 & 19.98 \\
\hline 852 & 33.105 & 436.758 & 24 & 443. & 0.495 & 8.10 & 0.180 & 21.07 & 20.14 & 20.10 \\
\hline 853 & 02.687 & 543.326 & 24 & 409. & 0.502 & 2.90 & 0.070 & 21.01 & 20.23 & 20.19 \\
\hline 856 & 23.108 & 645.115 & 24 & 376. & 0.328 & 2.30 & 0.050 & 21.38 & 20.46 & 19.91 \\
\hline 857 & 44.135 & 662.058 & 24 & 476. & .432 & 1.90 & 0.010 & 21.52 & 20.57 & 20.01 \\
\hline 858 & 39.946 & 524.230 & 21 & 489. & 0.490 & 2.80 & 0.080 & 21.82 & 21.24 & 20.03 \\
\hline 860 & 8.933 & 137.441 & 24 & 506. & 0.592 & 3.80 & 0.020 & 21.47 & 20.66 & 20.04 \\
\hline 861 & 49.0 & 372.270 & 24 & 408. & 0.256 & 0.90 & 0.030 & 21.29 & 20.25 & 19.91 \\
\hline 864 & 7.9 & 378.409 & 24 & 484. & 450 & 3.40 & 0.020 & 22.11 & 21.27 & 20.04 \\
\hline 869 & 72 & 301.476 & 24 & 480. & 0.392 & 38.60 & 1.000 & 20.94 & 20.25 & 19.88 \\
\hline 870 & 55.326 & 15.040 & 24 & 451. & 0.444 & 5.10 & 0.130 & 21.62 & 20.85 & 20.11 \\
\hline 872 & 43.791 & 359.843 & 24 & 427. & 0.362 & 1.00 & 0.020 & 22.00 & 20.98 & 20.09 \\
\hline 874 & 70.740 & 678.097 & 24 & 354. & 0.434 & 2.90 & 0.080 & 21.21 & 20.53 & 20.20 \\
\hline 876 & & 4.980 & 24 & 373. & $\$ 34$ & 2.80 & 020 & 21.15 & 20.09 & 20.03 \\
\hline 877 & 5.617 & 278.930 & 24 & 310. & 0.346 & 2.90 & 0.130 & 21.01 & 20.17 & 20.15 \\
\hline 883 & 6.275 & 129.456 & 24 & 757. & 0.440 & 6.60 & 0.850 & 21.20 & 20.25 & 20.07 \\
\hline 884 & 7.084 & 469.6 & 24 & 372. & 0.313 & 3.90 & 0.130 & 21.29 & 20.57 & 20.09 \\
\hline 95 & & & 24 & 288. & & 3.90 & & 21.30 & 20.24 & 20.03 \\
\hline 896 & & & 24 & 439. & 0.229 & 3.50 & & 21.17 & 20.24 & 19.94 \\
\hline 897 & 6.569 & 370.754 & 24 & 450. & & 6.70 & 0.050 & 22.20 & 20.85 & 20.15 \\
\hline 900 & 8.720 & 859.7 & 13 & 205. & 0.376 & 3.90 & 0.610 & 99.99 & 21.07 & 20.10 \\
\hline 901 & .901 & 6 & 24 & 433. & 478 & 3.60 & 0.040 & 21.41 & 20.66 & 20.14 \\
\hline 04 & & & 24 & 407. & & 4.60 & & 21.06 & 20.26 & 20.16 \\
\hline 905 & & 243. & 24 & 321. & 0.329 & 4.70 & 0.130 & 20.94 & 20.18 & 20.11 \\
\hline 906 & 17 & 38 & 24 & 341. & & 2.20 & & 21.09 & 20.39 & 20.05 \\
\hline 917 & & 13 & 24 & 458. & & 21.90 & 0.040 & 21.38 & 20.47 & 20.03 \\
\hline 18 & & & 24 & 335. & 57 & 50 & 00 & 21.05 & 20.30 & 20.01 \\
\hline 927 & & 47 & 24 & 430. & 0.694 & 5.10 & 0.020 & 23.05 & 21.74 & 20.35 \\
\hline 929 & 2.743 & 2 & 24 & 423. & 0.438 & 22.80 & 0.880 & 21.14 & 20.19 & 20.15 \\
\hline 935 & 6210 & 16 & 24 & 197. & & 4.80 & & 21.24 & & 20.00 \\
\hline 938 & & & 24 & 427. & & 6.60 & & 21.80 & 21.01 & 20.10 \\
\hline 942 & & 34 & 24 & 469. & 0.517 & 5.40 & 0.020 & 22.57 & 21.43 & 20.10 \\
\hline 944 & 4.171 & 204.963 & 22 & 475. & 0.429 & 9.10 & 0.760 & 21.92 & 20.46 & 19.97 \\
\hline 945 & & 44.377 & 24 & 405. & & 1.90 & & 21.28 & & 19.96 \\
\hline 946 & & & 24 & 398. & & 3.20 & 0.030 & 21.62 & 21. & 20.25 \\
\hline 954 & & & 24 & 421. & .369 & 3.30 & 0.240 & 22.31 & 20.57 & 19.90 \\
\hline 962 & 6.830 & 712.250 & 24 & 407. & 0.482 & 2.50 & 0.030 & 22.23 & 21.27 & 20.26 \\
\hline 964 & & & 24 & 387. & & 4.10 & & 21.29 & & 19.99 \\
\hline 966 & & 9.151 & 24 & 408. & & 5.60 & 0.170 & 21.68 & 20.93 & 19.90 \\
\hline 970 & & & 23 & 399. & 11 & 3.60 & 0.210 & 20.94 & 20.18 & 20.21 \\
\hline 973 & & & 24 & 358. & & 1.50 & 20 & 21.13 & 20.31 & 20.13 \\
\hline 975 & & & 23 & 410. & & 4.30 & & 21.74 & & 20.02 \\
\hline 976 & & 409.5 & 24 & 462. & & 12.40 & & 21.67 & 21.15 & 20.09 \\
\hline & & & 24 & 393. & & 4.60 & 0.170 & 21.46 & 20.37 & 19.96 \\
\hline 986 & 3.864 & 6 & 24 & 493. & 0.554 & 3.40 & 0.040 & 22.01 & 20.66 & 20.27 \\
\hline 988 & & & 24 & 360. & & 4.80 & & 21.24 & & 20.17 \\
\hline 991 & & 298.708 & 24 & 356. & & 2.60 & 0.040 & 21.22 & 20.60 & 20.14 \\
\hline 993 & & 806.4 & 23 & 277. & 47 & 7.60 & 0.950 & 21.30 & 20.32 & 20.08 \\
\hline 995 & & & 13 & 663. & & 16.20 & 0.880 & 21.10 & 20.36 & 20.13 \\
\hline 998 & 675.854 & 236.5 & 24 & 449. & 0.169 & 11.70 & 0.310 & 21.61 & 20.76 & 20.01 \\
\hline 1004 & & & 24 & 395. & & 2.50 & & 20.93 & 20.02 & 20.22 \\
\hline & 97 & 109.589 & 24 & 399. & 0.3 & 4.10 & 0.090 & 21.17 & 20.30 & 20.01 \\
\hline & & & 24 & 290. & & 4.50 & 0.230 & 21.45 & 20.52 & 20.09 \\
\hline 1017 & 501.398 & 309.847 & 23 & 449. & 0.623 & 3.20 & 0.020 & 21.32 & 20.39 & 20.32 \\
\hline 1019 & 145.017 & 297.283 & 24 & 416. & 0.450 & 7.10 & 0.300 & 21.67 & 20.48 & 19.93 \\
\hline
\end{tabular}


M. Rejkuba et al.: LPV catalogue in Cen A, Online Material p 18

Table 4. continuation.

\begin{tabular}{|c|c|c|c|c|c|c|c|c|c|c|}
\hline$\overline{\mathrm{F} 2 \mathrm{ID}}$ & $\mathrm{x}$ & $\mathrm{y}$ & $\overline{\mathrm{N}}$ & $\mathrm{P}$ & $\mathrm{A}$ & $\overline{\chi^{2}}$ & signif & $\mathrm{J}$ & $\overline{\mathrm{H}}$ & $<K>$ \\
\hline 1027 & 387.202 & 150.105 & 24 & 427. & 0.258 & 2.50 & 0.110 & 21.44 & 20.46 & 19.98 \\
\hline 1028 & 94.779 & 27.265 & 24 & 306. & & 2.20 & .040 & 20.96 & 0.04 & 20.17 \\
\hline 1032 & 86.150 & 72.583 & 24 & 302. & .390 & 3.30 & 0.050 & 21.42 & 20.43 & 20.25 \\
\hline 1037 & 41.930 & 653.414 & 24 & 313. & 267 & 4.50 & 0.250 & 21.19 & 20.33 & 20.03 \\
\hline 1039 & 2.432 & 1.454 & 24 & 416. & 526 & 3.50 & 040 & 21.04 & 0.24 & 20.25 \\
\hline 1044 & 66.510 & 748.804 & 24 & 182. & 0.242 & 6.40 & 0.360 & 21.09 & 20.28 & 19.99 \\
\hline 1045 & 47.012 & 590.337 & 24 & 479. & 0.653 & 4.70 & 0.020 & 24.13 & 21.99 & 20.11 \\
\hline 1059 & 45.611 & 319.988 & 24 & 605. & 0.704 & 4.40 & 0.020 & 23.32 & 21.60 & 20.36 \\
\hline & 24.933 & 56.899 & 24 & 474. & .408 & 9.60 & .210 & 23.54 & 1.61 & 20.05 \\
\hline 1065 & 7.791 & 178.163 & 24 & 407. & 354 & 5.50 & 050 & 21.24 & 20.21 & 20.07 \\
\hline 1074 & 1.034 & 88.744 & 24 & 464. & 0.231 & 5.00 & 0.540 & 21.95 & 20.92 & 20.07 \\
\hline 1081 & 4.049 & 818.506 & 24 & 412. & 0.440 & 5.90 & 0.300 & 21.32 & 20.55 & 20.25 \\
\hline 1082 & 63.847 & 428.322 & 23 & 416. & 0.463 & 2.40 & 0.050 & 21.07 & 20.23 & 20.24 \\
\hline & & 652.4 & 24 & 185. & 241 & 4.50 & 190 & 21.22 & 20.35 & 20.08 \\
\hline 1098 & 3.389 & 48.228 & 24 & 440. & 495 & 1.00 & 010 & 22.42 & 21.55 & 20.03 \\
\hline 1100 & 5.267 & 724.707 & 24 & 243. & 0.247 & 1.90 & 0.050 & 21.85 & 20.78 & 20.04 \\
\hline 04 & 30 & 212.496 & 19 & 483. & 0.5 & 6.20 & 900 & 99.99 & & 20.05 \\
\hline 1108 & & 595.427 & 24 & 434. & 416 & 2.00 & 040 & 21.59 & 20.58 & 20.12 \\
\hline 1110 & & 70 & 19 & 463. & & & 70 & 1.19 & & 20.13 \\
\hline 1111 & 174.542 & 430.648 & 24 & 458. & 0.536 & 4.20 & 0.030 & 21.93 & 20.76 & 20.25 \\
\hline 1112 & & 311.4 & 24 & 393. & & 3.80 & & 21.60 & & 19.97 \\
\hline 1115 & 3.077 & 161. & 24 & 514. & 419 & 2.40 & 0.030 & 21.80 & 20.75 & 20.26 \\
\hline & & & 24 & 598. & & & & 2.63 & & 0.36 \\
\hline 1122 & & 624. & 24 & 341. & & 3.20 & & 21.27 & 0.66 & 20.40 \\
\hline 1124 & & 98 & 22 & 393. & & 4.10 & & & & 20.25 \\
\hline 1125 & & & 24 & 453. & & & & & & 20.18 \\
\hline & & & 24 & 28 & & & & & & 20.08 \\
\hline & & 566 & 24 & 460. & & & 20 & & 0.64 & 20.29 \\
\hline 1134 & 690.081 & 79.8 & 24 & 409. & & 3.30 & & & & 20.26 \\
\hline 1135 & & 2.622 & 22 & 394. & & 4.90 & & & & 19.97 \\
\hline & & & 24 & 424. & & & & & & 20.10 \\
\hline & & & 24 & 35 & & & & & & 20.18 \\
\hline 1147 & & & 24 & 351. & & 1.40 & & & & 20.07 \\
\hline & & & 24 & 505. & & & & & & 20.12 \\
\hline & & & 24 & 494. & & & & 2.18 & & 20.05 \\
\hline & & & 24 & 33 & & & & 21.03 & 20.40 & 20.23 \\
\hline 1159 & & & 24 & 355. & & 60 & & 1.36 & 0.61 & 20.09 \\
\hline 1161 & & & 24 & 500. & & & & & & 20.42 \\
\hline & & & 24 & 436. & & & & & & 20.12 \\
\hline & & & 24 & 68 & & & & 23. & & 20.36 \\
\hline & & & 24 & 27 & & & & 20.85 & & 20.13 \\
\hline & & & 24 & 455. & & & & & & 20.27 \\
\hline & & & 24 & 381. & & & & & & 20.21 \\
\hline & & & 24 & 32 & & & & & & 20.06 \\
\hline & & & 24 & 373. & 0.4 & & & & 21.00 & 20.17 \\
\hline & & & 24 & 386. & & & & 21.73 & 20.50 & 20.04 \\
\hline & & & 24 & 382. & & & & & & 20.01 \\
\hline & & & & 369. & & & & & & 20.10 \\
\hline & & & 24 & 398. & & & & 21.81 & & 20.12 \\
\hline & & & 24 & 447. & & 1.70 & 0.020 & 21.79 & & 20.22 \\
\hline & & & 18 & 305. & & 6.30 & & & & 20.23 \\
\hline & & & 24 & 187. & & 2.30 & & 21.24 & 28 & 20.06 \\
\hline & & & 19 & 30 & & 7.20 & & 21.26 & & 20.25 \\
\hline 1224 & & & 24 & 476. & & 12.90 & & & 20.73 & 20.37 \\
\hline 1234 & & & 24 & 510. & & 4.00 & & 23.35 & 22.26 & 20.26 \\
\hline 1243 & & & 24 & 296. & & 3.10 & 0.020 & 21.61 & 20.82 & 20.22 \\
\hline & & 64 & 24 & 278. & & 9.50 & 0.4 & 21.42 & & 20.22 \\
\hline & & & 24 & 471. & & & & & & 20.38 \\
\hline 1258 & & & 24 & 396. & 0.360 & 4.30 & & 21.55 & 20.48 & 20.11 \\
\hline 1260 & 831.726 & 578.119 & 24 & 417. & 0.647 & 13.00 & 0.030 & 22.81 & 22.11 & 20.35 \\
\hline
\end{tabular}


Table 4. continuation.

\begin{tabular}{|c|c|c|c|c|c|c|c|c|c|c|}
\hline F2 ID & $\mathrm{x}$ & $\mathrm{y}$ & $\overline{\mathrm{N}}$ & $\mathrm{P}$ & $\mathrm{A}$ & $\chi^{2}$ & signif & $\bar{J}$ & $\overline{\mathrm{H}}$ & $<K>$ \\
\hline 1261 & $\overline{257.534}$ & $\overline{6881.248}$ & 24 & 427. & 0.538 & 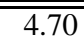 & $\overline{0.020}$ & 22.16 & $\overline{21.42}$ & 20.25 \\
\hline 1262 & 1.765 & & 24 & 379. & 471 & 3.10 & & 2.38 & 0.83 & 20.28 \\
\hline 1267 & 51.482 & 692.093 & 24 & 437. & 0.557 & 3.30 & 0.020 & 23.03 & 21.75 & 20.38 \\
\hline 1271 & 03.029 & 229.035 & 24 & 297. & 0.311 & 5.00 & 0.520 & 21.72 & 20.85 & 20.15 \\
\hline 1272 & 2.651 & & 24 & 373. & 0.383 & 4.50 & .090 & 21.28 & 20.44 & 20.24 \\
\hline 1282 & 63.396 & 656.010 & 21 & 351. & 0.586 & 18.00 & 0.970 & 21.04 & 20.46 & 20.43 \\
\hline 1283 & 3.011 & 491.855 & 22 & 403. & 0.435 & 2.30 & 0.060 & 23.57 & 22.60 & 20.39 \\
\hline 1294 & 71.705 & 600.459 & 24 & 323. & 0.335 & 6.60 & 0.660 & 21.33 & 20.68 & 20.03 \\
\hline 1295 & 9.530 & & 24 & 328. & 0.458 & 4.00 & 050 & 23.03 & 21.54 & 20.11 \\
\hline 1300 & 21.038 & 644.433 & 24 & 427. & 0.270 & 1.70 & 0.090 & 21.62 & 20.54 & 20.09 \\
\hline 1311 & 44.601 & 757.731 & 24 & 444. & 0.400 & 8.90 & 1.000 & 21.24 & 20.58 & 20.16 \\
\hline 1318 & 1.437 & 385.234 & 24 & 297. & 0.335 & 3.40 & 0.100 & 21.35 & 20.44 & 20.32 \\
\hline 1325 & 39.054 & 700.875 & 24 & 425. & 0.578 & 4.30 & 0.090 & 21.89 & 21.43 & 20.48 \\
\hline & 4.474 & 110. & 21 & 411. & 0.601 & 12.90 & .190 & 99.99 & 9.99 & 20.46 \\
\hline 1343 & .503 & & 24 & 319. & 0.448 & 3.90 & .040 & 21.13 & 20.45 & 20.30 \\
\hline 1349 & 872.937 & & 23 & 480. & & 30.60 & 1.000 & 20.90 & 20.29 & 20.11 \\
\hline 56 & 297.561 & 737.6 & 24 & 449. & & 4.90 & 020 & 21.76 & 20.59 & 20.41 \\
\hline 62 & 22 & 33 & 20 & 483. & 92 & 22.80 & .960 & 21.95 & 21.42 & 20.38 \\
\hline 64 & & & 24 & 180. & & & 620 & 21.29 & 0.34 & 20.17 \\
\hline 1367 & 6.052 & & 15 & 668. & & 30 & & 21.65 & 20.81 & 20.16 \\
\hline & & 253.5 & 24 & 366. & & & & 21.49 & & 20.22 \\
\hline 76 & & 571. & 24 & 380. & 0. & & 0.0 & 21.53 & 20.76 & 20.02 \\
\hline & & & 24 & 32 & & & & 21.20 & 0.58 & 20.24 \\
\hline & & & 24 & 180. & & & & 21.44 & 0.49 & 20.17 \\
\hline 80 & & & 24 & 274. & & & & 21.99 & & 20.22 \\
\hline & & & 24 & 452. & & & & 22.00 & 97 & 20.21 \\
\hline & & & 23 & 39 & & & & 21.92 & & 20.22 \\
\hline 89 & & & 24 & 302. & & & & 21.57 & 20.81 & 20.22 \\
\hline 1390 & & & 16 & 364. & & & .770 & 21.60 & & 20.10 \\
\hline 1401 & 96 & 258 & 24 & 401. & & & & 22.34 & & 20.29 \\
\hline & & 3 & 24 & & & & & 21.76 & & 20.10 \\
\hline & & & 24 & 39 & & & & & & 20.25 \\
\hline 16 & & 49 & 24 & 430. & & & & 22.86 & 21.60 & 20.22 \\
\hline & & & 24 & 400. & & & & & & 20.09 \\
\hline 1447 & & & 24 & 376. & & & & 21.71 & 21. & 20.20 \\
\hline & & & 24 & 56 & & & & 24.42 & 21. & 20.29 \\
\hline 1452 & & & 12 & 170. & & & & 20.89 & 21.65 & 21.09 \\
\hline & & & 23 & & & & & & & 19.98 \\
\hline & & & 24 & & & & & & & 20.25 \\
\hline & & & 24 & 17 & & & & 21.55 & & 20.23 \\
\hline & & & 24 & 337. & & & & 21.40 & 20.53 & 20.27 \\
\hline & & & 24 & 214. & & & & & & 20.20 \\
\hline & & & 23 & 468. & & & & & & 20.35 \\
\hline & & & 24 & 33 & & & & 21.39 & & 20.19 \\
\hline & & & 24 & 260. & 0.2 & & & 21.47 & 20.67 & 20.27 \\
\hline 1502 & 4 & & 24 & 308. & & & 0.080 & 21.30 & 20.47 & 20.61 \\
\hline & & & 24 & 436. & & & & 21.62 & & 20.18 \\
\hline & & & 24 & 324. & & & & 21.71 & 20.72 & 20.22 \\
\hline & & & 23 & 275. & & & & 23.03 & 21.47 & 20.33 \\
\hline & & & 24 & 446. & & & 0.040 & 21.87 & 21.46 & 20.49 \\
\hline & & & 24 & 289. & & & & & & 20.35 \\
\hline & & & 24 & 410. & & 2.30 & 0.2 & 22.01 & 20.60 & 20.12 \\
\hline & & & 24 & 31 & & & 0.0 & 21.85 & 21.01 & 20.27 \\
\hline & & & 24 & 428. & & & & 22.15 & & 20.25 \\
\hline & & & 24 & 295. & & & 0.020 & 21.60 & 20.71 & 20.35 \\
\hline & & 327.667 & 24 & 460. & & 2.40 & 0.020 & 21.50 & 20.58 & 20.30 \\
\hline & & & 24 & 328. & & & 0.0 & 21.28 & 20.80 & 20.52 \\
\hline & & & 2 & 33 & & & & & & 20.21 \\
\hline 1560 & & 357.201 & 24 & 297. & 0.479 & 2.90 & 0.020 & 21.87 & 20.91 & 20.47 \\
\hline 1579 & 359.919 & 637.705 & 24 & 369. & 0.409 & 7.00 & 0.480 & 21.76 & 21.01 & 20.16 \\
\hline
\end{tabular}


M. Rejkuba et al.: LPV catalogue in Cen A, Online Material p 20

Table 4. continuation.

\begin{tabular}{|c|c|c|c|c|c|c|c|c|c|c|}
\hline F2 ID & $\mathrm{x}$ & $\mathrm{y}$ & $\mathrm{N}$ & $\mathrm{P}$ & $\mathrm{A}$ & $x^{2}$ & signif & $\mathrm{J}$ & $\mathrm{H}$ & $<K>$ \\
\hline 1588 & 887.266 & 609.134 & 22 & 476. & 0.129 & 58.10 & 0.080 & 21.88 & 20.69 & 20.41 \\
\hline 1600 & 93.163 & 244.983 & 24 & 180. & 0.421 & 7.00 & 0.220 & 21.88 & 20.87 & 20.28 \\
\hline 1605 & 203.969 & 493.424 & 24 & 436. & 0.538 & 2.50 & 0.020 & 21.89 & 20.69 & 20.46 \\
\hline 1613 & 848.538 & 389.540 & 24 & 392. & 0.346 & 4.80 & 0.100 & 22.40 & 21.42 & 20.28 \\
\hline 1616 & 101.122 & 391.106 & 24 & 274. & 0.277 & 3.30 & 0.200 & 21.54 & 20.70 & 20.28 \\
\hline 1617 & 571.782 & 6.244 & 24 & 408. & 0.250 & 4.00 & 0.160 & 22.36 & 21.21 & 20.27 \\
\hline 1622 & 541.392 & 443.704 & 24 & 298. & 0.396 & 4.20 & 0.040 & 22.05 & 20.94 & 20.41 \\
\hline 1623 & 787.079 & 288.645 & 24 & 449. & 0.305 & 13.10 & 0.460 & 21.92 & 21.17 & 20.42 \\
\hline 1631 & 679.773 & 105.185 & 24 & 349. & 0.326 & 3.40 & 0.100 & 21.69 & 20.75 & 20.25 \\
\hline 1645 & 49.166 & 103.181 & 24 & 388. & 0.600 & 2.20 & 0.050 & 21.41 & 20.56 & 20.43 \\
\hline 1683 & 677.682 & 104.014 & 24 & 302. & 0.442 & 6.40 & 0.270 & 21.56 & 20.71 & 20.57 \\
\hline 1685 & 370.284 & 103.559 & 24 & 428. & 0.358 & 13.00 & 0.320 & 22.26 & 21.52 & 20.30 \\
\hline 1690 & 702.089 & 302.619 & 24 & 281. & 0.220 & 4.50 & 0.380 & 21.75 & 20.90 & 20.27 \\
\hline 1692 & 239.523 & 661.270 & 24 & 300. & 0.320 & 3.10 & 0.110 & 21.79 & 20.64 & 20.44 \\
\hline 1697 & 887.821 & 314.828 & 22 & 323. & 0.330 & 4.90 & 0.510 & 21.48 & 20.70 & 20.24 \\
\hline 1700 & 131.430 & 614.878 & 24 & 460. & 0.142 & 34.50 & 0.080 & 21.78 & 20.90 & 20.43 \\
\hline 1703 & 186.144 & 267.475 & 24 & 345. & 0.448 & 4.60 & 0.230 & 21.93 & 21.01 & 20.54 \\
\hline 1709 & 377.356 & 658.374 & 24 & 451. & 0.623 & 12.20 & 0.200 & 22.51 & 21.79 & 20.61 \\
\hline 1710 & 711.522 & 550.778 & 24 & 294. & 0.296 & 4.00 & 0.200 & 21.93 & 20.88 & 20.39 \\
\hline 1728 & 184.289 & 636.129 & 24 & 381. & 0.593 & 4.40 & 0.050 & 21.76 & 20.81 & 20.49 \\
\hline 1729 & 534.104 & 395.756 & 24 & 381. & 0.551 & 5.90 & 0.030 & 22.28 & 22.22 & 20.56 \\
\hline 1730 & 541.524 & 536.047 & 24 & 224. & 0.398 & 10.00 & 0.280 & 22.65 & 21.55 & 20.50 \\
\hline 1731 & 341.025 & 304.310 & 24 & 314. & 0.268 & 4.40 & 0.220 & 21.39 & 20.74 & 20.37 \\
\hline 1735 & 830.730 & 41.451 & 24 & 262. & 0.226 & 4.80 & 0.660 & 21.50 & 20.72 & 20.33 \\
\hline 1736 & 40.346 & 627.771 & 24 & 193. & 0.247 & 5.20 & 0.240 & 22.55 & 21.26 & 20.29 \\
\hline 1749 & 832.748 & 178.959 & 24 & 261. & 0.319 & 3.70 & 0.210 & 21.17 & 20.41 & 20.39 \\
\hline 1776 & 337.907 & -7.156 & 16 & 370. & 0.607 & 11.10 & 0.660 & 24.74 & 22.21 & 20.51 \\
\hline 1784 & 711.917 & 559.903 & 24 & 298. & 0.330 & 2.00 & 0.030 & 21.95 & 20.99 & 20.36 \\
\hline 1794 & 491.605 & 75.748 & 23 & 419. & 0.488 & 4.00 & 0.020 & 22.87 & 21.76 & 20.43 \\
\hline 1795 & 405.954 & 409.103 & 24 & 188. & 0.394 & 10.60 & 0.410 & 22.01 & 20.87 & 20.41 \\
\hline 1799 & 575.492 & 194.880 & 24 & 170. & 0.231 & 5.80 & 0.240 & 22.14 & 20.99 & 20.33 \\
\hline 1803 & 786.644 & 606.898 & 24 & 431. & 0.405 & 5.30 & 0.080 & 21.49 & 20.58 & 20.40 \\
\hline 1805 & 359.442 & 396.001 & 24 & 294. & 0.356 & 4.30 & 0.090 & 21.35 & 20.46 & 20.46 \\
\hline 1809 & 849.471 & 740.460 & 24 & 345. & 0.386 & 3.30 & 0.060 & 21.67 & 20.85 & 20.19 \\
\hline 1817 & 679.902 & 51.165 & 20 & 272. & 0.283 & 4.20 & 0.560 & 21.45 & 20.48 & 20.43 \\
\hline 1820 & 780.108 & 653.670 & 24 & 332. & 0.225 & 5.30 & 0.400 & 22.21 & 21.27 & 20.32 \\
\hline 1825 & 273.977 & 402.602 & 24 & 310. & 0.424 & 2.30 & 0.040 & 21.24 & 20.47 & 20.47 \\
\hline 1834 & 113.048 & 406.136 & 24 & 318. & 0.229 & 3.60 & 0.200 & 21.70 & 20.93 & 20.28 \\
\hline 1837 & 359.384 & 756.684 & 24 & 396. & 0.501 & 4.40 & 0.100 & 21.80 & 20.85 & 20.36 \\
\hline 1841 & 278.849 & 8.182 & 24 & 408. & 0.366 & 3.50 & 0.060 & 21.69 & 20.66 & 20.32 \\
\hline 1852 & 219.645 & 704.799 & 24 & 445. & 0.592 & 3.90 & 0.030 & 22.12 & 20.74 & 20.61 \\
\hline 1863 & 395.169 & 623.677 & 24 & 298. & 0.428 & 1.60 & 0.020 & 21.26 & 20.42 & 20.47 \\
\hline 1868 & 135.708 & 343.444 & 24 & 243. & 0.258 & 5.80 & 0.250 & 21.48 & 20.52 & 20.36 \\
\hline 1870 & 843.018 & 828.104 & 23 & 313. & 0.370 & 4.50 & 0.370 & 21.66 & 20.81 & 20.54 \\
\hline 1871 & 870.020 & 231.107 & 23 & 165. & 0.279 & 4.00 & 0.260 & 21.91 & 20.98 & 20.36 \\
\hline 1880 & 847.714 & 649.539 & 24 & 330. & 0.364 & 2.50 & 0.040 & 22.10 & 20.99 & 20.33 \\
\hline 1882 & 512.132 & 412.280 & 24 & 286. & 0.248 & 2.80 & 0.090 & 21.77 & 20.86 & 20.35 \\
\hline 1884 & 599.866 & 473.719 & 24 & 444. & 0.617 & 3.80 & 0.020 & 23.10 & 22.07 & 20.71 \\
\hline 1909 & 506.830 & 444.480 & 24 & 300. & 0.402 & 2.50 & 0.050 & 21.54 & 20.54 & 20.52 \\
\hline 1914 & 554.137 & 80.880 & 24 & 322. & 0.309 & 2.10 & 0.020 & 21.42 & 20.73 & 20.39 \\
\hline 1915 & 598.682 & 641.232 & 24 & 323. & 0.436 & 2.50 & 0.040 & 22.08 & 21.05 & 20.56 \\
\hline 1917 & 583.137 & 658.359 & 24 & 461. & 0.192 & 49.80 & 0.250 & 22.23 & 21.03 & 20.49 \\
\hline 1922 & 54.686 & 283.433 & 24 & 309. & 0.333 & 5.30 & 0.500 & 21.72 & 21.02 & 20.52 \\
\hline 1936 & 421.521 & 282.357 & 24 & 673. & 0.688 & 5.60 & 0.240 & 99.99 & 22.47 & 20.43 \\
\hline 1952 & 683.717 & 606.454 & 24 & 265. & 0.298 & 1.80 & 0.040 & 21.42 & 20.59 & 20.49 \\
\hline 1954 & 430.979 & 26.005 & 24 & 272. & 0.317 & 4.50 & 0.320 & 21.83 & 20.90 & 20.39 \\
\hline 1955 & 293.224 & 671.359 & 24 & 494. & 0.575 & 2.70 & 0.030 & 99.99 & 22.95 & 20.49 \\
\hline 1956 & 740.211 & 45.215 & 24 & 296. & 0.411 & 3.40 & 0.060 & 21.89 & 20.68 & 20.60 \\
\hline 1983 & 77.576 & 340.094 & 24 & 191. & 0.262 & 8.40 & 0.880 & 21.98 & 20.97 & 20.36 \\
\hline 1997 & 724.672 & 477.335 & 24 & 513. & 0.623 & 2.20 & 0.110 & 22.79 & 21.33 & 20.76 \\
\hline
\end{tabular}


M. Rejkuba et al.: LPV catalogue in Cen A, Online Material p 21

Table 4. continuation.

\begin{tabular}{|c|c|c|c|c|c|c|c|c|c|c|}
\hline F2 ID & $\mathrm{x}$ & $\mathrm{y}$ & $\overline{\mathrm{N}}$ & $\mathrm{P}$ & $\overline{\mathrm{A}}$ & $\chi^{2}$ & signif & $\bar{J}$ & $\overline{\mathrm{H}}$ & $<K>$ \\
\hline$\overline{2002}$ & 108.682 & 335.946 & 24 & 278. & $\overline{0.309}$ & $\overline{5.60}$ & $\overline{0.460}$ & 21.78 & $\overline{21.02}$ & 20.39 \\
\hline 2006 & 0.785 & 06.280 & 24 & 286. & 347 & 2.30 & 0.020 & 21.75 & 20.79 & 20.47 \\
\hline 2010 & 75.337 & 30.635 & 24 & 291. & .282 & 2.90 & 0.080 & 21.38 & 20.53 & 20.43 \\
\hline 2011 & 65.858 & 722.627 & 24 & 341. & 0.338 & 1.40 & 0.010 & 22.11 & 21.00 & 20.39 \\
\hline 2016 & & 76.538 & 24 & 307. & 249 & 10.20 & 510 & 21.67 & 0.73 & 20.53 \\
\hline 2028 & 7.790 & 519.523 & 24 & 312. & 0.296 & 2.10 & 0.040 & 21.46 & 20.74 & 20.47 \\
\hline 2042 & 62.821 & 421.725 & 24 & 172. & 0.290 & 3.50 & 0.110 & 21.94 & 20.88 & 20.36 \\
\hline 2043 & 75.293 & 718.491 & 24 & 378. & 0.419 & 1.80 & 0.020 & 21.68 & 20.79 & 20.50 \\
\hline 2047 & 404.297 & 72.172 & 24 & 290. & 0.321 & 3.80 & 0.050 & 21.71 & 20.78 & 20.53 \\
\hline 2053 & 00.940 & 249.853 & 24 & 292. & 0.270 & 4.80 & .170 & 21.81 & 20.79 & 20.51 \\
\hline 2089 & 56.694 & 173.340 & 24 & 306. & 0.331 & 4.00 & 0.210 & 21.27 & 20.52 & 20.33 \\
\hline 2097 & 9.588 & 266.447 & 24 & 178. & 0.495 & 10.20 & 0.260 & 21.80 & 20.90 & 20.45 \\
\hline 2100 & 56.260 & 252.061 & 24 & 450. & 0.097 & 36.00 & 0.300 & 22.37 & 21.12 & 20.47 \\
\hline 2104 & 3.1 & 364.457 & 24 & 272. & 365 & 2.00 & 050 & 21.61 & 0.93 & 20.50 \\
\hline 2109 & 57 & 8.619 & 24 & 164. & 201 & .40 & 740 & 21.58 & 20.44 & 20.37 \\
\hline 2111 & 40.356 & 523.504 & 24 & 330. & 0.332 & 6.10 & 0.360 & 21.44 & 20.80 & 20.35 \\
\hline 2112 & 6.300 & 208.554 & 24 & 290. & 0.264 & 1.70 & 0.040 & 21.94 & 21.14 & 20.47 \\
\hline 2115 & 468.654 & 668.942 & 24 & 298. & 0.385 & 3.10 & 0.050 & 21.86 & 21.06 & 20.61 \\
\hline 2116 & 8 & 47.312 & 24 & 288. & 341 & 3.20 & .220 & 21.41 & 0.55 & 20.51 \\
\hline 2128 & 651.229 & 824.181 & 24 & 290. & 0.339 & 2.90 & 0.040 & 21.83 & 21.06 & 20.34 \\
\hline 2130 & & 456.508 & 24 & 303. & & 6.70 & 0.230 & 21.43 & 20.56 & 20.47 \\
\hline 2131 & 26 & 701.2 & 24 & 496. & 0.618 & 4.60 & 0.030 & 22.45 & 21.17 & 20.71 \\
\hline 2139 & & & 24 & 292. & & 40 & & 21.94 & 87 & 20.49 \\
\hline 2143 & & & 24 & 342. & 360 & 8.60 & & 21.84 & 21.20 & 20.25 \\
\hline 2144 & 96 & & 24 & 283. & 0.292 & 3.50 & 0.180 & 21.65 & 20.97 & 20.39 \\
\hline 2145 & 50 & 96 & 24 & 292. & 69 & 6.50 & & 21.28 & 20.54 & 20.62 \\
\hline 2147 & 31 & 15 & 24 & 269. & 52 & 2.70 & & 21.54 & 20.73 & 20.48 \\
\hline 2149 & & & 24 & 36 & & & & 21.92 & 20.95 & 20.36 \\
\hline 2151 & 27 & 52 & 24 & 380. & 473 & 5.70 & 0.040 & 22.32 & 21.72 & 20.51 \\
\hline 2159 & & & 24 & 278. & & 2.80 & & 21.59 & & 20.49 \\
\hline 60 & & & 24 & 269. & & 2.00 & & .81 & 0.88 & 20.43 \\
\hline 2193 & & & 24 & 41 & 225 & & & 22.24 & 21.20 & 20.41 \\
\hline 2194 & & & 24 & 353. & 412 & 7.00 & 0.290 & 21.84 & 21.07 & 20.28 \\
\hline 2200 & & & 24 & 277. & 0.292 & 4.60 & 0.260 & 21.48 & 20.67 & 20.57 \\
\hline 2222 & & & 24 & 296. & & .70 & & 22.40 & & 20.77 \\
\hline 2228 & & & 24 & 272. & & 2. & & 21.40 & & 20.59 \\
\hline 2231 & & 99 & 24 & 355. & & 1.50 & 0.020 & 21.71 & 20.87 & 20.42 \\
\hline 2233 & & 244.348 & 24 & 296. & & 1.30 & 0.020 & 22.17 & 21.24 & 20.64 \\
\hline 2240 & & & 24 & 297. & & & & & & 20.54 \\
\hline 2247 & & & 24 & 400. & & 2.40 & & 22.52 & & 20.40 \\
\hline 2255 & & & 24 & 378. & & 4.00 & & 21.88 & 20.86 & 20.31 \\
\hline 2272 & & & 24 & 310. & 0.071 & 4.00 & 0.900 & 21.66 & 21.01 & 20.39 \\
\hline 2273 & & & 24 & 299. & & & & 21.91 & & 20.48 \\
\hline 2285 & -0 & 45 & 24 & 279. & & & & 21.81 & & 20.59 \\
\hline & & & 24 & 17 & & 4.30 & & 21.98 & 07 & 20.51 \\
\hline 2291 & & & 24 & 284. & & 2.40 & & 21.58 & & 20.56 \\
\hline & & & 24 & 292. & & & & & & 20.57 \\
\hline 2307 & 3 & 6 & 24 & 241. & & 3.80 & & 21.68 & & 20.48 \\
\hline & & & 24 & 312. & & 5.80 & & 21.66 & 20.97 & 20.50 \\
\hline 2312 & 53 & & 24 & 272. & 0.310 & 2.20 & 0.060 & 21.98 & 21.01 & 20.54 \\
\hline 2314 & & & 24 & 294. & & 6.10 & & 21.64 & 20.74 & 20.58 \\
\hline 2318 & & & 23 & 501. & & 2.30 & & 23.58 & 21.24 & 20.75 \\
\hline 2320 & & & 24 & 264. & & 10.20 & 0.630 & 21.85 & 21.02 & 20.58 \\
\hline & & & 24 & 291. & & & & 21.58 & & 20.42 \\
\hline 2324 & & & 24 & 556. & & 3.70 & 0.220 & 22.56 & 21.26 & 20.89 \\
\hline 2335 & & & 24 & 310. & & 4.50 & & 21.95 & 21.18 & 20.47 \\
\hline & 466.8 & 25 & 23 & 625. & & 9.00 & 0.240 & 22.97 & 21.64 & 20.82 \\
\hline & & & 18 & 54. & & 6.90 & & 99.99 & 21.61 & 20.82 \\
\hline & & & 24 & 527. & & 3.70 & 0.020 & 23.81 & 22.99 & 20.76 \\
\hline 2371 & 409.452 & 459.005 & 24 & 345. & 0.597 & 5.00 & 0.240 & 22.08 & 21.11 & 20.89 \\
\hline
\end{tabular}


Table 4. continuation.

\begin{tabular}{|c|c|c|c|c|c|c|c|c|c|c|}
\hline $\bar{F} 2$ ID & $\mathrm{x}$ & $\mathrm{y}$ & $\mathrm{N}$ & $\overline{\mathrm{P}}$ & $\overline{\mathrm{A}}$ & $\chi^{2}$ & signif & $\overline{\mathrm{J}}$ & $\overline{\mathrm{H}}$ & $<K>$ \\
\hline$\overline{2372}$ & 145.677 & 37.058 & $\overline{24}$ & 276. & 0.306 & $\bar{~} 6.70$ & 0.510 & 21.70 & 20.83 & 20.51 \\
\hline 2380 & 708.827 & 734.763 & 24 & 337. & 0.478 & 3.40 & 0.130 & 22.22 & 21.43 & 20.31 \\
\hline 2395 & 574.495 & 153.833 & 24 & 310. & 0.315 & 6.10 & 0.550 & 21.62 & 20.87 & 20.42 \\
\hline 2414 & 669.380 & 466.236 & 24 & 326. & 0.314 & 3.90 & 0.160 & 22.18 & 21.39 & 20.43 \\
\hline 2421 & 383.677 & 511.695 & 24 & 289. & 0.267 & 2.40 & 0.060 & 21.69 & 21.14 & 20.47 \\
\hline 2424 & 637.782 & 571.600 & 24 & 295. & 0.466 & 3.70 & 0.060 & 21.52 & 20.97 & 20.63 \\
\hline 2427 & 319.817 & 513.646 & 24 & 299. & 0.397 & 3.10 & 0.040 & 21.55 & 20.98 & 20.60 \\
\hline 2428 & 698.016 & 398.013 & 24 & 350. & 0.472 & 1.20 & 0.040 & 22.35 & 20.98 & 20.40 \\
\hline 2436 & 643.429 & 672.785 & 23 & 294. & 0.340 & 5.70 & 0.130 & 22.06 & 21.22 & 20.58 \\
\hline 2438 & 860.170 & 34.060 & 23 & 165. & 0.305 & 4.60 & 0.220 & 22.03 & 21.10 & 20.59 \\
\hline 2447 & 232.207 & 4.549 & 24 & 286. & 0.539 & 5.50 & 0.110 & 21.50 & 20.74 & 20.78 \\
\hline 2451 & 501.540 & 825.126 & 23 & 314. & 0.465 & 3.00 & 0.130 & 22.28 & 21.42 & 20.70 \\
\hline 2466 & 377.006 & 64.798 & 24 & 262. & 0.262 & 5.60 & 0.490 & 22.05 & 21.11 & 20.55 \\
\hline 2467 & 133.825 & 116.259 & 24 & 177. & 0.403 & 6.40 & 0.230 & 22.01 & 21.14 & 20.52 \\
\hline 2482 & 488.647 & 486.885 & 24 & 300. & 0.527 & 6.50 & 0.220 & 21.39 & 20.74 & 20.74 \\
\hline 2487 & 647.817 & 43.051 & 24 & 294. & 0.342 & 5.40 & 0.450 & 22.03 & 21.43 & 20.58 \\
\hline 2488 & 565.277 & 368.955 & 24 & 510. & 0.635 & 3.50 & 0.090 & 24.61 & 22.21 & 20.61 \\
\hline 2489 & 847.263 & 472.621 & 24 & 307. & 0.483 & 2.50 & 0.030 & 22.27 & 21.54 & 20.50 \\
\hline 2497 & 24.327 & 491.417 & 23 & 312. & 0.348 & 5.30 & 0.350 & 21.83 & 21.08 & 20.44 \\
\hline 2502 & 23.400 & 631.599 & 23 & 366. & 0.401 & 6.60 & 0.280 & 22.21 & 20.86 & 20.42 \\
\hline 2506 & 85.337 & 339.939 & 24 & 307. & 0.233 & 5.20 & 0.530 & 21.58 & 20.81 & 20.55 \\
\hline 2515 & 542.975 & 765.505 & 24 & 294. & 0.395 & 3.10 & 0.040 & 21.81 & 21.13 & 20.45 \\
\hline 2517 & 430.972 & 423.967 & 24 & 518. & 0.558 & 3.20 & 0.020 & 22.80 & 23.16 & 20.73 \\
\hline 2519 & 870.694 & 395.502 & 23 & 503. & 0.738 & 5.20 & 0.050 & 26.27 & 22.64 & 20.96 \\
\hline 2530 & 112.241 & 590.792 & 24 & 254. & 0.442 & 3.70 & 0.080 & 21.52 & 20.71 & 20.74 \\
\hline 2549 & 447.949 & 58.256 & 24 & 395. & 0.481 & 3.30 & 0.770 & 22.57 & 22.08 & 20.41 \\
\hline 2554 & 629.672 & 131.178 & 24 & 605. & 0.281 & 24.70 & 0.670 & 99.99 & 24.53 & 20.52 \\
\hline 2567 & 503.912 & 145.854 & 24 & 307. & 0.304 & 8.30 & 0.980 & 21.89 & 21.16 & 20.49 \\
\hline 2590 & 817.535 & 94.909 & 24 & 262. & 0.306 & 7.00 & 0.800 & 21.67 & 21.12 & 20.59 \\
\hline 2591 & 674.688 & 425.903 & 24 & 254. & 0.258 & 5.10 & 0.270 & 21.56 & 20.93 & 20.57 \\
\hline 2593 & 723.163 & 474.360 & 24 & 244. & 0.297 & 4.60 & 0.130 & 21.91 & 21.03 & 20.61 \\
\hline 2623 & 828.670 & 837.169 & 21 & 47. & 0.334 & 12.30 & 0.980 & 22.32 & 21.17 & 20.61 \\
\hline 2645 & 79.945 & 365.099 & 24 & 337. & 0.389 & 2.20 & 0.060 & 22.37 & 21.25 & 20.47 \\
\hline 2654 & 642.679 & 572.352 & 24 & 320. & 0.355 & 3.50 & 0.100 & 22.09 & 21.55 & 20.49 \\
\hline 2665 & 159.258 & 341.052 & 24 & 278. & 0.385 & 3.40 & 0.060 & 21.65 & 20.82 & 20.69 \\
\hline 2667 & 641.325 & 564.165 & 24 & 272. & 0.346 & 2.30 & 0.060 & 21.82 & 20.79 & 20.68 \\
\hline 2669 & 241.936 & 58.066 & 24 & 170. & 0.222 & 9.40 & 0.910 & 21.98 & 21.15 & 20.53 \\
\hline 2689 & 151.908 & 256.661 & 24 & 276. & 0.444 & 3.00 & 0.030 & 22.02 & 21.22 & 20.77 \\
\hline 2691 & 308.731 & 775.795 & 24 & 339. & 0.487 & 6.00 & 0.510 & 22.02 & 21.55 & 20.44 \\
\hline 2695 & 595.973 & 699.644 & 24 & 281. & 0.405 & 5.50 & 0.110 & 21.80 & 20.96 & 20.75 \\
\hline 2696 & 155.024 & 109.834 & 24 & 264. & 0.265 & 10.10 & 0.950 & 21.72 & 20.94 & 20.63 \\
\hline 2712 & 46.154 & 364.506 & 24 & 253. & 0.415 & 9.00 & 0.210 & 21.65 & 21.12 & 20.71 \\
\hline 2719 & 182.164 & 425.840 & 24 & 286. & 0.409 & 5.30 & 0.090 & 21.44 & 20.72 & 20.71 \\
\hline 2722 & 892.692 & 803.489 & 14 & 268. & 0.712 & 6.60 & 0.750 & 22.05 & 19.99 & 21.07 \\
\hline 2738 & 515.265 & 863.676 & 12 & 386. & 0.663 & 4.70 & 0.830 & 99.99 & 99.99 & 20.28 \\
\hline 2760 & 169.819 & 228.192 & 24 & 312. & 0.348 & 4.70 & 0.670 & 21.59 & 21.44 & 20.61 \\
\hline 2763 & 878.699 & 393.997 & 22 & 300. & 0.314 & 3.90 & 0.300 & 22.11 & 21.37 & 20.49 \\
\hline 2766 & 118.033 & 820.844 & 24 & 302. & 0.333 & 5.20 & 0.490 & 22.38 & 21.37 & 20.72 \\
\hline 2800 & 296.371 & 123.840 & 24 & 448. & 0.290 & 6.10 & 0.570 & 21.77 & 21.08 & 20.68 \\
\hline 2818 & 696.376 & 337.355 & 24 & 276. & 0.531 & 2.70 & 0.220 & 22.08 & 21.13 & 20.92 \\
\hline 2820 & 827.596 & 836.270 & 22 & 274. & 0.495 & 18.80 & 0.990 & 21.61 & 20.88 & 20.81 \\
\hline 2821 & 809.613 & 297.404 & 24 & 165. & 0.349 & 11.60 & 0.440 & 21.48 & 21.11 & 20.63 \\
\hline 2827 & 567.302 & 271.132 & 23 & 213. & 0.504 & 5.00 & 0.520 & 22.01 & 20.95 & 20.61 \\
\hline 2836 & 574.932 & 643.524 & 24 & 242. & 0.410 & 1.90 & 0.050 & 21.60 & 20.76 & 20.75 \\
\hline 2840 & 849.378 & 27.277 & 23 & 283. & 0.320 & 8.70 & 0.560 & 22.19 & 21.62 & 20.64 \\
\hline 2861 & 220.957 & 500.059 & 24 & 314. & 0.335 & 3.30 & 0.420 & 21.61 & 21.08 & 20.51 \\
\hline 2872 & 70.099 & 269.646 & 24 & 299. & 0.295 & 5.00 & 0.380 & 21.97 & 21.16 & 20.52 \\
\hline 2877 & 768.979 & 122.517 & 24 & 298. & 0.482 & 3.40 & 0.030 & 22.23 & 21.46 & 20.67 \\
\hline 2916 & 649.996 & 832.180 & 24 & 246. & 0.352 & 2.50 & 0.040 & 21.83 & 20.99 & 20.70 \\
\hline 2938 & 783.337 & 462.572 & 24 & 244. & 0.420 & 4.70 & 0.150 & 21.61 & 20.94 & 20.80 \\
\hline
\end{tabular}


M. Rejkuba et al.: LPV catalogue in Cen A, Online Material p 23

Table 4. continuation.

\begin{tabular}{|c|c|c|c|c|c|c|c|c|c|c|}
\hline F2 ID & $\mathrm{x}$ & $\mathrm{y}$ & $\overline{\mathrm{N}}$ & $\mathrm{P}$ & $\overline{\mathrm{A}}$ & $\overline{\chi^{2}}$ & signif & $\bar{J}$ & $\overline{\mathrm{H}}$ & $<K>$ \\
\hline 2942 & $\overline{403.046}$ & 806.695 & 24 & 291. & $\overline{0.311}$ & 2.70 & $\overline{0.060}$ & 21.70 & $\overline{21.01}$ & $\overline{20.66}$ \\
\hline 2960 & 67.599 & 15.115 & 23 & 370. & 506 & 3.60 & 0.280 & 22.02 & 1.10 & 20.56 \\
\hline 2964 & 15.637 & 137.195 & 24 & 467. & .423 & 4.00 & 0.080 & 22.35 & 21.31 & 20.73 \\
\hline 2966 & 92.007 & 641.779 & 24 & 440. & .564 & 2.20 & 0.030 & 23.77 & 22.62 & 20.94 \\
\hline 2981 & & & 24 & 289. & 268 & 3.30 & 590 & 21.96 & 1.20 & 20.61 \\
\hline 2988 & 1.439 & 630.638 & 24 & 254. & 0.284 & 2.40 & 0.340 & 21.71 & 20.79 & 20.74 \\
\hline 2998 & 07.871 & 427.580 & 24 & 297. & 0.339 & 6.40 & 0.350 & 22.03 & 21.47 & 20.59 \\
\hline 3021 & 07.149 & 169.294 & 24 & 350. & 0.270 & 5.60 & 0.960 & 22.25 & 21.43 & 20.61 \\
\hline 3042 & 82.359 & 678.613 & 24 & 456. & 0.538 & 9.70 & 0.080 & 22.67 & 22.09 & 20.91 \\
\hline 3048 & 87.507 & 56.560 & 24 & 239. & 306 & 2.90 & .130 & 21.67 & 21.14 & 20.69 \\
\hline 3059 & .935 & 645.011 & 24 & 241. & .386 & 4.00 & .250 & 21.60 & 20.69 & 20.81 \\
\hline 3116 & 98.267 & 39.442 & 24 & 264. & 0.419 & 3.70 & 0.070 & 21.49 & 20.82 & 20.76 \\
\hline 3118 & 31.157 & 843.8 & 23 & 278. & 0.432 & 3.60 & 0.070 & 22.09 & 21.31 & 20.88 \\
\hline 3121 & 07 & 260.865 & 24 & 273. & 444 & 4.40 & 140 & 22.03 & 21.01 & 20.90 \\
\hline 3152 & & 50.324 & 24 & 250. & & 6.00 & 310 & 21.75 & 0.82 & 20.81 \\
\hline 3159 & 96 & 25.273 & 23 & 303. & 0.338 & 9.90 & 0.970 & 21.78 & 21.05 & 20.82 \\
\hline 3167 & 97.800 & 834.029 & 24 & 192. & 0.329 & 2.10 & 0.220 & 21.59 & 20.68 & 20.72 \\
\hline 3169 & 545.314 & 695.390 & 24 & 237. & 0.291 & 4.50 & 0.250 & 22.03 & 21.04 & 20.75 \\
\hline 3173 & & 49 & 24 & 291. & & 6.10 & 710 & 21.99 & 1.21 & 20.64 \\
\hline 3201 & 73 & 34 & 24 & 242. & 347 & 11.50 & 0.920 & 24.05 & 22.90 & 20.68 \\
\hline 3231 & & 36.689 & 24 & 282. & & 4.20 & 0.310 & 22.21 & 1.35 & 20.68 \\
\hline 3238 & 1 & 222.6 & 24 & 288. & 12 & 4.00 & 0.260 & 21.65 & 20.76 & 20.76 \\
\hline & & & 24 & 828. & & 3 & & 22.08 & 1.19 & 20.72 \\
\hline 3364 & & & 24 & 264. & & 3.30 & 960 & 21.96 & 21.19 & 20.87 \\
\hline 3366 & 36 & 01 & 24 & 209. & & 2.20 & 0.200 & 22.17 & 21.40 & 20.81 \\
\hline 3378 & 95 & 09 & 24 & 259. & 71 & 21.30 & 480 & 21.89 & 21.15 & 20.72 \\
\hline 3390 & 16 & 971 & 24 & 232. & 436 & 2.40 & 0.060 & 21.90 & 21.10 & 20.88 \\
\hline & & & 23 & 274. & & 2.00 & & 22.56 & & 20.93 \\
\hline 3438 & & & 14 & 44. & & 3.20 & 1.000 & 21.25 & 0.64 & 20.98 \\
\hline 3460 & 32 & 5 & 24 & 228. & & 2.80 & 0.050 & 21.93 & & 20.99 \\
\hline 3474 & & & 24 & 262. & & 4.80 & & 22.30 & & 20.87 \\
\hline 91 & & & 24 & 28 & & 7.0 & & 22.01 & 30 & 20.90 \\
\hline 3509 & & & 19 & 456. & 732 & 8.50 & 0.520 & 23.75 & 99 & 21.11 \\
\hline 3519 & & & 24 & 234. & & 3.20 & & 21.70 & 21.03 & 20.90 \\
\hline 3524 & & & 24 & 271. & & & & 21.63 & & 20.88 \\
\hline & & & 24 & 249. & & 2.90 & & 21.83 & & 20.90 \\
\hline 3610 & & & 24 & 269. & & 3.60 & & 22.50 & & 20.86 \\
\hline 3622 & & 33 & 24 & 155. & & 3.80 & 0.160 & 21.76 & & 20.81 \\
\hline & & & 23 & 193. & & & & & & 20.88 \\
\hline 3668 & & & 24 & 261. & & 5.20 & & 21.83 & & 20.91 \\
\hline 3679 & & & 24 & 129. & 261 & .00 & & 22.30 & 1.78 & 20.88 \\
\hline 3682 & & & 24 & 302. & & 8.70 & & 21.96 & 21.37 & 20.83 \\
\hline 3712 & & & 24 & 231. & & & & 21.87 & & 20.96 \\
\hline 3722 & 9.2 & & 22 & 262. & & 4 & & 22.23 & & 20.81 \\
\hline & & & 24 & 203. & & & 0.8 & 21.96 & 21.18 & 20.78 \\
\hline 3792 & & & 24 & 247. & & 4.00 & & 21.73 & 21.03 & 20.95 \\
\hline & & & 24 & 254. & & & & & & 20.91 \\
\hline 3848 & & 45 & 24 & 260. & & 5.20 & & 22.38 & 21.77 & 20.91 \\
\hline & & & 24 & 254. & & 2.50 & 0.030 & 22.41 & 21.44 & 21.04 \\
\hline & & & 24 & 236. & & 2.70 & 0.060 & 22.15 & 21.35 & 20.96 \\
\hline 3914 & & & 24 & 476. & & 1.60 & & 23.08 & 22.28 & 20.89 \\
\hline 3928 & 790.322 & & 24 & 218. & & 4.30 & 0.400 & 21.65 & 21.14 & 20.84 \\
\hline & & 2 & 24 & 231. & & 5.20 & 0.680 & 21.72 & 21.00 & 20.98 \\
\hline & & & 24 & 276. & & & & & 20.98 & 20.99 \\
\hline 4017 & & & 24 & 245. & & 4.10 & 0.560 & 22.27 & 21.58 & 20.94 \\
\hline 4034 & & 229.530 & 24 & 259. & & 4.40 & 0.440 & 21.81 & 21.24 & 20.91 \\
\hline 4035 & & 30.280 & 24 & 214. & & 3.30 & 0.4 & 22.27 & 21.47 & 20.99 \\
\hline & & & 24 & 280. & & 1.90 & 0.090 & 21.91 & 21.01 & 20.92 \\
\hline 4054 & & 422.046 & 18 & 403. & & 12.40 & 1.000 & 22.42 & 21.95 & 21.22 \\
\hline 4097 & 526.847 & 688.469 & 24 & 243. & 0.042 & 13.40 & 0.090 & 22.17 & 21.33 & 20.87 \\
\hline
\end{tabular}


M. Rejkuba et al.: LPV catalogue in Cen A, Online Material p 24

Table 4. continuation.

\begin{tabular}{|c|c|c|c|c|c|c|c|c|c|c|}
\hline F2 ID & $\mathrm{x}$ & $\mathrm{y}$ & $\mathrm{N}$ & $\mathrm{P}$ & $\mathrm{A}$ & $\chi^{2}$ & signif & $\mathrm{J}$ & $\mathrm{H}$ & $<K>$ \\
\hline 4182 & 728.664 & 434.580 & 24 & 223. & 0.049 & 18.80 & 0.400 & 22.61 & 21.95 & $\overline{21.00}$ \\
\hline 4196 & 162.064 & 292.952 & 22 & 135. & 0.340 & 10.40 & 0.880 & 21.94 & 21.19 & 21.02 \\
\hline 4272 & 126.101 & 446.682 & 20 & 132. & 0.557 & 9.50 & 1.000 & 21.32 & 21.11 & 21.09 \\
\hline 4277 & 520.737 & 226.877 & 24 & 455. & 0.591 & 3.30 & 0.040 & 23.76 & 22.99 & 21.13 \\
\hline 4291 & 124.064 & 247.886 & 24 & 239. & 0.340 & 4.50 & 0.230 & 22.28 & 21.74 & 20.98 \\
\hline 4298 & 367.091 & 62.301 & 24 & 240. & 0.323 & 3.40 & 0.110 & 22.18 & 21.32 & 21.01 \\
\hline 4394 & 173.995 & 551.437 & 24 & 225. & 0.337 & 6.60 & 0.210 & 22.68 & 21.77 & 21.05 \\
\hline 4487 & 371.452 & 94.176 & 23 & 259. & 0.307 & 10.60 & 0.480 & 22.51 & 21.71 & 20.94 \\
\hline 4678 & 703.973 & 513.781 & 15 & 50. & 1.027 & 12.60 & 0.860 & 99.99 & 22.84 & 21.27 \\
\hline 4686 & 68.814 & 80.933 & 24 & 303. & 0.481 & 3.90 & 0.510 & 22.45 & 22.45 & 20.87 \\
\hline 4707 & 409.992 & 310.652 & 24 & 301. & 0.304 & 6.90 & 0.540 & 21.87 & 21.09 & 21.04 \\
\hline 4762 & 715.893 & 492.961 & 12 & 165. & 1.206 & 26.70 & 1.000 & 23.14 & 22.01 & 21.27 \\
\hline 4812 & 56.235 & 588.293 & 24 & 312. & 0.531 & 7.90 & 0.800 & 99.99 & 21.69 & 21.26 \\
\hline 4818 & 36.928 & 345.232 & 22 & 222. & 0.431 & 4.40 & 0.430 & 22.59 & 21.79 & 21.07 \\
\hline 4844 & 790.515 & 407.132 & 23 & 568. & 0.619 & 3.00 & 0.130 & 23.93 & 23.03 & 21.37 \\
\hline 4869 & 817.172 & 410.033 & 24 & 218. & 0.423 & 2.10 & 0.120 & 22.48 & 21.55 & 21.01 \\
\hline 4950 & 51.475 & 586.850 & 24 & 181. & 0.401 & 4.40 & 0.340 & 21.75 & 21.11 & 21.22 \\
\hline 5022 & 26.351 & 18.085 & 19 & 331. & 0.560 & 5.60 & 0.750 & 22.61 & 99.99 & 20.97 \\
\hline 5045 & 823.415 & 300.274 & 24 & 193. & 0.474 & 2.50 & 0.580 & 22.38 & 21.18 & 21.21 \\
\hline 5060 & .263 & 194.560 & 20 & 493. & 0.596 & 5.60 & 0.720 & 22.05 & 21.11 & 21.04 \\
\hline 5161 & 108.786 & 382.722 & 22 & 578. & 0.915 & 3.40 & 0.080 & 99.99 & 99.99 & 21.32 \\
\hline 5189 & 6.480 & 86.847 & 20 & 282. & 0.757 & 6.00 & 0.650 & 22.19 & 21.58 & 21.54 \\
\hline 5259 & 14.804 & 365.482 & 24 & 448. & 0.664 & 2.50 & 0.080 & 24.75 & 22.21 & 21.21 \\
\hline 5378 & 837.762 & 732.250 & 23 & 627. & 0.787 & 5.00 & 0.160 & 99.99 & 99.99 & 21.29 \\
\hline 5595 & 575.631 & 243.740 & 24 & 205. & 0.409 & 2.20 & 0.190 & 22.46 & 22.42 & 21.20 \\
\hline 5615 & 690.317 & 88.923 & 24 & 511. & 0.340 & 3.3 & 0.910 & 23.25 & 22.71 & 21.02 \\
\hline 5635 & 811.716 & 705.519 & 24 & 180. & 0.408 & 2.80 & 0.140 & 22.44 & 21.89 & 21.21 \\
\hline 5769 & .150 & 435.687 & 18 & 66. & 0.770 & 8.30 & 0.880 & 99.99 & 21.63 & 21.45 \\
\hline 5770 & 179.661 & 481.922 & 24 & 185. & 0.333 & 4.30 & 0.460 & 22.20 & 21.21 & 21.16 \\
\hline 5897 & 198.408 & 281.172 & 24 & 188. & 0.364 & 4.30 & 0.940 & 22.48 & 21.79 & 21.29 \\
\hline 5898 & 72.087 & 743.065 & 20 & 244. & 0.110 & 27.90 & 0.770 & 23.50 & 22.24 & 21.41 \\
\hline 6190 & 773.927 & 576.531 & 22 & 308. & 0.726 & 5.10 & 0.320 & 21.99 & 21.78 & 21.36 \\
\hline 6534 & 462.763 & 507.712 & 17 & 48. & 0.864 & 5.10 & 1.000 & 99.99 & 23.05 & 21.33 \\
\hline 6837 & 121.917 & 845.695 & 22 & 191. & 0.427 & 2.80 & 0.750 & 22.88 & 22.37 & 21.46 \\
\hline 6887 & 775.775 & 569.576 & 16 & 89. & 0.936 & 8.80 & 0.920 & 21.94 & 23.05 & 21.83 \\
\hline 7177 & 699.070 & 826.615 & 20 & 421. & 0.423 & 15.10 & 0.960 & 99.99 & 22.60 & 21.61 \\
\hline 7294 & 691.671 & 48.960 & 24 & 57. & 0.374 & 7.40 & 1.000 & 22.55 & 21.54 & 21.43 \\
\hline 7602 & 731.358 & 506.045 & 13 & 225. & 1.315 & 6.20 & 0.960 & 23.88 & 22.93 & 21.83 \\
\hline 7887 & & 684.0 & 20 & 213. & 0.607 & 8.60 & 0.950 & 22.22 & 21.25 & 21.54 \\
\hline 8718 & 595.039 & 198.886 & 19 & 209. & 0.759 & 3.90 & 0.760 & 99.99 & 21.94 & 21.45 \\
\hline 8837 & 204.386 & 427.624 & 11 & 105. & 0.693 & 54.80 & 1.000 & 24.17 & 99.99 & 22.13 \\
\hline 9299 & 579.266 & 356.438 & 12 & 219. & 1.096 & 3.80 & 1.000 & 22.54 & 22.89 & 22.01 \\
\hline 9668 & & -9.773 & 11 & 33. & 1.029 & 7.30 & 1.000 & 23.23 & 22.32 & 21.99 \\
\hline 10203 & 520.379 & 821.164 & 19 & 54. & 0.643 & 6.30 & 1.000 & 22.46 & 21.95 & 21.85 \\
\hline 11139 & 189.627 & 236.601 & 21 & 41. & 0.505 & 6.30 & 1.000 & 22.59 & 21.90 & 21.94 \\
\hline 12041 & 807.046 & 508.901 & 20 & 34. & 0.397 & 2.50 & 0.990 & 23.10 & 22.05 & 22.15 \\
\hline 12101 & 576.615 & 311.540 & 19 & 34. & 0.691 & 6.20 & 0.930 & 23.35 & 22.38 & 22.27 \\
\hline 12881 & & 266.481 & 17 & 92. & 0.758 & 9.90 & 0.990 & 22.82 & 22.71 & 22.37 \\
\hline 13468 & 253.199 & 381.898 & 16 & 48. & 0.678 & 4.40 & 1.000 & 23.85 & 23.11 & 22.32 \\
\hline
\end{tabular}


M. Rejkuba et al.: LPV catalogue in Cen A, Online Material p 25

Table 5. Additional variable stars for which periods could not be determined in Field 1. The columns from left to right list: star ID, $\mathrm{x}$ and $\mathrm{y}$ position with respect to the reference epoch, single epoch $J$ and $H$-band magnitudes and mean $K$-band magnitude. Magnitude 99.99 denotes that no measurement for that star was obtained.

\begin{tabular}{|c|c|c|c|c|c|c|}
\hline F1 ID & $x$ & $\mathrm{y}$ & $\mathrm{N}$ & $\mathrm{J}$ & $\mathrm{H}$ & $<K>$ \\
\hline 50 & $\overline{7763.941}$ & $\overline{462.473}$ & 20 & 19.70 & $\overline{18.76}$ & 18.23 \\
\hline 51 & 219.227 & 79.584 & 20 & 18.48 & 18.02 & 17.97 \\
\hline 69 & 113.406 & 403.575 & 12 & 99.99 & 99.99 & 21.80 \\
\hline 75 & 31.879 & 859.684 & 9 & 20.33 & 99.99 & 18.94 \\
\hline 76 & 48.965 & 862.669 & 7 & 19.58 & 99.99 & 19.16 \\
\hline 78 & 47.858 & 863.782 & 9 & 99.99 & 99.99 & 18.67 \\
\hline 101 & 792.787 & 471.547 & 20 & 20.91 & 19.87 & 19.11 \\
\hline 122 & 450.363 & 778.633 & 20 & 20.57 & 19.60 & 19.43 \\
\hline 132 & 610.861 & 804.691 & 13 & 99.99 & 99.99 & 20.19 \\
\hline 135 & 737.241 & 875.358 & 5 & 99.99 & 99.99 & 19.82 \\
\hline 158 & 41.740 & -29.076 & 8 & 99.99 & 21.26 & 20.14 \\
\hline 215 & 709.023 & 852.736 & 12 & 20.63 & 99.99 & 20.24 \\
\hline 220 & 129.748 & -29.810 & 12 & 99.99 & 20.36 & 20.16 \\
\hline 225 & 302.868 & 720.473 & 20 & 21.16 & 20.05 & 20.01 \\
\hline 251 & 138.639 & 691.085 & 20 & 22.27 & 20.62 & 19.84 \\
\hline 256 & 394.394 & -0.704 & 19 & 20.84 & 20.42 & 19.82 \\
\hline 282 & 460.999 & 864.607 & 9 & 99.99 & 99.99 & 19.89 \\
\hline 292 & 15.904 & 193.015 & 12 & 22.10 & 20.87 & 19.91 \\
\hline 365 & 518.253 & 737.2 & 20 & 21.32 & 20.57 & 20.01 \\
\hline 376 & 87.118 & 92.5 & 20 & 21.45 & 20.38 & 21.23 \\
\hline 414 & 106.809 & 397.028 & 20 & 21.62 & 20.95 & 20.06 \\
\hline 416 & 701.510 & 153.983 & 20 & 21.14 & 20.41 & 20.02 \\
\hline 554 & 41.029 & 357.6 & 20 & 23.97 & 22.87 & 20.44 \\
\hline 586 & 477.7 & 525.6 & 20 & 23.61 & 21.64 & 20.30 \\
\hline 626 & 192.645 & 54.7 & 15 & 99.99 & 22.27 & 20.25 \\
\hline 629 & 186.363 & 76.196 & 18 & 21.60 & 20.77 & 20.29 \\
\hline 631 & 153.405 & -12.272 & 17 & 21.36 & 20.60 & 20.29 \\
\hline 653 & 768.808 & -24.0 & 13 & 21.39 & 20.63 & 20.37 \\
\hline 686 & 399.3 & -14 & 17 & 21.77 & 20.80 & 20.77 \\
\hline 705 & 422.670 & 660.956 & 20 & 24.37 & 22.66 & 20.59 \\
\hline 711 & 601.609 & 371.452 & 13 & 21.23 & 21.48 & 21.00 \\
\hline 765 & 765.906 & 545.3 & 20 & 21.88 & 21.03 & 20.45 \\
\hline 893 & 57.376 & 36.5 & 18 & 22.39 & 21.21 & 20.58 \\
\hline 907 & 400.733 & 873. & 6 & 99.99 & 99.99 & 21.38 \\
\hline 990 & 373.261 & 304.521 & 19 & 22.92 & 22.37 & 20.80 \\
\hline 1006 & 399.938 & -0.339 & 12 & 21.64 & 23.25 & 20.60 \\
\hline 1033 & 493.8 & 824. & 20 & 21.82 & 21.32 & 20.56 \\
\hline 1072 & 125.9 & 710. & 19 & 21.94 & 20.92 & 20.62 \\
\hline 1073 & 58.723 & 531.8 & 20 & 21.90 & 20.99 & 20.55 \\
\hline 1079 & 362.169 & 871.716 & 4 & 99.99 & 99.99 & 14.69 \\
\hline 1109 & 425.117 & 501.833 & 20 & 22.15 & 21.65 & 20.62 \\
\hline 1162 & 325.0 & & 20 & 22.04 & 21.14 & 20.79 \\
\hline 1172 & 288.423 & 801.112 & 20 & 21.83 & 20.94 & 20.60 \\
\hline 1182 & 807.990 & 689.015 & 19 & 21.89 & 21.16 & 20.67 \\
\hline 1189 & 40.982 & 480.956 & 20 & 21.87 & 20.84 & 20.73 \\
\hline 1209 & 357.555 & 258.0 & 20 & 21.86 & 20.97 & 20.50 \\
\hline 1236 & 71.246 & 200.082 & 20 & 22.00 & 21.10 & 20.74 \\
\hline 1240 & 456.705 & 583.260 & 20 & 22.42 & 21.38 & 20.42 \\
\hline 1253 & 704.532 & 74.930 & 16 & 23.51 & 22.60 & 20.91 \\
\hline 1260 & 466.921 & 138.799 & 7 & 23.70 & 99.99 & 21.69 \\
\hline 1280 & & & 19 & 22.37 & 21.36 & 20.70 \\
\hline 1283 & 58.754 & & 18 & 21.98 & 20.85 & 20.80 \\
\hline 1307 & 139.077 & 307.559 & 20 & 21.68 & 20.81 & 20.55 \\
\hline 1310 & 862.948 & 24.903 & 18 & 21.92 & 20.78 & 20.71 \\
\hline 1359 & 709.803 & 853.631 & 8 & 99.99 & 99.99 & 20.89 \\
\hline 1422 & 165.721 & 818.853 & 20 & 22.25 & 21.65 & 20.88 \\
\hline 1452 & 368.674 & 313.404 & 19 & 21.85 & 23.16 & 21.20 \\
\hline 1464 & 56.592 & 35.701 & 16 & 21.94 & 21.20 & 20.68 \\
\hline 1488 & 500.939 & 277.511 & 20 & 22.16 & 21.20 & 20.90 \\
\hline
\end{tabular}


Table 5. continuation.

\begin{tabular}{|c|c|c|c|c|c|c|}
\hline F1 ID & $\mathrm{x}$ & $\mathrm{y}$ & $\overline{\mathrm{N}}$ & $\mathrm{J}$ & $\overline{\mathrm{H}}$ & $<K>$ \\
\hline 1624 & 853.974 & 765.488 & 18 & 23.86 & 22.89 & 20.65 \\
\hline 1639 & 489.291 & 863.474 & 9 & 22.10 & 99.99 & 21.21 \\
\hline 1689 & 404.355 & 7.315 & 16 & 21.93 & 20.88 & 21.00 \\
\hline 1705 & 816.287 & 857.316 & 8 & 21.78 & 99.99 & 21.09 \\
\hline 1800 & 111.960 & 699.811 & 20 & 22.87 & 21.41 & 21.25 \\
\hline 1804 & 713.258 & 872.670 & 7 & 99.99 & 99.99 & 20.99 \\
\hline 1827 & 753.817 & -0.236 & 15 & 24.96 & 24.14 & 21.05 \\
\hline 1838 & 693.158 & 75.868 & 18 & 23.55 & 21.84 & 21.05 \\
\hline 1859 & 18.449 & 33.166 & 9 & 21.85 & 21.53 & 21.30 \\
\hline 2027 & 836.758 & 722.754 & 18 & 22.13 & 21.14 & 21.21 \\
\hline 2037 & 245.570 & 872.812 & 6 & 99.99 & 99.99 & 21.65 \\
\hline 2057 & 802.054 & 815.425 & 15 & 99.99 & 23.81 & 21.40 \\
\hline 2059 & 392.803 & 868.560 & 7 & 99.99 & 99.99 & 21.42 \\
\hline 2201 & 877.361 & 311.087 & 3 & 25.45 & 23.80 & 20.78 \\
\hline 2269 & 687.439 & 872.047 & 4 & 99.99 & 99.99 & 21.84 \\
\hline 2429 & 853.792 & 28.540 & 7 & 99.99 & 24.08 & 22.24 \\
\hline 2443 & 60.021 & 697.0 & 20 & 23.88 & 22.56 & 21.86 \\
\hline 2445 & 116 & 409. & 20 & 22.68 & 22.07 & 21.78 \\
\hline 2460 & 700.330 & 51.969 & 4 & 26.13 & 23.79 & 21.99 \\
\hline 2470 & 594.966 & 872.656 & 6 & 99.99 & 99.99 & 21.54 \\
\hline 2630 & 19.447 & 12.171 & 9 & 22.10 & 21.30 & 21.53 \\
\hline 2867 & 116.083 & 688. & 16 & 21.77 & 22.73 & 21.57 \\
\hline 2978 & 582.002 & 660. & 19 & 23.21 & 22.56 & 22.93 \\
\hline 3025 & 854.730 & 760.0 & 13 & 99.99 & 99.99 & 21.60 \\
\hline 3047 & 472.7 & 394.4 & 14 & 23.59 & 21.50 & 21.73 \\
\hline 3070 & 508 & 8 & 7 & 27.18 & 99.99 & 22.23 \\
\hline 3075 & 676 & 7 & 6 & 24.66 & 22.83 & 22.87 \\
\hline 3095 & 634.2 & 276.660 & 19 & 22.51 & 21.87 & 21.57 \\
\hline 3134 & 34 & 89.6 & 7 & 21.53 & 99.99 & 21.21 \\
\hline 3137 & 437 & 426.709 & 13 & 22.46 & 21.96 & 21.61 \\
\hline 3161 & & 0 & 8 & 99.99 & 24.37 & 22.35 \\
\hline 3189 & 763.741 & 17.2 & 5 & 24.43 & 23.12 & 22.07 \\
\hline 3258 & 67.090 & 8727 & 5 & 99.99 & & 21.87 \\
\hline 3357 & 396.3 & 329.7 & 2 & 24.55 & 99.99 & 22.13 \\
\hline 3367 & & 710. & 19 & 22.75 & 21.66 & 21.54 \\
\hline 3397 & & 652.077 & 19 & 23.33 & 21.98 & 21.51 \\
\hline 3481 & & 872.6 & 6 & 99.99 & & 22.05 \\
\hline 3573 & & & 19 & 22.36 & 21 & 21.59 \\
\hline 3629 & & & 10 & 99.99 & 99.99 & 22.00 \\
\hline 3853 & 378 & 296.4 & 20 & 23.05 & 21.88 & 21.64 \\
\hline 3969 & & -8.356 & 6 & 23.12 & 23.23 & 22.20 \\
\hline 4007 & & 540260 & 18 & 23.02 & 22.82 & 21.79 \\
\hline 4045 & 486 & 836. & 8 & 24.18 & 23. & 22.34 \\
\hline 4088 & & 568. & 7 & 24.47 & 22.90 & 22.34 \\
\hline 4232 & 689.457 & 85.277 & 18 & 23.54 & 22.64 & 21.61 \\
\hline 4291 & 572.597 & 665165 & 11 & 25.93 & 22.87 & 21.91 \\
\hline 4311 & & 334.8 & 10 & 24.15 & 23.82 & 22.22 \\
\hline 4329 & 502.183 & 846.1 & 6 & 23.29 & 99.99 & 22.50 \\
\hline 4512 & 512.404 & 802.990 & 5 & 24.78 & 26.35 & 21.88 \\
\hline 4605 & 258.827 & 118.311 & 20 & 24.12 & 22.88 & 21.90 \\
\hline 4635 & 441.527 & 143.652 & 4 & 24.33 & 25.82 & 22.89 \\
\hline 4709 & & 682.8 & 18 & 23.22 & 21.94 & 21.98 \\
\hline 4773 & 121.215 & 718.489 & 9 & 24.07 & 23.97 & 22.40 \\
\hline 4846 & & 329.664 & 8 & 24.53 & 25.18 & 22.37 \\
\hline 4882 & & 342.975 & 7 & 24.85 & 22.84 & 22.23 \\
\hline 4921 & & & 18 & 23.66 & 22.68 & 21.92 \\
\hline 5054 & & 824.086 & 16 & 22.53 & 21.95 & 21.86 \\
\hline 5113 & 814.431 & 312.754 & 5 & 24.37 & 23.63 & 22.27 \\
\hline 5159 & 104.817 & 678.268 & 7 & 24.42 & 24.72 & 22.79 \\
\hline 5259 & 333.037 & 871.931 & 4 & 99.99 & 99.99 & 22.07 \\
\hline
\end{tabular}


Table 5. continuation.

\begin{tabular}{|c|c|c|c|c|c|c|}
\hline F1 ID & $\mathrm{x}$ & $\mathrm{y}$ & $\overline{\mathrm{N}}$ & $\overline{\mathrm{J}}$ & $\mathrm{H}$ & $<K>$ \\
\hline 5311 & 137.552 & 407.362 & 20 & 23.20 & $\overline{22.40}$ & 22.17 \\
\hline 5419 & 578.391 & 766.093 & 9 & 26.87 & 99.99 & 22.47 \\
\hline 5721 & 670.410 & 206.164 & 9 & 23.42 & 99.99 & 22.41 \\
\hline 5754 & 368.353 & 354.704 & 17 & 23.34 & 22.49 & 22.13 \\
\hline 5824 & 507.718 & 803.197 & 18 & 23.46 & 22.75 & 22.25 \\
\hline 5897 & 677.238 & 823.391 & 9 & 25.92 & 23.76 & 23.02 \\
\hline 6180 & 483.320 & 152.424 & 10 & 24.48 & 22.45 & 22.32 \\
\hline 6199 & 702.484 & 94.573 & 7 & 24.63 & 99.99 & 22.89 \\
\hline 6211 & 754.571 & 18.824 & 9 & 24.44 & 24.02 & 22.51 \\
\hline 6215 & 234.081 & 100.727 & 16 & 23.48 & 22.59 & 22.25 \\
\hline 6272 & 604.553 & 665.541 & 9 & 99.99 & 99.99 & 22.44 \\
\hline 6355 & 530.248 & 822.501 & 7 & 99.99 & 25.20 & 22.69 \\
\hline 6607 & 464.875 & 116.291 & 9 & 24.31 & 23.30 & 22.82 \\
\hline 6647 & 9.172 & 288.477 & 15 & 24.13 & 23.04 & 22.46 \\
\hline 6698 & 657.778 & 811.249 & 5 & 24.98 & 26.31 & 22.34 \\
\hline 6723 & 9.386 & 314.422 & 14 & 23.51 & 23.67 & 21.88 \\
\hline 6862 & & 534 & 17 & 23.84 & 22.84 & 22.34 \\
\hline 6884 & & 352 & 16 & 23.90 & 23.11 & 22.41 \\
\hline 6924 & 711.010 & 92.1 & 19 & 23.57 & 22.71 & 22.21 \\
\hline 7088 & 442.095 & 401.390 & 16 & 23.76 & 22.86 & 22.18 \\
\hline 7248 & & 60.7 & 8 & 24.00 & 24.18 & 22.62 \\
\hline 7285 & & 203 & 9 & 24.89 & 24.05 & 22.52 \\
\hline 7304 & 195.090 & 304.584 & 18 & 23.11 & 22.43 & 22.44 \\
\hline 7375 & 38.801 & & 14 & 23.59 & 22.98 & 22.40 \\
\hline 7401 & 6.830 & 636.969 & 14 & 23.63 & 22.26 & 22.36 \\
\hline 7560 & & 83 & 13 & 23.71 & 22.72 & 22.40 \\
\hline 7755 & 493.789 & 57 & 15 & 23.70 & 22.82 & 22.20 \\
\hline 7788 & 526.608 & 817. & 16 & 23.69 & 23.23 & 22.43 \\
\hline 7835 & 872.295 & 565.6 & 6 & 24.47 & 22.76 & 22.43 \\
\hline 8005 & 398.664 & 296.704 & 18 & 22.75 & 23.09 & 22.54 \\
\hline 8074 & 100 & 215 & 10 & 24.39 & 23.14 & 22.56 \\
\hline 8122 & 348.476 & 853.152 & 5 & 24.31 & 99.99 & 23.01 \\
\hline 8130 & 683.598 & 819.128 & 11 & 24.17 & 23.19 & 22.64 \\
\hline 8151 & & 829. & 14 & 23.77 & 23.02 & 22.53 \\
\hline 8273 & 500.841 & 239.201 & 4 & 24.80 & 23.26 & 23.04 \\
\hline 8619 & & 546.905 & 3 & 24.76 & 24.83 & 23.11 \\
\hline 8796 & 263.491 & 466.644 & 8 & 24.55 & 24.45 & 22.71 \\
\hline 8880 & & 720. & 6 & 24.75 & 24.77 & 22.81 \\
\hline 9329 & & 96.694 & 7 & 23.92 & 23.12 & 22.72 \\
\hline 9375 & 870.541 & 519.853 & 12 & 24.08 & 24.00 & 22.96 \\
\hline 9627 & 279.827 & 363.194 & 6 & 23.86 & 23.63 & 22.79 \\
\hline 9672 & 636.995 & 815.458 & 7 & 24.25 & 25.44 & 22.95 \\
\hline 9858 & & & 5 & 24.48 & 23.99 & 23.24 \\
\hline 10031 & 242.844 & 65.863 & 8 & 24.43 & 99.99 & 22.82 \\
\hline
\end{tabular}


Table 6. Additional variable stars for which periods could not be determined in Field 2. The columns from left to right list: star ID, $\mathrm{x}$ and $\mathrm{y}$ position with respect to the reference epoch, single epoch $J$ and $H$-band magnitudes and mean $K$-band magnitude. Magnitude 99.99 denotes that no measurement for that star was obtained.

\begin{tabular}{|c|c|c|c|c|c|c|}
\hline F2 ID & $\mathrm{x}$ & $\mathrm{y}$ & $\mathrm{N}$ & $\mathrm{J}$ & $\mathrm{H}$ & $<K>$ \\
\hline 5 & 110.132 & $\overline{451.042}$ & 17 & 99.99 & 18.68 & 15.51 \\
\hline 25 & 111.583 & -16.658 & 14 & 18.96 & 18.89 & 18.39 \\
\hline 63 & 133.142 & -27.505 & 9 & 19.13 & 18.49 & 18.96 \\
\hline 64 & 140.349 & -23.112 & 6 & 20.08 & 19.96 & 19.10 \\
\hline 69 & 139.234 & -31.121 & 8 & 99.99 & 99.99 & 19.71 \\
\hline 72 & 122.852 & -33.488 & 7 & 20.97 & 99.99 & 20.11 \\
\hline 76 & 139.031 & -28.038 & 9 & 99.99 & 20.04 & 19.24 \\
\hline 86 & 116.295 & -14.475 & 13 & 18.53 & 18.08 & 18.99 \\
\hline 96 & 259.271 & 269.331 & 16 & 20.91 & 21.35 & 19.11 \\
\hline 98 & 22.966 & 176.951 & 16 & 19.74 & 19.52 & 19.54 \\
\hline 110 & 129.337 & -24.781 & 6 & 99.99 & 99.99 & 18.69 \\
\hline 131 & 116.799 & -19.307 & 8 & 20.88 & 18.44 & 19.34 \\
\hline 139 & 122.165 & -28.415 & 8 & 99.99 & 19.90 & 19.56 \\
\hline 142 & 145.486 & -19.245 & 9 & 21.45 & 20.15 & 19.44 \\
\hline 146 & 271.826 & 268.954 & 23 & 22.10 & 19.72 & 19.86 \\
\hline 151 & 272.611 & 281.772 & 22 & 19.51 & 19.25 & 19.38 \\
\hline 162 & 66.212 & 384.706 & 24 & 19.98 & 19.43 & 19.19 \\
\hline 176 & 147.165 & -14.406 & 11 & 21.15 & 19.13 & 19.23 \\
\hline 192 & 276.007 & 276.599 & 19 & 22.35 & 19.95 & 20.66 \\
\hline 193 & 140.127 & -4.647 & 16 & 18.71 & 18.31 & 19.45 \\
\hline 200 & 249.412 & 273.123 & 15 & 99.99 & 99.99 & 19.73 \\
\hline 202 & 47.977 & 170.614 & 17 & 20.41 & 20.85 & 20.04 \\
\hline 203 & 48.521 & 165.258 & 21 & 20.28 & 19.79 & 19.48 \\
\hline 207 & 245.433 & 114.678 & 24 & 21.17 & 19.85 & 19.36 \\
\hline 216 & 128.889 & -29.875 & 7 & 18.61 & 18.80 & 19.00 \\
\hline 221 & 597.628 & 560.943 & 24 & 20.61 & 19.76 & 19.45 \\
\hline 223 & 43.347 & 736.172 & 24 & 20.74 & 19.80 & 19.41 \\
\hline 232 & 137.997 & -15.777 & 8 & 20.58 & 18.71 & 18.97 \\
\hline 245 & 268.374 & 265.404 & 20 & 20.00 & 19.79 & 19.68 \\
\hline 254 & 271.155 & 290.252 & 19 & 19.56 & 19.26 & 19.49 \\
\hline 275 & 52.212 & 173.756 & 16 & 99.99 & 20.29 & 19.37 \\
\hline 288 & 21.506 & 164.659 & 21 & 20.51 & 19.40 & 19.50 \\
\hline 291 & 44.895 & 163.187 & 21 & 21.16 & 21.76 & 19.22 \\
\hline 303 & 143.702 & -9.790 & 14 & 99.99 & 99.99 & 19.30 \\
\hline 350 & 251.404 & 283.837 & 17 & 19.74 & 20.50 & 19.75 \\
\hline 370 & 614.713 & 202.193 & 22 & 20.94 & 20.86 & 19.50 \\
\hline 411 & 47.289 & 175.205 & 11 & 99.99 & 19.05 & 19.37 \\
\hline 456 & 0.790 & 726.963 & 12 & 20.68 & 99.99 & 19.61 \\
\hline 460 & 243.893 & 274.440 & 18 & 20.42 & 19.85 & 19.63 \\
\hline 478 & 27.155 & 158.279 & 19 & 20.76 & 19.62 & 19.64 \\
\hline 484 & 861.987 & 481.214 & 23 & 21.22 & 20.15 & 19.77 \\
\hline 491 & 310.658 & 488.855 & 24 & 22.17 & 21.13 & 19.93 \\
\hline 494 & 870.000 & 39.193 & 22 & 20.71 & 20.11 & 19.76 \\
\hline 509 & 612.706 & 217.313 & 19 & 22.42 & 21.18 & 19.89 \\
\hline 527 & 610.340 & 199.084 & 23 & 21.79 & 20.03 & 19.77 \\
\hline 584 & 50.053 & 187.361 & 15 & 19.61 & 19.21 & 20.03 \\
\hline 792 & .439 & 161.490 & 15 & 19.46 & 19.71 & 19.98 \\
\hline 795 & 46.919 & 66.360 & 24 & 21.81 & 20.72 & 20.12 \\
\hline 802 & 604.547 & 212.523 & 22 & 21.38 & 22.24 & 20.45 \\
\hline 899 & 874.834 & 660.431 & 17 & 99.99 & 21.54 & 20.31 \\
\hline 920 & 894.150 & 151.063 & 15 & 21.39 & 99.99 & 20.01 \\
\hline 921 & 249.834 & 263.876 & 24 & 19.88 & 19.35 & 20.09 \\
\hline 924 & 251.805 & 266.782 & 15 & 99.99 & 20.09 & 20.14 \\
\hline 940 & 611.253 & 230.397 & 24 & 21.82 & 20.59 & 19.97 \\
\hline 941 & 471.261 & 171.558 & 24 & 22.53 & 20.47 & 20.05 \\
\hline 958 & 104.947 & -20.160 & 14 & 21.49 & 20.42 & 18.96 \\
\hline 981 & 1.907 & 76.444 & 13 & 21.15 & 20.30 & 20.09 \\
\hline 1023 & 131.064 & -34.965 & 8 & 19.62 & 20.54 & 19.49 \\
\hline 1167 & 895.388 & 660.961 & 9 & 21.07 & 99.99 & 19.85 \\
\hline 1194 & 149.128 & -28.142 & 12 & 21.36 & 21.13 & 20.20 \\
\hline
\end{tabular}


Table 6. continuation.

\begin{tabular}{|c|c|c|c|c|c|c|}
\hline F2 ID & $\mathrm{x}$ & $\mathrm{y}$ & $\overline{\mathrm{N}}$ & $\overline{\mathrm{J}}$ & $\mathrm{H}$ & 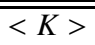 \\
\hline 1233 & 78.433 & 616.813 & 24 & 21.03 & $\overline{20.46}$ & 20.24 \\
\hline 1287 & 420.344 & 569.240 & 24 & 21.64 & 20.80 & 20.19 \\
\hline 1323 & 894.573 & 566.546 & 15 & 22.91 & 99.99 & 20.41 \\
\hline 1337 & 630.755 & 532.113 & 24 & 22.97 & 21.41 & 20.21 \\
\hline 1345 & 28.113 & 159.253 & 19 & 22.25 & 99.99 & 20.13 \\
\hline 1352 & 278.642 & 272.301 & 22 & 99.99 & 99.99 & 20.34 \\
\hline 1385 & 870.608 & 38.459 & 18 & 22.37 & 21.31 & 20.41 \\
\hline 1456 & 16.619 & 61.724 & 22 & 21.13 & 20.28 & 20.50 \\
\hline 1478 & 868.002 & 667.055 & 16 & 22.34 & 20.87 & 20.52 \\
\hline 1518 & 342.464 & 322.107 & 24 & 21.75 & 20.80 & 20.50 \\
\hline 1602 & 628.858 & 196.393 & 24 & 21.04 & 20.37 & 20.24 \\
\hline 1612 & 606.099 & 225.538 & 24 & 20.93 & 20.33 & 20.17 \\
\hline 1629 & 872.381 & 666.590 & 20 & 24.12 & 21.12 & 20.30 \\
\hline 1688 & 592.385 & 217.938 & 23 & 21.78 & 21.55 & 20.21 \\
\hline 1699 & 119.719 & 434.180 & 4 & 99.99 & 22.25 & 20.10 \\
\hline 1701 & 50.788 & 204.785 & 24 & 21.39 & 20.67 & 20.40 \\
\hline 1732 & 802.774 & 165. & 24 & 21.49 & 20.78 & 20.27 \\
\hline 1797 & 481. & -33 . & 12 & 21.69 & 21.20 & 20.44 \\
\hline 1804 & 605.698 & 879.8 & 8 & 99.99 & 99.99 & 20.54 \\
\hline 1881 & 245.502 & 278.014 & 20 & 19.53 & 20.81 & 20.55 \\
\hline 1979 & 697.5 & 522.166 & 7 & 22.54 & 21.95 & 21.18 \\
\hline 1999 & 671.946 & $537 .($ & 24 & 21.73 & 21.00 & 20.39 \\
\hline 2023 & 729.523 & -31.5 & 11 & 22.22 & 21.56 & 21.39 \\
\hline 2127 & 520.114 & 847.9 & 23 & 21.28 & 20.73 & 20.53 \\
\hline 2135 & 486.286 & 169.4 & 24 & 21.92 & 20.81 & 20.44 \\
\hline 2157 & 97.9 & & 24 & 21.19 & 20.57 & 20.41 \\
\hline 2175 & 99.192 & & 20 & 21.95 & 23.02 & 20.60 \\
\hline 2183 & 106.757 & & 23 & 22.13 & 21.06 & 20.57 \\
\hline 2185 & 611.151 & 223.807 & 16 & 99.99 & 20.49 & 20.69 \\
\hline 2206 & 511.707 & 825.415 & 24 & 21.57 & 20.76 & 20.41 \\
\hline 2251 & 64.773 & & 23 & 21.39 & 20.79 & 18.97 \\
\hline 2333 & 508.225 & & 18 & 22.10 & 21.70 & 20.78 \\
\hline 2413 & 9.806 & & 19 & 21.40 & 20.74 & 20.51 \\
\hline 2423 & 19 & 169.6 & 23 & 20.58 & 19.75 & 20.30 \\
\hline 2432 & 806. & & 20 & 22.22 & 21.09 & 20.44 \\
\hline 2471 & 600.781 & 221. & 21 & 21.05 & 20.26 & 20.56 \\
\hline 2510 & 1.269 & & 20 & 17 & 21.80 & 21.18 \\
\hline 2522 & 0.714 & & 24 & 21.76 & 20.87 & 20.60 \\
\hline 2535 & 482.608 & & 22 & 21.88 & 21.48 & 20.75 \\
\hline 2550 & 97.797 & 16 & 13 & 99.99 & 99.99 & 20.95 \\
\hline 2603 & & & 22 & 20.12 & 19.25 & 20.49 \\
\hline 2680 & 34.000 & & 13 & 21.46 & 99.99 & 21.14 \\
\hline 2745 & 56.166 & & 19 & 99.99 & 99.99 & 20.53 \\
\hline 2847 & 629.045 & & 24 & 22.27 & 21.44 & 20.80 \\
\hline 2878 & 41.405 & 736.545 & 23 & 22.10 & 20.96 & 20.83 \\
\hline 2894 & 642.332 & 39.686 & 24 & 22.20 & 21.56 & 20.53 \\
\hline 2977 & 534.652 & 425.8 & 24 & 21.89 & 21.12 & 20.74 \\
\hline 3017 & 806.094 & 833.476 & 20 & 22.24 & 21.26 & 20.87 \\
\hline 3058 & 897.064 & 203.126 & 9 & 99.99 & 99.99 & 20.69 \\
\hline 3074 & 473.478 & 183.960 & 19 & 21.29 & 21.56 & 20.74 \\
\hline 3077 & 215.977 & 359.867 & 23 & 21.77 & 21.06 & 20.67 \\
\hline 3094 & 755.8 & & 24 & 21.83 & 21.22 & 20.77 \\
\hline 3102 & 119.432 & 457.504 & 22 & 22.77 & 21.55 & 20.48 \\
\hline 3223 & 574.027 & 634.704 & 24 & 21.81 & 20.92 & 20.96 \\
\hline 3255 & 832.131 & 409.754 & 21 & 22.03 & 20.96 & 20.76 \\
\hline 3277 & & & 22 & 21.82 & 21.55 & 20.78 \\
\hline 3327 & & & 24 & 22.29 & 21.49 & 20.90 \\
\hline 3402 & 700.282 & 491.063 & 23 & 22.32 & 20.88 & 21.05 \\
\hline 3451 & 102.962 & 631.117 & 24 & 20.78 & 20.90 & 20.64 \\
\hline 3470 & 169.439 & 28.400 & 14 & 24.37 & 22.68 & 19.95 \\
\hline
\end{tabular}


Table 6. continuation.

\begin{tabular}{|c|c|c|c|c|c|c|}
\hline F2 ID & $\mathrm{x}$ & $\mathrm{y}$ & $\overline{\mathrm{N}}$ & $\mathrm{J}$ & $\overline{\mathrm{H}}$ & $<K>$ \\
\hline 3550 & 77.913 & 621.555 & 22 & 21.04 & 21.34 & 20.82 \\
\hline 3577 & 30.904 & 415.011 & 21 & 99.99 & 21.00 & 21.01 \\
\hline 3643 & 777.644 & 587.259 & 23 & 21.70 & 21.77 & 20.93 \\
\hline 3761 & 165.993 & 669.807 & 24 & 23.13 & 22.05 & 20.75 \\
\hline 3806 & 35.897 & 416.674 & 24 & 20.84 & 20.90 & 20.93 \\
\hline 3876 & 81.905 & 614.520 & 17 & 21.78 & 99.99 & 21.13 \\
\hline 3889 & 125.703 & 2.655 & 18 & 21.82 & 99.99 & 20.83 \\
\hline 3912 & 248.918 & 713.633 & 24 & 22.81 & 22.63 & 20.84 \\
\hline 3916 & 58.056 & 179.244 & 20 & 99.99 & 20.18 & 20.66 \\
\hline 4021 & 700.288 & 494.977 & 23 & 22.77 & 21.39 & 21.38 \\
\hline 4103 & 468.065 & 505.340 & 21 & 99.99 & 21.43 & 21.82 \\
\hline 4141 & 713.961 & 504.932 & 18 & 23.20 & 22.48 & 21.16 \\
\hline 4173 & 764.284 & 587.884 & 20 & 22.23 & 22.00 & 20.92 \\
\hline 4233 & 694.949 & 499.693 & 21 & 22.26 & 21.68 & 20.89 \\
\hline 4286 & 265.024 & 257.490 & 18 & 99.99 & 99.99 & 20.94 \\
\hline 4371 & 117.656 & 759.908 & 22 & 21.77 & 21.65 & 21.13 \\
\hline 4442 & 846.485 & 11.054 & 24 & 22.06 & 21.40 & 20.93 \\
\hline 4492 & 708.879 & 510.591 & 18 & 24.70 & 21.92 & 21.22 \\
\hline 4534 & 711.397 & 491.549 & 23 & 22.19 & 21.79 & 20.93 \\
\hline 4589 & 622.493 & 194.710 & 24 & 21.61 & 21.11 & 20.91 \\
\hline 4608 & 780.725 & 572.034 & 17 & 21.95 & 21.96 & 21.33 \\
\hline 4638 & 86.897 & 40.083 & 21 & 24.17 & 22.29 & 21.13 \\
\hline 4661 & 52.470 & 160.751 & 18 & 20.38 & 20.53 & 20.79 \\
\hline 4822 & 50.178 & 191.103 & 20 & 20.22 & 22.38 & 21.15 \\
\hline 5095 & 470.747 & 191.450 & 23 & 21.45 & 21.43 & 21.05 \\
\hline 5251 & 31.060 & 440.962 & 14 & 99.99 & 23.11 & 20.78 \\
\hline 5277 & 475.311 & 531.572 & 18 & 21.68 & 22.65 & 20.83 \\
\hline 5454 & 467.379 & 172.238 & 23 & 22.34 & 21.47 & 21.18 \\
\hline 5616 & 625.235 & 227.382 & 23 & 22.02 & 21.18 & 21.06 \\
\hline 5642 & 779.553 & 594.228 & 21 & 21.75 & 21.14 & 21.35 \\
\hline 5656 & 776.083 & 583.138 & 21 & 21.92 & 22.09 & 21.57 \\
\hline 5970 & 31.470 & 419.341 & 17 & 99.99 & 22.03 & 21.25 \\
\hline 6079 & 765.569 & 567.395 & 22 & 22.14 & 21.72 & 21.32 \\
\hline 6476 & 92.030 & 446.583 & 22 & 22.54 & 21.70 & 21.17 \\
\hline 6484 & 792.447 & 481.648 & 23 & 23.29 & 22.51 & 21.39 \\
\hline 6504 & 68.988 & 34.727 & 23 & 22.98 & 21.81 & 21.32 \\
\hline 6606 & 880.138 & 827.235 & 19 & 22.97 & 21.97 & 22.02 \\
\hline 7306 & 259.637 & 295.473 & 17 & 99.99 & 99.99 & 21.15 \\
\hline 7353 & 128.704 & 774.632 & 19 & 21.31 & 21.44 & 21.44 \\
\hline 7536 & 506.814 & 110.240 & 15 & 22.79 & 23.08 & 21.56 \\
\hline 7587 & 238.437 & 274.781 & 20 & 22.80 & 21.68 & 21.07 \\
\hline 7623 & 453.342 & 675.695 & 23 & 22.61 & 21.82 & 21.70 \\
\hline 7644 & 456.916 & 504.840 & 24 & 21.61 & 21.34 & 21.36 \\
\hline 7775 & 492.606 & 525.767 & 8 & 24.00 & 22.62 & 21.95 \\
\hline 7785 & 486.544 & 526.428 & 7 & 99.99 & 23.28 & 21.73 \\
\hline 7830 & 452.040 & 516.979 & 24 & 21.70 & 21.60 & 21.34 \\
\hline 8024 & 82.994 & 643.078 & 20 & 22.15 & 21.88 & 21.62 \\
\hline 8048 & 78.459 & 642.120 & 21 & 23.12 & 21.73 & 21.41 \\
\hline 8259 & 161.000 & 806.380 & 18 & 22.72 & 21.37 & 21.63 \\
\hline 8315 & 518.321 & 828.124 & 20 & 23.16 & 22.12 & 21.52 \\
\hline 8361 & 523.173 & 831.813 & 23 & 24.50 & 22.69 & 21.62 \\
\hline 8531 & 700.970 & 523.287 & 10 & 23.32 & 22.66 & 21.67 \\
\hline 8745 & 802.736 & 847.321 & 19 & 22.86 & 22.40 & 21.98 \\
\hline 8869 & 500.430 & 179.994 & 22 & 22.43 & 21.54 & 21.62 \\
\hline 8955 & 8.396 & 158.185 & 9 & 23.07 & 22.19 & 21.64 \\
\hline 9018 & 496.676 & 824.354 & 12 & 24.96 & 99.99 & 22.28 \\
\hline 9213 & 498.600 & 186.034 & 23 & 22.14 & 21.44 & 21.49 \\
\hline 9383 & 782.842 & 577.054 & 22 & 25.53 & 22.17 & 21.73 \\
\hline 9628 & 131.268 & 734.555 & 17 & 23.80 & 22.71 & 22.11 \\
\hline 9844 & 101.202 & 471.023 & 22 & 21.29 & 22.16 & 21.69 \\
\hline
\end{tabular}


M. Rejkuba et al.: LPV catalogue in Cen A, Online Material p 31

Table 6. continuation.

\begin{tabular}{cccrrrr}
\hline \hline F2 ID & $\mathrm{x}$ & $\mathrm{y}$ & $\mathrm{N}$ & $\mathrm{J}$ & $\mathrm{H}$ & $\langle K\rangle$ \\
\hline \hline 9979 & 46.537 & 423.611 & 9 & 23.61 & 23.05 & 22.43 \\
10761 & 227.931 & 439.216 & 21 & 22.45 & 22.59 & 21.91 \\
10854 & 49.091 & 429.543 & 11 & 23.60 & 24.91 & 22.14 \\
11088 & 456.068 & 541.129 & 7 & 23.31 & 23.22 & 22.19 \\
11139 & 189.627 & 236.601 & 21 & 22.59 & 21.90 & 21.94 \\
11513 & 74.208 & 191.714 & 20 & 23.36 & 22.25 & 22.21 \\
11635 & 166.246 & 208.354 & 15 & 23.58 & 22.88 & 22.11 \\
11698 & 536.324 & 845.998 & 19 & 23.51 & 23.22 & 21.97 \\
12003 & 453.077 & 522.497 & 17 & 21.62 & 21.75 & 21.80 \\
12529 & 145.762 & 779.651 & 19 & 23.32 & 22.41 & 22.05 \\
13140 & 273.655 & 820.992 & 8 & 24.04 & 23.52 & 22.93 \\
13620 & 728.246 & 491.702 & 13 & 24.09 & 23.74 & 22.27 \\
14598 & 274.310 & 313.415 & 8 & 23.78 & 23.32 & 22.81 \\
14638 & 841.787 & 474.766 & 9 & 23.97 & 22.57 & 22.47 \\
\hline
\end{tabular}


Table 7. Individual measurements of the K-band magnitudes for all the red variable stars detected in Field 1. The columns from left to right list: star ID, $\mathrm{x}$ and y position with respect to the reference epoch, the reference epoch K-band magnitudes and errors and all the other individual K-band magnitudes and the errors of the measurements as given by ALLFRAME to which 0.04 mag calibration error have been added in quadrature. Magnitude 99.99 and the error 9.99 denote that no measurement for that star was obtained.

\begin{tabular}{|c|c|c|c|c|c|c|c|c|c|}
\hline \multirow[t]{3}{*}{ F1 ID } & \multirow[t]{3}{*}{$\mathrm{x}$} & \multirow[t]{3}{*}{$\mathrm{y}$} & \multirow{3}{*}{$\begin{array}{l}1 \mathrm{Ks} 07(\sigma) \\
1 \mathrm{Ks} 08(\sigma) \\
1 \mathrm{Ks} 15(\sigma) \\
\end{array}$} & \multirow{3}{*}{$\begin{array}{l}1 \mathrm{Ks} 01(\sigma) \\
1 \mathrm{Ks} 09(\sigma) \\
1 \mathrm{Ks} 16(\sigma) \\
\end{array}$} & \multirow{3}{*}{$\begin{array}{l}1 \mathrm{Ks} 02(\sigma) \\
1 \mathrm{Ks} 10(\sigma) \\
1 \mathrm{Ks} 17(\sigma) \\
\end{array}$} & \multirow{3}{*}{$\begin{array}{l}1 \mathrm{Ks} 03(\sigma) \\
1 \mathrm{Ks} 11(\sigma) \\
1 \mathrm{Ks} 18(\sigma) \\
\end{array}$} & \multirow{3}{*}{$\begin{array}{l}1 \mathrm{Ks} 04(\sigma) \\
1 \mathrm{Ks} 12(\sigma) \\
1 \mathrm{Ks} 19(\sigma) \\
\end{array}$} & \multirow{3}{*}{$\begin{array}{l}1 \mathrm{Ks} 05(\sigma) \\
1 \mathrm{Ks} 13(\sigma) \\
1 \mathrm{Ks} 20(\sigma)\end{array}$} & \multirow{3}{*}{$\begin{array}{l}1 \mathrm{Ks} 06(\sigma) \\
1 \mathrm{Ks} 14(\sigma)\end{array}$} \\
\hline & & & & & & & & & \\
\hline & & & & & & & & & \\
\hline \multirow[t]{3}{*}{$\overline{31}$} & 460.926 & 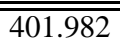 & $\overline{c 17.38(0.09)}$ & 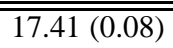 & $16.54(0.08)$ & $16.70(0.06)$ & 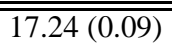 & $17.24(0.06)$ & $16.48(0.07)$ \\
\hline & & & $17.37(0.05)$ & $17.50(0.06)$ & $17.54(0.06)$ & $17.49(0.05)$ & $17.26(0.05)$ & $17.85(0.05)$ & $18.04(0.08)$ \\
\hline & & & $18.04(0.08)$ & $17.86(0.09)$ & $17.88(0.09)$ & $17.66(0.05)$ & $18.33(0.11)$ & $17.67(0.07)$ & \\
\hline \multirow[t]{3}{*}{50} & 763.941 & 462.473 & $18.00(0.08)$ & $18.12(0.05)$ & $17.98(0.05)$ & $17.94(0.04)$ & $17.92(0.07)$ & $18.00(0.05)$ & $17.80(0.05)$ \\
\hline & & & $17.85(0.04)$ & $18.04(0$ & $18.03(0$. & $18.09(0.0$ & $17.99(0.05)$ & $18.08(0.05)$ & $18.19(0.05)$ \\
\hline & & & $18.18(0.05)$ & $18.18(0.04)$ & $18.17(0.06)$ & $18.14(0.05)$ & $18.21(0.04)$ & $18.14(0.05)$ & \\
\hline \multirow[t]{3}{*}{51} & 219.227 & 79.584 & $17.91(0.04)$ & $17.83(0.04)$ & $17.71(0.06)$ & $17.78(0.05)$ & $17.71(0.05)$ & $17.90(0.04)$ & $17.76(0.05)$ \\
\hline & & & $18.12(0.04)$ & $18.06(0.04)$ & $18.07(0.04)$ & $18.23(0.04)$ & $18.18(0.04)$ & 18.24 & $18.29(0.04)$ \\
\hline & & & $18.23(0.05)$ & $18.18(0.04)$ & 18.18 & 18.06 & 18.25 & 18.13 & \\
\hline \multirow[t]{3}{*}{53} & 107.785 & 832.967 & $18.01(0.08)$ & $17.97(0.05)$ & 17.81 & 17.92 & 17.99 & 17.98 & $17.81(0.05)$ \\
\hline & & & $18.31(0.05)$ & $18.11(0.05)$ & 18.05 & 18.19 & 18.19 & 18.18 & $18.21(0.05)$ \\
\hline & & & $18.18(0.06)$ & $18.26(0.05)$ & 18.24 & $18.23(0.05)$ & 18.49 & 99.99 & \\
\hline \multirow[t]{3}{*}{64} & 610.320 & 803.826 & $18.41(0.05)$ & $18.30(0.04)$ & $18.95(0.05)$ & $18.42(0.04)$ & $18.42(0.04)$ & 18.39 & $19.01(0.04)$ \\
\hline & & & $18.50(0.04)$ & $18.32(0.04)$ & $18.31(0.05)$ & $18.22(0.04)$ & $18.27(0.04)$ & & $18.15(0.04)$ \\
\hline & & & 18.16 & $18.16(C$ & 18.21 & 18.22 & 18.25 & 18.25 & \\
\hline \multirow[t]{3}{*}{69} & 113.406 & 403.575 & 17.95 & 17.65 & 17.53 & 18.20 & 17.98 & 18.42 & $19.01(0.22)$ \\
\hline & & & $19.87(0.20)$ & 19.21 & 19.27 & 20.93 & 20.85 & 99.99 & 22.61 (1.14) \\
\hline & & & 99.99 (9.99) & 99.99 & 99.99 & 99.99 & 99.99 & 99.99 & \\
\hline 73 & 688.817 & 405.675 & 18.47 & 18.48 & 18.50 & 18.53 & 18.39 & 18.44 & $19.21(0.05)$ \\
\hline & & & 18.48 & 18.51 & 18.51 & 18.51 & 18.48 & 18.5 & 18.5 \\
\hline & & & 18.62 & 18.72 & 18.67 & 18.65 & 18.18 & 18.24 & \\
\hline 75 & 31.879 & 859.684 & $18.94(0$ & $18.97(C$ & 18.89 & 18.84 & 19.56 & 19.16 & $18.06(0.04)$ \\
\hline & & & 99.99 (9 & 99.99 (9 & 99.99 & 99.99 & 99.99 & 99.99 & $19.85(0.11)$ \\
\hline & & & & & & & & & \\
\hline 76 & 48.965 & 862.669 & $19.17(C$ & 19.16 & 19.10 & 19.08 & 19.91 & 19.60 & $18.22(0.04)$ \\
\hline & & & 99.99 (9 & 99.99 & 99.99 & 99.99 & 99.99 & 99.99 & 99.99 (9.99) \\
\hline & & & 99.99 & 99.99 & 99.99 & 99.99 & 99.99 & 99.99 & \\
\hline 77 & 859.402 & 627.796 & 18.7 & & & & 18.67 & & 99.9 \\
\hline & & & 18.4 & 18.5 & 18.5 & & 18.60 & 18.5 & $18.40(0.04)$ \\
\hline & & & 18.43 & 18.38 & 18.42 & 18.45 & 18.60 & 18.83 & \\
\hline 78 & 47.858 & 863.782 & $18.68(0.07)$ & $18.48(0.05)$ & 18.61 & $18.49(0.04)$ & $18.28(0.05)$ & 18.32 & $19.74(0.05)$ \\
\hline & & & & & & & & & $0.06)$ \\
\hline & & & & & & & & & \\
\hline 84 & 218.231 & 79.339 & $19.74(0.10)$ & $19.71(0$ & 99.99 & 99.99 & 99.99 & 19.66 & 99.99 (9.99) \\
\hline & & & $19.24(0.04)$ & $19.23(0.05)$ & 19.08 & 18.50 & 18.63 & 18.37 & $18.45(0.05)$ \\
\hline & & & & & & & & 18.74 & \\
\hline 91 & 860.939 & 628.112 & & & & & & & 99.9 \\
\hline & & & 19.30 & 19.01 & 18.71 & 19.10 & 18.82 & 18.93 & $19.15(0.05)$ \\
\hline & & & & $19.28(0.05)$ & $19.04(0.06)$ & $18.96(0.05)$ & $18.97(0.05)$ & $18.80(0.05)$ & \\
\hline 100 & 469.319 & 296.353 & $18.89(0.04)$ & $19.51(0.06)$ & $19.73(0.07)$ & $19.72(0.07)$ & $18.94(0.04)$ & $18.88(0.04)$ & $18.81(0.05)$ \\
\hline & & & & & & & & 18.99 & $18.96(0.05)$ \\
\hline & & & & & & & & & \\
\hline 101 & 792.787 & 471.547 & $19.44(0.16)$ & $19.31(0.12)$ & $18.92(0.10)$ & $18.94(0.08)$ & $19.28(0.15)$ & $19.24(0.13)$ & $18.44(0.07)$ \\
\hline & & & $18.91(0.08)$ & $19.19(0.10)$ & $19.44(0.15)$ & $19.07(0.09)$ & $19.10(0.09)$ & 19.31 & $19.32(0.10)$ \\
\hline & & & & & & & & & \\
\hline 104 & 860.140 & 629.895 & $19.19(0.10)$ & & & & & & 99.99 (9.99) \\
\hline & & & $18.98(0.05)$ & $19.13(0.05)$ & $19.23(0.09)$ & $19.11(0.05)$ & $19.11(0.05)$ & $19.18(0.05)$ & $19.33(0.05)$ \\
\hline & & & & & $19.43(0.07)$ & $19.30(0.06)$ & $19.09(0.05)$ & $18.96(0.05)$ & \\
\hline 122 & 450.363 & 778.633 & $19.28(0.05)$ & $18.78(0.05)$ & $18.84(0.05)$ & $18.94(0.05)$ & $19.98(0.05)$ & $19.93(0.04)$ & $19.51(0.04)$ \\
\hline & & & & & & & & & $19.15(0.04)$ \\
\hline & & & $19.09(0.04)$ & $19.05(0.04)$ & $18.99(0.04)$ & $19.04(0.04)$ & $19.04(0.04)$ & $19.11(0.04)$ & \\
\hline 129 & 366.442 & 678.245 & $19.22(0.04)$ & $19.54(0.05)$ & $19.62(0.06)$ & $19.73(0.05)$ & $18.82(0.04)$ & $18.91(0.04)$ & $19.02(0.05)$ \\
\hline & & & $19.70(0.05)$ & $19.39(0.04)$ & $19.18(0.04)$ & $19.48(0.05)$ & $19.57(0.05)$ & $19.62(0.05)$ & $19.61(0.04)$ \\
\hline & & & $19.61(0.04)$ & $19.55(0.04)$ & $19.46(0.04)$ & $19.43(0.04)$ & $19.50(0.05)$ & $19.49(0.05)$ & \\
\hline
\end{tabular}


Table 7. continuation.

\begin{tabular}{|c|c|c|c|c|c|c|c|c|c|}
\hline \multirow[t]{3}{*}{1 ID } & \multirow[t]{3}{*}{$\mathrm{x}$} & \multirow[t]{3}{*}{$\mathrm{y}$} & $1 \mathrm{Ks} 07(\sigma)$ & $1 \mathrm{Ks} 01(\sigma)$ & $1 \mathrm{Ks} 02(\sigma)$ & $1 \mathrm{Ks} 03(\sigma)$ & $1 \mathrm{Ks} 04(\sigma)$ & $\overline{1 \mathrm{Ks} 05(\sigma)}$ & \multirow{3}{*}{$\begin{array}{l}1 \mathrm{Ks} 06(\sigma) \\
1 \mathrm{Ks} 14(\sigma)\end{array}$} \\
\hline & & & $1 \mathrm{Ks} 08(\sigma)$ & $1 \mathrm{Ks} 09(\sigma)$ & $1 \mathrm{Ks} 10(\sigma)$ & $1 \mathrm{Ks} 11(\sigma)$ & $1 \mathrm{Ks} 12(\sigma)$ & $1 \mathrm{Ks} 13(\sigma)$ & \\
\hline & & & $1 \mathrm{Ks} 15(\sigma)$ & $1 \mathrm{Ks} 16(\sigma)$ & $1 \mathrm{Ks} 17(\sigma)$ & $1 \mathrm{Ks} 18(\sigma)$ & $1 \mathrm{Ks} 19(\sigma)$ & $1 \mathrm{Ks} 20(\sigma)$ & \\
\hline \multirow[t]{3}{*}{132} & 610.861 & 804.691 & $20.44(0.10)$ & $20.77(0.10)$ & $18.85(0.05)$ & $20.08(0.07)$ & $19.76(0.07)$ & $20.24(0.07)$ & $18.97(0.04)$ \\
\hline & & & $21.19(0.07)$ & $21.15(0.12)$ & $21.14(0.21)$ & $21.52(0.14)$ & $20.80(0.09)$ & $21.31(0.16)$ & 99.99 (9.99) \\
\hline & & & 99.99 (9.99) & 99.99 (9.99) & 99.99 (9.99) & 99.99 (9.99) & 99.99 (9.99) & 99.99 (9.99) & \\
\hline \multirow[t]{3}{*}{133} & 16.431 & 426.376 & $19.12(0.05)$ & $99.99(9.99)$ & $19.45(0.07)$ & $19.49(0.05)$ & $19.19(0.04)$ & $19.15(0.04)$ & $19.06(0.05)$ \\
\hline & & & 99.99 (9.99) & 99.99 (9.99) & 99.99 (9.99) & $19.49(0.05)$ & $19.52(0.05)$ & 99.99 (9.99) & $19.68(0.05)$ \\
\hline & & & $19.68(0.05)$ & $19.79(0.05)$ & 99.99 (9.99) & $19.70(0.05)$ & 99.99 (9.99) & 99.99 (9.99) & \\
\hline \multirow[t]{3}{*}{135} & 737.241 & 875.358 & $20.62(0.11)$ & $20.39(0.07)$ & 99.99 (9.99) & $21.69(6.49)$ & $18.80(0.05)$ & $20.44(0.14)$ & $20.51(0.08)$ \\
\hline & & & 99.99 (9.99) & 99.99 (9.99) & 99.99 (9.99) & 99.99 (9.99) & 99.99 (9.99) & 99.99 (9.99) & 99.99 (9.99) \\
\hline & & & 99.99 (9.99) & 99.99 (9.99) & 99.99 (9.99) & 99.99 (9.99) & 99.99 (9.99) & 99.99 (9.99) & \\
\hline \multirow[t]{3}{*}{146} & 241.723 & 710.178 & $19.37(0.04)$ & $19.46(0.05)$ & $19.53(0.05)$ & $19.90(0.06)$ & $19.09(0.04)$ & $19.13(0.04)$ & $19.24(0.04)$ \\
\hline & & & $19.46(0.04)$ & $19.26(0.04)$ & $19.06(0.04)$ & $19.86(0.05)$ & $19.83(0.05)$ & $19.84(0.05)$ & $19.59(0.04)$ \\
\hline & & & $19.52(0.05)$ & $19.58(0.05)$ & $19.60(0.05)$ & $19.62(0.04)$ & $19.66(0.05)$ & $19.65(0.05)$ & \\
\hline \multirow[t]{3}{*}{148} & 251.540 & -35.913 & 99.99 (9.99) & 99.99 (9.99) & $19.47(0.09)$ & 99.99 (9.99) & 99.99 (9.99) & 99.99 (9.99) & 99.99 (9.99) \\
\hline & & & $19.58(0.04)$ & $19.46(0.04)$ & $19.37(0.04)$ & $19.41(0.04)$ & $19.31(0.04)$ & $19.21(0.05)$ & 99.99 (9.99) \\
\hline & & & 99.99 (9.99) & $99.99(9.99)$ & $19.26(0.05)$ & $19.28(0.04)$ & $20.92(0.08)$ & $20.14(0.06)$ & \\
\hline \multirow[t]{3}{*}{151} & 383.324 & 278.858 & $19.76(0.05)$ & $19.33(0.05)$ & $19.24(0.07)$ & $19.31(0.06)$ & $19.80(0.05)$ & $19.82(0.05)$ & $19.87(0.05)$ \\
\hline & & & $19.92(0.05)$ & $20.01(0.05)$ & $19.95(0.05)$ & $19.28(0.05)$ & 19.30 & $19.28(0.05)$ & $19.19(0.04)$ \\
\hline & & & $19.19(0.04)$ & $19.18(0.04)$ & $19.24(0.04)$ & $19.30(0.04)$ & $19.42(0.05)$ & $19.60(0.05)$ & \\
\hline \multirow[t]{3}{*}{155} & 822.695 & 291.891 & $19.90(0.06)$ & $19.38(0.05)$ & $19.39(0.08)$ & $19.34(0.05)$ & $20.45(0.05)$ & $20.40(0.06)$ & 99.99 (9.99) \\
\hline & & & $19.25(0.04)$ & $19.60(0.05)$ & $19.75(0.05)$ & $20.18(0.06)$ & $20.15(0.06)$ & $20.04(0.06)$ & $19.77(0.05)$ \\
\hline & & & $19.71(0.05)$ & $19.56(0.05)$ & $19.39(0.04)$ & $19.29(0.05)$ & $19.09(0.04)$ & $18.99(0.04)$ & \\
\hline \multirow[t]{3}{*}{158} & 41.740 & -29.076 & 99.99 (9.99) & $99.99(9.99)$ & $17.95(0.06)$ & $99.99(9.99)$ & 99.99 (9.99) & $99.99(9.99)$ & 99.99 (9.99) \\
\hline & & & $20.30(0.06)$ & $19.77(0.06)$ & $19.82(0.10)$ & $20.08(0.06)$ & $19.25(0.05)$ & 99.99 (9.99) & 99.99 (9.99) \\
\hline & & & $99.99(9.99)$ & $22.12(0.27)$ & 99.99 (9.99) & $20.33(0.10)$ & 99.99 (9.99) & $99.99(9.99)$ & \\
\hline 160 & 157.838 & 174.017 & $19.59(0.08)$ & $19.39(0.06)$ & $19.73(0.07)$ & $19.74(0.05)$ & $19.50(0.06)$ & $19.53(0.05)$ & $19.98(0.05)$ \\
\hline & & & $19.36(C$ & 19.46( & 19.50 & 19.38 & 19.52 & $19.33(0.05)$ & $19.37(0.05)$ \\
\hline & & & $19.33(0.05)$ & $19.42(0.05)$ & $19.36(0.05)$ & $19.36(0.05)$ & $19.32(0.05)$ & $19.36(0.05)$ & \\
\hline 161 & 681.411 & 593.494 & $19.68(0.04)$ & $19.92(0.06)$ & $19.85(0.06)$ & $19.89(0.06)$ & $19.16(0.04)$ & $19.33(0.04)$ & $19.55(0.05)$ \\
\hline & & & $19.24(0.04)$ & $19.16(0.04)$ & $19.10(0.04)$ & $19.78(0.05)$ & $19.80(0.04)$ & $19.67(0.05)$ & $19.64(0.04)$ \\
\hline & & & $19.57(0$ & 19.63 & 19.72( & 19.69 & 19.81 & $19.61(0.05)$ & \\
\hline 164 & 645.768 & 675.400 & $19.86(0.05)$ & $19.68(0.05)$ & $19.74(0.06)$ & $19.47(0.05)$ & $19.92(0.04)$ & $20.02(0.05)$ & $19.98(0.05)$ \\
\hline & & & $19.14(0.04)$ & $19.12(0.04)$ & $19.24(0.04)$ & $19.46(0.05)$ & $19.49(0.05)$ & $19.48(0.04)$ & $19.57(0.05)$ \\
\hline & & & $19.62(0.05)$ & $19.63(0.04)$ & $19.62(0.05)$ & $19.36(0.04)$ & $19.20(0.05)$ & $19.04(0.04)$ & \\
\hline 166 & 476.846 & 747.158 & $19.75(0.05)$ & $19.71(0.05)$ & $19.53(0.05)$ & $19.54(0.05)$ & $19.43(0.04)$ & $19.62(0.04)$ & $19.71(0.05)$ \\
\hline & & & $19.34(0.04)$ & $19.21(0.04)$ & $19.31(0.04)$ & $19.53(0.05)$ & $19.64(0.05)$ & $19.65(0.04)$ & $19.72(0.05)$ \\
\hline & & & $19.68(0.04)$ & $19.65(0.05)$ & $19.47(0.05)$ & $19.36(0.05)$ & 19.22 & $19.12(0.04)$ & \\
\hline 168 & 63.609 & 662.896 & $19.31(0.05)$ & $19.19(0.05)$ & $19.21(0.06)$ & $19.23(0.05)$ & $19.21(0.04)$ & $19.20(0.04)$ & $19.19(0.05)$ \\
\hline & & & $19.48(0.04)$ & $19.37(0.04)$ & $19.25(0.04)$ & $20.11(0.05)$ & $20.12(0.05)$ & $20.03(0.05)$ & $19.88(0.05)$ \\
\hline & & & $19.84(0.05)$ & 19.94 & $19.94(0$. & $19.94(0.05)$ & $19.81(0$. & $19.51(0.05)$ & \\
\hline 170 & 280.584 & 257.958 & $19.24(0.04)$ & $19.28(0.05)$ & $19.39(0.05)$ & $19.62(0.05)$ & $19.42(0.04)$ & $19.30(0.04)$ & $19.23(0.04)$ \\
\hline & & & $19.65(0.05)$ & $19.70(0.04)$ & $19.70(0.04)$ & $19.43(0.05)$ & $19.39(0.05)$ & $19.45(0.04)$ & $19.59(0.04)$ \\
\hline & & & $19.67(0.04)$ & $19.72(0.05)$ & $19.77(0.05)$ & $19.76(0.05)$ & $19.75(0.05)$ & $19.71(0.06)$ & \\
\hline 171 & 205.283 & 725.663 & $19.23(0.05)$ & $19.08(0.05)$ & $19.15(0.05)$ & $19.22(0.05)$ & $19.35(0$. & $19.26(0$. & $19.17(0.04)$ \\
\hline & & & $19.74(0.05)$ & $19.62(0.04)$ & $19.53(0.04)$ & $19.49(0.04)$ & $19.55(0.04)$ & $19.60(0.05)$ & $19.68(0.05)$ \\
\hline & & & $19.75(0.04)$ & $19.85(0.04)$ & $19.77(0.05)$ & $19.74(0.04)$ & $19.72(0.05)$ & $19.70(0.05)$ & \\
\hline 172 & 651.896 & 747.256 & $20.09(0.15)$ & $19.98(0.08)$ & $19.95(0.13)$ & $19.81(0.07)$ & $19.88(0.11)$ & $19.85(0.07)$ & $19.85(0.07)$ \\
\hline & & & $19.51(0.05)$ & $19.54(0.06)$ & $19.83(0.11)$ & $19.48(0.05)$ & $19.44(0.06)$ & $19.52(0.07)$ & $19.36(0.06)$ \\
\hline & & & $19.46(0.07)$ & $19.37(0.05)$ & $19.44(0.09)$ & $19.42(0.07)$ & $19.38(0.05)$ & $19.50(0.06)$ & \\
\hline 173 & 132.979 & 205.212 & $20.21(0.05)$ & $20.32(0.06)$ & $20.42(0.07)$ & $20.68(0.08)$ & $20.31(0.06)$ & $20.37(0.06)$ & $20.35(0.06)$ \\
\hline & & & $19.75(0.05)$ & $19.91(0.05)$ & $20.09(0.04)$ & $19.66(0.05)$ & $19.60(0.05)$ & $19.39(0.05)$ & $19.31(0.04)$ \\
\hline & & & & $19.19(0.04)$ & & $19.14(0.04)$ & $19.26(0.04)$ & $19.40(0.05)$ & \\
\hline 174 & 876.368 & 141.793 & $19.90(0.07)$ & $19.82(0.05)$ & 99.99 (9.99) & $19.83(0.06)$ & $19.40(0.04)$ & $19.56(0.04)$ & 99.99 (9.99) \\
\hline & & & $19.13(0.04)$ & $19.37(0.04)$ & $19.50(0.05)$ & $19.77(0.05)$ & $19.81(0.05)$ & $19.77(0.05)$ & $19.82(0.05)$ \\
\hline & & & $19.78(0.05)$ & $19.67(0.05)$ & $19.54(0.05)$ & $19.42(0.04)$ & $19.42(0.05)$ & $19.44(0.05)$ & \\
\hline 177 & 387.634 & 211.691 & $19.27(0.05)$ & $19.47(0.05)$ & $19.49(0.06)$ & $19.54(0.06)$ & $19.49(0.04)$ & $19.36(0.04)$ & $19.30(0.05)$ \\
\hline & & & & & & & $19.79(0.04)$ & $19.72(0.05)$ & $19.62(0.04)$ \\
\hline & & & $19.64(0.04)$ & $19.65(0.04)$ & $19.64(0.05)$ & $19.65(0.05)$ & $19.58(0.05)$ & $19.39(0.05)$ & \\
\hline 178 & 281.918 & 543.030 & $19.74(0.06)$ & $19.78(0.06)$ & $19.78(0.06)$ & $19.54(0.06)$ & $19.59(0.06)$ & $19.66(0.05)$ & $19.63(0.05)$ \\
\hline & & & $19.07(0.04)$ & $19.08(0.05)$ & $19.25(0.06)$ & $19.93(0.05)$ & $19.93(0.05)$ & $19.90(0.05)$ & $19.82(0.05)$ \\
\hline & & & $19.90(0.05)$ & $19.83(0.05)$ & $19.72(0.06)$ & $19.67(0.05)$ & $19.52(0.05)$ & $19.35(0.05)$ & \\
\hline
\end{tabular}


Table 7. continuation.

\begin{tabular}{|c|c|c|c|c|c|c|c|c|c|}
\hline \multirow[t]{3}{*}{ F1 ID } & \multirow[t]{3}{*}{$\mathrm{x}$} & \multirow[t]{3}{*}{$\mathrm{y}$} & $1 \mathrm{Ks} 07(\sigma)$ & $1 \mathrm{Ks} 01(\sigma)$ & $1 \mathrm{Ks} 02(\sigma)$ & $1 \mathrm{Ks} 03(\sigma)$ & $1 \mathrm{Ks} 04(\sigma)$ & $1 \mathrm{Ks} 05(\sigma)$ & \multirow{3}{*}{$\begin{array}{l}1 \mathrm{Ks} 06(\sigma) \\
1 \mathrm{Ks} 14(\sigma)\end{array}$} \\
\hline & & & $1 \mathrm{Ks} 08(\sigma)$ & $1 \mathrm{Ks} 09(\sigma)$ & $1 \mathrm{Ks} 10(\sigma)$ & $1 \mathrm{Ks} 11(\sigma)$ & $1 \mathrm{Ks} 12(\sigma)$ & $1 \mathrm{Ks} 13(\sigma)$ & \\
\hline & & & $1 \mathrm{Ks} 15(\sigma)$ & $1 \mathrm{Ks} 16(\sigma)$ & $1 \mathrm{Ks} 17(\sigma)$ & $1 \mathrm{Ks} 18(\sigma)$ & $1 \mathrm{Ks} 19(\sigma)$ & $1 \mathrm{Ks} 20(\sigma)$ & \\
\hline \multirow[t]{3}{*}{182} & 679.090 & -5.543 & $99.99(9.99)$ & $19.46(0.05)$ & $19.41(0.05)$ & $19.45(0.05)$ & $19.37(0.06)$ & $19.28(0.06)$ & $99.99(9.99)$ \\
\hline & & & $19.49(0.04)$ & $19.37(0.05)$ & $19.31(0.06)$ & $19.60(0.04)$ & $19.70(0.05)$ & $19.64(0.05)$ & $19.69(0.05)$ \\
\hline & & & $19.74(0.05)$ & $19.66(0.05)$ & $19.61(0.05)$ & $19.57(0.05)$ & $19.50(0.05)$ & $19.41(0.05)$ & \\
\hline \multirow[t]{3}{*}{183} & 110.136 & 846.650 & 99.99 (9.99) & $19.28(0.05)$ & $19.33(0.06)$ & $19.33(0.05)$ & $19.61(0.05)$ & $19.41(0.05)$ & $19.30(0.06)$ \\
\hline & & & $19.65(2.95)$ & 99.99 (9.99) & 99.99 (9.99) & $19.50(0.05)$ & 99.99 (9.99) & $19.73(0.05)$ & 99.99 (9.99) \\
\hline & & & $19.81(0.06)$ & $19.86(0.06)$ & $20.18(0.06)$ & 99.99 (9.99) & 99.99 (9.99) & $99.99(9.99)$ & \\
\hline \multirow[t]{3}{*}{184} & 358.080 & 68.748 & $19.26(0.04)$ & $19.26(0.05)$ & $19.41(0.05)$ & $19.66(0.06)$ & $19.40(0.04)$ & $19.26(0.04)$ & $19.28(0.04)$ \\
\hline & & & $19.69(0.05)$ & $19.79(0.05)$ & $19.53(0.05)$ & $19.49(0.05)$ & $19.57(0.05)$ & $19.58(0.05)$ & $19.79(0.05)$ \\
\hline & & & $19.81(0.05)$ & $19.90(0.05)$ & $19.90(0.05)$ & $19.84(0.04)$ & $19.81(0.05)$ & $19.60(0.05)$ & \\
\hline \multirow[t]{3}{*}{185} & 144.836 & 180.467 & $19.22(0.06)$ & $19.24(0.05)$ & $19.47(0.06)$ & $19.72(0.06)$ & $19.32(0.05)$ & $19.24(0.05)$ & $19.20(0.05)$ \\
\hline & & & $19.76(0.05)$ & $20.02(0.05)$ & $19.92(0.05)$ & $19.54(0.05)$ & $19.58(0.05)$ & $19.55(0.05)$ & $19.68(0.05)$ \\
\hline & & & $19.58(0.05)$ & $19.64(0.05)$ & $19.78(0.06)$ & $19.80(0.05)$ & $19.89(0.05)$ & $20.01(0.05)$ & \\
\hline \multirow[t]{3}{*}{188} & 108.023 & 247.863 & $20.18(0.12)$ & $19.80(0.08)$ & $19.71(0.08)$ & $19.55(0.06)$ & $20.23(0.11)$ & $20.11(0.11)$ & $19.85(0.08)$ \\
\hline & & & $19.65(0.07)$ & $19.86(0.10)$ & $20.07(0.13)$ & $19.37(0.05)$ & $19.39(0.06)$ & $19.37(0.07)$ & $19.45(0.07)$ \\
\hline & & & $19.50(0.08)$ & $19.48(0.06)$ & $19.70(0.10)$ & $19.62(0.08)$ & $19.63(0.07)$ & $19.94(0.10)$ & \\
\hline \multirow[t]{3}{*}{190} & 489.946 & 400.930 & $19.95(0.05)$ & $20.15(0.05)$ & $20.07(0.08)$ & $19.89(0.06)$ & $19.98(0.05)$ & $20.09(0.05)$ & $20.00(0.05)$ \\
\hline & & & 20.47 (C & $20.07(0.05)$ & $19.88(0.04)$ & $19.29(0.05)$ & 19.25 & $19.25(0.04)$ & $19.32(0.04)$ \\
\hline & & & $19.35(0.04)$ & $19.40(0.04)$ & $19.52(0.05)$ & $19.64(0.04)$ & $19.81(0.05)$ & $19.96(0.05)$ & \\
\hline \multirow[t]{3}{*}{191} & 794.769 & 177.227 & $19.52(0.05)$ & $19.39(0.05)$ & $19.55(0.06)$ & $19.53(0.06)$ & $19.37(0.05)$ & $19.36(0.04)$ & $19.41(0.07)$ \\
\hline & & & $19.18(0.05)$ & $19.31(0.05)$ & $19.31(0.05)$ & $19.89(0.07)$ & $19.91(0.05)$ & $20.07(0.05)$ & $19.99(0.05)$ \\
\hline & & & & $19.86(0.05)$ & $20.00(0.05)$ & $19.96(0.05)$ & $19.70(0.05)$ & $19.54(0.05)$ & \\
\hline \multirow[t]{3}{*}{193} & 143.056 & 171.690 & $19.90(0.04)$ & $19.40(0.05)$ & $19.66(0.06)$ & $19.93(0.06)$ & $19.49(0.05)$ & $19.53(0.04)$ & $19.71(0.05)$ \\
\hline & & & $19.43(0.04)$ & $19.50(0.04)$ & $19.67(0.04)$ & $19.81(0.05)$ & $19.88(0.05)$ & $19.81(0.05)$ & $19.77(0.05)$ \\
\hline & & & $19.59(0.04)$ & $19.55(0.04)$ & $19.51(0.05)$ & $19.40(0.04)$ & $19.47(0.05)$ & $19.51(0.05)$ & \\
\hline 194 & 428.622 & 185.952 & 19.44 & $19.34(0.05)$ & $19.49(0.06)$ & $19.61(0.05)$ & $19.44(0.05)$ & $19.38(0.05)$ & 19.36 \\
\hline & & & 19.68 & 19.59 & 19.37 & 19.93 & 19.79 & $19.82(0.05)$ & $19.73(0.05)$ \\
\hline & & & $19.74(0.06)$ & $19.75(0.05)$ & $19.75(0.06)$ & $19.86(0.05)$ & $19.87(0.06)$ & $19.84(0.06)$ & \\
\hline 195 & 373.988 & 172.810 & $19.63(0.05)$ & $19.49(0.05)$ & $19.41(0.06)$ & $19.58(0.05)$ & $20.07(0.05)$ & $20.03(0.05)$ & $19.78(0.05)$ \\
\hline & & & $19.97(0.05)$ & $20.14(0.06)$ & $20.11(0.05)$ & $19.30(0.04)$ & $19.35(0.05)$ & $19.36(0$ & $19.42(0.05)$ \\
\hline & & & & 19.45 & & & $19.82(0$ & $20.01(0.07)$ & \\
\hline 197 & 129.658 & 501.311 & $19.38(0.05)$ & $19.49(0.05)$ & $19.55(0.05)$ & 19.50 & 19.63 & $19.52(0$ & $19.32(0.04)$ \\
\hline & & & $20.07(0.05)$ & $20.19(0.05)$ & $20.34(0.05)$ & 19.43 & $19.46(0$. & $19.51(0.05)$ & $19.46(0.04)$ \\
\hline & & & $19.53(0.05)$ & $19.59(0.05)$ & $19.69(0.05)$ & $19.77(0.04)$ & $19.89(0.05)$ & $20.02(0.05)$ & \\
\hline 199 & 52.553 & 723.548 & $19.72(0.14)$ & $19.32(0.07)$ & $19.29(0.10)$ & $19.24(0.06)$ & $19.67(0.10)$ & $19.53(0.08)$ & (0.07) \\
\hline & & & 19.49 & $19.57(0.07)$ & $19.77(0.13)$ & $19.72(0.07)$ & $19.87(0.08)$ & 19.84 & $19.87(0.09)$ \\
\hline & & & & 19.83 & 20.04 & 19.90 & & 19.82 & \\
\hline 200 & 447.286 & 399.913 & $19.44(0.04)$ & $19.39(0.05)$ & $19.51(0.06)$ & $19.64(0.07)$ & $19.60(0.05)$ & $19.48(0$ & $19.43(0.05)$ \\
\hline & & & & $19.63(0.05)$ & $19.41(0.05)$ & $20.38(0.09)$ & $20.13(0.07)$ & $20.10(0.09)$ & $20.29(0.07)$ \\
\hline & & & 20.46 & 20.10 & 20.09 & 20.26 & $20.28(0$ & $20.11(0.06)$ & \\
\hline 201 & 344.898 & 770.497 & $20.07(0.04)$ & $19.34(0.05)$ & $19.55(0.05)$ & $19.66(0.05)$ & $19.40(0.04)$ & $19.64(0.04)$ & $19.82(0.05)$ \\
\hline & & & & $19.62(0.04)$ & $19.85(0.05)$ & $20.25(0.05)$ & $20.16(0.05)$ & $19.93(0.05)$ & $19.59(0.04)$ \\
\hline & & & & $19.44(0.05)$ & $19.35(0.04)$ & $19.43(0.04)$ & $19.46(0.04)$ & $19.63(0.05)$ & \\
\hline 204 & 31.785 & 419.365 & $20.01(0.05)$ & $20.00(0.06)$ & $19.99(0.07)$ & $19.90(0.07)$ & 19.84 & $20.01(0.05)$ & $20.02(0.06)$ \\
\hline & & & $19.47(0.05)$ & $19.65(0.05)$ & $19.85(0.04)$ & $19.78(0.05)$ & $19.71(0.05)$ & $19.62(0.05)$ & $19.50(0.05)$ \\
\hline & & & $19.42(0.05)$ & $19.37(0.04)$ & $19.51(0.05)$ & $19.35(0.04)$ & $19.53(0.05)$ & $19.69(0.05)$ & \\
\hline 205 & 740.307 & 514.147 & $19.88(0.07)$ & $19.79(0.06)$ & $19.79(0.06)$ & $20.02(0.06)$ & $19.66(0.05)$ & $19.68(0.05)$ & $19.80(0.05)$ \\
\hline & & & & $19.38(0.05)$ & $19.53(0.05)$ & $20.17(0.06)$ & $20.20(0.07)$ & $20.21(0.06)$ & $20.06(0.06)$ \\
\hline & & & & $19.78(0.05)$ & $19.66(0.05)$ & $19.59(0.05)$ & $19.49(0.05)$ & $19.51(0.05)$ & \\
\hline 206 & 672.810 & 492.074 & $19.95(0.05)$ & $19.99(0.06)$ & $19.81(0.06)$ & $19.82(0.05)$ & $20.17(0.05)$ & $20.08(0.05)$ & $20.00(0.05)$ \\
\hline & & & $19.81(0.05)$ & $20.01(0.05)$ & $20.26(0.05)$ & $19.51(0.05)$ & $19.47(0.05)$ & $19.44(0.05)$ & $19.36(0.05)$ \\
\hline & & & $19.39(0.0$ & $19.32(0.05)$ & & $19.38(0.05)$ & $19.43(0.05)$ & $19.64(0.05)$ & \\
\hline 207 & 349.012 & 58.330 & $19.66(0.04)$ & $19.76(0.06)$ & $19.74(0.06)$ & $19.96(0.05)$ & $19.75(0.05)$ & $19.85(0.04)$ & $19.86(0.05)$ \\
\hline & & & $19.47(0.04)$ & $19.50(0.04)$ & $19.73(0.04)$ & $20.02(0.06)$ & $20.03(0.05)$ & $20.00(0.05)$ & $19.80(0.05)$ \\
\hline & & & $19.69(0.05)$ & $19.54(0.05)$ & $19.41(0.05)$ & $19.29(0.04)$ & $19.36(0.04)$ & $19.36(0.05)$ & \\
\hline 210 & 173.593 & 109.992 & $19.98(0.05)$ & $20.00(0.06)$ & $20.13(0.09)$ & $20.11(0.07)$ & $19.98(0.07)$ & $20.07(0.05)$ & $20.11(0.06)$ \\
\hline & & & & & & & & & $19.39(0.04)$ \\
\hline & & & $19.35(0.04)$ & $19.43(0.04)$ & $19.52(0.05)$ & $19.58(0.05)$ & $19.84(0.05)$ & $19.99(0.05)$ & \\
\hline 213 & 113.806 & 521.844 & $19.34(0.05)$ & $19.59(0.05)$ & $19.82(0.05)$ & $19.71(0.06)$ & $19.35(0.04)$ & $19.28(0.04)$ & $19.31(0.04)$ \\
\hline & & & $20.47(0.06)$ & $20.60(0.06)$ & $20.54(0.05)$ & $19.67(0.05)$ & $19.75(0.04)$ & $19.72(0.05)$ & $19.84(0.05)$ \\
\hline & & & $19.82(0.05)$ & $19.92(0.05)$ & $20.02(0.06)$ & $20.14(0.05)$ & $20.14(0.05)$ & $20.31(0.06)$ & \\
\hline
\end{tabular}


Table 7. continuation.

\begin{tabular}{|c|c|c|c|c|c|c|c|c|c|}
\hline \multirow[t]{3}{*}{1 ID } & \multirow[t]{3}{*}{$\mathrm{X}$} & \multirow[t]{3}{*}{$\mathrm{y}$} & 1Ks07 $(\sigma)$ & $1 \mathrm{Ks} 01(\sigma)$ & $1 \mathrm{Ks} 02(\sigma)$ & $1 \mathrm{Ks} 03(\sigma)$ & $1 \mathrm{Ks} 04(\sigma)$ & $1 \mathrm{Ks} 05(\sigma)$ & \multirow{3}{*}{$\begin{array}{l}1 \mathrm{Ks} 06(\sigma) \\
1 \mathrm{Ks} 14(\sigma)\end{array}$} \\
\hline & & & $1 \mathrm{Ks} 08(\sigma)$ & $1 \mathrm{Ks} 09(\sigma)$ & $1 \mathrm{Ks} 10(\sigma)$ & $1 \mathrm{Ks} 11(\sigma)$ & $1 \mathrm{Ks} 12(\sigma)$ & $1 \mathrm{Ks} 13(\sigma)$ & \\
\hline & & & $1 \mathrm{Ks} 15(\sigma)$ & $1 \mathrm{Ks} 16(\sigma)$ & $1 \mathrm{Ks} 17(\sigma)$ & $1 \mathrm{Ks} 18(\sigma)$ & $1 \mathrm{Ks} 19(\sigma)$ & $1 \mathrm{Ks} 20(\sigma)$ & \\
\hline \multirow[t]{3}{*}{215} & 709.023 & 852.736 & $20.47(0.04)$ & $20.00(0.05)$ & $21.74(0.20)$ & $20.42(0.07)$ & $20.67(0.06)$ & $20.81(0.06)$ & 999.99 (9.99) \\
\hline & & & 99.99 (9.99) & 99.99 (9.99) & 99.99 (9.99) & $19.92(0.13)$ & 99.99 (9.99) & $19.68(0.04)$ & $19.36(0.04)$ \\
\hline & & & $19.37(0.04)$ & $19.34(0.04)$ & $19.32(0.04)$ & 99.99 (9.99) & 99.99 (9.99) & 99.99 (9.99) & \\
\hline \multirow[t]{3}{*}{216} & 266.856 & 305.468 & $19.98(0.05)$ & $19.79(0.05)$ & $19.64(0.06)$ & $19.64(0.05)$ & $19.95(0.05)$ & $19.98(0.05)$ & $20.05(0.05)$ \\
\hline & & & $19.92(0.05)$ & $19.96(0.05)$ & $19.86(0.04)$ & $19.36(0.04)$ & $19.42(0.04)$ & $19.44(0.05)$ & $19.51(0.05)$ \\
\hline & & & $19.54(0.05)$ & $19.63(0.04)$ & $19.63(0.05)$ & $19.66(0.04)$ & $19.79(0.05)$ & $19.92(0.06)$ & \\
\hline \multirow[t]{3}{*}{218} & 506.923 & 159.390 & $19.88(0.05)$ & $19.64(0.05)$ & $19.48(0.05)$ & $19.50(0.06)$ & $20.05(0.05)$ & $20.02(0.05)$ & $20.09(0.05)$ \\
\hline & & & $20.05(0.05)$ & $20.21(0.05)$ & $20.27(0.05)$ & $19.67(0.05)$ & $19.58(0.05)$ & $19.51(0.04)$ & $19.51(0.04)$ \\
\hline & & & $19.50(0.04)$ & $19.49(0.05)$ & $19.58(0.04)$ & $19.63(0.04)$ & $19.60(0.05)$ & $19.86(0.06)$ & \\
\hline \multirow[t]{3}{*}{219} & 178.324 & 64.073 & $20.26(0.06)$ & $20.35(0.06)$ & $20.27(0.10)$ & $20.66(0.10)$ & $20.24(0.07)$ & $20.42(0.06)$ & $20.44(0.07)$ \\
\hline & & & $19.99(0.05)$ & $20.05(0.05)$ & $20.17(0.05)$ & $19.57(0.05)$ & $19.66(0.06)$ & $19.40(0.05)$ & $19.51(0.05)$ \\
\hline & & & $19.37(0.05)$ & $19.44(0.04)$ & $19.45(0.05)$ & $19.53(0.04)$ & $19.92(0.06)$ & $20.21(0.06)$ & \\
\hline \multirow[t]{3}{*}{220} & 129.748 & -29.810 & 99.99 (9.99) & 99.99 (9.99) & $22.00(0.43)$ & 99.99 (9.99) & 99.99 (9.99) & 99.99 (9.99) & 99.99 (9.99) \\
\hline & & & $19.99(0.05)$ & $19.79(0.05)$ & $19.77(0.05)$ & $19.85(0.05)$ & $20.02(0.05)$ & $19.54(0.05)$ & 99.99 (9.99) \\
\hline & & & 99.99 (9.99) & $19.71(0.05)$ & $19.49(0.05)$ & $19.54(0.05)$ & $19.17(0.05)$ & $19.29(0.06)$ & \\
\hline \multirow[t]{3}{*}{224} & 314.828 & 367.392 & $19.44(0.05)$ & $19.37(0.05)$ & $19.50(0.06)$ & $19.66(0.07)$ & $20.09(0.05)$ & $19.90(0.04)$ & $19.66(0.05)$ \\
\hline & & & $20.51(0.06)$ & $20.54(0.06)$ & $20.43(0.04)$ & $19.62(0.05)$ & 19.59 & $19.54(0.05)$ & $19.43(0.05)$ \\
\hline & & & $19.43(0.04)$ & $19.46(0.04)$ & $19.49(0.05)$ & $19.56(0.05)$ & $19.62(0.05)$ & $19.78(0.05)$ & \\
\hline \multirow[t]{3}{*}{225} & 302.868 & 720.473 & $19.92(0.05)$ & $19.38(0.05)$ & $19.42(0.05)$ & $19.45(0.06)$ & $20.53(0.04)$ & $20.54(0.05)$ & $20.20(0.05)$ \\
\hline & & & $20.44(0.05)$ & $20.39(0.05)$ & $20.51(0.05)$ & $19.75(0.05)$ & $19.60(0.04)$ & $19.54(0.04)$ & $19.42(0.04)$ \\
\hline & & & $19.40(0.04)$ & $19.41(0.04)$ & $19.40(0.04)$ & $19.45(0.04)$ & $19.47(0.05)$ & $19.55(0.05)$ & \\
\hline \multirow[t]{3}{*}{227} & 746.930 & 775.240 & $20.12(0.05)$ & $20.10(0.05)$ & $20.12(0.11)$ & $20.22(0.09)$ & $19.75(0.06)$ & $19.92(0.05)$ & $20.24(0.07)$ \\
\hline & & & $19.74(0.04)$ & $19.62(0.04)$ & $19.69(0.04)$ & $19.89(0.05)$ & $19.82(0.05)$ & $19.82(0.05)$ & $19.66(0.05)$ \\
\hline & & & $19.57(0.05)$ & $19.53(0.05)$ & $19.53(0.05)$ & $19.48(0.05)$ & $19.56(0.05)$ & $19.72(0.06)$ & \\
\hline 229 & 623.448 & 577.709 & $19.75(0.04)$ & $19.80(0.05)$ & $19.81(0.06)$ & $19.91(0.06)$ & $19.80(0.05)$ & $19.75(0.04)$ & $19.79(0.05)$ \\
\hline & & & $19.78(0.04)$ & 19.89 & 20.03 & 19.93 & 19.78 & $19.79(0.05)$ & $19.61(0.05)$ \\
\hline & & & $19.59(0.05)$ & $19.50(0.04)$ & $19.43(0.05)$ & $19.49(0.04)$ & $19.52(0.05)$ & $19.70(0.05)$ & \\
\hline 230 & 479.669 & 410.112 & $20.15(0.05)$ & $20.15(0.06)$ & $20.38(0.07)$ & $20.05(0.08)$ & $19.57(0.05)$ & $19.73(0.05)$ & $19.71(0.05)$ \\
\hline & & & $19.22(0.05)$ & $19.48(0.05)$ & $19.70(0.04)$ & $20.10(0.06)$ & $20.00(0.06)$ & $20.09(0.06)$ & $19.91(0.06)$ \\
\hline & & & $19.99(0.05)$ & 19.79 & 19.69 & 19.60( & $19.52(0.05)$ & $19.57(0.05)$ & \\
\hline 232 & 263.283 & 697.783 & $20.06(0.04)$ & $20.12(0.06)$ & $20.01(0.05)$ & $19.90(0.06)$ & $19.88(0.05)$ & $20.09(0.05)$ & $20.18(0.06)$ \\
\hline & & & $19.64(0.04)$ & $19.75(0.05)$ & $19.96(0.05)$ & $19.91(0.05)$ & $19.86(0.05)$ & $19.76(0.05)$ & $19.52(0.05)$ \\
\hline & & & $19.46(0.04)$ & $19.46(0.04)$ & $19.45(0.04)$ & $19.51(0.05)$ & $19.59(0.05)$ & $19.70(0.05)$ & \\
\hline 233 & 89.820 & 829.313 & $19.94(0.08)$ & $19.87(0.06)$ & $19.76(0.07)$ & $19.89(0.05)$ & $19.81(0.05)$ & $19.84(0.05)$ & 19.70 \\
\hline & & & $20.02(0.05)$ & $19.93(0.05)$ & $19.96(0.06)$ & $19.37(0.04)$ & $19.45(0.04)$ & $19.51(0.05)$ & $19.69(0.05)$ \\
\hline & & & $19.70(0.05)$ & $19.78(0.05)$ & $20.00(0.05)$ & $20.05(0.06)$ & $20.42(0.10)$ & 99.99 (9.99) & \\
\hline 234 & 871.714 & 142.695 & $20.15(0.07)$ & $19.94(0.06)$ & $19.86(0.09)$ & $19.72(0.06)$ & $20.27(0.05)$ & $20.23(0.05)$ & 99.99 (9.99) \\
\hline & & & $19.37(0.04)$ & $19.76(0.05)$ & $19.96(0.04)$ & $20.09(0.05)$ & $19.90(0.05)$ & $19.74(0.05)$ & $19.68(0.04)$ \\
\hline & & & $19.64(0.05)$ & $19.48(0$. & $19.45(0$. & $19.35(0.04)$ & 19.30( & $19.39(0.06)$ & \\
\hline 236 & 130.784 & 541.867 & $20.16(0.06)$ & $20.36(0.08)$ & $20.52(0.07)$ & $20.25(0.06)$ & $19.48(0.04)$ & $19.70(0.04)$ & $19.81(0.05)$ \\
\hline & & & $19.27(0.04)$ & $19.28(0.05)$ & $19.31(0.05)$ & $20.55(0.06)$ & $20.56(0.07)$ & $20.53(0.07)$ & $20.42(0.06)$ \\
\hline & & & $20.37(0.06)$ & $20.21(0.05)$ & $20.20(0.06)$ & $20.11(0.06)$ & $20.24(0.05)$ & $20.18(0.06)$ & \\
\hline 237 & 57.420 & 528.362 & $20.03(0.08)$ & $20.31(0.09)$ & $20.08(0.07)$ & $20.48(0.10)$ & $19.99(0.08)$ & $19.83(0.06)$ & 19.87 \\
\hline & & & $19.47(0.06)$ & $20.21(0.08)$ & $19.76(0.05)$ & $20.03(0.06)$ & $19.86(0.06)$ & $20.02(0.05)$ & $19.71(0.06)$ \\
\hline & & & $19.76(0.04)$ & $19.83(0.05)$ & $19.53(0.05)$ & $19.53(0.04)$ & $19.58(0.05)$ & $19.68(0.05)$ & \\
\hline 238 & 16.670 & 152.924 & $20.37(0.09)$ & $99.99(9.99)$ & $20.49(0.14)$ & $20.45(0.10)$ & $19.91(0.07)$ & $20.04(0.05)$ & $20.28(0.09)$ \\
\hline & & & 99.99 (9.99) & 99.99 (9.99) & 99.99 (9.99) & $19.88(0.05)$ & $19.83(0.05)$ & 99.99 (9.99) & $19.73(0.05)$ \\
\hline & & & $19.55(0.05)$ & $19.54(0.05)$ & 99.99 (9.99) & $19.49(0.05)$ & 99.99 (9.99) & 99.99 (9.99) & \\
\hline 240 & 139.735 & 474.178 & $20.00(0.04)$ & $19.81(0.05)$ & $19.83(0.05)$ & $19.96(0.06)$ & $19.77(0.05)$ & $19.91(0.04)$ & $20.00(0.05)$ \\
\hline & & & $19.49(0.04)$ & $19.56(0.05)$ & $19.73(0.04)$ & $19.76(0.05)$ & $19.81(0.05)$ & $19.83(0.05)$ & $19.77(0.05)$ \\
\hline & & & & $19.75(0.05)$ & $19.71(0.05)$ & $19.58(0.04)$ & $19.56(0.05)$ & $19.44(0.05)$ & \\
\hline 244 & 802.609 & 464.726 & $19.72(0.05)$ & $19.61(0.05)$ & $19.63(0.06)$ & $19.74(0.06)$ & $19.57(0.04)$ & $19.56(0.04)$ & $19.71(0.08)$ \\
\hline & & & $19.60(0.05)$ & $19.53(0.05)$ & $19.52(0.04)$ & $20.15(0.06)$ & $20.12(0.05)$ & $20.17(0.05)$ & $20.19(0.05)$ \\
\hline & & & $20.24(0.05)$ & $20.30(0.05)$ & $20.12(0.05)$ & $19.93(0.05)$ & $19.82(0.05)$ & $19.66(0.05)$ & \\
\hline 245 & 189.929 & 129.833 & $20.09(0.04)$ & $19.77(0.05)$ & $20.06(0.06)$ & $20.34(0.08)$ & $19.62(0.05)$ & $19.69(0.05)$ & $19.89(0.05)$ \\
\hline & & & $19.72(0.04)$ & & $19.79(0.04)$ & & $19.96(0.05)$ & $19.90(0.05)$ & $19.85(0.05)$ \\
\hline & & & $19.72(0.05)$ & $19.63(0.05)$ & $19.62(0.05)$ & $19.54(0.04)$ & $19.59(0.05)$ & $19.56(0.05)$ & \\
\hline 246 & 575.943 & 702.232 & $19.80(0.05)$ & $20.01(0.06)$ & $19.71(0.05)$ & $19.61(0.05)$ & $20.19(0.04)$ & $20.22(0.05)$ & $20.16(0.05)$ \\
\hline & & & $20.46(0.05)$ & $20.19(0.05)$ & $20.14(0.05)$ & $19.47(0.05)$ & $19.45(0.05)$ & $19.43(0.04)$ & $19.44(0.04)$ \\
\hline & & & $19.48(0.04)$ & $19.55(0.05)$ & $19.66(0.04)$ & $19.79(0.05)$ & $19.88(0.05)$ & $20.05(0.05)$ & \\
\hline
\end{tabular}


Table 7. continuation.

\begin{tabular}{|c|c|c|c|c|c|c|c|c|c|}
\hline \multirow[t]{3}{*}{ F1 ID } & \multirow[t]{3}{*}{$\mathrm{x}$} & \multirow[t]{3}{*}{$\mathrm{y}$} & $1 \mathrm{Ks} 07(\sigma)$ & $1 \mathrm{Ks} 01(\sigma)$ & $1 \mathrm{Ks} 02(\sigma)$ & $1 \mathrm{Ks} 03(\sigma)$ & $1 \mathrm{Ks} 04(\sigma)$ & $1 \mathrm{Ks} 05(\sigma)$ & \multirow{3}{*}{$\begin{array}{l}1 \mathrm{Ks} 06(\sigma) \\
1 \mathrm{Ks} 14(\sigma)\end{array}$} \\
\hline & & & $1 \mathrm{Ks} 08(\sigma)$ & $1 \mathrm{Ks} 09(\sigma)$ & $1 \mathrm{Ks} 10(\sigma)$ & $1 \mathrm{Ks} 11(\sigma)$ & $1 \mathrm{Ks} 12(\sigma)$ & $1 \mathrm{Ks} 13(\sigma)$ & \\
\hline & & & $1 \mathrm{Ks} 15(\sigma)$ & $1 \mathrm{Ks} 16(\sigma)$ & $1 \mathrm{Ks} 17(\sigma)$ & $1 \mathrm{Ks} 18(\sigma)$ & $1 \mathrm{Ks} 19(\sigma)$ & $1 \mathrm{Ks} 20(\sigma)$ & \\
\hline \multirow[t]{3}{*}{248} & 182.270 & 335.708 & $20.03(0.04)$ & $19.96(0.05)$ & $19.98(0.07)$ & $20.00(0.06)$ & $19.98(0.05)$ & $20.05(0.04)$ & $20.07(0.05)$ \\
\hline & & & $20.19(0.05)$ & $20.23(0.05)$ & $20.17(0.04)$ & $19.35(0.04)$ & $19.38(0.04)$ & $19.38(0.04)$ & $19.48(0.04)$ \\
\hline & & & $19.52(0.04)$ & $19.66(0.04)$ & $19.81(0.05)$ & $19.92(0.05)$ & $19.92(0.05)$ & $19.89(0.05)$ & \\
\hline \multirow[t]{3}{*}{250} & 889.374 & 396.430 & $19.85(0.05)$ & $19.41(0.05)$ & $99.99(9.99)$ & $19.51(0.05)$ & $20.21(0.05)$ & $19.94(0.05)$ & 99.99 (9.99) \\
\hline & & & $20.07(0.05)$ & $20.32(0.05)$ & $20.21(0.04)$ & $19.49(0.05)$ & $19.43(0.04)$ & $19.44(0.04)$ & $19.49(0.05)$ \\
\hline & & & $19.57(0.05)$ & $19.61(0.05)$ & $19.70(0.05)$ & $19.80(0.05)$ & $19.97(0.05)$ & $20.18(0.05)$ & \\
\hline \multirow[t]{3}{*}{251} & 138.639 & 691.085 & $19.45(0.05)$ & $19.31(0.06)$ & $19.10(0.05)$ & $19.93(0.07)$ & $19.86(0.07)$ & $19.86(0.07)$ & $19.87(0.07)$ \\
\hline & & & $21.31(0.15)$ & $20.22(0.15)$ & $19.56(0.06)$ & $19.89(0.05)$ & $19.44(0.06)$ & $19.59(0.06)$ & $19.48(0.08)$ \\
\hline & & & $19.94(0.05)$ & $21.04(0.10)$ & $20.02(0.06)$ & $20.22(0.05)$ & $20.12(0.06)$ & $20.33(0.06)$ & \\
\hline \multirow[t]{3}{*}{252} & 377.710 & 736.657 & $19.56(0.04)$ & $19.60(0.05)$ & $19.57(0.05)$ & $19.46(0.05)$ & $19.64(0.04)$ & $19.62(0.04)$ & $19.58(0.05)$ \\
\hline & & & $19.96(0.05)$ & $19.65(0.05)$ & $19.53(0.04)$ & $20.13(0.05)$ & $20.09(0.05)$ & $20.06(0.05)$ & $19.89(0.05)$ \\
\hline & & & $19.97(0.0$ & $19.98(0.04)$ & $20.04(0.05)$ & $19.98(0.04)$ & $19.98(0.05)$ & $19.79(0.05)$ & \\
\hline \multirow[t]{3}{*}{256} & 394.394 & -0.704 & $99.99(9.99)$ & $20.07(0.22)$ & $19.77(0.09)$ & $20.06(0.10)$ & $19.91(0.07)$ & $19.88(0.08)$ & $19.60(0.13)$ \\
\hline & & & $19.22(0.07)$ & $19.64(0.06)$ & $19.71(0.10)$ & $19.74(0.09)$ & $20.18(0.12)$ & $20.54(0.23)$ & $19.92(0.08)$ \\
\hline & & & $20.09(0.07)$ & $19.46(0.06)$ & $19.85(0.09)$ & $19.73(0.06)$ & $20.17(0.10)$ & $20.04(0.14)$ & \\
\hline \multirow[t]{3}{*}{258} & 584.651 & 646.161 & $20.02(0.05)$ & $20.14(0.05)$ & $20.39(0.10)$ & $20.27(0.07)$ & $20.21(0.05)$ & $20.11(0.05)$ & $20.28(0.06)$ \\
\hline & & & $19.13(0$ & $19.53(0.05)$ & $19.41(0.04)$ & $20.48(0.07)$ & 20.11 & $20.34(0.06)$ & $20.20(0.05)$ \\
\hline & & & $20.04(0.05)$ & $20.00(0.05)$ & $19.57(0.04)$ & $19.46(0.04)$ & $19.42(0.05)$ & $19.62(0.06)$ & \\
\hline \multirow[t]{3}{*}{259} & 564.125 & 580.467 & $19.53(0.06)$ & $19.69(0.05)$ & $19.51(0.05)$ & $19.62(0.05)$ & $20.04(0.05)$ & $19.86(0.04)$ & $19.70(0.05)$ \\
\hline & & & $20.28(0.05)$ & $20.38(0.06)$ & $20.39(0.06)$ & $19.61(0.05)$ & $19.60(0.04)$ & $19.62(0.05)$ & $19.59(0.05)$ \\
\hline & & & & $19.68(0.05)$ & $19.86(0.05)$ & $19.88(0.05)$ & $20.00(0.05)$ & $20.14(0.06)$ & \\
\hline \multirow[t]{3}{*}{260} & 550.035 & 697.299 & $19.78(0.04)$ & $19.92(0.05)$ & $20.07(0.06)$ & $20.37(0.07)$ & $19.57(0.04)$ & 19.59 (0. & $19.63(0.05)$ \\
\hline & & & $19.80(0.04)$ & $19.54(0.04)$ & $19.48(0.04)$ & $20.36(0.06)$ & $20.36(0.05)$ & $20.45(0.05)$ & $20.43(0.05)$ \\
\hline & & & $20.39(0.06)$ & $20.30(0.05)$ & $20.45(0.06)$ & $20.43(0.06)$ & $20.35(0.06)$ & $20.02(0.05)$ & \\
\hline 261 & 366.244 & 211.569 & $20.11(0.05)$ & $19.78(0.05)$ & $19.66(0.06)$ & $19.82(0.06)$ & $20.48(0.05)$ & $20.43(0.05)$ & $20.21(0.06)$ \\
\hline & & & 19.57 & 19.75 & 19.90 & 20.25 & $20.32(0.06)$ & $20.25(0$. & $20.04(0.06)$ \\
\hline & & & $19.92(0.05)$ & $19.66(0.04)$ & $19.51(0.04)$ & $19.43(0.04)$ & $19.36(0.05)$ & $19.34(0.05)$ & \\
\hline 263 & 194.740 & 832.315 & $19.64(0.05)$ & $19.40(0.05)$ & $19.51(0.06)$ & $19.40(0.05)$ & $19.77(0.05)$ & $19.64(0.05)$ & $19.41(0.05)$ \\
\hline & & & $19.93(0.04)$ & $19.75(0.05)$ & $19.81(0.05)$ & $19.91(0.05)$ & $19.91(0.05)$ & $19.95(0$. & $20.04(0.05)$ \\
\hline & & & & 20.29 & 20.10 & 20.09 & 21.19 & & \\
\hline 264 & 125.830 & 18.280 & $19.41(0.05)$ & $19.38(0.05)$ & $19.67(0.10)$ & $19.89(0.06)$ & $19.52(0.06)$ & $19.41(0$ & $19.59(0.06)$ \\
\hline & & & $20.18(0.05)$ & $19.88(0.05)$ & $19.61(0.05)$ & $20.01(0.05)$ & $20.17(0.05)$ & $20.02(0.06)$ & $20.12(0.06)$ \\
\hline & & & $20.06(0.05)$ & $19.95(0.05)$ & $19.88(0.06)$ & $19.79(0.05)$ & $19.82(0.05)$ & $19.87(0.07)$ & \\
\hline 269 & 783.385 & 778.874 & $20.16(0.0$ & $19.77(0.05)$ & $20.22(0.12)$ & $20.26(0.07)$ & $19.59(0.06)$ & $19.76(0.05)$ & 20.12 \\
\hline & & & 19.72 & $19.74(0.05)$ & $19.97(0.05)$ & $20.18(0.05)$ & $20.18(0.05)$ & $20.09(0.05)$ & $19.77(0.05)$ \\
\hline & & & $19.70(C$ & 19.59 & & 19.48 & & $19.74(0$ & \\
\hline 272 & 449.464 & 717.144 & $19.72(0.05)$ & $19.44(0.05)$ & $19.45(0.05)$ & $19.57(0.05)$ & $20.17(0.04)$ & $20.09(0.05)$ & $19.93(0.05)$ \\
\hline & & & $20.35(0.05)$ & $20.20(0.05)$ & $20.12(0.05)$ & $19.42(0.05)$ & $19.43(0.04)$ & $19.53(0.04)$ & $19.64(0.04)$ \\
\hline & & & 19.71 & 19.87 & 20.08 & 20.21 & 20.45 & $20.32(0$ & \\
\hline 275 & 97.280 & 801.841 & $20.22(0.07)$ & $20.11(0.06)$ & $20.14(0.07)$ & $20.03(0.07)$ & $19.82(0.04)$ & $20.06(0.05)$ & $20.57(0.07)$ \\
\hline & & & $19.60(0.05)$ & $20.14(0.05)$ & $20.11(0.05)$ & $20.04(0.05)$ & $20.02(0.05)$ & $19.89(0.05)$ & $19.73(0.05)$ \\
\hline & & & $19.73(0.05)$ & $19.46(0.04)$ & $19.70(0.05)$ & $19.65(0.05)$ & $19.33(0.05)$ & $19.41(0.06)$ & \\
\hline 282 & 460.999 & 864.607 & $19.65(0.05)$ & $19.53(0$ & $19.67(0$ & $19.71(0$ & 19.86 & 19.89 & $19.77(0.05)$ \\
\hline & & & 99.99 (9.99) & 99.99 (9.99) & 99.99 (9.99) & 99.99 (9.99) & 99.99 (9.99) & 99.99 (9.99) & $20.82(0.07)$ \\
\hline & & & $20.86(0.07)$ & 99.99 (9.99) & 99.99 (9.99) & 99.99 (9.99) & 99.99 (9.99) & 99.99 (9.99) & \\
\hline 283 & 347.964 & -3.955 & 99.99 (9.99) & $19.69(0.05)$ & $19.74(0.07)$ & $19.97(0.07)$ & $19.67(0.05)$ & $19.75(0.05)$ & $19.85(0.11)$ \\
\hline & & & & $19.89(0.05)$ & $19.99(0.04)$ & $19.99(0.05)$ & $20.00(0.05)$ & $19.76(0.05)$ & $19.68(0.04)$ \\
\hline & & & $19.66(0.04)$ & $19.67(0.05)$ & $19.71(0.05)$ & $19.73(0.05)$ & $19.90(0.05)$ & $20.13(0.05)$ & \\
\hline 284 & 193.238 & 500.106 & $20.19(0.05)$ & $20.94(0.09)$ & $20.62(0.09)$ & $20.64(0.07)$ & $20.30(0.05)$ & $20.37(0.05)$ & $20.30(0.06)$ \\
\hline & & & $19.89(0.05)$ & $20.07(0.05)$ & $20.35(0.05)$ & $19.74(0.05)$ & $19.73(0.05)$ & $19.69(0.05)$ & $19.51(0.04)$ \\
\hline & & & & $19.52(0.04)$ & $19.55(0.05)$ & $19.67(0.04)$ & $19.84(0.05)$ & $19.94(0.06)$ & \\
\hline 286 & 279.230 & 67.260 & $20.08(0.04)$ & $19.61(0.05)$ & $19.98(0.07)$ & $20.41(0.08)$ & $19.67(0.05)$ & $19.80(0.05)$ & $19.99(0.05)$ \\
\hline & & & $19.87(0.04)$ & $19.79(0.05)$ & $19.90(0.04)$ & $20.25(0.06)$ & $20.28(0.05)$ & $20.22(0.06)$ & $19.98(0.05)$ \\
\hline & & & $19.86(0.05)$ & $19.85(0.05)$ & $19.67(0.05)$ & $19.59(0.04)$ & $19.61(0.04)$ & $19.60(0.05)$ & \\
\hline 288 & 14.460 & 549.217 & $19.88(0.06)$ & 99.99 (9.99) & $19.80(0.07)$ & $20.16(0.08)$ & $19.65(0.04)$ & $19.66(0.05)$ & $19.78(0.05)$ \\
\hline & & & & & & & $20.37(0.17)$ & $99.99(9.99)$ & $20.52(0.07)$ \\
\hline & & & 99.99 (9.99) & $20.32(0.07)$ & 99.99 (9.99) & $20.22(0.05)$ & 99.99 (9.99) & 99.99 (9.99) & \\
\hline 292 & 15.904 & 193.015 & $20.13(0.08)$ & 99.99 (9.99) & $20.24(0.11)$ & $20.35(0.09)$ & $20.11(0.06)$ & $20.10(0.05)$ & $20.22(0.08)$ \\
\hline & & & 99.99 (9.99) & 99.99 (9.99) & 99.99 (9.99) & $19.62(0.05)$ & $19.60(0.05)$ & 99.99 (9.99) & $19.75(0.05)$ \\
\hline & & & $19.73(0.05)$ & $19.85(0.05)$ & 99.99 (9.99) & $19.88(0.05)$ & 99.99 (9.99) & 99.99 (9.99) & \\
\hline
\end{tabular}


Table 7. continuation.

\begin{tabular}{|c|c|c|c|c|c|c|c|c|c|}
\hline \multirow[t]{3}{*}{1 ID } & \multirow[t]{3}{*}{$\mathrm{X}$} & \multirow[t]{3}{*}{$\mathrm{y}$} & 1Ks07 $(\sigma)$ & $1 \mathrm{Ks} 01(\sigma)$ & $1 \mathrm{Ks} 02(\sigma)$ & $1 \mathrm{Ks} 03(\sigma)$ & $1 \mathrm{Ks} 04(\sigma)$ & $1 \mathrm{Ks} 05(\sigma)$ & \multirow{3}{*}{$\begin{array}{l}1 \mathrm{Ks} 06(\sigma) \\
1 \mathrm{Ks} 14(\sigma)\end{array}$} \\
\hline & & & $1 \mathrm{Ks} 08(\sigma)$ & $1 \mathrm{Ks} 09(\sigma)$ & $1 \mathrm{Ks} 10(\sigma)$ & $1 \mathrm{Ks} 11(\sigma)$ & $1 \mathrm{Ks} 12(\sigma)$ & $1 \mathrm{Ks} 13(\sigma)$ & \\
\hline & & & $1 \mathrm{Ks} 15(\sigma)$ & $1 \mathrm{Ks} 16(\sigma)$ & $1 \mathrm{Ks} 17(\sigma)$ & $1 \mathrm{Ks} 18(\sigma)$ & $1 \mathrm{Ks} 19(\sigma)$ & $1 \mathrm{Ks} 20(\sigma)$ & \\
\hline \multirow[t]{3}{*}{294} & 124.558 & 330.030 & $20.23(0.05)$ & $19.81(0.05)$ & $20.24(0.05)$ & $20.58(0.08)$ & $19.80(0.04)$ & $19.86(0.04)$ & $19.95(0.05)$ \\
\hline & & & $19.42(0.04)$ & $19.58(0.05)$ & $19.66(0.04)$ & $20.16(0.05)$ & $20.15(0.05)$ & $20.14(0.06)$ & $20.14(0.05)$ \\
\hline & & & $20.06(0.05)$ & $19.94(0.05)$ & $19.72(0.04)$ & $19.68(0.05)$ & $19.62(0.05)$ & $19.66(0.05)$ & \\
\hline \multirow[t]{3}{*}{295} & 445.246 & 622.988 & $20.04(0.05)$ & $20.22(0.08)$ & $20.44(0.11)$ & $20.67(0.11)$ & $19.65(0.05)$ & $19.75(0.04)$ & $19.96(0.05)$ \\
\hline & & & $19.57(0.04)$ & $19.46(0.04)$ & $19.48(0.04)$ & $20.46(0.08)$ & $20.55(0.05)$ & $20.36(0.05)$ & $20.45(0.05)$ \\
\hline & & & $20.43(0.05)$ & $20.39(0.05)$ & $20.13(0.05)$ & $19.93(0.05)$ & $19.73(0.05)$ & $19.60(0.05)$ & \\
\hline \multirow[t]{3}{*}{296} & 395.486 & 18.707 & $20.10(0.04)$ & $19.78(0.05)$ & $19.83(0.06)$ & $20.03(0.07)$ & $19.80(0.05)$ & $19.82(0.04)$ & $19.92(0.05)$ \\
\hline & & & $21.91(0.88)$ & $19.81(0.04)$ & $19.92(0.04)$ & $20.11(0.06)$ & $20.21(0.05)$ & $20.08(0.05)$ & $19.92(0.05)$ \\
\hline & & & $19.80(0.04)$ & $19.69(0.04)$ & $19.60(0.04)$ & $19.62(0.04)$ & $19.74(0.05)$ & $19.84(0.05)$ & \\
\hline \multirow[t]{3}{*}{301} & 364.732 & 570.560 & $20.04(0.05)$ & $20.16(0.06)$ & $20.16(0.08)$ & $20.00(0.06)$ & $20.13(0.05)$ & $20.16(0.05)$ & $19.97(0.05)$ \\
\hline & & & $19.95(0.05)$ & $20.03(0.05)$ & $20.14(0.05)$ & $19.67(0.05)$ & $19.56(0.05)$ & $19.56(0.05)$ & $19.53(0.05)$ \\
\hline & & & $19.58(0.04)$ & $19.63(0.05)$ & $19.78(0.05)$ & $19.85(0.05)$ & $19.98(0.05)$ & $20.01(0.06)$ & \\
\hline \multirow[t]{3}{*}{304} & 169.875 & 226.678 & $20.31(0.05)$ & $20.50(0.07)$ & $20.56(0.09)$ & $20.67(0.09)$ & $19.94(0.04)$ & $20.06(0.05)$ & $20.13(0.05)$ \\
\hline & & & $19.50(0.04)$ & $19.71(0.05)$ & $19.86(0.04)$ & $20.34(0.06)$ & $20.41(0.06)$ & $20.30(0.07)$ & $20.16(0.05)$ \\
\hline & & & $20.06(0.05)$ & $19.91(0.05)$ & $19.82(0.04)$ & $19.64(0.04)$ & $19.60(0.05)$ & $19.61(0.05)$ & \\
\hline \multirow[t]{3}{*}{311} & 51.930 & 187.867 & $20.26(0.05)$ & $20.17(0.07)$ & $20.55(0.11)$ & $20.95(0.11)$ & $19.64(0.05)$ & $19.78(0.05)$ & $20.05(0.05)$ \\
\hline & & & $19.59(0.05)$ & $19.66(0.05)$ & $19.66(0.04)$ & $20.47(0.07)$ & 20.51 & $20.45(0.07)$ & $20.59(0.06)$ \\
\hline & & & $20.44(0.07)$ & $20.40(0.05)$ & $20.10(0.06)$ & $19.88(0.05)$ & $19.90(0.06)$ & $19.76(0.06)$ & \\
\hline \multirow[t]{3}{*}{312} & 326.187 & 501.922 & $19.95(0.05)$ & $19.86(0.06)$ & $19.75(0.08)$ & $19.65(0.06)$ & $20.34(0.05)$ & $20.34(0.05)$ & $20.42(0.06)$ \\
\hline & & & $20.20(0.05)$ & $20.42(0.05)$ & $20.55(0.05)$ & $19.91(0.05)$ & $19.80(0.05)$ & $19.82(0.05)$ & $19.68(0.04)$ \\
\hline & & & $19.66(0.05)$ & $19.64(0.04)$ & $19.64(0.05)$ & $19.62(0.04)$ & $19.70(0.05)$ & $19.83(0.05)$ & \\
\hline \multirow[t]{3}{*}{314} & 108.418 & 332.330 & $20.27(0.04)$ & $20.07(0.06)$ & $20.34(0.06)$ & $20.37(0.06)$ & $19.71(0.04)$ & $19.85(0.04)$ & $19.99(0.05)$ \\
\hline & & & $19.43(0.04)$ & $19.57(0.04)$ & $19.82(0.04)$ & $20.49(0.06)$ & $20.51(0.06)$ & $20.43(0.05)$ & $20.24(0.05)$ \\
\hline & & & $20.13(0.05)$ & $20.00(0.05)$ & $19.86(0.05)$ & $19.76(0.05)$ & $19.66(0.05)$ & $19.61(0.05)$ & \\
\hline 315 & 483.406 & 293.818 & $20.06(0.04)$ & $20.14(0.07)$ & $20.48(0.09)$ & $20.27(0.07)$ & $19.69(0.04)$ & $19.79(0.05)$ & $19.78(0.05)$ \\
\hline & & & $19.73(0.05)$ & 19.67 & 19.65 & 20.10 & 20.15 & $20.16(0.05)$ & $20.28(0.05)$ \\
\hline & & & $20.28(0.05)$ & $20.39(0.05)$ & $20.09(0.06)$ & $19.92(0.05)$ & $19.72(0.05)$ & $19.53(0.05)$ & \\
\hline 316 & 721.808 & 688.040 & $19.66(0.04)$ & $19.79(0.05)$ & $19.75(0.08)$ & $19.93(0.06)$ & $19.60(0.04)$ & $19.56(0.04)$ & $19.60(0.05)$ \\
\hline & & & $19.99(0.05)$ & $19.83(0.05)$ & $19.73(0.04)$ & $20.36(0.06)$ & $20.30(0.05)$ & $20.38(0.05)$ & $20.27(0.05)$ \\
\hline & & & $20.34(0.05)$ & 20.29 & 20.30 & 20.26 & 20.09 & $19.89(0.05)$ & \\
\hline 317 & 400.985 & 356.026 & $20.33(0.05)$ & $19.99(0.06)$ & $20.18(0.08)$ & $20.56(0.09)$ & $19.82(0.05)$ & $19.97(0.05)$ & $20.18(0.06)$ \\
\hline & & & $19.70(0.05)$ & $19.81(0.05)$ & $20.06(0.04)$ & $20.14(0.05)$ & $20.08(0.05)$ & $20.01(0.05)$ & $19.81(0.05)$ \\
\hline & & & $19.76(0.05)$ & $19.69(0.05)$ & $19.66(0.05)$ & $19.66(0.04)$ & $19.70(0.05)$ & $20.03(0.06)$ & \\
\hline 319 & 83.836 & 591.370 & $20.71(0.05)$ & $20.48(0.08)$ & $20.22(0.05)$ & $19.99(0.06)$ & $20.76(0.05)$ & $20.71(0.06)$ & $20.70(0.08)$ \\
\hline & & & $20.03(0.05)$ & $20.26(0.06)$ & $20.56(0.05)$ & $19.99(0.05)$ & $19.98(0.05)$ & $19.89(0.05)$ & $19.71(0.05)$ \\
\hline & & & $19.59(0.05)$ & $19.57(0.04)$ & $19.51(0.05)$ & 19.56 & 19.68 & $19.74(0.05)$ & \\
\hline 322 & 574.036 & 475.742 & $19.47(0.05)$ & $19.82(0.06)$ & $19.60(0.05)$ & $19.61(0.05)$ & $19.80(0.04)$ & $19.65(0.04)$ & $19.48(0.05)$ \\
\hline & & & $20.15(0.05)$ & $19.91(0.05)$ & $19.79(0.04)$ & $20.40(0.07)$ & $20.54(0.06)$ & $20.67(0.07)$ & $20.53(0.07)$ \\
\hline & & & $20.55(0.05)$ & $20.45(0.05)$ & $20.36(0$. & $20.32(0.05)$ & $20.36(0.06)$ & $20.20(0.06)$ & \\
\hline 323 & 563.345 & 653.749 & $20.32(0.05)$ & $20.04(0.06)$ & $20.16(0.07)$ & $20.25(0.07)$ & $19.84(0.04)$ & $19.96(0.04)$ & $20.26(0.06)$ \\
\hline & & & $19.50(0.05)$ & $19.71(0.05)$ & $19.91(0.04)$ & $20.25(0.05)$ & $20.27(0.05)$ & $20.26(0.05)$ & $19.98(0.05)$ \\
\hline & & & $19.87(0.04)$ & $19.75(0.05)$ & $19.66(0.04)$ & $19.65(0.04)$ & $19.69(0.05)$ & $19.78(0.05)$ & \\
\hline 324 & 251.125 & -36.811 & 99.99 (9.99) & 99.99 (9.99) & 99.99 (9.99) & 99.99 (9.99) & 99.99 (9.99) & 99.99 (9.99) & 99.99 \\
\hline & & & $22.18(0.14)$ & 21.83 & $22.29(0$ & $21.46(0.11)$ & 99.99 (9.99) & 99.99 (9.99) & 99.99 (9.99) \\
\hline & & & $99.99(9.99)$ & 99.99 (9.99) & 99.99 (9.99) & $21.55(0.09)$ & $19.56(0.05)$ & $19.90(0.05)$ & \\
\hline 325 & 589.059 & 492.799 & $20.13(0.06)$ & $20.20(0.06)$ & $20.15(0.06)$ & $20.34(0.07)$ & $20.21(0.05)$ & $20.14(0.05)$ & $20.25(0.05)$ \\
\hline & & & & $20.08(0.05)$ & $20.13(0.06)$ & $19.41(0.05)$ & $19.49(0.05)$ & $19.58(0.04)$ & $19.73(0.04)$ \\
\hline & & & $19.84(0.05)$ & $19.92(0.05)$ & $20.15(0.06)$ & $20.28(0.05)$ & $20.22(0.06)$ & $20.14(0.06)$ & \\
\hline 327 & 853.249 & 736.471 & $19.58(0.05)$ & $19.54(0.05)$ & $19.73(0.14)$ & $19.67(0.06)$ & $19.56(0.05)$ & $19.48(0.05)$ & 99.99 (9.99) \\
\hline & & & $19.98(0.05)$ & $19.80(0.05)$ & $19.53(0.05)$ & $20.22(0.06)$ & $20.28(0.05)$ & $20.29(0.05)$ & $20.26(0.06)$ \\
\hline & & & & $20.16(0.06)$ & $20.11(0.06)$ & $20.23(0.05)$ & $20.16(0.05)$ & $20.22(0.07)$ & \\
\hline 330 & 636.715 & 523.009 & $19.55(0.04)$ & $19.70(0.06)$ & $19.73(0.05)$ & $19.77(0.06)$ & $19.83(0.05)$ & $19.74(0.04)$ & $19.60(0.05)$ \\
\hline & & & $22.08(0.14)$ & $21.61(0.12)$ & $21.71(0.07)$ & $19.89(0.05)$ & $19.83(0.05)$ & $19.93(0.05)$ & $20.12(0.05)$ \\
\hline & & & $20.16(0.05)$ & $20.26(0.05)$ & $20.49(0.06)$ & $20.72(0.05)$ & $20.91(0.08)$ & $21.33(0.12)$ & \\
\hline 331 & 480.523 & 569.934 & $20.35(0.05)$ & $20.53(0.08)$ & $20.50(0.06)$ & $20.45(0.08)$ & $19.84(0.05)$ & $20.05(0.04)$ & $20.17(0.06)$ \\
\hline & & & & & $19.42(0.04)$ & & $20.53(0.06)$ & $20.51(0.06)$ & $20.47(0.07)$ \\
\hline & & & $20.53(0.05)$ & $20.39(0.05)$ & $20.37(0.05)$ & $20.32(0.05)$ & $20.30(0.05)$ & $20.15(0.06)$ & \\
\hline 334 & 86.817 & 788.080 & $20.29(0.07)$ & $20.10(0.07)$ & $20.04(0.08)$ & $19.97(0.06)$ & $19.99(0.09)$ & $20.22(0.05)$ & $20.34(0.08)$ \\
\hline & & & $20.15(0.05)$ & $20.20(0.05)$ & $20.22(0.05)$ & $19.84(0.05)$ & $19.77(0.05)$ & $19.70(0.05)$ & $19.63(0.05)$ \\
\hline & & & $19.64(0.05)$ & $19.69(0.05)$ & $19.82(0.05)$ & $19.98(0.05)$ & $20.27(0.08)$ & $20.45(0.09)$ & \\
\hline
\end{tabular}


Table 7. continuation.

\begin{tabular}{|c|c|c|c|c|c|c|c|c|c|}
\hline \multirow[t]{3}{*}{ F1 ID } & \multirow[t]{3}{*}{$\mathrm{x}$} & \multirow[t]{3}{*}{$\mathrm{y}$} & $1 \mathrm{Ks} 07(\sigma)$ & $1 \mathrm{Ks} 01(\sigma)$ & $1 \mathrm{Ks} 02(\sigma)$ & $1 \mathrm{Ks} 03(\sigma)$ & $1 \mathrm{Ks} 04(\sigma)$ & $1 \mathrm{Ks} 05(\sigma)$ & \multirow{3}{*}{$\begin{array}{l}1 \mathrm{Ks} 06(\sigma) \\
1 \mathrm{Ks} 14(\sigma)\end{array}$} \\
\hline & & & $1 \mathrm{Ks} 08(\sigma)$ & $1 \mathrm{Ks} 09(\sigma)$ & $1 \mathrm{Ks} 10(\sigma)$ & $1 \mathrm{Ks} 11(\sigma)$ & $1 \mathrm{Ks} 12(\sigma)$ & $1 \mathrm{Ks} 13(\sigma)$ & \\
\hline & & & $1 \mathrm{Ks} 15(\sigma)$ & $1 \mathrm{Ks} 16(\sigma)$ & $1 \mathrm{Ks} 17(\sigma)$ & $1 \mathrm{Ks} 18(\sigma)$ & $1 \mathrm{Ks} 19(\sigma)$ & $1 \mathrm{Ks} 20(\sigma)$ & \\
\hline \multirow[t]{3}{*}{335} & 259.608 & 230.597 & $20.33(0.05)$ & $20.72(0.09)$ & $20.61(0.07)$ & $20.75(0.10)$ & $20.38(0.04)$ & $20.41(0.05)$ & $20.45(0.05)$ \\
\hline & & & $19.95(0.04)$ & $20.09(0.05)$ & $20.25(0.05)$ & $19.92(0.05)$ & $19.93(0.05)$ & $19.75(0.05)$ & $19.69(0.05)$ \\
\hline & & & $19.64(0.04)$ & $19.62(0.04)$ & $19.57(0.04)$ & $19.58(0.04)$ & $19.63(0.05)$ & $19.74(0.06)$ & \\
\hline \multirow[t]{3}{*}{338} & 87.810 & 792.326 & $19.64(0.06)$ & $20.87(0.11)$ & $20.83(0.09)$ & $20.74(0.09)$ & $19.41(0.05)$ & $19.41(0.04)$ & $19.52(0.06)$ \\
\hline & & & $21.23(0.08)$ & $21.21(0.09)$ & $21.28(0.07)$ & $21.30(0.12)$ & $21.32(0.09)$ & $21.35(0.09)$ & $21.49(0.12)$ \\
\hline & & & $21.29(0.10)$ & $21.30(0.09)$ & $21.02(0.08)$ & $20.61(0.06)$ & $20.29(0.08)$ & $20.26(0.09)$ & \\
\hline \multirow[t]{3}{*}{339} & 886.358 & 801.067 & $20.14(0.06)$ & $20.04(0.06)$ & 99.99 (9.99) & $20.15(0.07)$ & $19.96(0.06)$ & $19.89(0.06)$ & 99.99 (9.99) \\
\hline & & & $20.28(0.05)$ & $20.23(0.06)$ & $20.05(0.06)$ & $19.59(0.05)$ & $19.61(0.05)$ & $19.57(0.05)$ & $19.70(0.06)$ \\
\hline & & & $19.79(0.07)$ & $19.81(0.05)$ & $20.05(0.08)$ & $20.20(0.06)$ & $20.28(0.07)$ & $20.36(0.10)$ & \\
\hline \multirow[t]{3}{*}{342} & 359.105 & 464.911 & $20.07(0.05)$ & $20.05(0.05)$ & $20.33(0.07)$ & $20.50(0.08)$ & $19.57(0.05)$ & $19.69(0.05)$ & $19.86(0.05)$ \\
\hline & & & $19.65(0.05)$ & $19.58(0.04)$ & $19.58(0.04)$ & $20.59(0.08)$ & $20.54(0.05)$ & $20.63(0.07)$ & $20.46(0.06)$ \\
\hline & & & $20.45(0.05)$ & $20.43(0.05)$ & $20.31(0.05)$ & $20.11(0.06)$ & $19.97(0.05)$ & $19.83(0.05)$ & \\
\hline \multirow[t]{3}{*}{343} & 87.019 & 36.775 & $20.46(0.07)$ & $19.73(0.05)$ & $20.39(0.16)$ & $21.17(0.16)$ & $19.95(0.08)$ & $20.14(0.06)$ & $20.50(0.08)$ \\
\hline & & & $20.29(0.05)$ & $20.28(0.06)$ & $20.20(0.05)$ & $19.85(0.05)$ & $19.82(0.05)$ & $19.67(0.05)$ & $19.69(0.05)$ \\
\hline & & & $19.63(0.06)$ & $19.79(0.05)$ & $19.76(0.06)$ & $19.85(0.05)$ & $20.21(0.07)$ & $20.34(0.09)$ & \\
\hline \multirow[t]{3}{*}{350} & 895.862 & 228.496 & 99.99 (9.99) & $20.45(0.08)$ & 99.99 (9.99) & $21.16(0.10)$ & $20.01(0.04)$ & $20.07(0.05)$ & 99.99 (9.99) \\
\hline & & & $19.35(0$. & $19.60(0.05)$ & $19.74(0.04)$ & $20.81(0.07)$ & 20.95 & $20.92(0.09)$ & 99.99 (9.99) \\
\hline & & & $20.98(0.07)$ & $20.52(0.06)$ & $20.33(0.06)$ & $20.23(0.05)$ & $20.11(0.05)$ & $20.00(0.06)$ & \\
\hline \multirow[t]{3}{*}{352} & 249.665 & 451.350 & $20.08(0.05)$ & $20.34(0.07)$ & $20.32(0.08)$ & $20.63(0.09)$ & $20.28(0.05)$ & $20.27(0.05)$ & $20.02(0.05)$ \\
\hline & & & $20.26(0.05)$ & $20.36(0.05)$ & $20.36(0.05)$ & $19.87(0.05)$ & $19.85(0.04)$ & $19.76(0.05)$ & $19.62(0.04)$ \\
\hline & & & & $19.66(0.04)$ & $19.76(0.05)$ & $19.81(0.05)$ & $19.97(0.06)$ & $20.18(0.07)$ & \\
\hline \multirow[t]{3}{*}{353} & 900.235 & 821.974 & $99.99(9.99)$ & $19.75(0.06)$ & $99.99(9.99)$ & $19.75(0.21)$ & $20.58(0.09)$ & $20.56(0.06)$ & 99.99 (9.99) \\
\hline & & & $19.54(0.04)$ & $19.65(0.05)$ & $19.83(0.06)$ & $21.22(0.11)$ & $21.10(0.07)$ & $21.08(0.09)$ & 99.99 (9.99) \\
\hline & & & $21.36(0.12)$ & $21.08(0.08)$ & $21.23(0.11)$ & $21.12(0.08)$ & $20.81(0.10)$ & $20.37(0.10)$ & \\
\hline 355 & 66.638 & 549.981 & $20.29(0.05)$ & $20.44(0.07)$ & $20.32(0.07)$ & $20.27(0.07)$ & $19.86(0.04)$ & $20.11(0.05)$ & 20.16 \\
\hline & & & 19.47 & 19.85 & 20.08 & 20.32 & 20.17 & $20.14(0.05)$ & $19.99(0.05)$ \\
\hline & & & $19.88(0.05)$ & $19.75(0.04)$ & $19.64(0.05)$ & $19.67(0.05)$ & $19.78(0.05)$ & $19.81(0.05)$ & \\
\hline 357 & 757.542 & -12.799 & 99.99 (9.99) & $20.35(0.08)$ & $20.46(0.07)$ & $20.84(0.13)$ & $20.60(0.07)$ & 99.99 (9.99) & 99.99 (9.99) \\
\hline & & & $20.67(0.06)$ & $21.23(0.11)$ & $20.60(0.05)$ & $19.94(0.05)$ & $19.77(0.05)$ & $19.70(0.05)$ & $19.69(0.05)$ \\
\hline & & & & 19.85 & & & 19.90 & $20.11(0$ & \\
\hline 360 & 641.885 & 372.593 & $19.84(0.04)$ & $20.20(0.06)$ & $20.12(0.06)$ & 20.00 & 19.99 & 19.92 & $19.85(0.05)$ \\
\hline & & & $19.86(0.05)$ & $19.80(0.04)$ & $19.75(0.04)$ & $20.20(0.06)$ & $20.10(0$. & $20.20(0.05)$ & $20.22(0.06)$ \\
\hline & & & $20.21(0.05)$ & $20.13(0.05)$ & $20.01(0.05)$ & $19.89(0.04)$ & $19.81(0.05)$ & $19.89(0.05)$ & \\
\hline 361 & 266.155 & 53.191 & 20.09 & $20.05(0.06)$ & $20.08(0.07)$ & $20.48(0.07)$ & $20.07(0.05)$ & $20.14(0.05)$ & $0.05)$ \\
\hline & & & 20.15 & $20.12(0.05)$ & $20.18(0.05)$ & $19.83(0.05)$ & $19.82(0.05)$ & 19.73 & $19.78(0.05)$ \\
\hline & & & $19.74(0.0$ & $19.77(0.04)$ & $19.81(0.05)$ & 19.88 & 20.06 & 20.06 & \\
\hline 362 & 267.730 & 522.355 & $20.21(0.05)$ & $20.09(0.06)$ & $20.31(0.07)$ & $20.48(0.09)$ & $19.85(0.05)$ & $19.93(0.04)$ & $20.00(0.05)$ \\
\hline & & & & $19.85(0.05)$ & $20.21(0.05)$ & $20.09(0.06)$ & $20.02(0.05)$ & $20.01(0.05)$ & $19.80(0.05)$ \\
\hline & & & & 19.78 & & 19.95 & 20.12 & $20.28(0.06)$ & \\
\hline 364 & 461.075 & 786.365 & $20.60(0.05)$ & $20.29(0.07)$ & $20.38(0.07)$ & $20.15(0.06)$ & $19.95(0.05)$ & $20.25(0.05)$ & $20.37(0.06)$ \\
\hline & & & $19.91(0.04)$ & $19.79(0.05)$ & $19.94(0.05)$ & $20.40(0.07)$ & $20.35(0.06)$ & $20.24(0.06)$ & $20.02(0.05)$ \\
\hline & & & & $19.79(0.05)$ & $19.66(0.05)$ & $19.62(0.04)$ & $19.60(0.05)$ & $19.54(0.05)$ & \\
\hline 365 & 518.253 & 737.256 & $19.92(0.05)$ & 20.36 & & 20.36 & $20.17(0$ & 20.08 & 19.99 \\
\hline & & & $20.48(0.05)$ & $20.40(0.06)$ & $20.24(0.04)$ & $19.69(0.05)$ & $19.67(0.04)$ & $19.66(0.05)$ & $19.61(0.05)$ \\
\hline & & & $19.66(0.04)$ & $19.77(0.05)$ & $19.89(0.05)$ & $20.08(0.04)$ & $20.28(0.06)$ & $20.39(0.06)$ & \\
\hline 368 & 158.315 & 592.526 & $20.22(0.05)$ & $19.93(0.05)$ & $20.27(0.07)$ & $20.46(0.08)$ & $19.64(0.04)$ & $19.76(0.04)$ & $20.01(0.05)$ \\
\hline & & & $19.75(0.05)$ & $19.77(0.05)$ & $20.02(0.04)$ & $20.80(0.08)$ & $20.59(0.05)$ & $20.65(0.06)$ & $20.31(0.05)$ \\
\hline & & & $20.12(0.05)$ & $20.08(0.05)$ & $19.90(0.05)$ & $19.85(0.05)$ & $19.93(0.05)$ & $20.03(0.05)$ & \\
\hline 369 & 825.349 & 606.238 & $19.52(0.05)$ & $19.49(0.05)$ & $19.47(0.08)$ & $19.68(0.06)$ & $19.78(0.05)$ & $19.63(0.05)$ & 99.99 (9.99) \\
\hline & & & $20.13(0.05)$ & $19.93(0.05)$ & $19.67(0.04)$ & $20.28(0.05)$ & $20.36(0.06)$ & $20.37(0.05)$ & $20.47(0.06)$ \\
\hline & & & & $20.34(0.05)$ & $20.26(0.07)$ & $20.17(0.05)$ & $20.32(0.05)$ & $20.39(0.07)$ & \\
\hline 373 & 857.805 & 68.719 & $19.56(0.07)$ & $19.66(0.06)$ & $19.58(0.08)$ & $19.53(0.05)$ & $19.98(0.05)$ & $19.89(0.05)$ & 99.99 (9.99) \\
\hline & & & $20.15(0.05)$ & $20.31(0.05)$ & $20.31(0.05)$ & $19.73(0.05)$ & $19.73(0.04)$ & $19.79(0.04)$ & $20.02(0.05)$ \\
\hline & & & $20.07(0.05)$ & $20.04(0.05)$ & $20.29(0.06)$ & $20.23(0.05)$ & $20.28(0.06)$ & $20.35(0.07)$ & \\
\hline 375 & 394.411 & 672.023 & $20.24(0.05)$ & $20.03(0.06)$ & $20.24(0.07)$ & $20.36(0.08)$ & $19.73(0.04)$ & $19.96(0.05)$ & $20.16(0.05)$ \\
\hline & & & & $19.98(0.05)$ & & & & & $19.97(0.05)$ \\
\hline & & & $19.92(0.05)$ & $19.86(0.04)$ & $19.75(0.05)$ & $19.73(0.04)$ & $19.80(0.05)$ & $19.91(0.05)$ & \\
\hline 376 & 87.118 & 92.531 & $20.23(0.07)$ & $20.69(0.07)$ & $20.92(0.12)$ & $21.64(0.21)$ & $20.30(0.07)$ & $20.26(0.05)$ & $20.45(0.07)$ \\
\hline & & & $20.08(0.05)$ & $20.12(0.05)$ & $19.96(0.04)$ & $19.98(0.06)$ & $19.94(0.05)$ & $19.77(0.05)$ & $19.94(0.05)$ \\
\hline & & & $19.82(0.06)$ & $19.82(0.05)$ & $19.77(0.05)$ & $19.74(0.05)$ & $19.88(0.06)$ & $19.79(0.06)$ & \\
\hline
\end{tabular}


Table 7. continuation.

\begin{tabular}{|c|c|c|c|c|c|c|c|c|c|}
\hline \multirow[t]{3}{*}{1 ID } & \multirow[t]{3}{*}{$\mathrm{X}$} & \multirow[t]{3}{*}{$\mathrm{y}$} & 1Ks07 $(\sigma)$ & $1 \mathrm{Ks} 01(\sigma)$ & $1 \mathrm{Ks} 02(\sigma)$ & $1 \mathrm{Ks} 03(\sigma)$ & $1 \mathrm{Ks} 04(\sigma)$ & $1 \mathrm{Ks} 05(\sigma)$ & \multirow{3}{*}{$\begin{array}{l}1 \mathrm{Ks} 06(\sigma) \\
1 \mathrm{Ks} 14(\sigma)\end{array}$} \\
\hline & & & $1 \mathrm{Ks} 08(\sigma)$ & $1 \mathrm{Ks} 09(\sigma)$ & $1 \mathrm{Ks} 10(\sigma)$ & $1 \mathrm{Ks} 11(\sigma)$ & $1 \mathrm{Ks} 12(\sigma)$ & $1 \mathrm{Ks} 13(\sigma)$ & \\
\hline & & & $1 \mathrm{Ks} 15(\sigma)$ & $1 \mathrm{Ks} 16(\sigma)$ & $1 \mathrm{Ks} 17(\sigma)$ & $1 \mathrm{Ks} 18(\sigma)$ & $1 \mathrm{Ks} 19(\sigma)$ & $1 \mathrm{Ks} 20(\sigma)$ & \\
\hline \multirow[t]{3}{*}{377} & 506.196 & 698.151 & $20.65(0.05)$ & $20.72(0.09)$ & $20.22(0.06)$ & $19.96(0.06)$ & 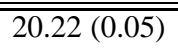 & $20.38(0.05)$ & $20.66(0.06)$ \\
\hline & & & $19.58(0.04)$ & $19.49(0.05)$ & $19.58(0.04)$ & $20.61(0.07)$ & $20.69(0.06)$ & $20.61(0.06)$ & $20.42(0.05)$ \\
\hline & & & $20.46(0.05)$ & $20.37(0.05)$ & $20.02(0.05)$ & $19.85(0.04)$ & $19.86(0.05)$ & $19.65(0.05)$ & \\
\hline \multirow[t]{3}{*}{382} & 147.260 & 308.850 & $19.92(0.04)$ & $20.01(0.06)$ & $20.33(0.08)$ & $20.34(0.07)$ & $19.59(0.04)$ & $19.65(0.04)$ & $19.81(0.05)$ \\
\hline & & & $20.07(0.05)$ & $20.08(0.05)$ & $19.87(0.04)$ & $20.85(0.08)$ & $21.02(0.08)$ & $20.98(0.09)$ & $21.10(0.07)$ \\
\hline & & & $21.02(0.08)$ & $20.99(0.07)$ & $20.99(0.08)$ & $20.96(0.06)$ & $20.87(0.06)$ & $20.53(0.06)$ & \\
\hline \multirow[t]{3}{*}{383} & 549.156 & 223.944 & $20.58(0.05)$ & $20.76(0.07)$ & $20.77(0.10)$ & $21.02(0.11)$ & $20.11(0.04)$ & $20.33(0.05)$ & $20.68(0.06)$ \\
\hline & & & $19.87(0.05)$ & $20.05(0.05)$ & $20.20(0.05)$ & $20.10(0.05)$ & $19.95(0.05)$ & $19.90(0.05)$ & $19.86(0.05)$ \\
\hline & & & $19.77(0.05)$ & $19.73(0.05)$ & $19.71(0.05)$ & $19.73(0.04)$ & $19.78(0.05)$ & $19.90(0.06)$ & \\
\hline \multirow[t]{3}{*}{386} & 844.386 & 709.244 & $20.56(0.06)$ & $21.12(0.11)$ & $21.12(0.15)$ & $20.91(0.11)$ & $20.11(0.06)$ & $20.30(0.05)$ & 99.99 (9.99) \\
\hline & & & $19.76(0.05)$ & $19.89(0.05)$ & $20.13(0.04)$ & $20.48(0.06)$ & $20.48(0.06)$ & $20.29(0.06)$ & $20.10(0.06)$ \\
\hline & & & $19.99(0.06)$ & $19.81(0.05)$ & $19.71(0.06)$ & $19.63(0.04)$ & $19.68(0.05)$ & $19.79(0.06)$ & \\
\hline \multirow[t]{3}{*}{388} & 310.293 & 220.148 & $20.36(0.05)$ & $19.93(0.05)$ & $20.05(0.06)$ & $20.29(0.09)$ & $19.93(0.05)$ & $20.15(0.05)$ & $20.36(0.05)$ \\
\hline & & & $20.04(0.05)$ & $20.11(0.05)$ & $20.19(0.05)$ & $19.63(0.05)$ & $19.71(0.05)$ & $19.70(0.05)$ & $19.75(0.05)$ \\
\hline & & & $19.78(0.05)$ & $19.85(0.05)$ & $20.01(0.05)$ & $20.13(0.05)$ & $20.22(0.07)$ & $20.43(0.07)$ & \\
\hline \multirow[t]{3}{*}{392} & 570.626 & 170.136 & $20.37(0.05)$ & $20.70(0.09)$ & $20.77(0.11)$ & $20.80(0.10)$ & $20.03(0.05)$ & $20.22(0.05)$ & $20.36(0.05)$ \\
\hline & & & $19.59(0.04)$ & $19.70(0.05)$ & $19.94(0.04)$ & $20.45(0.07)$ & 20.29 & $20.24(0.05)$ & $20.18(0.06)$ \\
\hline & & & $20.13(0.05)$ & $19.98(0.05)$ & $19.85(0.05)$ & $19.79(0.05)$ & $19.76(0.05)$ & $19.73(0.05)$ & \\
\hline \multirow[t]{3}{*}{393} & 434.904 & 18.061 & $20.17(0.05)$ & $19.78(0.05)$ & $19.90(0.06)$ & $20.31(0.07)$ & $19.90(0.05)$ & $19.86(0.04)$ & $20.02(0.05)$ \\
\hline & & & $20.07(0.05)$ & $19.94(0.05)$ & $20.10(0.04)$ & $20.46(0.06)$ & $20.44(0.06)$ & $20.31(0.05)$ & $20.09(0.05)$ \\
\hline & & & $19.99(0.05)$ & $19.83(0.05)$ & $19.75(0.05)$ & $19.78(0.04)$ & $19.83(0.05)$ & $19.94(0.05)$ & \\
\hline \multirow[t]{3}{*}{396} & 862.867 & 628.918 & $20.12(0.13)$ & $20.18(0.11)$ & $19.12(0.07)$ & $19.51(0.07)$ & $20.06(0.10)$ & $20.01(0.08)$ & 99.99 (9.99) \\
\hline & & & $19.91(0.06)$ & $20.00(0.07)$ & $20.44(0.13)$ & $19.84(0.07)$ & $19.85(0.07)$ & $20.13(0.07)$ & $20.64(0.07)$ \\
\hline & & & $20.69(0.09)$ & $20.38(0.08)$ & $20.79(0.11)$ & $20.33(0.08)$ & $19.85(0.06)$ & $19.65(0.06)$ & \\
\hline 397 & 519.331 & 615.644 & $20.46(0.05)$ & $20.14(0.05)$ & $20.15(0.07)$ & $20.51(0.07)$ & $19.85(0.04)$ & $20.00(0.05)$ & $20.26(0.06)$ \\
\hline & & & $19.99(0.05)$ & $19.99(0$. & $20.17(0.05)$ & 19.97( & 19.93 & $19.92(0.05)$ & $19.79(0.05)$ \\
\hline & & & $19.76(0.05)$ & $19.79(0.05)$ & $19.82(0.05)$ & $19.90(0.05)$ & $20.09(0.05)$ & $20.47(0.06)$ & \\
\hline 408 & 815.215 & 176.524 & $19.70(0.08)$ & $19.71(0.06)$ & $19.73(0.07)$ & $19.83(0.07)$ & $19.66(0.05)$ & $19.54(0.05)$ & 99.99 (9.99) \\
\hline & & & $19.79(0.06)$ & $19.86(0.06)$ & $19.68(0.06)$ & $20.16(0.07)$ & $20.23(0.06)$ & $20.36(0.07)$ & $20.48(0.07)$ \\
\hline & & & $20.57(0.07)$ & 20.42 & 20.42 & 20.37 & $20.23(0.06)$ & $20.28(0.07)$ & \\
\hline 410 & 15.327 & 271.408 & $20.51(0.08)$ & 99.99 (9.99) & $20.93(0.12)$ & $21.05(0.10)$ & $19.97(0.06)$ & $20.16(0.06)$ & $20.41(0.06)$ \\
\hline & & & $99.99(9.99)$ & 99.99 (9.99) & 99.99 (9.99) & $20.39(0.06)$ & $20.37(0.07)$ & 99.99 (9.99) & $20.15(0.05)$ \\
\hline & & & $20.06(0.07)$ & $19.98(0.05)$ & 99.99 (9.99) & $19.62(0.04)$ & 99.99 (9.99) & 99.99 (9.99) & \\
\hline 414 & 106.809 & 397.028 & $19.78(0.05)$ & $20.41(0.09)$ & $20.07(0.06)$ & $19.91(0.06)$ & $20.10(0.05)$ & $20.33(0.05)$ & $20.28(0.06)$ \\
\hline & & & $20.00(0.06)$ & $20.21(0.06)$ & $19.86(0.05)$ & $19.56(0.05)$ & $19.68(0.06)$ & $19.65(0.04)$ & $19.89(0.05)$ \\
\hline & & & $19.85(0.05)$ & $20.13(0.05)$ & $20.22(0.05)$ & $20.36(0.05)$ & 20.74 & $20.75(0.07)$ & \\
\hline 416 & 701.510 & 153.983 & $20.29(0.05)$ & $19.95(0.06)$ & $20.05(0.07)$ & $20.46(0.08)$ & $19.85(0.04)$ & $19.92(0.05)$ & $20.14(0.06)$ \\
\hline & & & $19.81(0.05)$ & $20.05(0.05)$ & $20.28(0.05)$ & $19.98(0.06)$ & $19.95(0.05)$ & $19.90(0.05)$ & $19.84(0.05)$ \\
\hline & & & $19.88(0.05)$ & $19.85(0$. & $19.94(0$. & $20.16(0.05)$ & 20.37 & $20.44(0.06)$ & \\
\hline 419 & 248.910 & 805.041 & $20.19(0.05)$ & $19.86(0.05)$ & $19.90(0.05)$ & $19.98(0.06)$ & $20.15(0.05)$ & $20.08(0.05)$ & $20.27(0.05)$ \\
\hline & & & $20.37(0.05)$ & $20.18(0.05)$ & $20.09(0.05)$ & $19.70(0.05)$ & $19.67(0.05)$ & $19.62(0.05)$ & $19.74(0.05)$ \\
\hline & & & $19.79(0.05)$ & $19.93(0.04)$ & $20.15(0.05)$ & $20.26(0.05)$ & $20.20(0.05)$ & $20.10(0.05)$ & \\
\hline 423 & 525.791 & -23.687 & 99.99 (9.99) & 99.99 (9.99) & $20.67(0.09)$ & 99.99 (9.99) & 99.99 (9.99) & 99.99 (9.99) & 99.99 \\
\hline & & & $20.32(0.05)$ & $20.29(0.05)$ & $20.49(0.05)$ & $19.73(0.05)$ & $19.65(0.05)$ & $19.61(0.05)$ & 99.99 (9.99) \\
\hline & & & $19.76(0.13)$ & $19.89(0.05)$ & $20.06(0.05)$ & $20.12(0.05)$ & $20.30(0.06)$ & $20.47(0.06)$ & \\
\hline 424 & 99.815 & 4.421 & $20.65(0.07)$ & $20.63(0.08)$ & $20.86(0.20)$ & $20.88(0.12)$ & $20.16(0.08)$ & $20.32(0.05)$ & $20.71(0.08)$ \\
\hline & & & & $19.82(0.05)$ & $19.99(0.05)$ & $20.22(0.05)$ & $20.28(0.06)$ & $20.13(0.06)$ & $20.19(0.05)$ \\
\hline & & & $20.09(0.05)$ & $20.01(0.05)$ & $19.77(0.05)$ & $19.62(0.05)$ & $19.78(0.08)$ & $19.64(0.08)$ & \\
\hline 425 & 608.033 & 515.828 & $19.89(0.05)$ & $20.44(0.07)$ & $20.03(0.06)$ & $19.98(0.07)$ & $20.25(0.05)$ & $20.06(0.05)$ & $19.96(0.05)$ \\
\hline & & & $19.90(0.05)$ & $19.80(0.05)$ & $19.80(0.05)$ & $20.33(0.06)$ & $20.45(0.06)$ & $20.31(0.06)$ & $20.14(0.05)$ \\
\hline & & & & $19.91(0.04)$ & $19.93(0.05)$ & $19.93(0.05)$ & $19.98(0.05)$ & $20.26(0.06)$ & \\
\hline 429 & 624.594 & 728.730 & $19.57(0.04)$ & $19.62(0.05)$ & $19.66(0.06)$ & $19.66(0.05)$ & $20.02(0.04)$ & $19.85(0.04)$ & $19.85(0.05)$ \\
\hline & & & $20.61(0.05)$ & $20.38(0.05)$ & $20.42(0.05)$ & $20.11(0.06)$ & $20.15(0.05)$ & $20.29(0.05)$ & $20.46(0.06)$ \\
\hline & & & $20.49(0.06)$ & $20.50(0.05)$ & $20.71(0.07)$ & $20.60(0.05)$ & $20.32(0.05)$ & $20.22(0.05)$ & \\
\hline 430 & 144.655 & 456.504 & $19.90(0.05)$ & $20.22(0.06)$ & $20.45(0.07)$ & $20.69(0.09)$ & $19.87(0.04)$ & $19.86(0.05)$ & $19.75(0.05)$ \\
\hline & & & $20.98(0.09)$ & & & & $19.82(0.05)$ & $19.85(0.05)$ & $19.96(0.05)$ \\
\hline & & & $20.03(0.05)$ & $20.09(0.05)$ & $20.31(0.05)$ & $20.36(0.05)$ & $20.54(0.07)$ & $20.76(0.08)$ & \\
\hline 431 & 399.194 & 565.095 & $20.09(0.05)$ & $20.08(0.06)$ & $19.92(0.07)$ & $19.95(0.06)$ & $19.82(0.04)$ & $19.83(0.04)$ & $20.03(0.05)$ \\
\hline & & & $20.01(0.05)$ & $20.01(0.05)$ & $20.21(0.05)$ & $20.58(0.07)$ & $20.36(0.05)$ & $20.38(0.06)$ & $20.14(0.06)$ \\
\hline & & & $20.06(0.05)$ & $19.95(0.05)$ & $19.89(0.05)$ & $19.90(0.05)$ & $19.97(0.06)$ & $20.15(0.06)$ & \\
\hline
\end{tabular}


Table 7. continuation.

\begin{tabular}{|c|c|c|c|c|c|c|c|c|c|}
\hline \multirow[t]{3}{*}{ F1 ID } & \multirow[t]{3}{*}{$\mathrm{x}$} & \multirow[t]{3}{*}{$\mathrm{y}$} & & $1 \mathrm{Ks} 01(\sigma)$ & $1 \mathrm{Ks} 02(\sigma)$ & $1 \mathrm{Ks} 03(\sigma)$ & $1 \mathrm{Ks} 04(\sigma)$ & $1 \mathrm{Ks} 05(\sigma)$ & \multirow{3}{*}{$\begin{array}{l}1 \mathrm{Ks} 06(\sigma) \\
1 \mathrm{Ks} 14(\sigma)\end{array}$} \\
\hline & & & & $1 \mathrm{Ks} 09(\sigma)$ & $1 \mathrm{Ks} 10(\sigma)$ & $1 \mathrm{Ks} 11(\sigma)$ & $1 \mathrm{Ks} 12(\sigma)$ & $1 \mathrm{Ks} 13(\sigma)$ & \\
\hline & & & & $1 \mathrm{Ks} 16(\sigma)$ & $1 \mathrm{Ks} 17(\sigma)$ & $1 \mathrm{Ks} 18(\sigma)$ & $1 \mathrm{Ks} 19(\sigma)$ & $1 \mathrm{Ks} 20(\sigma)$ & \\
\hline \multirow[t]{3}{*}{4435} & 361.769 & 514.580 & $20.28(0.06)$ & $19.83(0.06)$ & $19.95(0.06)$ & $19.97(0.07)$ & $19.97(0.05)$ & $20.13(0.05)$ & $20.16(0.06)$ \\
\hline & & & $20.05(0.05)$ & $20.09(0.05)$ & $20.39(0.05)$ & $19.94(0.05)$ & $19.94(0.05)$ & $19.84(0.05)$ & $19.76(0.05)$ \\
\hline & & & $19.83(0.05)$ & $19.90(0.05)$ & $20.05(0.06)$ & $20.20(0.05)$ & $20.44(0.06)$ & $20.51(0.07)$ & \\
\hline \multirow[t]{3}{*}{438} & 624.755 & 854.376 & $20.32(0.05)$ & $19.91(0.05)$ & $20.24(0.10)$ & $20.37(0.08)$ & $19.87(0.05)$ & $20.06(0.05)$ & $20.22(0.06)$ \\
\hline & & & 99.99 (9.99) & 99.99 (9.99) & 99.99 (9.99) & 99.99 (9.99) & 99.99 (9.99) & $19.97(0.06)$ & $19.80(0.05)$ \\
\hline & & & $19.82(0.05)$ & $19.87(0.05)$ & $20.16(0.81)$ & 99.99 (9.99) & 99.99 (9.99) & $99.99(9.99)$ & \\
\hline \multirow[t]{3}{*}{440} & 388.923 & 388.467 & $20.13(0.06)$ & $20.27(0.07)$ & $20.53(0.07)$ & $20.30(0.07)$ & $20.30(0.05)$ & $20.20(0.05)$ & $20.12(0.06)$ \\
\hline & & & $20.42(0.06)$ & $20.40(0.05)$ & $20.24(0.05)$ & $19.65(0.05)$ & $19.67(0.05)$ & $19.72(0.05)$ & $19.75(0.05)$ \\
\hline & & & $19.85(0.05)$ & $19.96(0.05)$ & $20.11(0.05)$ & $20.21(0.05)$ & $20.23(0.06)$ & $20.17(0.06)$ & \\
\hline \multirow[t]{3}{*}{441} & 414.633 & 108.811 & $20.23(0.05)$ & $19.90(0.06)$ & $19.84(0.05)$ & $19.96(0.06)$ & $19.87(0.05)$ & $19.85(0.04)$ & $20.09(0.05)$ \\
\hline & & & $19.97(0.05)$ & $20.27(0.05)$ & $20.44(0.05)$ & $19.99(0.05)$ & $20.03(0.05)$ & $19.89(0.05)$ & $19.87(0.05)$ \\
\hline & & & $19.92(0.05)$ & $20.01(0.05)$ & $20.16(0.05)$ & $20.19(0.05)$ & $20.40(0.05)$ & $20.40(0.06)$ & \\
\hline \multirow[t]{3}{*}{444} & 121.751 & 657.133 & $20.46(0.05)$ & $21.03(0.12)$ & $20.81(0.10)$ & $20.81(0.10)$ & $20.12(0.05)$ & $20.24(0.04)$ & $20.42(0.06)$ \\
\hline & & & $19.45(0.04)$ & $19.43(0.04)$ & $19.58(0.05)$ & $20.49(0.05)$ & $20.52(0.06)$ & $20.55(0.05)$ & $20.52(0.05)$ \\
\hline & & & $20.53(0.05)$ & $20.44(0.05)$ & $20.18(0.05)$ & $20.06(0.05)$ & $20.07(0.05)$ & $19.84(0.05)$ & \\
\hline \multirow[t]{3}{*}{449} & 341.643 & 462.908 & $19.74(0.04)$ & $20.29(0.06)$ & $19.98(0.07)$ & $20.12(0.07)$ & $20.18(0.05)$ & $19.97(0.04)$ & $19.80(0.05)$ \\
\hline & & & $20.05(0$ & $19.87(0.05)$ & $19.69(0.05)$ & $21.13(0.08)$ & 21.20 & $20.73(0.06)$ & $20.92(0.06)$ \\
\hline & & & $20.79(0.06)$ & $20.87(0.06)$ & $20.88(0.07)$ & $20.64(0.06)$ & $20.40(0.06)$ & $20.06(0.05)$ & \\
\hline \multirow[t]{3}{*}{451} & 126.261 & 525.443 & $19.69(0.05)$ & $19.70(0.05)$ & $19.62(0.05)$ & $19.66(0.05)$ & $20.13(0.04)$ & $19.92(0.05)$ & $19.73(0.05)$ \\
\hline & & & $20.35(0.05)$ & $20.25(0.05)$ & $19.97(0.04)$ & $20.41(0.06)$ & $20.51(0.06)$ & $20.41(0.05)$ & $20.26(0.05)$ \\
\hline & & & $20.29(0.05)$ & $20.17(0.05)$ & $20.12(0.05)$ & $20.09(0.05)$ & $20.21(0.05)$ & $19.99(0.05)$ & \\
\hline \multirow[t]{3}{*}{452} & 312.709 & 620.715 & $19.98(0.04)$ & $20.46(0.08)$ & $20.19(0.07)$ & $19.90(0.06)$ & $20.15(0.05)$ & $20.03(0.04)$ & $20.03(0.05)$ \\
\hline & & & $20.15(0.05)$ & $19.87(0.05)$ & $19.89(0.04)$ & $20.37(0.06)$ & $20.39(0.05)$ & $20.40(0.05)$ & $20.29(0.05)$ \\
\hline & & & $20.06(0.05)$ & $20.00(0.05)$ & $19.82(0.05)$ & $19.83(0.05)$ & $19.80(0.05)$ & $19.88(0.05)$ & \\
\hline 456 & 122.608 & 557.688 & $20.30(0.05)$ & $20.12(0.05)$ & $20.13(0.06)$ & $20.08(0.06)$ & $20.30(0.04)$ & $20.30(0.05)$ & $20.40(0.06)$ \\
\hline & & & 20.15 & 20.27 & 20.21 & 19.99 & 19.79 & $19.81(0$ & $19.72(0.05)$ \\
\hline & & & $19.65(0.05)$ & $19.80(0.05)$ & $19.78(0.05)$ & $19.97(0.05)$ & $20.10(0.05)$ & $20.18(0.05)$ & \\
\hline 459 & 233.563 & 541.986 & $20.17(0.04)$ & $20.20(0.05)$ & $20.36(0.10)$ & $20.42(0.08)$ & $20.12(0.05)$ & $20.19(0.04)$ & $20.23(0.06)$ \\
\hline & & & $20.22(0.05)$ & $20.04(0.05)$ & $20.12(0.04)$ & $19.64(0.05)$ & $19.71(0.04)$ & $19.70(0.05)$ & $19.91(0.05)$ \\
\hline & & & & 20.03 & & 20.17 & 20.15 & $20.15(0.06)$ & \\
\hline 462 & 605.738 & 856.930 & $19.89(0$ & $20.32(0.06)$ & $19.90(0.07)$ & $19.96(0.07)$ & 20.00 & 19.93 & $19.94(0.07)$ \\
\hline & & & 99.99 (9.99) & 99.99 (9.99) & 99.99 (9.99) & 99.99 (9.99) & 99.99 (9. & $20.45(0.18)$ & $20.29(0.06)$ \\
\hline & & & $20.28(0.05)$ & $20.27(0.05)$ & 99.99 (9.99) & 99.99 (9.99) & 99.99 (9.99) & $99.99(9.99)$ & \\
\hline 463 & 261.293 & 713.331 & 19.69 & $20.09(0.06)$ & $19.88(0.06)$ & $19.79(0.06)$ & $20.23(0.05)$ & $19.98(0.04)$ & 19.78 \\
\hline & & & 20.24 & 19.93 & $19.75(0.04)$ & $20.56(0.07)$ & $20.53(0.06)$ & 20.50 & $20.28(0.05)$ \\
\hline & & & 20.25 & 20.37 & 20.31 & 20.40 & 20.26 & 19.98 & \\
\hline 464 & 340.665 & 307.723 & $19.64(0.05)$ & $19.87(0.06)$ & $19.77(0.07)$ & $19.74(0.05)$ & $20.13(0.05)$ & $19.99(0.04)$ & $19.83(0.05)$ \\
\hline & & & $20.17(0.05)$ & $20.16(0.05)$ & $20.07(0.04)$ & $19.88(0.05)$ & $20.04(0.05)$ & $20.08(0.05)$ & $20.27(0.05)$ \\
\hline & & & & 20.22 & 20.15 & 20.07 & 20.12 & $20.24(0.06)$ & \\
\hline 465 & 202.571 & 650.247 & $20.14(0.08)$ & $20.18(0.08)$ & $20.31(0.07)$ & $20.05(0.06)$ & $20.16(0.06)$ & $20.03(0.06)$ & $20.00(0.05)$ \\
\hline & & & $19.87(0.05)$ & $20.02(0.06)$ & $20.36(0.09)$ & $19.99(0.05)$ & $19.95(0.05)$ & $19.89(0.07)$ & $19.92(0.05)$ \\
\hline & & & & $20.14(0.06)$ & & $20.28(0.06)$ & $20.31(0.06)$ & $20.31(0.06)$ & \\
\hline 466 & 382.225 & 561.735 & $19.53(0$ & & 19.68 & $19.71(0$ & 20.13 & 19.94 & 19.71 \\
\hline & & & $20.45(0.05)$ & $20.15(0.05)$ & $19.78(0.04)$ & $20.51(0.07)$ & $20.46(0.06)$ & $20.44(0.05)$ & $20.51(0.05)$ \\
\hline & & & & $20.50(0.05)$ & $20.28(0.05)$ & $20.41(0.05)$ & $20.50(0.06)$ & $20.30(0.05)$ & \\
\hline 468 & 776.724 & 505.933 & $19.99(0.04)$ & $20.21(0.05)$ & $20.24(0.07)$ & $20.62(0.09)$ & $20.20(0.05)$ & $20.14(0.05)$ & $20.18(0.06)$ \\
\hline & & & & $20.08(0.05)$ & $20.04(0.04)$ & $19.69(0.05)$ & $19.74(0.05)$ & $19.79(0.05)$ & $19.93(0.05)$ \\
\hline & & & & $20.12(0.05)$ & $20.33(0.06)$ & $20.50(0.05)$ & $20.49(0.05)$ & $20.37(0.06)$ & \\
\hline 469 & 744.998 & 822.162 & $20.75(0.07)$ & $20.11(0.06)$ & $20.62(0.15)$ & $20.49(0.08)$ & $20.16(0.07)$ & $20.30(0.05)$ & $21.07(0.11)$ \\
\hline & & & $20.14(0.05)$ & $20.18(0.05)$ & $20.30(0.05)$ & $20.15(0.06)$ & $20.05(0.05)$ & $19.97(0.05)$ & $19.81(0.05)$ \\
\hline & & & & & 19.76 & $19.84(0.05)$ & $19.92(0.05)$ & $20.27(0.06)$ & \\
\hline 472 & 637.803 & 504.423 & $20.13(0.04)$ & $20.37(0.05)$ & $20.34(0.06)$ & $20.18(0.07)$ & $20.31(0.05)$ & $20.21(0.05)$ & $20.17(0.05)$ \\
\hline & & & $20.45(0.05)$ & $20.61(0.06)$ & $20.59(0.05)$ & $19.73(0.05)$ & $19.71(0.05)$ & $19.76(0.05)$ & $19.75(0.05)$ \\
\hline & & & $19.78(0.05)$ & $19.78(0.05)$ & $19.90(0.05)$ & $19.99(0.05)$ & $20.23(0.06)$ & $20.46(0.06)$ & \\
\hline 474 & 557.936 & 724.268 & $20.16(0.05)$ & $19.99(0.06)$ & $20.11(0.07)$ & $20.24(0.06)$ & $20.02(0.04)$ & $20.04(0.05)$ & $20.12(0.05)$ \\
\hline & & & & & & & & & $20.21(0.05)$ \\
\hline & & & $20.02(0.05)$ & $19.94(0.05)$ & $19.95(0.05)$ & $19.98(0.05)$ & $19.99(0.05)$ & $20.17(0.05)$ & \\
\hline 476 & 650.086 & 380.669 & $20.80(0.05)$ & $19.76(0.05)$ & $19.77(0.05)$ & $19.73(0.05)$ & $20.71(0.05)$ & $20.77(0.05)$ & $20.91(0.06)$ \\
\hline & & & $19.49(0.04)$ & $19.53(0.04)$ & $19.70(0.04)$ & $20.60(0.07)$ & $20.48(0.05)$ & $20.63(0.06)$ & $20.70(0.06)$ \\
\hline & & & $20.61(0.07)$ & $20.79(0.06)$ & $20.69(0.07)$ & $20.65(0.05)$ & $20.52(0.06)$ & $20.29(0.06)$ & \\
\hline
\end{tabular}


Table 7. continuation.

\begin{tabular}{|c|c|c|c|c|c|c|c|c|c|}
\hline \multirow[t]{3}{*}{1 ID } & \multirow[t]{3}{*}{$\mathrm{X}$} & \multirow[t]{3}{*}{$\mathrm{y}$} & 1Ks07 $(\sigma)$ & $1 \mathrm{Ks} 01(\sigma)$ & $1 \mathrm{Ks} 02(\sigma)$ & $1 \mathrm{Ks} 03(\sigma)$ & $1 \mathrm{Ks} 04(\sigma)$ & $1 \mathrm{Ks} 05(\sigma)$ & \multirow{3}{*}{$\begin{array}{l}1 \mathrm{Ks} 06(\sigma) \\
1 \mathrm{Ks} 14(\sigma)\end{array}$} \\
\hline & & & $1 \mathrm{Ks} 08(\sigma)$ & $1 \mathrm{Ks} 09(\sigma)$ & $1 \mathrm{Ks} 10(\sigma)$ & $1 \mathrm{Ks} 11(\sigma)$ & $1 \mathrm{Ks} 12(\sigma)$ & $1 \mathrm{Ks} 13(\sigma)$ & \\
\hline & & & $1 \mathrm{Ks} 15(\sigma)$ & $1 \mathrm{Ks} 16(\sigma)$ & $1 \mathrm{Ks} 17(\sigma)$ & $1 \mathrm{Ks} 18(\sigma)$ & $1 \mathrm{Ks} 19(\sigma)$ & $1 \mathrm{Ks} 20(\sigma)$ & \\
\hline \multirow[t]{3}{*}{ 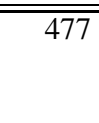 } & 752.549 & 36.633 & $20.27(0.05)$ & $20.44(0.08)$ & $20.30(0.06)$ & $20.34(0.08)$ & $20.31(0.05)$ & $20.31(0.05)$ & $20.58(0.10)$ \\
\hline & & & $20.34(0.05)$ & $20.38(0.05)$ & $20.41(0.04)$ & $19.78(0.05)$ & $19.76(0.05)$ & $19.75(0.05)$ & $19.82(0.05)$ \\
\hline & & & $19.82(0.05)$ & $19.77(0.05)$ & $19.94(0.05)$ & $20.06(0.05)$ & $20.32(0.05)$ & $20.30(0.06)$ & \\
\hline \multirow[t]{3}{*}{479} & 720.278 & 102.333 & $20.24(0.05)$ & $19.94(0.06)$ & $19.86(0.05)$ & $19.93(0.06)$ & $19.79(0.05)$ & $19.87(0.05)$ & $20.19(0.06)$ \\
\hline & & & $20.11(0.05)$ & $20.24(0.05)$ & $20.33(0.05)$ & $19.79(0.05)$ & $19.84(0.05)$ & $19.94(0.05)$ & $20.09(0.05)$ \\
\hline & & & $20.17(0.05)$ & $20.28(0.05)$ & $20.35(0.06)$ & $20.41(0.06)$ & $20.36(0.05)$ & $20.15(0.05)$ & \\
\hline \multirow[t]{3}{*}{483} & 120.610 & 235.796 & $20.03(0.06)$ & $20.28(0.07)$ & $20.36(0.07)$ & $20.28(0.06)$ & $20.55(0.06)$ & $20.33(0.05)$ & $20.17(0.06)$ \\
\hline & & & $20.01(0.05)$ & $19.94(0.05)$ & $19.83(0.05)$ & $20.44(0.07)$ & $20.43(0.05)$ & $20.33(0.06)$ & $20.33(0.06)$ \\
\hline & & & $20.21(0.05)$ & $20.06(0.05)$ & $19.97(0.05)$ & $19.78(0.05)$ & $19.81(0.05)$ & $19.90(0.05)$ & \\
\hline \multirow[t]{3}{*}{485} & 468.489 & 89.196 & $20.37(0.04)$ & $20.27(0.07)$ & $20.56(0.09)$ & $20.97(0.12)$ & $19.92(0.04)$ & $20.00(0.05)$ & $20.18(0.05)$ \\
\hline & & & $19.85(0.05)$ & $19.88(0.05)$ & $20.12(0.05)$ & $20.42(0.06)$ & $20.42(0.05)$ & $20.24(0.06)$ & $20.19(0.05)$ \\
\hline & & & $20.17(0.05)$ & $19.98(0.05)$ & $19.88(0.05)$ & $19.86(0.05)$ & $19.91(0.05)$ & $20.01(0.06)$ & \\
\hline \multirow[t]{3}{*}{494} & 380.188 & 672.565 & $20.56(0.05)$ & $20.22(0.07)$ & $20.56(0.08)$ & $20.65(0.07)$ & $20.01(0.05)$ & $20.35(0.05)$ & $20.68(0.06)$ \\
\hline & & & $20.16(0.05)$ & $20.31(0.05)$ & $20.54(0.04)$ & $19.92(0.05)$ & $19.91(0.05)$ & $19.86(0.05)$ & $19.79(0.04)$ \\
\hline & & & $19.81(0.05)$ & $19.80(0.04)$ & $19.80(0.05)$ & $19.85(0.05)$ & $20.00(0.05)$ & $20.28(0.05)$ & \\
\hline \multirow[t]{3}{*}{497} & 449.506 & 396.456 & $20.22(0.04)$ & $20.91(0.16)$ & $20.58(0.06)$ & $21.02(0.16)$ & $20.28(0.06)$ & $20.35(0.09)$ & $20.50(0.07)$ \\
\hline & & & $20.27(0.09)$ & $20.38(0.07)$ & $20.15(0.07)$ & $19.65(0.06)$ & 19.50 & $19.56(0.06)$ & $19.71(0.05)$ \\
\hline & & & $19.95(0.05)$ & $19.89(0.05)$ & $20.14(0.07)$ & $20.29(0.06)$ & $20.57(0.10)$ & $20.36(0.08)$ & \\
\hline \multirow[t]{3}{*}{503} & 78.869 & 286.741 & $20.44(0.05)$ & $20.50(0.08)$ & $20.45(0.06)$ & $20.69(0.08)$ & $20.58(0.05)$ & $20.45(0.05)$ & $20.43(0.06)$ \\
\hline & & & $20.62(0.06)$ & $20.75(0.07)$ & $20.59(0.05)$ & $19.75(0.05)$ & $19.81(0.05)$ & $19.71(0.05)$ & $19.80(0.04)$ \\
\hline & & & $19.82(0.05)$ & $19.95(0.05)$ & $20.04(0.05)$ & $20.18(0.05)$ & $20.39(0.06)$ & $20.65(0.07)$ & \\
\hline \multirow[t]{3}{*}{504} & 46.923 & 287.125 & $20.68(0.05)$ & $19.93(0.05)$ & $20.20(0.08)$ & $20.53(0.08)$ & $19.90(0.05)$ & $20.12(0.05)$ & $20.30(0.06)$ \\
\hline & & & $20.04(0.05)$ & $20.27(0.05)$ & $20.50(0.05)$ & $19.88(0.06)$ & $19.84(0.05)$ & $19.73(0.05)$ & $19.93(0.05)$ \\
\hline & & & $19.92(0.05)$ & $20.03(0.05)$ & $20.16(0.06)$ & $20.24(0.05)$ & $20.29(0.06)$ & $20.40(0.06)$ & \\
\hline 509 & 101.341 & 95.678 & $20.31(0.06)$ & $19.97(0.06)$ & $20.27(0.10)$ & $20.68(0.09)$ & $20.14(0.06)$ & $20.21(0.05)$ & $20.44(0.06)$ \\
\hline & & & $20.31(0.05)$ & $20.30(0.05)$ & 20.30 & 19.94 & 19.91 & $19.72(0.05)$ & $19.86(0.05)$ \\
\hline & & & $19.87(0.05)$ & $20.01(0.05)$ & $20.15(0.07)$ & $20.18(0.05)$ & $20.45(0.08)$ & $20.34(0.07)$ & \\
\hline 519 & 360.972 & 822.938 & $19.98(0.05)$ & $19.83(0.05)$ & $19.71(0.06)$ & $19.56(0.05)$ & $20.28(0.05)$ & $20.13(0.04)$ & $20.05(0.05)$ \\
\hline & & & $20.24(0.05)$ & $19.88(0.05)$ & $19.77(0.05)$ & $20.60(0.07)$ & $20.72(0.07)$ & $20.70(0.08)$ & $20.52(0.05)$ \\
\hline & & & $20.48(0.05)$ & 20.47 & $20.40(0$ & 20.33 & $20.15(0$. & $19.90(0.05)$ & \\
\hline 522 & 34.853 & 649.853 & $20.31(0.07)$ & $20.30(0.07)$ & $20.13(0.08)$ & $20.01(0.07)$ & $20.26(0.05)$ & $20.16(0.05)$ & $20.15(0.07)$ \\
\hline & & & $19.92(0.05)$ & $19.95(0.05)$ & $20.19(0.05)$ & $20.56(0.07)$ & $20.46(0.06)$ & $20.36(0.06)$ & $20.18(0.05)$ \\
\hline & & & $20.00(0.05)$ & $19.95(0.05)$ & $19.95(0.05)$ & $19.98(0.05)$ & $20.21(0.07)$ & $20.43(0.06)$ & \\
\hline 524 & 58.562 & 617.082 & $20.60(0.06)$ & $20.12(0.06)$ & $20.34(0.09)$ & $20.13(0.07)$ & $20.50(0.05)$ & $20.54(0.06)$ & $20.47(0.06)$ \\
\hline & & & $20.12(0.05)$ & $20.59(0.07)$ & 20.46 & 19.74( & 19.75 & $19.71(($ & $19.95(0.05)$ \\
\hline & & & $19.92(0.05)$ & $20.05(0.05)$ & $20.23(0.05)$ & $20.24(0.05)$ & $20.42(0.06)$ & $20.24(0.07)$ & \\
\hline 527 & 378.870 & 746.828 & $19.80(0.07)$ & $20.33(0.08)$ & $20.10(0.05)$ & $19.85(0.06)$ & $20.24(0.06)$ & $20.04(0.05)$ & $19.85(0.05)$ \\
\hline & & & $20.32(0.05)$ & $20.18(0.05)$ & $19.97(0.07)$ & $20.04(0.05)$ & $20.08(0.05)$ & $20.19(0.06)$ & $20.28(0.06)$ \\
\hline & & & $20.24(0.06)$ & $20.23(0.05)$ & $20.24(0.06)$ & $20.21(0.06)$ & 20.16 & $20.01(0.05)$ & \\
\hline 528 & 677.816 & 585.615 & $20.03(0.07)$ & $20.65(0.18)$ & $20.45(0.08)$ & $20.68(0.12)$ & $20.32(0.07)$ & $20.33(0.05)$ & $20.13(0.05)$ \\
\hline & & & $20.14(0.06)$ & $20.00(0.06)$ & $19.91(0.07)$ & $20.31(0.06)$ & $20.33(0.06)$ & $20.41(0.06)$ & $20.27(0.05)$ \\
\hline & & & $20.19(0.05)$ & $19.96(0.05)$ & $19.96(0.06)$ & $19.88(0.05)$ & $19.89(0.05)$ & $20.14(0.06)$ & \\
\hline 530 & 783.447 & 536.537 & $20.31(0.08)$ & $19.96(0.07)$ & $20.01(0.09)$ & $19.98(0.06)$ & $20.28(0.08)$ & $20.21(0.06)$ & $20.21(0.06)$ \\
\hline & & & $20.13(0.06)$ & $20.08(0.07)$ & $20.19(0.07)$ & $19.69(0.05)$ & $19.80(0.06)$ & $19.94(0.06)$ & $20.13(0.06)$ \\
\hline & & & $20.42(0.06)$ & $20.43(0.07)$ & $20.65(0.08)$ & $20.51(0.08)$ & $20.29(0.07)$ & $20.27(0.06)$ & \\
\hline 533 & 308.464 & 660.672 & $19.76(0.04)$ & $20.27(0.05)$ & $20.09(0.06)$ & $20.05(0.06)$ & $20.24(0.05)$ & $20.11(0.04)$ & $19.89(0.05)$ \\
\hline & & & $20.75(0.05)$ & $20.35(0.06)$ & $20.12(0.04)$ & $20.71(0.07)$ & $20.63(0.07)$ & $20.69(0.07)$ & $20.46(0.06)$ \\
\hline & & & $20.41(0.06)$ & $20.34(0.06)$ & $20.29(0.05)$ & $20.19(0.05)$ & $20.01(0.05)$ & $19.81(0.05)$ & \\
\hline 536 & 526.016 & 793.034 & $20.39(0.05)$ & $20.06(0.07)$ & $19.91(0.06)$ & $20.12(0.06)$ & $19.88(0.04)$ & $19.98(0.05)$ & $20.20(0.05)$ \\
\hline & & & $20.19(0.05)$ & $20.20(0.05)$ & $20.53(0.05)$ & $20.16(0.06)$ & $20.07(0.06)$ & $20.05(0.05)$ & $19.87(0.05)$ \\
\hline & & & & $19.93(0.05)$ & $20.11(0.05)$ & $20.33(0.05)$ & $20.62(0.06)$ & $20.68(0.08)$ & \\
\hline 541 & 384.603 & 709.448 & $20.62(0.05)$ & $20.37(0.07)$ & $20.51(0.07)$ & $20.81(0.11)$ & $20.25(0.05)$ & $20.32(0.05)$ & $20.49(0.05)$ \\
\hline & & & $20.05(0.05)$ & $20.04(0.05)$ & $20.12(0.05)$ & $20.53(0.06)$ & $20.47(0.06)$ & $20.42(0.06)$ & $20.20(0.05)$ \\
\hline & & & $20.08(0.05)$ & $20.14(0.05)$ & $19.85(0.05)$ & $19.88(0.04)$ & $19.81(0.05)$ & $19.86(0.05)$ & \\
\hline 548 & 436.416 & 426.247 & $20.12(0.05)$ & $20.00(0.06)$ & $19.93(0.08)$ & $20.07(0.07)$ & $20.05(0.05)$ & $20.13(0.05)$ & $20.04(0.05)$ \\
\hline & & & $20.74(0.06)$ & & $20.18(0.04)$ & $19.99(0.05)$ & $19.94(0.05)$ & $19.93(0.05)$ & $20.21(0.05)$ \\
\hline & & & $20.35(0.05)$ & $20.24(0.05)$ & $20.28(0.05)$ & $20.15(0.05)$ & $20.33(0.05)$ & $20.46(0.06)$ & \\
\hline 549 & 748.629 & 626.308 & $20.04(0.04)$ & $20.28(0.06)$ & $20.32(0.09)$ & $20.38(0.07)$ & $20.23(0.05)$ & $20.24(0.05)$ & $20.42(0.07)$ \\
\hline & & & $20.26(0.05)$ & $20.06(0.05)$ & $19.93(0.04)$ & $20.32(0.06)$ & $20.20(0.05)$ & $20.33(0.05)$ & $20.25(0.05)$ \\
\hline & & & $20.28(0.05)$ & $20.26(0.05)$ & $20.10(0.05)$ & $20.05(0.05)$ & $19.99(0.05)$ & $20.01(0.05)$ & \\
\hline
\end{tabular}


Table 7. continuation.

\begin{tabular}{|c|c|c|c|c|c|c|c|c|c|}
\hline \multirow[t]{3}{*}{ F1 ID } & \multirow[t]{3}{*}{$\mathrm{x}$} & \multirow[t]{3}{*}{$\mathrm{y}$} & $1 \mathrm{Ks} 07(\sigma)$ & $1 \mathrm{Ks} 01(\sigma)$ & $1 \mathrm{Ks} 02(\sigma)$ & $1 \mathrm{Ks} 03(\sigma)$ & $1 \mathrm{Ks} 04(\sigma)$ & $1 \mathrm{Ks} 05(\sigma)$ & \multirow{3}{*}{$\begin{array}{l}1 \mathrm{Ks} 06(\sigma) \\
1 \mathrm{Ks} 14(\sigma)\end{array}$} \\
\hline & & & $1 \mathrm{Ks} 08(\sigma)$ & $1 \mathrm{Ks} 09(\sigma)$ & $1 \mathrm{Ks} 10(\sigma)$ & $1 \mathrm{Ks} 11(\sigma)$ & $1 \mathrm{Ks} 12(\sigma)$ & $1 \mathrm{Ks} 13(\sigma)$ & \\
\hline & & & $1 \mathrm{Ks} 15(\sigma)$ & $1 \mathrm{Ks} 16(\sigma)$ & $1 \mathrm{Ks} 17(\sigma)$ & $1 \mathrm{Ks} 18(\sigma)$ & $1 \mathrm{Ks} 19(\sigma)$ & $1 \mathrm{Ks} 20(\sigma)$ & \\
\hline \multirow[t]{3}{*}{$\overline{552}$} & 4424.360 & 615.109 & $20.40(0.05)$ & $20.81(0.10)$ & $20.98(0.09)$ & $20.88(0.09)$ & $20.43(0.05)$ & $20.59(0.05)$ & $20.55(0.06)$ \\
\hline & & & $20.84(0.06)$ & $20.80(0.06)$ & $20.68(0.05)$ & $19.83(0.05)$ & $19.80(0.04)$ & $19.80(0.04)$ & $19.92(0.04)$ \\
\hline & & & $19.91(0.05)$ & $20.06(0.05)$ & $20.24(0.05)$ & $20.31(0.05)$ & $20.58(0.06)$ & $20.59(0.07)$ & \\
\hline \multirow[t]{3}{*}{554} & 41.029 & 357.642 & $20.46(0.05)$ & $20.69(0.08)$ & $20.94(0.10)$ & $21.08(0.09)$ & $19.89(0.04)$ & $20.04(0.05)$ & $20.21(0.05)$ \\
\hline & & & $20.03(0.05)$ & $20.06(0.05)$ & $19.94(0.04)$ & $20.49(0.07)$ & $20.49(0.06)$ & $20.59(0.07)$ & $20.91(0.08)$ \\
\hline & & & $20.96(0.06)$ & $21.04(0.06)$ & $21.26(0.08)$ & $21.21(0.09)$ & $21.74(0.14)$ & $21.58(0.17)$ & \\
\hline \multirow[t]{3}{*}{555} & 618.959 & 401.389 & $20.36(0.04)$ & $20.34(0.05)$ & $20.39(0.08)$ & $20.35(0.07)$ & $20.42(0.05)$ & $20.38(0.05)$ & $20.35(0.05)$ \\
\hline & & & $20.45(0.06)$ & $20.47(0.06)$ & $20.31(0.05)$ & $19.66(0.05)$ & $19.61(0.05)$ & $19.67(0.05)$ & $19.86(0.05)$ \\
\hline & & & $20.01(0.05)$ & $20.08(0.05)$ & $20.38(0.06)$ & $20.39(0.05)$ & $20.46(0.05)$ & $20.43(0.06)$ & \\
\hline \multirow[t]{3}{*}{559} & 109.478 & 737.089 & $20.45(0.06)$ & $20.17(0.07)$ & $20.22(0.06)$ & $19.99(0.05)$ & $20.59(0.06)$ & $20.56(0.06)$ & $20.64(0.07)$ \\
\hline & & & $20.50(0.06)$ & $20.49(0.06)$ & $20.57(0.06)$ & $19.99(0.05)$ & $19.91(0.05)$ & $19.84(0.05)$ & $19.91(0.05)$ \\
\hline & & & $19.96(0.06)$ & $20.05(0.05)$ & $20.14(0.06)$ & $20.19(0.05)$ & $20.38(0.07)$ & $20.54(0.07)$ & \\
\hline \multirow[t]{3}{*}{560} & 175.575 & 324.498 & $20.38(0.05)$ & $20.13(0.06)$ & $20.19(0.06)$ & $20.36(0.07)$ & $20.41(0.05)$ & $20.35(0.05)$ & $20.32(0.05)$ \\
\hline & & & $20.42(0.05)$ & $20.60(0.06)$ & $20.27(0.05)$ & $19.76(0.05)$ & $19.83(0.04)$ & $19.82(0.05)$ & $19.95(0.05)$ \\
\hline & & & $20.01(0.04)$ & $20.17(0.05)$ & $20.30(0.05)$ & $20.40(0.06)$ & $20.51(0.05)$ & $20.44(0.07)$ & \\
\hline \multirow[t]{3}{*}{561} & 159.943 & 560.438 & $20.49(0.05)$ & $20.28(0.07)$ & $20.62(0.07)$ & $20.66(0.09)$ & $20.20(0.05)$ & $20.54(0.06)$ & $20.79(0.07)$ \\
\hline & & & $20.29(0$ & $20.38(0.05)$ & $20.37(0.05)$ & $19.83(0.05)$ & 19.76 & $19.78(0.05)$ & $19.87(0.04)$ \\
\hline & & & $19.93(0.05)$ & $20.08(0.05)$ & $20.27(0.05)$ & $20.36(0.05)$ & $20.50(0.06)$ & $20.44(0.06)$ & \\
\hline \multirow[t]{3}{*}{564} & 70.924 & 715.081 & $20.89(0.06)$ & $20.95(0.10)$ & $21.33(0.15)$ & $21.07(0.13)$ & $20.64(0.06)$ & $20.81(0.05)$ & $21.17(0.09)$ \\
\hline & & & $20.25(0.06)$ & $20.44(0.06)$ & $20.73(0.06)$ & $19.96(0.05)$ & $19.94(0.05)$ & $19.93(0.05)$ & $19.86(0.05)$ \\
\hline & & & & $19.85(0.05)$ & $20.06(0.05)$ & $20.05(0.05)$ & $20.23(0.06)$ & $20.62(0.09)$ & \\
\hline \multirow[t]{3}{*}{566} & 143.276 & 386.319 & $19.85(0.07)$ & $20.13(0.07)$ & $20.37(0.07)$ & $20.46(0.07)$ & $20.31(0.05)$ & $20.28(0.05)$ & $19.94(0.05)$ \\
\hline & & & $20.00(0.05)$ & $20.26(0.05)$ & $20.44(0.06)$ & $20.04(0.06)$ & $20.10(0.06)$ & $20.08(0.05)$ & $20.10(0.05)$ \\
\hline & & & $20.19(0.06)$ & $20.14(0.05)$ & $20.19(0.07)$ & $20.23(0.06)$ & $20.27(0.06)$ & $20.34(0.07)$ & \\
\hline 567 & 796.243 & 303.054 & $20.58(0.05)$ & $20.50(0.07)$ & $20.73(0.09)$ & $20.81(0.09)$ & $20.27(0.05)$ & $20.43(0.05)$ & $20.60(0.07)$ \\
\hline & & & 20.52 & 20.33 & 20.57 & 19.87 & 19.81 & $19.81(0.05)$ & $19.91(0.05)$ \\
\hline & & & $19.93(0.04)$ & $20.01(0.05)$ & $20.10(0.05)$ & $20.25(0.05)$ & $20.27(0.05)$ & $20.67(0.07)$ & \\
\hline 571 & 204.220 & 129.730 & $20.31(0.04)$ & $20.01(0.05)$ & $20.01(0.07)$ & $20.36(0.08)$ & $20.19(0.05)$ & $20.21(0.05)$ & $20.12(0.05)$ \\
\hline & & & $20.38(0.05)$ & $20.30(0.05)$ & $20.28(0.05)$ & $19.90(0.06)$ & $19.90(0.05)$ & $19.84(0$ & $20.00(0.05)$ \\
\hline & & & & 20.20 & & 20.27 & 20.40 & $20.21(0$ & \\
\hline 572 & 831.839 & 266.819 & $20.29(0.05)$ & $20.41(0.06)$ & $20.60(0.10)$ & $20.75(0.08)$ & 20.05 & $20.06(0$ & 99.99 (9.99) \\
\hline & & & $19.74(0.05)$ & $19.92(0.05)$ & $20.03(0.05)$ & $21.07(0.08)$ & $21.13(0$. & $20.87(0.06)$ & $20.98(0.08)$ \\
\hline & & & $20.73(0.06)$ & $20.32(0.05)$ & $20.20(0.05)$ & $20.13(0.05)$ & $19.96(0.05)$ & $20.11(0.05)$ & \\
\hline 573 & 438.028 & 228.245 & $20.51(0.05)$ & $19.88(0.05)$ & $20.02(0.07)$ & $20.21(0.07)$ & $19.95(0.04)$ & $20.08(0.05)$ & 20.40 \\
\hline & & & 20.23 & $20.44(0.05)$ & $20.64(0.05)$ & $19.81(0.05)$ & $19.91(0.05)$ & $19.89(0.05)$ & $19.98(0.05)$ \\
\hline & & & $20.08(C$ & 20.26 & 2048 & 20.70 & $20.86(0.07)$ & $20.98(0.07)$ & \\
\hline 575 & 214.401 & 198.591 & $20.46(0.05)$ & $20.23(0.06)$ & $20.07(0.06)$ & $20.30(0.07)$ & $20.22(0.05)$ & $20.20(0$ & $20.35(0.06)$ \\
\hline & & & $20.07(0.05)$ & $20.16(0.05)$ & $20.48(0.05)$ & $19.97(0.05)$ & $20.04(0.05)$ & $19.91(0.05)$ & $19.89(0.05)$ \\
\hline & & & & 20.03 & 20.20 & 20.39 & 20.79 & $20.86(0.08)$ & \\
\hline 579 & 83.866 & 395.534 & $20.34(0.05)$ & $20.25(0.06)$ & $20.31(0.08)$ & $19.91(0.05)$ & $20.29(0.05)$ & $20.21(0.05)$ & $20.18(0.06)$ \\
\hline & & & $19.96(0.05)$ & $20.22(0.05)$ & $20.51(0.05)$ & $20.23(0.05)$ & $20.11(0.05)$ & $20.02(0.05)$ & $20.01(0.05)$ \\
\hline & & & & $20.09(0.05)$ & & $20.25(0.06)$ & $20.78(0.06)$ & $20.76(0.07)$ & \\
\hline 581 & 822.800 & 526.661 & 20.26 & 20.09 & 20.26 & $20.47(0$ & $20.02(0$ & 20.21 & 99.99 \\
\hline & & & $20.20(0.05)$ & $20.28(0.05)$ & $20.24(0.04)$ & $19.82(0.05)$ & $19.89(0.05)$ & $19.91(0.05)$ & $20.13(0.05)$ \\
\hline & & & $20.20(0.06)$ & $20.30(0.05)$ & $20.53(0.07)$ & $20.67(0.06)$ & $20.67(0.06)$ & $20.58(0.07)$ & \\
\hline 583 & 656.805 & 627.704 & $20.37(0.04)$ & $20.88(0.07)$ & $20.73(0.07)$ & $20.71(0.10)$ & $20.47(0.05)$ & $20.48(0.05)$ & $20.50(0.06)$ \\
\hline & & & $20.46(0.05)$ & $20.47(0.05)$ & $20.46(0.04)$ & $19.80(0.05)$ & $19.66(0.05)$ & $19.74(0.05)$ & $19.77(0.05)$ \\
\hline & & & $19.80(0.05)$ & $19.89(0.05)$ & $20.00(0.05)$ & $20.10(0.05)$ & $20.40(0.06)$ & $20.47(0.07)$ & \\
\hline 584 & 459.402 & 172.627 & $20.17(0.05)$ & $20.13(0.06)$ & $20.29(0.07)$ & $20.55(0.09)$ & $20.19(0.06)$ & $20.24(0.05)$ & $20.28(0.05)$ \\
\hline & & & $19.87(0.06)$ & $20.18(0.05)$ & $20.06(0.06)$ & $19.83(0.05)$ & $19.85(0.05)$ & $19.92(0.05)$ & $20.14(0.05)$ \\
\hline & & & & $20.45(0.05)$ & $20.47(0.05)$ & $20.34(0.05)$ & $20.35(0.06)$ & $20.44(0.07)$ & \\
\hline 585 & 70.548 & 307.259 & $20.25(0.06)$ & $20.12(0.06)$ & $20.18(0.08)$ & $20.31(0.06)$ & $20.34(0.05)$ & $20.36(0.05)$ & $20.37(0.06)$ \\
\hline & & & $20.23(0.06)$ & $20.32(0.06)$ & $19.94(0.05)$ & $20.33(0.06)$ & $20.35(0.05)$ & $20.29(0.07)$ & $20.25(0.06)$ \\
\hline & & & $20.28(0.05)$ & $20.28(0.05)$ & $20.18(0.05)$ & $20.04(0.05)$ & $19.94(0.05)$ & $19.84(0.06)$ & \\
\hline 586 & 477.751 & 525.601 & $19.92(0.04)$ & $20.27(0.07)$ & $20.13(0.07)$ & $20.10(0.06)$ & $20.10(0.05)$ & $20.00(0.05)$ & $19.93(0.05)$ \\
\hline & & & & $20.23(0.06)$ & & & & $20.49(0.06)$ & $20.51(0.06)$ \\
\hline & & & $20.68(0.07)$ & $20.57(0.06)$ & $20.70(0.07)$ & $20.81(0.07)$ & $20.50(0.06)$ & $20.39(0.07)$ & \\
\hline 587 & 90.366 & 126.397 & $19.88(0.06)$ & $20.26(0.07)$ & $20.43(0.10)$ & $20.29(0.07)$ & $20.35(0.05)$ & $20.28(0.05)$ & $20.03(0.06)$ \\
\hline & & & $20.34(0.05)$ & $20.07(0.05)$ & $19.91(0.04)$ & $20.45(0.06)$ & $20.43(0.05)$ & $20.40(0.06)$ & $20.62(0.06)$ \\
\hline & & & $20.36(0.07)$ & $20.33(0.05)$ & $20.10(0.06)$ & $19.99(0.05)$ & $20.00(0.06)$ & $19.87(0.07)$ & \\
\hline
\end{tabular}


Table 7. continuation.

\begin{tabular}{|c|c|c|c|c|c|c|c|c|c|}
\hline \multirow[t]{3}{*}{1 ID } & \multirow[t]{3}{*}{$\mathrm{X}$} & \multirow[t]{3}{*}{$\mathrm{y}$} & $1 \mathrm{Ks} 07(\sigma)$ & $1 \mathrm{Ks} 01(\sigma)$ & $1 \mathrm{Ks} 02(\sigma)$ & $1 \mathrm{Ks} 03(\sigma)$ & $1 \mathrm{Ks} 04(\sigma)$ & $1 \mathrm{Ks} 05(\sigma)$ & \multirow{3}{*}{$\begin{array}{l}1 \mathrm{Ks} 06(\sigma) \\
1 \mathrm{Ks} 14(\sigma)\end{array}$} \\
\hline & & & $1 \mathrm{Ks} 08(\sigma)$ & $1 \mathrm{Ks} 09(\sigma)$ & $1 \mathrm{Ks} 10(\sigma)$ & $1 \mathrm{Ks} 11(\sigma)$ & $1 \mathrm{Ks} 12(\sigma)$ & $1 \mathrm{Ks} 13(\sigma)$ & \\
\hline & & & $1 \mathrm{Ks} 15(\sigma)$ & $1 \mathrm{Ks} 16(\sigma)$ & $1 \mathrm{Ks} 17(\sigma)$ & $1 \mathrm{Ks} 18(\sigma)$ & $1 \mathrm{Ks} 19(\sigma)$ & $1 \mathrm{Ks} 20(\sigma)$ & \\
\hline \multirow[t]{3}{*}{$\overline{588}$} & 69.022 & 385.081 & $19.94(0.05)$ & $20.02(0.06)$ & $19.98(0.05)$ & $19.80(0.05)$ & $20.51(0.05)$ & $20.37(0.05)$ & $20.25(0.05)$ \\
\hline & & & $20.28(0.05)$ & $20.51(0.06)$ & $20.20(0.05)$ & $19.87(0.05)$ & $20.05(0.05)$ & $20.05(0.05)$ & $20.26(0.05)$ \\
\hline & & & $20.21(0.05)$ & $20.22(0.05)$ & $20.21(0.06)$ & $20.18(0.05)$ & $20.28(0.06)$ & $20.23(0.05)$ & \\
\hline \multirow[t]{3}{*}{590} & 147.305 & 746.683 & $20.54(0.06)$ & $20.11(0.07)$ & $20.03(0.06)$ & $19.94(0.06)$ & $19.93(0.06)$ & $20.06(0.05)$ & $20.11(0.06)$ \\
\hline & & & $20.12(0.06)$ & $20.28(0.06)$ & $20.43(0.05)$ & $20.03(0.07)$ & $20.02(0.05)$ & $20.12(0.06)$ & $20.11(0.06)$ \\
\hline & & & $20.23(0.06)$ & $20.25(0.05)$ & $20.45(0.07)$ & $20.44(0.07)$ & $20.44(0.07)$ & $20.36(0.07)$ & \\
\hline \multirow[t]{3}{*}{600} & 694.275 & 800.618 & $20.39(0.05)$ & $19.93(0.06)$ & $19.97(0.10)$ & $20.12(0.07)$ & $19.92(0.05)$ & $20.02(0.05)$ & $20.26(0.07)$ \\
\hline & & & $20.41(0.05)$ & $20.44(0.06)$ & $20.79(0.06)$ & $20.10(0.05)$ & $20.03(0.05)$ & $20.04(0.05)$ & $20.11(0.06)$ \\
\hline & & & $20.08(0.05)$ & $20.11(0.05)$ & $20.25(0.07)$ & $20.35(0.05)$ & $20.43(0.06)$ & $20.45(0.06)$ & \\
\hline \multirow[t]{3}{*}{603} & 122.595 & 494.816 & $20.55(0.07)$ & $20.28(0.08)$ & $20.30(0.08)$ & $20.35(0.07)$ & $20.08(0.06)$ & $20.29(0.05)$ & $20.41(0.06)$ \\
\hline & & & $20.23(0.05)$ & $20.40(0.06)$ & $20.45(0.06)$ & $20.04(0.05)$ & $19.99(0.05)$ & $19.97(0.05)$ & $20.02(0.05)$ \\
\hline & & & $20.06(0.05)$ & $20.27(0.05)$ & $20.25(0.05)$ & $20.32(0.05)$ & $20.38(0.05)$ & $20.31(0.06)$ & \\
\hline \multirow[t]{3}{*}{608} & 227.197 & 81.820 & $19.98(0.05)$ & $20.68(0.07)$ & $20.63(0.09)$ & $20.76(0.09)$ & $20.14(0.04)$ & $19.99(0.05)$ & $20.02(0.05)$ \\
\hline & & & $20.39(0.05)$ & $20.54(0.06)$ & $20.46(0.05)$ & $20.20(0.06)$ & $20.26(0.07)$ & $20.32(0.06)$ & $20.32(0.05)$ \\
\hline & & & $20.38(0.06)$ & $20.36(0.05)$ & $20.28(0.05)$ & $20.23(0.05)$ & $20.07(0.05)$ & $19.87(0.05)$ & \\
\hline \multirow[t]{3}{*}{610} & 875.921 & 243.440 & $20.27(0.06)$ & $20.19(0.07)$ & 99.99 (9.99) & $19.99(0.06)$ & $20.14(0.04)$ & $20.15(0.05)$ & 99.99 (9.99) \\
\hline & & & $19.91(0.05)$ & $20.09(0.05)$ & $20.11(0.04)$ & $20.33(0.05)$ & $20.34(0.06)$ & $20.27(0.05)$ & $20.38(0.05)$ \\
\hline & & & $20.47(0.05)$ & $20.40(0.06)$ & $20.39(0.06)$ & $20.31(0.05)$ & $20.18(0.06)$ & $20.10(0.06)$ & \\
\hline \multirow[t]{3}{*}{621} & 570.552 & 27.635 & $20.20(0.04)$ & $19.96(0.06)$ & $20.00(0.05)$ & $20.40(0.09)$ & $20.09(0.05)$ & $20.15(0.04)$ & $20.45(0.06)$ \\
\hline & & & $20.26(0.05)$ & $20.20(0.05)$ & $20.18(0.04)$ & $20.30(0.06)$ & $20.40(0.07)$ & $20.40(0.05)$ & $20.36(0.05)$ \\
\hline & & & $20.45(0.05)$ & $20.44(0.05)$ & $20.50(0.08)$ & $20.33(0.05)$ & $20.17(0.05)$ & $19.84(0.05)$ & \\
\hline \multirow[t]{3}{*}{623} & 445.251 & 779.298 & $20.18(0.05)$ & $20.35(0.07)$ & $20.23(0.07)$ & $20.20(0.06)$ & $20.42(0.05)$ & $20.31(0.05)$ & $20.16(0.05)$ \\
\hline & & & $20.63(0.05)$ & $20.48(0.06)$ & $20.43(0.05)$ & $19.85(0.05)$ & $19.90(0.05)$ & $19.86(0.05)$ & $20.02(0.05)$ \\
\hline & & & $20.03(0.05)$ & $20.16(0.05)$ & $20.45(0.06)$ & $20.49(0.05)$ & $20.70(0.07)$ & $20.56(0.06)$ & \\
\hline 624 & 63.818 & 31.241 & $20.10(0.05)$ & $20.42(0.07)$ & $20.13(0.10)$ & $20.50(0.10)$ & $20.02(0.05)$ & $20.08(0.05)$ & $20.21(0.07)$ \\
\hline & & & $20.34(0.06)$ & $20.21(0.06)$ & $20.27(0.05)$ & $20.12(0.06)$ & $20.04(0.06)$ & $20.10(0.06)$ & $20.37(0.06)$ \\
\hline & & & $20.50(0.05)$ & $20.30(0.05)$ & $20.43(0.06)$ & $20.08(0.05)$ & $24.45(2.05)$ & $20.84(0.08)$ & \\
\hline 626 & 192.645 & 54.732 & $20.07(0.08)$ & $19.92(0.06)$ & $19.33(0.22)$ & $19.41(0.15)$ & $19.90(0.12)$ & $19.90(0.10)$ & $19.35(0.13)$ \\
\hline & & & $21.57(0.15)$ & $20.69(0.09)$ & $20.45(0.10)$ & $21.44(0.18)$ & $20.57(0.09)$ & $21.17(0.35)$ & \\
\hline & & & $21.32(0.33)$ & $22.35(0.5$ & 21.70 & $20.88(0.18)$ & 99.99 (9.99) & $22.15(0.99)$ & \\
\hline 628 & 372.952 & 316.404 & $19.99(0.10)$ & $20.56(0.07)$ & $19.98(0.08)$ & $19.92(0.09)$ & $20.15(0.07)$ & $20.04(0.07)$ & $20.09(0.10)$ \\
\hline & & & $19.92(0.08)$ & $19.95(0.07)$ & $20.07(0.09)$ & $20.52(0.11)$ & $20.29(0.06)$ & $20.33(0.06)$ & $20.32(0.09)$ \\
\hline & & & $20.69(0.07)$ & $20.22(0.06)$ & $20.46(0.09)$ & $20.28(0.07)$ & $20.15(0.08)$ & $19.95(0.10)$ & \\
\hline 629 & 186.363 & 76.196 & $20.42(0.06)$ & $20.36(0.07)$ & $20.60(0.07)$ & $20.88(0.10)$ & $20.49(0.06)$ & $20.43(0.05)$ & $20.74(0.07)$ \\
\hline & & & $21.05(0.10)$ & $20.39(0.06)$ & 20.30 & 99.99 & $19.97(0.05)$ & $19.98(0.05)$ & 99.99 (9.99) \\
\hline & & & $20.16(0.05)$ & $20.22(0.05)$ & $20.17(0.05)$ & $20.08(0.05)$ & $20.04(0.05)$ & $20.13(0.05)$ & \\
\hline 631 & 153.405 & -12.272 & $99.99(9.99)$ & $20.39(0.08)$ & $20.32(0.13)$ & $20.38(0.11)$ & $19.87(0.08)$ & 99.99 (9.99) & 99.99 (9.99) \\
\hline & & & $20.33(0.05)$ & $20.48(0.06)$ & $20.61(0.05)$ & $20.10(0.06)$ & $20.18(0.06)$ & $19.94(0.05)$ & $20.05(0.05)$ \\
\hline & & & & $20.12(0.05)$ & $20.34(0.0$ & 20.31 & 20.61 & 20.59 & \\
\hline 632 & 687.298 & 541.673 & $20.07(0.04)$ & $20.25(0.07)$ & $20.31(0.07)$ & $20.27(0.06)$ & $20.19(0.05)$ & $20.09(0.05)$ & $20.12(0.05)$ \\
\hline & & & $20.23(0.05)$ & $20.32(0.05)$ & $20.27(0.04)$ & $19.95(0.05)$ & $20.00(0.05)$ & $20.13(0.05)$ & $20.48(0.05)$ \\
\hline & & & $20.52(0.05)$ & $20.48(0.05)$ & $20.49(0.07)$ & $20.48(0.05)$ & $20.41(0.05)$ & $20.41(0.05)$ & \\
\hline 633 & 765.892 & 33.885 & $20.06(0.06)$ & $20.44(0.06)$ & $20.08(0.08)$ & $19.96(0.06)$ & $20.53(0.06)$ & $20.45(0.05)$ & $20.87(0.11)$ \\
\hline & & & $20.57(0.06)$ & $20.54(0.06)$ & $20.59(0.05)$ & $19.82(0.05)$ & $19.86(0.05)$ & $19.85(0.05)$ & $20.01(0.05)$ \\
\hline & & & $20.15(0.05)$ & $20.18(0.05)$ & $20.38(0.06)$ & $20.41(0.05)$ & $20.48(0.06)$ & $20.69(0.07)$ & \\
\hline 641 & 198.166 & 560.710 & $20.34(0.04)$ & $20.08(0.05)$ & $20.05(0.06)$ & $20.10(0.06)$ & $20.22(0.05)$ & $20.21(0.05)$ & $20.17(0.06)$ \\
\hline & & & $20.25(0.05)$ & $20.19(0.05)$ & $20.48(0.04)$ & $20.79(0.09)$ & $20.52(0.05)$ & $20.34(0.06)$ & $20.12(0.05)$ \\
\hline & & & $20.03(0.05)$ & $20.04(0.05)$ & $20.05(0.05)$ & $20.08(0.05)$ & $20.17(0$. & 20.40 & \\
\hline 643 & 856.354 & 845.953 & $20.59(0.08)$ & $19.91(0.06)$ & $20.60(0.16)$ & $20.56(0.11)$ & $20.10(0.08)$ & $20.23(0.07)$ & 99.99 (9.99) \\
\hline & & & $19.92(1.61)$ & 99.99 (9.99) & $20.66(0.06)$ & $20.10(0.06)$ & $19.90(0.07)$ & $19.98(0.05)$ & $20.11(0.06)$ \\
\hline & & & $20.28(0.06)$ & $20.29(0.04)$ & $20.42(0.05)$ & 99.99 (9.99) & 99.99 (9.99) & 99.99 (9.99) & \\
\hline 648 & 323.482 & 229.953 & $20.10(0.05)$ & $20.89(0.09)$ & $20.91(0.10)$ & $21.03(0.10)$ & $20.89(0.06)$ & $20.59(0.05)$ & $20.32(0.05)$ \\
\hline & & & $20.38(0.05)$ & $20.17(0.05)$ & $19.99(0.04)$ & $20.81(0.08)$ & $20.80(0.06)$ & $20.70(0.08)$ & $20.45(0.06)$ \\
\hline & & & $20.40(0.05)$ & $20.16(0.05)$ & $20.04(0.05)$ & $20.01(0.05)$ & $20.00(0.05)$ & $20.16(0.07)$ & \\
\hline 652 & 522.784 & 730.552 & $20.18(0.04)$ & $20.40(0.07)$ & $20.34(0.07)$ & $20.30(0.07)$ & $20.14(0.05)$ & $20.24(0.05)$ & $20.19(0.05)$ \\
\hline & & & $20.37(0.05)$ & $20.19(0.05)$ & $20.13(0.05)$ & $20.05(0.05)$ & $20.17(0.05)$ & $20.17(0.05)$ & $20.44(0.06)$ \\
\hline & & & $20.53(0.06)$ & $20.49(0.05)$ & $20.43(0.05)$ & $20.33(0.05)$ & $20.41(0.06)$ & $20.34(0.06)$ & \\
\hline 653 & 768.808 & -24.089 & $99.99(9.99)$ & $99.99(9.99)$ & $20.60(0.09)$ & 99.99 (9.99) & $99.99(9.99)$ & $99.99(9.99)$ & 99.99 (9.99) \\
\hline & & & $20.14(0.05)$ & $20.06(0.05)$ & $20.12(0.05)$ & $20.77(0.08)$ & $20.64(0.06)$ & $20.61(0.06)$ & $20.60(0.18)$ \\
\hline & & & $20.65(1.51)$ & $20.28(0.06)$ & $20.26(0.06)$ & $20.18(0.06)$ & $20.45(0.07)$ & $20.67(0.09)$ & \\
\hline
\end{tabular}


Table 7. continuation.

\begin{tabular}{|c|c|c|c|c|c|c|c|c|c|}
\hline \multirow[t]{3}{*}{ F1 ID } & \multirow[t]{3}{*}{$\mathrm{X}$} & \multirow[t]{3}{*}{$\mathrm{y}$} & $1 \mathrm{Ks} 07(\sigma)$ & $1 \mathrm{Ks} 01(\sigma)$ & $1 \mathrm{Ks} 02(\sigma)$ & $1 \mathrm{Ks} 03(\sigma)$ & $1 \mathrm{Ks} 04(\sigma)$ & $1 \mathrm{Ks} 05(\sigma)$ & \multirow{3}{*}{$\begin{array}{l}1 \mathrm{Ks} 06(\sigma) \\
1 \mathrm{Ks} 14(\sigma)\end{array}$} \\
\hline & & & $1 \mathrm{Ks} 08(\sigma)$ & $1 \mathrm{Ks} 09(\sigma)$ & $1 \mathrm{Ks} 10(\sigma)$ & $1 \mathrm{Ks} 11(\sigma)$ & $1 \mathrm{Ks} 12(\sigma)$ & $1 \mathrm{Ks} 13(\sigma)$ & \\
\hline & & & $1 \mathrm{Ks} 15(\sigma)$ & $1 \mathrm{Ks} 16(\sigma)$ & $1 \mathrm{Ks} 17(\sigma)$ & $1 \mathrm{Ks} 18(\sigma)$ & $1 \mathrm{Ks} 19(\sigma)$ & $1 \mathrm{Ks} 20(\sigma)$ & \\
\hline \multirow[t]{3}{*}{$\overline{c 657}$} & 708.216 & 431.793 & $19.86(0.04)$ & $20.30(0.06)$ & $20.17(0.07)$ & $\bar{~} 19.96(0.06)$ & $20.27(0.05)$ & $20.18(0.05)$ & $19.97(0.05)$ \\
\hline & & & $20.80(0.07)$ & $20.76(0.05)$ & $20.87(0.05)$ & $20.19(0.06)$ & $20.16(0.05)$ & $20.28(0.05)$ & $20.47(0.06)$ \\
\hline & & & $20.62(0.06)$ & $20.71(0.05)$ & $20.87(0.07)$ & $21.04(0.06)$ & $20.85(0.08)$ & $21.16(0.09)$ & \\
\hline \multirow[t]{3}{*}{661} & 572.238 & 20.186 & $20.46(0.04)$ & $20.03(0.06)$ & $20.10(0.05)$ & $20.52(0.07)$ & $19.99(0.05)$ & $20.08(0.05)$ & $20.12(0.05)$ \\
\hline & & & $20.27(0.05)$ & $20.29(0.05)$ & $20.73(0.06)$ & $20.23(0.06)$ & $20.18(0.05)$ & $20.03(0.05)$ & $20.23(0.05)$ \\
\hline & & & $20.17(0.05)$ & $20.24(0.05)$ & $20.30(0.06)$ & $20.41(0.05)$ & $20.45(0.06)$ & $20.83(0.07)$ & \\
\hline \multirow[t]{3}{*}{663} & 49.071 & 207.327 & $20.63(0.06)$ & $19.96(0.06)$ & $20.25(0.11)$ & $20.53(0.08)$ & $20.43(0.05)$ & $20.66(0.06)$ & $20.46(0.07)$ \\
\hline & & & $20.38(0.05)$ & $20.46(0.06)$ & $20.27(0.05)$ & $20.48(0.07)$ & $20.49(0.06)$ & $20.24(0.06)$ & $20.43(0.05)$ \\
\hline & & & $20.29(0.05)$ & $20.41(0.05)$ & $20.19(0.06)$ & $20.01(0.05)$ & $19.93(0.05)$ & $19.88(0.05)$ & \\
\hline \multirow[t]{3}{*}{668} & 761.629 & 384.585 & $20.50(0.05)$ & $20.37(0.07)$ & $20.34(0.06)$ & $20.40(0.07)$ & $20.47(0.05)$ & $20.35(0.05)$ & $20.65(0.09)$ \\
\hline & & & $20.51(0.06)$ & $20.49(0.07)$ & $20.39(0.05)$ & $19.95(0.05)$ & $19.91(0.05)$ & $19.93(0.05)$ & $20.19(0.05)$ \\
\hline & & & $20.24(0.05)$ & $20.45(0.05)$ & $20.34(0.05)$ & $20.38(0.05)$ & $20.17(0.05)$ & $20.24(0.06)$ & \\
\hline \multirow[t]{3}{*}{669} & 819.291 & 784.309 & $20.61(0.05)$ & $19.93(0.06)$ & $20.50(0.12)$ & $20.79(0.11)$ & $20.11(0.06)$ & $20.30(0.05)$ & 99.99 (9.99) \\
\hline & & & $20.48(0.05)$ & $20.41(0.05)$ & $20.39(0.05)$ & $20.02(0.05)$ & $19.99(0.05)$ & $20.00(0.05)$ & $20.19(0.06)$ \\
\hline & & & $20.20(0.07)$ & $20.29(0.05)$ & $20.38(0.08)$ & $20.39(0.06)$ & $20.52(0.07)$ & $20.48(0.09)$ & \\
\hline \multirow[t]{3}{*}{670} & 437.063 & 810.658 & $20.33(0.05)$ & $20.02(0.06)$ & $20.30(0.07)$ & $20.14(0.07)$ & $20.26(0.05)$ & $20.24(0.05)$ & $20.36(0.06)$ \\
\hline & & & $20.69(0$ & $20.56(0.06)$ & $20.42(0.05)$ & $20.04(0.06)$ & 20.07 & $20.05(0.05)$ & $20.16(0.06)$ \\
\hline & & & $20.21(0.04)$ & $20.35(0.05)$ & $20.35(0.06)$ & $20.37(0.05)$ & $20.23(0.06)$ & $20.16(0.05)$ & \\
\hline \multirow[t]{3}{*}{682} & 361.295 & 534.566 & $20.72(0.05)$ & $20.68(0.09)$ & $20.51(0.08)$ & $20.55(0.10)$ & $20.89(0.06)$ & $20.97(0.06)$ & $21.22(0.09)$ \\
\hline & & & $20.60(0.06)$ & $20.57(0.05)$ & $20.50(0.05)$ & $19.76(0.05)$ & $19.79(0.05)$ & $19.87(0.05)$ & $19.90(0.05)$ \\
\hline & & & $20.02(0.05)$ & $20.16(0.05)$ & $20.23(0.05)$ & $20.46(0.05)$ & $20.65(0.06)$ & $20.66(0.07)$ & \\
\hline \multirow[t]{3}{*}{683} & 682.074 & 17.049 & $19.91(0.05)$ & $20.22(0.07)$ & $19.88(0.05)$ & $20.04(0.07)$ & $20.50(0.06)$ & $20.18(0.05)$ & $20.22(0.06)$ \\
\hline & & & $20.51(0.05)$ & $20.36(0.05)$ & $20.11(0.04)$ & $20.44(0.06)$ & $20.69(0.06)$ & $20.71(0.06)$ & $20.90(0.08)$ \\
\hline & & & $20.86(0.07)$ & $20.77(0.06)$ & $20.69(0.06)$ & $20.79(0.06)$ & $20.70(0.06)$ & $20.32(0.05)$ & \\
\hline 685 & 502.782 & 296.070 & $19.99(0.05)$ & $20.79(0.07)$ & $20.60(0.07)$ & $20.59(0.08)$ & $20.45(0.05)$ & $20.35(0.05)$ & 20.25 \\
\hline & & & 05) & 20.19 & 19.97 & 20.60 & 20.44 & $20.57(0.06)$ & $20.60(0.06)$ \\
\hline & & & $20.66(0.06)$ & $20.55(0.05)$ & $20.33(0.05)$ & $20.24(0.05)$ & $20.10(0.05)$ & $20.05(0.05)$ & \\
\hline 686 & 399.311 & -14.018 & 99.99 (9.99) & $19.90(0.18)$ & $20.10(0.09)$ & $20.52(0.18)$ & $20.29(0.21)$ & 99.99 (9.99) & 99.99 (9.99) \\
\hline & & & $19.81(0.08)$ & $20.23(0.10)$ & $20.12(0.11)$ & $20.73(0.22)$ & $20.86(0.24)$ & $20.59(0.15)$ & $21.22(0.33)$ \\
\hline & & & & 20.82 & & 21.49 & 21.32 & & \\
\hline 688 & 215.140 & 349.738 & $20.12(0.05)$ & $20.67(0.08)$ & $20.69(0.09)$ & 20.89 & 20.60 & 20.35 & $20.08(0.05)$ \\
\hline & & & $19.98(0.05)$ & $20.26(0.05)$ & $20.33(0.05)$ & $20.06(0.06)$ & 20.11 & $20.08(0.06)$ & $20.27(0.05)$ \\
\hline & & & $20.38(0.05)$ & $20.56(0.06)$ & $20.67(0.07)$ & $20.63(0.06)$ & $20.80(0.06)$ & $20.64(0.07)$ & \\
\hline 702 & 680.365 & 528.461 & 20.81 & $20.35(0.06)$ & $20.68(0.09)$ & $20.68(0.10)$ & $20.24(0.05)$ & $20.46(0.05)$ & 20.76 \\
\hline & & & 20.56 & $20.62(0.06)$ & $20.83(0.05)$ & $20.01(0.05)$ & 19.93 & 19.98 (0. & $20.04(0.05)$ \\
\hline & & & 20.06 ( & $20.04(0.05)$ & 20.21 & 20.38 & 20.55 & $20.92(0.09)$ & \\
\hline 703 & 801.073 & 764.353 & $20.03(0.05)$ & $20.25(0.06)$ & $20.11(0.16)$ & $19.99(0.07)$ & $20.44(0.06)$ & $20.41(0.05)$ & $20.54(0.11)$ \\
\hline & & & $20.60(0.05)$ & $20.28(0.05)$ & $20.07(0.04)$ & $20.45(0.06)$ & $20.63(0.06)$ & $20.36(0.08)$ & $20.61(0.08)$ \\
\hline & & & & 20.51 & 20.37 & 20.53 & 20.42 & $20.10(0.05)$ & \\
\hline 705 & 422.670 & 660.956 & $20.73(0.05)$ & $20.18(0.07)$ & $20.16(0.06)$ & $20.24(0.07)$ & $20.18(0.05)$ & $20.35(0.04)$ & $20.69(0.06)$ \\
\hline & & & $19.79(0.05)$ & $19.71(0.05)$ & $19.86(0.05)$ & $21.34(0.13)$ & $21.20(0.11)$ & $21.15(0.06)$ & $20.99(0.07)$ \\
\hline & & & & $20.98(0.06)$ & & $20.82(0.06)$ & $20.98(0.07)$ & $20.49(0.07)$ & \\
\hline 706 & 37.628 & 472.303 & 20. & 20.24 & 20.13 & 20.41 & & 20.12 & 20.37 \\
\hline & & & $20.43(0.06)$ & $20.40(0.05)$ & $20.55(0.05)$ & $20.23(0.06)$ & $20.22(0.05)$ & $20.29(0.06)$ & $20.22(0.05)$ \\
\hline & & & $20.18(0.05)$ & $20.38(0.05)$ & $20.36(0.07)$ & $20.40(0.05)$ & $20.59(0.07)$ & $20.52(0.07)$ & \\
\hline 707 & 511.431 & 752.320 & $20.70(0.05)$ & $20.15(0.05)$ & $20.50(0.07)$ & $20.49(0.09)$ & $19.96(0.07)$ & $20.27(0.05)$ & $20.47(0.06)$ \\
\hline & & & & $20.44(0.05)$ & $20.70(0.05)$ & & $20.18(0.05)$ & $20.22(0.05)$ & $20.11(0.05)$ \\
\hline & & & & $20.06(0.05)$ & $20.10(0.05)$ & $20.29(0.05)$ & $20.49(0.06)$ & $20.75(0.07)$ & \\
\hline 708 & 88.775 & 79.997 & $20.55(0.07)$ & $20.42(0.07)$ & $20.77(0.13)$ & $21.02(0.12)$ & $20.67(0.07)$ & $20.64(0.06)$ & $20.82(0.07)$ \\
\hline & & & $20.96(0.06)$ & $20.29(0.05)$ & $20.10(0.05)$ & $20.58(0.08)$ & $20.66(0.07)$ & $20.45(0.07)$ & $20.37(0.05)$ \\
\hline & & & & $20.16(0.05)$ & & $19.91(0.05)$ & $20.10(0.07)$ & $20.39(0.07)$ & \\
\hline 711 & 601.609 & 371.452 & $21.02(0.12)$ & 99.99 (9.99) & $99.99(9.99)$ & $21.83(0.42)$ & 99.99 (9.99) & $22.35(0.31)$ & 99.99 (9.99) \\
\hline & & & $22.25(0.26)$ & $21.80(0.18)$ & $20.83(0.12)$ & $21.20(0.11)$ & $20.62(0.07)$ & 99.99 (9.99) & $21.77(0.30)$ \\
\hline & & & $21.14(0.11)$ & $23.11(0.83)$ & $21.30(0.19)$ & 99.99 (9.99) & $19.97(0.06)$ & $19.95(0.05)$ & \\
\hline 733 & 705.344 & 239.892 & $20.52(0.05)$ & $20.50(0.07)$ & $20.31(0.06)$ & $20.14(0.07)$ & $20.00(0.04)$ & $20.02(0.04)$ & $20.26(0.06)$ \\
\hline & & & & & & & & $20.23(0.05)$ & $20.35(0.05)$ \\
\hline & & & $20.42(0.05)$ & $20.48(0.05)$ & $20.88(0.06)$ & $20.98(0.06)$ & $21.10(0.09)$ & $21.11(0.09)$ & \\
\hline 734 & 157.074 & 330.983 & $20.62(0.05)$ & $20.27(0.06)$ & $20.38(0.05)$ & $20.86(0.11)$ & $20.15(0.05)$ & $20.38(0.05)$ & $20.65(0.07)$ \\
\hline & & & $20.21(0.05)$ & $20.52(0.06)$ & $20.59(0.05)$ & $20.05(0.05)$ & $19.94(0.05)$ & $19.97(0.05)$ & $20.08(0.05)$ \\
\hline & & & $20.15(0.05)$ & $20.36(0.05)$ & $20.58(0.05)$ & $20.60(0.06)$ & $20.71(0.07)$ & $20.69(0.07)$ & \\
\hline
\end{tabular}


Table 7. continuation.

\begin{tabular}{|c|c|c|c|c|c|c|c|c|c|}
\hline \multirow[t]{3}{*}{1 ID } & \multirow[t]{3}{*}{$\mathrm{X}$} & \multirow[t]{3}{*}{$\mathrm{y}$} & 1Ks07 $(\sigma)$ & $1 \mathrm{Ks} 01(\sigma)$ & $1 \mathrm{Ks} 02(\sigma)$ & $1 \mathrm{Ks} 03(\sigma)$ & $1 \mathrm{Ks} 04(\sigma)$ & $\overline{1 \mathrm{Ks} 05(\sigma)}$ & \multirow{3}{*}{$\begin{array}{l}1 \mathrm{Ks} 06(\sigma) \\
1 \mathrm{Ks} 14(\sigma)\end{array}$} \\
\hline & & & $1 \mathrm{Ks} 08(\sigma)$ & $1 \mathrm{Ks} 09(\sigma)$ & $1 \mathrm{Ks} 10(\sigma)$ & $1 \mathrm{Ks} 11(\sigma)$ & $1 \mathrm{Ks} 12(\sigma)$ & $1 \mathrm{Ks} 13(\sigma)$ & \\
\hline & & & $1 \mathrm{Ks} 15(\sigma)$ & $1 \mathrm{Ks} 16(\sigma)$ & $1 \mathrm{Ks} 17(\sigma)$ & $1 \mathrm{Ks} 18(\sigma)$ & $1 \mathrm{Ks} 19(\sigma)$ & $1 \mathrm{Ks} 20(\sigma)$ & \\
\hline \multirow[t]{3}{*}{735} & 139.861 & 121.752 & $20.14(0.05)$ & $20.68(0.07)$ & $20.95(0.09)$ & $21.50(0.21)$ & $20.69(0.05)$ & $20.60(0.05)$ & $20.40(0.06)$ \\
\hline & & & $20.34(0.05)$ & $20.21(0.05)$ & $20.23(0.04)$ & $20.67(0.08)$ & $20.67(0.06)$ & $20.51(0.07)$ & $20.33(0.06)$ \\
\hline & & & $20.20(0.06)$ & $20.24(0.05)$ & $20.24(0.06)$ & $20.14(0.05)$ & $20.36(0.05)$ & $20.64(0.07)$ & \\
\hline \multirow[t]{3}{*}{737} & 508.883 & 516.055 & $20.81(0.07)$ & $20.34(0.06)$ & $20.45(0.08)$ & $21.02(0.11)$ & $20.38(0.05)$ & $20.62(0.06)$ & $20.70(0.06)$ \\
\hline & & & $20.55(0.06)$ & $20.83(0.07)$ & $20.91(0.06)$ & $19.95(0.05)$ & $19.98(0.05)$ & $20.04(0.05)$ & $20.07(0.05)$ \\
\hline & & & $20.23(0.05)$ & $20.27(0.05)$ & $20.49(0.06)$ & $20.64(0.06)$ & $20.74(0.06)$ & $20.64(0.07)$ & \\
\hline \multirow[t]{3}{*}{741} & 128.502 & 494.060 & $20.53(0.05)$ & $21.31(0.14)$ & $21.00(0.08)$ & $20.79(0.10)$ & $20.68(0.05)$ & $20.79(0.06)$ & $20.71(0.06)$ \\
\hline & & & $20.91(0.07)$ & $20.66(0.07)$ & $20.73(0.05)$ & $19.99(0.06)$ & $20.00(0.05)$ & $19.95(0.05)$ & $20.01(0.05)$ \\
\hline & & & $20.04(0.05)$ & $20.17(0.05)$ & $20.23(0.05)$ & $20.43(0.05)$ & $20.68(0.06)$ & $20.72(0.07)$ & \\
\hline \multirow[t]{3}{*}{744} & 904.262 & 474.976 & 99.99 (9.99) & $20.48(0.08)$ & 99.99 (9.99) & 99.99 (9.99) & $20.08(0.18)$ & 19.99 (1.04) & 99.99 (9.99) \\
\hline & & & $19.98(0.05)$ & $20.24(0.05)$ & $20.49(0.05)$ & $19.98(0.14)$ & $20.10(0.06)$ & $20.22(0.05)$ & 99.99 (9.99) \\
\hline & & & $20.45(0.06)$ & $20.55(0.06)$ & $20.69(0.07)$ & $20.28(0.11)$ & $20.59(0.06)$ & $20.52(0.06)$ & \\
\hline \multirow[t]{3}{*}{745} & 338.569 & 126.905 & $20.28(0.05)$ & $20.59(0.07)$ & $20.66(0.08)$ & $21.19(0.12)$ & $20.60(0.05)$ & $20.57(0.05)$ & $20.60(0.06)$ \\
\hline & & & $20.47(0.05)$ & $20.34(0.05)$ & $20.26(0.05)$ & $20.50(0.06)$ & $20.39(0.05)$ & $20.26(0.06)$ & $20.14(0.05)$ \\
\hline & & & $20.12(0.05)$ & $20.11(0.05)$ & $20.24(0.05)$ & $20.26(0.05)$ & $20.35(0.06)$ & $20.52(0.08)$ & \\
\hline \multirow[t]{3}{*}{746} & 225.747 & -14.905 & 99.99 (9.99) & $20.28(0.07)$ & $20.65(0.14)$ & $21.46(0.16)$ & $20.51(0.47)$ & 99.99 (9.99) & 99.99 (9.99) \\
\hline & & & $20.44(0.05)$ & $20.22(0.05)$ & $19.91(0.05)$ & $20.64(0.08)$ & $20.63(0.07)$ & $20.54(0.06)$ & $20.73(0.07)$ \\
\hline & & & $20.69(0.05)$ & $20.56(0.06)$ & $20.29(0.06)$ & $20.18(0.05)$ & $20.17(0.06)$ & $20.09(0.07)$ & \\
\hline \multirow[t]{3}{*}{751} & 105.709 & 309.710 & $20.22(0.05)$ & $20.96(0.11)$ & $20.87(0.08)$ & $21.06(0.11)$ & $20.49(0.05)$ & $20.30(0.05)$ & $20.12(0.05)$ \\
\hline & & & $20.34(0.06)$ & $20.34(0.06)$ & $20.62(0.05)$ & $20.15(0.07)$ & $20.20(0.05)$ & $20.01(0.06)$ & $20.15(0.05)$ \\
\hline & & & $20.12(0.05)$ & $20.25(0.05)$ & $20.43(0.05)$ & $20.62(0.06)$ & $20.80(0.07)$ & $20.71(0.06)$ & \\
\hline \multirow[t]{3}{*}{753} & 628.524 & 761.190 & $20.18(0.04)$ & $20.68(0.10)$ & $20.95(0.13)$ & $21.15(0.15)$ & $20.50(0.05)$ & $20.54(0.05)$ & $20.49(0.07)$ \\
\hline & & & $20.57(0.06)$ & $20.25(0.05)$ & $20.33(0.05)$ & $20.48(0.06)$ & $20.52(0.06)$ & $20.39(0.06)$ & $20.24(0.05)$ \\
\hline & & & $20.20(0.05)$ & $20.23(0.05)$ & $20.24(0.05)$ & $20.49(0.06)$ & $20.60(0.06)$ & $21.03(0.11)$ & \\
\hline 757 & 193.930 & 833.109 & $21.80(0.14)$ & 99.99 (9.99) & $21.72(0.26)$ & 99.99 (9.99) & $20.71(0.09)$ & $21.21(0.09)$ & 99.99 (9.99) \\
\hline & & & $20.96(0.06)$ & $20.77(0.06)$ & $20.75(0.06)$ & $20.91(0.08)$ & 20.88 & $20.71(0.07)$ & $20.33(0.05)$ \\
\hline & & & $20.53(0.06)$ & $20.23(0.05)$ & $20.44(0.06)$ & $20.53(0.05)$ & $19.82(0.04)$ & 99.99 (9.99) & \\
\hline 765 & 765.906 & 545.318 & $20.05(0.05)$ & $20.56(0.07)$ & $20.77(0.08)$ & $21.02(0.11)$ & $20.31(0.05)$ & $20.31(0.05)$ & $20.42(0.06)$ \\
\hline & & & $20.12(0.05)$ & $20.25(0.05)$ & $20.21(0.04)$ & $20.34(0.06)$ & $20.42(0.05)$ & $20.62(0.05)$ & $20.69(0.06)$ \\
\hline & & & $20.71(0.05)$ & 20.53 & 20.40 & 20.49 & 20.46 & $20.54(0.08)$ & \\
\hline 775 & 188.193 & 504.862 & $19.90(0.05)$ & $20.47(0.06)$ & $20.16(0.07)$ & $20.06(0.06)$ & 20.70 & $20.49(0.05)$ & $20.12(0.05)$ \\
\hline & & & $20.44(0.05)$ & $20.46(0.06)$ & $20.25(0.05)$ & $20.21(0.07)$ & $20.25(0.05)$ & $20.19(0.06)$ & $20.46(0.05)$ \\
\hline & & & $20.52(0.06)$ & $20.56(0.06)$ & $20.65(0.07)$ & $20.73(0.06)$ & $20.72(0.08)$ & $20.67(0.07)$ & \\
\hline 787 & 31.338 & 245.559 & $20.22(0.05)$ & $20.50(0.08)$ & $19.90(0.06)$ & $19.97(0.06)$ & $20.30(0.05)$ & $20.36(0.05)$ & $19.74(0.05)$ \\
\hline & & & $20.85(0.07)$ & $20.62(0.06)$ & $20.31(0.05)$ & $21.21(0.12)$ & $21.01(0.08)$ & $20.94(0.09)$ & $21.17(0.08)$ \\
\hline & & & $21.08(0.07)$ & $21.53(0.11)$ & $20.71(0.11)$ & $20.98(0.06)$ & $22.01(0.25)$ & $20.93(0.07)$ & \\
\hline 790 & 126.739 & 688.708 & $19.88(0.21)$ & $20.04(0.10)$ & $19.38(0.13)$ & $20.19(0.15)$ & $19.90(0.09)$ & $19.94(0.10)$ & $19.52(0.15)$ \\
\hline & & & $20.52(0.07)$ & $19.83(0.08)$ & $20.18(0.10)$ & $21.15(0.14)$ & $20.37(0.12)$ & $20.39(0.09)$ & $20.65(0.14)$ \\
\hline & & & $21.88(0.1$ & $21.09(0.1$ & $21.57(0.13)$ & $21.67(0.10)$ & 99.99 & 24.45 (3.99) & \\
\hline 793 & 205.793 & 127.571 & $20.08(0.04)$ & $20.54(0.07)$ & $20.29(0.07)$ & $20.34(0.07)$ & $21.04(0.05)$ & $20.90(0.06)$ & $20.60(0.06)$ \\
\hline & & & $20.99(0.07)$ & $21.12(0.08)$ & $21.31(0.07)$ & $19.98(0.05)$ & $20.06(0.05)$ & $20.00(0.05)$ & $20.28(0.05)$ \\
\hline & & & $20.39(0.05)$ & $20.43(0.05)$ & $20.60(0.07)$ & $20.80(0.06)$ & $20.83(0.08)$ & $21.03(0.09)$ & \\
\hline 797 & 706.228 & 562.504 & $20.15(0.04)$ & $20.84(0.08)$ & $20.55(0.08)$ & $20.78(0.12)$ & $20.84(0.06)$ & $20.61(0.05)$ & 20.40 \\
\hline & & & $20.21(0.05)$ & $20.22(0.05)$ & $20.52(0.05)$ & $20.11(0.05)$ & $20.08(0.05)$ & $20.21(0.06)$ & $20.35(0.06)$ \\
\hline & & & $20.42(0.05)$ & $20.51(0.06)$ & $20.71(0.06)$ & $20.78(0.06)$ & $20.75(0.06)$ & $20.80(0.07)$ & \\
\hline 799 & 398.332 & 38.844 & $20.42(0.05)$ & $20.29(0.07)$ & $20.22(0.06)$ & $21.08(0.09)$ & $20.41(0.05)$ & $20.45(0.05)$ & $20.51(0.06)$ \\
\hline & & & $20.69(0.06)$ & $20.71(0.06)$ & $20.56(0.05)$ & $20.28(0.05)$ & $20.29(0.06)$ & $20.31(0.06)$ & $20.39(0.06)$ \\
\hline & & & $20.34(0.05)$ & $20.33(0.05)$ & $20.17(0.05)$ & $20.17(0.05)$ & $20.17(0.06)$ & $20.04(0.05)$ & \\
\hline 802 & 773.116 & 77.463 & $20.51(0.06)$ & $20.30(0.06)$ & $20.30(0.06)$ & $20.78(0.10)$ & $20.58(0.05)$ & $20.68(0.05)$ & $20.82(0.10)$ \\
\hline & & & $20.42(0.05)$ & $20.53(0.06)$ & $20.32(0.05)$ & $20.52(0.06)$ & $20.47(0.05)$ & $20.46(0.06)$ & $20.40(0.05)$ \\
\hline & & & & $20.25(0.05)$ & $20.39(0.06)$ & $20.24(0.05)$ & $20.23(0.05)$ & $19.93(0.05)$ & \\
\hline 809 & 604.597 & 582.775 & $20.37(0.05)$ & $20.73(0.06)$ & $20.57(0.09)$ & $20.71(0.08)$ & $20.45(0.05)$ & $20.55(0.05)$ & $20.69(0.06)$ \\
\hline & & & $20.54(0.06)$ & $20.45(0.05)$ & $20.20(0.04)$ & $20.59(0.07)$ & $20.52(0.06)$ & $20.61(0.06)$ & $20.52(0.06)$ \\
\hline & & & $20.55(0.06)$ & $20.42(0.05)$ & $20.27(0.05)$ & $20.20(0.05)$ & $20.11(0.05)$ & $20.11(0.06)$ & \\
\hline 813 & 215.885 & 336.602 & $20.25(0.04)$ & $20.98(0.10)$ & $21.01(0.10)$ & $21.40(0.15)$ & $21.02(0.05)$ & $20.77(0.05)$ & $20.51(0.07)$ \\
\hline & & & $20.32(0.05)$ & $20.28(0.05)$ & & $20.72(0.08)$ & & $20.52(0.07)$ & $20.27(0.05)$ \\
\hline & & & $20.25(0.05)$ & $20.21(0.05)$ & $20.13(0.05)$ & $20.26(0.05)$ & $20.51(0.07)$ & $20.60(0.07)$ & \\
\hline 816 & 719.942 & 37.022 & $20.46(0.06)$ & $20.64(0.07)$ & $20.26(0.07)$ & $20.27(0.07)$ & $20.23(0.05)$ & $20.13(0.05)$ & $19.97(0.07)$ \\
\hline & & & $20.30(0.05)$ & $20.37(0.06)$ & $20.55(0.05)$ & $20.57(0.06)$ & $20.52(0.06)$ & $20.46(0.06)$ & $20.36(0.05)$ \\
\hline & & & $20.28(0.05)$ & $20.46(0.05)$ & $20.37(0.05)$ & $20.60(0.06)$ & $20.72(0.06)$ & $20.85(0.06)$ & \\
\hline
\end{tabular}


Table 7. continuation.

\begin{tabular}{|c|c|c|c|c|c|c|c|c|c|}
\hline \multirow[t]{3}{*}{ F1 ID } & \multirow[t]{3}{*}{$\mathrm{x}$} & \multirow[t]{3}{*}{$\mathrm{y}$} & $1 \mathrm{Ks} 07(\sigma)$ & $1 \mathrm{Ks} 01(\sigma)$ & $1 \mathrm{Ks} 02(\sigma)$ & $1 \mathrm{Ks} 03(\sigma)$ & $1 \mathrm{Ks} 04(\sigma)$ & $1 \mathrm{Ks} 05(\sigma)$ & \multirow{3}{*}{$\begin{array}{l}1 \mathrm{Ks} 06(\sigma) \\
1 \mathrm{Ks} 14(\sigma)\end{array}$} \\
\hline & & & $1 \mathrm{Ks} 08(\sigma)$ & $1 \mathrm{Ks} 09(\sigma)$ & $1 \mathrm{Ks} 10(\sigma)$ & $1 \mathrm{Ks} 11(\sigma)$ & $1 \mathrm{Ks} 12(\sigma)$ & $1 \mathrm{Ks} 13(\sigma)$ & \\
\hline & & & $1 \mathrm{Ks} 15(\sigma)$ & $1 \mathrm{Ks} 16(\sigma)$ & $1 \mathrm{Ks} 17(\sigma)$ & $1 \mathrm{Ks} 18(\sigma)$ & $1 \mathrm{Ks} 19(\sigma)$ & $1 \mathrm{Ks} 20(\sigma)$ & \\
\hline \multirow[t]{3}{*}{818} & 820.903 & 306.009 & $20.17(0.05)$ & $20.73(0.08)$ & $20.85(0.10)$ & $21.29(0.15)$ & $20.51(0.05)$ & $20.48(0.05)$ & $99.99(9.99)$ \\
\hline & & & $20.45(0.16)$ & $20.42(0.05)$ & $20.26(0.04)$ & $20.58(0.08)$ & $20.44(0.05)$ & $20.45(0.05)$ & $20.59(0.06)$ \\
\hline & & & $20.68(0.05)$ & $20.65(0.05)$ & $20.47(0.07)$ & $20.39(0.06)$ & $20.15(0.05)$ & $20.04(0.05)$ & \\
\hline \multirow[t]{3}{*}{821} & 801.008 & 814.429 & $20.59(0.05)$ & $20.85(0.08)$ & $22.28(0.32)$ & $20.96(0.13)$ & $20.83(0.06)$ & $20.68(0.05)$ & 99.99 (9.99) \\
\hline & & & $20.44(0.05)$ & $20.35(0.05)$ & $19.97(0.04)$ & $20.92(0.09)$ & $21.01(0.08)$ & $20.64(0.07)$ & $20.51(0.06)$ \\
\hline & & & $20.57(0.06)$ & $20.49(0.06)$ & $20.21(0.05)$ & $20.11(0.05)$ & $20.60(0.06)$ & $21.25(0.09)$ & \\
\hline \multirow[t]{3}{*}{828} & 881.336 & 780.161 & $20.36(0.09)$ & $20.36(0.07)$ & 99.99 (9.99) & $20.27(0.06)$ & $20.12(0.07)$ & $20.30(0.07)$ & 99.99 (9.99) \\
\hline & & & $20.85(0.06)$ & $20.46(0.06)$ & $20.42(0.09)$ & $20.57(0.07)$ & $20.17(0.05)$ & $20.48(0.07)$ & $20.65(0.06)$ \\
\hline & & & $20.64(0.08)$ & $20.63(0.06)$ & $20.47(0.09)$ & $20.39(0.07)$ & $20.28(0.06)$ & $20.01(0.06)$ & \\
\hline \multirow[t]{3}{*}{831} & 266.563 & 225.020 & $20.40(0.05)$ & $20.65(0.07)$ & $20.82(0.08)$ & $21.05(0.11)$ & $20.98(0.05)$ & $20.96(0.06)$ & $20.63(0.06)$ \\
\hline & & & $20.28(0.05)$ & $20.31(0.05)$ & $20.28(0.05)$ & $20.45(0.07)$ & $20.35(0.05)$ & $20.15(0.05)$ & $20.11(0.05)$ \\
\hline & & & $20.14(0.05)$ & $20.21(0.05)$ & $20.29(0.06)$ & $20.44(0.05)$ & $20.64(0.05)$ & $20.70(0.07)$ & \\
\hline \multirow[t]{3}{*}{838} & 246.087 & 213.945 & $20.30(0.04)$ & $21.10(0.08)$ & $21.06(0.08)$ & $21.05(0.11)$ & $20.66(0.04)$ & $20.74(0.06)$ & $20.60(0.06)$ \\
\hline & & & $20.40(0.05)$ & $20.18(0.05)$ & $20.27(0.04)$ & $20.39(0.06)$ & $20.50(0.05)$ & $20.46(0.05)$ & $20.25(0.05)$ \\
\hline & & & $20.25(0.05)$ & $20.11(0.05)$ & $20.26(0.05)$ & $20.33(0.05)$ & $20.76(0.06)$ & $20.80(0.07)$ & \\
\hline \multirow[t]{3}{*}{839} & 563.239 & 20.287 & $20.35(0.04)$ & $20.73(0.07)$ & $20.83(0.07)$ & $21.15(0.11)$ & $20.74(0.05)$ & $20.79(0.06)$ & $20.79(0.07)$ \\
\hline & & & $20.50(0$ & $20.28(0.05)$ & $20.16(0.05)$ & $20.95(0.08)$ & 21.01 & $20.83(0.07)$ & $20.45(0.07)$ \\
\hline & & & $20.34(0.05)$ & $20.22(0.05)$ & $20.24(0.06)$ & $20.20(0.05)$ & $20.29(0.06)$ & $20.46(0.06)$ & \\
\hline \multirow[t]{3}{*}{846} & 536.441 & 380.184 & $20.37(0.05)$ & $20.44(0.07)$ & $20.73(0.08)$ & $20.73(0.08)$ & $20.18(0.05)$ & $20.22(0.05)$ & $20.33(0.05)$ \\
\hline & & & $20.36(0.06)$ & $20.34(0.05)$ & $20.29(0.05)$ & $20.89(0.07)$ & $20.70(0.07)$ & $20.72(0.07)$ & $20.76(0.07)$ \\
\hline & & & $20.74(0.07)$ & $20.67(0.06)$ & $20.63(0.07)$ & $20.67(0.06)$ & $20.65(0.06)$ & $20.55(0.06)$ & \\
\hline \multirow[t]{3}{*}{847} & 563.904 & 47.281 & $20.25(0.04)$ & $20.67(0.09)$ & $20.53(0.06)$ & $20.98(0.12)$ & $20.34(0.05)$ & $20.28(0.05)$ & $20.33(0.05)$ \\
\hline & & & $20.55(0.05)$ & $20.48(0.05)$ & $20.55(0.04)$ & $20.11(0.06)$ & $20.13(0.05)$ & $20.19(0.05)$ & $20.40(0.05)$ \\
\hline & & & $20.43(0.05)$ & $20.52(0.05)$ & $20.53(0.07)$ & $20.60(0.05)$ & $20.50(0.06)$ & $20.61(0.06)$ & \\
\hline 854 & 97.899 & 800.704 & $20.19(0.07)$ & $20.12(0.05)$ & $20.12(0.07)$ & $20.24(0.08)$ & $21.01(0.07)$ & $20.34(0.05)$ & (0.05) \\
\hline & & & 21.23 & $20.44(0.06)$ & $20.77(0.06)$ & 20.75 & 20.75 & $20.94(0.09)$ & $20.78(0.07)$ \\
\hline & & & $20.51(0.07)$ & $21.11(0.08)$ & $20.40(0.05)$ & $20.50(0.07)$ & 99.99 (9.99) & 99.99 (9.99) & \\
\hline 870 & 838.892 & 828.252 & $20.70(0.05)$ & $20.42(0.07)$ & $99.99(9.99)$ & $22.36(0.35)$ & $20.85(0.05)$ & $20.69(0.05)$ & 99.99 (9.99) \\
\hline & & & $20.49(0.06)$ & $20.57(0.06)$ & $20.33(0.05)$ & $20.46(0.06)$ & $20.67(0.06)$ & $20.27(0.05)$ & $20.21(0.05)$ \\
\hline & & & & 20.12 & & 20.43 & 21.40 & $20.36(0.16)$ & \\
\hline 880 & 40.677 & -0.487 & $20.39(0.06)$ & $20.54(0.07)$ & $20.32(0.10)$ & $20.50(0.09)$ & 20.10 & 20.13 & $20.27(0.08)$ \\
\hline & & & $22.88(0.49)$ & $20.69(0.06)$ & $20.70(0.06)$ & $21.00(0.09)$ & $20.19(0.07)$ & $20.83(0.09)$ & $21.70(0.15)$ \\
\hline & & & $21.07(0.07)$ & $21.24(0.07)$ & $21.73(0.13)$ & $21.09(0.10)$ & 99.99 (9.99) & $99.99(9.99)$ & \\
\hline 890 & 809.620 & 316.646 & & $20.67(0.08)$ & $20.41(0.10)$ & $20.42(0.06)$ & $20.25(0.04)$ & $20.34(0.05)$ & 99.99 \\
\hline & & & 20.99 & $20.94(0.08)$ & $20.72(0.05)$ & $21.31(0.13)$ & $20.55(0.05)$ & $20.78(0.07)$ & $20.63(0.07)$ \\
\hline & & & 20.85 & 20.91 & 20.73 & 20.55 & 20.42 & $20.14(0.05)$ & \\
\hline 892 & 30.380 & 244.955 & $20.76(0.06)$ & $20.59(0.08)$ & $99.99(9.99)$ & 99.99 (9.99) & $21.05(0.07)$ & $20.62(0.05)$ & 99.99 (9.99) \\
\hline & & & $20.14(0.05)$ & $20.32(0.06)$ & $20.32(0.05)$ & $21.30(0.13)$ & $21.81(0.16)$ & $21.38(0.14)$ & $21.13(0.07)$ \\
\hline & & & & $20.90(0.0$ & 99.99 & 20.53 & 19.99 & $20.04(0.05)$ & \\
\hline 893 & 57.376 & 36.502 & $20.30(0.05)$ & $20.72(0.09)$ & $20.49(0.09)$ & $20.52(0.10)$ & $20.19(0.05)$ & $20.27(0.05)$ & $20.18(0.06)$ \\
\hline & & & $20.66(0.06)$ & $20.47(0.05)$ & $20.69(0.06)$ & $20.95(0.09)$ & $20.61(0.07)$ & $20.61(0.07)$ & $21.22(0.10)$ \\
\hline & & & & $21.26(0.08)$ & & $20.76(0.06)$ & 99.99 (9.99) & 99.99 (9.99) & \\
\hline 902 & 549.196 & 60.902 & 20.79 & 20.32 & 20.19 & $20.56(0$. & $20.21(0$ & $20.33(0$ & 20.92 \\
\hline & & & $20.49(0.05)$ & $20.71(0.05)$ & $20.75(0.05)$ & $20.05(0.05)$ & $20.07(0.05)$ & $20.09(0.06)$ & $20.38(0.05)$ \\
\hline & & & $20.46(0.05)$ & $20.62(0.05)$ & $20.81(0.08)$ & $20.78(0.06)$ & $20.79(0.07)$ & $20.67(0.07)$ & \\
\hline 903 & 654.386 & 370.159 & $20.39(0.04)$ & $20.32(0.06)$ & $20.32(0.07)$ & $20.48(0.08)$ & $20.57(0.05)$ & $20.49(0.05)$ & $20.48(0.06)$ \\
\hline & & & & $20.62(0.06)$ & $20.56(0.05)$ & $19.99(0.05)$ & $20.03(0.05)$ & $20.26(0.05)$ & $20.61(0.06)$ \\
\hline & & & $20.59(0.05)$ & $20.78(0.05)$ & $20.74(0.06)$ & $20.60(0.05)$ & $20.58(0.06)$ & $20.47(0.06)$ & \\
\hline 904 & 484.146 & 292.289 & $20.30(0.05)$ & $20.90(0.10)$ & $20.67(0.11)$ & $21.58(0.18)$ & $20.59(0.05)$ & $20.62(0.05)$ & $20.82(0.08)$ \\
\hline & & & $20.16(0.05)$ & $20.30(0.05)$ & $20.50(0.05)$ & $20.32(0.05)$ & $20.28(0.05)$ & $20.44(0.06)$ & $20.52(0.06)$ \\
\hline & & & & $20.49(0.05)$ & & $20.79(0.06)$ & $20.76(0.06)$ & $21.08(0.08)$ & \\
\hline 907 & 400.733 & 873.163 & $22.98(0.21)$ & $22.61(0.45)$ & $19.58(0.12)$ & & $22.44(0.33)$ & 99.99 (9.99) & $23.04(0.42)$ \\
\hline & & & 99.99 (9.99) & 99.99 (9.99) & 99.99 (9.99) & 99.99 (9.99) & 99.99 (9.99) & 99.99 (9.99) & 99.99 (9.99) \\
\hline & & & 99.99 (9.99) & 99.99 (9.99) & $99.99(9.99)$ & 99.99 (9.99) & 99.99 (9.99) & $99.99(9.99)$ & \\
\hline 908 & 108.452 & 540.397 & $20.69(0.05)$ & $20.88(0.10)$ & $21.14(0.10)$ & $21.29(0.14)$ & $20.84(0.06)$ & $20.84(0.06)$ & $20.79(0.07)$ \\
\hline & & & & & & & & $20.51(0.06)$ & $20.41(0.05)$ \\
\hline & & & $20.31(0.05)$ & $20.31(0.05)$ & $20.25(0.05)$ & $20.24(0.05)$ & $20.32(0.05)$ & $20.33(0.06)$ & \\
\hline 910 & 230.526 & 829.521 & $20.70(0.05)$ & $20.37(0.06)$ & $20.51(0.08)$ & $20.73(0.10)$ & $20.38(0.06)$ & $20.55(0.05)$ & $20.76(0.06)$ \\
\hline & & & $20.58(0.05)$ & $20.70(0.06)$ & $20.67(0.05)$ & $20.09(0.06)$ & $20.12(0.05)$ & $20.14(0.05)$ & $20.20(0.05)$ \\
\hline & & & $20.23(0.06)$ & $20.41(0.05)$ & $20.58(0.06)$ & $20.59(0.06)$ & $20.80(0.07)$ & 99.99 (9.99) & \\
\hline
\end{tabular}


Table 7. continuation.

\begin{tabular}{|c|c|c|c|c|c|c|c|c|c|}
\hline \multirow[t]{3}{*}{1 ID } & \multirow[t]{3}{*}{$\mathrm{X}$} & \multirow[t]{3}{*}{$\mathrm{y}$} & $1 \mathrm{Ks} 07(\sigma)$ & $1 \mathrm{Ks} 01(\sigma)$ & $1 \mathrm{Ks} 02(\sigma)$ & $1 \mathrm{Ks} 03(\sigma)$ & $1 \mathrm{Ks} 04(\sigma)$ & $\overline{1 \mathrm{Ks} 05(\sigma)}$ & \multirow{3}{*}{$\begin{array}{l}1 \mathrm{Ks} 06(\sigma) \\
1 \mathrm{Ks} 14(\sigma)\end{array}$} \\
\hline & & & $1 \mathrm{Ks} 08(\sigma)$ & $1 \mathrm{Ks} 09(\sigma)$ & $1 \mathrm{Ks} 10(\sigma)$ & $1 \mathrm{Ks} 11(\sigma)$ & $1 \mathrm{Ks} 12(\sigma)$ & $1 \mathrm{Ks} 13(\sigma)$ & \\
\hline & & & $1 \mathrm{Ks} 15(\sigma)$ & $1 \mathrm{Ks} 16(\sigma)$ & $1 \mathrm{Ks} 17(\sigma)$ & $1 \mathrm{Ks} 18(\sigma)$ & $1 \mathrm{Ks} 19(\sigma)$ & $1 \mathrm{Ks} 20(\sigma)$ & \\
\hline \multirow[t]{3}{*}{9911} & 96.725 & 45.664 & $20.39(0.06)$ & $20.13(0.07)$ & $20.57(0.15)$ & $20.84(0.13)$ & $20.34(0.07)$ & $20.40(0.05)$ & $20.77(0.07)$ \\
\hline & & & $21.05(0.07)$ & $20.60(0.06)$ & $20.20(0.06)$ & $20.26(0.06)$ & $20.28(0.05)$ & $20.16(0.06)$ & $20.46(0.06)$ \\
\hline & & & $20.53(0.05)$ & $20.83(0.07)$ & $20.58(0.05)$ & $20.43(0.05)$ & $20.70(0.08)$ & $20.57(0.08)$ & \\
\hline \multirow[t]{3}{*}{924} & 725.735 & 704.143 & $20.85(0.05)$ & $19.97(0.06)$ & $20.29(0.09)$ & $20.47(0.09)$ & $20.25(0.05)$ & $20.41(0.05)$ & $20.64(0.07)$ \\
\hline & & & $20.58(0.05)$ & $20.86(0.06)$ & $21.07(0.06)$ & $20.27(0.06)$ & $20.19(0.05)$ & $20.21(0.05)$ & $20.27(0.05)$ \\
\hline & & & $20.37(0.06)$ & $20.50(0.06)$ & $20.62(0.06)$ & $20.77(0.06)$ & $20.83(0.08)$ & $20.74(0.07)$ & \\
\hline \multirow[t]{3}{*}{928} & 100.283 & 437.042 & $20.45(0.05)$ & $20.46(0.06)$ & $20.85(0.09)$ & $20.97(0.11)$ & $20.66(0.05)$ & $20.69(0.05)$ & $20.49(0.06)$ \\
\hline & & & $20.84(0.05)$ & $20.66(0.07)$ & $20.41(0.05)$ & $20.83(0.08)$ & $20.68(0.07)$ & $20.55(0.06)$ & $20.47(0.06)$ \\
\hline & & & $20.50(0.06)$ & $20.38(0.05)$ & $20.31(0.06)$ & $20.18(0.05)$ & $20.10(0.06)$ & $19.95(0.05)$ & \\
\hline \multirow[t]{3}{*}{930} & 656.499 & 229.322 & $20.30(0.07)$ & $20.04(0.09)$ & $19.99(0.12)$ & $19.96(0.07)$ & $20.30(0.07)$ & $20.22(0.06)$ & $19.15(0.09)$ \\
\hline & & & $20.76(0.07)$ & $20.63(0.06)$ & $20.38(0.05)$ & $20.80(0.08)$ & $20.80(0.10)$ & $20.76(0.07)$ & $20.58(0.08)$ \\
\hline & & & $20.67(0.05)$ & $20.76(0.07)$ & $20.59(0.07)$ & $20.71(0.07)$ & 99.99 (9.99) & 99.99 (9.99) & \\
\hline \multirow[t]{3}{*}{932} & 395.348 & 403.649 & $20.68(0.05)$ & $21.10(0.08)$ & $20.74(0.08)$ & $20.51(0.07)$ & $20.08(0.04)$ & $20.20(0.05)$ & $20.44(0.05)$ \\
\hline & & & $20.61(0.06)$ & $20.66(0.08)$ & $20.67(0.05)$ & $20.30(0.05)$ & $20.32(0.05)$ & $20.58(0.06)$ & $20.79(0.06)$ \\
\hline & & & $21.08(0.08)$ & $21.18(0.08)$ & $21.06(0.07)$ & $20.97(0.06)$ & $20.96(0.09)$ & $20.78(0.08)$ & \\
\hline \multirow[t]{3}{*}{942} & 337.809 & 155.365 & $20.52(0.05)$ & $20.08(0.05)$ & $20.06(0.06)$ & $20.17(0.08)$ & $20.22(0.04)$ & $20.32(0.05)$ & $20.48(0.06)$ \\
\hline & & & $20.60(0.06)$ & $20.60(0.06)$ & $20.66(0.05)$ & $20.52(0.06)$ & $20.57(0.06)$ & $20.70(0.06)$ & $20.91(0.06)$ \\
\hline & & & $20.99(0.06)$ & $21.04(0.06)$ & $20.98(0.09)$ & $20.83(0.07)$ & $20.79(0.07)$ & $20.80(0.07)$ & \\
\hline \multirow[t]{3}{*}{945} & 369.763 & 611.692 & $20.75(0.05)$ & $21.56(0.16)$ & $21.28(0.11)$ & $21.80(0.18)$ & $20.54(0.05)$ & $20.71(0.05)$ & $20.86(0.07)$ \\
\hline & & & $20.24(0.05)$ & $20.10(0.05)$ & $20.19(0.05)$ & $20.82(0.07)$ & $20.76(0.07)$ & $20.70(0.06)$ & $20.64(0.05)$ \\
\hline & & & $20.59(0.06)$ & $20.55(0.05)$ & $20.41(0.05)$ & $20.45(0.05)$ & $20.34(0.06)$ & $20.19(0.05)$ & \\
\hline \multirow[t]{3}{*}{966} & 852.050 & 611.405 & $20.26(0.05)$ & $20.91(0.10)$ & $21.20(0.16)$ & $20.61(0.08)$ & $20.52(0.05)$ & $20.35(0.05)$ & 99.99 (9.99) \\
\hline & & & $20.32(0.06)$ & $20.41(0.05)$ & $20.78(0.06)$ & $20.31(0.06)$ & $20.33(0.06)$ & $20.38(0.06)$ & $20.75(0.07)$ \\
\hline & & & $20.85(0.08)$ & $21.04(0.06)$ & $20.95(0.08)$ & $21.23(0.08)$ & $21.23(0.10)$ & $21.23(0.10)$ & \\
\hline 972 & 145.285 & 96.698 & $20.68(0.05)$ & $20.11(0.07)$ & $20.11(0.07)$ & $20.45(0.09)$ & $20.22(0.05)$ & $20.33(0.05)$ & $20.61(0.06)$ \\
\hline & & & $20.59(0.05)$ & $20.62(0.05)$ & 20.51 & $20.37(0.07)$ & 20.65 & $20.40(0.06)$ & $20.57(0.05)$ \\
\hline & & & $20.56(0.06)$ & $20.57(0.06)$ & $20.63(0.07)$ & $20.61(0.06)$ & $20.70(0.09)$ & $20.64(0.06)$ & \\
\hline 983 & 140.809 & 632.952 & $20.89(0.06)$ & $20.35(0.08)$ & $20.24(0.06)$ & $20.52(0.08)$ & $20.64(0.05)$ & $20.90(0.06)$ & $20.82(0.07)$ \\
\hline & & & $20.62(0.05)$ & $20.50(0.06)$ & $20.32(0.05)$ & $21.05(0.10)$ & $20.76(0.07)$ & $20.53(0.07)$ & $20.40(0.06)$ \\
\hline & & & $20.28(0.05)$ & 20.29 & $20.27(0$. & 20.40 & 20.68 & $21.13(0.10)$ & \\
\hline 985 & 671.709 & 132.349 & $20.63(0.05)$ & $20.30(0.05)$ & $20.59(0.08)$ & $20.78(0.10)$ & $20.67(0.05)$ & $20.75(0.05)$ & $20.83(0.07)$ \\
\hline & & & $20.65(0.06)$ & $20.51(0.06)$ & $20.22(0.05)$ & $20.54(0.07)$ & $20.58(0.07)$ & $20.61(0.06)$ & $20.66(0.07)$ \\
\hline & & & $20.53(0.05)$ & $20.51(0.05)$ & $20.54(0.06)$ & $20.54(0.06)$ & $20.28(0.05)$ & $20.13(0.05)$ & \\
\hline 987 & 157.953 & 314.607 & $20.57(0.05)$ & $20.94(0.12)$ & $20.91(0.07)$ & $20.75(0.09)$ & $20.36(0.04)$ & $20.39(0.05)$ & $20.45(0.05)$ \\
\hline & & & $20.93(0.07)$ & $20.74(0.06)$ & $20.85(0.06)$ & $20.69(0.09)$ & $20.60(0.06)$ & $20.61(0.06)$ & $20.79(0.06)$ \\
\hline & & & $20.76(0.06)$ & $20.78(0.06)$ & $20.51(C$ & $20.30(0.05)$ & 20.18 & $20.28(0.05)$ & \\
\hline 988 & 903.969 & 362.990 & $99.99(9.99)$ & $20.34(0.06)$ & 99.99 (9.99) & 99.99 (9.99) & $21.06(0.07)$ & $20.81(1.07)$ & 99.99 (9.99) \\
\hline & & & $19.95(0.06)$ & $20.26(0.05)$ & $20.62(0.05)$ & $20.39(0.16)$ & $20.31(0.05)$ & $20.38(0.06)$ & 99.99 (9.99) \\
\hline & & & 20.88 & $20.93(0.06)$ & $21.17(0.08)$ & $21.21(0.16)$ & 21.15 & $20.91(0.08)$ & \\
\hline 990 & 373.261 & 304.521 & $20.30(0.09)$ & $20.93(0.12)$ & $20.03(0.10)$ & $19.81(0.15)$ & $20.18(0.11)$ & $19.90(0.10)$ & $19.90(0.14)$ \\
\hline & & & $19.91(0.09)$ & $20.47(0.12)$ & $20.42(0.11)$ & $21.06(0.18)$ & $20.58(0.09)$ & $21.06(0.13)$ & $21.52(0.18)$ \\
\hline & & & $21.26(0.09)$ & $20.57(0.07)$ & $21.07(0.12)$ & $20.87(0.10)$ & 99.99 (9.99) & $21.78(0.49)$ & \\
\hline 995 & 582.106 & 56.439 & $20.69(0.05)$ & $20.05(0.06)$ & $20.09(0.06)$ & $20.61(0.08)$ & $20.27(0.05)$ & $20.56(0.06)$ & 20.75 \\
\hline & & & $20.71(0.06)$ & $20.58(0.05)$ & $20.47(0.05)$ & $20.16(0.05)$ & $20.34(0.05)$ & $20.42(0.06)$ & $20.71(0.07)$ \\
\hline & & & $20.75(0.07)$ & $20.72(0.06)$ & $20.72(0.07)$ & $20.72(0.06)$ & $20.78(0.07)$ & $20.72(0.06)$ & \\
\hline 996 & 451.072 & 192.105 & $20.56(0.05)$ & $20.11(0.06)$ & $20.26(0.05)$ & $20.48(0.06)$ & $20.38(0.05)$ & $20.51(0.05)$ & $20.71(0.06)$ \\
\hline & & & & $20.78(0.06)$ & $20.60(0.05)$ & $20.84(0.08)$ & $20.90(0.07)$ & $20.96(0.07)$ & $20.88(0.06)$ \\
\hline & & & $20.80(0.07)$ & $20.79(0.06)$ & $20.51(0.05)$ & $20.33(0.06)$ & $20.16(0.05)$ & $20.07(0.06)$ & \\
\hline 1001 & 238.444 & 759.722 & $20.57(0.05)$ & $20.64(0.07)$ & $20.73(0.09)$ & $20.67(0.09)$ & $20.65(0.05)$ & $20.72(0.06)$ & $20.54(0.06)$ \\
\hline & & & $20.75(0.06)$ & $20.59(0.06)$ & $20.44(0.05)$ & $20.15(0.06)$ & $20.20(0.05)$ & $20.29(0.06)$ & $20.36(0.05)$ \\
\hline & & & & $20.68(0.05)$ & $20.76(0.06)$ & $20.79(0.07)$ & $20.68(0.06)$ & $20.48(0.06)$ & \\
\hline 1002 & 438.041 & 97.386 & $20.40(0.05)$ & $20.84(0.09)$ & $21.45(0.13)$ & $21.47(0.16)$ & $20.87(0.06)$ & $20.73(0.06)$ & $20.49(0.05)$ \\
\hline & & & $20.54(0.06)$ & $20.50(0.06)$ & $20.57(0.05)$ & $20.32(0.07)$ & $20.35(0.06)$ & $20.22(0.05)$ & $20.23(0.06)$ \\
\hline & & & $20.38(0.05)$ & $20.58(0.06)$ & $20.77(0.06)$ & $20.94(0.06)$ & $20.78(0.07)$ & $20.84(0.08)$ & \\
\hline 1006 & 399.938 & -0.339 & $21.30(0.14)$ & $99.99(9.99)$ & $20.56(0.16)$ & $20.43(0.16)$ & $21.33(0.19)$ & $23.13(1.09)$ & $19.70(0.13)$ \\
\hline & & & $19.94(0.10)$ & & & & & 99.99 (9.99) & $22.08(0.74)$ \\
\hline & & & $22.43(0.41)$ & $21.14(0.23)$ & $22.46(0.99)$ & $22.70(0.67)$ & 24.09 (4.24) & 99.99 (9.99) & \\
\hline 1017 & 882.341 & 628.039 & $20.86(0.04)$ & $20.40(0.07)$ & $99.99(9.99)$ & $20.78(0.11)$ & $20.60(0.05)$ & $20.71(0.05)$ & 99.99 (9.99) \\
\hline & & & $20.29(0.06)$ & $20.53(0.05)$ & $20.35(0.05)$ & $19.99(0.05)$ & $20.13(0.05)$ & $20.23(0.05)$ & $20.41(0.06)$ \\
\hline & & & $20.45(0.05)$ & $20.63(0.06)$ & $20.85(0.07)$ & $20.89(0.06)$ & $21.37(0.09)$ & $20.66(0.07)$ & \\
\hline
\end{tabular}


Table 7. continuation.

\begin{tabular}{|c|c|c|c|c|c|c|c|c|c|}
\hline \multirow[t]{3}{*}{ F1 ID } & \multirow[t]{3}{*}{$\mathrm{x}$} & \multirow[t]{3}{*}{$\mathrm{y}$} & $1 \mathrm{Ks} 07(\sigma)$ & $1 \mathrm{Ks} 01(\sigma)$ & $1 \mathrm{Ks} 02(\sigma)$ & $1 \mathrm{Ks} 03(\sigma)$ & $1 \mathrm{Ks} 04(\sigma)$ & $1 \mathrm{Ks} 05(\sigma)$ & \multirow{3}{*}{$\begin{array}{l}1 \mathrm{Ks} 06(\sigma) \\
1 \mathrm{Ks} 14(\sigma)\end{array}$} \\
\hline & & & $1 \mathrm{Ks} 08(\sigma)$ & $1 \mathrm{Ks} 09(\sigma)$ & $1 \mathrm{Ks} 10(\sigma)$ & $1 \mathrm{Ks} 11(\sigma)$ & $1 \mathrm{Ks} 12(\sigma)$ & $1 \mathrm{Ks} 13(\sigma)$ & \\
\hline & & & $1 \mathrm{Ks} 15(\sigma)$ & $1 \mathrm{Ks} 16(\sigma)$ & $1 \mathrm{Ks} 17(\sigma)$ & $1 \mathrm{Ks} 18(\sigma)$ & $1 \mathrm{Ks} 19(\sigma)$ & $1 \mathrm{Ks} 20(\sigma)$ & \\
\hline \multirow[t]{3}{*}{1019} & 852.987 & $\overline{7764.701}$ & $20.81(0.05)$ & $20.85(0.10)$ & $99.99(9.99)$ & $21.49(0.12)$ & $21.12(0.07)$ & $21.05(0.07)$ & $99.99(9.99)$ \\
\hline & & & $20.45(0.06)$ & $20.39(0.05)$ & $20.44(0.05)$ & $21.05(0.10)$ & $21.07(0.07)$ & $20.56(0.06)$ & $20.33(0.05)$ \\
\hline & & & $20.42(0.05)$ & $20.52(0.05)$ & $20.30(0.05)$ & $20.29(0.05)$ & $21.01(0.09)$ & $21.75(0.19)$ & \\
\hline \multirow[t]{3}{*}{1024} & 501.442 & 359.190 & $20.71(0.06)$ & $20.49(0.06)$ & $20.72(0.10)$ & $20.88(0.10)$ & $20.72(0.05)$ & $20.74(0.05)$ & $20.79(0.07)$ \\
\hline & & & $20.74(0.06)$ & $20.73(0.06)$ & $20.41(0.05)$ & $20.65(0.07)$ & $20.62(0.06)$ & $20.62(0.06)$ & $20.50(0.05)$ \\
\hline & & & $20.47(0.05)$ & $20.45(0.05)$ & $20.28(0.05)$ & $20.35(0.05)$ & $20.38(0.05)$ & $20.54(0.07)$ & \\
\hline \multirow[t]{3}{*}{1029} & 864.494 & 341.648 & $20.51(0.05)$ & $20.72(0.10)$ & $20.89(0.11)$ & $20.68(0.10)$ & $20.27(0.04)$ & $20.25(0.05)$ & 99.99 (9.99) \\
\hline & & & $20.64(0.06)$ & $21.05(0.08)$ & $21.04(0.05)$ & $20.68(0.07)$ & $20.63(0.05)$ & $20.92(0.07)$ & $20.77(0.07)$ \\
\hline & & & $20.86(0.06)$ & $20.72(0.06)$ & $20.76(0.08)$ & $20.76(0.05)$ & $20.42(0.06)$ & $20.29(0.05)$ & \\
\hline \multirow[t]{3}{*}{1033} & 493.834 & 824.223 & $20.23(0.07)$ & $20.52(0.10)$ & $20.45(0.11)$ & $20.82(0.08)$ & $20.49(0.11)$ & $20.43(0.09)$ & $20.89(0.07)$ \\
\hline & & & $20.41(0.07)$ & $21.08(0.07)$ & $20.34(0.07)$ & $21.27(0.12)$ & $20.60(0.10)$ & $21.02(0.12)$ & $20.56(0.05)$ \\
\hline & & & $20.30(0.06)$ & $20.80(0.07)$ & $20.18(0.07)$ & $20.63(0.07)$ & $20.56(0.05)$ & $20.24(0.06)$ & \\
\hline \multirow[t]{3}{*}{1048} & 594.172 & 346.425 & $20.34(0.05)$ & $20.92(0.09)$ & $20.78(0.10)$ & $21.24(0.14)$ & $20.89(0.05)$ & $20.95(0.06)$ & $20.52(0.06)$ \\
\hline & & & $20.40(0.05)$ & $20.28(0.05)$ & $20.40(0.05)$ & $20.51(0.06)$ & $20.49(0.05)$ & $20.40(0.05)$ & $20.41(0.05)$ \\
\hline & & & $20.37(0.05)$ & $20.58(0.06)$ & $20.77(0.06)$ & $20.92(0.06)$ & $21.07(0.08)$ & $21.14(0.08)$ & \\
\hline \multirow[t]{3}{*}{1053} & 606.274 & 349.345 & $20.31(0.05)$ & $20.39(0.06)$ & $20.42(0.08)$ & $20.40(0.10)$ & $21.53(0.07)$ & $21.23(0.07)$ & $20.84(0.07)$ \\
\hline & & & $21.54(0$ & $21.60(0.12)$ & $21.32(0.06)$ & 20.19 & 20.19 & $20.20(0.05)$ & $20.27(0.05)$ \\
\hline & & & $20.43(0.05)$ & $20.61(0.06)$ & $20.77(0.07)$ & $20.97(0.06)$ & $21.05(0.08)$ & $21.13(0.07)$ & \\
\hline \multirow[t]{3}{*}{1055} & 677.455 & 807.190 & $20.87(0.06)$ & $20.64(0.09)$ & $20.68(0.11)$ & $20.62(0.09)$ & $20.67(0.05)$ & $20.85(0.06)$ & $21.15(0.09)$ \\
\hline & & & $20.86(0.06)$ & $20.43(0.06)$ & $20.25(0.05)$ & $20.44(0.06)$ & $20.47(0.05)$ & $20.41(0.05)$ & $20.43(0.06)$ \\
\hline & & & $20.42(0.06)$ & $20.53(0.05)$ & $20.65(0.07)$ & $20.69(0.06)$ & $20.69(0.06)$ & $20.86(0.08)$ & \\
\hline \multirow[t]{3}{*}{1064} & 308.596 & 749.128 & $20.47(0.06)$ & $20.69(0.08)$ & $20.78(0.10)$ & $20.33(0.09)$ & $20.35(0.05)$ & $20.33(0.05)$ & $20.32(0.06)$ \\
\hline & & & $20.74(0.06)$ & $20.90(0.06)$ & $21.33(0.09)$ & $20.44(0.06)$ & $20.67(0.06)$ & $20.66(0.07)$ & $20.84(0.06)$ \\
\hline & & & $20.81(0.07)$ & $20.87(0.07)$ & $20.91(0.08)$ & $20.78(0.07)$ & $20.46(0.06)$ & $20.30(0.05)$ & \\
\hline 1068 & 83.742 & 289.019 & 20.96 & $20.18(0.07)$ & $20.28(0.06)$ & $20.76(0.10)$ & $20.42(0.04)$ & $20.59(0.06)$ & 20.88 \\
\hline & & & 20.30 & 20.74 & 20.83 & 20.31 & 20.33 & $20.28(0.05)$ & $20.54(0.06)$ \\
\hline & & & $20.52(0.06)$ & $20.72(0.06)$ & $20.77(0.06)$ & $20.79(0.06)$ & $20.84(0.08)$ & $20.82(0.08)$ & \\
\hline 1071 & 58.112 & 315.068 & $20.67(0.05)$ & $19.92(0.06)$ & $20.12(0.07)$ & $20.33(0.08)$ & $20.53(0.05)$ & $20.77(0.05)$ & $21.05(0.09)$ \\
\hline & & & $20.86(0.07)$ & $20.91(0.06)$ & $20.73(0.05)$ & $20.81(0.09)$ & $20.97(0.10)$ & 20.93 (0. & $20.89(0.07)$ \\
\hline & & & & 20.72 & & 20.49 & 20.29 & $20.04(0$ & \\
\hline 1072 & 125.904 & 710.688 & $21.01(0.06)$ & $20.78(0.09)$ & $20.56(0.08)$ & 21.57 & 20.43 & 20.67 & $21.91(0.19)$ \\
\hline & & & $20.58(0.09)$ & $20.55(0.15)$ & $20.46(0.16)$ & 99.99 (9.99) & $20.44(0$ & $20.76(0.15)$ & $20.17(0.08)$ \\
\hline & & & $20.52(0.06)$ & $20.83(0.09)$ & $20.19(0.05)$ & $20.35(0.06)$ & $21.74(0.13)$ & $20.80(0.07)$ & \\
\hline 1073 & 58.723 & 531.854 & $21.51(0.47)$ & $20.78(0.09)$ & $20.05(0.07)$ & $20.37(0.08)$ & 20.82 & $20.68(0.07)$ & $0.07)$ \\
\hline & & & 20.98 & $20.88(0.07)$ & $20.57(0.06)$ & $20.51(0.07)$ & $20.48(0.07)$ & 20.09 (0. & $20.46(0.06)$ \\
\hline & & & & 20.74 & 2042 & 20.48 & 20.64 & $21.02(0$ & \\
\hline 1079 & 362.169 & 871.716 & $23.66(0.40)$ & $22.87(0.58)$ & $21.77(3.18)$ & $19.97(0.09)$ & 22.71 & 99.99 (9.99) & $22.71(0.26)$ \\
\hline & & & & 99.99 (9.99) & & 99.99 (9.99) & $99.99(9.99)$ & $99.99(9.99)$ & $99.99(9.99)$ \\
\hline & & & & 99.99 & & 99.95 & 99.99 & 99.99 (9. & \\
\hline 1082 & 75.009 & 169.117 & $20.37(0.06)$ & $20.78(0.08)$ & $20.80(0.07)$ & $21.21(0.16)$ & $20.53(0.05)$ & $20.41(0.05)$ & $20.46(0.06)$ \\
\hline & & & $20.84(0.06)$ & $20.89(0.07)$ & $20.94(0.05)$ & $20.60(0.07)$ & $20.62(0.06)$ & $20.57(0.08)$ & $20.48(0.05)$ \\
\hline & & & & $20.44(0.06)$ & & $20.33(0.05)$ & $20.46(0.07)$ & $20.50(0.08)$ & \\
\hline 1085 & 175.552 & 762.948 & 21.00 & 20.00 & & & 20.38 & & 21.02 \\
\hline & & & $20.89(0.07)$ & $20.84(0.07)$ & $20.84(0.06)$ & $20.33(0.05)$ & $20.46(0.05)$ & $20.49(0.06)$ & $20.70(0.06)$ \\
\hline & & & $20.87(0.05)$ & $20.98(0.08)$ & $20.85(0.07)$ & $20.74(0.07)$ & $20.69(0.07)$ & $20.74(0.07)$ & \\
\hline 1086 & 779.232 & 544.839 & $20.33(0.07)$ & $20.70(0.08)$ & $20.74(0.09)$ & $21.29(0.12)$ & $20.79(0.08)$ & $20.64(0.06)$ & $20.58(0.08)$ \\
\hline & & & & $20.51(0.06)$ & & $20.35(0.06)$ & $20.27(0.05)$ & $20.41(0.07)$ & $20.55(0.06)$ \\
\hline & & & & $20.80(0.07)$ & $20.97(0.09)$ & $20.88(0.08)$ & $20.85(0.09)$ & $20.82(0.08)$ & \\
\hline 1089 & 737.949 & 479.971 & $20.83(0.05)$ & $20.73(0.09)$ & $20.48(0.07)$ & $20.34(0.07)$ & $20.30(0.04)$ & $20.35(0.05)$ & $20.70(0.08)$ \\
\hline & & & $20.91(0.09)$ & $21.08(0.07)$ & $20.68(0.05)$ & $20.56(0.07)$ & $20.55(0.07)$ & $20.64(0.06)$ & $20.92(0.08)$ \\
\hline & & & & & & $20.66(0.06)$ & $20.51(0.06)$ & $20.53(0.07)$ & \\
\hline 1097 & 331.354 & 394.755 & $20.84(0.06)$ & $20.59(0.07)$ & $20.57(0.10)$ & $20.45(0.08)$ & $20.42(0.05)$ & $20.69(0.05)$ & $20.67(0.06)$ \\
\hline & & & $20.83(0.06)$ & $20.88(0.07)$ & $20.85(0.05)$ & $20.83(0.08)$ & $20.77(0.06)$ & $20.83(0.06)$ & $20.82(0.07)$ \\
\hline & & & $20.72(0.07)$ & $20.65(0.06)$ & $20.46(0.05)$ & $20.23(0.05)$ & $20.17(0.06)$ & $20.21(0.05)$ & \\
\hline 1109 & 425.117 & 501.833 & $20.61(0.18)$ & $20.60(0.09)$ & $20.30(0.13)$ & $20.54(0.11)$ & $20.71(0.13)$ & $20.58(0.09)$ & $20.03(0.06)$ \\
\hline & & & & & & & & & $20.72(0.08)$ \\
\hline & & & $20.83(0.09)$ & $20.87(0.09)$ & & $20.69(0.09)$ & $20.96(0.09)$ & $21.11(0.10)$ & \\
\hline 1112 & 455.042 & 37.267 & $20.66(0.05)$ & $20.50(0.07)$ & $20.73(0.06)$ & $21.43(0.13)$ & $21.07(0.07)$ & $21.07(0.06)$ & $20.98(0.08)$ \\
\hline & & & $20.51(0.05)$ & $20.29(0.05)$ & $20.35(0.04)$ & $20.71(0.07)$ & $20.63(0.06)$ & $20.50(0.06)$ & $20.47(0.05)$ \\
\hline & & & $20.51(0.05)$ & $20.58(0.06)$ & $20.78(0.06)$ & $20.73(0.06)$ & $20.87(0.08)$ & $20.79(0.07)$ & \\
\hline
\end{tabular}


Table 7. continuation.

\begin{tabular}{|c|c|c|c|c|c|c|c|c|c|}
\hline \multirow[t]{3}{*}{$\overline{\text { F1 ID }}$} & \multirow[t]{3}{*}{$\mathrm{X}$} & \multirow[t]{3}{*}{$\mathrm{y}$} & $1 \mathrm{Ks} 07(\sigma)$ & $1 \mathrm{Ks} 01(\sigma)$ & $1 \mathrm{Ks} 02(\sigma)$ & $1 \mathrm{Ks} 03(\sigma)$ & $1 \mathrm{Ks} 04(\sigma)$ & $1 \mathrm{Ks} 05(\sigma)$ & \multirow{3}{*}{$\begin{array}{l}1 \mathrm{Ks} 06(\sigma) \\
1 \mathrm{Ks} 14(\sigma)\end{array}$} \\
\hline & & & $1 \mathrm{Ks} 08(\sigma)$ & $1 \mathrm{Ks} 09(\sigma)$ & $1 \mathrm{Ks} 10(\sigma)$ & $1 \mathrm{Ks} 11(\sigma)$ & $1 \mathrm{Ks} 12(\sigma)$ & $1 \mathrm{Ks} 13(\sigma)$ & \\
\hline & & & $1 \mathrm{Ks} 15(\sigma)$ & $1 \mathrm{Ks} 16(\sigma)$ & $1 \mathrm{Ks} 17(\sigma)$ & $1 \mathrm{Ks} 18(\sigma)$ & $1 \mathrm{Ks} 19(\sigma)$ & $1 \mathrm{Ks} 20(\sigma)$ & \\
\hline \multirow[t]{3}{*}{1113} & 347.323 & $\overline{344.220}$ & $20.49(0.06)$ & $20.67(0.07)$ & $20.84(0.08)$ & $20.72(0.09)$ & $20.64(0.05)$ & $20.78(0.05)$ & $20.54(0.06)$ \\
\hline & & & $20.87(0.07)$ & $20.86(0.07)$ & $20.77(0.05)$ & $20.27(0.05)$ & $20.21(0.05)$ & $20.43(0.06)$ & $20.66(0.06)$ \\
\hline & & & $20.54(0.06)$ & $20.69(0.05)$ & $20.60(0.06)$ & $20.67(0.06)$ & $20.58(0.06)$ & $20.43(0.07)$ & \\
\hline \multirow[t]{3}{*}{1115} & 172.184 & 215.968 & $20.68(0.06)$ & $20.55(0.08)$ & $20.54(0.06)$ & $21.21(0.14)$ & $21.66(0.07)$ & $21.21(0.08)$ & $21.26(0.08)$ \\
\hline & & & $21.13(0.07)$ & $21.63(0.11)$ & $21.56(0.08)$ & $20.57(0.07)$ & $20.57(0.06)$ & $20.38(0.06)$ & $20.40(0.05)$ \\
\hline & & & $20.39(0.05)$ & $20.39(0.05)$ & $20.31(0.06)$ & $20.34(0.06)$ & $20.43(0.06)$ & $20.57(0.06)$ & \\
\hline \multirow[t]{3}{*}{1125} & 451.718 & 376.012 & $20.68(0.05)$ & $20.35(0.06)$ & $20.56(0.10)$ & $20.92(0.11)$ & $20.66(0.05)$ & $20.81(0.05)$ & $20.78(0.07)$ \\
\hline & & & $21.05(0.13)$ & $20.67(0.07)$ & $20.46(0.05)$ & $21.06(0.10)$ & $20.80(0.05)$ & $20.79(0.07)$ & $20.79(0.07)$ \\
\hline & & & $20.86(0.06)$ & $20.61(0.06)$ & $20.44(0.06)$ & $20.37(0.05)$ & $20.29(0.05)$ & $20.33(0.05)$ & \\
\hline \multirow[t]{3}{*}{1133} & 579.644 & 774.993 & $20.64(0.05)$ & $20.42(0.06)$ & $20.68(0.08)$ & $20.53(0.07)$ & $20.44(0.05)$ & $20.49(0.05)$ & $20.81(0.06)$ \\
\hline & & & $21.01(0.06)$ & $20.69(0.06)$ & $20.55(0.05)$ & $20.80(0.07)$ & $20.87(0.07)$ & $20.73(0.08)$ & $20.72(0.06)$ \\
\hline & & & $20.76(0.06)$ & $20.56(0.05)$ & $20.47(0.06)$ & $20.47(0.05)$ & $20.40(0.06)$ & $20.45(0.07)$ & \\
\hline \multirow[t]{3}{*}{1143} & 754.050 & 659.413 & $20.86(0.05)$ & $20.95(0.10)$ & $20.74(0.13)$ & $20.67(0.10)$ & $20.75(0.06)$ & $20.95(0.05)$ & $21.20(0.10)$ \\
\hline & & & $20.66(0.06)$ & $20.53(0.05)$ & $20.45(0.04)$ & $20.27(0.05)$ & $20.40(0.05)$ & $20.31(0.05)$ & $20.49(0.05)$ \\
\hline & & & $20.77(0.06)$ & $20.81(0.06)$ & $21.12(0.08)$ & $20.90(0.07)$ & $21.00(0.08)$ & $20.86(0.07)$ & \\
\hline \multirow[t]{3}{*}{1151} & 393.950 & 764.899 & $20.65(0.04)$ & $20.24(0.07)$ & $20.43(0.08)$ & $20.68(0.09)$ & $20.89(0.07)$ & $20.89(0.06)$ & $20.75(0.06)$ \\
\hline & & & $21.16(0.07)$ & $20.68(0.06)$ & $20.60(0.04)$ & $20.78(0.06)$ & $20.67(0.06)$ & $20.80(0.07)$ & $20.66(0.06)$ \\
\hline & & & $20.83(0.06)$ & $20.82(0.06)$ & $20.46(0.06)$ & $20.42(0.05)$ & $20.26(0.05)$ & $20.05(0.05)$ & \\
\hline \multirow[t]{3}{*}{1159} & 409.233 & 469.687 & $20.70(0.06)$ & $20.50(0.07)$ & $20.66(0.10)$ & $20.99(0.09)$ & $20.80(0.05)$ & $20.92(0.05)$ & $20.85(0.07)$ \\
\hline & & & $20.98(0.06)$ & $20.72(0.07)$ & $20.62(0.05)$ & $20.28(0.06)$ & $20.24(0.05)$ & $20.41(0.06)$ & $20.49(0.05)$ \\
\hline & & & $20.58(0.05)$ & $20.74(0.06)$ & $20.53(0.07)$ & $20.52(0.05)$ & $20.44(0.06)$ & $20.60(0.06)$ & \\
\hline \multirow[t]{3}{*}{1162} & 325.069 & 77.062 & $20.81(0.07)$ & $20.71(0.08)$ & $20.99(0.09)$ & $21.60(0.17)$ & $20.80(0.06)$ & $20.75(0.05)$ & $20.64(0.06)$ \\
\hline & & & $21.14(0.07)$ & $20.98(0.08)$ & $20.62(0.07)$ & $20.58(0.08)$ & $20.54(0.06)$ & $20.46(0.06)$ & $20.37(0.06)$ \\
\hline & & & $20.36(0.06)$ & $20.35(0.05)$ & $20.46(0.07)$ & $20.55(0.07)$ & $20.62(0.06)$ & $20.53(0.06)$ & \\
\hline 1167 & 176.566 & 702.620 & $20.76(0.05)$ & $20.26(0.07)$ & $20.78(0.07)$ & $20.51(0.07)$ & $20.85(0.05)$ & $20.89(0.06)$ & $20.89(0.07)$ \\
\hline & & & $20.68(0.06)$ & $20.60(0.06)$ & $20.48(0.05)$ & $20.97(0.09)$ & $21.02(0.07)$ & 20.80 & $20.83(0.06)$ \\
\hline & & & $20.74(0.06)$ & $20.71(0.06)$ & $20.58(0.06)$ & $20.48(0.05)$ & $20.33(0.05)$ & $20.13(0.05)$ & \\
\hline 1172 & 288.423 & 801.112 & $20.67(0.05)$ & $20.42(0.06)$ & $20.38(0.07)$ & $20.34(0.07)$ & $20.40(0.05)$ & $20.52(0.05)$ & $20.47(0.05)$ \\
\hline & & & $21.05(0.06)$ & $20.85(0.06)$ & $20.68(0.05)$ & $20.69(0.06)$ & $20.73(0.06)$ & $20.70(0.07)$ & $20.72(0.07)$ \\
\hline & & & $20.68(0.0$ & $20.69(0$. & & $20.69(0.05)$ & $20.54(0.07)$ & $20.41(0.06)$ & \\
\hline 1177 & 854.540 & 771.523 & $20.77(0.05)$ & $20.98(0.13)$ & $99.99(9.99)$ & $21.67(0.18)$ & $21.24(0.06)$ & $21.19(0.06)$ & 99.99 (9.99) \\
\hline & & & $20.57(0.05)$ & $20.58(0.06)$ & $20.53(0.05)$ & $20.93(0.11)$ & $20.96(0.08)$ & $20.40(0.05)$ & $20.30(0.06)$ \\
\hline & & & $20.38(0.05)$ & $20.38(0.05)$ & $20.65(0.06)$ & $21.12(0.07)$ & $21.81(0.14)$ & $22.79(0.37)$ & \\
\hline 1178 & 87.904 & 336.986 & $20.92(0.05)$ & $20.21(0.08)$ & $20.40(0.08)$ & $20.48(0.07)$ & $20.27(0.05)$ & $20.48(0.05)$ & $20.78(0.08)$ \\
\hline & & & $20.61(0.07)$ & $20.89(0.07)$ & 20.81 & 20.27 & $20.34(0.06)$ & 20.42 & $20.67(0.06)$ \\
\hline & & & $20.73(0.06)$ & $20.88(0.06)$ & $20.76(0.06)$ & $20.87(0.06)$ & $20.95(0.07)$ & $20.73(0.07)$ & \\
\hline 1182 & 807.990 & 689.015 & $20.81(0.05)$ & $20.92(0.09)$ & $20.99(0.17)$ & $20.95(0.13)$ & $20.14(0.05)$ & $20.25(0.05)$ & 99.99 (9.99) \\
\hline & & & $20.91(0.06)$ & $21.01(0.07)$ & $20.88(0.05)$ & $20.55(0.06)$ & $20.74(0.06)$ & $20.82(0.07)$ & $20.79(0.08)$ \\
\hline & & & $20.99(0.08)$ & 20.73 & 20.66 & & 20.64 & 20.59 & \\
\hline 1189 & 40.982 & 480.956 & $20.64(0.06)$ & $21.04(0.11)$ & $20.84(0.10)$ & $21.32(0.10)$ & $20.62(0.05)$ & $20.42(0.05)$ & $20.37(0.06)$ \\
\hline & & & $20.62(0.06)$ & $21.25(0.09)$ & $21.20(0.07)$ & $20.86(0.08)$ & $20.82(0.07)$ & $20.81(0.08)$ & $20.78(0.07)$ \\
\hline & & & $20.82(0.08)$ & $20.78(0.06)$ & $20.63(0.06)$ & $20.39(0.05)$ & $20.36(0.06)$ & $20.28(0.06)$ & \\
\hline 1195 & 723.969 & 300.127 & $20.96(0.05)$ & $20.40(0.07)$ & $20.51(0.05)$ & $20.86(0.10)$ & $20.68(0.05)$ & $20.67(0.06)$ & $21.06(0.07)$ \\
\hline & & & $20.70(0.06)$ & $20.93(0.08)$ & 21.00 & $20.13(0.05)$ & 20.07 & $20.21(0.05)$ & $20.55(0.05)$ \\
\hline & & & $20.62(0.06)$ & $20.75(0.05)$ & $21.10(0.07)$ & $21.07(0.08)$ & $21.00(0.08)$ & $21.06(0.08)$ & \\
\hline 1198 & 793.095 & 281.772 & $20.72(0.05)$ & $20.23(0.06)$ & $20.32(0.08)$ & $20.53(0.10)$ & $20.19(0.05)$ & $20.29(0.05)$ & $20.62(0.06)$ \\
\hline & & & $20.82(0.06)$ & $21.07(0.08)$ & $20.95(0.05)$ & $20.64(0.07)$ & $20.76(0.06)$ & $20.66(0.06)$ & $21.00(0.08)$ \\
\hline & & & $21.00(0.06)$ & $21.14(0.08)$ & $21.05(0.07)$ & $21.00(0.08)$ & $21.07(C$ & 20.77 & \\
\hline 1199 & 808.362 & 642.118 & $20.45(0.04)$ & $20.86(0.11)$ & $21.45(0.20)$ & $21.23(0.10)$ & $20.69(0.05)$ & $20.55(0.06)$ & 99.99 (9.99) \\
\hline & & & $22.03(0.16)$ & $22.12(0.16)$ & $21.52(0.06)$ & $20.40(0.06)$ & $20.52(0.05)$ & $20.52(0.07)$ & $20.57(0.06)$ \\
\hline & & & $20.59(0.05)$ & $20.64(0.06)$ & $20.68(0.08)$ & $20.75(0.06)$ & $21.12(0.09)$ & $21.24(0.10)$ & \\
\hline 1202 & 130.634 & 718.495 & $20.78(0.06)$ & $20.37(0.06)$ & $20.36(0.07)$ & $20.37(0.07)$ & $20.74(0.05)$ & $20.78(0.06)$ & $20.84(0.07)$ \\
\hline & & & $20.76(0.08)$ & $20.75(0.07)$ & $20.68(0.05)$ & $21.08(0.10)$ & $20.88(0.08)$ & $20.70(0.06)$ & $20.65(0.05)$ \\
\hline & & & $20.52(0.05)$ & $20.80(0.07)$ & $20.67(0.06)$ & $20.54(0.06)$ & $20.41(0.06)$ & $20.22(0.06)$ & \\
\hline 1207 & 252.832 & 598.495 & $21.19(0.05)$ & $21.61(0.15)$ & $21.51(0.15)$ & $20.97(0.12)$ & $20.51(0.05)$ & $20.72(0.05)$ & $20.79(0.07)$ \\
\hline & & & $20.44(0.05)$ & $20.32(0.05)$ & $20.28(0.05)$ & $21.31(0.14)$ & $21.60(0.11)$ & $21.87(0.14)$ & $21.35(0.09)$ \\
\hline & & & $21.54(0.10)$ & $21.39(0.09)$ & $21.14(0.08)$ & $21.19(0.08)$ & $20.98(0.08)$ & $21.27(0.12)$ & \\
\hline 1209 & 357.555 & 258.060 & $20.78(0.05)$ & $20.22(0.06)$ & $20.48(0.09)$ & $20.89(0.09)$ & $20.71(0.05)$ & $20.95(0.07)$ & $21.09(0.09)$ \\
\hline & & & $21.02(0.08)$ & $20.74(0.07)$ & $20.78(0.05)$ & $20.29(0.06)$ & $20.40(0.06)$ & $20.40(0.06)$ & $20.72(0.06)$ \\
\hline & & & $20.68(0.06)$ & $20.58(0.06)$ & $20.28(0.05)$ & $20.48(0.06)$ & $20.59(0.08)$ & $20.59(0.06)$ & \\
\hline
\end{tabular}


Table 7. continuation.

\begin{tabular}{|c|c|c|c|c|c|c|c|c|c|}
\hline \multirow[t]{3}{*}{ F1 ID } & \multirow[t]{3}{*}{$\mathrm{x}$} & \multirow[t]{3}{*}{$\mathrm{y}$} & $1 \mathrm{Ks} 07(\sigma)$ & $1 \mathrm{Ks} 01(\sigma)$ & $1 \mathrm{Ks} 02(\sigma)$ & $1 \mathrm{Ks} 03(\sigma)$ & $1 \mathrm{Ks} 04(\sigma)$ & $1 \mathrm{Ks} 05(\sigma)$ & \multirow{3}{*}{$\begin{array}{l}1 \mathrm{Ks} 06(\sigma) \\
1 \mathrm{Ks} 14(\sigma)\end{array}$} \\
\hline & & & $1 \mathrm{Ks} 08(\sigma)$ & $1 \mathrm{Ks} 09(\sigma)$ & $1 \mathrm{Ks} 10(\sigma)$ & $1 \mathrm{Ks} 11(\sigma)$ & $1 \mathrm{Ks} 12(\sigma)$ & $1 \mathrm{Ks} 13(\sigma)$ & \\
\hline & & & $1 \mathrm{Ks} 15(\sigma)$ & $1 \mathrm{Ks} 16(\sigma)$ & $1 \mathrm{Ks} 17(\sigma)$ & $1 \mathrm{Ks} 18(\sigma)$ & $1 \mathrm{Ks} 19(\sigma)$ & $1 \mathrm{Ks} 20(\sigma)$ & \\
\hline \multirow[t]{3}{*}{1216} & 694.102 & 639.796 & $20.64(0.04)$ & "21.01(0.09) & $21.08(0.14)$ & $20.90(0.14)$ & $20.49(0.05)$ & $20.48(0.05)$ & $20.47(0.06)$ \\
\hline & & & $20.94(0.07)$ & $20.80(0.06)$ & $20.77(0.05)$ & $20.93(0.08)$ & $20.86(0.07)$ & $20.89(0.07)$ & $20.85(0.07)$ \\
\hline & & & $20.79(0.07)$ & $20.73(0.06)$ & $20.60(0.07)$ & $20.48(0.06)$ & $20.50(0.05)$ & $20.60(0.07)$ & \\
\hline \multirow[t]{3}{*}{1222} & 283.927 & 656.067 & $20.84(0.05)$ & $20.71(0.07)$ & $20.70(0.08)$ & $20.81(0.10)$ & $20.53(0.05)$ & $20.75(0.06)$ & $20.87(0.07)$ \\
\hline & & & $20.86(0.06)$ & $20.92(0.07)$ & $20.56(0.05)$ & $20.84(0.08)$ & $20.73(0.07)$ & $20.66(0.06)$ & $20.53(0.06)$ \\
\hline & & & $20.47(0.05)$ & $20.49(0.05)$ & $20.48(0.06)$ & $20.56(0.05)$ & $20.83(0.08)$ & $20.84(0.07)$ & \\
\hline \multirow[t]{3}{*}{1227} & 123.256 & 499.771 & $20.57(0.06)$ & $20.66(0.10)$ & $20.81(0.09)$ & $20.58(0.10)$ & $21.35(0.07)$ & $20.69(0.07)$ & $20.31(0.06)$ \\
\hline & & & $20.68(0.06)$ & $21.41(0.10)$ & $20.81(0.07)$ & $20.64(0.08)$ & $20.89(0.08)$ & $20.84(0.08)$ & $20.45(0.06)$ \\
\hline & & & $20.42(0.05)$ & $20.45(0.06)$ & $20.58(0.06)$ & $20.85(0.07)$ & $21.11(0.09)$ & $21.34(0.12)$ & \\
\hline \multirow[t]{3}{*}{1232} & 62.177 & 65.747 & $20.97(0.06)$ & $20.37(0.09)$ & $20.71(0.16)$ & $20.75(0.11)$ & $20.41(0.06)$ & $20.70(0.06)$ & $21.21(0.10)$ \\
\hline & & & $21.45(0.08)$ & $20.80(0.07)$ & $20.58(0.05)$ & $20.87(0.08)$ & $21.16(0.09)$ & $21.02(0.09)$ & $20.95(0.07)$ \\
\hline & & & $20.75(0.06)$ & $20.73(0.06)$ & $20.49(0.08)$ & $20.27(0.05)$ & $20.51(0.08)$ & $20.68(0.09)$ & \\
\hline \multirow[t]{3}{*}{1236} & 71.246 & 200.082 & $20.67(0.05)$ & $21.01(0.11)$ & $20.89(0.10)$ & $20.91(0.10)$ & $20.96(0.05)$ & $20.90(0.05)$ & $20.96(0.07)$ \\
\hline & & & $20.48(0.05)$ & $20.58(0.06)$ & $20.53(0.05)$ & $20.25(0.06)$ & $20.54(0.06)$ & $20.46(0.06)$ & $20.90(0.06)$ \\
\hline & & & $20.77(0.06)$ & $20.59(0.05)$ & $20.79(0.05)$ & $20.52(0.05)$ & $20.66(0.06)$ & $20.39(0.06)$ & \\
\hline \multirow[t]{3}{*}{1240} & 456.705 & 583.260 & $20.79(0.07)$ & $20.39(0.07)$ & $20.34(0.07)$ & $20.26(0.07)$ & $21.24(0.07)$ & $21.18(0.06)$ & $21.11(0.08)$ \\
\hline & & & 21.00 & $20.94(0.06)$ & $21.12(0.07)$ & $20.54(0.07)$ & 20.53 & $20.60(0.06)$ & $20.60(0.05)$ \\
\hline & & & $20.53(0.06)$ & $20.48(0.05)$ & $20.53(0.05)$ & $20.55(0.05)$ & $20.55(0.07)$ & $20.72(0.08)$ & \\
\hline \multirow[t]{3}{*}{1241} & 252.580 & 54.482 & $21.20(0.05)$ & $20.67(0.08)$ & $20.68(0.07)$ & $21.14(0.12)$ & $20.94(0.06)$ & $21.21(0.07)$ & $21.47(0.08)$ \\
\hline & & & $21.00(0.06)$ & $20.90(0.07)$ & $20.42(0.05)$ & $20.92(0.08)$ & $20.96(0.06)$ & $21.00(0.10)$ & $20.86(0.07)$ \\
\hline & & & $20.69(0.06)$ & $20.54(0.05)$ & $20.22(0.05)$ & $20.33(0.05)$ & $20.36(0.06)$ & $20.59(0.07)$ & \\
\hline \multirow[t]{3}{*}{1242} & 593.086 & 698.145 & $20.51(0.05)$ & $20.90(0.12)$ & $21.24(0.11)$ & $21.42(0.17)$ & $21.06(0.05)$ & $20.91(0.06)$ & $20.69(0.06)$ \\
\hline & & & $20.73(0.06)$ & $20.59(0.05)$ & $20.95(0.05)$ & $20.31(0.06)$ & $20.26(0.05)$ & $20.29(0.05)$ & $20.56(0.06)$ \\
\hline & & & $20.64(0.05)$ & $20.86(0.06)$ & $20.77(0.08)$ & $20.79(0.06)$ & $20.61(0.06)$ & $20.71(0.07)$ & \\
\hline 1253 & 704.532 & 74.930 & $20.92(0.19)$ & $20.67(0.17)$ & $20.05(0.15)$ & $20.46(0.19)$ & $20.34(0.10)$ & $19.96(0.09)$ & 19.12 \\
\hline & & & 21.13 & 20.96 & 21.28 & 22.63 & & 99.99 (9.99) & 99.99 \\
\hline & & & $22.85(0.35)$ & $21.40(0.14)$ & $23.60(0.91)$ & 99.99 (9.99) & $21.68(0.19)$ & $22.52(0.37)$ & \\
\hline 1254 & 323.129 & 308.024 & $20.73(0.05)$ & $20.61(0.08)$ & $21.01(0.08)$ & $21.79(0.17)$ & $20.96(0.06)$ & $20.87(0.07)$ & $20.79(0.07)$ \\
\hline & & & $20.81(0.06)$ & $20.91(0.07)$ & $20.51(0.05)$ & $21.02(0.10)$ & $20.91(0.06)$ & $21.00(0$. & $20.91(0.06)$ \\
\hline & & & & 20.74 & & & & 20.19 (0. & \\
\hline 1260 & 466.921 & 138.799 & 22.18 & $99.99(9.99)$ & $20.45(0.30)$ & $99.99(9.99)$ & 99.99 & $99.99(9.99)$ & $20.22(0.09)$ \\
\hline & & & 99.99 (9.99) & $22.87(0.68)$ & $22.16(0.45)$ & $21.74(0.18)$ & $20.97(0$ & $21.77(0.30)$ & 99.99 (9.99) \\
\hline & & & 99.99 (9.99) & 99.99 (9.99) & $0000 / 00$ & 99.99 (9.99) & 99.99 (9.99) & $99.99(9.99)$ & \\
\hline 1280 & 849.583 & 593.666 & $20.82(0.06)$ & $20.86(0.08)$ & $20.67(0.0$ & $21.13(0.12)$ & $20.82(0.05)$ & $20.90(0.06)$ & 99.99 \\
\hline & & & 20.59 & $20.67(0.06)$ & $20.43(0.05)$ & $20.89(0.09)$ & $20.88(0.05)$ & 21.15 & $21.06(0.09)$ \\
\hline & & & 21.02 & $21.12(0.0$ & 21066 & $20.79(0.05)$ & 20.51 & 20.18 & \\
\hline 1283 & 58.754 & 533.571 & 99.99 (9.99) & $21.36(0.14)$ & $21.26(0.22)$ & $21.04(0.11)$ & $21.23(0.10)$ & $20.80(0$ & $20.77(0.09)$ \\
\hline & & & & $21.38(0.09)$ & & $20.83(0.10)$ & $20.72(0.11)$ & $99.99(9.99)$ & $21.09(0.11)$ \\
\hline & & & & 20.63 & & 20.77 & 20.62 & $20.48(0.06)$ & \\
\hline 1291 & 455.682 & 392.499 & $21.02(0.08)$ & $20.92(0.09)$ & $20.84(0.12)$ & $20.43(0.09)$ & $20.34(0.07)$ & $20.44(0.07)$ & $20.60(0.08)$ \\
\hline & & & $20.91(0.16)$ & $20.99(0.12)$ & $20.72(0.09)$ & $21.25(0.18)$ & $21.11(0.14)$ & $20.88(0.09)$ & $21.17(0.11)$ \\
\hline & & & & $21.24(0.12)$ & & $20.59(0.07)$ & $20.53(0.07)$ & $20.54(0.08)$ & \\
\hline 1298 & 390.213 & -6.731 & 99 & 22.36 & & 99.99 & & 22.13 & 99.99 \\
\hline & & & $20.08(0.11)$ & $20.62(0.15)$ & $21.12(0.33)$ & $20.60(0.17)$ & $21.32(0.37)$ & $21.66(0.51)$ & $99.99(9.99)$ \\
\hline & & & $21.92(0.15)$ & $20.26(0.11)$ & $20.71(0.18)$ & $22.23(0.46)$ & $23.44(1.61)$ & $99.99(9.99)$ & \\
\hline 1304 & 380.244 & 314.110 & $20.47(0.15)$ & $21.37(0.21)$ & $20.72(0.17)$ & $21.60(0.59)$ & $20.56(0.12)$ & $20.66(0.11)$ & 99.99 (9.99) \\
\hline & & & & $21.01(0.12)$ & $20.50(0.14)$ & $21.92(0.33)$ & $20.73(0.12)$ & $24.07(2.09)$ & $20.58(0.11)$ \\
\hline & & & $21.52(0.14)$ & $20.50(0.09)$ & $21.60(0.17)$ & $21.62(0.16)$ & $20.43(0.24)$ & $20.41(0.17)$ & \\
\hline 1307 & 139.077 & 307.559 & $21.10(0.05)$ & $20.75(0.09)$ & $20.96(0.09)$ & $20.69(0.09)$ & $20.76(0.05)$ & $20.95(0.06)$ & $21.36(0.10)$ \\
\hline & & & $21.04(0.07)$ & $21.07(0.07)$ & $20.72(0.05)$ & $20.81(0.08)$ & $20.93(0.06)$ & $20.62(0.07)$ & $20.72(0.05)$ \\
\hline & & & & & & $20.40(0.05)$ & $20.38(0.05)$ & $20.63(0.06)$ & \\
\hline 1310 & 862.948 & 24.903 & $20.99(0.05)$ & $21.21(0.13)$ & $21.56(0.20)$ & $21.74(0.26)$ & $20.49(0.05)$ & $20.77(0.06)$ & 99.99 (9.99) \\
\hline & & & $20.36(0.06)$ & $20.84(0.09)$ & $20.95(0.05)$ & $20.68(0.07)$ & $20.97(0.07)$ & $20.73(0.06)$ & $20.53(0.06)$ \\
\hline & & & $20.61(0.06)$ & $20.43(0.05)$ & $20.78(0.06)$ & $20.58(0.05)$ & $23.06(0.40)$ & 99.99 (9.99) & \\
\hline 1327 & 320.303 & 693.920 & $20.81(0.05)$ & $20.90(0.09)$ & $20.92(0.11)$ & $21.05(0.14)$ & $20.55(0.05)$ & $20.70(0.05)$ & $20.80(0.06)$ \\
\hline & & & & & & & & $20.90(0.06)$ & $20.84(0.07)$ \\
\hline & & & $20.97(0.06)$ & $20.95(0.06)$ & $21.03(0.06)$ & $20.81(0.07)$ & $20.55(0.06)$ & $20.50(0.06)$ & \\
\hline 1333 & 705.585 & 752.688 & $21.14(0.05)$ & $20.58(0.09)$ & $20.62(0.10)$ & $20.86(0.11)$ & $20.85(0.05)$ & $21.25(0.06)$ & $21.50(0.11)$ \\
\hline & & & $20.97(0.06)$ & $20.82(0.06)$ & $20.46(0.04)$ & $21.46(0.17)$ & $21.15(0.09)$ & $21.47(0.11)$ & $21.08(0.08)$ \\
\hline & & & $20.98(0.07)$ & $20.71(0.05)$ & $20.53(0.09)$ & $20.37(0.05)$ & $20.39(0.05)$ & $20.64(0.06)$ & \\
\hline
\end{tabular}


Table 7. continuation.

\begin{tabular}{|c|c|c|c|c|c|c|c|c|c|}
\hline \multirow[t]{3}{*}{ F1 ID } & \multirow[t]{3}{*}{$\mathrm{X}$} & \multirow[t]{3}{*}{$\mathrm{y}$} & $1 \mathrm{Ks} 07(\sigma)$ & 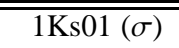 & $1 \mathrm{Ks} 02(\sigma)$ & $1 \mathrm{Ks} 03(\sigma)$ & $1 \mathrm{Ks} 04(\sigma)$ & $1 \mathrm{K \textrm {Ks } 0 5 ( \sigma )}$ & \multirow{3}{*}{$\begin{array}{l}1 \mathrm{Ks} 06(\sigma) \\
1 \mathrm{Ks} 14(\sigma)\end{array}$} \\
\hline & & & $1 \mathrm{Ks} 08(\sigma)$ & $1 \mathrm{Ks} 09(\sigma)$ & $1 \mathrm{Ks} 10(\sigma)$ & $1 \mathrm{Ks} 11(\sigma)$ & $1 \mathrm{Ks} 12(\sigma)$ & $1 \mathrm{Ks} 13(\sigma)$ & \\
\hline & & & $1 \mathrm{Ks} 15(\sigma)$ & $1 \mathrm{Ks} 16(\sigma)$ & $1 \mathrm{Ks} 17(\sigma)$ & $1 \mathrm{Ks} 18(\sigma)$ & $1 \mathrm{Ks} 19(\sigma)$ & $1 \mathrm{Ks} 20(\sigma)$ & \\
\hline \multirow[t]{3}{*}{1356} & 275.836 & 724.749 & $20.99(0.05)$ & $20.10(0.06)$ & $20.51(0.09)$ & $20.89(0.10)$ & $20.81(0.05)$ & $21.09(0.06)$ & $21.12(0.08)$ \\
\hline & & & $20.86(0.06)$ & $20.59(0.06)$ & $20.63(0.05)$ & $20.45(0.06)$ & $20.64(0.06)$ & $20.65(0.07)$ & $20.83(0.06)$ \\
\hline & & & $20.87(0.07)$ & $20.89(0.07)$ & $20.77(0.06)$ & $20.76(0.06)$ & $20.68(0.06)$ & $20.32(0.06)$ & \\
\hline \multirow[t]{3}{*}{1359} & 709.803 & 853.631 & $21.42(0.06)$ & $22.27(0.29)$ & $19.93(0.06)$ & $20.86(0.10)$ & $21.36(0.08)$ & $21.38(0.07)$ & $20.34(0.05)$ \\
\hline & & & 99.99 (9.99) & 99.99 (9.99) & 99.99 (9.99) & 99.99 (9.99) & 99.99 (9.99) & $21.25(0.08)$ & 99.99 (9.99) \\
\hline & & & $99.99(9.99)$ & 99.99 (9.99) & 99.99 (9.99) & 99.99 (9.99) & 99.99 (9.99) & 99.99 (9.99) & \\
\hline \multirow[t]{3}{*}{1361} & 62.584 & 30.180 & 99.99 (9.99) & $21.13(0.10)$ & 99.99 (9.99) & 99.99 (9.99) & 99.99 (9.99) & 99.99 (9.99) & 99.99 (9.99) \\
\hline & & & $21.56(0.13)$ & $22.00(0.19)$ & $21.47(0.10)$ & $22.29(0.28)$ & $23.04(0.61)$ & $21.31(0.12)$ & $21.10(0.09)$ \\
\hline & & & $20.71(0.06)$ & $21.44(0.11)$ & $20.92(0.08)$ & $21.78(0.13)$ & $19.99(0.06)$ & $20.46(0.07)$ & \\
\hline \multirow[t]{3}{*}{1370} & 119.517 & 697.211 & $21.30(0.45)$ & $20.89(0.20)$ & $20.21(0.08)$ & 99.99 (9.99) & $20.42(0.14)$ & $21.10(0.17)$ & 99.99 (9.99) \\
\hline & & & $20.91(0.14)$ & $20.51(0.12)$ & $20.81(0.15)$ & 99.99 (9.99) & $21.58(0.31)$ & $21.41(0.27)$ & $21.40(0.26)$ \\
\hline & & & $21.62(0.16)$ & $20.87(0.13)$ & $22.82(0.65)$ & $21.97(0.20)$ & $20.78(0.25)$ & $21.06(0.23)$ & \\
\hline \multirow[t]{3}{*}{1381} & 412.053 & 131.619 & $20.42(0.06)$ & $20.51(0.07)$ & $20.90(0.10)$ & $21.59(0.15)$ & $20.59(0.05)$ & $20.54(0.05)$ & $20.56(0.06)$ \\
\hline & & & $20.86(0.06)$ & $20.90(0.08)$ & $20.87(0.05)$ & $20.60(0.07)$ & $20.87(0.07)$ & $20.74(0.06)$ & $21.07(0.07)$ \\
\hline & & & $21.12(0.09)$ & $21.12(0.07)$ & $21.07(0.09)$ & $20.96(0.05)$ & $21.05(0.08)$ & $20.82(0.08)$ & \\
\hline \multirow[t]{3}{*}{1390} & 252.473 & 92.608 & $20.90(0.06)$ & $21.01(0.10)$ & $21.08(0.11)$ & $21.66(0.22)$ & $20.67(0.05)$ & $20.52(0.06)$ & $20.67(0.06)$ \\
\hline & & & $21.00(0.06)$ & $21.07(0.08)$ & $20.93(0.06)$ & $20.93(0.09)$ & $20.96(0.07)$ & $20.82(0.08)$ & $20.96(0.07)$ \\
\hline & & & $21.07(0.07)$ & $20.85(0.05)$ & $20.56(0.06)$ & $20.42(0.05)$ & $20.56(0.05)$ & $20.72(0.08)$ & \\
\hline \multirow[t]{3}{*}{1394} & 324.783 & 739.677 & $20.91(0.05)$ & $20.60(0.09)$ & $20.50(0.07)$ & $20.66(0.09)$ & $20.93(0.05)$ & $20.94(0.05)$ & $20.90(0.06)$ \\
\hline & & & $20.66(0.05)$ & $20.40(0.05)$ & $20.60(0.05)$ & $20.39(0.06)$ & $20.36(0.06)$ & $20.53(0.06)$ & $20.70(0.06)$ \\
\hline & & & $20.72(0.06)$ & $20.78(0.06)$ & $21.00(0.07)$ & $21.06(0.06)$ & $21.10(0.08)$ & $20.94(0.08)$ & \\
\hline \multirow[t]{3}{*}{1396} & 305.918 & 295.709 & $20.48(0.04)$ & $20.92(0.07)$ & $21.65(0.16)$ & $22.35(0.27)$ & $20.90(0.05)$ & $20.71(0.05)$ & $20.56(0.05)$ \\
\hline & & & $20.94(0.07)$ & $21.12(0.07)$ & $20.99(0.06)$ & $20.87(0.09)$ & $21.09(0.08)$ & $21.20(0.10)$ & $20.85(0.08)$ \\
\hline & & & $21.11(0.07)$ & $21.05(0.06)$ & $21.04(0.09)$ & $20.79(0.06)$ & $20.61(0.06)$ & $20.43(0.07)$ & \\
\hline 1416 & 719.443 & 827.022 & $21.02(0.06)$ & $20.96(0.08)$ & $21.22(0.13)$ & $21.00(0.13)$ & $20.51(0.06)$ & $20.66(0.05)$ & 21.27 \\
\hline & & & $21.28(0.07)$ & $21.19(0.07)$ & $20.75(0.05)$ & 21.14 & 20.95 & $20.91(0.07)$ & $20.73(0.06)$ \\
\hline & & & $20.55(0.07)$ & $20.46(0.05)$ & $20.47(0.07)$ & $20.55(0.05)$ & $20.81(0.07)$ & $20.58(0.79)$ & \\
\hline 1422 & 165.721 & 818.853 & $20.50(0.05)$ & $21.21(0.13)$ & $21.32(0.12)$ & $20.83(0.13)$ & $20.73(0.05)$ & $20.49(0.05)$ & $20.53(0.07)$ \\
\hline & & & $20.88(0.06)$ & $20.73(0.06)$ & $20.75(0.05)$ & $20.67(0.07)$ & $20.83(0.06)$ & $21.09(0.07)$ & $21.09(0.09)$ \\
\hline & & & $21.05(0.07)$ & 21.18 & 21.07 & 20.94 & $21.18(0.09)$ & $21.14(0.11)$ & \\
\hline 1445 & 53.525 & 768.300 & $21.18(0.07)$ & $21.05(0.12)$ & $21.28(0.13)$ & 99.99 (9.99) & $20.65(0.05)$ & $20.90(0.07)$ & 99.99 (9.99) \\
\hline & & & $21.10(0.06)$ & $20.97(0.07)$ & $20.82(0.08)$ & $21.40(0.15)$ & $21.56(0.11)$ & $20.62(0.07)$ & $20.92(0.06)$ \\
\hline & & & $20.59(0.05)$ & $20.71(0.07)$ & $20.76(0.06)$ & $20.59(0.06)$ & $20.71(0.08)$ & $20.76(0.08)$ & \\
\hline 1450 & 331.171 & 253.491 & $21.35(0.07)$ & $20.59(0.10)$ & $20.47(0.06)$ & $20.67(0.10)$ & $21.72(0.08)$ & $21.62(0.11)$ & $21.82(0.14)$ \\
\hline & & & $20.78(0.06)$ & $21.36(0.08)$ & $21.25(0.05)$ & $20.96(0.08)$ & $21.00(0.08)$ & $20.93(0.09)$ & $20.69(0.06)$ \\
\hline & & & $20.72(0.06)$ & $20.58(0.05)$ & $20.52(0.06)$ & $20.48(0.05)$ & $20.51(0.06)$ & $20.37(0.07)$ & \\
\hline 1452 & 368.674 & 313.404 & $20.54(0.18)$ & $21.40(0.22)$ & $20.42(0.16)$ & $20.30(0.17)$ & $20.99(0.18)$ & $20.55(0.12)$ & $19.87(0.14)$ \\
\hline & & & $20.30(0.09)$ & $20.81(0.13)$ & $20.70(0.12)$ & $21.49(0.22)$ & $21.14(0.18)$ & $22.25(0.37)$ & $21.95(0.28)$ \\
\hline & & & $22.02(0.23)$ & 20.87 & $21.77(C$ & $21.92(0.2$ & 99.99 & $21.50(0$. & \\
\hline 1464 & 56.592 & 35.701 & $22.00(0.11)$ & $21.30(0.13)$ & $99.99(9.99)$ & $99.99(9.99)$ & $99.99(9.99)$ & $22.54(0.16)$ & 99.99 (9.99) \\
\hline & & & $21.39(0.08)$ & $22.08(0.14)$ & $21.05(0.07)$ & $20.81(0.07)$ & $21.38(0.12)$ & $21.06(0.10)$ & $20.85(0.08)$ \\
\hline & & & $20.78(0.07)$ & $20.67(0.05)$ & $20.67(0.07)$ & $21.04(0.07)$ & $20.37(0.06)$ & $20.31(0.06)$ & \\
\hline 1485 & 65.611 & 460.413 & $20.78(0.06)$ & $20.53(0.09)$ & $20.70(0$. & $21.07(0.12)$ & 21.07 & $20.81(0$. & 20.79 \\
\hline & & & $21.05(0.09)$ & $20.98(0.06)$ & $20.97(0.05)$ & $20.85(0.09)$ & $20.91(0.06)$ & $20.60(0.07)$ & $20.48(0.06)$ \\
\hline & & & $20.50(0.05)$ & $20.60(0.06)$ & $20.85(0.07)$ & $21.13(0.11)$ & $21.16(0.08)$ & $20.96(0.07)$ & \\
\hline 1488 & 500.939 & 277.511 & $20.95(0.06)$ & $20.63(0.07)$ & $21.31(0.16)$ & $21.14(0.14)$ & $21.41(0.08)$ & $21.19(0.07)$ & $21.29(0.08)$ \\
\hline & & & & $20.65(0.07)$ & $20.63(0.05)$ & $20.66(0.07)$ & $20.88(0.06)$ & $20.64(0.07)$ & $20.89(0.06)$ \\
\hline & & & $20.77(0.06)$ & $20.75(0.05)$ & $20.77(0.07)$ & $20.75(0.06)$ & $20.92(0.09)$ & $20.97(0.09)$ & \\
\hline 1495 & 839.865 & 829.356 & $20.94(0.06)$ & $21.18(0.12)$ & $20.15(0.11)$ & $20.37(0.07)$ & $20.70(0.04)$ & $20.83(0.05)$ & 99.99 (9.99) \\
\hline & & & $22.20(0.19)$ & $21.40(0.09)$ & $21.60(0.09)$ & $21.39(0.13)$ & $21.01(0.08)$ & $21.96(0.15)$ & $22.88(0.39)$ \\
\hline & & & 99.99 (9.99) & $22.41(0.24)$ & 99.99 (9.99) & $21.39(0.10)$ & $20.55(0.06)$ & 99.99 (9.99) & \\
\hline 1513 & 281.031 & 309.679 & $20.59(0.05)$ & $21.45(0.13)$ & $21.13(0.10)$ & $20.93(0.12)$ & $21.26(0.07)$ & $21.36(0.07)$ & $21.12(0.08)$ \\
\hline & & & $21.08(0.09)$ & $21.46(0.10)$ & $21.16(0.06)$ & $21.02(0.08)$ & $20.84(0.06)$ & $20.70(0.07)$ & $20.46(0.05)$ \\
\hline & & & $20.51(0.05)$ & $20.82(0.06)$ & $20.97(0.08)$ & $21.18(0.09)$ & $21.36(0.11)$ & $21.19(0.09)$ & \\
\hline 1533 & 412.255 & -22.898 & $99.99(9.99)$ & $99.99(9.99)$ & $21.64(0.19)$ & 99.99 (9.99) & 99.99 (9.99) & 99.99 (9.99) & 99.99 (9.99) \\
\hline & & & & & & $20.99(0.24)$ & $22.77(1.25)$ & $20.45(0.15)$ & $20.29(3.51)$ \\
\hline & & & $21.51(0.13)$ & $99.99(9.99)$ & $21.65(0.14)$ & $22.49(0.43)$ & $20.84(0.13)$ & $21.58(0.35)$ & \\
\hline 1539 & 89.590 & 146.109 & $20.61(0.05)$ & $20.79(0.09)$ & $20.59(0.10)$ & $21.05(0.11)$ & $21.31(0.07)$ & $20.98(0.06)$ & $20.74(0.07)$ \\
\hline & & & $21.05(0.07)$ & $21.25(0.08)$ & $20.75(0.05)$ & $21.05(0.11)$ & $21.01(0.09)$ & $20.66(0.06)$ & $20.86(0.07)$ \\
\hline & & & $20.76(0.06)$ & $21.05(0.07)$ & $21.17(0.07)$ & $21.30(0.10)$ & $21.30(0.11)$ & $21.01(0.12)$ & \\
\hline
\end{tabular}


Table 7. continuation.

\begin{tabular}{|c|c|c|c|c|c|c|c|c|c|}
\hline \multirow[t]{3}{*}{ F1 ID } & \multirow[t]{3}{*}{$\mathrm{x}$} & \multirow[t]{3}{*}{$\mathrm{y}$} & & $1 \mathrm{Ks} 01(\sigma)$ & $1 \mathrm{Ks} 02(\sigma)$ & $1 \mathrm{Ks} 03(\sigma)$ & $1 \mathrm{Ks} 04(\sigma)$ & $1 \mathrm{Ks} 05(\sigma)$ & \multirow{3}{*}{$\begin{array}{l}1 \mathrm{Ks} 06(\sigma) \\
1 \mathrm{Ks} 14(\sigma)\end{array}$} \\
\hline & & & & $1 \mathrm{Ks} 09(\sigma)$ & $1 \mathrm{Ks} 10(\sigma)$ & $1 \mathrm{Ks} 11(\sigma)$ & $1 \mathrm{Ks} 12(\sigma)$ & $1 \mathrm{Ks} 13(\sigma)$ & \\
\hline & & & & $1 \mathrm{Ks} 16(\sigma)$ & $1 \mathrm{Ks} 17(\sigma)$ & $1 \mathrm{Ks} 18(\sigma)$ & $1 \mathrm{Ks} 19(\sigma)$ & $1 \mathrm{Ks} 20(\sigma)$ & \\
\hline \multirow[t]{3}{*}{1554} & 164.071 & 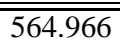 & $21.03(0.05)$ & $21.29(0.11)$ & $21.40(0.12)$ & $21.19(0.12)$ & $21.42(0.07)$ & $21.48(0.07)$ & $21.29(0.09)$ \\
\hline & & & $21.14(0.08)$ & $21.59(0.11)$ & $21.76(0.11)$ & $21.52(0.11)$ & $21.27(0.10)$ & $21.21(0.09)$ & $21.01(0.08)$ \\
\hline & & & $20.62(0.05)$ & $20.54(0.05)$ & $20.60(0.05)$ & $20.72(0.07)$ & $21.13(0.08)$ & $21.19(0.09)$ & \\
\hline \multirow[t]{3}{*}{1564} & 300.273 & 611.715 & $21.17(0.05)$ & $20.76(0.09)$ & $20.91(0.11)$ & $20.73(0.10)$ & $20.65(0.05)$ & $20.84(0.06)$ & $21.51(0.09)$ \\
\hline & & & $21.07(0.07)$ & $20.97(0.07)$ & $20.77(0.05)$ & $21.20(0.12)$ & $21.27(0.09)$ & $21.26(0.09)$ & $20.97(0.06)$ \\
\hline & & & $20.81(0.06)$ & $20.75(0.06)$ & $20.57(0.06)$ & $20.63(0.05)$ & $20.83(0.07)$ & $21.01(0.08)$ & \\
\hline \multirow[t]{3}{*}{1624} & 853.974 & 765.488 & $20.93(0.05)$ & $21.68(0.22)$ & $20.38(0.08)$ & $20.88(0.08)$ & $20.92(0.05)$ & $20.97(0.06)$ & $99.99(9.99)$ \\
\hline & & & $21.99(0.17)$ & $22.26(0.17)$ & $21.08(0.06)$ & $20.97(0.09)$ & $20.96(0.07)$ & $22.14(0.17)$ & $23.20(0.51)$ \\
\hline & & & $22.03(0.15)$ & $21.81(0.10)$ & $23.08(0.36)$ & $21.64(0.10)$ & $20.56(0.07)$ & $20.19(0.06)$ & \\
\hline \multirow[t]{3}{*}{1629} & 768.512 & 220.448 & $21.00(0.06)$ & $21.23(0.11)$ & $21.47(0.12)$ & $21.46(0.15)$ & $20.71(0.05)$ & $20.78(0.05)$ & $20.87(0.07)$ \\
\hline & & & $20.88(0.07)$ & $20.84(0.07)$ & $20.82(0.05)$ & $20.72(0.08)$ & $20.96(0.07)$ & $20.83(0.06)$ & $21.06(0.08)$ \\
\hline & & & $21.18(0.06)$ & $21.23(0.08)$ & $21.37(0.11)$ & $21.44(0.08)$ & $21.21(0.09)$ & $21.06(0.09)$ & \\
\hline \multirow[t]{3}{*}{1639} & 489.291 & 863.474 & $21.33(0.05)$ & $21.50(0.14)$ & $21.94(0.32)$ & $20.57(0.09)$ & $20.76(0.21)$ & $21.11(0.07)$ & $22.64(0.25)$ \\
\hline & & & 99.99 (9.99) & 99.99 (9.99) & 99.99 (9.99) & 99.99 (9.99) & 99.99 (9.99) & 99.99 (9.99) & $20.55(0.05)$ \\
\hline & & & $20.56(0.06)$ & 99.99 (9.99) & 99.99 (9.99) & 99.99 (9.99) & 99.99 (9.99) & $99.99(9.99)$ & \\
\hline \multirow[t]{3}{*}{1649} & 777.550 & 813.482 & $21.24(0.06)$ & $21.20(0.10)$ & $22.32(0.26)$ & $21.58(0.20)$ & $20.67(0.06)$ & $20.64(0.06)$ & $21.21(0.11)$ \\
\hline & & & 21.55 & $21.27(0.10)$ & $20.86(0.05)$ & $21.43(0.13)$ & 21.00 & $20.80(0.06)$ & $20.76(0.05)$ \\
\hline & & & $20.70(0.08)$ & $20.71(0.06)$ & $20.92(0.06)$ & $21.29(0.08)$ & $21.50(0.14)$ & $21.35(0.10)$ & \\
\hline \multirow[t]{3}{*}{1670} & 534.016 & 605.585 & $21.01(0.06)$ & $21.00(0.11)$ & $20.69(0.09)$ & $20.44(0.07)$ & $21.10(0.06)$ & $21.15(0.06)$ & $21.28(0.08)$ \\
\hline & & & $20.70(0.06)$ & $20.72(0.07)$ & $20.85(0.05)$ & $20.69(0.07)$ & $20.73(0.06)$ & $20.88(0.06)$ & $21.22(0.07)$ \\
\hline & & & $21.16(0.07)$ & $21.07(0.07)$ & $21.03(0.07)$ & $21.25(0.09)$ & $21.19(0.09)$ & $21.22(0.11)$ & \\
\hline \multirow[t]{3}{*}{1671} & 74.539 & 825.258 & $21.28(0.07)$ & $21.08(0.10)$ & $21.58(0.15)$ & $21.60(0.17)$ & $20.86(0.05)$ & $20.75(0.06)$ & $20.85(0.09)$ \\
\hline & & & $21.02(0.08)$ & $21.13(0.07)$ & $20.90(0.05)$ & $20.77(0.08)$ & $21.12(0.07)$ & $20.99(0.08)$ & $20.74(0.06)$ \\
\hline & & & $20.64(0.06)$ & $20.85(0.06)$ & $21.19(0.09)$ & $21.31(0.10)$ & $22.25(0.17)$ & $21.49(0.13)$ & \\
\hline 1682 & 206.538 & 46.923 & $21.21(0.05)$ & $20.82(0.13)$ & $21.30(0.11)$ & $22.13(0.30)$ & $20.59(0.06)$ & $20.79(0.06)$ & 21.01 \\
\hline & & & 21.18 & $20.67(0.07)$ & $20.84(0.06)$ & $20.98(0.10)$ & $21.18(0.11)$ & $20.94(0.12)$ & 21.24 \\
\hline & & & $21.10(0.06)$ & $21.35(0.15)$ & $21.33(0.13)$ & $20.84(0.07)$ & $20.66(0.08)$ & $20.76(0.09)$ & \\
\hline 1689 & 404.355 & 7.315 & $22.19(0.12)$ & $22.17(0.90)$ & $21.47(0.33)$ & $24.86(7.34)$ & $21.39(0.26)$ & $21.00(0.21)$ & $21.91(0.21)$ \\
\hline & & & $19.65(0.10)$ & $20.80(0.21)$ & $21.60(0.43)$ & $21.05(0.25)$ & $20.62(0.13)$ & $22.27(0.86)$ & $20.86(0.31)$ \\
\hline & & & & 20.58 & & & & $22.67(0.94)$ & \\
\hline 1705 & 816.287 & 857.316 & $21.78(0$ & $20.99(0.10)$ & 99.99 (9. & 99.99 & 21.43 & 21.53 & 99.99 (9.99) \\
\hline & & & 99.99 (9.99) & 99.99 (9.99) & $0000 / 0$ & 99.99 (9.99) & 99.99 (9.99) & $19.94(0.18)$ & $20.62(0.06)$ \\
\hline & & & $20.78(0.06)$ & $20.66(0.06)$ & $0000 / 00$ & 99.99 (9.99) & 99.99 (9.99) & $99.99(9.99)$ & \\
\hline 1735 & 511.708 & -8.212 & 99.99 & $20.81(0.08)$ & $20.75(0.09)$ & $21.15(0.11)$ & $21.13(0.05)$ & $20.81(0.14)$ & 99.99 \\
\hline & & & 21.16 & $20.99(0.06)$ & $20.76(0.05)$ & $20.76(0.09)$ & $20.79(0.07)$ & $20.79(0.07)$ & $21.12(0.08)$ \\
\hline & & & $21.26(0.08)$ & $21.25(0.08)$ & $21.12(0.07)$ & $21.17(0.09)$ & $21.31(0.11)$ & $20.83(0.07)$ & \\
\hline 1775 & 763.695 & 286.694 & $20.80(0.05)$ & $20.71(0.08)$ & $21.27(0.09)$ & $21.52(0.16)$ & $21.38(0.06)$ & $21.17(0.06)$ & $20.95(0.07)$ \\
\hline & & & & $21.34(0.11)$ & $20.95(0.05)$ & $21.43(0.13)$ & $21.06(0.07)$ & $20.89(0.06)$ & $20.94(0.07)$ \\
\hline & & & & 20.85 & & 21.24 & 21.36 & $21.40(0.11)$ & \\
\hline 1800 & 111.960 & 699.811 & $22.40(0.12)$ & $20.58(0.20)$ & $21.15(0.17)$ & $21.35(0.15)$ & $21.28(0.21)$ & 21.28 & $20.73(0.08)$ \\
\hline & & & $20.52(0.07)$ & $20.64(0.19)$ & $20.80(0.26)$ & $21.08(0.15)$ & $21.08(0.21)$ & $21.53(0.33)$ & $21.78(0.30)$ \\
\hline & & & & $20.83(0.09)$ & & $22.75(0.43)$ & $20.90(0.12)$ & $21.23(0.09)$ & \\
\hline 1804 & 713.258 & 872.670 & & & & & & & 21.94 \\
\hline & & & 99.99 (9.99) & 99.99 (9.99) & 99.99 (9.99) & 99.99 (9.99) & 99.99 (9.99) & 99.99 (9.99) & 99.99 (9.99) \\
\hline & & & 99.99 (9.99) & 99.99 (9.99) & $99.99(9.99)$ & 99.99 (9.99) & $99.99(9.99)$ & $99.99(9.99)$ & \\
\hline 1806 & 65.875 & 646.566 & $21.53(0.07)$ & $21.12(0.10)$ & $21.58(0.25)$ & $21.63(0.19)$ & $21.15(0.08)$ & $21.11(0.06)$ & $20.82(0.09)$ \\
\hline & & & & & & $21.77(0.18)$ & $21.51(0.13)$ & $21.06(0.08)$ & $20.99(0.06)$ \\
\hline & & & & $20.69(0.06)$ & $20.92(0.06)$ & $20.95(0.06)$ & $21.26(0.11)$ & $22.04(0.24)$ & \\
\hline 1810 & 606.950 & -17.238 & $99.99(9.99)$ & $20.36(0.14)$ & $20.88(0.09)$ & $21.28(0.16)$ & $99.99(9.99)$ & $99.99(9.99)$ & 99.99 (9.99) \\
\hline & & & $21.54(0.09)$ & $21.35(0.09)$ & $21.17(0.07)$ & $21.73(0.17)$ & $21.55(0.10)$ & $21.49(0.10)$ & $21.26(0.11)$ \\
\hline & & & & $21.18(0.07)$ & $21.03(0.08)$ & $20.86(0.07)$ & $20.60(0.06)$ & $20.42(0.06)$ & \\
\hline 1827 & 753.817 & -0.236 & $99.99(9.99)$ & $20.71(0.13)$ & $20.40(0.18)$ & $20.63(0.21)$ & $20.71(0.11)$ & $20.35(0.11)$ & $18.77(0.26)$ \\
\hline & & & $22.12(0.16)$ & $21.35(0.13)$ & $21.91(0.24)$ & $22.08(0.31)$ & $22.01(0.18)$ & $23.34(0.57)$ & $23.81(0.94)$ \\
\hline & & & $22.37(0.23)$ & $23.02(0.75)$ & $23.15(0.68)$ & $23.34(0.40)$ & $20.66(0.18)$ & $20.98(0.21)$ & \\
\hline 1832 & 105.314 & 515.071 & $21.45(0.07)$ & $21.24(0.12)$ & $21.40(0.13)$ & $21.89(0.21)$ & $21.22(0.05)$ & $21.40(0.08)$ & $21.17(0.08)$ \\
\hline & & & & & & & & $21.50(0.11)$ & $20.90(0.06)$ \\
\hline & & & $20.77(0.05)$ & $20.63(0.06)$ & $20.63(0.08)$ & $20.74(0.06)$ & $21.05(0.07)$ & $21.24(0.12)$ & \\
\hline 1838 & 693.158 & 75.868 & $21.00(0.19)$ & $21.94(0.38)$ & $20.12(0.14)$ & $99.99(9.99)$ & $20.74(0.15)$ & $21.17(0.26)$ & $99.99(9.99)$ \\
\hline & & & $20.68(0.08)$ & $20.63(0.09)$ & $20.89(0.11)$ & $21.99(0.18)$ & $21.18(0.10)$ & $22.07(0.20)$ & $21.49(0.13)$ \\
\hline & & & $21.95(0.16)$ & $21.20(0.12)$ & $21.87(0.21)$ & $21.82(0.19)$ & $21.17(0.14)$ & $21.01(0.15)$ & \\
\hline
\end{tabular}


Table 7. continuation.

\begin{tabular}{|c|c|c|c|c|c|c|c|c|c|}
\hline \multirow[t]{3}{*}{ F1 ID } & \multirow[t]{3}{*}{$\mathrm{X}$} & \multirow[t]{3}{*}{$\mathrm{y}$} & $1 \mathrm{Ks} 07(\sigma)$ & 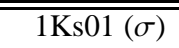 & $1 \mathrm{Ks} 02(\sigma)$ & $1 \mathrm{Ks} 03(\sigma)$ & $1 \mathrm{Ks} 04(\sigma)$ & $1 \mathrm{K \textrm {Ks } 0 5 ( \sigma )}$ & \multirow{3}{*}{$\begin{array}{l}1 \mathrm{Ks} 06(\sigma) \\
1 \mathrm{Ks} 14(\sigma)\end{array}$} \\
\hline & & & $1 \mathrm{Ks} 08(\sigma)$ & $1 \mathrm{Ks} 09(\sigma)$ & $1 \mathrm{Ks} 10(\sigma)$ & $1 \mathrm{Ks} 11(\sigma)$ & $1 \mathrm{Ks} 12(\sigma)$ & $1 \mathrm{Ks} 13(\sigma)$ & \\
\hline & & & $1 \mathrm{Ks} 15(\sigma)$ & $1 \mathrm{Ks} 16(\sigma)$ & $1 \mathrm{Ks} 17(\sigma)$ & $1 \mathrm{Ks} 18(\sigma)$ & $1 \mathrm{Ks} 19(\sigma)$ & $1 \mathrm{Ks} 20(\sigma)$ & \\
\hline \multirow[t]{3}{*}{ 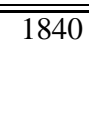 } & 263.550 & 751.289 & $21.27(0.07)$ & $21.23(0.12)$ & $21.87(0.18)$ & $21.67(0.23)$ & $20.83(0.06)$ & $21.03(0.06)$ & $21.36(0.09)$ \\
\hline & & & $21.49(0.08)$ & $21.35(0.07)$ & $20.86(0.05)$ & $21.12(0.10)$ & $21.01(0.06)$ & $20.76(0.07)$ & $20.75(0.06)$ \\
\hline & & & $20.95(0.06)$ & $20.98(0.07)$ & $21.22(0.07)$ & $21.27(0.08)$ & $21.34(0.09)$ & $21.20(0.09)$ & \\
\hline \multirow[t]{3}{*}{1851} & 227.825 & 255.322 & $20.98(0.05)$ & $21.18(0.10)$ & $21.06(0.13)$ & $21.66(0.17)$ & $21.49(0.07)$ & $21.41(0.08)$ & $21.27(0.09)$ \\
\hline & & & $21.14(0.07)$ & $21.31(0.08)$ & $20.95(0.05)$ & $20.91(0.10)$ & $20.71(0.06)$ & $20.67(0.07)$ & $20.80(0.06)$ \\
\hline & & & $21.27(0.08)$ & $21.23(0.08)$ & $21.32(0.09)$ & $21.29(0.08)$ & $21.94(0.20)$ & $21.27(0.09)$ & \\
\hline \multirow[t]{3}{*}{1859} & 18.449 & 33.166 & $21.85(0.11)$ & 99.99 (9.99) & 99.99 (9.99) & 99.99 (9.99) & $22.31(0.18)$ & $21.61(0.08)$ & 99.99 (9.99) \\
\hline & & & 99.99 (9.99) & 99.99 (9.99) & 99.99 (9.99) & $22.05(0.22)$ & $21.86(0.18)$ & 99.99 (9.99) & $20.99(0.07)$ \\
\hline & & & $20.87(0.07)$ & $20.71(0.06)$ & 99.99 (9.99) & $20.84(0.06)$ & 99.99 (9.99) & 99.99 (9.99) & \\
\hline \multirow[t]{3}{*}{1867} & 878.280 & 140.257 & $20.98(0.11)$ & $21.16(0.11)$ & $99.99(9.99)$ & $21.12(0.12)$ & $21.56(0.08)$ & $20.92(0.07)$ & 99.99 (9.99) \\
\hline & & & $20.80(0.08)$ & $21.09(0.07)$ & $21.20(0.09)$ & $20.99(0.12)$ & $21.00(0.07)$ & $21.38(0.14)$ & $21.49(0.11)$ \\
\hline & & & $21.72(0.12)$ & $21.64(0.13)$ & $21.55(0.17)$ & $21.56(0.13)$ & $20.61(0.08)$ & $20.35(0.07)$ & \\
\hline \multirow[t]{3}{*}{1888} & 151.663 & 580.097 & $20.80(0.05)$ & $21.16(0.10)$ & $20.75(0.06)$ & $20.76(0.07)$ & $21.72(0.07)$ & $21.53(0.07)$ & $21.15(0.08)$ \\
\hline & & & $21.70(0.12)$ & $21.48(0.09)$ & $21.44(0.06)$ & $20.72(0.07)$ & $20.88(0.06)$ & $21.02(0.09)$ & $21.22(0.07)$ \\
\hline & & & $21.32(0.07)$ & $21.29(0.08)$ & $21.91(0.19)$ & $21.35(0.08)$ & $21.77(0.14)$ & $21.76(0.18)$ & \\
\hline \multirow[t]{3}{*}{1893} & 447.366 & 406.122 & $21.60(0.11)$ & $21.14(0.17)$ & $22.33(0.46)$ & $21.44(0.22)$ & $21.46(0.09)$ & $20.95(0.10)$ & $20.89(0.09)$ \\
\hline & & & $20.28(0.09)$ & $21.00(0.16)$ & $21.17(0.16)$ & $21.24(0.19)$ & $20.75(0.11)$ & $21.25(0.13)$ & $20.93(0.11)$ \\
\hline & & & $21.58(0.15)$ & $20.83(0.11)$ & $21.51(0.18)$ & $21.03(0.10)$ & $21.36(0.12)$ & $21.21(0.21)$ & \\
\hline \multirow[t]{3}{*}{1897} & 357.604 & 318.509 & $21.63(0.10)$ & $22.02(0.26)$ & 99.99 (9.99) & $22.57(0.54)$ & $21.46(0.09)$ & $21.72(0.08)$ & 99.99 (9.99) \\
\hline & & & $20.14(0.09)$ & $20.56(0.10)$ & $21.38(0.09)$ & $20.84(0.11)$ & $21.47(0.13)$ & $21.07(0.11)$ & $20.95(0.08)$ \\
\hline & & & $21.08(0.08)$ & $21.05(0.10)$ & $21.13(0.08)$ & $21.02(0.07)$ & $20.63(0.07)$ & $20.65(0.10)$ & \\
\hline \multirow[t]{3}{*}{1903} & 251.772 & 690.018 & $21.09(0.05)$ & $21.56(0.16)$ & $22.19(0.31)$ & $22.56(0.41)$ & $21.13(0.06)$ & $21.33(0.09)$ & $21.37(0.08)$ \\
\hline & & & $22.04(0.14)$ & $22.17(0.20)$ & $21.82(0.09)$ & $22.73(0.36)$ & $21.21(0.08)$ & $21.07(0.07)$ & $20.83(0.05)$ \\
\hline & & & $20.66(0.05)$ & $21.03(0.07)$ & $21.39(0.10)$ & $21.61(0.09)$ & $21.64(0.12)$ & $21.69(0.12)$ & \\
\hline 1911 & 865.938 & 292.732 & $20.91(0.06)$ & $21.18(0.10)$ & $21.24(0.13)$ & $21.51(0.15)$ & $21.39(0.07)$ & $21.31(0.06)$ & 99.99 (9.99) \\
\hline & & & $21.57(0.25)$ & 21.86( & 21.06( & $20.77(0.08)$ & 20.70 & $20.73(0.06)$ & $21.00(0.07)$ \\
\hline & & & $21.24(0.07)$ & $21.22(0.08)$ & $21.34(0.09)$ & $21.24(0.08)$ & $20.82(0.06)$ & $21.04(0.08)$ & \\
\hline 1943 & 652.101 & 471.075 & $20.80(0.05)$ & $21.60(0.15)$ & $21.12(0.13)$ & $21.04(0.12)$ & $21.43(0.06)$ & $21.40(0.07)$ & $21.43(0.08)$ \\
\hline & & & $21.24(0.09)$ & $21.19(0.09)$ & $21.32(0.06)$ & $21.27(0.11)$ & $21.45(0.10)$ & $21.28(0.09)$ & $20.86(0.06)$ \\
\hline & & & 20.95 & 20.69 & 20.80 & 20.87 & 21.01 & $21.42(0.10)$ & \\
\hline 2014 & 862.100 & 33.721 & $21.79(0.11)$ & $21.65(0.17)$ & $22.28(0.34)$ & $21.84(0.23)$ & 21.30 & $21.40(0.08)$ & 99.99 (9.99) \\
\hline & & & $20.69(0.07)$ & $20.91(0.08)$ & $20.97(0.06)$ & $21.30(0.12)$ & $21.43(0.09)$ & $21.42(0.10)$ & $21.63(0.10)$ \\
\hline & & & $21.22(0.07)$ & $21.08(0.07)$ & $20.91(0.08)$ & $20.74(0.07)$ & $20.88(0.09)$ & $21.12(0.13)$ & \\
\hline 2015 & 644.197 & 327.874 & $21.74(0.08)$ & $21.24(0.10)$ & $21.15(0.10)$ & $21.56(0.17)$ & $20.90(0.05)$ & $21.00(0.06)$ & $0.11)$ \\
\hline & & & $21.15(0.08)$ & $20.95(0.07)$ & $21.32(0.06)$ & $21.97(0.17)$ & $21.55(0.09)$ & $21.45(0.10)$ & $21.23(0.08)$ \\
\hline & & & $21.10(0.06)$ & $20.91(0.07)$ & $20.82(0.0$ & $21.13(0.07)$ & $21.19(0.08)$ & $21.23(0.11)$ & \\
\hline 2027 & 836.758 & 722.754 & $21.34(0.05)$ & $21.46(0.19)$ & 99.99 (9.99) & $21.54(0.17)$ & $21.25(0.05)$ & $21.55(0.08)$ & 99.99 (9.99) \\
\hline & & & $21.57(0.09)$ & $20.82(0.06)$ & $21.15(0.05)$ & $21.33(0.11)$ & $21.15(0.07)$ & $20.75(0.07)$ & $20.82(0.06)$ \\
\hline & & & $20.75(0.06)$ & $20.74(0$. & $20.83(0$. & 21.05 & 21.38 & $21.98(0.16)$ & \\
\hline 2037 & 245.570 & 872.812 & $22.01(0.07)$ & $22.87(0.49)$ & $19.48(0.11)$ & $20.10(0.10)$ & $22.09(0.17)$ & $22.44(0.98)$ & $22.38(0.27)$ \\
\hline & & & 99.99 (9.99) & 99.99 (9.99) & 99.99 (9.99) & 99.99 (9.99) & 99.99 (9.99) & 99.99 (9.99) & 99.99 (9.99) \\
\hline & & & $99.99(9.99)$ & $99.99(9.99)$ & 99.99 (9.99) & 99.99 (9.99) & 99.99 (9.99) & 99.99 (9.99) & \\
\hline 2050 & 54.552 & 767.989 & $21.58(0.10)$ & $21.16(0.13)$ & $21.13(0.12)$ & $20.65(0.08)$ & $22.69(0.1$ & $21.78(0.10)$ & 20.51 \\
\hline & & & $21.75(0.10)$ & $21.76(0.14)$ & $22.08(0.19)$ & $21.97(0.26)$ & $21.42(0.09)$ & 99.99 (9.99) & $22.63(0.26)$ \\
\hline & & & $23.57(0.45)$ & 99.99 (9.99) & $22.74(0.35)$ & 99.99 (9.99) & 99.99 (9.99) & $99.99(9.99)$ & \\
\hline 2057 & 802.054 & 815.425 & $21.48(0.06)$ & $22.05(0.19)$ & $20.96(0.08)$ & 99.99 (9.99) & $21.57(0.09)$ & $21.66(0.08)$ & $20.52(0.09)$ \\
\hline & & & & $21.93(0.14)$ & & $21.86(0.19)$ & $21.89(0.14)$ & $22.28(0.23)$ & $99.99(9.99)$ \\
\hline & & & 99.99 (9.99) & $23.08(0.43)$ & $23.52(0.64)$ & $22.36(0.19)$ & $20.73(0.07)$ & $20.37(0.06)$ & \\
\hline 2059 & 392.803 & 868.560 & $21.84(0.11)$ & $21.60(0.18)$ & $20.32(0.09)$ & $22.64(1.14)$ & $20.95(0.10)$ & $22.64(0.25)$ & $21.98(0.15)$ \\
\hline & & & 99.99 (9.99) & 99.99 (9.99) & 99.99 (9.99) & 99.99 (9.99) & 99.99 (9.99) & 99.99 (9.99) & $22.02(0.48)$ \\
\hline & & & 99.99 (9.99) & 99.99 (9.99) & 99.99 (9.99) & 99.99 (9.99) & 99.99 (9.99) & 99.99 (9.99) & \\
\hline 2093 & 719.435 & 768.779 & $21.48(0.06)$ & $21.28(0.13)$ & $21.98(0.18)$ & $21.63(0.19)$ & $20.88(0.05)$ & $20.91(0.06)$ & $21.43(0.09)$ \\
\hline & & & $21.57(0.10)$ & $21.58(0.09)$ & $21.20(0.05)$ & $21.46(0.13)$ & $21.36(0.08)$ & $21.45(0.09)$ & $21.24(0.11)$ \\
\hline & & & $21.01(0.06)$ & $21.09(0.07)$ & $20.88(0.06)$ & $20.85(0.06)$ & $21.14(0.08)$ & $21.26(0.10)$ & \\
\hline 2104 & 728.300 & 354.414 & $21.48(0.06)$ & $21.50(0.15)$ & $23.07(0.56)$ & $22.64(0.31)$ & $20.95(0.05)$ & $21.08(0.06)$ & $21.14(0.07)$ \\
\hline & & & & & & $21.04(0.09)$ & & $21.05(0.07)$ & $21.41(0.11)$ \\
\hline & & & $21.63(0.10)$ & $21.60(0.11)$ & $21.62(0.10)$ & $21.65(0.11)$ & $21.57(0.15)$ & $21.62(0.12)$ & \\
\hline 2108 & 722.411 & 633.752 & $20.94(0.05)$ & $21.37(0.11)$ & $21.28(0.15)$ & $21.72(0.21)$ & $21.61(0.07)$ & $21.67(0.09)$ & $21.46(0.11)$ \\
\hline & & & $21.87(0.13)$ & $21.41(0.09)$ & $20.97(0.05)$ & $20.97(0.09)$ & $21.04(0.09)$ & $20.98(0.09)$ & $21.21(0.09)$ \\
\hline & & & $21.61(0.10)$ & $21.49(0.09)$ & $21.55(0.09)$ & $21.36(0.07)$ & $21.45(0.11)$ & $20.91(0.08)$ & \\
\hline
\end{tabular}


Table 7. continuation.

\begin{tabular}{|c|c|c|c|c|c|c|c|c|c|}
\hline \multirow[t]{3}{*}{ F1 ID } & \multirow[t]{3}{*}{$\mathrm{x}$} & \multirow[t]{3}{*}{$\mathrm{y}$} & $1 \mathrm{Ks} 07(\sigma)$ & $1 \mathrm{Ks} 01(\sigma)$ & $1 \mathrm{Ks} 02(\sigma)$ & $1 \mathrm{Ks} 03(\sigma)$ & $1 \mathrm{Ks} 04(\sigma)$ & $1 \mathrm{Ks} 05(\sigma)$ & \multirow{3}{*}{$\begin{array}{l}1 \mathrm{Ks} 06(\sigma) \\
1 \mathrm{Ks} 14(\sigma)\end{array}$} \\
\hline & & & $1 \mathrm{Ks} 08(\sigma)$ & $1 \mathrm{Ks} 09(\sigma)$ & $1 \mathrm{Ks} 10(\sigma)$ & $1 \mathrm{Ks} 11(\sigma)$ & $1 \mathrm{Ks} 12(\sigma)$ & $1 \mathrm{Ks} 13(\sigma)$ & \\
\hline & & & $1 \mathrm{Ks} 15(\sigma)$ & $1 \mathrm{Ks} 16(\sigma)$ & $1 \mathrm{Ks} 17(\sigma)$ & $1 \mathrm{Ks} 18(\sigma)$ & $1 \mathrm{Ks} 19(\sigma)$ & $1 \mathrm{Ks} 20(\sigma)$ & \\
\hline \multirow[t]{3}{*}{2144} & 863.752 & 23.934 & $21.37(0.06)$ & $21.19(0.13)$ & $21.33(0.18)$ & $21.59(0.20)$ & $22.91(0.19)$ & $21.68(0.11)$ & $99.99(9.99)$ \\
\hline & & & 99.99 (9.99) & $22.07(0.21)$ & $21.79(0.07)$ & $21.80(0.19)$ & $21.41(0.11)$ & $21.79(0.12)$ & 99.99 (9.99) \\
\hline & & & $22.86(0.29)$ & 99.99 (9.99) & $22.07(0.14)$ & $22.63(0.19)$ & $20.56(0.06)$ & $20.56(0.06)$ & \\
\hline \multirow[t]{3}{*}{2201} & 877.361 & 311.087 & $99.99(9.99)$ & $22.92(0.54)$ & $99.99(9.99)$ & $23.25(0.91)$ & $23.13(0.54)$ & $23.37(0.39)$ & 99.99 (9.99) \\
\hline & & & $20.34(0.11)$ & 99.99 (9.99) & 99.99 (9.99) & $23.00(0.59)$ & 99.99 (9.99) & $23.49(0.53)$ & $23.45(0.62)$ \\
\hline & & & $23.86(0.68)$ & $23.67(0.44)$ & $24.32(2.02)$ & 99.99 (9.99) & $23.19(0.57)$ & $99.99(9.99)$ & \\
\hline \multirow[t]{3}{*}{2223} & 760.724 & 516.453 & $22.45(0.11)$ & $22.07(0.16)$ & $21.72(0.17)$ & $21.75(0.24)$ & $22.37(0.11)$ & $22.91(0.27)$ & $22.70(0.27)$ \\
\hline & & & $21.17(0.08)$ & $21.42(0.11)$ & $21.58(0.07)$ & $21.98(0.18)$ & $22.11(0.22)$ & $21.63(0.11)$ & $21.03(0.08)$ \\
\hline & & & $21.35(0.08)$ & $20.93(0.07)$ & $21.06(0.08)$ & $20.84(0.07)$ & $20.79(0.07)$ & $20.79(0.06)$ & \\
\hline \multirow[t]{3}{*}{2266} & 324.743 & 541.049 & $21.15(0.05)$ & $21.79(0.19)$ & $21.81(0.22)$ & $21.62(0.13)$ & $21.54(0.07)$ & $21.73(0.08)$ & $21.55(0.09)$ \\
\hline & & & $21.56(0.09)$ & $21.28(0.09)$ & $21.32(0.06)$ & $21.66(0.15)$ & $21.32(0.10)$ & $21.26(0.08)$ & $20.84(0.06)$ \\
\hline & & & $20.98(0.08)$ & $20.91(0.08)$ & $21.06(0.11)$ & $21.00(0.07)$ & $21.28(0.09)$ & $21.22(0.09)$ & \\
\hline \multirow[t]{3}{*}{2269} & 687.439 & 872.047 & $23.28(0.20)$ & $22.41(0.30)$ & $20.24(0.98)$ & $19.96(0.13)$ & $22.06(0.23)$ & $23.74(0.96)$ & 99.99 (9.99) \\
\hline & & & 99.99 (9.99) & 99.99 (9.99) & 99.99 (9.99) & 99.99 (9.99) & 99.99 (9.99) & 99.99 (9.99) & 99.99 (9.99) \\
\hline & & & 99.99 (9.99) & 99.99 (9.99) & 99.99 (9.99) & 99.99 (9.99) & 99.99 (9.99) & $99.99(9.99)$ & \\
\hline \multirow[t]{3}{*}{2297} & 114.922 & 685.100 & $22.18(0.15)$ & $20.98(0.19)$ & $22.93(0.50)$ & $21.53(0.19)$ & $21.02(0.25)$ & $20.86(0.18)$ & $21.54(0.14)$ \\
\hline & & & 20.67 & 20.45 & $21.56(0.37)$ & $21.63(0.16)$ & 21.03 & $21.05(0.17)$ & $20.90(0.20)$ \\
\hline & & & $21.32(0.12)$ & $21.14(0.10)$ & $21.89(0.11)$ & $21.37(0.11)$ & $22.11(0.23)$ & $21.42(0.12)$ & \\
\hline \multirow[t]{3}{*}{2306} & 140.396 & 703.310 & $23.80(0.26)$ & $23.96(1.30)$ & $99.99(9.99)$ & $21.13(0.14)$ & 99.99 (9.99) & $25.31(2.48)$ & $21.51(0.17)$ \\
\hline & & & $21.08(0.11)$ & $21.29(0.20)$ & $99.99(9.99)$ & $20.80(0.07)$ & $20.72(0.17)$ & $99.99(9.99)$ & $21.82(0.46)$ \\
\hline & & & $22.88(0.31)$ & $21.72(0.16)$ & $23.55(0.76)$ & $23.37(0.53)$ & $21.71(0.15)$ & $22.05(0.20)$ & \\
\hline \multirow[t]{3}{*}{2364} & 521.966 & 828.285 & 21.28 & $20.90(0.17)$ & $21.09(0.12)$ & $21.55(0.17)$ & 21.89 & $21.59(0.11)$ & $22.30(0.23)$ \\
\hline & & & $20.63(0.08)$ & $21.55(0.15)$ & $21.27(0.15)$ & $23.31(0.66)$ & 21.62 & $21.92(0.22)$ & $21.16(0.11)$ \\
\hline & & & $21.89(0.16)$ & $21.56(0.10)$ & $21.97(0.21)$ & $22.10(0.14)$ & $21.19(0.10)$ & $20.51(0.11)$ & \\
\hline 2394 & 514.065 & 834.808 & 21.29 & 99.99 (9.9 & $21.37(0.23)$ & $21.77(0.31)$ & $22.24(0.25)$ & $21.92(0.23)$ & .14) \\
\hline & & & 20.67 & 21.46 & 21.82 & 23.43 & 22.25 & 99.99 (9.99) & 99.99 \\
\hline & & & 99.99 & $22.79(0.30)$ & 99.99 (9.99) & $22.79(0.39)$ & 23.35 & 99.99 (9.99) & \\
\hline 2429 & 853.792 & 28.540 & $21.65(0.09)$ & $23.25(0.75)$ & $99.99(9.99)$ & 99.99 (9.99) & 99.99 (9.99) & $22.45(0.21)$ & $99.99(9.99)$ \\
\hline & & & $22.51(0.23)$ & $22.89(0.31)$ & $22.92(0.26)$ & 99.99 (9.99) & $23.37(0.59)$ & $99.99(9.99)$ & $23.35(0.66)$ \\
\hline & & & 99.99 & 99.99 & 99.99 & 24.12 & 21.18 & $20.73(0.07)$ & \\
\hline 2443 & 60.021 & 697.063 & $21.28(0.07)$ & $21.53(0.16)$ & $21.79(0.14)$ & $21.99(0.19)$ & 21.01 & $21.03(0.06)$ & $21.33(0.10)$ \\
\hline & & & $21.24(0.09)$ & $21.01(0.07)$ & $21.15(0.06)$ & $21.43(0.13)$ & $21.60(0.1$ & $21.76(0.14)$ & $21.80(0.11)$ \\
\hline & & & $21.74(0.12)$ & $21.99(0.13)$ & $21.63(0.16)$ & $21.82(0.16)$ & $22.08(0.17)$ & $21.88(0.14)$ & \\
\hline 2445 & 116.053 & 409.325 & $20.72(0.15)$ & $22.89(0.50)$ & $21.48(0.15)$ & $21.50(0.17)$ & $21.47(0.14)$ & $21.45(0.13)$ & 20.71 \\
\hline & & & 20.69 & 21.36 & $20.93(0.14)$ & 22.15 & $21.21(0.14)$ & $22.40(0.26)$ & $21.12(0.11)$ \\
\hline & & & $21.67(0$ & 21.31 & 21.80 & $22.27(0.19)$ & 21.77 & $22.13(0.17)$ & \\
\hline 2460 & 700.330 & 51.969 & 99.99 (9.99) & 99.99 (9.99) & $24.23(1.85)$ & $23.00(0.67)$ & $23.99(0.35)$ & 99.99 (9.99) & $24.29(1.14)$ \\
\hline & & & & 99.99 (9.99) & $99.99(9.99)$ & $22.85(0.45)$ & $99.99(9.99)$ & $23.43(0.60)$ & $24.88(2.02)$ \\
\hline & & & & 99.99 & 1) & 99.99 & 25.03 & 22.75 & \\
\hline 2461 & 855.807 & 772.237 & $21.03(0.06)$ & $22.06(0.33)$ & $20.55(0.09)$ & $21.42(0.14)$ & $21.36(0.06)$ & $21.40(0.08)$ & 99.99 \\
\hline & & & $21.54(0.08)$ & $21.41(0.09)$ & $21.57(0.09)$ & $21.26(0.13)$ & $21.11(0.10)$ & $21.78(0.13)$ & $23.41(0.67)$ \\
\hline & & & & 99.99 (9.99) & & $21.89(0.14)$ & $21.36(0.09)$ & $20.92(0.08)$ & \\
\hline 2470 & 594.966 & 872.656 & 22.10 & & & & & 99.99 & 22.84 \\
\hline & & & 99.99 (9 & 99.99 ( & 99.99 & 99.99 (9.99) & 99.99 (9.99) & 99.99 (9.99) & 99.99 (9.99) \\
\hline & & & 99.99 (9.99) & 99.99 (9.99) & & 99.99 (9.99) & $99.99(9.99)$ & $99.99(9.99)$ & \\
\hline 2630 & 19.447 & 12.171 & $21.78(0.07)$ & 99.99 (9.99) & $99.99(9.99)$ & $24.63(3.61)$ & $21.98(0.15)$ & $22.19(0.14)$ & 99.99 (9.99) \\
\hline & & & & & & & $22.09(0.24)$ & 99.99 (9.99) & $21.04(0.08)$ \\
\hline & & & & & & & 99.99 (9.99) & & \\
\hline 2860 & 206.907 & 513.450 & $21.14(0.05)$ & $21.70(0.14)$ & $21.68(0.12)$ & $21.96(0.21)$ & $21.70(0.08)$ & $21.74(0.12)$ & $21.31(0.09)$ \\
\hline & & & $21.26(0.09)$ & $21.05(0.08)$ & $21.47(0.06)$ & $22.47(0.32)$ & $22.00(0.18)$ & $22.38(0.19)$ & $21.99(0.20)$ \\
\hline & & & & $21.64(0.08)$ & $21.40(0.10)$ & $21.36(0.11)$ & $21.59(0.14)$ & $21.79(0.14)$ & \\
\hline 2867 & 116.083 & 688.670 & $22.88(1.39)$ & $20.97(0.16)$ & $21.37(0.34)$ & $23.33(0.93)$ & $21.27(0.20)$ & $20.77(0.18)$ & $22.28(0.22)$ \\
\hline & & & $21.37(0.14)$ & $21.60(0.35)$ & $20.96(0.25)$ & $22.79(0.48)$ & $21.75(0.54)$ & $21.97(0.43)$ & $21.39(0.30)$ \\
\hline & & & $21.67(0.21)$ & $21.68(0.10)$ & $21.09(0.08)$ & $22.35(0.24)$ & $22.56(0.24)$ & 99.99 (9.99) & \\
\hline 2872 & 576.165 & 524.680 & $21.70(0.06)$ & $21.55(0.15)$ & $21.39(0.13)$ & $21.35(0.14)$ & $21.20(0.09)$ & $21.53(0.09)$ & $21.18(0.08)$ \\
\hline & & & & & & & & $21.53(0.09)$ & $21.14(0.09)$ \\
\hline & & & $22.43(0.19)$ & $22.02(0.14)$ & $21.57(0.09)$ & $21.28(0.10)$ & $21.71(0.15)$ & $22.29(0.20)$ & \\
\hline 2896 & 700.140 & 68.674 & $21.44(0.29)$ & $21.95(0.27)$ & $20.65(0.22)$ & $22.36(0.32)$ & $21.36(0.17)$ & $99.99(9.99)$ & $20.68(0.17)$ \\
\hline & & & $20.78(0.10)$ & $21.44(0.12)$ & $21.45(0.16)$ & $23.64(0.81)$ & $23.29(0.52)$ & $23.46(0.57)$ & $22.21(0.24)$ \\
\hline & & & $23.11(0.33)$ & $21.99(0.16)$ & $23.80(0.97)$ & $22.89(0.35)$ & $23.31(0.65)$ & $23.76(0.91)$ & \\
\hline
\end{tabular}


Table 7. continuation.

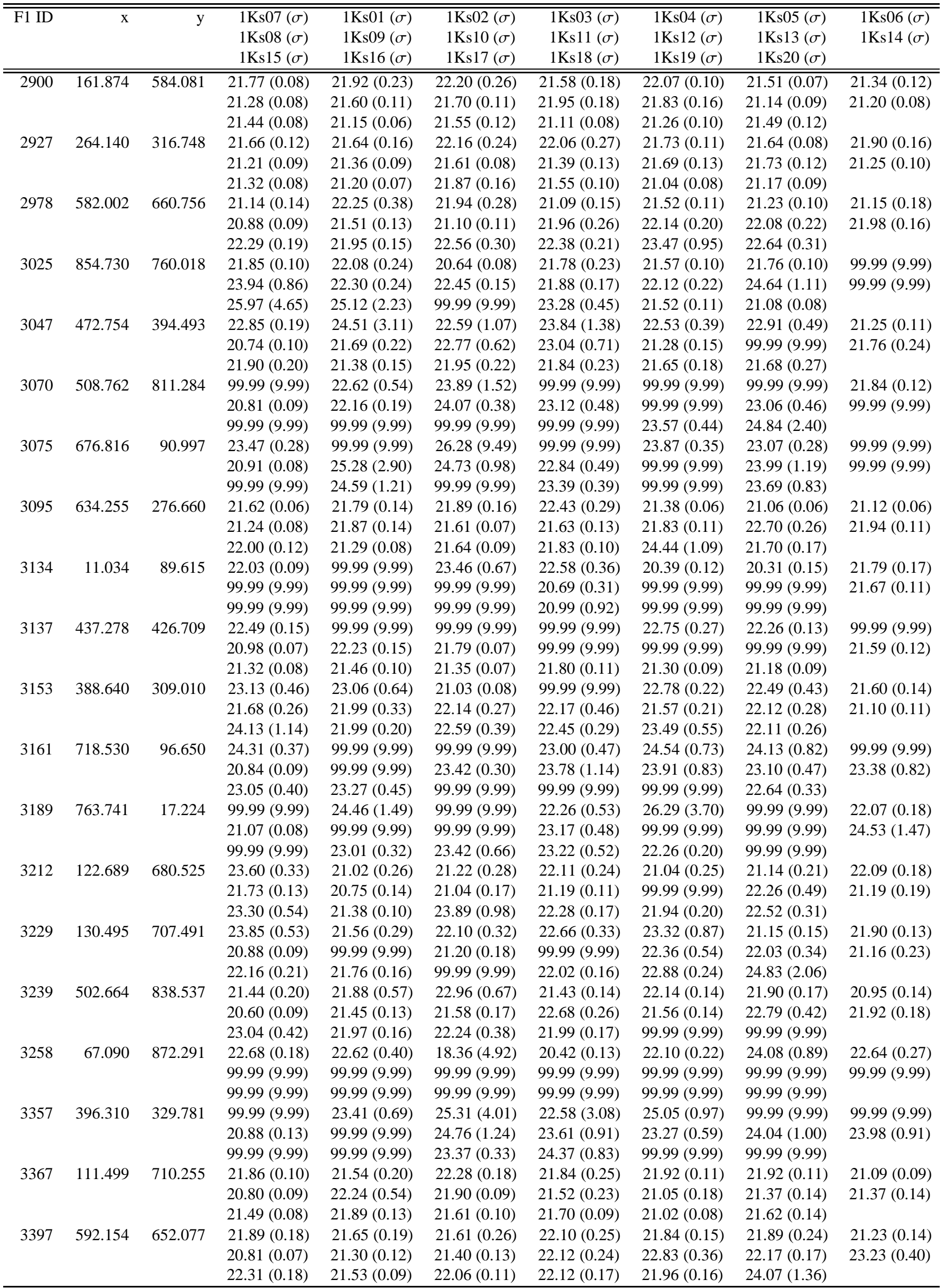


Table 7. continuation.

\begin{tabular}{|c|c|c|c|c|c|c|c|c|c|}
\hline \multirow[t]{3}{*}{ F1 ID } & \multirow[t]{3}{*}{$\mathrm{x}$} & \multirow[t]{3}{*}{$\mathrm{y}$} & $1 \mathrm{Ks} 07(\sigma)$ & $1 \mathrm{Ks} 01(\sigma)$ & $1 \mathrm{Ks} 02(\sigma)$ & $1 \mathrm{Ks} 03(\sigma)$ & $1 \mathrm{Ks} 04(\sigma)$ & $1 \mathrm{Ks} 05(\sigma)$ & \multirow{3}{*}{$\begin{array}{l}1 \mathrm{Ks} 06(\sigma) \\
1 \mathrm{Ks} 14(\sigma)\end{array}$} \\
\hline & & & $1 \mathrm{Ks} 08(\sigma)$ & $1 \mathrm{Ks} 09(\sigma)$ & $1 \mathrm{Ks} 10(\sigma)$ & $1 \mathrm{Ks} 11(\sigma)$ & $1 \mathrm{Ks} 12(\sigma)$ & $1 \mathrm{Ks} 13(\sigma)$ & \\
\hline & & & $1 \mathrm{Ks} 15(\sigma)$ & $1 \mathrm{Ks} 16(\sigma)$ & $1 \mathrm{Ks} 17(\sigma)$ & $1 \mathrm{Ks} 18(\sigma)$ & $1 \mathrm{Ks} 19(\sigma)$ & $1 \mathrm{Ks} 20(\sigma)$ & \\
\hline \multirow[t]{3}{*}{3478} & 345.472 & 220.106 & $21.89(0.06)$ & $21.95(0.19)$ & $21.01(0.12)$ & 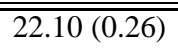 & $21.71(0.07)$ & $21.72(0.08)$ & $25.32(3.17)$ \\
\hline & & & $21.63(0.10)$ & $21.14(0.07)$ & $21.48(0.06)$ & $22.05(0.17)$ & $21.46(0.09)$ & $22.08(0.16)$ & $21.10(0.07)$ \\
\hline & & & $21.44(0.09)$ & $21.52(0.11)$ & $21.39(0.08)$ & $22.04(0.13)$ & $22.01(0.13)$ & $21.60(0.10)$ & \\
\hline \multirow[t]{3}{*}{3481} & 668.202 & 872.628 & $21.89(0.07)$ & $22.92(0.44)$ & $18.95(2.08)$ & $19.90(0.12)$ & $20.31(0.28)$ & $22.70(0.21)$ & $21.99(0.17)$ \\
\hline & & & 99.99 (9.99) & 99.99 (9.99) & 99.99 (9.99) & 99.99 (9.99) & 99.99 (9.99) & 99.99 (9.99) & 99.99 (9.99) \\
\hline & & & 99.99 (9.99) & 99.99 (9.99) & 99.99 (9.99) & 99.99 (9.99) & 99.99 (9.99) & $99.99(9.99)$ & \\
\hline \multirow[t]{3}{*}{3510} & 470.457 & 392.256 & $22.37(0.12)$ & $22.08(0.29)$ & $22.19(0.27)$ & $23.07(0.90)$ & $22.00(0.16)$ & $21.42(0.10)$ & $21.15(0.10)$ \\
\hline & & & $20.95(0.10)$ & $22.24(0.36)$ & $21.32(0.09)$ & 99.99 (9.99) & $22.55(0.39)$ & $21.93(0.28)$ & $21.99(0.29)$ \\
\hline & & & $22.51(0.29)$ & $21.66(0.17)$ & $22.40(0.45)$ & $22.41(0.33)$ & $22.88(0.52)$ & 99.99 (9.99) & \\
\hline \multirow[t]{3}{*}{3515} & 565.427 & 841.839 & $21.72(0.06)$ & $21.24(0.12)$ & $21.94(0.25)$ & $23.35(0.78)$ & $21.97(0.10)$ & $21.94(0.10)$ & $21.83(0.14)$ \\
\hline & & & $21.65(0.11)$ & $21.66(0.11)$ & $21.19(0.07)$ & $21.23(0.11)$ & $21.12(0.07)$ & $21.34(0.10)$ & $21.28(0.09)$ \\
\hline & & & $21.56(0.11)$ & $21.91(0.15)$ & $22.01(0.14)$ & $21.83(0.11)$ & 99.99 (9.99) & 99.99 (9.99) & \\
\hline \multirow[t]{3}{*}{3545} & 899.173 & 0.910 & $99.99(9.99)$ & $21.38(0.12)$ & $99.99(9.99)$ & $99.99(9.99)$ & $23.59(0.49)$ & $22.14(0.15)$ & 99.99 (9.99) \\
\hline & & & $21.64(0.14)$ & $23.29(0.43)$ & $22.70(0.23)$ & $21.46(0.13)$ & $22.07(0.18)$ & $23.23(0.62)$ & 99.99 (9.99) \\
\hline & & & $22.70(0.22)$ & $23.85(0.71)$ & $22.76(0.33)$ & $24.04(0.82)$ & $21.07(0.09)$ & $21.08(0.10)$ & \\
\hline \multirow[t]{3}{*}{3573} & 465.414 & 155.014 & $22.00(0.18)$ & $21.72(0.21)$ & $22.18(0.28)$ & $23.12(0.53)$ & $21.99(0.13)$ & $21.87(0.11)$ & $21.14(0.08)$ \\
\hline & & & $20.85(0.10)$ & $21.87(0.13)$ & $21.72(0.12)$ & $21.42(0.14)$ & 21.81 & $21.72(0.13)$ & $21.43(0.09)$ \\
\hline & & & $21.93(0.16)$ & $21.77(0.10)$ & $21.70(0.12)$ & $21.46(0.10)$ & $21.33(0.11)$ & $21.90(0.17)$ & \\
\hline \multirow[t]{3}{*}{3586} & 51.917 & 654.580 & $21.94(0.09)$ & $21.78(0.26)$ & $23.42(0.67)$ & $22.56(0.38)$ & $21.98(0.08)$ & $22.45(0.18)$ & 99.99 (9.99) \\
\hline & & & $21.34(0.10)$ & $21.61(0.10)$ & $21.65(0.08)$ & $22.69(0.30)$ & $21.66(0.15)$ & $22.11(0.17)$ & $21.65(0.11)$ \\
\hline & & & & $21.33(0.08)$ & $21.53(0.11)$ & $21.67(0.10)$ & $21.03(0.07)$ & $21.28(0.09)$ & \\
\hline \multirow[t]{3}{*}{3609} & 714.557 & 74.640 & $23.91(0.36)$ & $99.99(9.99)$ & $22.85(0.71)$ & $22.31(0.23)$ & $24.71(0.97)$ & $22.97(0.45)$ & $21.03(0.08)$ \\
\hline & & & $21.39(0.10)$ & $22.35(0.28)$ & $24.42(0.74)$ & $22.17(0.25)$ & $21.80(0.12)$ & $24.01(1.06)$ & $21.64(0.12)$ \\
\hline & & & $25.98(6$ & $22.22(0.18)$ & 24.09 (1.35) & $25.93(4.11)$ & $23.39(0.67)$ & $22.47(0.25)$ & \\
\hline 3629 & 869.122 & 765.743 & 22.03 & 99.99 (9.9 & $20.99(0.14)$ & 99.99 (9.99) & $21.97(0.09)$ & $21.84(0.10)$ & 99.99 \\
\hline & & & 9) & 22.98 & 23.05 & 24.11 & 22.77 & $22.90(0.26)$ & 99.99 \\
\hline & & & 99.99 & 99.99 (9.99) & 99.99 (9.99) & $23.99(0.73)$ & 21.57 & $20.90(0.07)$ & \\
\hline 3853 & 378.402 & 296.451 & $22.05(0.08)$ & $22.02(0.28)$ & $22.11(0.29)$ & $21.83(0.25)$ & $21.90(0.10)$ & $21.67(0.12)$ & $21.42(0.14)$ \\
\hline & & & $21.57(0.18)$ & $21.98(0.29)$ & $21.28(0.14)$ & $21.50(0.26)$ & $21.07(0.19)$ & $21.78(0.26)$ & $21.17(0.13)$ \\
\hline & & & & 21.33 & & & & & \\
\hline 3969 & 98.334 & -8.356 & $99.99(9.99)$ & $99.99(9.99)$ & $99.99(9.99)$ & 99.99 & 99.99 & $99.99(9.99)$ & 99.99 (9.99) \\
\hline & & & 99.99 (9.99) & 99.99 (9.99) & $23.39(0.72)$ & $22.87(0.41)$ & $25.63(4.46)$ & 24.44 (1.77) & $23.45(0.80)$ \\
\hline & & & $22.35(0.19)$ & $22.96(0.33)$ & $22.56(0.17)$ & 24.24 (1.19) & $21.20(0.11)$ & $21.29(0.10)$ & \\
\hline 4007 & 49.133 & 540.260 & $20.92(0.17)$ & $22.42(0.32)$ & $21.99(0.20)$ & $21.94(0.26)$ & $22.12(0.14)$ & $21.30(0.10)$ & 22.92 \\
\hline & & & 21.06 & $21.76(0.12)$ & $21.59(0.15)$ & $22.59(0.40)$ & $21.85(0.20)$ & $22.99(0.41)$ & $22.46(0.35)$ \\
\hline & & & $23.14(0.34)$ & $22.12(0.15)$ & $22.50(0.28)$ & $23.33(0.46)$ & $23.48(0.67)$ & $24.00(1.07)$ & \\
\hline 4036 & 681.597 & 88.905 & $25.50(1.60)$ & $23.59(0.78)$ & $23.41(1.01)$ & $22.28(0.21)$ & $26.14(2.03)$ & $24.28(1.11)$ & $21.35(0.15)$ \\
\hline & & & & $21.36(0.20)$ & $23.38(0.33)$ & $22.13(0.28)$ & $22.42(0.25)$ & $23.36(0.61)$ & $21.83(0.14)$ \\
\hline & & & & 99.99 & & 22.67 & 22.17 & $22.92(0.35)$ & \\
\hline 4045 & 486.630 & 836.254 & $23.17(0.27)$ & $25.74(6.42)$ & $99.99(9.99)$ & $24.32(2.41)$ & 24.63 & $23.23(0.30)$ & $22.47(0.40)$ \\
\hline & & & $20.88(0.09)$ & $22.15(0.25)$ & $24.32(0.63)$ & $22.34(0.29)$ & 99.99 (9.99) & 23.96 (1.09) & $23.72(0.88)$ \\
\hline & & & & $23.31(0.40)$ & & $23.71(0.64)$ & $22.79(0.60)$ & 99.99 (9.99) & \\
\hline 4052 & 523.697 & 838.905 & 24.8 & 22.42 & 23.07 & 22.13 & 24.20 & 23.41 & 22.00 \\
\hline & & & $21.05(0.13)$ & $21.56(0.15)$ & $23.49(0.34)$ & $21.15(0.08)$ & $21.64(0.18)$ & $22.04(0.22)$ & $22.23(0.26)$ \\
\hline & & & $23.03(0.40)$ & $22.76(0.42)$ & $23.44(0.50)$ & $23.15(0.35)$ & 99.99 (9.99) & $99.99(9.99)$ & \\
\hline 4088 & 885.361 & 568.917 & $24.47(0.63)$ & 24.41 (1.94) & $99.99(9.99)$ & $22.04(0.25)$ & $23.96(0.34)$ & $24.26(0.67)$ & 99.99 (9.99) \\
\hline & & & $19.90(0.14)$ & & $24.62(1.53)$ & $23.49(0.65)$ & $99.99(9.99)$ & $24.90(2.69)$ & $25.64(3.93)$ \\
\hline & & & $23.07(0.29)$ & $23.62(0.61)$ & $23.08(0.46)$ & & $22.73(0.26)$ & 99.99 (9.99) & \\
\hline 4098 & 103.000 & 615.344 & $22.60(0.13)$ & $22.44(0.32)$ & $99.99(9.99)$ & $99.99(9.99)$ & $23.73(0.47)$ & $22.02(0.10)$ & 99.99 (9.99) \\
\hline & & & $21.22(0.08)$ & $21.80(0.17)$ & $22.28(0.11)$ & $21.54(0.13)$ & $21.21(0.08)$ & $21.32(0.09)$ & $21.88(0.16)$ \\
\hline & & & & $22.02(0.17)$ & 䧑 & $21.81(0.13)$ & $23.28(0.49)$ & $21.94(0.18)$ & \\
\hline 4107 & 507.828 & 816.482 & $24.20(0.52)$ & $21.20(0.25)$ & $21.86(0.30)$ & $23.36(0.86)$ & $22.85(0.19)$ & $22.50(0.19)$ & $21.74(0.14)$ \\
\hline & & & $20.60(0.10)$ & $22.67(0.43)$ & $21.95(0.18)$ & 99.99 (9.99) & 99.99 (9.99) & $22.59(0.39)$ & 99.99 (9.99) \\
\hline & & & $23.02(0.38)$ & $23.55(0.68)$ & $23.92(1.17)$ & $22.05(0.14)$ & $23.76(0.84)$ & $21.91(0.20)$ & \\
\hline 4211 & 519.771 & 822.923 & $22.96(0.27)$ & 99.99 (9.99) & $22.27(0.29)$ & $22.27(0.37)$ & $22.41(0.15)$ & $22.27(0.16)$ & $21.52(0.11)$ \\
\hline & & & & & & & $23.22(0.46)$ & 99.99 (9.99) & $22.38(0.19)$ \\
\hline & & & $22.56(0.30)$ & $22.16(0.24)$ & $22.83(0.25)$ & $23.00(0.49)$ & $22.56(0.24)$ & $22.37(0.32)$ & \\
\hline 4232 & 689.457 & 85.277 & $21.30(0.13)$ & $22.22(0.33)$ & $21.81(0.49)$ & $99.99(9.99)$ & $22.09(0.42)$ & $22.01(0.37)$ & 99.99 (9.99) \\
\hline & & & $21.38(0.13)$ & $21.26(0.10)$ & $21.05(0.14)$ & $22.36(0.26)$ & $22.18(0.19)$ & $22.22(0.18)$ & $22.46(0.20)$ \\
\hline & & & $22.74(0.31)$ & $21.74(0.15)$ & $22.59(0.26)$ & $22.53(0.30)$ & $22.32(0.18)$ & $21.99(0.17)$ & \\
\hline
\end{tabular}


Table 7. continuation.

\begin{tabular}{|c|c|c|c|c|c|c|c|c|c|}
\hline \multirow[t]{3}{*}{ F1 ID } & \multirow[t]{3}{*}{$\mathrm{X}$} & \multirow[t]{3}{*}{$\mathrm{y}$} & $1 \mathrm{Ks} 07(\sigma)$ & 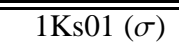 & $1 \mathrm{Ks} 02(\sigma)$ & $1 \mathrm{Ks} 03(\sigma)$ & $1 \mathrm{Ks} 04(\sigma)$ & $1 \mathrm{K \textrm {Ks } 0 5 ( \sigma )}$ & \multirow{3}{*}{$\begin{array}{l}1 \mathrm{Ks} 06(\sigma) \\
1 \mathrm{Ks} 14(\sigma)\end{array}$} \\
\hline & & & $1 \mathrm{Ks} 08(\sigma)$ & $1 \mathrm{Ks} 09(\sigma)$ & $1 \mathrm{Ks} 10(\sigma)$ & $1 \mathrm{Ks} 11(\sigma)$ & $1 \mathrm{Ks} 12(\sigma)$ & $1 \mathrm{Ks} 13(\sigma)$ & \\
\hline & & & $1 \mathrm{Ks} 15(\sigma)$ & $1 \mathrm{Ks} 16(\sigma)$ & $1 \mathrm{Ks} 17(\sigma)$ & $1 \mathrm{Ks} 18(\sigma)$ & $1 \mathrm{Ks} 19(\sigma)$ & $1 \mathrm{Ks} 20(\sigma)$ & \\
\hline \multirow[t]{3}{*}{4237} & 119.598 & 678.105 & $22.40(0.17)$ & $22.09(0.36)$ & $22.51(0.34)$ & $22.21(0.29)$ & $23.01(0.27)$ & $22.66(0.28)$ & $21.50(0.11)$ \\
\hline & & & $21.01(0.08)$ & $20.73(0.20)$ & $22.27(0.10)$ & 99.99 (9.99) & $21.07(0.26)$ & $21.53(0.20)$ & $21.20(0.26)$ \\
\hline & & & $22.18(0.23)$ & $21.94(0.18)$ & $22.18(0.25)$ & $22.23(0.20)$ & $22.30(0.34)$ & $22.20(0.21)$ & \\
\hline \multirow[t]{3}{*}{4291} & 572.597 & 665.165 & $23.96(0.63)$ & $23.27(0.73)$ & $23.54(1.20)$ & $22.65(0.39)$ & $25.75(2.07)$ & $24.00(0.69)$ & $21.53(0.11)$ \\
\hline & & & $20.92(0.09)$ & $21.48(0.12)$ & $24.66(0.92)$ & $22.09(0.22)$ & $21.75(0.13)$ & 99.99 (9.99) & $22.62(0.28)$ \\
\hline & & & $22.48(0.22)$ & $22.21(0.15)$ & 25.91 (4.64) & $22.77(0.20)$ & $24.64(1.88)$ & $22.35(0.17)$ & \\
\hline \multirow[t]{3}{*}{4311} & 97.971 & 334.840 & $22.95(0.17)$ & $22.72(0.39)$ & 99.99 (9.99) & 24.49 (5.59) & $23.77(0.45)$ & $23.40(0.22)$ & $23.03(0.35)$ \\
\hline & & & $20.59(0.09)$ & $23.94(0.73)$ & $22.83(0.25)$ & $23.03(0.58)$ & 99.99 (9.99) & 99.99 (9.99) & $23.54(0.71)$ \\
\hline & & & $23.03(0.36)$ & $26.09(7.63)$ & $23.15(0.38)$ & $23.77(0.70)$ & $22.79(0.33)$ & $23.19(0.55)$ & \\
\hline \multirow[t]{3}{*}{4329} & 502.183 & 846.113 & $24.24(0.91)$ & $25.32(6.15)$ & $24.18(1.56)$ & $22.89(0.44)$ & $23.69(0.38)$ & $23.58(0.36)$ & $21.23(0.10)$ \\
\hline & & & 99.99 (9.99) & 99.99 (9.99) & $23.81(1.17)$ & $23.90(1.12)$ & 99.99 (9.99) & $23.37(0.61)$ & $21.60(0.14)$ \\
\hline & & & $23.53(0.54)$ & $22.28(0.22)$ & $25.10(2.18)$ & 99.99 (9.99) & 99.99 (9.99) & 99.99 (9.99) & \\
\hline \multirow[t]{3}{*}{4374} & 806.535 & 341.542 & $21.89(0.08)$ & $22.05(0.25)$ & $22.24(0.26)$ & $22.11(0.23)$ & $21.97(0.07)$ & $21.98(0.08)$ & 99.99 (9.99) \\
\hline & & & $19.94(0.15)$ & $21.55(0.10)$ & $21.98(0.08)$ & $21.79(0.23)$ & $21.35(0.11)$ & $21.36(0.08)$ & $21.93(0.17)$ \\
\hline & & & $21.56(0.10)$ & $21.91(0.14)$ & $21.89(0.18)$ & $21.72(0.14)$ & $21.39(0.11)$ & $21.44(0.10)$ & \\
\hline \multirow[t]{3}{*}{4512} & 512.404 & 802.990 & $23.51(0.19)$ & $24.63(2.32)$ & $23.97(1.68)$ & $22.74(0.54)$ & 99.99 (9.99) & $24.81(1.14)$ & $24.01(0.85)$ \\
\hline & & & $20.91(0.10)$ & $25.74(5.62)$ & 26.59 (4.58) & $23.41(0.93)$ & $25.94(6.79)$ & $23.88(0.79)$ & $23.82(0.71)$ \\
\hline & & & 99.99 (9.99) & 99.99 (9.99) & $22.78(0.33)$ & $23.47(0.38)$ & $23.61(0.66)$ & $23.39(0.46)$ & \\
\hline \multirow[t]{3}{*}{4586} & 407.657 & 8.980 & $22.57(0.11)$ & $22.36(0.60)$ & 99.99 (9.99) & $21.66(0.41)$ & $22.91(0.58)$ & $22.77(0.47)$ & $21.82(0.16)$ \\
\hline & & & $20.85(0.24)$ & $99.99(9.99)$ & $23.82(2.75)$ & $22.96(1.39)$ & 99.99 (9.99) & $23.04(1.82)$ & $20.66(0.22)$ \\
\hline & & & $22.91(0.34)$ & $22.97(0.78)$ & $22.20(0.34)$ & $23.67(2.31)$ & $20.59(0.13)$ & $21.91(0.43)$ & \\
\hline \multirow[t]{3}{*}{4605} & 258.827 & 118.311 & $22.52(0.13)$ & $21.91(0.20)$ & $22.82(0.25)$ & $22.99(0.38)$ & $21.88(0.07)$ & $22.07(0.13)$ & $21.99(0.15)$ \\
\hline & & & $21.31(0.08)$ & $21.31(0.09)$ & $21.53(0.08)$ & $21.96(0.26)$ & $21.52(0.11)$ & $21.73(0.10)$ & $22.30(0.21)$ \\
\hline & & & $22.47(0.28)$ & $21.98(0.18)$ & $22.35(0.30)$ & $22.29(0.19)$ & $22.30(0.24)$ & $22.35(0.27)$ & \\
\hline 4635 & 441.527 & 143.652 & 99.99 (9.99) & $25.36(4.83)$ & $23.29(0.93)$ & $23.56(1.16)$ & $25.76(1.44)$ & $25.66(3.25)$ & 99.99 \\
\hline & & & $21.16(0.12)$ & 23.05 & 25.06 & 99.99 & 23.23 & $24.42(1.31)$ & $23.75(0.60)$ \\
\hline & & & $23.62(0.43)$ & 99.99 (9.99) & 99.99 (9.99) & $23.46(0.58)$ & 99.99 (9.99) & $23.34(0.53)$ & \\
\hline 4709 & 101.783 & 682.848 & $22.37(0.14)$ & $21.93(0.22)$ & $24.07(0.99)$ & $23.00(0.45)$ & $22.78(0.18)$ & $22.18(0.15)$ & $23.10(0.40)$ \\
\hline & & & $20.71(0.09)$ & $99.99(9.99)$ & $22.09(0.09)$ & $22.08(0.18)$ & $21.92(0.21)$ & $22.24(0.21)$ & $21.40(0.11)$ \\
\hline & & & 21.91 & 21.97 & 21.72 & 22.31 & 22.39 & $21.88(0.19)$ & \\
\hline 4712 & 781.172 & 313.477 & $24.29(0.46)$ & $99.99(9.99)$ & $22.42(0.49)$ & $99.99(9.99)$ & $26.14(2.75)$ & $99.99(9.99)$ & $20.98(0.16)$ \\
\hline & & & $20.45(0.17)$ & $22.68(0.42)$ & $25.16(1.51)$ & $23.06(0.38)$ & $22.79(0.27)$ & $22.87(0.38)$ & $23.68(0.82)$ \\
\hline & & & $99.99(9.99)$ & $24.50(1.13)$ & $23.16(0.41)$ & $23.09(0.38)$ & $23.55(0.64)$ & $23.33(0.60)$ & \\
\hline 4773 & 121.215 & 718.489 & $23.81(0.30)$ & 99.99 (9.99) & $23.01(0.43)$ & $23.07(0.58)$ & $23.34(0.29)$ & $23.10(0.27)$ & 23.39 \\
\hline & & & $20.67(0.10)$ & $24.58(2.17)$ & $22.93(0.32)$ & $23.47(0.75)$ & $22.93(0.44)$ & $23.71(0.74)$ & $24.02(0.94)$ \\
\hline & & & $24.72(1.61)$ & 99.99 (9.99) & $23.51(0.39)$ & $24.49(1.23)$ & $23.22(0.52)$ & $22.27(0.24)$ & \\
\hline 4795 & 424.331 & -17.963 & $99.99(9.99)$ & 99.99 (9.99) & $24.14(1.12)$ & $22.55(0.39)$ & 99.99 (9.99) & 99.99 (9.99) & 99.99 (9.99) \\
\hline & & & $20.49(0.14)$ & $21.74(0.31)$ & $22.97(0.22)$ & $20.95(0.31)$ & $23.57(1.23)$ & $23.45(0.73)$ & $22.38(0.36)$ \\
\hline & & & 23.05 & 22.76 & 24.00 & 22.89 & 22.38 & $23.64(1.36)$ & \\
\hline 4809 & 490.033 & 839.255 & $24.02(0.49)$ & $22.96(0.49)$ & $99.99(9.99)$ & $22.51(0.37)$ & $24.26(0.68)$ & $23.19(0.32)$ & $21.54(0.16)$ \\
\hline & & & $20.70(0.11)$ & $21.88(0.16)$ & 99.99 (9.99) & $22.02(0.23)$ & $22.35(0.17)$ & 99.99 (9.99) & $22.31(0.23)$ \\
\hline & & & $24.12(0.56)$ & $22.89(0.31)$ & $23.13(0.27)$ & $24.23(1.13)$ & 99.99 (9.99) & 99.99 (9.99) & \\
\hline 4846 & 633.993 & 329.664 & $23.27(0.26)$ & $22.70(0.33)$ & $24.35(1.58)$ & $24.57(2.18)$ & 22.68 & $25.73(2.92)$ & 24.03 \\
\hline & & & $21.23(0.07)$ & 99.99 (9.99) & $22.71(0.14)$ & $24.28(1.53)$ & $22.63(0.26)$ & $22.40(0.23)$ & $23.01(0.36)$ \\
\hline & & & $24.32(1.32)$ & $23.66(0.55)$ & 99.99 (9.99) & $23.41(0.60)$ & 99.99 (9.99) & $23.34(0.64)$ & \\
\hline 4856 & 583.757 & 677.830 & $22.71(0.18)$ & $22.55(0.29)$ & $22.29(0.25)$ & $21.27(0.13)$ & $22.71(0.15)$ & $22.58(0.13)$ & $21.58(0.12)$ \\
\hline & & & & $23.16(0.62)$ & $22.84(0.16)$ & $21.98(0.22)$ & $22.62(0.27)$ & $21.79(0.14)$ & $22.11(0.20)$ \\
\hline & & & $22.21(0.17)$ & $22.05(0.17)$ & $22.73(0.26)$ & & $21.97(0.14)$ & $21.92(0.18)$ & \\
\hline 4882 & 835.915 & 342.975 & $24.53(0.38)$ & $23.36(0.77)$ & $20.32(0.16)$ & $99.99(9.99)$ & $23.13(0.28)$ & $22.56(0.24)$ & 99.99 (9.99) \\
\hline & & & $21.08(0.20)$ & 99.99 (9.99) & $22.09(0.19)$ & 99.99 (9.99) & $22.77(0.25)$ & $25.30(2.89)$ & 99.99 (9.99) \\
\hline & & & $24.23(0.99)$ & 99.99 (9.99) & 99.99 (9.99) & 99.99 (9.99) & 99.99 (9.99) & 99.99 (9.99) & \\
\hline 4895 & 526.297 & 825.770 & $22.81(0.17)$ & $22.15(0.26)$ & $24.23(2.61)$ & $21.63(0.21)$ & $23.46(0.31)$ & $23.00(0.20)$ & $22.50(0.33)$ \\
\hline & & & $20.66(0.09)$ & $21.66(0.19)$ & $24.23(0.73)$ & $22.36(0.29)$ & $21.92(0.17)$ & $22.65(0.32)$ & $22.12(0.17)$ \\
\hline & & & 99.99 (9.99) & $22.46(0.26)$ & $23.14(0.38)$ & $24.34(0.85)$ & $22.49(0.23)$ & $23.38(0.62)$ & \\
\hline 4919 & 813.520 & 332.950 & $22.38(0.07)$ & $23.58(0.89)$ & $24.42(2.67)$ & $22.15(0.28)$ & $22.24(0.12)$ & $22.44(0.18)$ & 99.99 (9.99) \\
\hline & & & & $22.22(0.20)$ & $22.39(0.13)$ & $21.23(0.13)$ & $21.06(0.07)$ & $21.97(0.18)$ & $21.53(0.10)$ \\
\hline & & & $22.01(0.17)$ & $21.74(0.15)$ & $22.64(0.29)$ & $22.76(0.18)$ & $22.15(0.23)$ & $22.21(0.21)$ & \\
\hline 4921 & 886.259 & 616.180 & $22.16(0.14)$ & $22.10(0.26)$ & $99.99(9.99)$ & $22.10(0.30)$ & $22.20(0.11)$ & $22.32(0.17)$ & 99.99 (9.99) \\
\hline & & & $21.01(0.07)$ & $21.81(0.11)$ & $22.08(0.09)$ & $22.22(0.27)$ & $22.00(0.14)$ & $22.05(0.16)$ & $22.19(0.22)$ \\
\hline & & & $22.18(0.14)$ & $22.18(0.17)$ & $22.47(0.21)$ & $22.65(0.25)$ & $21.94(0.22)$ & $22.12(0.22)$ & \\
\hline
\end{tabular}


Table 7. continuation.

\begin{tabular}{|c|c|c|c|c|c|c|c|c|c|}
\hline \multirow[t]{3}{*}{ F1 ID } & \multirow[t]{3}{*}{$\mathrm{X}$} & \multirow[t]{3}{*}{$\mathrm{y}$} & $1 \mathrm{Ks} 07(\sigma)$ & $1 \mathrm{Ks} 01(\sigma)$ & $1 \mathrm{Ks} 02(\sigma)$ & $1 \mathrm{Ks} 03(\sigma)$ & $1 \mathrm{Ks} 04(\sigma)$ & $1 \mathrm{Ks} 05(\sigma)$ & \multirow{3}{*}{$\begin{array}{l}1 \mathrm{Ks} 06(\sigma) \\
1 \mathrm{Ks} 14(\sigma)\end{array}$} \\
\hline & & & $1 \mathrm{Ks} 08(\sigma)$ & $1 \mathrm{Ks} 09(\sigma)$ & $1 \mathrm{Ks} 10(\sigma)$ & $1 \mathrm{Ks} 11(\sigma)$ & $1 \mathrm{Ks} 12(\sigma)$ & $1 \mathrm{Ks} 13(\sigma)$ & \\
\hline & & & $1 \mathrm{Ks} 15(\sigma)$ & $1 \mathrm{Ks} 16(\sigma)$ & $1 \mathrm{Ks} 17(\sigma)$ & $1 \mathrm{Ks} 18(\sigma)$ & $1 \mathrm{Ks} 19(\sigma)$ & $1 \mathrm{Ks} 20(\sigma)$ & \\
\hline \multirow[t]{3}{*}{44973} & 106.173 & 705.263 & $25.17(1.28)$ & $222.80(0.43)$ & $22.64(0.34)$ & $222.88(0.71)$ & $22.57(0.20)$ & $222.74(0.36)$ & $21.88(0.22)$ \\
\hline & & & $20.97(0.11)$ & $21.41(0.26)$ & $24.01(0.95)$ & $21.91(0.19)$ & $23.54(1.26)$ & $23.71(0.75)$ & $21.72(0.14)$ \\
\hline & & & $23.09(0.36)$ & $23.30(0.50)$ & 24.34 (1.44) & $22.97(0.28)$ & $21.86(0.19)$ & $23.72(0.91)$ & \\
\hline \multirow[t]{3}{*}{4980} & 388.986 & 69.780 & $22.23(0.20)$ & $22.12(0.24)$ & $22.26(0.31)$ & $23.02(0.56)$ & $22.31(0.13)$ & $22.25(0.17)$ & $21.46(0.09)$ \\
\hline & & & $21.38(0.09)$ & $22.03(0.21)$ & $22.38(0.27)$ & $22.23(0.24)$ & $21.92(0.18)$ & $22.32(0.20)$ & $22.39(0.35)$ \\
\hline & & & $22.58(0.29)$ & $21.58(0.10)$ & $22.10(0.17)$ & $22.19(0.19)$ & $22.15(0.19)$ & $21.92(0.22)$ & \\
\hline \multirow[t]{3}{*}{5054} & 795.501 & 824.086 & $22.31(0.07)$ & $21.66(0.19)$ & $23.51(0.78)$ & $22.36(0.34)$ & $22.31(0.10)$ & $22.45(0.16)$ & 99.99 (9.99) \\
\hline & & & $21.76(0.10)$ & $22.65(0.22)$ & $22.19(0.12)$ & $21.33(0.11)$ & $21.48(0.13)$ & $22.84(0.27)$ & $21.36(0.09)$ \\
\hline & & & $21.52(0.10)$ & $21.50(0.09)$ & $21.49(0.11)$ & $21.83(0.09)$ & $23.40(0.66)$ & $23.15(0.59)$ & \\
\hline \multirow[t]{3}{*}{5113} & 814.431 & 312.754 & $23.65(0.36)$ & $23.46(1.08)$ & $23.67(1.30)$ & 99.99 (9.99) & $24.07(0.52)$ & 99.99 (9.99) & 99.99 (9.99) \\
\hline & & & $20.12(0.16)$ & 99.99 (9.99) & $25.78(2.12)$ & $22.22(0.21)$ & $23.54(0.54)$ & $22.88(0.34)$ & $24.21(1.18)$ \\
\hline & & & 25.34 (1.89) & $23.38(0.42)$ & $23.50(0.63)$ & $23.86(0.84)$ & 99.99 (9.99) & $23.11(0.63)$ & \\
\hline \multirow[t]{3}{*}{5123} & 572.458 & 659.620 & $22.96(0.13)$ & $23.41(0.68)$ & $22.61(0.36)$ & $22.33(0.32)$ & $23.06(0.21)$ & $22.34(0.20)$ & $21.61(0.13)$ \\
\hline & & & $20.81(0.08)$ & $21.94(0.24)$ & $23.40(0.65)$ & $22.70(0.42)$ & $22.08(0.18)$ & $22.00(0.18)$ & $22.18(0.20)$ \\
\hline & & & $23.09(0.43)$ & $22.05(0.14)$ & $22.91(0.35)$ & $22.46(0.21)$ & $22.83(0.35)$ & $22.62(0.30)$ & \\
\hline \multirow[t]{3}{*}{5159} & 104.817 & 678.268 & $24.04(0.34)$ & $22.54(0.30)$ & $23.50(1.15)$ & 99.99 (9.99) & $24.60(0.97)$ & $23.58(0.36)$ & $23.46(0.60)$ \\
\hline & & & 20.98 & 23.27 & 26.02 & 99.99 (9.99) & 22.77 & $23.16(0.48)$ & 99.99 (9.99) \\
\hline & & & $23.48(0.42)$ & $24.20(0.98)$ & $25.30(3.81)$ & $23.45(0.61)$ & $23.47(0.68)$ & $24.57(1.62)$ & \\
\hline \multirow[t]{3}{*}{5225} & 97.012 & 404.027 & $22.25(0.07)$ & $22.03(0.27)$ & $22.90(0.32)$ & $22.77(0.36)$ & $22.18(0.10)$ & $22.52(0.21)$ & $21.69(0.12)$ \\
\hline & & & $21.34(0.13)$ & $22.06(0.15)$ & $22.40(0.29)$ & $21.45(0.15)$ & $21.77(0.24)$ & $22.19(0.22)$ & $22.13(0.20)$ \\
\hline & & & $22.83(0.28)$ & $21.77(0.11)$ & $21.28(0.07)$ & $21.81(0.12)$ & $22.53(0.21)$ & $22.06(0.18)$ & \\
\hline \multirow[t]{3}{*}{5259} & 333.037 & 871.931 & $23.27(0.14)$ & $22.51(0.40)$ & $20.67(0.90)$ & $20.50(0.13)$ & 22.40 & $99.99(9.99)$ & $24.84(1.68)$ \\
\hline & & & 99.99 (9.99) & 99.99 (9.99) & 99.99 (9.99) & 99.99 (9.99) & 99.99 (9. & 99.99 (9.99) & 99.99 (9.99) \\
\hline & & & 99.99 (9.99) & 99.99 (9.99) & 99.99 (9.99) & 99.99 (9.99) & 99.99 (9.99) & 99.99 (9.99) & \\
\hline 5266 & 714.185 & 407.121 & $22.16(0.11)$ & $22.70(0.55)$ & $23.88(1.04)$ & $22.52(0.39)$ & $22.08(0.08)$ & $22.18(0.11)$ & 22.65 \\
\hline & & & 20.58 & 21.70 & 22.15 & 21.86 & 21.93 & $21.76(0.14)$ & 22.15 \\
\hline & & & $22.58(0.25)$ & $22.16(0.16)$ & $22.32(0.16)$ & $22.07(0.13)$ & $21.82(0.16)$ & $22.44(0.24)$ & \\
\hline 5311 & 137.552 & 407.362 & $22.44(0.13)$ & $22.01(0.25)$ & $22.91(0.48)$ & $22.30(0.26)$ & $22.34(0.10)$ & $22.50(0.15)$ & $22.36(0.22)$ \\
\hline & & & $19.38(0.10)$ & $22.18(0.22)$ & $22.26(0.09)$ & $22.26(0.24)$ & $22.76(0.34)$ & $22.51(0.22)$ & $22.37(0.25)$ \\
\hline & & & & 22.17 & 22.70 & & 22.27 & $22.16(0.17)$ & \\
\hline 5333 & 389.254 & 296.955 & $27.50(9.92)$ & $22.24(0.25)$ & $22.57(0.96)$ & 99.99 & 99.99 & 23.76 & $21.99(0.19)$ \\
\hline & & & $20.99(0.13)$ & $21.99(0.17)$ & $23.19(0.27)$ & 23.01 & 21.37 & 99.99 (9.99) & $22.34(0.27)$ \\
\hline & & & $23.86(0.85)$ & $22.48(0.30)$ & $23.17(0.44)$ & $23.77(0.68)$ & $23.54(0.72)$ & $23.13(0.48)$ & \\
\hline 5340 & 346.614 & 200.477 & 22.78 & $22.04(0.23)$ & $23.11(0.48)$ & $22.73(0.36)$ & $23.30(0.31)$ & $22.47(0.14)$ & 24.47 \\
\hline & & & 21.08 & 22.70 & 23.07 & 21.56 & 21.79 & $21.96(0.17)$ & $22.80(0.29)$ \\
\hline & & & 22.63 & 22.73 & $22.78(0.3$ & 99.99 (9.99) & 22.07 & $23.77(0.74)$ & \\
\hline 5341 & 481.378 & 831.793 & $23.06(0.15)$ & $23.10(0.68)$ & $99.99(9.99)$ & $21.95(0.28)$ & $23.17(0.26)$ & $23.03(0.39)$ & (1.04) \\
\hline & & & $20.65(0.09)$ & $24.76(1.71)$ & $23.40(0.35)$ & $25.07(3.35)$ & $23.21(0.59)$ & $99.99(9.99)$ & $23.29(0.40)$ \\
\hline & & & & 23.07 & 22.62 & 22.66 & 24.77 & 99.99 (9.99) & \\
\hline 5419 & 578.391 & 766.093 & $23.86(0.38)$ & $22.26(0.30)$ & $23.00(0.46)$ & $21.56(0.17)$ & $24.48(0.97)$ & $22.78(0.20)$ & 99.99 (9.99) \\
\hline & & & $21.52(0.08)$ & $24.84(1.65)$ & & $25.20(3.95)$ & $24.79(2.17)$ & 99.99 (9.99) & $22.28(0.16)$ \\
\hline & & & & $24.18(0.93)$ & & $22.82(0.29)$ & 99.99 (9.99) & 99.99 (9.99) & \\
\hline 5721 & 670.410 & 206.164 & 23.53 & 99.99 & 23.55 & 22.07 & 99.99 & 99.99 (9. & 21.94 \\
\hline & & & 99.99 (9.99) & $22.66(0.29)$ & $22.60(0.12)$ & $21.19(0.09)$ & $23.61(0.57)$ & 99.99 (9.99) & $22.22(0.21)$ \\
\hline & & & & $24.56(1.20)$ & $23.34(0.48)$ & 99.99 (9.99) & 99.99 (9.99) & 99.99 (9.99) & \\
\hline 5754 & 368.353 & 354.704 & $22.26(0.12)$ & $21.75(0.18)$ & $21.46(0.16)$ & $23.10(0.52)$ & $22.21(0.10)$ & $22.82(0.23)$ & $21.35(0.10)$ \\
\hline & & & & $23.18(0.58)$ & $22.09(0.08)$ & $23.09(0.62)$ & $22.40(0.25)$ & $21.63(0.11)$ & $22.49(0.22)$ \\
\hline & & & & $22.03(0.12)$ & & & $22.94(0.43)$ & $22.28(0.28)$ & \\
\hline 5824 & 507.718 & 803.197 & $23.13(0.20)$ & $22.47(0.40)$ & $22.91(0.43)$ & $23.40(1.13)$ & $22.67(0.17)$ & $23.24(0.27)$ & $23.94(0.92)$ \\
\hline & & & $20.58(0.09)$ & $22.45(0.18)$ & $22.66(0.13)$ & $22.12(0.21)$ & $22.34(0.25)$ & $22.89(0.35)$ & $22.48(0.26)$ \\
\hline & & & & $22.50(0.26)$ & $22.61(0.29)$ & $22.57(0.21)$ & $22.72(0.25)$ & $21.94(0.16)$ & \\
\hline 5897 & 677.238 & 823.391 & $24.16(0.50)$ & $23.55(1.22)$ & $22.87(0.55)$ & $99.99(9.99)$ & $25.14(0.92)$ & $24.78(1.55)$ & 21.85 \\
\hline & & & $21.42(0.09)$ & $22.27(0.15)$ & 99.99 (9.99) & $24.22(1.22)$ & $23.36(0.39)$ & $22.93(0.48)$ & $24.70(1.26)$ \\
\hline & & & $24.82(2.37)$ & $22.66(0.17)$ & $23.99(0.76)$ & $23.16(0.28)$ & $23.33(0.43)$ & $23.93(1.46)$ & \\
\hline 5906 & 513.170 & 810.870 & $22.60(0.19)$ & $22.70(0.35)$ & $22.77(0.30)$ & $23.81(1.38)$ & $22.30(0.12)$ & $22.99(0.25)$ & $22.73(0.27)$ \\
\hline & & & & & & & & $22.86(0.47)$ & 99.99 (9.99) \\
\hline & & & $22.62(0.22)$ & $22.33(0.16)$ & $22.91(0.50)$ & $22.03(0.15)$ & $22.15(0.13)$ & $22.25(0.21)$ & \\
\hline 6180 & 483.320 & 152.424 & $24.06(0.61)$ & $25.94(6.29)$ & $23.94(0.96)$ & $23.15(0.56)$ & $24.99(1.46)$ & $99.99(9.99)$ & $21.44(0.10)$ \\
\hline & & & $21.91(0.19)$ & $22.34(0.26)$ & $23.63(0.38)$ & $22.36(0.30)$ & $22.46(0.21)$ & $22.59(0.41)$ & $22.59(0.22)$ \\
\hline & & & 99.99 (9.99) & $23.85(0.90)$ & $23.82(0.65)$ & $23.52(0.61)$ & $22.37(0.24)$ & $22.86(0.36)$ & \\
\hline
\end{tabular}


Table 7. continuation.

\begin{tabular}{|c|c|c|c|c|c|c|c|c|c|}
\hline \multirow[t]{3}{*}{ F1 ID } & \multirow[t]{3}{*}{$\mathrm{x}$} & \multirow[t]{3}{*}{$\mathrm{y}$} & $1 \mathrm{Ks} 07(\sigma)$ & $1 \mathrm{Ks} 01(\sigma)$ & $1 \mathrm{Ks} 02(\sigma)$ & $1 \mathrm{Ks} 03(\sigma)$ & $1 \mathrm{Ks} 04(\sigma)$ & $1 \mathrm{Ks} 05(\sigma)$ & \multirow{3}{*}{$\begin{array}{l}1 \mathrm{Ks} 06(\sigma) \\
1 \mathrm{Ks} 14(\sigma)\end{array}$} \\
\hline & & & $1 \mathrm{Ks} 08(\sigma)$ & $1 \mathrm{Ks} 09(\sigma)$ & $1 \mathrm{Ks} 10(\sigma)$ & $1 \mathrm{Ks} 11(\sigma)$ & $1 \mathrm{Ks} 12(\sigma)$ & $1 \mathrm{Ks} 13(\sigma)$ & \\
\hline & & & $1 \mathrm{Ks} 15(\sigma)$ & $1 \mathrm{Ks} 16(\sigma)$ & $1 \mathrm{Ks} 17(\sigma)$ & $1 \mathrm{Ks} 18(\sigma)$ & $1 \mathrm{Ks} 19(\sigma)$ & $1 \mathrm{Ks} 20(\sigma)$ & \\
\hline \multirow[t]{3}{*}{6199} & 702.484 & 94.573 & $24.43(0.43)$ & "99.99(9.99) & "99.99(9.99) & $23.78(1.06)$ & "99.99(9.99) & $24.45(1.52)$ & $99.99(9.99)$ \\
\hline & & & $21.46(0.10)$ & $23.03(0.74)$ & $26.64(7.67)$ & $23.66(0.73)$ & $23.41(0.49)$ & $23.45(0.47)$ & $22.33(0.18)$ \\
\hline & & & 99.99 (9.99) & 99.99 (9.99) & $23.43(0.41)$ & $22.49(0.17)$ & $99.99(9.99)$ & 99.99 (9.99) & \\
\hline \multirow[t]{3}{*}{6211} & 754.571 & 18.824 & 99.99 (9.99) & $23.30(0.64)$ & 99.99 (9.99) & $21.52(0.19)$ & $23.60(0.39)$ & $22.96(0.41)$ & $24.74(2.31)$ \\
\hline & & & $21.71(0.09)$ & $22.45(0.25)$ & 99.99 (9.99) & $22.30(0.22)$ & $23.48(0.35)$ & $23.84(0.73)$ & $23.18(0.42)$ \\
\hline & & & 99.99 (9.99) & $22.94(0.28)$ & 24.05 (1.22) & $25.67(3.27)$ & 99.99 (9.99) & 23.37 (0.59) & \\
\hline \multirow[t]{3}{*}{6215} & 234.081 & 100.727 & $22.49(0.13)$ & $21.91(0.18)$ & $22.81(0.46)$ & $23.93(0.99)$ & $22.68(0.13)$ & $22.15(0.10)$ & $22.69(0.20)$ \\
\hline & & & 99.99 (9.99) & $23.62(0.71)$ & $22.76(0.16)$ & $21.67(0.15)$ & $22.78(0.37)$ & $22.42(0.33)$ & $21.64(0.11)$ \\
\hline & & & $23.04(0.38)$ & $23.61(0.55)$ & $22.59(0.30)$ & $22.89(0.28)$ & $21.32(0.09)$ & $21.60(0.15)$ & \\
\hline \multirow[t]{3}{*}{6272} & 604.553 & 665.541 & $25.35(1.78)$ & $22.73(0.35)$ & $24.96(3.96)$ & $22.03(0.20)$ & $24.57(0.69)$ & $23.48(0.40)$ & $21.51(0.13)$ \\
\hline & & & $21.51(0.16)$ & $23.07(C$ & 99.99 (9.99) & $24.43(1.8$ & 22.38 & 25.43 (2.97) & $22.82(0.36)$ \\
\hline & & & $23.96(0$ & 22.78 & 24.61 & 24.47 & 23.89 & 99.99 (9.99) & \\
\hline \multirow[t]{3}{*}{6355} & 530.248 & 822.501 & $22.95(0.19)$ & 99.99 & 99.99 & 99.99 (9.99) & 23.95 & $23.24(0.27)$ & 99.99 (9.99) \\
\hline & & & $21.13(0.11)$ & 99.99 & $23.54(C$ & $22.67(0.40)$ & 99.99 & 99.99 (9.99) & $25.01(2.12)$ \\
\hline & & & $23.45(0.36)$ & $24.32(1.07)$ & 99.99 (9.99) & $23.79(0.53)$ & 99.99 (9.99) & $23.45(0.68)$ & \\
\hline \multirow[t]{3}{*}{6480} & 845.449 & 630.594 & $22.53(0.09)$ & 99.99 (9.99) & 24.16( & $22.82(0.58)$ & 22.51 & $22.38(0.12)$ & 99.99 (9.99) \\
\hline & & & 20.95 & 22.21( & 22.91 & 22.50 & 21.81 & $22.42(0.23)$ & $23.07(0.40)$ \\
\hline & & & 99.99 (9.99) & $22.57(0.29)$ & $23.00(0.24)$ & $22.32(0.17)$ & 22.25 & $22.98(0.63)$ & \\
\hline \multirow[t]{3}{*}{6514} & 521.932 & 816.652 & $22.88(0.17)$ & $22.38(0.35)$ & $23.45(0.61)$ & $22.29(0.36)$ & 22.73 & $22.85(0.22)$ & $21.88(0.16)$ \\
\hline & & & 20.97 & 22.92 & 22.89 & 22.54 & 22.79 & 22.39 & 22.08 \\
\hline & & & 22.09 & 23.00 & 22.68 & 24.15 & 22.28 & 22.50 & \\
\hline \multirow[t]{3}{*}{6516} & 244.538 & 395.868 & $22.51(0.14)$ & 22.54 & 22.92 & 22.06 & 22.27 & 22.17 & $22.75(0.25)$ \\
\hline & & & $21.67(0.11)$ & 23.59 & 22.67 & 23.07 & 22.67 & $21.32(C$ & $23.43(0.45)$ \\
\hline & & & $22.55(0.23)$ & 21.95 & 22.73 & 22.86 & 23.86 & $23.22(C$ & \\
\hline 6540 & 567.744 & 639.884 & 22.73 & 99.99 & 24.02 & 21.93 & 22.67 & 23.42 & $22.52(0.20)$ \\
\hline & & & 20.58 & 23.32 & 22.32 & 23.29 & 21.91 & 23.29 & $22.33(0.25)$ \\
\hline & & & $22.91(0.26)$ & 22.29 & 22.79 & 22.68 & 23.40 & 22.27 & \\
\hline 6607 & 464.875 & 116.291 & $23.29(0.23)$ & $23.59(C$ & 24.57 & $22.64(0.5$ & 23.12 & 23.91 & (9.99) \\
\hline & & & 21.49 & & & & & & $9.99)$ \\
\hline & & & 22.79 & 25.20 & 25.91 & 24.08 & 23.25 & 23.29 & \\
\hline 6647 & 369.172 & 288.477 & $23.38(($ & 23.39 & 23.47 & 22.54 & 23.46 & 24.07 & $22.57(0.40)$ \\
\hline & & & 20.73 & 22.49 & 23.57 & 22.14 & 23.86 & 22.96 & $23.60(0.51)$ \\
\hline & & & 23.21 & 2202 & & & 22.69 & & \\
\hline 6698 & 657.778 & 811.249 & & & 99.99 & & & & $0.31)$ \\
\hline & & & 21.67 & 24.58 & 24.82 & 99.99 & 23.46 & 99.99 & $23.67(0.68)$ \\
\hline & & & 24.93 & 2374 & 22.54 & 24.90 & 22.78 & 23.43 & \\
\hline 6710 & 858.503 & 549.942 & $23.45(0.19)$ & 99.99 & 23.25 & 22.03 & 99.99 & & (9.99) \\
\hline & & & & & & & & & $22.63(0.24)$ \\
\hline & & & 99.99 & 22.27 & 23.20 & 99.99 & 23.37 & 99.99 & \\
\hline 6723 & 349.386 & 314.422 & $22.88(0.16)$ & $22.88(0.50)$ & $24.81(1.70)$ & 24.27 (1.86) & 22.85 & $23.50(0.32)$ & $22.80(0.22)$ \\
\hline & & & & 2343 & $22.76(0.18)$ & & 23.14 & $22.23(0.23)$ & $22.81(0.24)$ \\
\hline & & & & & & & 25.50 & & \\
\hline 6733 & 475.878 & 385.235 & $8(0.12)$ & $22.15(0.18)$ & $23.49(0.72)$ & 23.54 & 24.02 & 24.49 (1.17) & $22.99(0.57)$ \\
\hline & & & 20.55 & 22.63 & 23.03 & 23.43 & 22.56 & 23.43 & $22.42(0.15)$ \\
\hline & & & & $22.75(0.22)$ & $24.90(2.07)$ & $22.03(0.16)$ & 23.91( & $22.20(0.24)$ & \\
\hline 6862 & 857.979 & & $23.07(0.13)$ & $22.53(0.36)$ & $22.57(0.28)$ & $22.45(0.35)$ & 23.70 & $22.98(0.24)$ & 99.99 (9.99) \\
\hline & & & & & & & & & $22.85(0.36)$ \\
\hline & & & $22.79(0.25)$ & $22.45(0.17)$ & $22.99(0.31)$ & $23.21(0.48)$ & $21.96(0.18)$ & $22.52(0.31)$ & \\
\hline 6884 & 131.389 & 352.233 & $22.71(0.13)$ & $23.17(0.51)$ & $22.20(0.32)$ & $21.31(0.12)$ & $22.53(0.16)$ & $23.75(0.64)$ & $23.07(0.49)$ \\
\hline & & & $22.65(0.24)$ & $22.71(0.44)$ & $22.38(0.11)$ & $23.82(0.89)$ & $21.75(0.14)$ & $21.59(0.12)$ & $22.58(0.18)$ \\
\hline & & & & & & & & & \\
\hline 6924 & 711.010 & 92.149 & $23.06(0.18)$ & $22.28(0.30)$ & $22.52(0.35)$ & & $22.96(0.14)$ & & $21.74(0.16)$ \\
\hline & & & $21.44(0.12)$ & $22.39(0.27)$ & $22.41(0.18)$ & $22.71(0.36)$ & $22.31(0.18)$ & $23.23(0.55)$ & $22.41(0.18)$ \\
\hline & & & $22.97(0.37)$ & $22.26(0.24)$ & $22.76(0.35)$ & $22.43(0.15)$ & $21.54(0.10)$ & $22.09(0.16)$ & \\
\hline 6994 & 41.908 & 241.307 & & $99.99(9.99)$ & $22.72(0.35)$ & $22.66(0.51)$ & $22.36(0.16)$ & $23.98(0.75)$ & $21.76(0.13)$ \\
\hline & & & $23.94(0.85)$ & & & & & $22.98(0.36)$ & $22.39(0.25)$ \\
\hline & & & $23.38(0.44)$ & $23.92(0.56)$ & $23.55(0.50)$ & $21.62(0.11)$ & 99.99 (9.99) & $22.43(0.23)$ & \\
\hline 7088 & 442.095 & 401.390 & $22.80(0.13)$ & 99.99 (9.99) & 23.84 (1.25) & $23.03(0.79)$ & $23.27(0.19)$ & $22.36(0.11)$ & $21.18(0.12)$ \\
\hline & & & $20.93(0.12)$ & $21.86(0.25)$ & $22.59(0.09)$ & $21.88(0.35)$ & $22.71(0.41)$ & $22.76(0.42)$ & $22.16(0.18)$ \\
\hline & & & $22.22(0.19)$ & $22.50(0.16)$ & $22.87(0.34)$ & $22.52(0.27)$ & $22.58(0.39)$ & $23.50(0.87)$ & \\
\hline
\end{tabular}


Table 7. continuation.

\begin{tabular}{|c|c|c|c|c|c|c|c|c|c|}
\hline \multirow[t]{3}{*}{ F1 ID } & \multirow[t]{3}{*}{$\mathrm{X}$} & \multirow[t]{3}{*}{$\mathrm{y}$} & $1 \mathrm{Ks} 07(\sigma)$ & $1 \mathrm{Ks} 01(\sigma)$ & $1 \mathrm{Ks} 02(\sigma)$ & $1 \mathrm{Ks} 03(\sigma)$ & $1 \mathrm{Ks} 04(\sigma)$ & $1 \mathrm{Ks} 05(\sigma)$ & \multirow{3}{*}{$\begin{array}{l}1 \mathrm{Ks} 06(\sigma) \\
1 \mathrm{Ks} 14(\sigma)\end{array}$} \\
\hline & & & $1 \mathrm{Ks} 08(\sigma)$ & $1 \mathrm{Ks} 09(\sigma)$ & $1 \mathrm{Ks} 10(\sigma)$ & $1 \mathrm{Ks} 11(\sigma)$ & $1 \mathrm{Ks} 12(\sigma)$ & $1 \mathrm{Ks} 13(\sigma)$ & \\
\hline & & & $1 \mathrm{Ks} 15(\sigma)$ & $1 \mathrm{Ks} 16(\sigma)$ & $1 \mathrm{Ks} 17(\sigma)$ & $1 \mathrm{Ks} 18(\sigma)$ & $1 \mathrm{Ks} 19(\sigma)$ & $1 \mathrm{Ks} 20(\sigma)$ & \\
\hline \multirow[t]{3}{*}{7098} & 567.725 & 6555.871 & $23.39(0.26)$ & $23.10(0.53)$ & $23.10(0.54)$ & 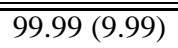 & $23.27(0.27)$ & 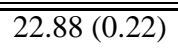 & $22.30(0.26)$ \\
\hline & & & $21.07(0.11)$ & $22.26(0.23)$ & $23.70(0.45)$ & $21.88(0.13)$ & $22.33(0.23)$ & $23.24(0.42)$ & $23.10(0.49)$ \\
\hline & & & $22.48(0.18)$ & $22.85(0.26)$ & $22.45(0.25)$ & $22.84(0.20)$ & $22.31(0.22)$ & $23.91(0.85)$ & \\
\hline \multirow[t]{3}{*}{7165} & 97.672 & 421.263 & $23.22(0.22)$ & $21.91(0.17)$ & $22.91(0.41)$ & $21.49(0.14)$ & $23.12(0.23)$ & $22.73(0.19)$ & $23.88(0.89)$ \\
\hline & & & $22.54(0.44)$ & $22.94(0.39)$ & $24.17(0.59)$ & $21.62(0.13)$ & $22.83(0.38)$ & $22.57(0.30)$ & $22.87(0.28)$ \\
\hline & & & $22.58(0.18)$ & $22.56(0.18)$ & $22.19(0.19)$ & $22.72(0.33)$ & $21.78(0.11)$ & $22.17(0.22)$ & \\
\hline \multirow[t]{3}{*}{7219} & 394.372 & 341.060 & $22.37(0.11)$ & $21.66(0.12)$ & 99.99 (9.99) & 99.99 (9.99) & $23.31(0.30)$ & $22.78(0.17)$ & $22.90(0.33)$ \\
\hline & & & $23.64(0.59)$ & $22.92(0.21)$ & $22.54(0.14)$ & $22.51(0.29)$ & $23.43(0.55)$ & $22.83(0.49)$ & $22.50(0.20)$ \\
\hline & & & $21.94(0.10)$ & $21.79(0.12)$ & $22.68(0.21)$ & $22.70(0.27)$ & $21.82(0.13)$ & $22.46(0.27)$ & \\
\hline \multirow[t]{3}{*}{7248} & 679.430 & 60.764 & $22.90(0.10)$ & $22.67(0.34)$ & $26.01(6.96)$ & $25.50(5.35)$ & $23.75(0.43)$ & $23.54(0.52)$ & 99.99 (9.99) \\
\hline & & & $20.84(0.10)$ & $24.78(1.77)$ & $23.25(0.26)$ & $23.23(0.66)$ & $23.53(0.54)$ & $22.41(0.17)$ & $23.10(0.53)$ \\
\hline & & & $23.32(0.36)$ & $23.80(0.63)$ & 25.39 (3.20) & $24.28(0.87)$ & $22.92(0.39)$ & $23.23(0.57)$ & \\
\hline \multirow[t]{3}{*}{7285} & 819.717 & 203.536 & $24.94(1.07)$ & $99.99(9.99)$ & $24.19(1.41)$ & $23.04(0.52)$ & $26.57(5.28)$ & $23.55(0.47)$ & 99.99 (9.99) \\
\hline & & & $21.34(0.11)$ & $22.76(0.31)$ & $23.23(0.25)$ & $23.55(0.88)$ & 23.99 (1.07) & $22.74(0.36)$ & $23.13(0.36)$ \\
\hline & & & $23.93(0.71)$ & $23.42(0.45)$ & $23.12(0.41)$ & $23.03(0.39)$ & $23.56(0.65)$ & $23.54(0.83)$ & \\
\hline \multirow[t]{3}{*}{7304} & 195.090 & 304.584 & $22.95(0.22)$ & $22.93(0.43)$ & $23.01(0.58)$ & $22.56(0.37)$ & $22.56(0.19)$ & $22.69(0.21)$ & $25.38(2.95)$ \\
\hline & & & $21.73(0.11)$ & $22.68(0.26)$ & $22.77(0.17)$ & 21.91 & 22.74 & 21.99 & $21.74(0.11)$ \\
\hline & & & $22.97(0.39)$ & $22.73(0.26)$ & $22.99(0.35)$ & $22.60(0.23)$ & $22.16(0.18)$ & $22.67(0.30)$ & \\
\hline \multirow[t]{3}{*}{7345} & 119.065 & 839.147 & $22.86(0.12)$ & $22.55(0.49)$ & $22.85(0.54)$ & 99.99 (9.99) & $22.67(0.13)$ & $22.62(0.23)$ & $21.73(0.17)$ \\
\hline & & & $22.19(0.20)$ & $22.97(0.39)$ & $23.33(0.38)$ & $21.94(0.20)$ & $21.87(0.22)$ & $22.24(0.23)$ & $21.75(0.09)$ \\
\hline & & & $23.58(0.77)$ & $22.12(0.17)$ & $23.39(0.48)$ & $23.75(0.91)$ & $21.36(0.62)$ & 99.99 (9.99) & \\
\hline \multirow[t]{3}{*}{7375} & 738.801 & 18.194 & 23.08 & $24.05(1.46)$ & $23.22(0.68)$ & $23.35(0.77)$ & 23.86 & $23.00(0.43)$ & $22.54(0.25)$ \\
\hline & & & $21.60(0.11)$ & $22.01(0.13)$ & $23.58(0.42)$ & $22.52(0.20)$ & $21.85(0.11)$ & $22.56(0.24)$ & $22.38(0.24)$ \\
\hline & & & $22.84(0.31)$ & $22.06(0.17)$ & $24.09(0.84)$ & $22.62(0.30)$ & $24.00(1.05)$ & $22.68(0.32)$ & \\
\hline 7401 & 116.830 & 636.969 & $22.35(0.07)$ & $21.51(0.1$ & $22.81(0.45)$ & $23.07(0.57)$ & $22.81(0.16)$ & $21.91(0.11)$ & 22.41 \\
\hline & & & 22.46 & 23.03 & $22.95(0.23)$ & 22.18 & 23.53 & $21.73(0.11)$ & 21.90 \\
\hline & & & $22.92(0.27)$ & $22.76(0.31)$ & $24.43(1.15)$ & $24.37(1.02)$ & 24.17 (1.08) & $23.15(0.60)$ & \\
\hline 7416 & 474.764 & 416.396 & $23.17(0.15)$ & 99.99 (9.99) & $23.77(1.33)$ & $22.34(0.39)$ & $23.71(0.31)$ & 99.99 (9.99) & $21.68(0.18)$ \\
\hline & & & $22.14(0.36)$ & 99.99 (9.99) & $23.61(0.42)$ & $21.63(0.28)$ & 21.51 (0. & $22.65(0.55)$ & $22.59(0.54)$ \\
\hline & & & & 22.95 & 22.71 & 23.50 & 21.68 & 21.95 & \\
\hline 7432 & 709.054 & 98.738 & $23.19(0.19)$ & $22.37(0.24)$ & $22.37(0.37)$ & 22.81 & 23.84 & 23.27 & $21.95(0.17)$ \\
\hline & & & $21.25(0.11)$ & $23.95(0.79)$ & $23.19(0.28)$ & $22.52(0.31)$ & $22.87(0.3$ & $23.42(0.66)$ & $23.10(0.37)$ \\
\hline & & & $22.98(0.34)$ & $23.12(0.38)$ & $25.30(3.80)$ & $23.12(0.43)$ & $22.24(0.17)$ & $23.67(0.86)$ & \\
\hline 7560 & 671.435 & 407.083 & $23.12(0.18)$ & 99.99 (9.99) & 23.46 & $24.08(1.82)$ & $24.01(0.53)$ & $22.30(0.14)$ & 22.53 \\
\hline & & & 21.35 & 22.54 & $23.04(0.25)$ & $22.07(0.24)$ & 22.86 & 22.82 & $23.16(0.52)$ \\
\hline & & & $24.30(0.78)$ & $22.76(0.29)$ & $23.42(0.63)$ & $22.75(0.34)$ & 22.39 & 22.98 & \\
\hline 7608 & 695.119 & 51.824 & $23.59(0.31)$ & $24.18(1.32)$ & $23.24(0.67)$ & $24.62(2.04)$ & 23.65 & 22.97 (0. & $22.83(0.36)$ \\
\hline & & & & $23.47(0.62)$ & $23.36(0.28)$ & $24.33(1.24)$ & $23.42(0.3$ & $22.68(0.34)$ & $24.31(1.49)$ \\
\hline & & & & 23.65 & & 22.99 & 23.47 & $22.12(0.24)$ & \\
\hline 7630 & 731.075 & 3.369 & $99.99(9.99)$ & $23.27(0.54)$ & $23.52(0.87)$ & $99.99(9.99)$ & $23.01(0$ & 22.87 (0) & $22.70(0.40)$ \\
\hline & & & $23.51(0.55)$ & $23.05(0.41)$ & 24.79 (1.22) & $22.71(0.23)$ & $21.76(0.09)$ & 99.99 (9.99) & $22.83(0.28)$ \\
\hline & & & & $22.68(0.21)$ & $22.67(0.21)$ & $23.35(0.37)$ & $23.96(0.76)$ & $22.98(0.55)$ & \\
\hline 7689 & 532.141 & 813.352 & 22.58 & 22.89 & 23.19 & 22.69 & 22.86 & 22.69 & 22.16 \\
\hline & & & $20.99(0.12)$ & $22.68(0.29)$ & $23.05(0.20)$ & $21.51(0.13)$ & $22.54(0.27)$ & $22.47(0.22)$ & $22.75(0.27)$ \\
\hline & & & $22.81(0.36)$ & $22.47(0.16)$ & $22.26(0.12)$ & $23.17(0.46)$ & $22.58(0.27)$ & $22.77(0.29)$ & \\
\hline 7740 & 751.847 & 22.710 & $23.06(0.21)$ & 99.99 (9.99) & $23.17(0.58)$ & 99.99 (9.99) & $23.00(0.19)$ & $22.92(0.22)$ & $22.69(0.39)$ \\
\hline & & & & & $23.32(0.25)$ & 99.99 (9.99) & $23.29(0.40)$ & $23.58(0.77)$ & $22.72(0.24)$ \\
\hline & & & & & & $23.59(0.72)$ & $23.19(0.39)$ & $24.13(1.33)$ & \\
\hline 7753 & 684.468 & 63.865 & $22.69(0.14)$ & $22.46(0.26)$ & $22.66(0.37)$ & $22.58(0.34)$ & $22.54(0.12)$ & $23.29(0.37)$ & $21.35(0.15)$ \\
\hline & & & $21.23(0.16)$ & $21.83(0.22)$ & $22.66(0.17)$ & $23.82(1.06)$ & $23.20(0.50)$ & $22.86(0.31)$ & $21.82(0.16)$ \\
\hline & & & & $22.73(0.27)$ & 24.35 (1.36) & $22.74(0.21)$ & $23.35(0.67)$ & $22.64(0.25)$ & \\
\hline 7755 & 493.789 & 805.957 & $22.53(0.10)$ & $22.33(0.25)$ & $23.17(0.58)$ & $24.95(4.33)$ & $22.54(0.10)$ & $22.93(0.21)$ & $23.43(0.57)$ \\
\hline & & & $20.98(0.10)$ & $22.56(0.20)$ & $22.58(0.17)$ & $22.83(0.43)$ & $22.95(0.26)$ & $22.65(0.25)$ & $24.03(1.33)$ \\
\hline & & & $22.83(0.20)$ & $24.50(1.53)$ & $23.24(0.50)$ & $23.20(0.44)$ & $23.14(0.34)$ & $22.45(0.23)$ & \\
\hline 7788 & 526.608 & 817.757 & $23.07(0.13)$ & 99.99 (9.99) & $23.08(0.56)$ & $22.97(0.45)$ & $23.41(0.23)$ & $23.16(0.28)$ & $22.80(0.32)$ \\
\hline & & & & & & $25.80(8.61)$ & & $23.08(0.35)$ & $22.60(0.31)$ \\
\hline & & & $23.29(0.35)$ & $23.49(0.64)$ & $22.47(0.18)$ & $22.26(0.13)$ & $23.10(0.37)$ & $23.12(0.48)$ & \\
\hline 7835 & 872.295 & 565.614 & $24.70(0.55)$ & $99.99(9.99)$ & $22.98(0.65)$ & $23.23(0.68)$ & $25.17(1.52)$ & $25.89(4.21)$ & 99.99 (9.99) \\
\hline & & & 99.99 (9.99) & $22.40(0.24)$ & 99.99 (9.99) & $23.39(0.44)$ & $21.81(0.10)$ & $23.54(0.58)$ & $22.85(0.22)$ \\
\hline & & & 99.99 (9.99) & 24.21 (1.19) & 99.99 (9.99) & $23.02(0.27)$ & $23.70(0.77)$ & $23.01(0.25)$ & \\
\hline
\end{tabular}


Table 7. continuation.

\begin{tabular}{|c|c|c|c|c|c|c|c|c|c|}
\hline \multirow[t]{3}{*}{ F1 ID } & \multirow[t]{3}{*}{$\mathrm{X}$} & \multirow[t]{3}{*}{$\mathrm{y}$} & $1 \mathrm{Ks} 07(\sigma)$ & 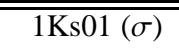 & $1 \mathrm{Ks} 02(\sigma)$ & $1 \mathrm{Ks} 03(\sigma)$ & $1 \mathrm{Ks} 04(\sigma)$ & $1 \mathrm{K \textrm {Ks } 0 5 ( \sigma )}$ & \multirow{3}{*}{$\begin{array}{l}1 \mathrm{Ks} 06(\sigma) \\
1 \mathrm{Ks} 14(\sigma)\end{array}$} \\
\hline & & & $1 \mathrm{Ks} 08(\sigma)$ & $1 \mathrm{Ks} 09(\sigma)$ & $1 \mathrm{Ks} 10(\sigma)$ & $1 \mathrm{Ks} 11(\sigma)$ & $1 \mathrm{Ks} 12(\sigma)$ & $1 \mathrm{Ks} 13(\sigma)$ & \\
\hline & & & $1 \mathrm{Ks} 15(\sigma)$ & $1 \mathrm{Ks} 16(\sigma)$ & $1 \mathrm{Ks} 17(\sigma)$ & $1 \mathrm{Ks} 18(\sigma)$ & $1 \mathrm{Ks} 19(\sigma)$ & $1 \mathrm{Ks} 20(\sigma)$ & \\
\hline \multirow[t]{3}{*}{7843} & 690.220 & $\bar{~} 56.601$ & $24.10(0.58)$ & $24.89(3.60)$ & $22.91(0.41)$ & $22.32(0.31)$ & $23.94(0.48)$ & $23.40(0.34)$ & $23.52(0.70)$ \\
\hline & & & $21.49(0.12)$ & $22.79(0.29)$ & $23.31(0.28)$ & $22.28(0.27)$ & $23.63(0.52)$ & $24.34(1.51)$ & $23.80(0.61)$ \\
\hline & & & $22.93(0.24)$ & $24.30(1.05)$ & $26.07(5.10)$ & $22.82(0.24)$ & $22.04(0.16)$ & $24.32(1.17)$ & \\
\hline \multirow[t]{3}{*}{8005} & 398.664 & 296.704 & $22.96(0.16)$ & $22.82(0.49)$ & $24.48(1.24)$ & $23.99(2.03)$ & $23.18(0.16)$ & $23.07(0.28)$ & $23.06(0.37)$ \\
\hline & & & $20.79(0.12)$ & $22.69(0.26)$ & $23.19(0.30)$ & $22.44(0.31)$ & $23.01(0.43)$ & $22.46(0.21)$ & $22.76(0.27)$ \\
\hline & & & $22.26(0.16)$ & $23.00(0.35)$ & $22.29(0.19)$ & $22.63(0.29)$ & $22.89(0.37)$ & $22.33(0.19)$ & \\
\hline \multirow[t]{3}{*}{8034} & 117.399 & 721.861 & $23.07(0.15)$ & $23.65(1.00)$ & $23.08(0.45)$ & $22.41(0.39)$ & $23.85(0.36)$ & $24.29(0.61)$ & $23.41(0.47)$ \\
\hline & & & $20.87(0.13)$ & 99.99 (9.99) & $23.01(0.26)$ & $23.26(0.67)$ & $22.61(0.25)$ & $23.51(0.63)$ & $25.94(4.36)$ \\
\hline & & & $23.11(0.52)$ & $23.21(0.42)$ & $22.76(0.34)$ & $23.14(0.45)$ & $24.69(1.83)$ & $23.33(0.61)$ & \\
\hline \multirow[t]{3}{*}{8074} & 374.100 & 215.678 & $23.62(0.26)$ & $22.70(0.40)$ & $25.80(6.68)$ & 99.99 (9.99) & $24.41(0.61)$ & $25.52(2.03)$ & $22.88(0.40)$ \\
\hline & & & $99.99(9.99)$ & $22.34(0.29)$ & $23.20(0.36)$ & $22.39(0.32)$ & $22.86(0.41)$ & $99.99(9.99)$ & $21.71(0.11)$ \\
\hline & & & $24.22(0.71)$ & $22.92(0.37)$ & $23.50(0.81)$ & $22.86(0.34)$ & $23.42(0.61)$ & $23.64(0.88)$ & \\
\hline \multirow[t]{3}{*}{8122} & 348.476 & 853.152 & $24.36(0.38)$ & $99.99(9.99)$ & $21.56(0.14)$ & $24.82(2.60)$ & $24.13(0.49)$ & $24.67(0.92)$ & $23.02(0.35)$ \\
\hline & & & 99.99 (9.99) & 99.99 (9.99) & 99.99 (9.99) & 99.99 (9.99) & 99.99 (9.99) & 99.99 (9.99) & $23.87(0.80)$ \\
\hline & & & $24.36(0.78)$ & $23.19(0.35)$ & $23.54(0.97)$ & 99.99 (9.99) & 99.99 (9.99) & 99.99 (9.99) & \\
\hline \multirow[t]{3}{*}{8130} & 683.598 & 819.128 & $23.22(0.15)$ & 99.99 (9.99) & $23.88(1.40)$ & $23.09(0.49)$ & $23.38(0.26)$ & $23.41(0.41)$ & $23.13(0.37)$ \\
\hline & & & $21.55(0.10)$ & $25.19(2.57)$ & $23.09(0.38)$ & $22.53(0.29)$ & 23.70 & $24.16(1.27)$ & $23.72(0.73)$ \\
\hline & & & $22.59(0.24)$ & $22.47(0.23)$ & $23.52(0.68)$ & $22.73(0.24)$ & 99.99 (9.99) & 24.09 (1.08) & \\
\hline \multirow[t]{3}{*}{8151} & 644.569 & 829.938 & $23.01(0.26)$ & $22.46(0.24)$ & 99.99 (9.99) & $21.99(0.27)$ & $22.84(0.14)$ & $23.02(0.21)$ & $22.75(0.21)$ \\
\hline & & & $21.80(0.10)$ & $23.04(0.31)$ & $23.17(0.30)$ & $21.96(0.16)$ & $22.00(0.15)$ & $25.20(3.27)$ & $22.52(0.25)$ \\
\hline & & & $23.75(0.68)$ & $22.73(0.31)$ & $23.23(0.58)$ & $23.83(0.74)$ & $22.95(0.28)$ & 99.99 (9.99) & \\
\hline \multirow[t]{3}{*}{8208} & 578.446 & 642.597 & $23.28(0.24)$ & $23.88(1.02)$ & $23.47(0.76)$ & $21.98(0.21)$ & 23.39 & 23.49 & $21.77(0.17)$ \\
\hline & & & $21.30(0.14)$ & $22.65(0.33)$ & $23.20(0.20)$ & $22.32(0.20)$ & $24.72(2.26)$ & $23.57(0.83)$ & $23.00(0.38)$ \\
\hline & & & $22.69(0.26)$ & $23.23(0.50)$ & $23.37(0.38)$ & $22.64(0.20)$ & $22.67(0.26)$ & 99.99 (9.99) & \\
\hline \multirow[t]{3}{*}{8273} & 500.841 & 239.201 & $23.50(0.24)$ & 99.99 (9. & $23.91(1.40)$ & 99.99 (9.99) & $23.83(0.26)$ & 99.99 (9.99) & 23.95 \\
\hline & & & 21.49 & 99.99 & 24.90 & 22.99 & 23.32 & 99.99 & 99.99 \\
\hline & & & 99.99 & 99.99 & 23.65 & $25.28(2.49)$ & $24.63(1.68)$ & $99.99(9.99)$ & \\
\hline 8319 & 28.685 & 532.373 & $23.02(0.11)$ & $99.99(9.99)$ & $23.59(0.46)$ & $22.46(0.46)$ & $22.65(0.13)$ & $22.86(0.19)$ & $22.72(0.38)$ \\
\hline & & & $21.60(0.17)$ & $21.87(0.17)$ & $22.84(0.20)$ & $22.14(0.28)$ & $22.11(0.36)$ & $22.17(0.20)$ & $21.44(0.18)$ \\
\hline & & & & 22.87 & 99.99 & 22.22 & 21.79 & $22.64(0$. & \\
\hline 8343 & 442.410 & 137.706 & $23.01(0.17)$ & $23.51(0.89)$ & $99.99(9.99)$ & $99.99(9.99)$ & 23.30 & $23.54(0.57)$ & 99.99 (9.99) \\
\hline & & & $21.60(0.11)$ & $22.86(0.27)$ & $23.22(0.38)$ & $22.63(0.39)$ & 24.49 & $22.84(0.32)$ & 99.99 (9.99) \\
\hline & & & $22.84(0.29)$ & $23.45(0.47)$ & $22.24(0.17)$ & $22.95(0.33)$ & $22.51(0.22)$ & $22.84(0.43)$ & \\
\hline 8575 & 455.378 & 425.579 & $23.04(0.18)$ & $23.45(0.91)$ & $23.28(0.56)$ & $23.36(1.00)$ & $23.28(0.25)$ & $23.04(0.23)$ & $23.70(0.80)$ \\
\hline & & & 21.19 & 22.17 & 23.39 & 21.94 & 22.64 & $21.91(0.15)$ & $23.62(0.80)$ \\
\hline & & & $22.76(0.27)$ & $23.22(0.36)$ & 23.25 & $23.17(0.41)$ & 22.18 & $22.38(0.26)$ & \\
\hline 8619 & 59.405 & 546.905 & $23.93(0.27)$ & $99.99(9.99)$ & $23.10(0.47)$ & 99.99 (9.99) & 24.85 (1.19) & $26.05(4.77)$ & $23.40(0.79)$ \\
\hline & & & $21.47(0.17)$ & $25.23(3.52)$ & 99.99 (9.99) & 99.99 (9.99) & $23.12(0.78)$ & $23.47(0.69)$ & $23.57(0.79)$ \\
\hline & & & & 99.99 & 23.27 & & 23.60 & 99.99 & \\
\hline 8796 & 263.491 & 466.644 & $23.45(0.28)$ & $24.80(3.38)$ & $21.69(0.11)$ & $25.46(7.76)$ & $23.97(0.53)$ & $24.04(0.73)$ & $22.63(0.26)$ \\
\hline & & & 99.99 (9.99) & $24.32(1.79)$ & $23.65(0.36)$ & $23.68(0.98)$ & $22.75(0.38)$ & 99.99 (9.99) & $23.33(0.55)$ \\
\hline & & & $23.09(0.34)$ & $23.00(0.29)$ & $23.37(0.64)$ & $23.18(0.31)$ & $23.53(0.61)$ & $23.28(0.57)$ & \\
\hline 8880 & 814.379 & 720.644 & 23.48 & $22.14(0.2$ & $21.84(0.13)$ & 99.99 (9. & $23.20(0.28)$ & $24.11(0.71)$ & 99.99 (9.99) \\
\hline & & & 24.10 & $99.99(9.99)$ & 24.16 & 99.99 (9.99) & 22.96 & $23.37(0.58)$ & $24.12(1.37)$ \\
\hline & & & $24.08(0.76)$ & $23.86(0.70)$ & $23.53(0.61)$ & $23.32(0.48)$ & 99.99 (9.99) & $24.19(1.31)$ & \\
\hline 9271 & 482.027 & 808.044 & $23.20(0.16)$ & $22.78(0.39)$ & $23.45(0.75)$ & $22.24(0.35)$ & $23.19(0.19)$ & $22.85(0.17)$ & $22.18(0.13)$ \\
\hline & & & & $23.64(0.68)$ & $22.90(0.26)$ & $21.99(0.21)$ & $22.23(0.17)$ & $23.91(0.82)$ & $24.46(1.19)$ \\
\hline & & & & $24.02(0.75)$ & $25.04(2.20)$ & $22.12(0.11)$ & 22.56 & 23.75 & \\
\hline 9329 & 696.917 & 96.694 & $23.78(0.21)$ & $23.26(0.62)$ & $24.52(2.73)$ & $23.11(0.61)$ & $99.99(9.99)$ & $23.45(0.37)$ & 99.99 (9.99) \\
\hline & & & 99.99 (9.99) & $22.32(0.38)$ & $23.31(0.29)$ & $23.68(0.76)$ & $24.12(0.90)$ & $24.63(1.40)$ & $21.86(0.11)$ \\
\hline & & & $23.99(0.85)$ & $22.41(0.19)$ & $23.36(0.40)$ & $23.53(0.58)$ & 99.99 (9.99) & $23.33(0.53)$ & \\
\hline 9375 & 870.541 & 519.853 & $23.58(0.22)$ & $23.01(0.46)$ & $22.58(0.34)$ & $24.31(1.83)$ & $24.16(0.49)$ & $24.45(0.85)$ & 99.99 (9.99) \\
\hline & & & $20.85(0.16)$ & $22.89(0.28)$ & $24.23(0.48)$ & $22.91(0.46)$ & 99.99 (9.99) & $22.92(0.35)$ & $23.24(0.48)$ \\
\hline & & & $23.20(0.42)$ & $24.92(1.67)$ & $23.27(0.52)$ & $23.51(0.51)$ & $23.06(0.41)$ & $25.31(3.36)$ & \\
\hline 9627 & 279.827 & 363.194 & $23.28(0.26)$ & $24.83(3.44)$ & $23.65(0.51)$ & $22.67(1.12)$ & $23.75(0.52)$ & $23.93(0.51)$ & $23.74(0.70)$ \\
\hline & & & $23.12(0.32)$ & $22.83(0.31)$ & $22.91(0.20)$ & $22.89(0.54)$ & $23.69(0.93)$ & 99.99 (9.99) & $23.63(0.69)$ \\
\hline & & & $21.91(0.12)$ & $24.00(0.84)$ & $24.03(1.11)$ & $23.53(0.51)$ & $24.95(1.80)$ & $22.47(0.30)$ & \\
\hline
\end{tabular}


Table 7. continuation.

\begin{tabular}{|c|c|c|c|c|c|c|c|c|c|}
\hline \multirow[t]{3}{*}{$\overline{\text { F1 ID }}$} & $\mathrm{x}$ & $\mathrm{y}$ & $1 \mathrm{Ks} 07(\sigma)$ & $1 \mathrm{Ks} 01(\sigma)$ & $1 \mathrm{Ks} 02(\sigma)$ & $1 \mathrm{Ks} 03(\sigma)$ & $1 \mathrm{Ks} 04(\sigma)$ & $1 \mathrm{Ks} 05(\sigma)$ & $1 \mathrm{Ks} 06(\sigma)$ \\
\hline & & & $1 \mathrm{Ks} 08(\sigma)$ & $1 \mathrm{Ks} 09(\sigma)$ & $1 \mathrm{Ks} 10(\sigma)$ & $1 \mathrm{Ks} 11(\sigma)$ & $1 \mathrm{Ks} 12(\sigma)$ & $1 \mathrm{Ks} 13(\sigma)$ & $1 \mathrm{Ks} 14(\sigma)$ \\
\hline & & & $1 \mathrm{Ks} 15(\sigma)$ & $1 \mathrm{Ks} 16(\sigma)$ & $1 \mathrm{Ks} 17(\sigma)$ & $1 \mathrm{Ks} 18(\sigma)$ & $1 \mathrm{Ks} 19(\sigma)$ & $1 \mathrm{Ks} 20(\sigma)$ & \\
\hline \multirow[t]{3}{*}{9672} & 636.995 & 815.458 & $23.90(0.33)$ & $23.36(0.74)$ & $24.41(1.80)$ & $23.08(0.85)$ & $23.61(0.27)$ & $23.51(0.56)$ & $22.79(0.27)$ \\
\hline & & & $21.92(0.12)$ & $23.87(0.91)$ & $23.73(0.37)$ & $22.84(0.50)$ & $24.61(1.43)$ & $22.42(0.23)$ & $24.06(0.87)$ \\
\hline & & & 99.99 (9.99) & $23.56(0.52)$ & $24.39(1.47)$ & $22.98(0.27)$ & $24.29(1.26)$ & 99.99 (9.99) & \\
\hline \multirow[t]{3}{*}{9858} & 356.205 & 81.613 & $24.13(0.48)$ & $22.04(0.12)$ & 99.99 (9.99) & 99.99 (9.99) & $23.26(0.26)$ & $23.37(0.37)$ & $24.41(1.61)$ \\
\hline & & & 99.99 (9.99) & $24.44(0.73)$ & $23.83(0.34)$ & 99.99 (9.99) & 99.99 (9.99) & 99.99 (9.99) & $23.83(0.94)$ \\
\hline & & & 99.99 (9.99) & $24.11(1.13)$ & $23.86(1.23)$ & $23.95(0.89)$ & $23.23(0.50)$ & $23.87(0.99)$ & \\
\hline \multirow[t]{3}{*}{10031} & 242.844 & 65.863 & $23.43(0.23)$ & $22.63(0.43)$ & $24.28(2.24)$ & $23.07(0.58)$ & $25.15(1.39)$ & $24.46(0.93)$ & $23.26(0.45)$ \\
\hline & & & $21.93(0.14)$ & $24.26(1.50)$ & $23.47(0.36)$ & 24.07 (1.49) & $23.75(0.81)$ & $24.52(2.20)$ & $22.50(0.27)$ \\
\hline & & & $23.40(0.52)$ & $24.86(1.55)$ & $23.85(0.96)$ & $23.08(0.44)$ & $23.64(0.75)$ & $23.09(0.44)$ & \\
\hline \multirow[t]{3}{*}{10375} & 623.502 & 566.500 & $23.32(0.25)$ & $23.23(0.64)$ & $24.31(1.54)$ & $22.81(0.54)$ & $23.77(0.28)$ & $23.05(0.12)$ & $23.23(0.30)$ \\
\hline & & & $21.91(0.11)$ & $22.70(0.30)$ & $23.97(0.45)$ & $23.93(1.15)$ & 99.99 (9.99) & $22.70(0.27)$ & $24.84(2.34)$ \\
\hline & & & $23.33(0.39)$ & $23.72(0.65)$ & $23.33(0.50)$ & $23.08(0.48)$ & $22.61(0.27)$ & $23.04(0.38)$ & \\
\hline
\end{tabular}


Table 8. Individual measurements of the K-band magnitudes for all the red variable stars detected in Field 2. The columns from left to right list: star ID, $\mathrm{x}$ and y position with respect to the reference epoch, the reference epoch K-band magnitudes and errors and all the other individual $\mathrm{K}$-band magnitudes and the errors of the measurements as given by ALLFRAME to which 0.04 mag calibration error have been added in quadrature. Magnitude 99.99 and the error 9.99 denote that no measurement for that star was obtained.

\begin{tabular}{|c|c|c|c|c|c|c|c|c|}
\hline \multirow[t]{4}{*}{ F2 ID } & \multirow[t]{4}{*}{$\mathrm{x}$} & \multirow[t]{4}{*}{$\mathrm{y}$} & $2 \mathrm{Ks} 10(\sigma)$ & $2 \mathrm{Ks} 01(\sigma)$ & $2 \mathrm{Ks} 02(\sigma)$ & $2 \mathrm{Ks} 03(\sigma)$ & $2 \mathrm{Ks} 05(\sigma)$ & $2 \mathrm{Ks06}(\sigma)$ \\
\hline & & & $2 \mathrm{Ks} 07(\sigma)$ & $2 \mathrm{Ks} 08(\sigma)$ & $2 \mathrm{Ks} 09(\sigma)$ & $2 \mathrm{Ks} 11(\sigma)$ & $2 \mathrm{Ks} 12(\sigma)$ & $2 \mathrm{Ks} 13(\sigma)$ \\
\hline & & & $2 \mathrm{Ks} 14(\sigma)$ & $2 \mathrm{Ks} 15(\sigma)$ & $2 \mathrm{Ks} 16(\sigma)$ & $2 \mathrm{Ks} 17(\sigma)$ & $2 \mathrm{Ks} 18(\sigma)$ & $2 \mathrm{Ks} 19(\sigma)$ \\
\hline & & & $2 \mathrm{Ks} 20(\sigma)$ & $2 \mathrm{Ks} 21(\sigma)$ & $2 \mathrm{Ks} 22(\sigma)$ & $2 \mathrm{Ks} 23(\sigma)$ & $2 \mathrm{Ks} 24(\sigma)$ & $2 \mathrm{Ks} 04(\sigma)$ \\
\hline \multirow[t]{4}{*}{5} & \multirow[t]{4}{*}{110.132} & \multirow[t]{4}{*}{4451.042} & $14.76(0.04)$ & $15.33(0.14)$ & $\bar{~} 15.04(0.15)$ & $15.00(0.12)$ & "14.66(0.05) & 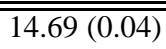 \\
\hline & & & $14.68(0.04)$ & $14.68(0.04)$ & $14.69(0.04)$ & $15.37(0.06)$ & $15.41(0.07)$ & $15.50(0.13)$ \\
\hline & & & $16.69(0.13)$ & $17.02(0.16)$ & $15.97(0.28)$ & $16.08(0.18)$ & 99.99 (9.99) & 99.99 (9.99) \\
\hline & & & 99.99 (9.99) & 99.99 (9.99) & 99.99 (9.99) & 99.99 (9.99) & 99.99 (9.99) & $14.73(0.07)$ \\
\hline \multirow[t]{4}{*}{25} & \multirow[t]{4}{*}{111.583} & \multirow[t]{4}{*}{-16.658} & 99.99 (9.99) & $22.55(0.25)$ & 99.99 (9.99) & $99.99(9.99)$ & 99.99 (9.99) & 99.99 (9.99) \\
\hline & & & 99.99 (9.99) & 99.99 (9.99) & 99.99 (9.99) & $17.57(0.07)$ & $18.26(0.11)$ & $16.74(0.04)$ \\
\hline & & & $18.18(0.21)$ & $17.87(0.11)$ & 99.99 (9.99) & 99.99 (9.99) & $21.48(0.34)$ & $19.79(0.24)$ \\
\hline & & & $20.17(0.09)$ & $21.46(0.18)$ & $18.18(0.16)$ & $19.47(0.09)$ & $19.93(0.10)$ & $19.98(0.22)$ \\
\hline \multirow[t]{4}{*}{35} & \multirow{4}{*}{115.155} & \multirow[t]{4}{*}{-29.046} & 99.99 (9.99) & 99.99 (9.99) & 99.99 (9.99) & 99.99 (9.99) & 99.99 (9.99) & 99.99 (9.99) \\
\hline & & & 99.99 (9.99) & 99.99 (9.99) & 99.99 (9.99) & $17.02(0.06)$ & 99.99 (9.99) & $16.71(0.06)$ \\
\hline & & & $19.02(0.27)$ & $17.75(0.18)$ & $24.37(6.32)$ & 99.99 (9.99) & $22.20(0.49)$ & 99.99 (9.99) \\
\hline & & & $20.37(0.10)$ & $21.65(0.31)$ & $18.49(0.19)$ & 99.99 (9.99) & $21.58(0.34)$ & $19.99(0.23)$ \\
\hline \multirow[t]{4}{*}{49} & \multirow[t]{4}{*}{41.708} & \multirow[t]{4}{*}{172.990} & $17.49(0.31)$ & $17.02(0.34)$ & $17.29(0.24)$ & $16.68(0.20)$ & $17.33(0.20)$ & 99.99 (9.99) \\
\hline & & & $17.85(0$ & $17.24(0$ & $18.00(0$ & 99.99 (9. & $19.18(0.11)$ & 99.99 (9.99) \\
\hline & & & 99.99 (9 & 99.99 & 99.99 & 99.99 (9.99) & 99.99 & 99.99 \\
\hline & & & 99.99 (9.99) & 99.99 (9.99) & 99.99 (9.99) & 99.99 (9.99) & 99.99 (9.99) & $16.21(0.49)$ \\
\hline \multirow[t]{4}{*}{61} & \multirow[t]{4}{*}{591.542} & 445.812 & $18.26(0.04)$ & $18.56(0.04)$ & $18.57(0.05)$ & $18.60(0.05)$ & $18.28(0.04)$ & $18.29(0.04)$ \\
\hline & & & $18.26(0.04)$ & $18.28(0.04)$ & $18.23(0.04)$ & $18.52(0.04)$ & $18.50(0.04)$ & $18.57(0.04)$ \\
\hline & & & $18.34(0.04)$ & $18.31(0.04)$ & $18.23(0.04)$ & $18.21(0.05)$ & 18.25 & $18.21(0.04)$ \\
\hline & & & $18.20(0.04)$ & $18.16(0.04)$ & $18.19(0.04)$ & $18.23(0.04)$ & $18.27(0.04)$ & $18.57(0.06)$ \\
\hline 63 & 133.142 & -27.505 & 99.99 (9.99) & 99.99 (9.99) & 99.99 (9.99) & 99.99 (9.99) & 99.99 (9.99) & 99.99 (9.99) \\
\hline & & & 99.99 (9.99) & 99.99 (9.99) & 99.99 (9.99) & $17.41(0.06)$ & $20.01(0.34)$ & $17.56(0.14)$ \\
\hline & & & $18.28(0.21)$ & $20.15(1.39)$ & 99.99 (9. & $99.99(9$. & 18.39 & $99.99(9.99)$ \\
\hline & & & $20.37(0.09)$ & $20.74(0.18)$ & $19.51(0.33)$ & 99.99 (9.99) & 99.99 (9.99) & $19.35(0.19)$ \\
\hline 64 & 140.349 & -23.112 & 99.99 (9.99) & 99.99 (9.99) & 99.99 (9.99) & 99.99 (9.99) & 99.99 (9.99) & 99.99 (9.99) \\
\hline & & & 99.99 (9.99) & 99.99 (9.99) & 99.99 (9.99) & $17.34(0.06)$ & $19.01(0.15)$ & $22.22(5.15)$ \\
\hline & & & $18.85(0.29)$ & 99.99 (9.99) & 99.99 & 99.99 (9.99) & 99.99 & $99.99(9.99)$ \\
\hline & & & $19.72(0.08)$ & $21.07(0.21)$ & 99.99 (9.99) & $23.52(1.48)$ & $22.17(0.56)$ & $19.14(0.21)$ \\
\hline 69 & 139.234 & -31.121 & 99.99 (9.99) & 99.99 (9.99) & $99.99(9.99)$ & 99.99 (9.99) & 99.99 (9.99) & 99.99 (9.99) \\
\hline & & & $99.99(9.99)$ & 99.99 (9.99) & 99.99 (9.99) & $18.27(0.13)$ & $99.99(9.99)$ & $17.80(0.08)$ \\
\hline & & & $19.00(0.32)$ & $18.80(0.37)$ & 99.99 (9.99) & 99.99 (9.99) & 99.99 (9.99) & $99.99(9.99)$ \\
\hline & & & $21.44(0$ & 21.91 & 19.60 & 99.99 & 99.99 & $20.73(0.32)$ \\
\hline 72 & 122.852 & -33.488 & $99.99(9.99)$ & $99.99(9.99)$ & 99.99 (9.99) & 99.99 (9.99) & 99.99 (9.99) & 99.99 (9.99) \\
\hline & & & 99.99 (9.99) & 99.99 (9.99) & 99.99 (9.99) & 99.99 (9.99) & 99.99 (9.99) & $17.42(0.07)$ \\
\hline & & & 99.99 (9.99) & $17.92(0.16)$ & 99.99 (9.99) & 99.99 (9.99) & 99.99 (9.99) & 99.99 (9.99) \\
\hline & & & $20.88(0.14)$ & $21.23(0.1$ & $19.68(0.31)$ & $21.33(0.22)$ & 99.99 (9.99) & $20.15(0.19)$ \\
\hline 75 & 33.955 & 173.506 & $18.47(0.18)$ & 99.99 (9.99) & $19.46(0.32)$ & 99.99 (9.99) & $17.66(0.09)$ & 99.99 (9.99) \\
\hline & & & $99.99(9.99)$ & $99.99(9.99)$ & $18.67(0.14)$ & $18.25(0.14)$ & $20.29(0.13)$ & $17.98(0.13)$ \\
\hline & & & $20.70(1.38)$ & 99.99 (9.99) & 99.99 (9.99) & 99.99 (9.99) & $19.39(0.44)$ & $19.20(0.21)$ \\
\hline & & & $20.23(0.19)$ & $19.67(0.26)$ & $18.29(0.15)$ & $17.38(0.19)$ & $17.60(0.20)$ & 99.99 (9.99) \\
\hline 76 & 139.031 & -28.038 & 99.99 (9.99) & $99.99(9.99)$ & 99.99 (9.99) & 99.99 (9.99) & 99.99 (9.99) & 99.99 (9.99) \\
\hline & & & 99.99 (9.99) & 99.99 (9.99) & 99.99 (9.99) & $17.88(0.10)$ & $19.06(0.16)$ & 99.99 (9.99) \\
\hline & & & 99.99 (9.99) & $18.63(0.32)$ & $17.30(0.05)$ & 99.99 (9.99) & 99.99 (9.99) & 99.99 (9.99) \\
\hline & & & $20.18(0.09)$ & $20.94(0.07)$ & 99.99 (9.99) & $21.60(0.17)$ & $21.52(0.25)$ & $19.99(0.18)$ \\
\hline 86 & 116.295 & -14.475 & 99.99 (9.99) & $21.74(3.15)$ & $99.99(9.99)$ & 99.99 (9.99) & 99.99 (9.99) & 99.99 (9.99) \\
\hline & & & 99.99 (9.99) & 99.99 (9.99) & 99.99 (9.99) & $17.74(0.11)$ & $18.40(0.08)$ & $17.78(0.10)$ \\
\hline & & & $18.35(0.15)$ & 99.99 (9.99) & $17.48(0.12)$ & 99.99 (9.99) & $18.21(0.14)$ & $19.63(0.29)$ \\
\hline & & & $19.70(0.08)$ & $19.47(0.11)$ & $18.01(0.10)$ & $20.85(0.19)$ & $20.30(0.14)$ & $19.07(0.14)$ \\
\hline
\end{tabular}


Table 8. continuation.

\begin{tabular}{|c|c|c|c|c|c|c|c|c|}
\hline \multirow[t]{4}{*}{$\bar{F}$ F ID } & $\mathrm{x}$ & \multirow[t]{4}{*}{$\mathrm{y}$} & $2 \mathrm{Ks} 10(\sigma)$ & $2 \mathrm{Ks} 01(\sigma)$ & $2 \mathrm{Ks} 02(\sigma)$ & $2 \mathrm{Ks} 03(\sigma)$ & $2 \mathrm{Ks} 05(\sigma)$ & $2 \mathrm{Ks} 06(\sigma)$ \\
\hline & & & $2 \mathrm{Ks} 07(\sigma)$ & $2 \mathrm{Ks} 08(\sigma)$ & $2 \mathrm{Ks} 09(\sigma)$ & $2 \mathrm{Ks} 11(\sigma)$ & $2 \mathrm{Ks} 12(\sigma)$ & $2 \mathrm{Ks} 13(\sigma)$ \\
\hline & & & $2 \mathrm{Ks} 14(\sigma)$ & $2 \mathrm{Ks} 15(\sigma)$ & $2 \mathrm{Ks} 16(\sigma)$ & $2 \mathrm{Ks} 17(\sigma)$ & $2 \mathrm{Ks} 18(\sigma)$ & $2 \mathrm{Ks} 19(\sigma)$ \\
\hline & & & $2 \mathrm{Ks} 20(\sigma)$ & $2 \mathrm{Ks} 21(\sigma)$ & $2 \mathrm{Ks} 22(\sigma)$ & $2 \mathrm{Ks} 23(\sigma)$ & $2 \mathrm{Ks} 24(\sigma)$ & $2 \mathrm{Ks} 04(\sigma)$ \\
\hline \multirow[t]{4}{*}{94} & 143.469 & -15.349 & 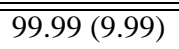 & $21.94(2.01)$ & 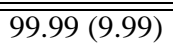 & 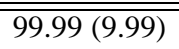 & 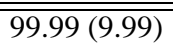 & 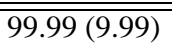 \\
\hline & & & 99.99 (9.99) & $99.99(9.99)$ & 99.99 (9.99) & $19.19(0.30)$ & $20.24(0.38)$ & $22.59(8.01)$ \\
\hline & & & $19.62(0.42)$ & $19.36(1.19)$ & $17.70(0.07)$ & 99.99 (9.99) & 99.99 (9.99) & $19.84(0.38)$ \\
\hline & & & $20.58(0.13)$ & $21.82(0.26)$ & $21.11(1.90)$ & $22.28(0.34)$ & $20.84(0.16)$ & $20.49(0.20)$ \\
\hline \multirow[t]{4}{*}{96} & 259.271 & 269.331 & $19.33(0.14)$ & $20.12(0.43)$ & $19.03(0.12)$ & $18.18(0.18)$ & $18.01(0.08)$ & 99.99 (9.99) \\
\hline & & & $20.13(0.21)$ & $99.99(9.99)$ & $19.01(0.15)$ & $19.01(0.22)$ & $20.38(0.35)$ & $19.03(0.21)$ \\
\hline & & & $20.13(0.30)$ & $99.99(9.99)$ & $18.63(0.13)$ & $20.27(0.35)$ & $19.97(0.26)$ & $21.79(0.67)$ \\
\hline & & & 99.99 (9.99) & $99.99(9.99)$ & $20.48(0.44)$ & $99.99(9.99)$ & 99.99 (9.99) & $18.91(0.29)$ \\
\hline \multirow[t]{4}{*}{98} & 22.966 & 176.951 & $21.94(0.53)$ & $22.26(0.59)$ & $22.21(1.05)$ & 22.75 (3.09) & $21.60(0.15)$ & $16.95(0.07)$ \\
\hline & & & $22.14(0.49)$ & $17.62(0.12)$ & 99.99 (9.99) & $18.13(0.08)$ & $19.95(0.09)$ & $17.79(0.10)$ \\
\hline & & & $19.11(0.24)$ & $20.34(0.92)$ & 99.99 (9.99) & $99.99(9.99)$ & $21.56(0.39)$ & $21.27(0.12)$ \\
\hline & & & $21.22(0.20)$ & $21.40(0.27)$ & $18.90(0.13)$ & $21.86(0.19)$ & $20.62(0.12)$ & $19.79(0.19)$ \\
\hline \multirow[t]{4}{*}{104} & 875.231 & 67.293 & $18.94(0.04)$ & 99.99 (9.99) & $19.27(0.05)$ & $19.25(0.05)$ & $18.51(0.05)$ & $18.68(0.04)$ \\
\hline & & & $18.70(0.04)$ & $18.77(0.04)$ & $18.77(0.05)$ & $19.44(0.04)$ & $19.56(0.05)$ & $19.16(0.05)$ \\
\hline & & & $18.57(0.04)$ & $18.67(0.04)$ & $18.68(0.05)$ & $18.73(0.07)$ & $18.86(0.07)$ & $18.67(0.05)$ \\
\hline & & & $18.92(0.04)$ & $18.90(0.06)$ & $18.87(0.05)$ & $19.01(0.05)$ & $19.23(0.05)$ & $18.67(0.13)$ \\
\hline \multirow[t]{4}{*}{105} & 119.195 & -22.547 & 99.99 (9.99) & 99.99 (9.99) & 99.99 (9.99) & 99.99 (9.99) & 99.99 (9.99) & 99.99 (9.99) \\
\hline & & & 99.99 (9.99) & $99.99(9.99)$ & 99.99 (9.99) & $18.43(0.22)$ & $18.68(0.13)$ & $18.64(0.28)$ \\
\hline & & & $19.21(0.44)$ & $99.99(9.99)$ & 99.99 (9.99) & 99.99 & 17.58 & 19.43 \\
\hline & & & $19.91(0.08)$ & $20.09(0.17)$ & $21.11(1.73)$ & $19.38(0.17)$ & $19.96(0.22)$ & $20.21(0.27)$ \\
\hline \multirow[t]{4}{*}{106} & 34.529 & 166.407 & $18.77(0.14)$ & $18.50(0.17)$ & $18.76(0.17)$ & $17.50(0.08)$ & $18.08(0.09)$ & $17.54(0.12)$ \\
\hline & & & $20.00(0.11)$ & $18.29(0.19)$ & $18.53(0.11)$ & $19.25(0.21)$ & $20.06(0.10)$ & $17.61(0.07)$ \\
\hline & & & 99.99 (9.99) & $99.99(9.99)$ & $18.47(0.26)$ & 99.99 (9.99) & 99.99 (9.99) & $21.48(0.17)$ \\
\hline & & & $20.02(0.06)$ & $20.00(0.20)$ & $19.61(0.25)$ & $20.51(0.21)$ & $20.88(0.24)$ & $20.18(0.23)$ \\
\hline \multirow[t]{4}{*}{110} & 129.337 & -24.781 & 99.99 (9.99) & 99.99 (9.99) & 99.99 (9.99) & 99.99 (9.99) & 99.99 (9.99) & 99.99 (9.99) \\
\hline & & & 99.99 (9.99) & 99.99 (9.99) & 99.99 (9.99) & $17.49(0.11)$ & $18.79(0.13)$ & 99.99 (9.99) \\
\hline & & & 99.99 (9.99) & $99.99(9.99)$ & 99.99 (9.99) & 99.99 (9.99) & 99.99 (9.99) & 99.99 (9.99) \\
\hline & & & $19.71(0.07)$ & $21.55(0.34)$ & 99.99 (9.99) & $17.14(0.19)$ & $17.34(0.19)$ & 99.99 (9.99) \\
\hline \multirow[t]{4}{*}{111} & 586.548 & 279.567 & $19.32(0.06)$ & $19.29(0.08)$ & $19.15(0.06)$ & $19.06(0.06)$ & $18.82(0.05)$ & $18.93(0.05)$ \\
\hline & & & $19.03(0.05)$ & $19.10(0.05)$ & $19.18(0.06)$ & $18.62(0.04)$ & $18.62(0.05)$ & $18.73(0.06)$ \\
\hline & & & $19.17(0.05)$ & $19.25(0.06)$ & $19.34(0.07)$ & $19.36(0.06)$ & $19.43(0.07)$ & $19.33(0.05)$ \\
\hline & & & & $19.43(0.06)$ & $19.21(0.05)$ & $18.99(0.05)$ & $18.80(0.05)$ & $18.65(0.06)$ \\
\hline \multirow[t]{4}{*}{112} & 42.165 & 180.308 & $19.40(0.30)$ & $18.90(0.15)$ & $18.81(0.27)$ & $18.17(0.21)$ & $18.60(0.23)$ & $18.67(0.28)$ \\
\hline & & & $19.83(0.12)$ & $17.29(0.12)$ & $18.34(0.08)$ & $20.42(0.64)$ & $20.45(0.13)$ & 99.99 (9.99) \\
\hline & & & 99.99 (9.99) & $99.99(9.99)$ & $19.39(0.33)$ & 99.99 (9.99) & 99.99 (9.99) & 99.99 (9.99) \\
\hline & & & $20.49(0.13)$ & $20.83(0.44)$ & $20.30(0.38)$ & 99.99 (9.99) & 99.99 (9.99) & $18.25(0.30)$ \\
\hline \multirow[t]{4}{*}{118} & 736.158 & 751.081 & $19.06(0.04)$ & $19.18(0.07)$ & $19.16(0.05)$ & $19.13(0.05)$ & $18.66(0.04)$ & $18.93(0.04)$ \\
\hline & & & $18.81(0.04)$ & $19.00(0.04)$ & $18.92(0.04)$ & $19.31(0.04)$ & $19.05(0.04)$ & $18.78(0.04)$ \\
\hline & & & $18.78(0.04)$ & $18.77(0.04)$ & $18.88(0.05)$ & $19.02(0.08)$ & $19.00(0.05)$ & $19.18(0.05)$ \\
\hline & & & $19.21(0.05)$ & $19.12(0.05)$ & $19.31(0.05)$ & $19.27(0.04)$ & $19.19(0.04)$ & $18.71(0.05)$ \\
\hline \multirow[t]{4}{*}{123} & 255.404 & 276.950 & $18.83(0.08)$ & $19.35(0.29)$ & $19.68(0.20)$ & $19.71(0.29)$ & $18.16(0.11)$ & $20.48(1.23)$ \\
\hline & & & 99.99 (9.99) & $20.49(1.38)$ & $19.07(0.11)$ & $18.99(0.13)$ & $20.08(0.16)$ & $18.56(0.12)$ \\
\hline & & & $21.54(1.29)$ & 99.99 (9.99) & $20.21(0.66)$ & $19.10(0.20)$ & $21.04(0.55)$ & 99.99 (9.99) \\
\hline & & & $20.79(0.52)$ & $21.81(1.08)$ & $19.77(0.13)$ & 99.99 (9.99) & 99.99 (9.99) & $18.85(0.26)$ \\
\hline \multirow[t]{4}{*}{124} & 51.280 & 179.498 & $18.65(0.18)$ & $18.57(0.16)$ & $19.02(0.19)$ & $18.02(0.11)$ & $18.53(0.13)$ & $17.49(0.10)$ \\
\hline & & & $19.96(0.10)$ & $17.78(0.12)$ & $18.50(0.11)$ & $18.94(0.16)$ & $20.29(0.12)$ & $19.42(0.36)$ \\
\hline & & & $18.94(0.14)$ & $18.14(0.13)$ & $17.64(0.09)$ & $19.30(0.33)$ & 99.99 (9.99) & $20.01(0.14)$ \\
\hline & & & $20.36(0.10)$ & $20.02(0.20)$ & $19.33(0.21)$ & $22.04(0.25)$ & $20.21(0.08)$ & $19.43(0.18)$ \\
\hline
\end{tabular}


Table 8. continuation.

\begin{tabular}{|c|c|c|c|c|c|c|c|c|}
\hline \multirow[t]{4}{*}{$\bar{F}$ F ID } & \multirow[t]{4}{*}{$\mathrm{x}$} & \multirow[t]{4}{*}{$\mathrm{y}$} & $2 \mathrm{Ks} 10(\sigma)$ & $2 \mathrm{Ks} 01(\sigma)$ & $2 \mathrm{Ks} 02(\sigma)$ & $2 \mathrm{Ks} 03(\sigma)$ & $2 \mathrm{Ks} 05(\sigma)$ & $2 \mathrm{Ks} 06(\sigma)$ \\
\hline & & & $2 \mathrm{Ks} 07(\sigma)$ & $2 \mathrm{Ks} 08(\sigma)$ & $2 \mathrm{Ks} 09(\sigma)$ & $2 \mathrm{Ks} 11(\sigma)$ & $2 \mathrm{Ks} 12(\sigma)$ & $2 \mathrm{Ks} 13(\sigma)$ \\
\hline & & & $2 \mathrm{Ks} 14(\sigma)$ & $2 \mathrm{Ks} 15(\sigma)$ & $2 \mathrm{Ks} 16(\sigma)$ & $2 \mathrm{Ks} 17(\sigma)$ & $2 \mathrm{Ks} 18(\sigma)$ & $2 \mathrm{Ks} 19(\sigma)$ \\
\hline & & & $2 \mathrm{Ks} 20(\sigma)$ & $2 \mathrm{Ks} 21(\sigma)$ & $2 \mathrm{Ks} 22(\sigma)$ & $2 \mathrm{Ks} 23(\sigma)$ & $2 \mathrm{Ks} 24(\sigma)$ & $2 \mathrm{Ks} 04(\sigma)$ \\
\hline \multirow[t]{4}{*}{131} & 116.799 & -19.307 & $99.99(9.99)$ & 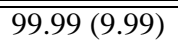 & $99.99(9.99)$ & $999.99(9.99)$ & $\bar{~} 99.99(9.99)$ & $\overline{999.99(9.99)}$ \\
\hline & & & 99.99 (9.99) & 99.99 (9.99) & 99.99 (9.99) & 99.99 (9.99) & $18.31(0.08)$ & 99.99 (9.99) \\
\hline & & & $17.91(0.14)$ & $20.24(1.08)$ & $19.13(0.16)$ & 99.99 (9.99) & 99.99 (9.99) & 99.99 (9.99) \\
\hline & & & $20.13(0.07)$ & $19.95(0.21)$ & $18.19(0.10)$ & $21.84(0.55)$ & $21.34(0.34)$ & $20.13(0.21)$ \\
\hline \multirow[t]{4}{*}{137} & 802.342 & 660.341 & $19.29(0.05)$ & $19.43(0.07)$ & $19.28(0.06)$ & $19.13(0.06)$ & $19.21(0.07)$ & $19.25(0.05)$ \\
\hline & & & $19.21(0.06)$ & $19.26(0.06)$ & $19.30(0.06)$ & $19.12(0.05)$ & $19.18(0.05)$ & $19.16(0.06)$ \\
\hline & & & $18.91(0.05)$ & $18.85(0.05)$ & $18.91(0.06)$ & $18.92(0.07)$ & $18.96(0.05)$ & $18.95(0.05)$ \\
\hline & & & $18.95(0.05)$ & $18.99(0.05)$ & $19.05(0.05)$ & $19.06(0.05)$ & $19.20(0.05)$ & $19.21(0.08)$ \\
\hline \multirow[t]{4}{*}{139} & 122.165 & -28.415 & 99.99 (9.99) & 99.99 (9.99) & 99.99 (9.99) & 99.99 (9.99) & 99.99 (9.99) & 99.99 (9.99) \\
\hline & & & 99.99 (9.99) & 99.99 (9.99) & 99.99 (9.99) & 99.99 (9.99) & $19.98(0.32)$ & $21.18(2.37)$ \\
\hline & & & 99.99 (9.99) & $18.17(0.26)$ & 17.50 & 99.99 (9.99) & 99.99 (9.99) & $99.99(9.99)$ \\
\hline & & & $20.91(0.11)$ & $21.28(0.17)$ & $19.73(0.51)$ & $20.71(0.20)$ & $20.49(0.15)$ & $20.06(0.25)$ \\
\hline \multirow[t]{4}{*}{140} & 500.951 & 593.051 & $19.13(0.04)$ & $19.08(0.06)$ & $19.22(0.05)$ & $19.46(0.05)$ & $19.70(0.04)$ & $19.38(0.05)$ \\
\hline & & & $19.25(0.04)$ & $19.24(0.05)$ & $19.16(0$. & $19.79(0.05)$ & $19.78(0$ & $19.75(0.05)$ \\
\hline & & & $19.09(0.04)$ & $19.03(0.04)$ & $18.94(C$ & 18.84 & 18.84 & 18.84 \\
\hline & & & $18.78(0.04)$ & 18.71 & $18.76(0.04)$ & 18.84 & 18.91 & 19.86 \\
\hline \multirow[t]{4}{*}{141} & 789.272 & 224.574 & $19.33(0.04)$ & $19.12(0.05)$ & $18.92(0.05)$ & $18.84(0.04)$ & $19.25(0.04)$ & $19.22(0.05)$ \\
\hline & & & $19.26(0.04)$ & $19.34(0.05)$ & $19.25(0.04)$ & $19.06(0.04)$ & $19.21(0.05)$ & $19.29(0.04)$ \\
\hline & & & $19.22(0$ & 19.28 & 19.26 & 19.24 & 19.28 & 19.06 \\
\hline & & & $18.96(0.04)$ & 19.00 & 18.89 & 18.75 & 18.76 & $19.37(0.09)$ \\
\hline \multirow[t]{4}{*}{142} & 145.486 & -19.245 & 99.99 (9.99) & $22.63(1.15)$ & 99.99 (9.99) & 99.99 (9.99) & 99.99 (9.99) & 99.99 (9.99) \\
\hline & & & 99.99 (9.99) & 99.99 (9.99) & 99.99 (9.99) & $17.75(0.10)$ & $18.55(0.13)$ & 99.99 (9.99) \\
\hline & & & $18.09(0.13)$ & 99.99 (9.99) & 99.99 (9.99) & 99.99 (9.99) & $21.59(0.75)$ & $18.93(0.13)$ \\
\hline & & & $20.02(0.11)$ & $21.61(0.26)$ & 20.09 & $22.02(0.27)$ & 20.54 & $19.72(0.22)$ \\
\hline \multirow[t]{4}{*}{143} & 613.780 & 471.722 & $18.77(0.04)$ & $19.14(0.05)$ & $19.35(0.05)$ & $19.55(0.05)$ & $18.58(0.05)$ & $18.66(0.04)$ \\
\hline & & & $18.67(0.04)$ & $18.72(0.04)$ & $18.67(0.04)$ & $19.62(0.04)$ & $19.66(0.05)$ & $19.32(0.04)$ \\
\hline & & & $19.02(0.04)$ & $19.12(0.04)$ & $19.23(0.05)$ & $19.37(0.05)$ & $19.53(0.05)$ & $19.62(0.05)$ \\
\hline & & & $19.76(0$ & 19.68 & 19.87 & 19.91 & 19.76 & 18.96 \\
\hline \multirow[t]{4}{*}{146} & 271.826 & 268.954 & $19.02(0.15)$ & $18.73(0.16)$ & $19.09(0.15)$ & $18.23(0.12)$ & $18.07(0.13)$ & $18.09(0.13)$ \\
\hline & & & $19.51(0.16)$ & $18.46(0.25)$ & $18.73(0.12)$ & $18.51(0.10)$ & $19.82(0.13)$ & $18.82(0.16)$ \\
\hline & & & $19.86(0.29)$ & $19.10(0.17)$ & $18.65(0.18)$ & $19.92(0.34)$ & $19.56(0.09)$ & $19.91(0.13)$ \\
\hline & & & & & & $21.79(0.66)$ & 21.50 & $19.28(0.21)$ \\
\hline \multirow[t]{4}{*}{148} & 850.071 & -34.921 & & & & 99.99 (9.99) & 99.99 (9.99) & $99.99(9.99)$ \\
\hline & & & 99.99 (9.99) & 99.99 (9.99) & 99.99 (9.99) & $19.10(0.04)$ & $19.34(0.05)$ & $19.47(0.05)$ \\
\hline & & & $19.47(0.28)$ & $19.54(0.05)$ & $19.63(0.07)$ & 99.99 (9.99) & 99.99 (9.99) & 99.99 (9.99) \\
\hline & & & & & 18.86 & & & \\
\hline \multirow[t]{4}{*}{151} & 272.611 & 281.772 & $19.28(0.19)$ & $18.98(0.17)$ & $18.83(0.14)$ & $18.45(0.14)$ & $18.43(0.13)$ & $18.59(0.16)$ \\
\hline & & & $19.12(0.13)$ & $19.62(0.63)$ & $18.62(0.13)$ & $18.52(0.11)$ & $19.31(0.09)$ & $18.56(0.12)$ \\
\hline & & & $19.05(0.12)$ & $19.24(0.14)$ & $20.29(0.77)$ & $19.21(0.09)$ & $19.72(0.23)$ & $19.58(0.13)$ \\
\hline & & & & $19.98(0.26)$ & $19.07(0.12)$ & $20.11(0.10)$ & $19.91(0.10)$ & $19.05(0.19)$ \\
\hline \multirow[t]{4}{*}{154} & 834.447 & 691.914 & $18.97(0.05)$ & $19.04(0.07)$ & $19.12(0.05)$ & $19.22(0.06)$ & $18.94(0.06)$ & $18.91(0.05)$ \\
\hline & & & $18.87(0.05)$ & $19.10(0.05)$ & $18.90(0.06)$ & $19.39(0.05)$ & $19.39(0.05)$ & $19.20(0.06)$ \\
\hline & & & $19.28(0.05)$ & $19.32(0.06)$ & $19.40(0.08)$ & $19.52(0.11)$ & $19.49(0.08)$ & $19.38(0.06)$ \\
\hline & & & $19.24(0.05)$ & $19.45(0.06)$ & $19.31(0.06)$ & $19.20(0.05)$ & $19.30(0.05)$ & $19.18(0.08)$ \\
\hline \multirow[t]{4}{*}{156} & 679.925 & 778.012 & $19.73(0.05)$ & $19.47(0.07)$ & $19.42(0.05)$ & $19.15(0.05)$ & $19.74(0.05)$ & $19.71(0.05)$ \\
\hline & & & $19.64(0.05)$ & $19.70(0.05)$ & $19.74(0.06)$ & $19.38(0.05)$ & $19.52(0.05)$ & $19.75(0.05)$ \\
\hline & & & $19.38(0.05)$ & $19.23(0.05)$ & $19.13(0.05)$ & $19.09(0.05)$ & $18.91(0.04)$ & $18.98(0.04)$ \\
\hline & & & $18.90(0.04)$ & $18.85(0.05)$ & $18.88(0.04)$ & $18.86(0.04)$ & $18.95(0.04)$ & $19.72(0.10)$ \\
\hline
\end{tabular}


Table 8. continuation.

\begin{tabular}{|c|c|c|c|c|c|c|c|c|}
\hline \multirow[t]{4}{*}{$\overline{\mathrm{F} 2 \mathrm{ID}}$} & \multirow[t]{4}{*}{$\mathrm{x}$} & \multirow[t]{4}{*}{$\mathrm{y}$} & $2 \mathrm{Ks} 10(\sigma)$ & $2 \mathrm{Ks} 01(\sigma)$ & $2 \mathrm{Ks} 02(\sigma)$ & $2 \mathrm{Ks} 03(\sigma)$ & $2 \mathrm{Ks} 05(\sigma)$ & $\overline{2 \mathrm{Ks} 06(\sigma)}$ \\
\hline & & & $2 \mathrm{Ks} 07(\sigma)$ & $2 \mathrm{Ks} 08(\sigma)$ & $2 \mathrm{Ks} 09(\sigma)$ & $2 \mathrm{Ks} 11(\sigma)$ & $2 \mathrm{Ks} 12(\sigma)$ & $2 \mathrm{Ks} 13(\sigma)$ \\
\hline & & & $2 \mathrm{Ks} 14(\sigma)$ & $2 \mathrm{Ks} 15(\sigma)$ & $2 \mathrm{Ks} 16(\sigma)$ & $2 \mathrm{Ks} 17(\sigma)$ & $2 \mathrm{Ks} 18(\sigma)$ & $2 \mathrm{Ks} 19(\sigma)$ \\
\hline & & & $2 \mathrm{Ks} 20(\sigma)$ & $2 \mathrm{Ks} 21(\sigma)$ & $2 \mathrm{Ks} 22(\sigma)$ & $2 \mathrm{Ks} 23(\sigma)$ & $2 \mathrm{Ks} 24(\sigma)$ & $2 \mathrm{Ks} 04(\sigma)$ \\
\hline \multirow[t]{4}{*}{157} & 257.797 & $\overline{-11.193}$ & $99.99(9.99)$ & $19.80(0.06)$ & $999.99(9.99)$ & $99.99(9.99)$ & $19.16(3.17)$ & 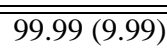 \\
\hline & & & 99.99 (9.99) & 99.99 (9.99) & 99.99 (9.99) & $19.14(0.06)$ & $19.26(0.06)$ & $19.68(0.08)$ \\
\hline & & & $19.51(0.07)$ & $19.32(0.07)$ & $19.54(0.08)$ & $99.99(9.99)$ & $19.27(0.07)$ & $19.05(0.06)$ \\
\hline & & & $18.96(0.06)$ & $19.22(0.07)$ & $18.95(0.06)$ & $18.87(0.05)$ & $18.93(0.05)$ & $19.51(0.17)$ \\
\hline \multirow[t]{4}{*}{162} & 66.212 & 384.706 & $18.99(0.06)$ & $18.79(0.10)$ & $18.84(0.07)$ & $18.82(0.07)$ & $19.00(0.07)$ & $18.96(0.05)$ \\
\hline & & & $18.94(0.05)$ & $18.94(0.05)$ & $18.91(0.05)$ & $19.15(0.04)$ & $19.08(0.05)$ & $19.07(0.05)$ \\
\hline & & & $19.18(0.04)$ & $19.18(0.04)$ & $19.36(0.04)$ & $19.54(0.05)$ & $19.26(0.04)$ & $19.26(0.05)$ \\
\hline & & & $19.19(0.05)$ & $19.33(0.05)$ & $19.27(0.05)$ & $19.35(0.05)$ & $19.30(0.05)$ & $18.81(0.08)$ \\
\hline \multirow[t]{4}{*}{163} & 45.964 & 179.276 & $19.26(0.25)$ & $19.27(0.34)$ & $19.07(0.18)$ & $18.44(0.16)$ & $19.31(0.31)$ & $18.59(0.30)$ \\
\hline & & & $20.15(0.15)$ & $18.16(0.21)$ & $18.78(0.16)$ & $18.55(0.11)$ & $20.33(0.11)$ & $19.43(0.58)$ \\
\hline & & & $18.74(0.14)$ & 99.99 (9.99) & $18.03(0.15)$ & 99.99 (9.99) & $19.90(0.39)$ & $22.56(0.78)$ \\
\hline & & & $22.38(0.53)$ & $20.58(0.20)$ & $19.32(0.14)$ & 99.99 (9.99) & $99.99(9.99)$ & $19.62(0.49)$ \\
\hline \multirow[t]{4}{*}{164} & 744.544 & 648.632 & $18.96(0.04)$ & $19.35(0.05)$ & $19.57(0.06)$ & $19.64(0.06)$ & $18.81(0.04)$ & $18.87(0.04)$ \\
\hline & & & $18.79(0.05)$ & $18.91(0.05)$ & $18.86(0.04)$ & $19.73(0.05)$ & $19.47(0.05)$ & $19.14(0.05)$ \\
\hline & & & $19.11(0.04)$ & $19.18(0.04)$ & $19.32(0.05)$ & $19.41(0.06)$ & $19.49(0.04)$ & $19.54(0.05)$ \\
\hline & & & $19.72(0.06)$ & $19.64(0.05)$ & $19.74(0.05)$ & $19.57(0.05)$ & $19.64(0.05)$ & $19.08(0.06)$ \\
\hline \multirow[t]{4}{*}{166} & 538.063 & 121.359 & $19.57(0.04)$ & $19.42(0.05)$ & $19.27(0.06)$ & $19.18(0.05)$ & $19.41(0.04)$ & $19.57(0.04)$ \\
\hline & & & $19.45(0.05)$ & $19.35(0.05)$ & $19.48(0.04)$ & $19.41(0.05)$ & $19.51(0.05)$ & $19.42(0.04)$ \\
\hline & & & $19.05(0.04)$ & $18.96(0.04)$ & $18.96(0.05)$ & $18.94(0.04)$ & $18.96(0.04)$ & $18.87(0.04)$ \\
\hline & & & $19.00(0.04)$ & $18.96(0.04)$ & $19.02(0.04)$ & $19.09(0.04)$ & $19.23(0.04)$ & $19.61(0.06)$ \\
\hline \multirow[t]{4}{*}{167} & 679.250 & 59.629 & $19.48(0.06)$ & $19.62(0.06)$ & $19.62(0.07)$ & $19.54(0.06)$ & $19.54(0.07)$ & $19.56(0.06)$ \\
\hline & & & $19.65(0.07)$ & $19.39(0.06)$ & $19.55(0.07)$ & $18.95(0.05)$ & $19.13(0.05)$ & $19.32(0.06)$ \\
\hline & & & $19.29(0.06)$ & $19.45(0.06)$ & $19.66(0.07)$ & $19.47(0.07)$ & $19.51(0.07)$ & $19.17(0.05)$ \\
\hline & & & $19.08(0.05)$ & $19.27(0.06)$ & $18.97(0.05)$ & $18.86(0.05)$ & $18.86(0.05)$ & $19.19(0.07)$ \\
\hline \multirow[t]{4}{*}{169} & 298.943 & 642.226 & $19.13(0.04)$ & $19.67(0.06)$ & $19.75(0.05)$ & $19.70(0.06)$ & $18.78(0.04)$ & $18.92(0.04)$ \\
\hline & & & $18.86(0.04)$ & $18.94(0.05)$ & $18.96(0.04)$ & $19.45(0.04)$ & $19.19(0.04)$ & $19.00(0.04)$ \\
\hline & & & $19.29(0.05)$ & $19.39(0.04)$ & $19.36(0.04)$ & $19.40(0.05)$ & $19.45(0.04)$ & $19.51(0.04)$ \\
\hline & & & $19.38(0.05)$ & $19.40(0.05)$ & $19.35(0.04)$ & $19.36(0.05)$ & $19.31(0.05)$ & $18.97(0.07)$ \\
\hline \multirow[t]{4}{*}{173} & 66.678 & 695.443 & $19.38(0.05)$ & $19.62(0.05)$ & $19.76(0.05)$ & $19.73(0.06)$ & $19.47(0.05)$ & $19.58(0.05)$ \\
\hline & & & $19.63(0.05)$ & $19.54(0.05)$ & $19.54(0.05)$ & $18.65(0.04)$ & $18.68(0.04)$ & $18.82(0.04)$ \\
\hline & & & $19.38(0.05)$ & $19.43(0.05)$ & $19.35(0.04)$ & $19.37(0.06)$ & $19.39(0.06)$ & $19.42(0.05)$ \\
\hline & & & $19.53(0.05)$ & $19.46(0.05)$ & $19.51(0.05)$ & $19.37(0.05)$ & $19.12(0.05)$ & $19.37(0.18)$ \\
\hline \multirow[t]{4}{*}{176} & 147.165 & -14.406 & $99.99(9.99)$ & $99.99(9.99)$ & $99.99(9.99)$ & 99.99 (9.99) & $99.99(9.99)$ & $99.99(9.99)$ \\
\hline & & & 99.99 (9.99) & 99.99 (9.99) & 99.99 (9.99) & $17.79(0.11)$ & $18.14(0.10)$ & $21.14(0.77)$ \\
\hline & & & $19.00(0.21)$ & $18.99(0.30)$ & 99.99 (9.99) & 99.99 (9.99) & $21.49(0.19)$ & $19.68(0.22)$ \\
\hline & & & $20.93(0.22)$ & 99.99 (9.99) & $18.51(0.16)$ & $21.24(0.18)$ & $20.35(0.13)$ & $20.39(0.15)$ \\
\hline \multirow[t]{4}{*}{177} & 526.510 & 8.394 & $19.66(0.06)$ & $19.16(0.07)$ & $19.11(0.05)$ & $19.01(0.05)$ & $19.58(0.07)$ & $19.76(0.06)$ \\
\hline & & & $19.66(0.06)$ & $19.62(0.06)$ & $19.64(0.08)$ & $19.13(0.05)$ & $19.25(0.05)$ & $19.38(0.07)$ \\
\hline & & & $19.53(0.05)$ & $19.50(0.05)$ & $19.45(0.08)$ & $19.38(0.06)$ & $19.37(0.06)$ & $19.16(0.05)$ \\
\hline & & & $19.14(0.05)$ & $19.30(0.05)$ & $19.08(0.05)$ & $19.02(0.04)$ & $18.96(0.05)$ & $19.48(0.10)$ \\
\hline \multirow[t]{4}{*}{178} & 687.346 & 575.031 & $19.68(0.04)$ & $18.94(0.05)$ & $18.82(0.05)$ & $18.80(0.04)$ & $19.37(0.04)$ & $19.61(0.05)$ \\
\hline & & & $19.62(0.05)$ & $19.82(0.05)$ & $19.69(0.05)$ & $18.67(0.04)$ & $18.59(0.04)$ & $18.51(0.04)$ \\
\hline & & & $19.38(0.05)$ & $19.43(0.04)$ & $19.54(0.04)$ & $19.67(0.05)$ & $19.86(0.05)$ & $20.04(0.05)$ \\
\hline & & & $20.27(0.06)$ & $20.11(0.07)$ & $20.21(0.05)$ & $20.33(0.05)$ & $20.27(0.06)$ & $19.15(0.09)$ \\
\hline \multirow[t]{4}{*}{182} & 206.565 & 721.171 & $19.23(0.04)$ & $19.43(0.05)$ & $19.26(0.05)$ & $19.23(0.05)$ & $18.74(0.04)$ & $18.93(0.04)$ \\
\hline & & & $18.93(0.04)$ & $19.01(0.04)$ & $19.01(0.04)$ & $19.13(0.04)$ & $19.03(0.04)$ & $18.84(0.04)$ \\
\hline & & & $19.39(0.05)$ & $19.55(0.04)$ & $19.62(0.04)$ & $19.69(0.04)$ & $19.75(0.05)$ & $19.82(0.05)$ \\
\hline & & & $19.56(0.05)$ & $19.58(0.05)$ & $19.42(0.04)$ & $19.36(0.04)$ & $19.33(0.04)$ & $18.79(0.06)$ \\
\hline
\end{tabular}


Table 8. continuation.

\begin{tabular}{|c|c|c|c|c|c|c|c|c|}
\hline \multirow[t]{4}{*}{$\overline{\mathrm{F} 2 \mathrm{ID}}$} & \multirow[t]{4}{*}{$\mathrm{x}$} & \multirow[t]{4}{*}{$\mathrm{y}$} & $2 \mathrm{Ks} 10(\sigma)$ & $2 \mathrm{Ks} 01(\sigma)$ & $2 \mathrm{Ks} 02(\sigma)$ & $2 \mathrm{Ks} 03(\sigma)$ & $2 \mathrm{Ks} 05(\sigma)$ & $2 \mathrm{Ks06}(\sigma)$ \\
\hline & & & $2 \mathrm{Ks} 07(\sigma)$ & $2 \mathrm{Ks} 08(\sigma)$ & $2 \mathrm{Ks} 09(\sigma)$ & $2 \mathrm{Ks} 11(\sigma)$ & $2 \mathrm{Ks} 12(\sigma)$ & $2 \mathrm{Ks} 13(\sigma)$ \\
\hline & & & $2 \mathrm{Ks} 14(\sigma)$ & $2 \mathrm{Ks} 15(\sigma)$ & $2 \mathrm{Ks} 16(\sigma)$ & $2 \mathrm{Ks} 17(\sigma)$ & $2 \mathrm{Ks} 18(\sigma)$ & $2 \mathrm{Ks} 19(\sigma)$ \\
\hline & & & $2 \mathrm{Ks} 20(\sigma)$ & $2 \mathrm{Ks} 21(\sigma)$ & $2 \mathrm{Ks} 22(\sigma)$ & $2 \mathrm{Ks} 23(\sigma)$ & $2 \mathrm{Ks} 24(\sigma)$ & $2 \mathrm{Ks} 04(\sigma)$ \\
\hline \multirow[t]{4}{*}{$\overline{183}$} & 118.156 & 302.625 & $19.15(0.05)$ & $19.58(0.06)$ & $19.58(0.07)$ & $19.70(0.06)$ & $19.40(0.06)$ & 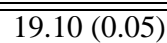 \\
\hline & & & $19.22(0.05)$ & $19.03(0.05)$ & $19.16(0.05)$ & $19.83(0.07)$ & $19.86(0.07)$ & $20.14(0.10)$ \\
\hline & & & $19.24(0.05)$ & $19.16(0.05)$ & $19.25(0.06)$ & $19.18(0.06)$ & $19.13(0.06)$ & $19.08(0.05)$ \\
\hline & & & $19.08(0.05)$ & $19.18(0.05)$ & $19.19(0.05)$ & $19.22(0.05)$ & $19.31(0.05)$ & $19.64(0.09)$ \\
\hline \multirow[t]{4}{*}{192} & 276.007 & 276.599 & $19.06(0.13)$ & $19.29(0.17)$ & $19.08(0.16)$ & $19.34(0.18)$ & $18.29(0.12)$ & 99.99 (9.99) \\
\hline & & & $19.48(0.15)$ & 99.99 (9.99) & $19.07(0.12)$ & $18.45(0.09)$ & $19.86(0.17)$ & 99.99 (9.99) \\
\hline & & & $19.77(0.25)$ & $19.62(0.18)$ & 99.99 (9.99) & $19.67(0.19)$ & $20.26(0.27)$ & $19.52(0.14)$ \\
\hline & & & $19.09(0.13)$ & $20.00(0.19)$ & $23.59(7.65)$ & $21.31(0.20)$ & $20.84(0.15)$ & $19.29(0.22)$ \\
\hline \multirow[t]{4}{*}{193} & 140.127 & -4.647 & 99.99 (9.99) & $22.52(0.30)$ & 99.99 (9.99) & 99.99 (9.99) & $21.39(0.12)$ & $20.53(0.45)$ \\
\hline & & & $99.99(9.99)$ & 99.99 (9.99) & 99.99 (9.99) & $18.21(0.11)$ & $19.25(0.22)$ & $20.16(0.43)$ \\
\hline & & & $17.84(0.10)$ & $19.90(0.74)$ & $21.41(0.21)$ & 99.99 (9.99) & $21.14(0.13)$ & $19.03(0.16)$ \\
\hline & & & $20.88(0.10)$ & $21.04(0.14)$ & $17.35(0.07)$ & $20.25(0.17)$ & $20.11(0.09)$ & $20.04(0.21)$ \\
\hline \multirow[t]{4}{*}{194} & 587.021 & 98.805 & $19.05(0.04)$ & $19.42(0.05)$ & $19.60(0.05)$ & $19.77(0.05)$ & $18.79(0.04)$ & $18.94(0.04)$ \\
\hline & & & $18.86(0.04)$ & $18.94(0.04)$ & $18.89(0.04)$ & $19.65(0.05)$ & $19.65(0.05)$ & $19.26(0.04)$ \\
\hline & & & $19.20(0.04)$ & $19.32(0.04)$ & $19.53(0.04)$ & $19.65(0.05)$ & $19.76(0.05)$ & $19.73(0.05)$ \\
\hline & & & $19.79(0.05)$ & $19.84(0.05)$ & $19.71(0.05)$ & $19.62(0.05)$ & $19.45(0.05)$ & $18.99(0.06)$ \\
\hline \multirow[t]{4}{*}{197} & 100.756 & 704.343 & $19.55(0.05)$ & $19.75(0.08)$ & $19.84(0.06)$ & $19.71(0.07)$ & $19.61(0.05)$ & $19.60(0.05)$ \\
\hline & & & $19.64(0.06)$ & $19.49(0.06)$ & $19.62(0.06)$ & $19.09(0.05)$ & $19.23(0.05)$ & $19.41(0.05)$ \\
\hline & & & $19.48(0.05)$ & $19.42(0.05)$ & $19.28(0.06)$ & $19.20(0.06)$ & $19.10(0.06)$ & $19.04(0.05)$ \\
\hline & & & $18.93(0.05)$ & $19.06(0.05)$ & $18.98(0.05)$ & $19.02(0.05)$ & $19.05(0.05)$ & $19.47(0.15)$ \\
\hline \multirow[t]{4}{*}{199} & 229.905 & 503.969 & $19.71(0.05)$ & $19.38(0.07)$ & $19.13(0.06)$ & $19.11(0.05)$ & $19.06(0.05)$ & $19.28(0.04)$ \\
\hline & & & $19.31(0.05)$ & $19.55(0.05)$ & $19.49(0.05)$ & $18.98(0.04)$ & $18.98(0.04)$ & $19.03(0.05)$ \\
\hline & & & $19.56(0.05)$ & $19.47(0.05)$ & $19.40(0.05)$ & $19.30(0.05)$ & $19.33(0.05)$ & $19.34(0.05)$ \\
\hline & & & $19.34(0.05)$ & $19.39(0.05)$ & $19.32(0.05)$ & $19.36(0.05)$ & $19.13(0.05)$ & $18.81(0.08)$ \\
\hline \multirow[t]{4}{*}{200} & 249.412 & 273.123 & $20.39(0.23)$ & $22.17(2.54)$ & $19.34(0.20)$ & $22.58(6.25)$ & $19.24(0.22)$ & 17.71 \\
\hline & & & $19.63(0.11)$ & 99.99 (9.99) & $19.75(0.20)$ & 99.99 (9.99) & $19.69(0.10)$ & 99.99 (9.99) \\
\hline & & & $19.80(0.18)$ & $20.16(0.42)$ & $20.45(0.73)$ & $19.29(0.22)$ & $20.22(0.22)$ & $20.98(0.24)$ \\
\hline & & & 99.99 (9.99) & 99.99 (9.99) & $20.05(0.29)$ & 99.99 (9.99) & $21.21(0.18)$ & $19.64(0.21)$ \\
\hline \multirow[t]{4}{*}{202} & 47.977 & 170.614 & $20.69(0.42)$ & $18.66(0.18)$ & $18.43(0.14)$ & $18.01(0.12)$ & $18.35(0.09)$ & 99.99 \\
\hline & & & $20.13(0.10)$ & 99.99 (9.99) & $18.79(0.14)$ & $18.55(0.12)$ & $20.05(0.08)$ & $19.82(2.02)$ \\
\hline & & & $19.51(0.21)$ & 99.99 (9.99) & $18.30(0.13)$ & $19.53(0.27)$ & 99.99 (9.99) & $22.11(0.33)$ \\
\hline & & & $20.92(0.15)$ & $20.59(0.24)$ & 99.99 (9.99) & $24.26(3.88)$ & $21.31(0.25)$ & $20.02(0.32)$ \\
\hline \multirow[t]{4}{*}{203} & 48.521 & 165.258 & $18.57(0.14)$ & $18.44(0.17)$ & $18.95(0.14)$ & $18.12(0.12)$ & $17.78(0.10)$ & $17.73(0.19)$ \\
\hline & & & $20.17(0.10)$ & $19.82(1.25)$ & $18.30(0.11)$ & $18.25(0.10)$ & $20.15(0.10)$ & $18.52(0.26)$ \\
\hline & & & $20.74(0.72)$ & $18.72(0.24)$ & $18.26(0.18)$ & $19.34(0.27)$ & $18.75(0.17)$ & $20.65(0.10)$ \\
\hline & & & $20.79(0.15)$ & $19.76(0.17)$ & 99.99 (9.99) & $21.58(0.16)$ & $20.32(0.09)$ & $19.78(0.16)$ \\
\hline \multirow[t]{4}{*}{204} & 832.484 & 675.812 & $19.11(0.05)$ & $19.06(0.07)$ & $19.20(0.05)$ & $19.31(0.06)$ & $19.26(0.05)$ & $19.19(0.05)$ \\
\hline & & & $19.12(0.05)$ & $19.05(0.05)$ & $19.10(0.05)$ & $19.41(0.05)$ & $19.53(0.06)$ & $19.47(0.06)$ \\
\hline & & & $19.13(0.05)$ & $19.14(0.05)$ & $19.32(0.06)$ & $19.51(0.10)$ & $19.55(0.08)$ & $19.53(0.06)$ \\
\hline & & & $19.53(0.06)$ & $19.66(0.06)$ & $19.75(0.06)$ & $19.82(0.06)$ & $19.80(0.07)$ & $19.65(0.09)$ \\
\hline \multirow[t]{4}{*}{207} & 245.433 & 114.678 & $19.34(0.05)$ & $18.98(0.07)$ & $18.90(0.05)$ & $18.97(0.05)$ & $19.53(0.06)$ & $19.60(0.05)$ \\
\hline & & & $19.65(0.06)$ & $19.63(0.05)$ & $19.68(0.06)$ & $19.71(0.05)$ & $19.77(0.05)$ & $19.81(0.06)$ \\
\hline & & & $19.19(0.05)$ & $19.07(0.05)$ & $19.20(0.06)$ & $19.12(0.07)$ & $19.09(0.06)$ & $19.06(0.05)$ \\
\hline & & & $19.04(0.04)$ & $19.12(0.05)$ & $19.09(0.05)$ & $19.13(0.04)$ & $19.15(0.05)$ & $19.36(0.05)$ \\
\hline \multirow[t]{4}{*}{208} & 691.197 & 210.117 & $19.48(0.05)$ & $19.32(0.06)$ & $19.18(0.05)$ & $19.05(0.05)$ & $19.54(0.05)$ & $19.60(0.05)$ \\
\hline & & & $19.63(0.05)$ & $19.58(0.05)$ & $19.56(0.05)$ & $19.66(0.05)$ & $19.62(0.05)$ & $19.49(0.05)$ \\
\hline & & & $19.08(0.05)$ & $19.07(0.05)$ & $19.11(0.06)$ & $19.12(0.04)$ & $19.16(0.05)$ & $19.09(0.04)$ \\
\hline & & & $19.14(0.04)$ & $19.20(0.05)$ & $19.19(0.04)$ & $19.20(0.05)$ & $19.25(0.05)$ & $19.64(0.09)$ \\
\hline
\end{tabular}


Table 8. continuation.

\begin{tabular}{|c|c|c|c|c|c|c|c|c|}
\hline \multirow[t]{4}{*}{$\bar{F}$ F2 ID } & \multirow[t]{4}{*}{$\mathrm{x}$} & \multirow[t]{4}{*}{$\mathrm{y}$} & $2 \mathrm{Ks} 10(\sigma)$ & $2 \mathrm{Ks} 01(\sigma)$ & $2 \mathrm{Ks} 02(\sigma)$ & $2 \mathrm{Ks} 03(\sigma)$ & $2 \mathrm{Ks} 05(\sigma)$ & $\overline{2 \mathrm{Ks} 06(\sigma)}$ \\
\hline & & & $2 \mathrm{Ks} 07(\sigma)$ & $2 \mathrm{Ks} 08(\sigma)$ & $2 \mathrm{Ks} 09(\sigma)$ & $2 \mathrm{Ks} 11(\sigma)$ & $2 \mathrm{Ks} 12(\sigma)$ & $2 \mathrm{Ks} 13(\sigma)$ \\
\hline & & & $2 \mathrm{Ks} 14(\sigma)$ & $2 \mathrm{Ks} 15(\sigma)$ & $2 \mathrm{Ks} 16(\sigma)$ & $2 \mathrm{Ks} 17(\sigma)$ & $2 \mathrm{Ks} 18(\sigma)$ & $2 \mathrm{Ks} 19(\sigma)$ \\
\hline & & & $2 \mathrm{Ks} 20(\sigma)$ & $2 \mathrm{Ks} 21(\sigma)$ & $2 \mathrm{Ks} 22(\sigma)$ & $2 \mathrm{Ks} 23(\sigma)$ & $2 \mathrm{Ks} 24(\sigma)$ & $2 \mathrm{Ks} 04(\sigma)$ \\
\hline \multirow[t]{4}{*}{210} & $\overline{789.583}$ & 543.906 & $18.96(0.04)$ & $19.11(0.05)$ & $\overline{19.28(0.05)}$ & $19.42(0.05)$ & $19.11(0.04)$ & 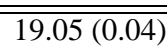 \\
\hline & & & $18.98(0.05)$ & $19.10(0.05)$ & $18.97(0.04)$ & $19.57(0.05)$ & $19.54(0.05)$ & $19.42(0.04)$ \\
\hline & & & $19.27(0.04)$ & $19.32(0.04)$ & $19.36(0.04)$ & $19.48(0.05)$ & $19.56(0.05)$ & $19.62(0.04)$ \\
\hline & & & $19.63(0.05)$ & $19.62(0.05)$ & $19.64(0.05)$ & $19.58(0.05)$ & $19.51(0.05)$ & $19.61(0.07)$ \\
\hline \multirow[t]{4}{*}{214} & 60.696 & 435.243 & $19.64(0.04)$ & $18.77(0.06)$ & $18.67(0.05)$ & $18.76(0.05)$ & $19.72(0.05)$ & $19.91(0.06)$ \\
\hline & & & $19.66(0.05)$ & $19.92(0.06)$ & $19.58(0.05)$ & $19.13(0.04)$ & $19.18(0.05)$ & $19.74(0.06)$ \\
\hline & & & $20.53(0.06)$ & $20.38(0.05)$ & $20.21(0.05)$ & $19.92(0.06)$ & $19.61(0.05)$ & $19.38(0.04)$ \\
\hline & & & $19.14(0.04)$ & $19.13(0.05)$ & $19.04(0.05)$ & $19.01(0.04)$ & $18.87(0.04)$ & $20.10(0.10)$ \\
\hline \multirow[t]{4}{*}{216} & 128.889 & -29.875 & 99.99 (9.99) & 99.99 (9.99) & $99.99(9.99)$ & 99.99 (9.99) & 99.99 (9.99) & 99.99 (9.99) \\
\hline & & & 99.99 (9.99) & 99.99 (9.99) & 99.99 (9.99) & $18.52(0.13)$ & $18.97(0.14)$ & 99.99 (9.99) \\
\hline & & & $18.64(0.16)$ & 99.99 (9.99) & 99.99 (9.99) & 99.99 (9.99) & 99.99 (9.99) & 99.99 (9.99) \\
\hline & & & $20.35(0.08)$ & $19.77(0.16)$ & $17.92(0.10)$ & 99.99 (9.99) & $20.86(0.57)$ & $19.61(0.19)$ \\
\hline \multirow[t]{4}{*}{219} & 329.618 & 569.642 & $19.12(0.04)$ & $19.35(0.05)$ & $19.58(0.05)$ & $19.72(0.06)$ & $19.23(0.04)$ & $19.18(0.04)$ \\
\hline & & & $19.17(0.05)$ & $19.09(0.05)$ & $19.12(0.04)$ & $19.54(0.05)$ & $19.59(0.05)$ & $19.62(0.04)$ \\
\hline & & & $19.14(0.04)$ & $19.19(0.04)$ & $19.20(0.04)$ & $19.21(0.05)$ & $19.34(0.05)$ & $19.47(0.05)$ \\
\hline & & & $19.59(0.05)$ & $19.52(0.05)$ & $19.67(0.05)$ & $19.79(0.05)$ & $19.85(0.05)$ & $19.72(0.09)$ \\
\hline \multirow[t]{4}{*}{221} & 597.628 & 560.943 & $19.28(0.05)$ & $19.53(0.06)$ & $19.36(0.05)$ & $19.37(0.05)$ & $19.18(0.05)$ & $19.20(0.04)$ \\
\hline & & & $19.06(0.05)$ & $19.17(0.05)$ & $19.14(0.05)$ & $19.18(0.04)$ & $19.18(0.04)$ & $19.46(0.05)$ \\
\hline & & & $19.54(0.05)$ & $19.52(0.05)$ & $19.43(0.05)$ & $19.36(0.06)$ & $19.40(0.06)$ & $19.36(0.05)$ \\
\hline & & & $19.48(0.05)$ & $19.31(0.05)$ & $19.43(0.05)$ & $19.55(0.05)$ & $19.70(0.05)$ & $19.42(0.06)$ \\
\hline \multirow[t]{4}{*}{223} & 43.347 & 736.172 & $19.45(0.05)$ & $19.03(0.04)$ & $18.91(0.05)$ & $18.90(0.05)$ & $19.33(0.05)$ & $19.41(0.05)$ \\
\hline & & & $19.43(0.05)$ & $19.41(0.05)$ & $19.39(0.05)$ & $19.62(0.05)$ & $19.66(0.05)$ & $19.73(0.05)$ \\
\hline & & & $19.51(0.05)$ & $19.42(0.04)$ & $19.20(0.05)$ & $19.42(0.05)$ & $19.32(0.05)$ & $19.26(0.05)$ \\
\hline & & & $19.11(0.04)$ & $19.23(0.05)$ & $19.27(0.04)$ & $19.73(0.05)$ & $19.68(0.05)$ & $19.91(0.25)$ \\
\hline \multirow[t]{4}{*}{224} & 483.740 & 722.181 & $19.35(0.05)$ & $19.24(0.05)$ & $19.44(0.05)$ & $19.50(0.05)$ & $19.11(0.04)$ & $19.25(0.04)$ \\
\hline & & & $19.17(0.05)$ & $19.13(0.04)$ & $19.18(0.04)$ & $19.27(0.04)$ & $19.22(0.04)$ & $19.20(0.04)$ \\
\hline & & & $19.57(0.05)$ & $19.61(0.05)$ & $19.55(0.05)$ & $19.56(0.05)$ & $19.49(0.05)$ & $19.66(0.05)$ \\
\hline & & & $19.58(0.05)$ & $19.54(0.05)$ & $19.46(0.05)$ & $19.38(0.04)$ & $19.24(0.05)$ & $19.31(0.08)$ \\
\hline \multirow[t]{4}{*}{226} & 89.463 & 42.892 & $19.53(0.05)$ & $18.85(0.05)$ & $18.81(0.06)$ & $18.91(0.05)$ & $19.67(0.06)$ & $19.58(0.06)$ \\
\hline & & & $19.44(0.05)$ & $19.60(0.07)$ & $19.89(0.06)$ & $18.96(0.04)$ & $18.90(0.04)$ & $19.29(0.05)$ \\
\hline & & & $19.73(0.05)$ & $19.58(0.07)$ & $19.81(0.08)$ & $19.90(0.09)$ & $19.54(0.05)$ & $19.48(0.05)$ \\
\hline & & & $19.63(0.05)$ & $19.50(0.06)$ & $19.70(0.05)$ & $19.74(0.05)$ & $19.58(0.05)$ & $19.89(0.19)$ \\
\hline \multirow[t]{4}{*}{231} & 811.270 & 254.707 & $18.99(0.04)$ & $19.02(0.05)$ & $19.01(0.05)$ & $19.12(0.05)$ & $19.33(0.05)$ & $19.13(0.05)$ \\
\hline & & & $19.20(0.05)$ & $19.08(0.05)$ & $19.07(0.04)$ & $19.59(0.05)$ & $19.76(0.06)$ & $19.75(0.05)$ \\
\hline & & & $19.29(0.05)$ & $19.51(0.04)$ & $19.66(0.05)$ & $19.83(0.05)$ & $19.88(0.05)$ & $19.87(0.05)$ \\
\hline & & & $20.01(0.06)$ & $20.05(0.06)$ & $20.14(0.05)$ & $20.03(0.06)$ & $19.90(0.06)$ & $19.83(0.11)$ \\
\hline \multirow[t]{4}{*}{232} & 137.997 & -15.777 & $99.99(9.99)$ & $99.99(9.99)$ & $99.99(9.99)$ & 99.99 (9.99) & $99.99(9.99)$ & $99.99(9.99)$ \\
\hline & & & 99.99 (9.99) & 99.99 (9.99) & 99.99 (9.99) & $18.54(0.21)$ & $19.02(0.13)$ & $18.39(0.14)$ \\
\hline & & & $17.92(0.16)$ & $99.99(9.99)$ & 19.69 (1.64) & 99.99 (9.99) & $99.99(9.99)$ & 99.99 (9.99) \\
\hline & & & $19.94(0.10)$ & $19.68(0.06)$ & $18.30(0.13)$ & $99.99(9.99)$ & $99.99(9.99)$ & $18.43(0.30)$ \\
\hline \multirow[t]{4}{*}{233} & 596.110 & 808.644 & $19.32(0.04)$ & $18.99(0.05)$ & $18.92(0.05)$ & $19.01(0.05)$ & $19.95(0.05)$ & $20.06(0.05)$ \\
\hline & & & $19.82(0.05)$ & $19.52(0.05)$ & $19.45(0.04)$ & $20.14(0.05)$ & $20.00(0.05)$ & $19.82(0.04)$ \\
\hline & & & $19.10(0.04)$ & $19.06(0.04)$ & $19.05(0.04)$ & $19.04(0.06)$ & $19.00(0.04)$ & $19.20(0.04)$ \\
\hline & & & $19.24(0.04)$ & $19.11(0.04)$ & $19.36(0.05)$ & $19.53(0.05)$ & $19.63(0.05)$ & $20.11(0.09)$ \\
\hline \multirow[t]{4}{*}{237} & 693.643 & 383.014 & $19.89(0.05)$ & $19.78(0.05)$ & $19.77(0.06)$ & $19.82(0.06)$ & $19.36(0.05)$ & $19.47(0.05)$ \\
\hline & & & $19.64(0.06)$ & $19.76(0.05)$ & $19.73(0.05)$ & $18.90(0.04)$ & $18.90(0.04)$ & $18.93(0.04)$ \\
\hline & & & $19.56(0.05)$ & $19.58(0.05)$ & $19.56(0.05)$ & $19.54(0.05)$ & $19.56(0.05)$ & $19.45(0.05)$ \\
\hline & & & $19.54(0.05)$ & $19.54(0.05)$ & $19.54(0.05)$ & $19.50(0.05)$ & $19.25(0.05)$ & $19.05(0.07)$ \\
\hline
\end{tabular}


Table 8. continuation.

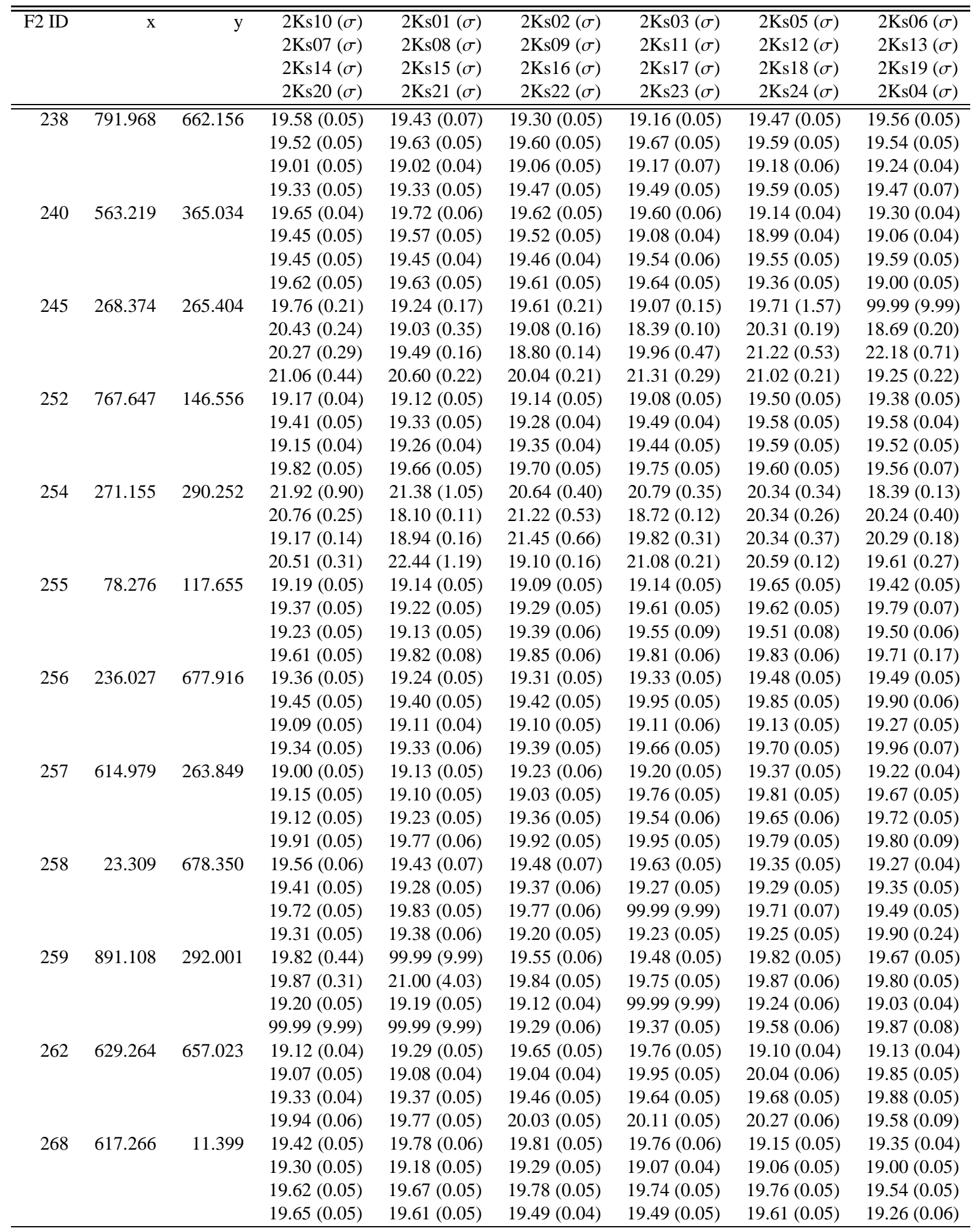


Table 8. continuation.

\begin{tabular}{|c|c|c|c|c|c|c|c|c|}
\hline \multirow[t]{4}{*}{ F2 ID } & \multirow[t]{4}{*}{$\mathrm{x}$} & \multirow[t]{4}{*}{$\mathrm{y}$} & $2 \mathrm{Ks} 10(\sigma)$ & $2 \mathrm{Ks} 01(\sigma)$ & $2 \mathrm{Ks} 02(\sigma)$ & $2 \mathrm{Ks} 03(\sigma)$ & $2 \mathrm{Ks} 05(\sigma)$ & $2 \mathrm{Ks} 06(\sigma)$ \\
\hline & & & $2 \mathrm{Ks} 07(\sigma)$ & $2 \mathrm{Ks} 08(\sigma)$ & $2 \mathrm{Ks} 09(\sigma)$ & $2 \mathrm{Ks} 11(\sigma)$ & $2 \mathrm{Ks} 12(\sigma)$ & $2 \mathrm{Ks} 13(\sigma)$ \\
\hline & & & $2 \mathrm{Ks} 14(\sigma)$ & $2 \mathrm{Ks} 15(\sigma)$ & $2 \mathrm{Ks} 16(\sigma)$ & $2 \mathrm{Ks} 17(\sigma)$ & $2 \mathrm{Ks} 18(\sigma)$ & $2 \mathrm{Ks} 19(\sigma)$ \\
\hline & & & $2 \mathrm{Ks} 20(\sigma)$ & $2 \mathrm{Ks} 21(\sigma)$ & $2 \mathrm{Ks} 22(\sigma)$ & $2 \mathrm{Ks} 23(\sigma)$ & $2 \mathrm{Ks} 24(\sigma)$ & $2 \mathrm{Ks} 04(\sigma)$ \\
\hline \multirow[t]{4}{*}{269} & \multirow[t]{4}{*}{7.301} & \multirow[t]{4}{*}{183.796} & 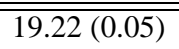 & 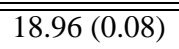 & 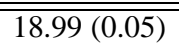 & 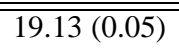 & 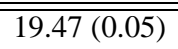 & 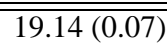 \\
\hline & & & $19.36(0.05)$ & $19.28(0.05)$ & $19.27(0.05)$ & 99.99 (9.99) & 99.99 (9.99) & 99.99 (9.99) \\
\hline & & & $19.60(0.05)$ & $19.49(0.05)$ & $19.63(0.07)$ & 99.99 (9.99) & $19.59(0.07)$ & $19.71(0.05)$ \\
\hline & & & $19.60(0.05)$ & $19.63(0.05)$ & $19.63(0.05)$ & 99.99 (9.99) & $19.78(0.13)$ & $19.85(0.26)$ \\
\hline \multirow[t]{4}{*}{272} & \multirow[t]{4}{*}{725.302} & \multirow[t]{4}{*}{96.781} & $19.40(0.04)$ & $19.66(0.05)$ & $19.69(0.06)$ & $19.60(0.06)$ & $19.45(0.04)$ & $19.48(0.05)$ \\
\hline & & & $19.45(0.05)$ & $19.52(0.05)$ & $19.37(0.05)$ & $19.75(0.05)$ & $19.80(0.05)$ & $19.86(0.05)$ \\
\hline & & & $19.77(0.05)$ & $19.63(0.04)$ & $19.50(0.05)$ & $19.39(0.04)$ & $19.38(0.05)$ & $19.17(0.04)$ \\
\hline & & & $19.27(0.05)$ & $19.21(0.05)$ & $19.13(0.04)$ & $19.16(0.04)$ & $19.22(0.05)$ & $19.59(0.06)$ \\
\hline \multirow[t]{4}{*}{273} & \multirow[t]{4}{*}{217.894} & \multirow[t]{4}{*}{526.151} & $19.73(0.05)$ & $19.78(0.05)$ & $19.74(0.05)$ & $19.68(0.05)$ & $19.56(0.05)$ & $19.69(0.04)$ \\
\hline & & & $19.68(0.05)$ & $19.64(0.05)$ & $19.67(0.05)$ & $19.44(0.04)$ & $19.51(0.05)$ & $19.66(0.05)$ \\
\hline & & & $19.36(0.05)$ & $19.35(0.04)$ & $19.23(0.05)$ & $19.09(0.05)$ & $19.13(0.05)$ & $19.16(0.04)$ \\
\hline & & & $19.19(0.05)$ & $19.20(0.05)$ & $19.28(0.04)$ & $19.51(0.05)$ & $19.56(0.05)$ & $19.32(0.05)$ \\
\hline \multirow[t]{4}{*}{274} & \multirow[t]{4}{*}{829.010} & \multirow[t]{4}{*}{545.445} & $19.70(0.05)$ & $19.63(0.07)$ & $19.69(0.07)$ & $19.69(0.05)$ & $19.34(0.04)$ & $19.52(0.05)$ \\
\hline & & & $19.51(0.05)$ & $19.88(0.05)$ & $19.66(0.05)$ & $19.14(0.04)$ & $19.14(0.05)$ & $19.23(0.04)$ \\
\hline & & & $19.63(0.05)$ & $19.57(0.05)$ & $19.59(0.04)$ & $19.66(0.06)$ & $19.71(0.05)$ & $19.71(0.05)$ \\
\hline & & & $19.52(0.05)$ & $19.54(0.05)$ & $19.39(0.05)$ & $19.30(0.05)$ & $19.16(0.04)$ & $19.17(0.06)$ \\
\hline \multirow[t]{4}{*}{275} & \multirow[t]{4}{*}{52.212} & \multirow[t]{4}{*}{173.756} & $18.76(0.16)$ & $18.58(0.17)$ & $19.15(0.15)$ & $18.84(0.14)$ & $18.33(0.10)$ & 99.99 (9.99) \\
\hline & & & $20.19(0.11)$ & 99.99 (9.99) & $18.80(0.12)$ & $18.83(0.11)$ & $20.07(0.11)$ & $19.43(0.44)$ \\
\hline & & & $20.38(0.49)$ & 99.99 (9.99) & 99.99 (9. & $20.38(0.59)$ & $20.08(0.30)$ & $20.45(0.16)$ \\
\hline & & & $20.64(0.11)$ & $21.02(0.34)$ & 99.99 (9.99) & 99.99 (9.99) & 99.99 (9.99) & $19.54(0.17)$ \\
\hline 276 & 308.469 & 327.348 & $19.62(0.04)$ & $19.83(0.06)$ & $19.63(0.06)$ & $19.49(0.05)$ & $19.78(0.04)$ & $19.65(0.04)$ \\
\hline & & & $19.73(0.05)$ & $19.66(0.05)$ & $19.63(0.05)$ & $19.42(0.04)$ & $19.58(0.05)$ & $19.84(0.04)$ \\
\hline & & & $19.76(0.05)$ & $19.59(0.04)$ & $19.46(0.04)$ & $19.28(0.05)$ & $19.26(0.05)$ & $19.19(0.04)$ \\
\hline & & & $19.13(0.05)$ & $19.09(0.05)$ & $19.08(0.04)$ & $19.13(0.04)$ & $19.25(0.05)$ & $19.81(0.09)$ \\
\hline 282 & 433.087 & 63.769 & $19.27(0.04)$ & $19.15(0.05)$ & $19.07(0.05)$ & $19.11(0.05)$ & $19.73(0.04)$ & $19.75(0.04)$ \\
\hline & & & $19.61(0.05)$ & $19.34(0.05)$ & $19.35(0.04)$ & $19.89(0.05)$ & $19.80(0.05)$ & $19.80(0.04)$ \\
\hline & & & $19.15(0.04)$ & $19.11(0.04)$ & $19.29(0.04)$ & $19.32(0.05)$ & $19.32(0.04)$ & $19.39(0.04)$ \\
\hline & & & $19.70(0.05)$ & $19.62(0.06)$ & $19.68(0.05)$ & $19.61(0.05)$ & $19.59(0.05)$ & $19.76(0.08)$ \\
\hline 285 & 564.710 & 488.882 & $19.64(0.04)$ & $19.77(0.05)$ & $19.78(0.07)$ & $19.87(0.06)$ & $19.81(0.05)$ & $19.90(0.05)$ \\
\hline & & & $19.81(0.05)$ & $19.98(0.06)$ & $19.79(0.05)$ & $19.01(0.04)$ & $19.12(0.05)$ & $19.33(0.04)$ \\
\hline & & & $19.51(0.05)$ & $19.56(0.04)$ & $19.60(0.05)$ & $19.63(0.05)$ & $19.69(0.05)$ & $19.67(0.05)$ \\
\hline & & & $19.39(0.05)$ & $19.43(0.06)$ & $19.24(0.04)$ & $19.13(0.04)$ & $19.04(0.05)$ & $19.57(0.07)$ \\
\hline 288 & 21.506 & 164.659 & $19.71(0.06)$ & $19.86(0.10)$ & $19.90(0.08)$ & $20.25(0.06)$ & $19.26(0.05)$ & 21.77 (1.70) \\
\hline & & & $19.44(0.05)$ & $18.18(0.22)$ & $19.61(0.05)$ & $17.85(0.08)$ & $20.40(0.11)$ & $19.74(0.10)$ \\
\hline & & & $20.28(0.92)$ & $19.59(0.16)$ & $19.65(0.06)$ & 99.99 (9.99) & $19.52(0.07)$ & $19.35(0.05)$ \\
\hline & & & $19.34(0.05)$ & $19.64(0.06)$ & $19.20(0.17)$ & $19.30(0.05)$ & $19.22(0.06)$ & $19.19(0.20)$ \\
\hline 289 & 624.248 & 201.533 & $19.22(0.07)$ & $19.21(0.07)$ & $19.55(0.08)$ & $19.43(0.07)$ & $19.13(0.08)$ & $20.20(0.24)$ \\
\hline & & & $19.26(0.08)$ & $18.93(0.13)$ & $19.20(0.06)$ & 99.99 (9.99) & $20.18(0.10)$ & $19.35(0.07)$ \\
\hline & & & $19.75(0.16)$ & $19.37(0.07)$ & $19.59(0.09)$ & $19.78(0.14)$ & $19.99(0.10)$ & $19.98(0.11)$ \\
\hline & & & $20.72(0.12)$ & $19.89(0.14)$ & $19.87(0.13)$ & $21.48(0.20)$ & $21.89(0.25)$ & $19.69(0.17)$ \\
\hline 291 & 44.895 & 163.187 & $18.77(0.20)$ & $18.39(0.17)$ & $18.81(0.16)$ & $18.32(0.11)$ & $17.76(0.09)$ & 99.99 (9.99) \\
\hline & & & $20.03(0.10)$ & 99.99 (9.99) & $18.14(0.11)$ & $18.44(0.09)$ & $20.53(0.12)$ & $17.62(0.26)$ \\
\hline & & & $18.93(0.12)$ & $18.43(0.18)$ & $19.18(0.23)$ & $19.31(0.40)$ & $21.32(1.30)$ & $19.77(0.05)$ \\
\hline & & & $20.72(0.15)$ & $20.67(0.29)$ & $20.07(0.22)$ & $20.45(0.08)$ & $21.17(0.19)$ & $19.42(0.11)$ \\
\hline 296 & 843.817 & 426.917 & $19.33(0.04)$ & $19.82(0.06)$ & $20.03(0.06)$ & $20.22(0.07)$ & $19.15(0.04)$ & $19.16(0.05)$ \\
\hline & & & $19.21(0.05)$ & $19.34(0.05)$ & $19.26(0.05)$ & $19.73(0.05)$ & $19.78(0.05)$ & $19.68(0.04)$ \\
\hline & & & $19.21(0.04)$ & $19.34(0.04)$ & $19.39(0.04)$ & $19.57(0.06)$ & $19.69(0.05)$ & $19.77(0.05)$ \\
\hline & & & $19.89(0.05)$ & $19.97(0.05)$ & $20.16(0.06)$ & $20.26(0.05)$ & $20.48(0.06)$ & $19.48(0.08)$ \\
\hline
\end{tabular}


Table 8. continuation.

\begin{tabular}{|c|c|c|c|c|c|c|c|c|}
\hline \multirow[t]{4}{*}{ F2 ID } & $\mathrm{x}$ & \multirow[t]{4}{*}{$\mathrm{y}$} & $2 \mathrm{Ks} 10(\sigma)$ & $2 \mathrm{Ks} 01(\sigma)$ & $2 \mathrm{Ks} 02(\sigma)$ & $2 \mathrm{Ks} 03(\sigma)$ & $2 \mathrm{Ks} 05(\sigma)$ & $2 \mathrm{Ks} 06(\sigma)$ \\
\hline & & & $2 \mathrm{Ks} 07(\sigma)$ & $2 \mathrm{Ks} 08(\sigma)$ & $2 \mathrm{Ks} 09(\sigma)$ & $2 \mathrm{Ks} 11(\sigma)$ & $2 \mathrm{Ks} 12(\sigma)$ & $2 \mathrm{Ks} 13(\sigma)$ \\
\hline & & & $2 \mathrm{Ks} 14(\sigma)$ & $2 \mathrm{Ks} 15(\sigma)$ & $2 \mathrm{Ks} 16(\sigma)$ & $2 \mathrm{Ks} 17(\sigma)$ & $2 \mathrm{Ks} 18(\sigma)$ & $2 \mathrm{Ks} 19(\sigma)$ \\
\hline & & & $2 \mathrm{Ks} 20(\sigma)$ & $2 \mathrm{Ks} 21(\sigma)$ & $2 \mathrm{Ks} 22(\sigma)$ & $2 \mathrm{Ks} 23(\sigma)$ & $2 \mathrm{Ks} 24(\sigma)$ & $2 \mathrm{Ks} 04(\sigma)$ \\
\hline \multirow[t]{4}{*}{299} & 358.945 & 470.513 & 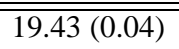 & 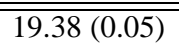 & 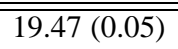 & 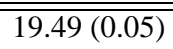 & 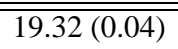 & 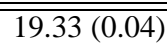 \\
\hline & & & $19.30(0.05)$ & $19.30(0.05)$ & $19.29(0.04)$ & $19.25(0.04)$ & $19.22(0.05)$ & $19.39(0.05)$ \\
\hline & & & $20.15(0.06)$ & $20.11(0.05)$ & $19.96(0.05)$ & $19.90(0.05)$ & $19.89(0.06)$ & $19.84(0.05)$ \\
\hline & & & $19.68(0.05)$ & $19.67(0.06)$ & $19.57(0.05)$ & $19.57(0.05)$ & $19.56(0.05)$ & $19.61(0.10)$ \\
\hline \multirow[t]{4}{*}{302} & 398.003 & 141.525 & $19.56(0.05)$ & $19.59(0.06)$ & $19.40(0.05)$ & $19.35(0.05)$ & $19.85(0.05)$ & $19.85(0.05)$ \\
\hline & & & $19.90(0.05)$ & $19.75(0.05)$ & $19.81(0.05)$ & $19.76(0.05)$ & $19.67(0.05)$ & $19.73(0.05)$ \\
\hline & & & $19.12(0.04)$ & $19.11(0.04)$ & $19.29(0.06)$ & $19.31(0.04)$ & $19.37(0.05)$ & $19.40(0.05)$ \\
\hline & & & $19.56(0.05)$ & $19.62(0.05)$ & $19.63(0.05)$ & $19.68(0.06)$ & $19.83(0.05)$ & $19.85(0.08)$ \\
\hline \multirow[t]{4}{*}{303} & 143.702 & -9.790 & 99.99 (9.99) & $20.79(0.12)$ & 99.99 (9.99) & 99.99 (9.99) & $20.74(0.15)$ & 99.99 (9.99) \\
\hline & & & 99.99 (9.99) & 99.99 (9.99) & 99.99 (9.99) & $17.47(0.07)$ & $19.74(0.31)$ & 99.99 (9.99) \\
\hline & & & $18.87(0.24)$ & $18.76(0.33)$ & $20.80(0.15)$ & 99.99 (9.99) & $20.38(0.23)$ & $19.36(0.28)$ \\
\hline & & & $19.98(0.09)$ & $20.65(0.11)$ & $20.37(0.70)$ & $20.91(0.13)$ & $20.08(0.10)$ & $19.80(0.19)$ \\
\hline \multirow[t]{4}{*}{307} & 482.659 & 165.534 & $19.65(0.05)$ & $19.67(0.06)$ & $19.79(0.06)$ & $19.91(0.06)$ & $19.82(0.05)$ & $19.72(0.08)$ \\
\hline & & & $19.79(0.06)$ & $19.78(0.16)$ & $19.63(0.06)$ & $19.40(0.06)$ & $19.59(0.05)$ & $20.13(0.08)$ \\
\hline & & & $19.67(0.06)$ & $19.52(0.05)$ & $19.38(0.05)$ & $19.36(0.04)$ & $19.35(0.04)$ & $19.41(0.05)$ \\
\hline & & & $19.39(0.05)$ & $19.31(0.05)$ & $19.36(0.05)$ & $19.47(0.05)$ & $19.55(0.05)$ & $19.65(0.08)$ \\
\hline \multirow[t]{4}{*}{309} & 702.863 & 824.379 & $19.66(0.04)$ & $19.86(0.06)$ & $19.80(0.06)$ & $19.84(0.06)$ & $19.24(0.04)$ & $19.56(0.04)$ \\
\hline & & & $19.39(0.05)$ & $19.67(0.05)$ & $19.51(0.05)$ & $19.20(0.04)$ & $19.08(0.04)$ & $19.07(0.04)$ \\
\hline & & & $19.91(0.05)$ & $19.77(0$. & 19.75 & 19.79 & 19.64 & $19.69(0.05)$ \\
\hline & & & $19.70(0.05)$ & $19.66(0.06)$ & $19.68(0.05)$ & $19.60(0.05)$ & $19.50(0.05)$ & $19.19(0.09)$ \\
\hline \multirow[t]{4}{*}{312} & 479.483 & 204.810 & $19.59(0.04)$ & $19.86(0.07)$ & $19.88(0.06)$ & $19.92(0.05)$ & $19.21(0.04)$ & $19.38(0.04)$ \\
\hline & & & $19.33(0.05)$ & $19.61(0.06)$ & $19.40(0.05)$ & $18.98(0.05)$ & $19.25(0.05)$ & $19.10(0.04)$ \\
\hline & & & $19.40(0.05)$ & $19.49(0.04)$ & $19.63(0.05)$ & $19.68(0.05)$ & $19.78(0.05)$ & $19.83(0.05)$ \\
\hline & & & $19.95(0.05)$ & $19.80(0.05)$ & $19.99(0.05)$ & $19.91(0.05)$ & $19.84(0.05)$ & $19.18(0.05)$ \\
\hline \multirow[t]{4}{*}{313} & 65.465 & 671.823 & $19.51(0.05)$ & $19.54(0.05)$ & $19.63(0.06)$ & $19.87(0.07)$ & $19.34(0.05)$ & $19.30(0.04)$ \\
\hline & & & $19.40(0.05)$ & $19.33(0.05)$ & $19.38(0.05)$ & $19.29(0.04)$ & $19.18(0.05)$ & $19.16(0.05)$ \\
\hline & & & $19.93(0.06)$ & $19.88(0.05)$ & $19.81(0.05)$ & $19.85(0.06)$ & $19.83(0.06)$ & $19.82(0.05)$ \\
\hline & & & $19.82(0.05)$ & $19.95(0.0$ & $19.75(0.05)$ & $19.65(0.05)$ & $19.43(0.05)$ & $19.78(0.20)$ \\
\hline \multirow[t]{4}{*}{315} & 119.328 & 571.688 & $19.94(0.05)$ & $19.65(0.06)$ & $19.49(0.05)$ & $19.54(0.06)$ & $20.46(0.05)$ & $20.35(0.06)$ \\
\hline & & & $20.26(0.06)$ & $20.37(0.07)$ & $20.34(0.06)$ & $20.23(0.06)$ & $20.25(0.06)$ & $20.32(0.06)$ \\
\hline & & & $19.39(0.04)$ & $19.35(0.04)$ & $19.23(0.04)$ & $19.15(0.05)$ & $19.18(0.05)$ & $19.21(0.04)$ \\
\hline & & & $19.39(0.05)$ & & $19.41(0.04)$ & $19.54(0.05)$ & $19.80(0.05)$ & $20.62(0.11)$ \\
\hline \multirow[t]{4}{*}{319} & 241.857 & 410.714 & $19.87(0.05)$ & $19.69(0.06)$ & $19.89(0.05)$ & $19.86(0.05)$ & $19.48(0.05)$ & $19.45(0.05)$ \\
\hline & & & $19.64(0.05)$ & $19.56(0.05)$ & $19.75(0.05)$ & $19.19(0.05)$ & $19.27(0.05)$ & $19.54(0.05)$ \\
\hline & & & $19.79(0.05)$ & $19.79(0.05)$ & $19.83(0.05)$ & $19.70(0.05)$ & $19.68(0.06)$ & $19.52(0.05)$ \\
\hline & & & $19.30(0.05)$ & & $19.30(0.04)$ & $19.33(0.05)$ & $19.36(0.05)$ & $19.37(0.09)$ \\
\hline \multirow[t]{4}{*}{322} & 466.585 & 163.313 & $19.61(0.05)$ & $19.47(0.05)$ & $19.45(0.06)$ & $19.34(0.05)$ & $19.60(0.05)$ & $19.74(0.05)$ \\
\hline & & & $19.70(0.06)$ & $19.67(0.05)$ & $19.69(0.06)$ & $19.24(0.05)$ & $19.82(0.05)$ & $19.71(0.05)$ \\
\hline & & & $19.18(0.05)$ & $19.19(0.05)$ & $19.30(0.06)$ & $19.30(0.06)$ & $19.37(0.05)$ & $19.36(0.05)$ \\
\hline & & & $19.54(0.05)$ & $19.51(0.06)$ & $19.60(0.05)$ & $19.69(0.05)$ & $19.65(0.05)$ & $19.79(0.08)$ \\
\hline \multirow[t]{4}{*}{324} & 891.658 & 765.781 & $20.01(3.08)$ & 99.99 (9.99) & $19.70(0.06)$ & $19.83(0.08)$ & $19.38(0.05)$ & $19.56(0.05)$ \\
\hline & & & $20.86(8.32)$ & 99.99 (9.99) & $19.79(0.05)$ & $19.29(0.04)$ & $19.23(0.05)$ & $19.34(0.05)$ \\
\hline & & & $19.88(0.06)$ & $19.84(0.05)$ & $19.97(0.07)$ & $99.99(9.99)$ & $19.79(0.09)$ & $19.60(0.06)$ \\
\hline & & & 99.99 (9.99) & 99.99 (9.99) & $19.45(0.23)$ & $19.29(0.06)$ & $19.34(0.06)$ & $19.61(0.26)$ \\
\hline \multirow[t]{4}{*}{326} & 18.559 & 274.274 & $20.02(0.06)$ & $20.02(0.09)$ & $20.02(0.07)$ & $20.27(0.10)$ & $20.15(0.05)$ & $20.11(0.05)$ \\
\hline & & & $20.27(0.07)$ & $20.47(0.08)$ & $20.21(0.06)$ & $19.46(0.04)$ & $19.45(0.05)$ & $19.73(0.05)$ \\
\hline & & & $20.01(0.05)$ & $19.84(0.05)$ & $19.79(0.05)$ & 99.99 (9.99) & $19.47(0.05)$ & $19.39(0.04)$ \\
\hline & & & $19.25(0.05)$ & $19.22(0.05)$ & $19.18(0.05)$ & $19.21(0.05)$ & $19.13(0.05)$ & $19.61(0.14)$ \\
\hline
\end{tabular}


Table 8. continuation.

\begin{tabular}{|c|c|c|c|c|c|c|c|c|}
\hline \multirow[t]{4}{*}{ F2 ID } & \multirow[t]{4}{*}{$\mathrm{x}$} & \multirow[t]{4}{*}{$\mathrm{y}$} & \multirow{4}{*}{$\begin{array}{l}(\sigma) \\
\sigma) \\
\sigma) \\
\sigma)\end{array}$} & \multirow{4}{*}{$\begin{array}{l}2 \mathrm{Ks} 01(\sigma) \\
2 \mathrm{Ks} 08(\sigma) \\
2 \mathrm{Ks} 15(\sigma) \\
2 \mathrm{Ks} 21(\sigma)\end{array}$} & \multirow{4}{*}{$\begin{array}{l}2 \mathrm{Ks} 02(\sigma) \\
2 \mathrm{Ks} 09(\sigma) \\
2 \mathrm{Ks} 16(\sigma) \\
2 \mathrm{Ks} 22(\sigma)\end{array}$} & \multirow{4}{*}{$\begin{array}{l}2 \mathrm{Ks} 03(\sigma) \\
2 \mathrm{Ks} 11(\sigma) \\
2 \mathrm{Ks} 17(\sigma) \\
2 \mathrm{Ks} 23(\sigma)\end{array}$} & \multirow{4}{*}{$\begin{array}{l}2 \mathrm{Ks} 05(\sigma) \\
2 \mathrm{Ks} 12(\sigma) \\
2 \mathrm{Ks} 18(\sigma) \\
2 \mathrm{Ks} 24(\sigma)\end{array}$} & \multirow{4}{*}{$\begin{array}{l}\text { 2Ks06 }(\sigma) \\
2 \mathrm{Ks} 13(\sigma) \\
2 \mathrm{Ks} 19(\sigma) \\
2 \mathrm{Ks} 04(\sigma)\end{array}$} \\
\hline & & & & & & & & \\
\hline & & & & & & & & \\
\hline & & & & & & & & \\
\hline \multirow[t]{4}{*}{338} & \multirow[t]{4}{*}{774.833} & \multirow[t]{4}{*}{518.023} & $19.65(0.05)$ & $19.29(0.06)$ & $19.20(0.05)$ & $19.15(0.05)$ & $19.87(0.05)$ & $19.82(0.05)$ \\
\hline & & & $19.88(0.06)$ & $19.93(0.06)$ & $19.95(0.05)$ & $19.93(0.05)$ & $19.96(0.05)$ & $19.96(0.05)$ \\
\hline & & & $19.40(0.05)$ & $19.35(0.04)$ & $19.32(0.05)$ & $19.35(0.05)$ & $19.43(0.05)$ & $19.37(0.04)$ \\
\hline & & & $19.39(0.05)$ & $19.36(0.05)$ & $19.40(0.04)$ & $19.51(0.05)$ & $19.63(0.05)$ & $20.24(0.13)$ \\
\hline \multirow[t]{4}{*}{343} & \multirow[t]{4}{*}{29.215} & \multirow[t]{4}{*}{391.401} & $20.13(0.05)$ & $19.75(0.06)$ & $19.86(0.05)$ & $19.89(0.06)$ & $20.02(0.05)$ & $20.07(0.05)$ \\
\hline & & & $20.14(0.06)$ & $20.30(0.07)$ & $20.22(0.06)$ & $19.39(0.04)$ & $19.32(0.05)$ & $19.58(0.05)$ \\
\hline & & & $19.94(0.05)$ & $19.85(0.05)$ & $19.76(0.05)$ & 99.99 (9.99) & $19.46(0.06)$ & $19.34(0.04)$ \\
\hline & & & $19.22(0.05)$ & $19.26(0.05)$ & $19.19(0.05)$ & $19.19(0.04)$ & $19.14(0.04)$ & $19.73(0.12)$ \\
\hline \multirow[t]{4}{*}{346} & \multirow[t]{4}{*}{608.595} & \multirow[t]{4}{*}{215.089} & $19.52(0.11)$ & $20.15(0.18)$ & $19.93(0.15)$ & $19.67(0.15)$ & $18.80(0.13)$ & $19.32(0.10)$ \\
\hline & & & $19.71(0.15)$ & $19.06(0.16)$ & $19.57(0.15)$ & $18.95(0.07)$ & $20.35(0.11)$ & $18.91(0.12)$ \\
\hline & & & $19.70(0.11)$ & $19.61(0.09)$ & $20.23(0.21)$ & $21.43(0.63)$ & $20.93(0.30)$ & $21.14(0.32)$ \\
\hline & & & $20.96(0.14)$ & 99.99 (9.99) & $20.87(0.21)$ & $19.57(0.21)$ & $19.79(0.22)$ & 99.99 (9.99) \\
\hline 348 & 880.854 & 305.110 & $19.82(0.05)$ & 99.99 (9.99) & $19.57(0.05)$ & $19.60(0.06)$ & $19.81(0.05)$ & $19.90(0.06)$ \\
\hline & & & $20.01(0.06)$ & $20.23(0.07)$ & $19.86(0.05)$ & $19.45(0.04)$ & $19.65(0.05)$ & $19.62(0.05)$ \\
\hline & & & $19.74(0.05)$ & $19.81(0.05)$ & $19.71(0.05)$ & $19.60(0.07)$ & $19.60(0.06)$ & $19.34(0.05)$ \\
\hline & & & 99.99 (9.99) & $19.39(0.05)$ & $19.23(0.05)$ & $19.24(0.05)$ & $19.23(0.04)$ & $19.56(0.07)$ \\
\hline 350 & 251.404 & 283.837 & $19.35(0.14)$ & $19.50(0.23)$ & $19.71(0.16)$ & $20.77(0.85)$ & $18.55(0.14)$ & $18.18(0.13)$ \\
\hline & & & $19.64(0.13)$ & 99.99 (9.99) & $19.12(0.09)$ & $20.77(0.61)$ & $19.67(0.11)$ & $21.12(1.20)$ \\
\hline & & & $20.34(0.38)$ & $20.23(0.32)$ & $19.86(0.51)$ & $19.81(0.17)$ & $19.37(0.15)$ & $19.57(0.09)$ \\
\hline & & & 99.99 (9.99) & $19.59(0.18)$ & $19.84(0.18)$ & $22.50(1.25)$ & $20.58(0.09)$ & $19.29(0.19)$ \\
\hline 351 & 335.357 & 155.840 & $19.51(0.04)$ & $19.43(0.05)$ & $19.46(0.05)$ & $19.50(0.05)$ & $19.94(0.05)$ & $19.85(0.04)$ \\
\hline & & & $19.72(0.05)$ & $19.49(0.05)$ & $19.59(0.04)$ & $20.36(0.05)$ & $20.60(0.07)$ & $20.49(0.05)$ \\
\hline & & & $19.38(0.04)$ & $19.30(0.04)$ & $19.37(0.04)$ & $19.32(0.05)$ & $19.31(0.04)$ & $19.34(0.04)$ \\
\hline & & & $19.43(0.05)$ & $19.48(0.06)$ & $19.47(0.04)$ & $19.88(0.05)$ & $19.88(0.05)$ & $20.29(0.08)$ \\
\hline 352 & 26.321 & 178.747 & $19.50(0.12)$ & $19.59(0.42)$ & $19.36(0.16)$ & $19.29(0.32)$ & $20.72(0.79)$ & $20.23(1.11)$ \\
\hline & & & $20.19(0.11)$ & 20.45 (1.29) & $19.08(0.17)$ & $18.83(0.12)$ & $19.99(0.09)$ & $18.65(0.20)$ \\
\hline & & & $19.27(0.19)$ & $19.06(0.28)$ & $17.87(0.11)$ & 99.99 (9.99) & $19.48(0.21)$ & $21.33(0.17)$ \\
\hline & & & $21.37(0.22)$ & $21.46(0.59)$ & $18.90(0.12)$ & $22.58(0.30)$ & $21.35(0.17)$ & $20.24(0.21)$ \\
\hline 357 & 783.957 & 585.890 & $19.97(0.07)$ & $19.90(0.07)$ & $19.71(0.06)$ & $19.58(0.07)$ & $19.95(0.05)$ & $21.92(0.34)$ \\
\hline & & & $19.86(0.06)$ & $19.52(0.11)$ & $19.98(0.06)$ & $20.52(0.07)$ & $19.84(0.05)$ & $21.04(0.12)$ \\
\hline & & & $19.52(0.05)$ & $19.46(0.05)$ & $19.23(0.05)$ & $19.20(0.06)$ & $19.17(0.05)$ & $19.43(0.05)$ \\
\hline & & & $19.79(0.06)$ & $19.26(0.05)$ & $19.42(0.04)$ & $19.44(0.05)$ & $19.81(0.05)$ & $19.88(0.08)$ \\
\hline 362 & 43.414 & 166.625 & 99.99 (9.99) & $18.70(0.24)$ & $18.85(0.17)$ & $18.55(0.14)$ & $19.68(0.48)$ & $18.36(0.20)$ \\
\hline & & & $20.25(0.11)$ & $18.85(0.30)$ & $18.71(0.12)$ & $18.51(0.12)$ & $20.28(0.10)$ & $18.19(0.14)$ \\
\hline & & & $19.23(0.21)$ & $21.11(1.83)$ & $20.72(1.67)$ & $19.78(0.37)$ & $19.51(0.25)$ & $21.27(0.13)$ \\
\hline & & & $21.54(0.28)$ & $20.64(0.33)$ & $20.07(0.24)$ & $22.31(0.51)$ & $21.29(0.21)$ & $20.22(0.41)$ \\
\hline 363 & 191.899 & 355.583 & $20.11(0.05)$ & $20.12(0.07)$ & $19.90(0.06)$ & $19.97(0.06)$ & $19.53(0.05)$ & $19.70(0.05)$ \\
\hline & & & $19.71(0.05)$ & $19.89(0.05)$ & $19.94(0.05)$ & $19.57(0.04)$ & $19.61(0.05)$ & $19.79(0.05)$ \\
\hline & & & $19.98(0.05)$ & $19.87(0.04)$ & $19.71(0.05)$ & $19.52(0.05)$ & $19.44(0.05)$ & $19.39(0.04)$ \\
\hline & & & $19.29(0.05)$ & $19.29(0.05)$ & $19.26(0.04)$ & $19.30(0.05)$ & $19.33(0.05)$ & $19.36(0.08)$ \\
\hline 370 & 614.713 & 202.193 & $19.30(0.09)$ & $19.11(0.13)$ & $19.55(0.11)$ & $18.83(0.08)$ & $18.96(0.13)$ & $19.35(0.24)$ \\
\hline & & & $19.15(0.10)$ & $19.42(0.43)$ & $19.31(0.11)$ & $19.45(0.19)$ & $19.89(0.10)$ & $19.53(0.12)$ \\
\hline & & & $19.73(0.12)$ & $19.90(0.13)$ & $19.77(0.23)$ & $20.21(0.20)$ & $19.83(0.14)$ & $19.88(0.10)$ \\
\hline & & & $20.52(0.10)$ & $19.92(0.16)$ & $19.84(0.06)$ & 99.99 (9.99) & 99.99 (9.99) & $18.82(0.27)$ \\
\hline 372 & 523.553 & 598.487 & $19.33(0.04)$ & $19.57(0.05)$ & $19.70(0.07)$ & $20.04(0.06)$ & $19.26(0.04)$ & $19.27(0.04)$ \\
\hline & & & $19.23(0.04)$ & $19.37(0.05)$ & $19.23(0.04)$ & $19.99(0.05)$ & $19.83(0.05)$ & $19.64(0.04)$ \\
\hline & & & $19.93(0.05)$ & $20.00(0.05)$ & $20.16(0.05)$ & $20.16(0.06)$ & $20.17(0.05)$ & $20.18(0.05)$ \\
\hline & & & $20.27(0.07)$ & $20.12(0.06)$ & $20.00(0.05)$ & $20.05(0.05)$ & $20.07(0.05)$ & $19.69(0.12)$ \\
\hline
\end{tabular}


Table 8. continuation.

\begin{tabular}{|c|c|c|c|c|c|c|c|c|}
\hline \multirow[t]{4}{*}{$\bar{F} 2$ ID } & \multirow[t]{4}{*}{$\mathrm{x}$} & \multirow[t]{4}{*}{$\mathrm{y}$} & $2 \mathrm{Ks} 10(\sigma)$ & $2 \mathrm{Ks} 01(\sigma)$ & $2 \mathrm{Ks} 02(\sigma)$ & $2 \mathrm{Ks} 03(\sigma)$ & $2 \mathrm{Ks} 05(\sigma)$ & $2 \mathrm{Ks} 06(\sigma)$ \\
\hline & & & $2 \mathrm{Ks} 07(\sigma)$ & $2 \mathrm{Ks} 08(\sigma)$ & $2 \mathrm{Ks} 09(\sigma)$ & $2 \mathrm{Ks} 11(\sigma)$ & $2 \mathrm{Ks} 12(\sigma)$ & $2 \mathrm{Ks} 13(\sigma)$ \\
\hline & & & $2 \mathrm{Ks} 14(\sigma)$ & $2 \mathrm{Ks} 15(\sigma)$ & $2 \mathrm{Ks} 16(\sigma)$ & $2 \mathrm{Ks} 17(\sigma)$ & $2 \mathrm{Ks} 18(\sigma)$ & $2 \mathrm{Ks} 19(\sigma)$ \\
\hline & & & $2 \mathrm{Ks} 20(\sigma)$ & $2 \mathrm{Ks} 21(\sigma)$ & $2 \mathrm{Ks} 22(\sigma)$ & $2 \mathrm{Ks} 23(\sigma)$ & $2 \mathrm{Ks} 24(\sigma)$ & $2 \mathrm{Ks} 04(\sigma)$ \\
\hline \multirow[t]{4}{*}{373} & $\overline{603.818}$ & 657.368 & $19.56(0.04)$ & $19.80(0.05)$ & $20.05(0.06)$ & $19.95(0.07)$ & $19.13(0.04)$ & $19.34(0.04)$ \\
\hline & & & $19.30(0.05)$ & $19.46(0.05)$ & $19.39(0.04)$ & $19.48(0.04)$ & $19.39(0.05)$ & $19.26(0.04)$ \\
\hline & & & $20.12(0.06)$ & $20.27(0.05)$ & $20.36(0.05)$ & $20.45(0.06)$ & $20.43(0.05)$ & $20.48(0.06)$ \\
\hline & & & $20.25(0.06)$ & $20.13(0.06)$ & $20.30(0.05)$ & $20.31(0.06)$ & $20.47(0.06)$ & $19.18(0.08)$ \\
\hline \multirow[t]{4}{*}{375} & 328.261 & 403.974 & $19.59(0.04)$ & $19.78(0.06)$ & $19.76(0.06)$ & $19.86(0.06)$ & $19.69(0.04)$ & $19.72(0.04)$ \\
\hline & & & $19.76(0.05)$ & $19.67(0.05)$ & $19.65(0.05)$ & $19.83(0.05)$ & $19.77(0.05)$ & $19.83(0.04)$ \\
\hline & & & $19.56(0.04)$ & $19.49(0.04)$ & $19.43(0.04)$ & $19.31(0.05)$ & $19.35(0.05)$ & $19.39(0.04)$ \\
\hline & & & $19.38(0.05)$ & $19.33(0.05)$ & $19.47(0.04)$ & $19.62(0.05)$ & $19.69(0.05)$ & $19.59(0.08)$ \\
\hline \multirow[t]{4}{*}{389} & 804.286 & 348.623 & $19.71(0.05)$ & $19.48(0.05)$ & $19.65(0.06)$ & $19.82(0.06)$ & $19.31(0.04)$ & $19.35(0.05)$ \\
\hline & & & $19.48(0.05)$ & $19.73(0.05)$ & $19.59(0.05)$ & $19.52(0.04)$ & $19.44(0.05)$ & $19.38(0.04)$ \\
\hline & & & $19.95(0.05)$ & $19.90(0.05)$ & $19.95(0.05)$ & $20.04(0.05)$ & $20.04(0.05)$ & $20.01(0.05)$ \\
\hline & & & $19.75(0.05)$ & $19.90(0.07)$ & $19.65(0.05)$ & $19.51(0.05)$ & $19.40(0.05)$ & $19.53(0.07)$ \\
\hline \multirow[t]{4}{*}{390} & 765.461 & 395.915 & $19.75(0.05)$ & $20.14(0.07)$ & $20.18(0.08)$ & $19.90(0.06)$ & $19.45(0.04)$ & $19.61(0.05)$ \\
\hline & & & $19.63(0.05)$ & $20.01(0.05)$ & $19.80(0.04)$ & $19.19(0.04)$ & $19.22(0.05)$ & $19.37(0.04)$ \\
\hline & & & $19.86(0.05)$ & $19.85(0.04)$ & $19.90(0.05)$ & $19.87(0.05)$ & $19.98(0.05)$ & $19.81(0.05)$ \\
\hline & & & $19.66(0.05)$ & $19.76(0.05)$ & $19.55(0.04)$ & $19.43(0.04)$ & $19.37(0.05)$ & $19.31(0.08)$ \\
\hline \multirow[t]{4}{*}{392} & 231.989 & 714.767 & $19.93(0.05)$ & $20.03(0.06)$ & $19.96(0.05)$ & $19.86(0.06)$ & $19.95(0.05)$ & $20.08(0.05)$ \\
\hline & & & $20.10(0.05)$ & $20.10(0.07)$ & $20.00(0.05)$ & $19.52(0.04)$ & $19.63(0.05)$ & $19.89(0.05)$ \\
\hline & & & $19.71(0.05)$ & $19.67(0.04)$ & $19.49(0.04)$ & $19.42(0.05)$ & $19.38(0.05)$ & $19.38(0.04)$ \\
\hline & & & $19.30(0.05)$ & $19.34(0.05)$ & $19.34(0.04)$ & $19.42(0.05)$ & $19.45(0.05)$ & $19.78(0.07)$ \\
\hline \multirow[t]{4}{*}{393} & 222.743 & 659.931 & $19.76(0.04)$ & $19.92(0.06)$ & $19.82(0.05)$ & $19.75(0.05)$ & $19.50(0.04)$ & $19.68(0.05)$ \\
\hline & & & $19.71(0.05)$ & $19.74(0.05)$ & $19.70(0.04)$ & $19.16(0.04)$ & $19.22(0.05)$ & $19.40(0.04)$ \\
\hline & & & $19.91(0.05)$ & $19.92(0.05)$ & $19.85(0.05)$ & $19.83(0.05)$ & $19.84(0.05)$ & $19.85(0.05)$ \\
\hline & & & $19.66(0.05)$ & $19.72(0.05)$ & $19.61(0.04)$ & $19.51(0.05)$ & $19.47(0.05)$ & $19.40(0.06)$ \\
\hline \multirow[t]{4}{*}{395} & 105.867 & 375.836 & $20.02(0.05)$ & $19.56(0.05)$ & $19.82(0.06)$ & $20.21(0.06)$ & $19.43(0.04)$ & $19.54(0.05)$ \\
\hline & & & $19.71(0.06)$ & $19.87(0.06)$ & $19.80(0.05)$ & $19.48(0.04)$ & $19.53(0.05)$ & $19.68(0.05)$ \\
\hline & & & $20.22(0.06)$ & $20.20(0.05)$ & $20.06(0.06)$ & $19.83(0.06)$ & $19.73(0.05)$ & $19.71(0.05)$ \\
\hline & & & $19.49(0.05)$ & $19.48(0.05)$ & $19.43(0.04)$ & $19.39(0.04)$ & $19.38(0.05)$ & $19.39(0.06)$ \\
\hline \multirow[t]{4}{*}{397} & 753.563 & 17.565 & $19.64(0.04)$ & $19.90(0.07)$ & $20.05(0.07)$ & $20.27(0.07)$ & $19.20(0.05)$ & $19.43(0.04)$ \\
\hline & & & $19.40(0.04)$ & $19.48(0.05)$ & $19.49(0.04)$ & $19.40(0.04)$ & $19.38(0.05)$ & $19.26(0.04)$ \\
\hline & & & $19.80(0.05)$ & $19.93(0.05)$ & $20.08(0.05)$ & $20.17(0.06)$ & $20.21(0.06)$ & $20.00(0.05)$ \\
\hline & & & $20.02(0.05)$ & $20.16(0.06)$ & $19.96(0.05)$ & $20.00(0.06)$ & $19.73(0.05)$ & $19.19(0.07)$ \\
\hline \multirow[t]{4}{*}{398} & 382.470 & 153.388 & $19.37(0.04)$ & $19.36(0.05)$ & $19.46(0.05)$ & $19.72(0.05)$ & $19.73(0.05)$ & $19.67(0.04)$ \\
\hline & & & $19.52(0.05)$ & $19.44(0.05)$ & $19.42(0.04)$ & $19.90(0.05)$ & $20.02(0.06)$ & $19.89(0.05)$ \\
\hline & & & $19.37(0.04)$ & $19.40(0.04)$ & $19.57(0.05)$ & $19.67(0.05)$ & $19.74(0.05)$ & $19.81(0.05)$ \\
\hline & & & $19.95(0.06)$ & $19.95(0.06)$ & $20.13(0.05)$ & $20.21(0.06)$ & $20.15(0.06)$ & $20.39(0.15)$ \\
\hline \multirow[t]{4}{*}{406} & 267.293 & -12.372 & $99.99(9.99)$ & $20.17(0.07)$ & 99.99 (9.99) & 99.99 (9.99) & 99.99 (9.99) & 99.99 (9.99) \\
\hline & & & $99.99(9.99)$ & 99.99 (9.99) & $99.99(9.99)$ & $20.13(0.06)$ & $20.22(0.06)$ & $20.45(0.06)$ \\
\hline & & & $19.49(0.05)$ & $19.32(0.05)$ & $19.52(0.06)$ & 99.99 (9.99) & $19.42(0.05)$ & $19.46(0.04)$ \\
\hline & & & $19.53(0.05)$ & $19.62(0.05)$ & $19.64(0.05)$ & $19.72(0.05)$ & $19.96(0.06)$ & $20.11(0.11)$ \\
\hline \multirow[t]{4}{*}{408} & 489.302 & 607.923 & $19.86(0.04)$ & $20.22(0.06)$ & $20.28(0.07)$ & $20.03(0.07)$ & $20.00(0.05)$ & $20.14(0.05)$ \\
\hline & & & $20.02(0.05)$ & $20.22(0.06)$ & $20.01(0.05)$ & $19.40(0.04)$ & $19.55(0.05)$ & $19.78(0.04)$ \\
\hline & & & $19.85(0.05)$ & $19.75(0.05)$ & $19.63(0.05)$ & $19.51(0.05)$ & $19.45(0.05)$ & $19.38(0.04)$ \\
\hline & & & $19.35(0.05)$ & $19.23(0.05)$ & $19.27(0.04)$ & $19.34(0.05)$ & $19.35(0.05)$ & $19.81(0.09)$ \\
\hline \multirow[t]{4}{*}{410} & 38.202 & 496.561 & $19.70(0.05)$ & $19.79(0.07)$ & $19.97(0.06)$ & $20.00(0.06)$ & $19.43(0.05)$ & $19.28(0.05)$ \\
\hline & & & $19.42(0.05)$ & $19.45(0.05)$ & $19.53(0.05)$ & $19.55(0.05)$ & $19.47(0.05)$ & $19.41(0.05)$ \\
\hline & & & $20.03(0.05)$ & $20.07(0.05)$ & $20.08(0.05)$ & $20.19(0.09)$ & $20.10(0.07)$ & $19.97(0.06)$ \\
\hline & & & $19.86(0.06)$ & $20.07(0.07)$ & $19.78(0.05)$ & $19.64(0.05)$ & $19.50(0.05)$ & $19.63(0.12)$ \\
\hline
\end{tabular}


Table 8. continuation.

\begin{tabular}{|c|c|c|c|c|c|c|c|c|}
\hline \multirow[t]{4}{*}{$\bar{F} 2$ ID } & \multirow[t]{4}{*}{$\mathrm{x}$} & \multirow[t]{4}{*}{$\mathrm{y}$} & $2 \mathrm{Ks} 10(\sigma)$ & $2 \mathrm{Ks} 01(\sigma)$ & $2 \mathrm{Ks} 02(\sigma)$ & $2 \mathrm{Ks} 03(\sigma)$ & $2 \mathrm{Ks} 05(\sigma)$ & $2 \mathrm{Ks} 06(\sigma)$ \\
\hline & & & $2 \mathrm{Ks} 07(\sigma)$ & $2 \mathrm{Ks} 08(\sigma)$ & $2 \mathrm{Ks} 09(\sigma)$ & $2 \mathrm{Ks} 11(\sigma)$ & $2 \mathrm{Ks} 12(\sigma)$ & $2 \mathrm{Ks} 13(\sigma)$ \\
\hline & & & $2 \mathrm{Ks} 14(\sigma)$ & $2 \mathrm{Ks} 15(\sigma)$ & $2 \mathrm{Ks} 16(\sigma)$ & $2 \mathrm{Ks} 17(\sigma)$ & $2 \mathrm{Ks} 18(\sigma)$ & $2 \mathrm{Ks} 19(\sigma)$ \\
\hline & & & $2 \mathrm{Ks} 20(\sigma)$ & $2 \mathrm{Ks} 21(\sigma)$ & $2 \mathrm{Ks} 22(\sigma)$ & $2 \mathrm{Ks} 23(\sigma)$ & $2 \mathrm{Ks} 24(\sigma)$ & $2 \mathrm{Ks} 04(\sigma)$ \\
\hline \multirow[t]{4}{*}{ 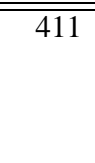 } & 47.289 & 175.205 & 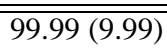 & $20.79(0.93)$ & 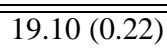 & 99.99 (9.99) & $\bar{~} 18.10(0.15)$ & 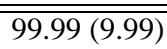 \\
\hline & & & $19.95(0.10)$ & 99.99 (9.99) & $19.00(0.16)$ & 99.99 (9.99) & $19.98(0.07)$ & $20.01(0.49)$ \\
\hline & & & $18.59(0.10)$ & $20.08(0.75)$ & $99.99(9.99)$ & $20.15(0.79)$ & $19.57(0.44)$ & $22.12(0.97)$ \\
\hline & & & $22.05(0.40)$ & $19.97(0.09)$ & 99.99 (9.99) & 99.99 (9.99) & 99.99 (9.99) & $19.05(0.27)$ \\
\hline \multirow[t]{4}{*}{415} & 557.163 & 429.572 & $19.54(0.04)$ & $19.64(0.05)$ & $19.46(0.05)$ & $19.45(0.06)$ & $19.87(0.05)$ & $19.94(0.05)$ \\
\hline & & & $19.88(0.05)$ & $19.88(0.05)$ & $19.72(0.05)$ & $20.17(0.05)$ & $20.14(0.05)$ & $20.09(0.05)$ \\
\hline & & & $19.34(0.05)$ & $19.38(0.04)$ & $19.35(0.04)$ & $19.35(0.05)$ & $19.45(0.04)$ & $19.51(0.04)$ \\
\hline & & & $19.63(0.05)$ & $19.57(0.05)$ & $19.77(0.05)$ & $19.92(0.05)$ & $20.12(0.05)$ & $20.05(0.09)$ \\
\hline \multirow[t]{4}{*}{416} & 428.793 & 781.882 & $19.46(0.05)$ & $20.10(0.07)$ & $20.23(0.08)$ & $20.33(0.09)$ & $19.17(0.05)$ & $19.44(0.04)$ \\
\hline & & & $19.30(0.05)$ & $19.36(0.05)$ & $19.34(0.05)$ & $20.07(0.05)$ & $19.95(0.05)$ & $19.48(0.05)$ \\
\hline & & & $19.63(0.05)$ & $19.67(0.05)$ & $19.73(0.05)$ & $19.76(0.06)$ & $19.77(0.05)$ & $19.96(0.05)$ \\
\hline & & & $20.16(0.06)$ & $20.22(0.08)$ & $20.25(0.06)$ & $20.25(0.05)$ & $20.29(0.06)$ & $19.26(0.06)$ \\
\hline \multirow[t]{4}{*}{419} & 375.338 & 596.128 & $19.38(0.05)$ & $19.34(0.06)$ & $19.35(0.05)$ & $19.32(0.05)$ & $19.60(0.09)$ & $19.67(0.05)$ \\
\hline & & & $19.56(0.05)$ & $19.52(0.05)$ & $19.45(0.05)$ & $19.74(0.05)$ & $19.73(0.05)$ & $19.65(0.05)$ \\
\hline & & & $19.67(0.05)$ & $19.79(0.05)$ & $19.81(0.05)$ & $19.77(0.06)$ & $19.82(0.05)$ & $19.78(0.05)$ \\
\hline & & & $19.72(0.05)$ & $19.72(0.06)$ & $19.73(0.05)$ & $19.73(0.05)$ & $19.74(0.05)$ & $19.80(0.10)$ \\
\hline \multirow[t]{4}{*}{420} & 528.382 & 34.913 & $19.84(0.05)$ & $20.14(0.07)$ & $20.21(0.06)$ & $20.22(0.07)$ & $19.36(0.04)$ & $19.65(0.04)$ \\
\hline & & & $19.48(0.05)$ & $19.77(0.05)$ & $19.67(0.05)$ & $19.19(0.04)$ & $19.16(0.04)$ & $19.22(0.05)$ \\
\hline & & & $20.38(0.06)$ & $20.21(0.05)$ & $20.39(0.06)$ & $20.34(0.06)$ & $20.36(0.07)$ & $20.21(0.06)$ \\
\hline & & & $20.44(0.09)$ & $20.39(0.08)$ & $20.42(0.07)$ & $20.35(0.06)$ & $20.01(0.05)$ & $19.22(0.07)$ \\
\hline \multirow[t]{4}{*}{422} & 45.001 & 693.969 & $19.60(0.05)$ & $19.45(0.05)$ & $19.69(0.05)$ & $19.94(0.05)$ & $19.33(0.05)$ & $19.35(0.05)$ \\
\hline & & & $19.38(0.04)$ & $19.36(0.05)$ & $19.38(0.05)$ & $19.52(0.05)$ & $19.35(0.05)$ & $19.16(0.04)$ \\
\hline & & & $20.20(0.06)$ & $20.29(0.05)$ & $20.14(0.05)$ & $20.18(0.07)$ & $20.18(0.07)$ & $20.06(0.05)$ \\
\hline & & & $20.18(0.06)$ & $20.18(0.06)$ & $20.29(0.05)$ & $20.36(0.06)$ & $20.03(0.06)$ & $19.76(0.19)$ \\
\hline \multirow[t]{4}{*}{425} & 191.625 & 365.705 & $19.35(0.04)$ & $19.58(0.05)$ & $19.55(0.05)$ & $19.67(0.05)$ & $19.66(0.04)$ & $19.48(0.04)$ \\
\hline & & & $19.55(0.05)$ & $19.34(0.05)$ & $19.39(0.05)$ & $19.81(0.05)$ & $19.79(0.05)$ & $19.70(0.05)$ \\
\hline & & & $19.56(0.05)$ & $19.59(0.05)$ & $19.72(0.04)$ & $19.82(0.05)$ & $19.86(0.05)$ & $19.96(0.05)$ \\
\hline & & & $20.05(0.05)$ & $20.05(0.05)$ & $20.01(0.05)$ & $20.02(0.05)$ & $19.88(0.05)$ & $19.83(0.11)$ \\
\hline \multirow[t]{4}{*}{427} & 263.874 & 41.438 & $19.72(0.05)$ & $19.82(0.06)$ & $19.84(0.06)$ & $19.61(0.06)$ & $19.88(0.05)$ & $19.83(0.06)$ \\
\hline & & & $19.82(0.05)$ & $19.89(0.07)$ & $19.83(0.05)$ & $19.85(0.06)$ & $19.98(0.06)$ & $20.05(0.06)$ \\
\hline & & & $19.36(0.05)$ & $19.24(0.05)$ & $19.43(0.05)$ & $19.51(0.07)$ & $19.37(0.05)$ & $19.42(0.05)$ \\
\hline & & & $19.54(0.05)$ & $19.56(0.06)$ & $19.65(0.05)$ & $19.85(0.05)$ & $19.92(0.05)$ & $19.82(0.10)$ \\
\hline
\end{tabular}


Table 8. continuation.

\begin{tabular}{|c|c|c|c|c|c|c|c|c|}
\hline \multirow[t]{4}{*}{ F2 ID } & $\mathrm{x}$ & \multirow[t]{4}{*}{$\mathrm{y}$} & $2 \mathrm{Ks} 10(\sigma)$ & $2 \mathrm{Ks} 01(\sigma)$ & $2 \mathrm{Ks} 02(\sigma)$ & $2 \mathrm{Ks} 03(\sigma)$ & $2 \mathrm{Ks} 05(\sigma)$ & $2 \mathrm{Ks} 06(\sigma)$ \\
\hline & & & $2 \mathrm{Ks} 07(\sigma)$ & $2 \mathrm{Ks} 08(\sigma)$ & $2 \mathrm{Ks} 09(\sigma)$ & $2 \mathrm{Ks} 11(\sigma)$ & $2 \mathrm{Ks} 12(\sigma)$ & $2 \mathrm{Ks} 13(\sigma)$ \\
\hline & & & $2 \mathrm{Ks} 14(\sigma)$ & $2 \mathrm{Ks} 15(\sigma)$ & $2 \mathrm{Ks} 16(\sigma)$ & $2 \mathrm{Ks} 17(\sigma)$ & $2 \mathrm{Ks} 18(\sigma)$ & $2 \mathrm{Ks} 19(\sigma)$ \\
\hline & & & $2 \mathrm{Ks} 20(\sigma)$ & $2 \mathrm{Ks} 21(\sigma)$ & $2 \mathrm{Ks} 22(\sigma)$ & $2 \mathrm{Ks} 23(\sigma)$ & $2 \mathrm{Ks} 24(\sigma)$ & $2 \mathrm{Ks} 04(\sigma)$ \\
\hline \multirow[t]{4}{*}{$\overline{4429}$} & 353.452 & 442.537 & 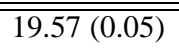 & 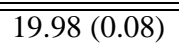 & 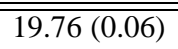 & $19.64(0.05)$ & 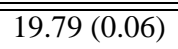 & 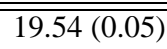 \\
\hline & & & $19.55(0.06)$ & $19.47(0.05)$ & $19.58(0.06)$ & $19.49(0.05)$ & $19.51(0.06)$ & $19.58(0.06)$ \\
\hline & & & $19.86(0.07)$ & $19.95(0.06)$ & $20.02(0.05)$ & $19.92(0.08)$ & $19.92(0.07)$ & $19.75(0.06)$ \\
\hline & & & $19.58(0.06)$ & $19.75(0.06)$ & $19.56(0.05)$ & $19.50(0.05)$ & $19.45(0.05)$ & $19.82(0.11)$ \\
\hline \multirow[t]{4}{*}{430} & 150.273 & 309.684 & $19.90(0.04)$ & $20.25(0.07)$ & $20.14(0.05)$ & $20.20(0.07)$ & $19.43(0.04)$ & $19.54(0.05)$ \\
\hline & & & $19.70(0.05)$ & $19.70(0.05)$ & $19.73(0.05)$ & $19.14(0.04)$ & $19.11(0.05)$ & $19.16(0.04)$ \\
\hline & & & $20.17(0.05)$ & $20.14(0.05)$ & $20.15(0.05)$ & $20.07(0.05)$ & $20.00(0.05)$ & $20.07(0.05)$ \\
\hline & & & $20.07(0.06)$ & $20.14(0.07)$ & $20.24(0.06)$ & $20.09(0.05)$ & $19.57(0.05)$ & $19.16(0.07)$ \\
\hline \multirow[t]{4}{*}{432} & 735.275 & 52.508 & $19.84(0.05)$ & $20.01(0.06)$ & $20.07(0.07)$ & $20.00(0.06)$ & $19.97(0.06)$ & $19.91(0.05)$ \\
\hline & & & $19.83(0.05)$ & $19.84(0.05)$ & $19.83(0.06)$ & $20.06(0.05)$ & $20.27(0.07)$ & $20.34(0.07)$ \\
\hline & & & $19.45(0.05)$ & $19.46(0.05)$ & $19.49(0.06)$ & $19.44(0.06)$ & $19.47(0.06)$ & $19.31(0.05)$ \\
\hline & & & $19.41(0.05)$ & $19.48(0.05)$ & $19.46(0.05)$ & $19.53(0.05)$ & $19.76(0.05)$ & $20.01(0.09)$ \\
\hline \multirow[t]{4}{*}{436} & 550.447 & 731.093 & $19.92(0.05)$ & $19.78(0.05)$ & $20.09(0.07)$ & $20.38(0.08)$ & $19.41(0.04)$ & $19.61(0.05)$ \\
\hline & & & $19.55(0.05)$ & $19.84(0.05)$ & $19.71(0.05)$ & $19.38(0.04)$ & $19.31(0.04)$ & $19.31(0.04)$ \\
\hline & & & $20.15(0.05)$ & $20.07(0.05)$ & $20.02(0.05)$ & $20.02(0.05)$ & $19.96(0.04)$ & $20.05(0.05)$ \\
\hline & & & $19.85(0.05)$ & $19.99(0.06)$ & $19.75(0.05)$ & $19.63(0.05)$ & $19.39(0.05)$ & $19.51(0.06)$ \\
\hline \multirow[t]{4}{*}{438} & 394.952 & 609.696 & $19.40(0.04)$ & $19.45(0.05)$ & $19.38(0.05)$ & $19.49(0.05)$ & $19.94(0.04)$ & $19.98(0.05)$ \\
\hline & & & $19.81(0.05)$ & $19.61(0.05)$ & $19.52(0.05)$ & $20.04(0.05)$ & $19.97(0.05)$ & $20.01(0.05)$ \\
\hline & & & $19.36(0.05)$ & $19.39(C$ & 19.41 & $19.46(0$ & $19.54(0.0$ & 19.71 \\
\hline & & & $19.89(0.06)$ & $19.76(0.05)$ & $19.88(0.05)$ & $20.05(0.05)$ & $20.16(0.06)$ & $20.20(0.11)$ \\
\hline \multirow[t]{4}{*}{440} & 603.394 & 170.867 & $19.65(0.05)$ & $19.38(0.05)$ & $19.34(0.05)$ & $19.43(0.06)$ & $20.14(0.07)$ & $20.00(0.05)$ \\
\hline & & & $19.93(0.05)$ & $19.92(0.06)$ & $19.78(0.05)$ & $19.96(0.05)$ & $20.07(0.05)$ & $20.24(0.07)$ \\
\hline & & & $19.55(0.05)$ & $19.56(0.05)$ & $19.64(0.06)$ & $19.59(0.05)$ & $19.60(0.06)$ & $19.44(0.05)$ \\
\hline & & & $19.44(0.05)$ & $19.59(0.06)$ & $19.53(0.05)$ & $19.60(0.05)$ & $19.64(0.05)$ & $20.36(0.13)$ \\
\hline \multirow[t]{4}{*}{441} & 541.100 & 317.749 & $19.63(0.05)$ & $19.59(0.07)$ & $19.75(0.05)$ & $19.78(0.06)$ & $20.06(0.05)$ & $19.76(0.05)$ \\
\hline & & & $19.81(0.07)$ & $19.87(0.05)$ & $19.73(0.05)$ & $20.52(0.06)$ & $20.53(0.07)$ & $20.37(0.06)$ \\
\hline & & & $19.22(0.04)$ & $19.28(0.04)$ & $19.34(0.05)$ & $19.42(0.05)$ & $19.47(0.05)$ & $19.62(0.05)$ \\
\hline & & & $19.74(0$. & 19.69 & $19.81(0.05)$ & $19.94(0.05)$ & $20.20(0.06)$ & $20.47(0.13)$ \\
\hline \multirow[t]{4}{*}{442} & 185.512 & 530.924 & $19.84(0.05)$ & $19.49(0.06)$ & $19.72(0.05)$ & $19.90(0.06)$ & $19.37(0.04)$ & $19.42(0.04)$ \\
\hline & & & $19.46(0.05)$ & $19.59(0.05)$ & $19.60(0.05)$ & $19.44(0.04)$ & $19.42(0.05)$ & $19.52(0.04)$ \\
\hline & & & $20.03(0.05)$ & $20.01(0.05)$ & $20.05(0.05)$ & $20.04(0.05)$ & $20.08(0.05)$ & $20.10(0.05)$ \\
\hline & & & $19.89(0.06)$ & & $19.65(0.05)$ & $19.66(0.05)$ & $19.52(0.05)$ & $19.55(0.06)$ \\
\hline \multirow[t]{4}{*}{445} & 553.145 & 180.724 & $19.30(0.04)$ & & $19.41(0.05)$ & $19.63(0.05)$ & $19.50(0.04)$ & $19.40(0.05)$ \\
\hline & & & $19.38(0.05)$ & $19.33(0.05)$ & $19.27(0.04)$ & $20.46(0.05)$ & $20.36(0.06)$ & $19.96(0.05)$ \\
\hline & & & $20.06(0.05)$ & $20.09(0.05)$ & $20.19(0.05)$ & $20.26(0.07)$ & $20.32(0.06)$ & $20.16(0.05)$ \\
\hline & & & $20.10(0.06)$ & $20.19(0.07)$ & $20.04(0.05)$ & $19.94(0.05)$ & $20.10(0.06)$ & $19.88(0.07)$ \\
\hline \multirow[t]{4}{*}{447} & 503.621 & 655.923 & $19.37(0.05)$ & $19.39(0.05)$ & $19.45(0.05)$ & $19.38(0.05)$ & $19.72(0.05)$ & $19.54(0.05)$ \\
\hline & & & $19.53(0.05)$ & $19.45(0.05)$ & $19.36(0.05)$ & $19.91(0.05)$ & $20.02(0.06)$ & $20.03(0.06)$ \\
\hline & & & $19.47(0.05)$ & $19.62(0.05)$ & $19.75(0.05)$ & $19.86(0.06)$ & $19.87(0.06)$ & $20.01(0.05)$ \\
\hline & & & $20.04(0.07)$ & $20.06(0.07)$ & $20.02(0.06)$ & $19.92(0.05)$ & $19.81(0.05)$ & $19.95(0.08)$ \\
\hline \multirow[t]{4}{*}{448} & 612.347 & 301.497 & $19.82(0.04)$ & $19.95(0.05)$ & $19.95(0.06)$ & $19.89(0.06)$ & $19.87(0.05)$ & $20.02(0.05)$ \\
\hline & & & $19.98(0.05)$ & $19.91(0.05)$ & $19.87(0.05)$ & $19.53(0.04)$ & $19.74(0.05)$ & $19.88(0.04)$ \\
\hline & & & $19.83(0.05)$ & $19.78(0.04)$ & $19.69(0.04)$ & $19.61(0.05)$ & $19.60(0.04)$ & $19.46(0.04)$ \\
\hline & & & $19.43(0.05)$ & $19.44(0.05)$ & $19.41(0.04)$ & $19.37(0.05)$ & $19.45(0.05)$ & $19.58(0.06)$ \\
\hline \multirow[t]{4}{*}{449} & 563.957 & 505.081 & $19.99(0.05)$ & $19.59(0.06)$ & $19.43(0.06)$ & $19.38(0.05)$ & $20.33(0.06)$ & $20.31(0.05)$ \\
\hline & & & $20.15(0.06)$ & $20.48(0.06)$ & $20.35(0.05)$ & $19.98(0.05)$ & $20.18(0.07)$ & $20.25(0.05)$ \\
\hline & & & $19.73(0.05)$ & $19.61(0.04)$ & $19.51(0.04)$ & $19.43(0.05)$ & $19.44(0.04)$ & $19.37(0.04)$ \\
\hline & & & $19.40(0.05)$ & $19.36(0.05)$ & $19.38(0.04)$ & $19.50(0.05)$ & $19.61(0.05)$ & $20.45(0.11)$ \\
\hline
\end{tabular}


Table 8. continuation.

\begin{tabular}{|c|c|c|c|c|c|c|c|c|}
\hline \multirow[t]{4}{*}{$\bar{F}$ F2 ID } & \multirow[t]{4}{*}{$\mathrm{x}$} & \multirow[t]{4}{*}{$\mathrm{y}$} & $2 \mathrm{Ks} 10(\sigma)$ & $2 \mathrm{Ks} 01(\sigma)$ & $2 \mathrm{Ks} 02(\sigma)$ & $2 \mathrm{Ks} 03(\sigma)$ & $2 \mathrm{Ks} 05(\sigma)$ & $\overline{2 \mathrm{Ks} 06(\sigma)}$ \\
\hline & & & $2 \mathrm{Ks} 07(\sigma)$ & $2 \mathrm{Ks} 08(\sigma)$ & $2 \mathrm{Ks} 09(\sigma)$ & $2 \mathrm{Ks} 11(\sigma)$ & $2 \mathrm{Ks} 12(\sigma)$ & $2 \mathrm{Ks} 13(\sigma)$ \\
\hline & & & $2 \mathrm{Ks} 14(\sigma)$ & $2 \mathrm{Ks} 15(\sigma)$ & $2 \mathrm{Ks} 16(\sigma)$ & $2 \mathrm{Ks} 17(\sigma)$ & $2 \mathrm{Ks} 18(\sigma)$ & $2 \mathrm{Ks} 19(\sigma)$ \\
\hline & & & $2 \mathrm{Ks} 20(\sigma)$ & $2 \mathrm{Ks} 21(\sigma)$ & $2 \mathrm{Ks} 22(\sigma)$ & $2 \mathrm{Ks} 23(\sigma)$ & $2 \mathrm{Ks} 24(\sigma)$ & $2 \mathrm{Ks} 04(\sigma)$ \\
\hline \multirow[t]{4}{*}{$\overline{450}$} & $\overline{405.718}$ & 554.259 & $19.35(0.04)$ & $19.49(0.05)$ & $\overline{19.46(0.05)}$ & $19.63(0.06)$ & $19.62(0.05)$ & 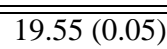 \\
\hline & & & $19.51(0.05)$ & $19.38(0.05)$ & $19.34(0.04)$ & $19.76(0.05)$ & $19.75(0.05)$ & $19.81(0.05)$ \\
\hline & & & $19.57(0.05)$ & $19.62(0.04)$ & $19.74(0.04)$ & $19.79(0.06)$ & $19.90(0.05)$ & $20.11(0.05)$ \\
\hline & & & $20.09(0.06)$ & $20.16(0.06)$ & $20.23(0.05)$ & $20.29(0.05)$ & $20.30(0.06)$ & $19.82(0.06)$ \\
\hline \multirow[t]{4}{*}{452} & 266.825 & 166.526 & $19.38(0.04)$ & $19.28(0.05)$ & $19.49(0.06)$ & $19.63(0.06)$ & $19.62(0.04)$ & $19.47(0.04)$ \\
\hline & & & $19.42(0.05)$ & $19.38(0.05)$ & $19.36(0.05)$ & $20.08(0.05)$ & $19.84(0.06)$ & $19.53(0.04)$ \\
\hline & & & $19.84(0.05)$ & $19.84(0.05)$ & $20.00(0.05)$ & $20.05(0.05)$ & $20.05(0.05)$ & $20.04(0.05)$ \\
\hline & & & $19.86(0.06)$ & $19.94(0.06)$ & $19.87(0.05)$ & $19.98(0.06)$ & $20.06(0.06)$ & $20.12(0.11)$ \\
\hline \multirow[t]{4}{*}{454} & 875.939 & 35.790 & $19.89(0.05)$ & 99.99 (9.99) & $19.88(0.07)$ & $20.02(0.06)$ & $19.46(0.05)$ & $19.59(0.05)$ \\
\hline & & & $19.51(0.05)$ & $19.53(0.05)$ & $19.72(0.05)$ & $19.46(0.04)$ & $19.45(0.05)$ & $19.32(0.04)$ \\
\hline & & & $19.95(0.05)$ & $19.90(0.05)$ & $19.94(0.05)$ & $20.01(0.06)$ & $20.08(0.06)$ & $19.82(0.05)$ \\
\hline & & & $19.89(0.06)$ & $19.90(0.06)$ & $19.67(0.05)$ & $19.61(0.05)$ & $19.52(0.06)$ & $19.75(0.19)$ \\
\hline \multirow[t]{4}{*}{455} & 409.322 & 736.005 & $19.85(0.06)$ & $19.97(0.08)$ & $19.88(0.06)$ & $19.83(0.06)$ & $19.89(0.06)$ & $19.95(0.05)$ \\
\hline & & & $19.91(0.07)$ & $19.93(0.06)$ & $19.95(0.06)$ & $19.55(0.05)$ & $19.57(0.06)$ & $19.71(0.07)$ \\
\hline & & & $19.75(0.05)$ & $19.71(0.05)$ & $19.70(0.08)$ & $19.59(0.08)$ & $19.54(0.08)$ & $19.51(0.06)$ \\
\hline & & & $19.42(0.05)$ & $19.57(0.08)$ & $19.45(0.06)$ & $19.42(0.05)$ & $19.35(0.05)$ & $19.68(0.07)$ \\
\hline \multirow[t]{4}{*}{456} & 0.790 & 726.963 & $19.91(0.08)$ & $20.15(0.06)$ & $19.62(0.10)$ & $20.29(0.06)$ & 99.99 (9.99) & $19.78(0.44)$ \\
\hline & & & $19.98(0.05)$ & $19.90(0.06)$ & $19.84(0.05)$ & 99.99 (9.99) & 99.99 (9.99) & 99.99 (9.99) \\
\hline & & & $99.99(9.99)$ & 99.99 (9.99) & 99.99 (9.99) & $99.99(9.99)$ & $99.99(9.99)$ & $19.36(0.05)$ \\
\hline & & & $19.29(0.05)$ & $19.40(0.05)$ & $19.12(0.83)$ & 99.99 (9.99) & 99.99 (9.99) & $20.10(0.34)$ \\
\hline \multirow[t]{4}{*}{458} & 376.220 & 655.209 & $19.41(0.04)$ & $19.39(0.05)$ & $19.29(0.05)$ & $19.33(0.05)$ & $19.61(0.04)$ & $19.60(0.05)$ \\
\hline & & & $19.52(0.05)$ & $19.51(0.05)$ & $19.45(0.04)$ & $19.85(0.05)$ & $19.69(0.05)$ & $19.65(0.04)$ \\
\hline & & & $19.89(0.05)$ & $20.00(0.05)$ & $19.99(0.05)$ & $19.99(0.05)$ & $19.97(0.05)$ & $19.97(0.05)$ \\
\hline & & & $19.83(0.05)$ & $19.79(0.05)$ & $19.79(0.04)$ & $19.82(0.05)$ & $19.81(0.06)$ & $20.01(0.08)$ \\
\hline \multirow[t]{4}{*}{460} & 243.893 & 274.440 & $22.55(0.36)$ & $24.23(2.41)$ & $23.46(1.19)$ & $22.23(0.44)$ & $22.65(0.26)$ & $17.93(0.15)$ \\
\hline & & & 99.99 (9.99) & $17.57(0.12)$ & $22.53(0.56)$ & $18.90(0.09)$ & $19.17(0.12)$ & $21.23(0.75)$ \\
\hline & & & $19.17(0.16)$ & $21.20(0.44)$ & $23.34(0.59)$ & $22.48(0.27)$ & $22.02(0.26)$ & $20.54(0.22)$ \\
\hline & & & $20.41(0.17)$ & $22.00(0.32)$ & $19.51(0.17)$ & $20.13(0.09)$ & $20.74(0.10)$ & $19.75(0.15)$ \\
\hline \multirow[t]{4}{*}{463} & 217.575 & 43.394 & $20.10(0.05)$ & $19.95(0.08)$ & $20.28(0.08)$ & $20.06(0.05)$ & $19.64(0.05)$ & $19.81(0.05)$ \\
\hline & & & $19.87(0.06)$ & $20.33(0.07)$ & $19.94(0.05)$ & $19.48(0.04)$ & $19.53(0.05)$ & $19.67(0.05)$ \\
\hline & & & $20.04(0.05)$ & $19.89(0.06)$ & $19.88(0.05)$ & $19.90(0.06)$ & $19.66(0.05)$ & $19.59(0.05)$ \\
\hline & & & $19.65(0.05)$ & $19.50(0.06)$ & $19.46(0.04)$ & $19.49(0.05)$ & $19.33(0.05)$ & $19.43(0.09)$ \\
\hline \multirow[t]{4}{*}{465} & 834.136 & 505.911 & $19.94(0.05)$ & $20.32(0.09)$ & $20.29(0.08)$ & $20.18(0.06)$ & $20.11(0.05)$ & $19.95(0.05)$ \\
\hline & & & $19.87(0.06)$ & $20.02(0.05)$ & $19.87(0.05)$ & $19.68(0.05)$ & $19.83(0.05)$ & $19.87(0.05)$ \\
\hline & & & $19.63(0.05)$ & $19.63(0.05)$ & $19.53(0.06)$ & $19.56(0.05)$ & $19.58(0.05)$ & $19.43(0.05)$ \\
\hline & & & $19.50(0.05)$ & $19.47(0.05)$ & $19.41(0.04)$ & $19.50(0.05)$ & $19.55(0.05)$ & $19.92(0.07)$ \\
\hline \multirow[t]{4}{*}{466} & 497.668 & 115.838 & $19.68(0.05)$ & $19.69(0.06)$ & $19.79(0.05)$ & $19.98(0.06)$ & $19.38(0.04)$ & $19.90(0.05)$ \\
\hline & & & $19.37(0.05)$ & $19.49(0.09)$ & $19.51(0.05)$ & $19.62(0.05)$ & $19.44(0.05)$ & $19.29(0.05)$ \\
\hline & & & $20.28(0.08)$ & $20.33(0.06)$ & $20.09(0.05)$ & $20.10(0.06)$ & $20.03(0.05)$ & $20.05(0.07)$ \\
\hline & & & $20.33(0.07)$ & $20.15(0.07)$ & $19.96(0.06)$ & $20.11(0.06)$ & $19.72(0.06)$ & $19.53(0.06)$ \\
\hline \multirow[t]{4}{*}{467} & 219.420 & 287.679 & $19.53(0.04)$ & $19.56(0.05)$ & $19.90(0.06)$ & $20.17(0.06)$ & $19.39(0.04)$ & $19.29(0.04)$ \\
\hline & & & $19.46(0.05)$ & $19.35(0.05)$ & $19.41(0.05)$ & $19.78(0.05)$ & $19.71(0.05)$ & $19.54(0.04)$ \\
\hline & & & $20.03(0.05)$ & $20.08(0.05)$ & $20.27(0.05)$ & $20.36(0.05)$ & $20.52(0.05)$ & $20.58(0.06)$ \\
\hline & & & $20.75(0.11)$ & $20.67(0.10)$ & $20.49(0.06)$ & $20.46(0.06)$ & $20.50(0.06)$ & $19.48(0.06)$ \\
\hline \multirow[t]{4}{*}{470} & 413.810 & 765.776 & $20.34(0.05)$ & $20.35(0.07)$ & $20.19(0.07)$ & $20.53(0.08)$ & $20.08(0.05)$ & $20.36(0.05)$ \\
\hline & & & $20.44(0.06)$ & $20.51(0.07)$ & $20.45(0.05)$ & $19.80(0.05)$ & $19.89(0.06)$ & $20.14(0.05)$ \\
\hline & & & $19.61(0.05)$ & $19.52(0.04)$ & $19.41(0.04)$ & $19.39(0.05)$ & $19.32(0.04)$ & $19.47(0.04)$ \\
\hline & & & $19.47(0.05)$ & $19.40(0.05)$ & $19.55(0.05)$ & $19.63(0.05)$ & $19.75(0.05)$ & $19.79(0.09)$ \\
\hline
\end{tabular}


Table 8. continuation.

\begin{tabular}{|c|c|c|c|c|c|c|c|c|}
\hline \multirow[t]{4}{*}{$\bar{F}$ F ID } & \multirow[t]{4}{*}{$\mathrm{x}$} & \multirow[t]{4}{*}{$\mathrm{y}$} & $2 \mathrm{Ks} 10(\sigma)$ & $2 \mathrm{Ks} 01(\sigma)$ & $2 \mathrm{Ks} 02(\sigma)$ & $2 \mathrm{Ks} 03(\sigma)$ & $2 \mathrm{Ks} 05(\sigma)$ & $2 \mathrm{Ks} 06(\sigma)$ \\
\hline & & & $2 \mathrm{Ks} 07(\sigma)$ & $2 \mathrm{Ks} 08(\sigma)$ & $2 \mathrm{Ks} 09(\sigma)$ & $2 \mathrm{Ks} 11(\sigma)$ & $2 \mathrm{Ks} 12(\sigma)$ & $2 \mathrm{Ks} 13(\sigma)$ \\
\hline & & & $2 \mathrm{Ks} 14(\sigma)$ & $2 \mathrm{Ks} 15(\sigma)$ & $2 \mathrm{Ks} 16(\sigma)$ & $2 \mathrm{Ks} 17(\sigma)$ & $2 \mathrm{Ks} 18(\sigma)$ & $2 \mathrm{Ks} 19(\sigma)$ \\
\hline & & & $2 \mathrm{Ks} 20(\sigma)$ & $2 \mathrm{Ks} 21(\sigma)$ & $2 \mathrm{Ks} 22(\sigma)$ & $2 \mathrm{Ks} 23(\sigma)$ & $2 \mathrm{Ks} 24(\sigma)$ & $2 \mathrm{Ks} 04(\sigma)$ \\
\hline \multirow[t]{4}{*}{4771} & 654.271 & 480.820 & $19.99(0.05)$ & $20.49(0.08)$ & $20.82(0.10)$ & $20.32(0.07)$ & $19.46(0.04)$ & $19.69(0.05)$ \\
\hline & & & $19.72(0.05)$ & $19.91(0.06)$ & $19.80(0.05)$ & $19.19(0.04)$ & $19.17(0.05)$ & $19.22(0.04)$ \\
\hline & & & $20.19(0.06)$ & $20.14(0.06)$ & $20.25(0.05)$ & $20.34(0.05)$ & $20.31(0.05)$ & $20.19(0.05)$ \\
\hline & & & $20.20(0.06)$ & $20.23(0.07)$ & $20.24(0.06)$ & $20.14(0.06)$ & $19.66(0.05)$ & $19.35(0.10)$ \\
\hline \multirow[t]{4}{*}{475} & 388.723 & 379.739 & $19.99(0.05)$ & $19.98(0.08)$ & $20.01(0.06)$ & $20.08(0.07)$ & $19.99(0.04)$ & $20.00(0.05)$ \\
\hline & & & $20.02(0.05)$ & $20.06(0.05)$ & $19.89(0.05)$ & $19.87(0.05)$ & $19.99(0.05)$ & $20.22(0.05)$ \\
\hline & & & $19.74(0.05)$ & $19.65(0.04)$ & $19.54(0.04)$ & $19.44(0.05)$ & $19.40(0.05)$ & $19.41(0.04)$ \\
\hline & & & $19.38(0.05)$ & $19.37(0.05)$ & $19.46(0.05)$ & $19.48(0.04)$ & $19.66(0.05)$ & $19.97(0.09)$ \\
\hline \multirow[t]{4}{*}{478} & 27.155 & 158.279 & $20.21(0.05)$ & $20.49(0.10)$ & $19.86(0.05)$ & $20.04(0.11)$ & $20.65(0.06)$ & 99.99 (9.99) \\
\hline & & & $20.34(0.06)$ & 99.99 (9.99) & 20.46 & $18.13(0.08)$ & $21.65(0.23)$ & $19.70(0.08)$ \\
\hline & & & $19.24(0.19)$ & $19.25(0.11)$ & $19.40(0.06)$ & 99.99 (9.99) & $19.40(0.06)$ & $19.14(0.05)$ \\
\hline & & & $19.58(0.05)$ & $19.65(0.05)$ & 99.99 (9.99) & $20.59(0.08)$ & $19.40(0.05)$ & 99.99 (9.99) \\
\hline \multirow[t]{4}{*}{484} & 861.987 & 481.214 & $19.79(0.05)$ & 99.99 (9.99) & $19.86(0.05)$ & $19.84(0.06)$ & $19.83(0.04)$ & $19.82(0.05)$ \\
\hline & & & $19.88(0.06)$ & $20.03(0.05)$ & 19.85 & $19.81(0.04)$ & $19.75(0$ & $19.67(0.04)$ \\
\hline & & & $19.36(0.04)$ & $19.36(0.04)$ & 19.40 & 19.55 & 19.69 & 19.74 \\
\hline & & & $19.86(0.05)$ & $19.89(0.08)$ & 19.97 & 20.04 & 19.89 & 20.01 \\
\hline \multirow[t]{4}{*}{486} & 12.504 & 328.661 & $20.33(0.06)$ & $20.10(0.08)$ & $20.00(0.06)$ & $20.00(0.07)$ & $19.92(0.05)$ & $19.98(0.05)$ \\
\hline & & & $20.16(0.07)$ & $20.58(0.08)$ & $20.22(0.06)$ & $19.39(0.44)$ & $19.67(0.05)$ & $19.72(0.06)$ \\
\hline & & & 20.03 & 19.90 & 19.73 & 99.99 & 19.45 & 19.49 \\
\hline & & & $19.38(0.05)$ & $19.39(0.05)$ & 19.37 & $19.14(0.39)$ & 19.46 & 19.56 \\
\hline \multirow[t]{4}{*}{487} & 215.930 & 745.931 & $20.18(0.05)$ & $20.07(0.05)$ & $19.95(0.06)$ & $19.92(0.06)$ & $19.62(0.05)$ & $19.82(0.05)$ \\
\hline & & & $19.95(0.06)$ & $20.07(0.05)$ & $19.98(0.05)$ & $19.34(0.04)$ & $19.32(0.04)$ & $19.45(0.04)$ \\
\hline & & & $20.14(0.06)$ & $20.13(0.05)$ & $20.03(0.05)$ & $20.11(0.05)$ & $20.00(0$ & 20.05 \\
\hline & & & $19.67(0.05)$ & $19.69(0.05)$ & $19.53(0.04)$ & $19.49(0.04)$ & $19.44(0.05)$ & 19.38 \\
\hline \multirow[t]{4}{*}{488} & 409.287 & 716.276 & $20.03(0.05)$ & $19.85(0.06)$ & $20.05(0.06)$ & $20.09(0.06)$ & $19.42(0.05)$ & $19.73(0.04)$ \\
\hline & & & $19.71(0.05)$ & $19.84(0.05)$ & $19.85(0.05)$ & $19.37(0.04)$ & $19.52(0.05)$ & $19.76(0.04)$ \\
\hline & & & $20.22(0.05)$ & $20.17(0.05)$ & $19.96(0.05)$ & $19.85(0.05)$ & $19.72(0.05)$ & $19.68(0.05)$ \\
\hline & & & & & 19.55 & & 19.67 & 19.32 \\
\hline \multirow[t]{4}{*}{490} & 597.413 & 11.477 & $19.41(0.04)$ & $19.29(0.05)$ & $19.40(0.05)$ & $19.43(0.05)$ & $19.57(0.05)$ & $19.68(0.05)$ \\
\hline & & & $19.54(0.05)$ & $19.50(0.06)$ & $19.48(0.05)$ & $19.91(0.05)$ & $19.68(0.05)$ & $19.48(0.04)$ \\
\hline & & & $20.24(0.06)$ & $20.18(0.05)$ & $20.21(0.05)$ & $20.08(0.05)$ & $20.04(0.05)$ & $19.98(0.05)$ \\
\hline & & & & & $20.08(0.06)$ & $20.09(0.05)$ & & $20.14(0.11)$ \\
\hline \multirow[t]{4}{*}{491} & 310.658 & 488.855 & & & $20.67(0.09)$ & & & $19.61(0.05)$ \\
\hline & & & $19.62(0.05)$ & $19.76(0.05)$ & $19.64(0.05)$ & $19.40(0.04)$ & $19.26(0.04)$ & $19.23(0.04)$ \\
\hline & & & $19.99(0.05)$ & $20.09(0.05)$ & $20.19(0.05)$ & $20.11(0.05)$ & $20.17(0.05)$ & $20.21(0.05)$ \\
\hline & & & & & $19.97(0.05)$ & $20.09(0.05)$ & $20.09(0.06)$ & $19.43(0.10)$ \\
\hline \multirow[t]{4}{*}{494} & 870.000 & 39.193 & $19.86(0.05)$ & 99.99 (9.99) & $20.11(0.08)$ & $20.18(0.07)$ & $19.49(0.05)$ & $19.57(0.05)$ \\
\hline & & & $19.99(0.09)$ & $19.53(0.05)$ & $19.98(0.05)$ & $19.51(0.05)$ & $19.87(0.05)$ & $19.57(0.05)$ \\
\hline & & & $20.32(0.06)$ & $20.27(0.05)$ & $20.23(0.06)$ & $19.61(0.07)$ & $19.66(0.06)$ & $19.23(0.05)$ \\
\hline & & & & $19.45(0.05)$ & $19.50(0.05)$ & $21.22(0.12)$ & 99.99 (9.99) & $19.60(0.14)$ \\
\hline \multirow[t]{4}{*}{496} & 442.349 & 576.357 & $19.40(0.04)$ & $19.46(0.06)$ & $19.35(0.06)$ & $19.33(0.05)$ & $19.83(0.04)$ & $19.72(0.05)$ \\
\hline & & & $19.70(0.05)$ & $19.52(0.06)$ & $19.46(0.04)$ & $19.82(0.05)$ & $19.88(0.05)$ & $19.80(0.04)$ \\
\hline & & & $19.56(0.04)$ & $19.59(0.04)$ & $19.61(0.05)$ & $19.69(0.05)$ & $19.79(0.05)$ & $19.94(0.05)$ \\
\hline & & & $19.89(0.05)$ & $19.81(0.05)$ & $19.96(0.05)$ & $19.94(0.05)$ & $19.86(0.05)$ & $20.04(0.07)$ \\
\hline \multirow[t]{4}{*}{503} & 791.055 & 118.358 & $19.41(0.04)$ & $19.50(0.05)$ & $19.38(0.05)$ & $19.38(0.05)$ & $19.74(0.05)$ & $19.65(0.05)$ \\
\hline & & & $19.73(0.05)$ & $19.51(0.05)$ & $19.57(0.05)$ & $19.89(0.05)$ & $19.93(0.05)$ & $19.83(0.04)$ \\
\hline & & & $19.60(0.05)$ & $19.75(0.04)$ & $19.86(0.05)$ & $19.89(0.06)$ & $19.94(0.05)$ & $19.80(0.05)$ \\
\hline & & & $19.98(0.06)$ & $19.82(0.06)$ & $19.80(0.05)$ & $19.85(0.05)$ & $19.91(0.05)$ & $19.95(0.06)$ \\
\hline
\end{tabular}


Table 8. continuation.

\begin{tabular}{|c|c|c|c|c|c|c|c|c|}
\hline \multirow[t]{4}{*}{ F2 ID } & \multirow[t]{4}{*}{$\mathrm{x}$} & \multirow[t]{4}{*}{$\mathrm{y}$} & $2 \mathrm{Ks} 10(\sigma)$ & $2 \mathrm{Ks} 01(\sigma)$ & $2 \mathrm{Ks} 02(\sigma)$ & $2 \mathrm{Ks} 03(\sigma)$ & $2 \mathrm{Ks} 05(\sigma)$ & $\overline{2 \mathrm{Ks} 06(\sigma)}$ \\
\hline & & & $2 \mathrm{Ks} 07(\sigma)$ & $2 \mathrm{Ks} 08(\sigma)$ & $2 \mathrm{Ks} 09(\sigma)$ & $2 \mathrm{Ks} 11(\sigma)$ & $2 \mathrm{Ks} 12(\sigma)$ & $2 \mathrm{Ks} 13(\sigma)$ \\
\hline & & & $2 \mathrm{Ks} 14(\sigma)$ & $2 \mathrm{Ks} 15(\sigma)$ & $2 \mathrm{Ks} 16(\sigma)$ & $2 \mathrm{Ks} 17(\sigma)$ & $2 \mathrm{Ks} 18(\sigma)$ & $2 \mathrm{Ks} 19(\sigma)$ \\
\hline & & & $2 \mathrm{Ks} 20(\sigma)$ & $2 \mathrm{Ks} 21(\sigma)$ & $2 \mathrm{Ks} 22(\sigma)$ & $2 \mathrm{Ks} 23(\sigma)$ & $2 \mathrm{Ks} 24(\sigma)$ & $2 \mathrm{Ks} 04(\sigma)$ \\
\hline \multirow[t]{4}{*}{$\overline{504}$} & \multirow[t]{4}{*}{433.530} & \multirow[t]{4}{*}{766.677} & $19.50(0.05)$ & $19.45(0.05)$ & $19.56(0.06)$ & $19.49(0.05)$ & $19.52(0.05)$ & 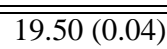 \\
\hline & & & $19.50(0.05)$ & $19.46(0.05)$ & $19.44(0.05)$ & $19.99(0.05)$ & $19.77(0.05)$ & $19.59(0.05)$ \\
\hline & & & $20.06(0.06)$ & $20.09(0.05)$ & $19.98(0.06)$ & $20.15(0.09)$ & $20.11(0.07)$ & $20.00(0.06)$ \\
\hline & & & $19.93(0.05)$ & $20.02(0.06)$ & $19.91(0.05)$ & $20.11(0.07)$ & $19.91(0.07)$ & $20.66(0.47)$ \\
\hline \multirow[t]{4}{*}{505} & \multirow[t]{4}{*}{724.158} & \multirow[t]{4}{*}{628.283} & $19.54(0.04)$ & $19.52(0.05)$ & $19.60(0.05)$ & $19.72(0.07)$ & $19.42(0.04)$ & $19.48(0.05)$ \\
\hline & & & $19.38(0.05)$ & $19.66(0.05)$ & $19.43(0.05)$ & $19.78(0.05)$ & $19.61(0.05)$ & $19.45(0.04)$ \\
\hline & & & $20.58(0.07)$ & $20.45(0.05)$ & $20.41(0.05)$ & $20.47(0.06)$ & $20.54(0.06)$ & $20.57(0.06)$ \\
\hline & & & $20.27(0.06)$ & $20.40(0.08)$ & $20.03(0.05)$ & $19.78(0.05)$ & $19.61(0.05)$ & $20.00(0.08)$ \\
\hline \multirow[t]{4}{*}{508} & \multirow[t]{4}{*}{199.389} & \multirow[t]{4}{*}{-20.741} & 99.99 (9.99) & $20.24(0.10)$ & 99.99 (9.99) & 99.99 (9.99) & 99.99 (9.99) & 99.99 (9.99) \\
\hline & & & 99.99 (9.99) & $99.99(9.99)$ & 99.99 (9.99) & $19.82(0.06)$ & $20.06(0.06)$ & $20.22(0.05)$ \\
\hline & & & $19.54(0.05)$ & $19.24(0.05)$ & $19.46(0.05)$ & 99.99 (9.99) & $19.41(0.05)$ & $19.52(0.05)$ \\
\hline & & & $19.68(0.06)$ & $19.73(0.05)$ & $19.84(0.05)$ & $19.88(0.05)$ & $19.93(0.05)$ & $19.96(0.17)$ \\
\hline \multirow[t]{4}{*}{509} & \multirow[t]{4}{*}{612.706} & \multirow[t]{4}{*}{217.313} & $19.26(0.10)$ & $19.89(0.17)$ & $19.86(0.12)$ & $19.63(0.14)$ & $18.92(0.12)$ & 99.99 (9.99) \\
\hline & & & $19.42(0.08)$ & 99.99 (9.99) & $19.50(0.15)$ & $19.20(0.09)$ & $19.84(0.08)$ & $19.68(0.16)$ \\
\hline & & & 99.99 (9.99) & $20.33(0.22)$ & $19.75(0.16)$ & $20.44(0.35)$ & $20.50(0.18)$ & $20.57(0.18)$ \\
\hline & & & $20.26(0.10)$ & $20.61(0.22)$ & $20.06(0.09)$ & 99.99 (9.99) & 99.99 (9.99) & $20.01(0.17)$ \\
\hline \multirow[t]{4}{*}{510} & \multirow[t]{4}{*}{553.145} & \multirow[t]{4}{*}{557.418} & $20.05(0.05)$ & $19.27(0.06)$ & $19.26(0.06)$ & $19.24(0.05)$ & $20.31(0.05)$ & $20.43(0.06)$ \\
\hline & & & $20.43(0.07)$ & $20.61(0.08)$ & $20.53(0.06)$ & $19.84(0.05)$ & $19.87(0.05)$ & $20.14(0.05)$ \\
\hline & & & $20.04(0.05)$ & $19.90(0.05)$ & $19.71(0.05)$ & $19.60(0.04)$ & $19.58(0.05)$ & $19.57(0.04)$ \\
\hline & & & $19.51(0.05)$ & $19.43(0.05)$ & $19.41(0.04)$ & $19.49(0.05)$ & $19.46(0.05)$ & $20.73(0.17)$ \\
\hline 511 & 559.647 & 843.494 & $19.50(0.04)$ & $19.40(0.06)$ & $19.58(0.05)$ & $19.65(0.06)$ & $19.50(0.05)$ & $19.65(0.04)$ \\
\hline & & & $19.43(0.04)$ & $19.52(0.05)$ & $19.46(0.04)$ & $19.99(0.04)$ & $19.74(0.05)$ & $19.51(0.04)$ \\
\hline & & & $20.10(0.05)$ & $20.10(0.05)$ & $20.21(0.05)$ & $20.21(0.06)$ & $20.10(0.05)$ & $20.03(0.05)$ \\
\hline & & & $19.96(0.05)$ & $19.93(0.07)$ & $19.93(0.05)$ & $19.97(0.05)$ & 99.99 (9.99) & $19.73(0.09)$ \\
\hline 514 & 621.504 & 185.202 & $19.99(0.05)$ & $19.94(0.07)$ & $20.01(0.08)$ & $20.10(0.06)$ & $19.47(0.05)$ & $19.63(0.05)$ \\
\hline & & & $19.70(0.05)$ & $19.88(0.05)$ & $19.81(0.05)$ & $19.90(0.29)$ & $19.44(0.05)$ & $19.58(0.05)$ \\
\hline & & & $19.85(0.05)$ & $19.91(0.05)$ & $19.97(0.05)$ & $19.98(0.05)$ & $19.96(0.05)$ & $19.70(0.04)$ \\
\hline & & & $19.61(0.05)$ & $19.70(0.05)$ & $19.49(0.04)$ & $19.40(0.05)$ & $19.45(0.05)$ & $19.53(0.08)$ \\
\hline 515 & 217.426 & 544.319 & $20.02(0.04)$ & $20.06(0.06)$ & $20.31(0.07)$ & $20.45(0.09)$ & $19.47(0.04)$ & $19.61(0.05)$ \\
\hline & & & $19.70(0.04)$ & $19.82(0.06)$ & $19.80(0.05)$ & $19.44(0.04)$ & $19.53(0.05)$ & $19.75(0.04)$ \\
\hline & & & $20.15(0.06)$ & $20.13(0.05)$ & $20.05(0.05)$ & $19.93(0.06)$ & $19.80(0.05)$ & $19.71(0.05)$ \\
\hline & & & $19.57(0.05)$ & $19.57(0.05)$ & $19.49(0.05)$ & $19.45(0.05)$ & $19.47(0.05)$ & $19.49(0.09)$ \\
\hline 517 & 711.276 & 414.502 & $19.70(0.05)$ & $19.75(0.06)$ & $19.89(0.07)$ & $20.32(0.09)$ & $19.35(0.05)$ & $19.39(0.04)$ \\
\hline & & & $19.44(0.05)$ & $19.50(0.05)$ & $19.49(0.04)$ & $19.66(0.05)$ & $19.64(0.05)$ & $19.54(0.05)$ \\
\hline & & & $20.18(0.06)$ & $20.31(0.05)$ & $20.24(0.05)$ & $20.34(0.06)$ & $20.48(0.06)$ & $20.19(0.06)$ \\
\hline & & & $20.36(0.07)$ & $20.27(0.06)$ & $20.18(0.06)$ & $19.85(0.05)$ & $19.68(0.05)$ & $19.52(0.09)$ \\
\hline 518 & 288.647 & 647.864 & $20.14(0.05)$ & $19.62(0.06)$ & $19.47(0.05)$ & $19.57(0.05)$ & $20.14(0.06)$ & $20.21(0.05)$ \\
\hline & & & $20.35(0.06)$ & $20.45(0.07)$ & $20.20(0.06)$ & $19.29(0.04)$ & $19.36(0.05)$ & $19.56(0.05)$ \\
\hline & & & $20.17(0.05)$ & $20.12(0.05)$ & $20.06(0.05)$ & $19.94(0.05)$ & $19.88(0.05)$ & $19.76(0.05)$ \\
\hline & & & $19.55(0.05)$ & $19.57(0.05)$ & $19.49(0.04)$ & $19.46(0.05)$ & $19.39(0.05)$ & $19.87(0.09)$ \\
\hline 519 & 116.502 & 331.936 & $20.40(0.05)$ & $20.33(0.06)$ & $20.18(0.08)$ & $20.24(0.06)$ & $19.91(0.05)$ & $20.03(0.05)$ \\
\hline & & & $20.19(0.07)$ & $20.28(0.07)$ & $20.28(0.05)$ & $19.52(0.04)$ & $19.60(0.05)$ & $19.82(0.05)$ \\
\hline & & & $20.24(0.05)$ & $20.01(0.05)$ & $19.94(0.05)$ & $19.78(0.06)$ & $19.63(0.05)$ & $19.57(0.05)$ \\
\hline & & & $19.39(0.04)$ & $19.46(0.05)$ & $19.42(0.05)$ & $19.42(0.05)$ & $19.43(0.04)$ & $19.61(0.06)$ \\
\hline 525 & 827.538 & 654.031 & $20.34(0.06)$ & $20.39(0.10)$ & $20.35(0.08)$ & $20.28(0.10)$ & $20.26(0.07)$ & $20.45(0.08)$ \\
\hline & & & $20.51(0.09)$ & $20.78(0.12)$ & $20.56(0.08)$ & $19.70(0.05)$ & $19.87(0.06)$ & $20.07(0.05)$ \\
\hline & & & $19.72(0.05)$ & $19.61(0.05)$ & $19.54(0.05)$ & $19.64(0.08)$ & $19.56(0.06)$ & $19.45(0.05)$ \\
\hline & & & $19.44(0.05)$ & $19.48(0.05)$ & $19.47(0.05)$ & $19.53(0.05)$ & $19.69(0.05)$ & $19.89(0.09)$ \\
\hline
\end{tabular}


Table 8. continuation.

\begin{tabular}{|c|c|c|c|c|c|c|c|c|}
\hline \multirow[t]{4}{*}{$\overline{\mathrm{F} 2 \mathrm{ID}}$} & \multirow[t]{4}{*}{$\mathrm{x}$} & \multirow[t]{4}{*}{$\mathrm{y}$} & $2 \mathrm{Ks} 10(\sigma)$ & $2 \mathrm{Ks} 01(\sigma)$ & $2 \mathrm{Ks} 02(\sigma)$ & $2 \mathrm{Ks} 03(\sigma)$ & $2 \mathrm{Ks} 05(\sigma)$ & $2 \mathrm{Ks06}(\sigma)$ \\
\hline & & & $2 \mathrm{Ks} 07(\sigma)$ & $2 \mathrm{Ks} 08(\sigma)$ & $2 \mathrm{Ks} 09(\sigma)$ & $2 \mathrm{Ks} 11(\sigma)$ & $2 \mathrm{Ks} 12(\sigma)$ & $2 \mathrm{Ks} 13(\sigma)$ \\
\hline & & & $2 \mathrm{Ks} 14(\sigma)$ & $2 \mathrm{Ks} 15(\sigma)$ & $2 \mathrm{Ks} 16(\sigma)$ & $2 \mathrm{Ks} 17(\sigma)$ & $2 \mathrm{Ks} 18(\sigma)$ & $2 \mathrm{Ks} 19(\sigma)$ \\
\hline & & & $2 \mathrm{Ks} 20(\sigma)$ & $2 \mathrm{Ks} 21(\sigma)$ & $2 \mathrm{Ks} 22(\sigma)$ & $2 \mathrm{Ks} 23(\sigma)$ & $2 \mathrm{Ks} 24(\sigma)$ & $2 \mathrm{Ks} 04(\sigma)$ \\
\hline \multirow[t]{4}{*}{$\overline{527}$} & $\overline{610.340}$ & $\begin{array}{l}199.084 \\
\end{array}$ & $19.36(0.09)$ & $19.41(0.12)$ & $19.69(0.11)$ & $19.07(0.08)$ & $18.94(0.11)$ & 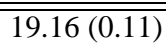 \\
\hline & & & $19.20(0.08)$ & 99.99 (9.99) & $19.09(0.09)$ & $19.10(0.09)$ & $20.19(0.11)$ & $19.59(0.09)$ \\
\hline & & & $19.34(0.09)$ & $19.81(0.08)$ & $19.77(0.19)$ & $20.12(0.17)$ & $19.83(0.12)$ & $19.79(0.09)$ \\
\hline & & & $19.92(0.07)$ & $20.04(0.13)$ & $19.83(0.07)$ & $20.44(0.06)$ & $20.02(0.06)$ & $19.40(0.15)$ \\
\hline \multirow[t]{4}{*}{528} & 810.748 & 227.502 & $19.58(0.05)$ & $19.79(0.05)$ & $19.57(0.06)$ & $19.67(0.06)$ & $19.73(0.05)$ & $19.52(0.05)$ \\
\hline & & & $19.54(0.05)$ & $19.58(0.05)$ & $19.54(0.05)$ & $19.64(0.05)$ & $19.65(0.05)$ & $19.55(0.06)$ \\
\hline & & & $19.92(0.06)$ & $20.00(0.06)$ & $20.19(0.06)$ & $20.16(0.06)$ & $20.26(0.07)$ & $19.92(0.06)$ \\
\hline & & & $19.77(0.05)$ & $19.99(0.07)$ & $19.77(0.05)$ & $19.69(0.05)$ & $19.65(0.05)$ & $20.08(0.07)$ \\
\hline \multirow[t]{4}{*}{529} & 446.288 & 331.541 & $19.51(0.04)$ & $19.85(0.05)$ & $19.87(0.06)$ & $19.82(0.06)$ & $19.68(0.04)$ & $19.57(0.04)$ \\
\hline & & & $19.64(0.05)$ & $19.49(0.05)$ & $19.52(0.05)$ & $19.58(0.05)$ & $19.82(0.05)$ & $20.23(0.05)$ \\
\hline & & & $20.11(0.05)$ & $20.20(0.05)$ & $20.10(0.04)$ & $19.96(0.06)$ & $19.84(0.05)$ & $19.67(0.05)$ \\
\hline & & & $19.60(0.05)$ & $19.59(0.05)$ & $19.56(0.05)$ & $19.50(0.05)$ & $19.51(0.05)$ & $19.60(0.07)$ \\
\hline \multirow[t]{4}{*}{531} & 141.483 & 398.036 & $20.24(0.05)$ & $19.80(0.05)$ & $20.10(0.07)$ & $20.18(0.06)$ & $19.55(0.04)$ & $19.74(0.05)$ \\
\hline & & & $19.79(0.05)$ & $20.09(0.06)$ & $19.98(0.05)$ & $19.42(0.04)$ & $19.43(0.05)$ & $19.58(0.04)$ \\
\hline & & & $20.09(0.05)$ & $20.01(0.05)$ & $19.99(0.05)$ & $19.92(0.05)$ & $19.82(0.05)$ & $19.69(0.05)$ \\
\hline & & & $19.64(0.05)$ & $19.56(0.05)$ & $19.48(0.04)$ & $19.51(0.05)$ & $19.42(0.05)$ & $19.46(0.05)$ \\
\hline \multirow[t]{4}{*}{532} & 787.989 & 400.477 & $19.93(0.04)$ & $19.93(0.05)$ & $19.69(0.06)$ & $19.54(0.05)$ & $20.09(0.05)$ & $19.95(0.05)$ \\
\hline & & & $20.20(0.05)$ & $20.13(0.06)$ & $20.02(0.05)$ & $20.10(0.05)$ & $20.10(0.05)$ & $19.99(0.05)$ \\
\hline & & & $19.39(0.04)$ & $19.41(0.04)$ & $19.40(0.04)$ & $19.43(0.05)$ & $19.56(0.04)$ & $19.58(0.05)$ \\
\hline & & & $19.75(0.05)$ & $19.68(0.05)$ & $19.77(0.05)$ & $19.88(0.05)$ & $20.07(0.06)$ & $20.21(0.10)$ \\
\hline \multirow[t]{4}{*}{542} & 854.465 & 430.490 & $19.79(0.05)$ & $19.88(0.08)$ & $19.92(0.05)$ & $20.08(0.07)$ & $19.50(0.05)$ & $19.47(0.05)$ \\
\hline & & & $19.53(0.05)$ & $19.65(0.05)$ & $19.62(0.06)$ & $19.49(0.04)$ & $19.48(0.05)$ & $19.44(0.05)$ \\
\hline & & & $20.29(0.07)$ & $20.24(0.05)$ & $20.22(0.06)$ & $20.27(0.06)$ & $20.35(0.06)$ & $20.27(0.05)$ \\
\hline & & & $19.93(0.05)$ & $20.16(0.07)$ & $19.83(0.05)$ & $19.77(0.05)$ & $19.61(0.05)$ & $19.59(0.07)$ \\
\hline \multirow[t]{4}{*}{543} & 446.794 & 197.546 & $19.42(0.04)$ & $19.57(0.05)$ & $19.50(0.05)$ & $19.51(0.06)$ & $19.71(0.05)$ & $19.58(0.05)$ \\
\hline & & & $19.54(0.05)$ & $19.39(0.05)$ & $19.42(0.05)$ & $20.19(0.05)$ & $20.07(0.06)$ & $19.79(0.04)$ \\
\hline & & & $20.16(0.06)$ & $20.04(0.05)$ & $19.98(0.04)$ & $19.94(0.05)$ & $19.99(0.06)$ & $19.98(0.05)$ \\
\hline & & & $19.88(0.06)$ & $20.01(0.05)$ & $20.00(0.05)$ & $19.83(0.05)$ & $19.60(0.05)$ & $20.22(0.12)$ \\
\hline \multirow[t]{4}{*}{545} & 319.482 & 579.227 & $19.72(0.05)$ & $19.60(0.06)$ & $19.61(0.06)$ & $19.93(0.07)$ & $20.25(0.05)$ & $20.21(0.05)$ \\
\hline & & & $20.25(0.06)$ & $19.98(0.05)$ & $19.84(0.05)$ & $20.59(0.06)$ & $20.61(0.07)$ & $20.58(0.06)$ \\
\hline & & & $19.90(0.05)$ & $19.74(0.05)$ & $19.60(0.04)$ & $19.52(0.05)$ & $19.52(0.04)$ & $19.51(0.04)$ \\
\hline & & & $19.49(0.05)$ & $19.46(0.05)$ & $19.52(0.04)$ & $19.64(0.05)$ & $19.70(0.05)$ & $20.20(0.12)$ \\
\hline \multirow[t]{4}{*}{546} & 487.030 & 631.010 & $19.45(0.04)$ & $19.58(0.05)$ & $19.41(0.05)$ & $19.44(0.05)$ & $19.79(0.05)$ & $19.88(0.05)$ \\
\hline & & & $19.69(0.05)$ & $19.68(0.05)$ & $19.62(0.05)$ & $19.89(0.05)$ & $19.81(0.05)$ & $19.92(0.05)$ \\
\hline & & & $19.51(0.05)$ & $19.56(0.04)$ & $19.59(0.05)$ & $19.76(0.05)$ & $19.84(0.05)$ & $19.98(0.05)$ \\
\hline & & & $20.19(0.05)$ & $19.98(0.06)$ & $20.15(0.05)$ & $20.18(0.06)$ & $20.12(0.06)$ & $19.98(0.09)$ \\
\hline \multirow[t]{4}{*}{548} & 892.660 & 672.237 & $99.99(9.99)$ & 99.99 (9.99) & $20.74(0.19)$ & $20.40(0.14)$ & $19.73(0.09)$ & $19.04(0.07)$ \\
\hline & & & $20.59(0.17)$ & $18.48(0.33)$ & $20.55(2.89)$ & $21.08(0.20)$ & $21.69(0.20)$ & $20.69(0.21)$ \\
\hline & & & $99.99(9.99)$ & 99.99 (9.99) & $18.99(0.10)$ & 99.99 (9.99) & $20.62(0.13)$ & $20.62(0.20)$ \\
\hline & & & 99.99 (9.99) & 99.99 (9.99) & $20.13(5.03)$ & 99.99 (9.99) & $22.19(0.41)$ & $20.49(0.24)$ \\
\hline \multirow[t]{4}{*}{551} & 376.536 & 391.737 & $19.44(0.05)$ & $19.78(0.05)$ & $19.90(0.06)$ & $20.08(0.06)$ & $19.70(0.06)$ & $19.63(0.04)$ \\
\hline & & & $19.64(0.05)$ & $19.54(0.05)$ & $19.49(0.05)$ & $20.04(0.05)$ & $20.11(0.06)$ & $20.43(0.07)$ \\
\hline & & & $20.68(0.07)$ & $20.52(0.06)$ & $20.55(0.06)$ & $20.24(0.06)$ & $20.06(0.06)$ & $19.75(0.05)$ \\
\hline & & & $19.56(0.05)$ & $19.63(0.05)$ & $19.55(0.05)$ & $19.58(0.05)$ & $19.48(0.05)$ & $20.34(0.16)$ \\
\hline \multirow[t]{4}{*}{554} & 276.199 & 581.529 & $20.30(0.05)$ & $20.14(0.07)$ & $20.12(0.06)$ & $19.91(0.05)$ & $20.08(0.05)$ & $20.39(0.05)$ \\
\hline & & & $20.57(0.08)$ & $20.50(0.09)$ & $20.38(0.06)$ & $19.85(0.05)$ & $19.90(0.05)$ & $20.11(0.05)$ \\
\hline & & & $19.97(0.05)$ & $19.86(0.05)$ & $19.64(0.04)$ & $19.50(0.04)$ & $19.48(0.04)$ & $19.51(0.05)$ \\
\hline & & & $19.37(0.05)$ & $19.36(0.05)$ & $19.47(0.05)$ & $19.60(0.05)$ & $19.78(0.05)$ & $19.69(0.08)$ \\
\hline
\end{tabular}


Table 8. continuation.

\begin{tabular}{|c|c|c|c|c|c|c|c|c|}
\hline \multirow[t]{4}{*}{ F2 ID } & \multirow[t]{4}{*}{$\mathrm{x}$} & \multirow[t]{4}{*}{$\mathrm{y}$} & $2 \mathrm{Ks} 10(\sigma)$ & $2 \mathrm{Ks} 01(\sigma)$ & $2 \mathrm{Ks} 02(\sigma)$ & $2 \mathrm{Ks} 03(\sigma)$ & $2 \mathrm{Ks} 05(\sigma)$ & 2Ks06 $(\sigma)$ \\
\hline & & & $2 \mathrm{Ks} 07(\sigma)$ & $2 \mathrm{Ks} 08(\sigma)$ & $2 \mathrm{Ks} 09(\sigma)$ & $2 \mathrm{Ks} 11(\sigma)$ & $2 \mathrm{Ks} 12(\sigma)$ & $2 \mathrm{Ks} 13(\sigma)$ \\
\hline & & & $2 \mathrm{Ks} 14(\sigma)$ & $2 \mathrm{Ks} 15(\sigma)$ & $2 \mathrm{Ks} 16(\sigma)$ & $2 \mathrm{Ks} 17(\sigma)$ & $2 \mathrm{Ks} 18(\sigma)$ & $2 \mathrm{Ks} 19(\sigma)$ \\
\hline & & & $2 \mathrm{Ks} 20(\sigma)$ & $2 \mathrm{Ks} 21(\sigma)$ & $2 \mathrm{Ks} 22(\sigma)$ & $2 \mathrm{Ks} 23(\sigma)$ & $2 \mathrm{Ks} 24(\sigma)$ & $2 \mathrm{Ks} 04(\sigma)$ \\
\hline \multirow[t]{4}{*}{$\overline{5667}$} & 828.328 & 263.400 & $20.18(0.05)$ & $20.12(0.07)$ & $20.08(0.06)$ & $20.08(0.07)$ & $20.05(0.05)$ & 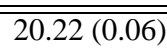 \\
\hline & & & $20.29(0.06)$ & $20.43(0.07)$ & $20.22(0.06)$ & $19.70(0.05)$ & $19.78(0.05)$ & $20.11(0.06)$ \\
\hline & & & $19.97(0.05)$ & $19.94(0.05)$ & $19.80(0.05)$ & $19.71(0.07)$ & $19.65(0.06)$ & $19.47(0.05)$ \\
\hline & & & $19.47(0.05)$ & $19.46(0.05)$ & $19.42(0.05)$ & $19.42(0.05)$ & $19.53(0.05)$ & $19.71(0.07)$ \\
\hline \multirow[t]{4}{*}{570} & 331.109 & 213.328 & $20.33(0.06)$ & $20.23(0.08)$ & $20.42(0.07)$ & $20.30(0.08)$ & $19.78(0.06)$ & $19.89(0.06)$ \\
\hline & & & $19.91(0.07)$ & $20.10(0.06)$ & $20.15(0.06)$ & $19.58(0.05)$ & $19.73(0.06)$ & $19.95(0.06)$ \\
\hline & & & $19.92(0.06)$ & $19.82(0.05)$ & $19.90(0.06)$ & $19.74(0.05)$ & $19.72(0.05)$ & $19.58(0.05)$ \\
\hline & & & $19.46(0.05)$ & $19.57(0.05)$ & $19.49(0.05)$ & $19.49(0.05)$ & $19.63(0.05)$ & $19.51(0.12)$ \\
\hline \multirow[t]{4}{*}{577} & 10.390 & 631.845 & $20.14(0.07)$ & $20.10(0.08)$ & $20.12(0.09)$ & $20.16(0.09)$ & $19.50(0.11)$ & $19.88(0.05)$ \\
\hline & & & $19.95(0.06)$ & $19.93(0.06)$ & $20.13(0.06)$ & 99.99 (9.99) & $19.48(0.39)$ & 99.99 (9.99) \\
\hline & & & $19.92(0.06)$ & $20.05(0.06)$ & $19.83(0.07)$ & 99.99 (9.99) & $19.66(0.09)$ & $19.76(0.05)$ \\
\hline & & & $19.86(0.06)$ & $19.78(0.07)$ & $19.79(0.05)$ & $17.79(0.06)$ & $19.45(0.05)$ & 99.99 (9.99) \\
\hline \multirow[t]{4}{*}{579} & 464.394 & 206.324 & $20.25(0.06)$ & $19.81(0.06)$ & $19.85(0.06)$ & $19.87(0.06)$ & $20.32(0.05)$ & $20.23(0.06)$ \\
\hline & & & $20.23(0.07)$ & $20.23(0.07)$ & $20.23(0.07)$ & $19.57(0.07)$ & $20.00(0.05)$ & $20.10(0.06)$ \\
\hline & & & $19.85(0.05)$ & $19.78(0.05)$ & $19.79(0.05)$ & $19.67(0.06)$ & $19.64(0.06)$ & $19.53(0.05)$ \\
\hline & & & $19.49(0.05)$ & $19.52(0.06)$ & $19.54(0.05)$ & $19.50(0.05)$ & $19.59(0.05)$ & $20.22(0.09)$ \\
\hline \multirow[t]{4}{*}{584} & 50.053 & 187.361 & $22.05(0.42)$ & $22.64(0.45)$ & $22.92(1.79)$ & $21.88(0.64)$ & $22.26(0.48)$ & $17.82(0.16)$ \\
\hline & & & $23.32(1.22)$ & $18.43(0.23)$ & 99.99 (9.99) & $18.71(0.15)$ & $20.48(0.11)$ & $21.98(2.51)$ \\
\hline & & & $18.58(0.13)$ & $20.90(1.51)$ & $21.39(0.27)$ & $99.99(9.99)$ & 24.75 (5.64) & $21.14(0.17)$ \\
\hline & & & $20.43(0.09)$ & $23.81(4.31)$ & $18.32(0.08)$ & $21.49(0.16)$ & $20.17(0.07)$ & $20.31(0.19)$ \\
\hline \multirow[t]{4}{*}{593} & 826.095 & 702.382 & $20.13(0.05)$ & $20.27(0.10)$ & $20.23(0.09)$ & $20.28(0.08)$ & $20.04(0.05)$ & $20.27(0.05)$ \\
\hline & & & $20.09(0.06)$ & $20.55(0.08)$ & $20.27(0.06)$ & $20.01(0.05)$ & $19.90(0.05)$ & $20.13(0.06)$ \\
\hline & & & $19.61(0.05)$ & $19.52(0.04)$ & $19.54(0.06)$ & $19.69(0.08)$ & $19.52(0.05)$ & $19.53(0.05)$ \\
\hline & & & $19.48(0.05)$ & $19.52(0.06)$ & $19.62(0.05)$ & $19.60(0.05)$ & $19.89(0.05)$ & $19.71(0.09)$ \\
\hline \multirow[t]{4}{*}{594} & 250.736 & 727.544 & $20.18(0.05)$ & $19.84(0.05)$ & $19.94(0.06)$ & $19.99(0.05)$ & $19.64(0.04)$ & $19.89(0.05)$ \\
\hline & & & $19.89(0.05)$ & $19.92(0.05)$ & $19.99(0.04)$ & $19.52(0.04)$ & $19.56(0.05)$ & $19.86(0.05)$ \\
\hline & & & $20.02(0.05)$ & $20.05(0.05)$ & $19.88(0.05)$ & $19.71(0.04)$ & $19.65(0.05)$ & $19.62(0.04)$ \\
\hline & & & $19.57(0.05)$ & $19.47(0.05)$ & $19.53(0.05)$ & $19.65(0.04)$ & $19.72(0.05)$ & $19.66(0.06)$ \\
\hline \multirow[t]{4}{*}{597} & 137.681 & 476.851 & $20.26(0.05)$ & $19.56(0.05)$ & $19.60(0.05)$ & $19.52(0.05)$ & $19.97(0.05)$ & $20.01(0.05)$ \\
\hline & & & $20.23(0.06)$ & $20.25(0.06)$ & $20.22(0.05)$ & $19.28(0.04)$ & $19.26(0.04)$ & $19.44(0.04)$ \\
\hline & & & $20.38(0.07)$ & $20.47(0.05)$ & $20.41(0.06)$ & $20.40(0.06)$ & $20.41(0.05)$ & $20.48(0.06)$ \\
\hline & & & $20.46(0.07)$ & $20.41(0.07)$ & $20.25(0.05)$ & $19.95(0.05)$ & $19.67(0.05)$ & $19.84(0.07)$ \\
\hline \multirow[t]{4}{*}{598} & 576.358 & 405.224 & $19.92(0.05)$ & $19.70(0.05)$ & $19.93(0.06)$ & $20.28(0.07)$ & $19.51(0.05)$ & $19.65(0.05)$ \\
\hline & & & $19.75(0.05)$ & $19.99(0.06)$ & $19.79(0.05)$ & $19.51(0.04)$ & $19.48(0.05)$ & $19.59(0.05)$ \\
\hline & & & $20.38(0.06)$ & $20.30(0.06)$ & $20.20(0.05)$ & $20.10(0.06)$ & $20.09(0.06)$ & $19.99(0.05)$ \\
\hline & & & $19.90(0.06)$ & $19.94(0.06)$ & $19.81(0.05)$ & $19.69(0.05)$ & $19.72(0.05)$ & $19.57(0.06)$ \\
\hline \multirow[t]{4}{*}{601} & 227.961 & 389.674 & $20.02(0.04)$ & $19.81(0.07)$ & $20.06(0.06)$ & $20.24(0.06)$ & $19.52(0.05)$ & $19.64(0.05)$ \\
\hline & & & $19.75(0.05)$ & $19.92(0.06)$ & $19.81(0.05)$ & $19.51(0.04)$ & $19.48(0.05)$ & $19.68(0.04)$ \\
\hline & & & $20.22(0.05)$ & $20.26(0.05)$ & $20.18(0.04)$ & $20.03(0.05)$ & $19.90(0.05)$ & $19.73(0.04)$ \\
\hline & & & $19.51(0.05)$ & $19.57(0.05)$ & $19.47(0.05)$ & $19.48(0.05)$ & $19.47(0.05)$ & $19.46(0.08)$ \\
\hline \multirow[t]{4}{*}{602} & 327.154 & 294.349 & $20.01(0.05)$ & $19.88(0.06)$ & $20.06(0.06)$ & $20.24(0.08)$ & $19.61(0.05)$ & $19.68(0.05)$ \\
\hline & & & $19.79(0.05)$ & $19.79(0.05)$ & $19.85(0.05)$ & $19.50(0.05)$ & $19.52(0.05)$ & $19.73(0.05)$ \\
\hline & & & $20.15(0.06)$ & $20.15(0.05)$ & $20.16(0.05)$ & $20.07(0.05)$ & $20.00(0.06)$ & $19.81(0.05)$ \\
\hline & & & $19.63(0.05)$ & $19.68(0.05)$ & $19.66(0.04)$ & $19.70(0.05)$ & $19.65(0.05)$ & $19.34(0.07)$ \\
\hline \multirow[t]{4}{*}{603} & 200.621 & 102.823 & $19.46(0.04)$ & $19.54(0.05)$ & $19.54(0.05)$ & $19.60(0.05)$ & $19.96(0.05)$ & $19.63(0.05)$ \\
\hline & & & $19.73(0.05)$ & $19.56(0.05)$ & $19.52(0.05)$ & $19.95(0.05)$ & $19.68(0.05)$ & $19.59(0.05)$ \\
\hline & & & $20.06(0.05)$ & $19.96(0.05)$ & $20.18(0.06)$ & $20.19(0.08)$ & $20.07(0.06)$ & $19.96(0.05)$ \\
\hline & & & $19.96(0.06)$ & $19.96(0.06)$ & $19.95(0.05)$ & $20.03(0.05)$ & $19.98(0.05)$ & $20.15(0.10)$ \\
\hline
\end{tabular}


Table 8. continuation.

\begin{tabular}{|c|c|c|c|c|c|c|c|c|}
\hline \multirow[t]{4}{*}{$\overline{\mathrm{F} 2 \mathrm{ID}}$} & \multirow[t]{4}{*}{$\mathrm{x}$} & \multirow[t]{4}{*}{$\mathrm{y}$} & $2 \mathrm{Ks} 10(\sigma)$ & $2 \mathrm{Ks} 01(\sigma)$ & $2 \mathrm{Ks} 02(\sigma)$ & $2 \mathrm{Ks} 03(\sigma)$ & $2 \mathrm{Ks} 05(\sigma)$ & $2 \mathrm{Ks} 06(\sigma)$ \\
\hline & & & $2 \mathrm{Ks} 07(\sigma)$ & $2 \mathrm{Ks} 08(\sigma)$ & $2 \mathrm{Ks} 09(\sigma)$ & $2 \mathrm{Ks} 11(\sigma)$ & $2 \mathrm{Ks} 12(\sigma)$ & $2 \mathrm{Ks} 13(\sigma)$ \\
\hline & & & $2 \mathrm{Ks} 14(\sigma)$ & $2 \mathrm{Ks} 15(\sigma)$ & $2 \mathrm{Ks} 16(\sigma)$ & $2 \mathrm{Ks} 17(\sigma)$ & $2 \mathrm{Ks} 18(\sigma)$ & $2 \mathrm{Ks} 19(\sigma)$ \\
\hline & & & $2 \mathrm{Ks} 20(\sigma)$ & $2 \mathrm{Ks} 21(\sigma)$ & $2 \mathrm{Ks} 22(\sigma)$ & $2 \mathrm{Ks} 23(\sigma)$ & $2 \mathrm{Ks} 24(\sigma)$ & $2 \mathrm{Ks} 04(\sigma)$ \\
\hline \multirow[t]{4}{*}{$\overline{6605}$} & 593.389 & 604.382 & $19.67(0.04)$ & $20.08(0.07)$ & $20.43(0.07)$ & $19.98(0.08)$ & $20.08(0.05)$ & 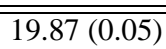 \\
\hline & & & $19.79(0.05)$ & $19.70(0.05)$ & $19.66(0.04)$ & $19.62(0.04)$ & $19.63(0.05)$ & $19.68(0.05)$ \\
\hline & & & $20.28(0.05)$ & $20.09(0.05)$ & $19.89(0.05)$ & $19.76(0.05)$ & $19.75(0.05)$ & $19.64(0.05)$ \\
\hline & & & $19.64(0.05)$ & $19.52(0.05)$ & $19.66(0.05)$ & $19.78(0.05)$ & $20.00(0.05)$ & $20.31(0.08)$ \\
\hline \multirow[t]{4}{*}{607} & 140.220 & 119.771 & $19.45(0.04)$ & $19.65(0.06)$ & $19.51(0.05)$ & $19.41(0.05)$ & $19.99(0.05)$ & $19.78(0.05)$ \\
\hline & & & $19.83(0.05)$ & $19.63(0.06)$ & $19.70(0.05)$ & $19.93(0.05)$ & $19.91(0.05)$ & $19.88(0.05)$ \\
\hline & & & $19.67(0.05)$ & $19.66(0.05)$ & $19.90(0.06)$ & $19.96(0.08)$ & $19.87(0.06)$ & $19.93(0.05)$ \\
\hline & & & $19.99(0.06)$ & $19.94(0.07)$ & $19.98(0.05)$ & $19.81(0.05)$ & $19.89(0.06)$ & $19.80(0.11)$ \\
\hline \multirow[t]{4}{*}{614} & 260.170 & 752.254 & $19.58(0.04)$ & $19.51(0.05)$ & $19.49(0.05)$ & $19.65(0.05)$ & $19.68(0.04)$ & $19.69(0.05)$ \\
\hline & & & $19.51(0.05)$ & $19.54(0.05)$ & $19.52(0.04)$ & $20.07(0.05)$ & $19.78(0.05)$ & $19.55(0.04)$ \\
\hline & & & $20.28(0.05)$ & $20.24(0.05)$ & $20.04(0.05)$ & $20.00(0.05)$ & $19.98(0.05)$ & $20.17(0.05)$ \\
\hline & & & $20.19(0.06)$ & $20.14(0.05)$ & $20.17(0.05)$ & $20.21(0.05)$ & $19.87(0.05)$ & $20.33(0.11)$ \\
\hline \multirow[t]{4}{*}{616} & 74.593 & 149.486 & $19.53(0.05)$ & $19.46(0.06)$ & $19.59(0.05)$ & $19.71(0.05)$ & $19.76(0.05)$ & $19.57(0.05)$ \\
\hline & & & $19.56(0.05)$ & $19.52(0.05)$ & $19.56(0.05)$ & $20.13(0.06)$ & $19.87(0.05)$ & $19.67(0.05)$ \\
\hline & & & $19.84(0.05)$ & $19.84(0.05)$ & $20.08(0.05)$ & $20.29(0.08)$ & $20.11(0.06)$ & $20.16(0.05)$ \\
\hline & & & $20.12(0.06)$ & $20.21(0.06)$ & $20.15(0.05)$ & $20.13(0.05)$ & $20.03(0.06)$ & $19.95(0.15)$ \\
\hline \multirow[t]{4}{*}{620} & 373.103 & 646.696 & $19.53(0.04)$ & $19.40(0.05)$ & $19.42(0.05)$ & $19.49(0.05)$ & $19.79(0.05)$ & $19.75(0.04)$ \\
\hline & & & $19.75(0.05)$ & $19.60(0.05)$ & $19.61(0.05)$ & $20.19(0.05)$ & $20.10(0.06)$ & $20.13(0.05)$ \\
\hline & & & $19.64(0.05)$ & $19.70(0.05)$ & $19.82(0.05)$ & $19.88(0.05)$ & $19.95(0.05)$ & $20.10(0.05)$ \\
\hline & & & $20.24(0.06)$ & $20.25(0.07)$ & $20.22(0.05)$ & $20.14(0.05)$ & $20.08(0.06)$ & $20.03(0.07)$ \\
\hline \multirow[t]{4}{*}{625} & 216.687 & 846.436 & $20.07(0.05)$ & $19.84(0.05)$ & $20.02(0.06)$ & $20.05(0.06)$ & $19.39(0.05)$ & $19.77(0.05)$ \\
\hline & & & $19.69(0.05)$ & $19.61(0.05)$ & $19.78(0.05)$ & $19.63(0.04)$ & $19.55(0.05)$ & $19.50(0.05)$ \\
\hline & & & $20.33(0.06)$ & $20.12(0.05)$ & $19.98(0.06)$ & $20.00(0.05)$ & $19.96(0.06)$ & $20.05(0.05)$ \\
\hline & & & $19.92(0.05)$ & $20.13(0.06)$ & $19.87(0.05)$ & $19.67(0.05)$ & 99.99 (9.99) & $19.54(0.16)$ \\
\hline \multirow[t]{4}{*}{631} & 867.045 & 551.661 & $20.18(0.05)$ & $99.99(9.99)$ & $20.32(0.09)$ & $20.13(0.06)$ & $19.82(0.05)$ & $19.96(0.05)$ \\
\hline & & & $19.94(0.06)$ & $20.14(0.06)$ & $20.15(0.06)$ & $19.31(0.04)$ & $19.29(0.05)$ & $19.31(0.04)$ \\
\hline & & & $20.21(0.06)$ & $20.30(0.05)$ & $20.36(0.07)$ & $20.46(0.07)$ & $20.42(0.06)$ & $20.30(0.05)$ \\
\hline & & & $20.30(0.08)$ & $20.34(0.06)$ & $20.17(0.05)$ & $19.95(0.05)$ & $19.78(0.05)$ & $19.60(0.08)$ \\
\hline \multirow[t]{4}{*}{632} & 684.770 & 31.204 & $20.00(0.05)$ & $20.22(0.06)$ & $19.89(0.06)$ & $19.74(0.06)$ & $20.21(0.05)$ & $20.21(0.05)$ \\
\hline & & & $20.16(0.05)$ & $20.12(0.06)$ & $20.13(0.05)$ & $20.18(0.05)$ & $20.25(0.07)$ & $20.19(0.05)$ \\
\hline & & & $19.50(0.05)$ & $19.52(0.04)$ & $19.55(0.05)$ & $19.54(0.04)$ & $19.58(0.04)$ & $19.49(0.05)$ \\
\hline & & & $19.77(0.05)$ & $19.65(0.05)$ & $19.69(0.05)$ & $19.75(0.05)$ & $20.07(0.06)$ & $20.09(0.10)$ \\
\hline \multirow[t]{4}{*}{639} & 449.303 & 20.019 & $19.40(0.04)$ & $19.83(0.05)$ & $19.66(0.06)$ & $19.49(0.05)$ & $20.10(0.05)$ & $20.10(0.05)$ \\
\hline & & & $19.88(0.05)$ & $19.50(0.05)$ & $19.73(0.05)$ & $20.38(0.06)$ & $20.08(0.05)$ & $20.05(0.05)$ \\
\hline & & & $19.60(0.05)$ & $19.64(0.05)$ & $19.90(0.05)$ & $19.97(0.07)$ & $19.95(0.05)$ & $19.95(0.05)$ \\
\hline & & & $20.05(0.06)$ & $20.09(0.07)$ & $19.92(0.05)$ & $20.00(0.05)$ & $20.08(0.06)$ & $19.96(0.08)$ \\
\hline \multirow[t]{4}{*}{640} & 823.333 & 255.813 & $19.93(0.04)$ & $20.19(0.07)$ & $20.32(0.07)$ & $19.94(0.07)$ & $20.14(0.06)$ & $20.19(0.06)$ \\
\hline & & & $20.09(0.05)$ & $20.16(0.07)$ & $19.98(0.05)$ & $20.19(0.05)$ & $20.08(0.06)$ & $20.20(0.06)$ \\
\hline & & & $19.53(0.05)$ & $19.55(0.04)$ & $19.50(0.04)$ & $19.58(0.05)$ & $19.63(0.05)$ & $19.59(0.05)$ \\
\hline & & & $19.73(0.05)$ & $19.76(0.05)$ & $19.81(0.05)$ & $19.96(0.05)$ & $20.16(0.06)$ & $19.90(0.10)$ \\
\hline \multirow[t]{4}{*}{641} & 863.081 & 172.932 & $19.63(0.05)$ & $99.99(9.99)$ & $19.46(0.05)$ & $19.46(0.06)$ & $19.88(0.05)$ & $19.67(0.05)$ \\
\hline & & & $19.75(0.05)$ & $19.62(0.05)$ & $19.63(0.05)$ & $19.73(0.06)$ & $19.75(0.05)$ & $19.52(0.05)$ \\
\hline & & & $20.25(0.07)$ & $20.54(0.06)$ & $20.56(0.06)$ & $20.52(0.07)$ & $20.61(0.07)$ & $20.28(0.06)$ \\
\hline & & & $20.48(0.08)$ & $20.71(0.09)$ & $20.38(0.06)$ & $20.63(0.08)$ & $20.35(0.08)$ & $20.16(0.10)$ \\
\hline \multirow[t]{4}{*}{644} & 68.446 & 709.041 & $20.11(0.05)$ & $19.96(0.06)$ & $20.04(0.07)$ & $20.16(0.07)$ & $19.68(0.05)$ & $19.84(0.05)$ \\
\hline & & & $19.87(0.05)$ & $20.05(0.06)$ & $19.93(0.05)$ & $19.46(0.04)$ & $19.51(0.05)$ & $19.69(0.04)$ \\
\hline & & & $20.21(0.07)$ & $20.26(0.06)$ & $20.06(0.05)$ & $20.12(0.07)$ & $20.11(0.06)$ & $20.01(0.05)$ \\
\hline & & & $19.80(0.05)$ & $19.82(0.05)$ & $19.67(0.05)$ & $19.68(0.05)$ & $19.61(0.06)$ & $19.84(0.23)$ \\
\hline
\end{tabular}


Table 8. continuation.

\begin{tabular}{|c|c|c|c|c|c|c|c|c|}
\hline \multirow[t]{4}{*}{$\bar{F}$ F2 ID } & \multirow[t]{4}{*}{$\mathrm{x}$} & \multirow[t]{4}{*}{$\mathrm{y}$} & $2 \mathrm{Ks} 10(\sigma)$ & $2 \mathrm{Ks} 01(\sigma)$ & $2 \mathrm{Ks} 02(\sigma)$ & $2 \mathrm{Ks} 03(\sigma)$ & $2 \mathrm{Ks} 05(\sigma)$ & $\overline{2 \mathrm{Ks} 06(\sigma)}$ \\
\hline & & & $2 \mathrm{Ks} 07(\sigma)$ & $2 \mathrm{Ks} 08(\sigma)$ & $2 \mathrm{Ks} 09(\sigma)$ & $2 \mathrm{Ks} 11(\sigma)$ & $2 \mathrm{Ks} 12(\sigma)$ & $2 \mathrm{Ks} 13(\sigma)$ \\
\hline & & & $2 \mathrm{Ks} 14(\sigma)$ & $2 \mathrm{Ks} 15(\sigma)$ & $2 \mathrm{Ks} 16(\sigma)$ & $2 \mathrm{Ks} 17(\sigma)$ & $2 \mathrm{Ks} 18(\sigma)$ & $2 \mathrm{Ks} 19(\sigma)$ \\
\hline & & & $2 \mathrm{Ks} 20(\sigma)$ & $2 \mathrm{Ks} 21(\sigma)$ & $2 \mathrm{Ks} 22(\sigma)$ & $2 \mathrm{Ks} 23(\sigma)$ & $2 \mathrm{Ks} 24(\sigma)$ & $2 \mathrm{Ks} 04(\sigma)$ \\
\hline \multirow[t]{4}{*}{$\overline{645}$} & $\overline{753.390}$ & 308.952 & $19.90(0.04)$ & $19.54(0.05)$ & $\overline{19.78(0.06)}$ & $20.03(0.07)$ & $19.54(0.04)$ & 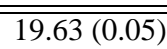 \\
\hline & & & $19.68(0.05)$ & $19.87(0.05)$ & $19.68(0.05)$ & $19.57(0.04)$ & $19.45(0.05)$ & $19.59(0.04)$ \\
\hline & & & $20.49(0.07)$ & $20.57(0.05)$ & $20.58(0.06)$ & $20.65(0.06)$ & $20.70(0.06)$ & $20.67(0.06)$ \\
\hline & & & $20.18(0.06)$ & $20.41(0.07)$ & $20.24(0.05)$ & $20.00(0.05)$ & $19.87(0.05)$ & $19.76(0.08)$ \\
\hline \multirow[t]{4}{*}{647} & 442.445 & -0.474 & $19.97(1.23)$ & $20.64(0.08)$ & $20.88(0.14)$ & $21.53(0.17)$ & $19.53(0.05)$ & $19.73(0.05)$ \\
\hline & & & $19.45(0.47)$ & $20.77(0.51)$ & 99.99 (9.99) & $21.49(0.07)$ & $21.07(0.09)$ & $20.96(0.06)$ \\
\hline & & & $19.64(0.05)$ & $19.54(0.05)$ & $19.67(0.05)$ & 99.99 (9.99) & $19.65(0.04)$ & $19.73(0.05)$ \\
\hline & & & $19.81(0.05)$ & $19.78(0.06)$ & $19.90(0.05)$ & $20.08(0.05)$ & $20.17(0.05)$ & $19.66(0.07)$ \\
\hline \multirow[t]{4}{*}{651} & 501.926 & 11.122 & $19.65(0.04)$ & $19.60(0.06)$ & $19.78(0.05)$ & $19.83(0.06)$ & $19.66(0.05)$ & $19.73(0.05)$ \\
\hline & & & $19.51(0.05)$ & $19.53(0.05)$ & $19.57(0.05)$ & $19.83(0.05)$ & $19.76(0.05)$ & $19.61(0.04)$ \\
\hline & & & $20.57(0.08)$ & $20.47(0.05)$ & $20.65(0.06)$ & $20.60(0.06)$ & $20.62(0.06)$ & $20.56(0.07)$ \\
\hline & & & $20.54(0.08)$ & $20.55(0.08)$ & $20.59(0.06)$ & $20.31(0.06)$ & $20.00(0.05)$ & $19.95(0.07)$ \\
\hline \multirow[t]{4}{*}{653} & 761.328 & 478.053 & $19.72(0.05)$ & $19.59(0.05)$ & $19.56(0.06)$ & $19.64(0.05)$ & $19.67(0.04)$ & $19.64(0.05)$ \\
\hline & & & $19.66(0.05)$ & $19.85(0.05)$ & $19.66(0.05)$ & $19.63(0.04)$ & $19.55(0.05)$ & $19.56(0.04)$ \\
\hline & & & $20.18(0.06)$ & $20.18(0.05)$ & $20.19(0.05)$ & $20.26(0.05)$ & $20.29(0.05)$ & $20.11(0.05)$ \\
\hline & & & $19.94(0.05)$ & $19.87(0.05)$ & $19.85(0.05)$ & $19.80(0.05)$ & $19.71(0.05)$ & $20.22(0.10)$ \\
\hline \multirow[t]{4}{*}{654} & 75.946 & 540.901 & $19.99(0.05)$ & $19.71(0.05)$ & $19.95(0.05)$ & $20.15(0.07)$ & $19.87(0.05)$ & $19.86(0.05)$ \\
\hline & & & $20.04(0.06)$ & $20.09(0.06)$ & $19.99(0.05)$ & $19.81(0.05)$ & $19.84(0.05)$ & $19.87(0.05)$ \\
\hline & & & $19.49(0.05)$ & $19.52(0.05)$ & $19.47(0.05)$ & $19.46(0.06)$ & $19.58(0.05)$ & $19.76(0.05)$ \\
\hline & & & $19.79(0.05)$ & $19.80(0.05)$ & $19.94(0.05)$ & $20.19(0.05)$ & $20.02(0.05)$ & $19.66(0.08)$ \\
\hline \multirow[t]{4}{*}{655} & 45.450 & 186.668 & $99.99(9.99)$ & $19.30(0.36)$ & $19.70(0.23)$ & 99.99 (9.99) & $20.30(0.58)$ & $18.44(0.27)$ \\
\hline & & & $20.33(0.13)$ & 99.99 (9.99) & $19.68(0.24)$ & 99.99 (9.99) & $20.21(0.09)$ & $19.22(0.26)$ \\
\hline & & & $19.47(0.24)$ & $18.17(0.10)$ & 99.99 (9.99) & $19.56(0.35)$ & $18.89(0.12)$ & $20.36(0.13)$ \\
\hline & & & $20.35(0.11)$ & $20.17(0.28)$ & $19.49(0.21)$ & $22.63(0.37)$ & $21.18(0.14)$ & $19.98(0.20)$ \\
\hline \multirow[t]{4}{*}{656} & 607.630 & 715.505 & $20.20(0.05)$ & $20.21(0.09)$ & $20.58(0.09)$ & $20.43(0.08)$ & $19.62(0.05)$ & $19.82(0.05)$ \\
\hline & & & $19.83(0.05)$ & $19.94(0.06)$ & $19.96(0.05)$ & $19.53(0.04)$ & $19.58(0.05)$ & $19.65(0.05)$ \\
\hline & & & $20.18(0.05)$ & $20.09(0.05)$ & $20.08(0.05)$ & $20.06(0.05)$ & $20.04(0.04)$ & $20.03(0.05)$ \\
\hline & & & $19.73(0.05)$ & $19.70(0.05)$ & $19.59(0.05)$ & $19.53(0.05)$ & $19.41(0.05)$ & $19.54(0.06)$ \\
\hline \multirow[t]{4}{*}{660} & 121.747 & 653.525 & $19.88(0.04)$ & $19.51(0.05)$ & $19.53(0.06)$ & $19.62(0.05)$ & $20.47(0.05)$ & $20.34(0.05)$ \\
\hline & & & $20.57(0.06)$ & $20.21(0.08)$ & $20.23(0.06)$ & $20.45(0.06)$ & $20.63(0.07)$ & $20.73(0.06)$ \\
\hline & & & $20.01(0.05)$ & $20.06(0.05)$ & $19.73(0.05)$ & $19.58(0.05)$ & $19.55(0.05)$ & $19.58(0.04)$ \\
\hline & & & $19.72(0.05)$ & $19.50(0.05)$ & $19.56(0.04)$ & $19.69(0.05)$ & $19.75(0.05)$ & $21.09(0.18)$ \\
\hline \multirow[t]{4}{*}{663} & 497.577 & 237.269 & $20.06(0.05)$ & $19.72(0.07)$ & $19.83(0.06)$ & $20.00(0.07)$ & $19.59(0.04)$ & $19.86(0.05)$ \\
\hline & & & $19.88(0.05)$ & $20.09(0.05)$ & $19.98(0.05)$ & $19.63(0.05)$ & $19.72(0.05)$ & $19.90(0.04)$ \\
\hline & & & $20.06(0.06)$ & $20.06(0.05)$ & $20.00(0.04)$ & $19.89(0.05)$ & $19.81(0.05)$ & $19.70(0.05)$ \\
\hline & & & $19.71(0.05)$ & $19.65(0.05)$ & $19.58(0.04)$ & $19.65(0.05)$ & $19.72(0.05)$ & $19.62(0.07)$ \\
\hline \multirow[t]{4}{*}{664} & 624.931 & 400.023 & $19.86(0.04)$ & $20.22(0.06)$ & $20.34(0.09)$ & $20.72(0.11)$ & $19.36(0.05)$ & $19.39(0.04)$ \\
\hline & & & $19.46(0.05)$ & $19.65(0.05)$ & $19.60(0.04)$ & $19.85(0.05)$ & $19.75(0.05)$ & $19.67(0.04)$ \\
\hline & & & $20.23(0.06)$ & $20.35(0.05)$ & $20.50(0.05)$ & $20.54(0.07)$ & $20.59(0.06)$ & $20.57(0.05)$ \\
\hline & & & $20.44(0.08)$ & $20.32(0.07)$ & $20.32(0.05)$ & $20.13(0.06)$ & $20.12(0.05)$ & $19.40(0.07)$ \\
\hline \multirow[t]{4}{*}{665} & 612.844 & 108.361 & $19.61(0.05)$ & $19.61(0.05)$ & $19.41(0.05)$ & $19.36(0.05)$ & $20.08(0.06)$ & $19.81(0.05)$ \\
\hline & & & $19.78(0.06)$ & $19.61(0.06)$ & $19.68(0.05)$ & $19.93(0.05)$ & $19.76(0.05)$ & $19.67(0.05)$ \\
\hline & & & $20.44(0.07)$ & $20.55(0.08)$ & $20.58(0.08)$ & $20.49(0.06)$ & $20.49(0.08)$ & $20.30(0.06)$ \\
\hline & & & $20.26(0.06)$ & $20.30(0.08)$ & $20.15(0.05)$ & $19.91(0.05)$ & $19.69(0.05)$ & $20.51(0.14)$ \\
\hline \multirow[t]{4}{*}{666} & 770.632 & 463.310 & $20.18(0.05)$ & $19.79(0.05)$ & $20.18(0.07)$ & $19.95(0.07)$ & $19.65(0.04)$ & $19.85(0.05)$ \\
\hline & & & $19.88(0.05)$ & $20.02(0.05)$ & $20.05(0.05)$ & $19.93(0.05)$ & $19.96(0.05)$ & $20.08(0.05)$ \\
\hline & & & $19.58(0.05)$ & $19.66(0.05)$ & $19.60(0.05)$ & $19.66(0.04)$ & $19.69(0.04)$ & $19.67(0.05)$ \\
\hline & & & $19.77(0.05)$ & $19.87(0.06)$ & $19.96(0.05)$ & $20.11(0.06)$ & $20.20(0.05)$ & $19.63(0.09)$ \\
\hline
\end{tabular}


Table 8. continuation.

\begin{tabular}{|c|c|c|c|c|c|c|c|c|}
\hline \multirow[t]{4}{*}{$\overline{\mathrm{F} 2 \mathrm{ID}}$} & \multirow[t]{4}{*}{$\mathrm{x}$} & \multirow[t]{4}{*}{$\mathrm{y}$} & $2 \mathrm{Ks} 10(\sigma)$ & $2 \mathrm{Ks} 01(\sigma)$ & $2 \mathrm{Ks} 02(\sigma)$ & $2 \mathrm{Ks} 03(\sigma)$ & $2 \mathrm{Ks} 05(\sigma)$ & $2 \mathrm{Ks06}(\sigma)$ \\
\hline & & & $2 \mathrm{Ks} 07(\sigma)$ & $2 \mathrm{Ks} 08(\sigma)$ & $2 \mathrm{Ks} 09(\sigma)$ & $2 \mathrm{Ks} 11(\sigma)$ & $2 \mathrm{Ks} 12(\sigma)$ & $2 \mathrm{Ks} 13(\sigma)$ \\
\hline & & & $2 \mathrm{Ks} 14(\sigma)$ & $2 \mathrm{Ks} 15(\sigma)$ & $2 \mathrm{Ks} 16(\sigma)$ & $2 \mathrm{Ks} 17(\sigma)$ & $2 \mathrm{Ks} 18(\sigma)$ & $2 \mathrm{Ks} 19(\sigma)$ \\
\hline & & & $2 \mathrm{Ks} 20(\sigma)$ & $2 \mathrm{Ks} 21(\sigma)$ & $2 \mathrm{Ks} 22(\sigma)$ & $2 \mathrm{Ks} 23(\sigma)$ & $2 \mathrm{Ks} 24(\sigma)$ & $2 \mathrm{Ks} 04(\sigma)$ \\
\hline \multirow[t]{4}{*}{$\overline{676}$} & 265.506 & 126.520 & $20.04(0.07)$ & $20.34(0.08)$ & $20.70(0.12)$ & $20.53(0.08)$ & $19.92(0.05)$ & 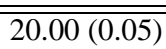 \\
\hline & & & $20.00(0.06)$ & $20.08(0.06)$ & $20.11(0.06)$ & $19.75(0.05)$ & $19.78(0.05)$ & $19.86(0.06)$ \\
\hline & & & $20.00(0.05)$ & $19.89(0.05)$ & $19.93(0.05)$ & $19.82(0.06)$ & $19.76(0.06)$ & $19.67(0.05)$ \\
\hline & & & $19.64(0.05)$ & $19.69(0.06)$ & $19.60(0.05)$ & $19.72(0.05)$ & $19.53(0.05)$ & $19.78(0.11)$ \\
\hline \multirow[t]{4}{*}{677} & 535.476 & 226.278 & $19.41(0.04)$ & $19.57(0.05)$ & $19.65(0.05)$ & $19.85(0.06)$ & $19.56(0.05)$ & $19.49(0.05)$ \\
\hline & & & $19.43(0.05)$ & $19.42(0.05)$ & $19.37(0.04)$ & $20.44(0.05)$ & $20.36(0.06)$ & $20.02(0.04)$ \\
\hline & & & $20.17(0.05)$ & $20.19(0.05)$ & $20.25(0.05)$ & $20.43(0.06)$ & $20.43(0.05)$ & $20.39(0.06)$ \\
\hline & & & $20.30(0.07)$ & $20.38(0.07)$ & $20.22(0.05)$ & $20.05(0.06)$ & $20.06(0.06)$ & $20.11(0.13)$ \\
\hline \multirow[t]{4}{*}{679} & 672.370 & 757.677 & $19.81(0.05)$ & $19.87(0.05)$ & $20.12(0.07)$ & $20.05(0.08)$ & $19.60(0.05)$ & $19.72(0.05)$ \\
\hline & & & $19.67(0.05)$ & $19.83(0.05)$ & $19.67(0.05)$ & $19.67(0.05)$ & $19.55(0.05)$ & $19.45(0.06)$ \\
\hline & & & $20.20(0.07)$ & $20.17(0.05)$ & $20.30(0.07)$ & $20.24(0.07)$ & $20.09(0.07)$ & $20.24(0.06)$ \\
\hline & & & $20.18(0.06)$ & $20.30(0.06)$ & $20.06(0.05)$ & $19.83(0.05)$ & $19.72(0.05)$ & $19.85(0.08)$ \\
\hline \multirow[t]{4}{*}{683} & 168.621 & 199.772 & $19.72(0.05)$ & $20.10(0.08)$ & $19.78(0.06)$ & $19.66(0.05)$ & $20.11(0.05)$ & $19.83(0.05)$ \\
\hline & & & $19.88(0.06)$ & $19.63(0.05)$ & $19.78(0.05)$ & $20.38(0.06)$ & $19.64(0.05)$ & $19.53(0.05)$ \\
\hline & & & $20.16(0.06)$ & $20.12(0.05)$ & $20.22(0.06)$ & $20.23(0.07)$ & $20.21(0.06)$ & $20.08(0.06)$ \\
\hline & & & $19.77(0.05)$ & $20.06(0.06)$ & $19.77(0.05)$ & $19.64(0.05)$ & $19.57(0.05)$ & $20.15(0.11)$ \\
\hline \multirow[t]{4}{*}{685} & 547.669 & 746.478 & $19.63(0.04)$ & $19.59(0.06)$ & $19.68(0.06)$ & $19.64(0.05)$ & $19.53(0.04)$ & $19.62(0.05)$ \\
\hline & & & $19.60(0.05)$ & $19.73(0.05)$ & $19.51(0.04)$ & $19.96(0.05)$ & $19.77(0.05)$ & $19.68(0.04)$ \\
\hline & & & $20.62(0.07)$ & $20.38(0.05)$ & $20.34(0.05)$ & $20.33(0.05)$ & $20.24(0.05)$ & $20.47(0.06)$ \\
\hline & & & $20.22(0.06)$ & $20.10(0.07)$ & $20.00(0.05)$ & $19.84(0.05)$ & $19.80(0.05)$ & $20.04(0.09)$ \\
\hline \multirow[t]{4}{*}{691} & 161.947 & 593.063 & $20.34(0.05)$ & $20.61(0.08)$ & $20.61(0.10)$ & $20.60(0.06)$ & $19.82(0.04)$ & $19.99(0.05)$ \\
\hline & & & $20.08(0.05)$ & $20.10(0.07)$ & $20.19(0.05)$ & $19.37(0.04)$ & $19.49(0.05)$ & $19.66(0.04)$ \\
\hline & & & $20.19(0.06)$ & $20.29(0.05)$ & $20.23(0.05)$ & $20.09(0.05)$ & $20.11(0.05)$ & $20.01(0.05)$ \\
\hline & & & $19.81(0.05)$ & $19.77(0.05)$ & $19.77(0.05)$ & $19.76(0.05)$ & $19.58(0.05)$ & $19.59(0.06)$ \\
\hline \multirow[t]{4}{*}{692} & 164.060 & 165.640 & $20.30(0.05)$ & $19.94(0.05)$ & $20.17(0.07)$ & $20.35(0.06)$ & $19.92(0.05)$ & 20.00 \\
\hline & & & $19.98(0.06)$ & $20.19(0.06)$ & $20.17(0.05)$ & $19.90(0.05)$ & $19.95(0.05)$ & $20.19(0.05)$ \\
\hline & & & $19.75(0.05)$ & $19.59(0.05)$ & $19.65(0.04)$ & $19.62(0.07)$ & $19.47(0.05)$ & $19.54(0.04)$ \\
\hline & & & $19.58(0.06)$ & $19.67(0.06)$ & $19.80(0.05)$ & $19.84(0.05)$ & $20.15(0.05)$ & $19.66(0.06)$ \\
\hline \multirow[t]{4}{*}{700} & 810.154 & 784.950 & $20.00(0.05)$ & $19.87(0.08)$ & $20.14(0.07)$ & $20.05(0.07)$ & $19.68(0.05)$ & $19.92(0.05)$ \\
\hline & & & $19.74(0.05)$ & $20.07(0.06)$ & $19.93(0.05)$ & $19.68(0.05)$ & $19.65(0.05)$ & $19.83(0.04)$ \\
\hline & & & $20.17(0.05)$ & $20.03(0.04)$ & $20.06(0.05)$ & $20.19(0.11)$ & $19.90(0.07)$ & $19.83(0.05)$ \\
\hline & & & $19.70(0.05)$ & $19.61(0.05)$ & $19.64(0.06)$ & $19.60(0.06)$ & $19.73(0.06)$ & $19.94(0.21)$ \\
\hline \multirow[t]{4}{*}{701} & 695.499 & 245.672 & $19.77(0.04)$ & $19.57(0.05)$ & $19.84(0.06)$ & $19.96(0.06)$ & $19.63(0.05)$ & $19.55(0.04)$ \\
\hline & & & $19.62(0.05)$ & $19.74(0.05)$ & $19.61(0.05)$ & $19.97(0.04)$ & $19.78(0.05)$ & $19.73(0.05)$ \\
\hline & & & $20.41(0.07)$ & $20.40(0.06)$ & $20.36(0.05)$ & $20.29(0.05)$ & $20.36(0.05)$ & $20.28(0.06)$ \\
\hline & & & $20.09(0.06)$ & $20.18(0.06)$ & $19.89(0.05)$ & $19.76(0.05)$ & $19.69(0.05)$ & $20.15(0.12)$ \\
\hline \multirow[t]{4}{*}{715} & 356.303 & 157.950 & $20.17(0.05)$ & $20.00(0.06)$ & $20.14(0.07)$ & $20.24(0.06)$ & $19.81(0.05)$ & $19.87(0.05)$ \\
\hline & & & $19.91(0.05)$ & $19.95(0.05)$ & $20.03(0.05)$ & $19.71(0.05)$ & $19.84(0.06)$ & $19.88(0.05)$ \\
\hline & & & $20.03(0.06)$ & $20.05(0.06)$ & $20.13(0.05)$ & $20.02(0.05)$ & $19.93(0.05)$ & $19.70(0.05)$ \\
\hline & & & $19.57(0.05)$ & $19.61(0.05)$ & $19.58(0.04)$ & $19.67(0.05)$ & $19.68(0.05)$ & $19.85(0.13)$ \\
\hline \multirow[t]{4}{*}{720} & 490.606 & 232.419 & $20.65(0.06)$ & $20.61(0.08)$ & $20.52(0.10)$ & $20.74(0.11)$ & $20.33(0.05)$ & $20.49(0.07)$ \\
\hline & & & $20.49(0.06)$ & $20.89(0.08)$ & $20.76(0.08)$ & $19.98(0.05)$ & $20.05(0.05)$ & $20.27(0.05)$ \\
\hline & & & $19.71(0.05)$ & $19.69(0.05)$ & $19.65(0.04)$ & $19.56(0.05)$ & $19.61(0.05)$ & $19.62(0.05)$ \\
\hline & & & $19.74(0.05)$ & $19.67(0.05)$ & $19.80(0.05)$ & $19.90(0.05)$ & $20.01(0.06)$ & $20.15(0.15)$ \\
\hline \multirow[t]{4}{*}{721} & 478.034 & 712.025 & $19.68(0.04)$ & $19.76(0.05)$ & $19.87(0.06)$ & $19.97(0.06)$ & $20.13(0.04)$ & $20.10(0.05)$ \\
\hline & & & $19.95(0.05)$ & $19.94(0.06)$ & $19.87(0.05)$ & $20.59(0.06)$ & $20.78(0.08)$ & $20.77(0.06)$ \\
\hline & & & $20.01(0.05)$ & $19.95(0.05)$ & $19.84(0.04)$ & $19.77(0.05)$ & $19.74(0.05)$ & $19.74(0.05)$ \\
\hline & & & $19.73(0.05)$ & $19.71(0.05)$ & $19.67(0.04)$ & $19.70(0.05)$ & $19.74(0.05)$ & $21.00(0.17)$ \\
\hline
\end{tabular}


Table 8. continuation.

\begin{tabular}{|c|c|c|c|c|c|c|c|c|}
\hline \multirow[t]{4}{*}{ F2 ID } & \multirow[t]{4}{*}{$\mathrm{x}$} & \multirow[t]{4}{*}{$\mathrm{y}$} & $2 \mathrm{Ks} 10(\sigma)$ & $2 \mathrm{Ks} 01(\sigma)$ & $2 \mathrm{Ks} 02(\sigma)$ & $2 \mathrm{Ks} 03(\sigma)$ & $2 \mathrm{Ks} 05(\sigma)$ & $2 \mathrm{Ks} 06(\sigma)$ \\
\hline & & & $2 \mathrm{Ks} 07(\sigma)$ & $2 \mathrm{Ks} 08(\sigma)$ & $2 \mathrm{Ks} 09(\sigma)$ & $2 \mathrm{Ks} 11(\sigma)$ & $2 \mathrm{Ks} 12(\sigma)$ & $2 \mathrm{Ks} 13(\sigma)$ \\
\hline & & & $2 \mathrm{Ks} 14(\sigma)$ & $2 \mathrm{Ks} 15(\sigma)$ & $2 \mathrm{Ks} 16(\sigma)$ & $2 \mathrm{Ks} 17(\sigma)$ & $2 \mathrm{Ks} 18(\sigma)$ & $2 \mathrm{Ks} 19(\sigma)$ \\
\hline & & & $2 \mathrm{Ks} 20(\sigma)$ & $2 \mathrm{Ks} 21(\sigma)$ & $2 \mathrm{Ks} 22(\sigma)$ & $2 \mathrm{Ks} 23(\sigma)$ & $2 \mathrm{Ks} 24(\sigma)$ & $2 \mathrm{Ks} 04(\sigma)$ \\
\hline \multirow[t]{4}{*}{722} & 214.223 & 359.629 & 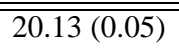 & 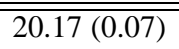 & 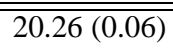 & 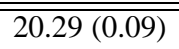 & 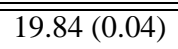 & $\overline{20.09(0.05)}$ \\
\hline & & & $20.19(0.06)$ & $20.12(0.07)$ & $20.11(0.05)$ & $19.60(0.05)$ & $19.91(0.05)$ & $19.97(0.05)$ \\
\hline & & & $20.06(0.05)$ & $19.95(0.05)$ & $19.88(0.05)$ & $19.72(0.05)$ & $19.70(0.04)$ & $19.75(0.05)$ \\
\hline & & & $19.64(0.05)$ & $19.67(0.06)$ & $19.65(0.05)$ & $19.73(0.05)$ & $19.85(0.05)$ & $19.46(0.07)$ \\
\hline \multirow[t]{4}{*}{723} & 234.762 & 350.070 & $20.24(0.05)$ & $20.66(0.09)$ & $20.71(0.14)$ & $20.48(0.06)$ & $20.00(0.05)$ & $20.31(0.06)$ \\
\hline & & & $20.46(0.08)$ & $20.45(0.07)$ & $20.31(0.05)$ & $19.85(0.05)$ & $19.93(0.06)$ & $20.23(0.05)$ \\
\hline & & & $19.98(0.05)$ & $19.85(0.05)$ & $19.70(0.04)$ & $19.63(0.05)$ & $19.57(0.05)$ & $19.57(0.05)$ \\
\hline & & & $19.53(0.05)$ & $19.49(0.05)$ & $19.67(0.04)$ & $19.91(0.05)$ & $20.08(0.06)$ & $19.74(0.09)$ \\
\hline \multirow[t]{4}{*}{724} & 771.042 & 384.463 & $19.55(0.04)$ & $19.89(0.06)$ & $19.95(0.06)$ & $20.32(0.08)$ & $19.92(0.05)$ & $19.82(0.05)$ \\
\hline & & & $19.69(0.05)$ & $19.76(0.05)$ & $19.62(0.05)$ & $19.81(0.04)$ & $19.85(0.05)$ & $19.99(0.04)$ \\
\hline & & & $21.02(0.09)$ & $21.12(0.05)$ & $21.00(0.06)$ & $20.82(0.07)$ & $20.79(0.06)$ & $20.13(0.05)$ \\
\hline & & & $19.80(0.05)$ & $19.74(0.05)$ & $19.61(0.05)$ & $19.66(0.05)$ & $19.67(0.05)$ & $20.59(0.14)$ \\
\hline \multirow[t]{4}{*}{726} & 6.964 & 347.804 & $20.11(0.05)$ & $20.45(0.07)$ & $20.83(0.12)$ & $20.47(0.06)$ & $24.25(3.33)$ & $20.79(0.07)$ \\
\hline & & & $20.43(0.06)$ & $20.59(0.08)$ & $20.44(0.06)$ & $99.99(9.99)$ & $99.99(9.99)$ & 99.99 (9.99) \\
\hline & & & $19.64(0.05)$ & $19.45(0.04)$ & $19.55(0.06)$ & 99.99 (9.99) & $19.59(0.05)$ & $19.72(0.05)$ \\
\hline & & & $20.14(0.07)$ & $19.81(0.05)$ & $20.00(0.05)$ & 99.99 (9.99) & $19.89(0.31)$ & $20.02(0.07)$ \\
\hline \multirow[t]{4}{*}{727} & 384.770 & 349.745 & $19.95(0.04)$ & $19.86(0.07)$ & $19.72(0.06)$ & $19.66(0.05)$ & $20.27(0.05)$ & $20.33(0.06)$ \\
\hline & & & $20.55(0.07)$ & $20.39(0.07)$ & $20.19(0.06)$ & $20.47(0.05)$ & $20.32(0.06)$ & $20.34(0.05)$ \\
\hline & & & $19.45(0.05)$ & $19.46(0.04)$ & $19.51(0.04)$ & $19.55(0.05)$ & $19.64(0.05)$ & 19.70 \\
\hline & & & $19.91(0.05)$ & $19.82(0.05)$ & $20.08(0.05)$ & $20.28(0.05)$ & $20.39(0.06)$ & $20.42(0.11)$ \\
\hline \multirow[t]{4}{*}{730} & 237.848 & 279.713 & $20.01(0.05)$ & $20.40(0.07)$ & $20.45(0.08)$ & $20.17(0.07)$ & $19.81(0.04)$ & $19.95(0.06)$ \\
\hline & & & $19.91(0.05)$ & $20.43(0.11)$ & $20.03(0.04)$ & $18.75(0.11)$ & $19.52(0.12)$ & $19.87(0.05)$ \\
\hline & & & $20.21(0.11)$ & $20.04(0.05)$ & $20.09(0.05)$ & $19.91(0.05)$ & $19.79(0.05)$ & $19.70(0.05)$ \\
\hline & & & $19.55(0.05)$ & $19.63(0.06)$ & $19.58(0.05)$ & $19.52(0.06)$ & $19.45(0.06)$ & $19.52(0.09)$ \\
\hline \multirow[t]{4}{*}{734} & 20.150 & 269.139 & $19.75(0.05)$ & $19.52(0.06)$ & $19.63(0.05)$ & $19.76(0.06)$ & $19.90(0.05)$ & $19.66(0.06)$ \\
\hline & & & $19.75(0.05)$ & $19.59(0.05)$ & $19.66(0.05)$ & $19.77(0.05)$ & $19.58(0.05)$ & $19.50(0.05)$ \\
\hline & & & $20.78(0.07)$ & $20.53(0.07)$ & $20.56(0.06)$ & 99.99 (9.99) & $20.45(0.06)$ & $20.55(0.06)$ \\
\hline & & & $20.50(0.08)$ & $20.50(0.09)$ & $20.43(0.06)$ & $20.44(0.06)$ & $20.00(0.05)$ & $20.31(0.19)$ \\
\hline \multirow[t]{4}{*}{735} & 763.260 & 172.846 & $20.35(0.05)$ & $20.68(0.07)$ & $20.50(0.06)$ & $20.75(0.10)$ & $20.08(0.04)$ & $20.10(0.05)$ \\
\hline & & & $20.24(0.06)$ & $20.28(0.06)$ & $20.36(0.06)$ & $19.67(0.05)$ & $19.78(0.05)$ & $19.98(0.05)$ \\
\hline & & & $20.29(0.06)$ & $20.08(0.05)$ & $20.04(0.05)$ & $19.99(0.05)$ & $19.89(0.05)$ & $19.64(0.05)$ \\
\hline & & & $19.52(0.05)$ & $19.63(0.05)$ & $19.54(0.04)$ & $19.52(0.05)$ & $19.55(0.05)$ & $19.81(0.08)$ \\
\hline \multirow[t]{4}{*}{738} & 729.100 & 572.033 & $19.87(0.05)$ & $19.51(0.06)$ & $19.76(0.06)$ & $19.75(0.05)$ & $19.67(0.05)$ & $19.80(0.05)$ \\
\hline & & & $19.82(0.05)$ & $20.08(0.06)$ & $19.90(0.05)$ & $19.96(0.05)$ & $19.99(0.05)$ & $20.09(0.06)$ \\
\hline & & & $19.66(0.05)$ & $19.65(0.04)$ & $19.73(0.05)$ & $19.84(0.05)$ & $19.90(0.06)$ & $19.97(0.05)$ \\
\hline & & & $19.94(0.05)$ & $20.07(0.08)$ & $20.09(0.05)$ & $20.14(0.05)$ & $20.21(0.05)$ & $19.62(0.08)$ \\
\hline \multirow[t]{4}{*}{743} & 49.043 & 131.927 & $20.34(0.05)$ & $19.99(0.09)$ & $20.34(0.06)$ & $20.47(0.09)$ & $19.83(0.04)$ & $19.75(0.05)$ \\
\hline & & & $20.07(0.05)$ & $20.06(0.06)$ & $20.18(0.06)$ & $19.82(0.05)$ & $19.80(0.05)$ & $19.83(0.05)$ \\
\hline & & & $20.19(0.05)$ & $20.05(0.06)$ & $19.98(0.05)$ & $20.17(0.09)$ & $19.86(0.05)$ & $19.89(0.05)$ \\
\hline & & & $19.75(0.05)$ & $19.71(0.05)$ & $19.63(0.04)$ & $19.79(0.05)$ & $19.70(0.05)$ & $19.89(0.14)$ \\
\hline \multirow[t]{4}{*}{745} & 764.869 & 387.731 & $19.93(0.05)$ & $19.60(0.05)$ & $19.72(0.06)$ & $19.96(0.05)$ & $19.78(0.04)$ & $19.76(0.05)$ \\
\hline & & & $19.92(0.05)$ & $20.05(0.06)$ & $19.80(0.05)$ & $19.62(0.05)$ & $19.58(0.05)$ & $19.65(0.04)$ \\
\hline & & & $20.12(0.06)$ & $20.17(0.05)$ & $20.20(0.05)$ & $20.27(0.06)$ & $20.43(0.05)$ & $20.24(0.06)$ \\
\hline & & & $19.99(0.06)$ & $20.06(0.06)$ & $19.96(0.05)$ & $19.84(0.05)$ & $19.79(0.05)$ & $20.16(0.10)$ \\
\hline \multirow[t]{4}{*}{746} & 128.006 & 596.062 & $19.69(0.05)$ & $19.61(0.05)$ & $19.63(0.06)$ & $19.73(0.05)$ & $19.88(0.05)$ & $19.64(0.05)$ \\
\hline & & & $19.73(0.05)$ & $19.63(0.05)$ & $19.66(0.05)$ & $19.92(0.05)$ & $19.80(0.06)$ & $19.76(0.05)$ \\
\hline & & & $20.39(0.07)$ & $20.46(0.06)$ & $20.58(0.07)$ & $20.44(0.07)$ & $20.43(0.06)$ & $20.34(0.06)$ \\
\hline & & & $20.25(0.06)$ & $20.38(0.09)$ & $20.09(0.05)$ & $20.03(0.05)$ & $19.86(0.06)$ & $20.04(0.08)$ \\
\hline
\end{tabular}


Table 8. continuation.

\begin{tabular}{|c|c|c|c|c|c|c|c|c|}
\hline \multirow[t]{4}{*}{$\overline{\mathrm{F} 2 \mathrm{ID}}$} & \multirow[t]{4}{*}{$\mathrm{x}$} & \multirow[t]{4}{*}{$\mathrm{y}$} & $2 \mathrm{Ks} 10(\sigma)$ & $2 \mathrm{Ks} 01(\sigma)$ & $2 \mathrm{Ks} 02(\sigma)$ & $2 \mathrm{Ks} 03(\sigma)$ & $2 \mathrm{Ks} 05(\sigma)$ & $2 \mathrm{Ks} 06(\sigma)$ \\
\hline & & & $2 \mathrm{Ks} 07(\sigma)$ & $2 \mathrm{Ks} 08(\sigma)$ & $2 \mathrm{Ks} 09(\sigma)$ & $2 \mathrm{Ks} 11(\sigma)$ & $2 \mathrm{Ks} 12(\sigma)$ & $2 \mathrm{Ks} 13(\sigma)$ \\
\hline & & & $2 \mathrm{Ks} 14(\sigma)$ & $2 \mathrm{Ks} 15(\sigma)$ & $2 \mathrm{Ks} 16(\sigma)$ & $2 \mathrm{Ks} 17(\sigma)$ & $2 \mathrm{Ks} 18(\sigma)$ & $2 \mathrm{Ks} 19(\sigma)$ \\
\hline & & & $2 \mathrm{Ks} 20(\sigma)$ & $2 \mathrm{Ks} 21(\sigma)$ & $2 \mathrm{Ks} 22(\sigma)$ & $2 \mathrm{Ks} 23(\sigma)$ & $2 \mathrm{Ks} 24(\sigma)$ & $2 \mathrm{Ks} 04(\sigma)$ \\
\hline \multirow[t]{4}{*}{748} & 532.077 & 342.634 & $\begin{array}{l}19.83(0.05)\end{array}$ & $19.64(0.05)$ & 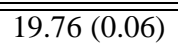 & $19.77(0.05)$ & $19.80(0.05)$ & $19.71(0.05)$ \\
\hline & & & $19.70(0.05)$ & $19.81(0.05)$ & $19.66(0.05)$ & $19.64(0.04)$ & $19.62(0.05)$ & $19.67(0.04)$ \\
\hline & & & $20.63(0.07)$ & $20.84(0.07)$ & $20.73(0.05)$ & $20.90(0.06)$ & $20.81(0.06)$ & $20.55(0.06)$ \\
\hline & & & $20.28(0.06)$ & $20.27(0.08)$ & $20.25(0.05)$ & $20.04(0.05)$ & $19.83(0.05)$ & $20.32(0.09)$ \\
\hline \multirow[t]{4}{*}{751} & 374.288 & 560.951 & $19.95(0.05)$ & $20.19(0.09)$ & $20.06(0.06)$ & $20.11(0.07)$ & $20.09(0.05)$ & $20.15(0.05)$ \\
\hline & & & $20.14(0.06)$ & $20.12(0.06)$ & $20.02(0.05)$ & $20.13(0.05)$ & $20.08(0.05)$ & $20.06(0.05)$ \\
\hline & & & $19.53(0.05)$ & $19.56(0.05)$ & $19.57(0.04)$ & $19.58(0.05)$ & $19.69(0.05)$ & $19.83(0.05)$ \\
\hline & & & $19.94(0.06)$ & $19.82(0.06)$ & $19.96(0.05)$ & $20.10(0.06)$ & $20.36(0.06)$ & $19.82(0.10)$ \\
\hline \multirow[t]{4}{*}{754} & 815.200 & 569.692 & $20.30(0.05)$ & $20.42(0.09)$ & $20.38(0.09)$ & $20.57(0.08)$ & $20.03(0.05)$ & $20.26(0.06)$ \\
\hline & & & $20.26(0.06)$ & $20.79(0.09)$ & $20.40(0.06)$ & $19.56(0.04)$ & $19.74(0.05)$ & $19.97(0.05)$ \\
\hline & & & $20.26(0.06)$ & $20.15(0.05)$ & $19.87(0.05)$ & $19.88(0.06)$ & $19.81(0.05)$ & $19.77(0.05)$ \\
\hline & & & $19.63(0.05)$ & $19.62(0.06)$ & $19.64(0.04)$ & $19.70(0.05)$ & $19.76(0.05)$ & $19.98(0.08)$ \\
\hline \multirow[t]{4}{*}{762} & 868.161 & 351.759 & $19.63(0.05)$ & 99.99 (9.99) & $19.76(0.06)$ & $19.63(0.06)$ & $19.94(0.05)$ & $19.63(0.05)$ \\
\hline & & & $19.75(0.06)$ & $19.67(0.05)$ & $19.62(0.05)$ & $19.88(0.05)$ & $19.85(0.06)$ & $19.65(0.05)$ \\
\hline & & & $20.29(0.07)$ & $20.34(0.06)$ & $20.43(0.06)$ & $20.53(0.09)$ & $20.64(0.07)$ & $20.31(0.06)$ \\
\hline & & & $20.20(0.07)$ & $20.35(0.07)$ & $20.01(0.05)$ & $19.77(0.05)$ & $19.74(0.05)$ & $20.54(0.09)$ \\
\hline \multirow[t]{4}{*}{766} & 578.610 & 714.755 & $19.82(0.05)$ & $19.79(0.05)$ & $19.80(0.05)$ & $19.80(0.06)$ & $20.43(0.05)$ & $20.70(0.07)$ \\
\hline & & & $20.41(0.06)$ & $20.37(0.07)$ & $20.15(0.05)$ & $20.70(0.06)$ & $20.61(0.07)$ & $20.61(0.05)$ \\
\hline & & & $19.72(0.05)$ & $19.66(0.04)$ & $19.57(0.05)$ & $19.58(0.05)$ & $19.57(0.05)$ & $19.68(0.05)$ \\
\hline & & & $19.73(0.05)$ & $19.62(0.05)$ & $19.86(0.05)$ & $20.04(0.05)$ & $20.18(0.05)$ & $20.59(0.10)$ \\
\hline \multirow[t]{4}{*}{767} & 565.067 & 437.930 & $20.09(0.05)$ & $20.12(0.07)$ & $20.02(0.06)$ & $20.03(0.06)$ & $19.99(0.05)$ & $19.95(0.05)$ \\
\hline & & & $19.95(0.05)$ & $20.05(0.06)$ & $20.02(0.05)$ & $20.14(0.05)$ & $20.00(0.05)$ & $19.99(0.05)$ \\
\hline & & & $19.47(0.05)$ & $19.51(0.04)$ & $19.64(0.05)$ & $19.75(0.06)$ & $19.86(0.05)$ & $20.03(0.05)$ \\
\hline & & & $19.99(0.06)$ & $20.08(0.06)$ & $20.17(0.05)$ & $20.25(0.05)$ & $20.21(0.06)$ & $19.90(0.07)$ \\
\hline \multirow[t]{4}{*}{768} & 86.564 & 427.453 & $20.08(0.05)$ & $19.80(0.06)$ & $19.68(0.06)$ & $19.63(0.06)$ & $20.43(0.05)$ & $20.20(0.06)$ \\
\hline & & & $20.37(0.06)$ & $20.54(0.08)$ & $20.41(0.06)$ & $20.43(0.06)$ & $20.56(0.08)$ & $20.46(0.05)$ \\
\hline & & & $19.57(0.05)$ & $19.57(0.05)$ & $19.48(0.06)$ & $19.47(0.06)$ & $19.53(0.05)$ & $19.62(0.05)$ \\
\hline & & & $19.76(0.06)$ & $19.72(0.06)$ & $19.84(0.05)$ & $20.11(0.06)$ & $20.34(0.06)$ & $20.49(0.10)$ \\
\hline \multirow[t]{4}{*}{769} & 192.557 & 554.662 & $19.73(0.05)$ & $19.64(0.06)$ & $19.66(0.06)$ & $19.76(0.06)$ & $19.83(0.05)$ & $19.80(0.05)$ \\
\hline & & & $19.72(0.05)$ & $19.71(0.05)$ & $19.70(0.05)$ & $19.90(0.05)$ & $19.77(0.05)$ & $19.64(0.04)$ \\
\hline & & & $20.28(0.05)$ & $20.17(0.05)$ & $20.06(0.05)$ & $20.00(0.05)$ & $19.97(0.05)$ & $20.14(0.05)$ \\
\hline & & & $20.08(0.06)$ & $20.08(0.05)$ & $20.06(0.05)$ & $19.90(0.05)$ & $19.71(0.05)$ & $20.29(0.09)$ \\
\hline \multirow[t]{4}{*}{771} & 21.368 & 211.332 & $19.86(0.05)$ & $20.14(0.08)$ & $20.15(0.08)$ & $20.45(0.10)$ & $19.82(0.04)$ & $19.65(0.05)$ \\
\hline & & & $19.69(0.05)$ & $19.89(0.06)$ & $19.78(0.05)$ & $19.85(0.05)$ & $19.73(0.05)$ & $19.66(0.05)$ \\
\hline & & & $20.47(0.06)$ & $20.29(0.06)$ & $20.35(0.06)$ & 99.99 (9.99) & $20.21(0.06)$ & $20.23(0.06)$ \\
\hline & & & $20.07(0.06)$ & $20.15(0.06)$ & $20.02(0.05)$ & $19.89(0.05)$ & $19.66(0.05)$ & $20.42(0.27)$ \\
\hline \multirow[t]{4}{*}{773} & 860.026 & 519.779 & $19.84(0.05)$ & 99.99 (9.99) & $19.66(0.05)$ & $19.65(0.05)$ & $19.73(0.04)$ & $19.68(0.05)$ \\
\hline & & & $19.59(0.05)$ & $19.74(0.05)$ & $19.64(0.05)$ & $19.78(0.04)$ & $19.77(0.05)$ & $19.80(0.04)$ \\
\hline & & & $20.24(0.06)$ & $20.27(0.05)$ & $20.18(0.04)$ & $20.30(0.06)$ & $20.12(0.05)$ & $19.96(0.05)$ \\
\hline & & & $19.99(0.05)$ & $19.86(0.05)$ & $19.95(0.05)$ & $19.83(0.05)$ & $20.05(0.05)$ & $20.26(0.11)$ \\
\hline \multirow[t]{4}{*}{775} & 442.662 & 159.731 & $19.52(0.04)$ & $19.81(0.06)$ & $19.78(0.05)$ & $19.57(0.05)$ & $19.80(0.05)$ & $19.76(0.05)$ \\
\hline & & & $19.69(0.05)$ & $19.62(0.05)$ & $19.59(0.04)$ & $19.98(0.05)$ & $19.95(0.05)$ & $19.74(0.05)$ \\
\hline & & & $20.13(0.05)$ & $20.16(0.06)$ & $20.31(0.05)$ & $20.29(0.05)$ & $20.32(0.05)$ & $20.27(0.05)$ \\
\hline & & & $20.27(0.06)$ & $20.31(0.06)$ & $20.19(0.05)$ & $20.24(0.06)$ & $20.11(0.06)$ & $20.13(0.10)$ \\
\hline \multirow[t]{4}{*}{777} & 526.587 & 462.444 & $20.13(0.05)$ & $19.66(0.06)$ & $19.81(0.06)$ & $20.07(0.06)$ & $19.60(0.04)$ & $19.78(0.05)$ \\
\hline & & & $19.77(0.05)$ & $20.04(0.06)$ & $19.86(0.05)$ & $19.79(0.04)$ & $19.82(0.05)$ & $19.89(0.05)$ \\
\hline & & & $20.49(0.06)$ & $20.49(0.05)$ & $20.39(0.05)$ & $20.19(0.06)$ & $20.14(0.05)$ & $19.99(0.05)$ \\
\hline & & & $19.85(0.05)$ & $19.70(0.05)$ & $19.68(0.05)$ & $19.72(0.05)$ & $19.69(0.05)$ & $19.75(0.07)$ \\
\hline
\end{tabular}


Table 8. continuation.

\begin{tabular}{|c|c|c|c|c|c|c|c|c|}
\hline \multirow[t]{4}{*}{$\overline{\mathrm{F} 2 \mathrm{ID}}$} & \multirow[t]{4}{*}{$\mathrm{x}$} & \multirow[t]{4}{*}{$\mathrm{y}$} & $\overline{2 \mathrm{Ks} 10(\sigma)}$ & $\overline{2 \mathrm{Ks} 01(\sigma)}$ & $2 \mathrm{Ks} 02(\sigma)$ & $2 \mathrm{Ks} 03(\sigma)$ & $2 \mathrm{Ks} 05(\sigma)$ & $2 \mathrm{Ks} 06(\sigma)$ \\
\hline & & & $2 \mathrm{Ks} 07(\sigma)$ & $2 \mathrm{Ks} 08(\sigma)$ & $2 \mathrm{Ks} 09(\sigma)$ & $2 \mathrm{Ks} 11(\sigma)$ & $2 \mathrm{Ks} 12(\sigma)$ & $2 \mathrm{Ks} 13(\sigma)$ \\
\hline & & & $2 \mathrm{Ks} 14(\sigma)$ & $2 \mathrm{Ks} 15(\sigma)$ & $2 \mathrm{Ks} 16(\sigma)$ & $2 \mathrm{Ks} 17(\sigma)$ & $2 \mathrm{Ks} 18(\sigma)$ & $2 \mathrm{Ks} 19(\sigma)$ \\
\hline & & & $2 \mathrm{Ks} 20(\sigma)$ & $2 \mathrm{Ks} 21(\sigma)$ & $2 \mathrm{Ks} 22(\sigma)$ & $2 \mathrm{Ks} 23(\sigma)$ & $2 \mathrm{Ks} 24(\sigma)$ & $2 \mathrm{Ks} 04(\sigma)$ \\
\hline \multirow[t]{4}{*}{778} & \multirow[t]{4}{*}{583.304} & \multirow[t]{4}{*}{118.457} & $19.52(0.05)$ & $\begin{array}{l}19.60(0.06) \\
\end{array}$ & $19.85(0.06)$ & $19.92(0.06)$ & $19.39(0.05)$ & $\begin{array}{c}19.69(0.05) \\
\end{array}$ \\
\hline & & & $19.49(0.05)$ & $19.41(0.06)$ & $19.39(0.05)$ & $20.27(0.07)$ & $19.94(0.05)$ & $19.81(0.06)$ \\
\hline & & & $20.11(0.06)$ & $20.11(0.05)$ & $20.25(0.06)$ & $20.30(0.05)$ & $20.38(0.06)$ & $20.30(0.06)$ \\
\hline & & & $20.21(0.07)$ & $20.38(0.07)$ & $20.15(0.06)$ & $20.34(0.06)$ & $20.11(0.06)$ & $19.58(0.07)$ \\
\hline \multirow[t]{4}{*}{779} & \multirow[t]{4}{*}{621.909} & \multirow[t]{4}{*}{244.180} & $20.38(0.06)$ & $20.31(0.08)$ & $20.07(0.07)$ & $19.95(0.06)$ & $20.24(0.05)$ & $20.39(0.05)$ \\
\hline & & & $20.34(0.06)$ & $20.60(0.07)$ & $20.36(0.05)$ & $19.63(0.04)$ & $19.80(0.05)$ & $19.93(0.05)$ \\
\hline & & & $20.36(0.06)$ & $20.24(0.05)$ & $20.08(0.04)$ & $19.93(0.04)$ & $19.91(0.05)$ & $19.68(0.05)$ \\
\hline & & & $19.52(0.05)$ & $19.60(0.05)$ & $19.52(0.04)$ & $19.48(0.05)$ & $19.48(0.05)$ & $19.90(0.10)$ \\
\hline \multirow[t]{4}{*}{786} & \multirow[t]{4}{*}{25.180} & \multirow[t]{4}{*}{214.175} & $20.48(0.05)$ & $20.51(0.10)$ & $20.71(0.09)$ & $20.44(0.07)$ & $20.33(0.05)$ & $20.20(0.06)$ \\
\hline & & & $20.53(0.08)$ & $20.49(0.07)$ & $20.57(0.06)$ & $20.03(0.05)$ & $20.13(0.07)$ & $20.34(0.06)$ \\
\hline & & & $19.73(0.05)$ & $19.57(0.05)$ & $19.62(0.06)$ & $99.99(9.99)$ & $19.53(0.05)$ & $19.58(0.05)$ \\
\hline & & & $19.61(0.05)$ & $19.60(0.05)$ & $19.65(0.05)$ & $19.83(0.05)$ & $20.03(0.06)$ & $19.94(0.15)$ \\
\hline \multirow[t]{4}{*}{788} & \multirow[t]{4}{*}{437.687} & \multirow[t]{4}{*}{95.468} & $20.14(0.04)$ & $20.42(0.08)$ & $20.82(0.08)$ & $20.74(0.09)$ & $19.65(0.04)$ & $19.90(0.05)$ \\
\hline & & & $19.80(0.05)$ & $20.09(0.06)$ & $19.99(0.05)$ & $19.58(0.04)$ & $19.43(0.04)$ & $19.45(0.04)$ \\
\hline & & & $20.39(0.06)$ & $20.45(0.05)$ & $20.30(0.05)$ & $20.26(0.05)$ & $20.21(0.05)$ & $20.19(0.06)$ \\
\hline & & & $20.33(0.06)$ & $20.30(0.05)$ & $20.23(0.05)$ & $20.08(0.06)$ & $20.00(0.05)$ & $19.68(0.09)$ \\
\hline \multirow[t]{4}{*}{789} & \multirow[t]{4}{*}{723.670} & 55.691 & $20.31(0.05)$ & $20.33(0.06)$ & $20.46(0.08)$ & $19.87(0.06)$ & $20.51(0.06)$ & $20.46(0.06)$ \\
\hline & & & $20.39(0.08)$ & $20.64(0.08)$ & $20.44(0.07)$ & $20.02(0.05)$ & $20.06(0.05)$ & $20.27(0.05)$ \\
\hline & & & $19.85(0.05)$ & $19.80(0.05)$ & $19.83(0.05)$ & $19.84(0.05)$ & $19.87(0.05)$ & $19.62(0.05)$ \\
\hline & & & $19.67(0.05)$ & $19.74(0.05)$ & $19.62(0.05)$ & $19.64(0.05)$ & $19.71(0.05)$ & $20.41(0.09)$ \\
\hline 792 & 39.439 & 161.490 & 99.99 (9.99) & $19.25(0.25)$ & $19.13(0.13)$ & 99.99 (9.99) & 99.99 (9.99) & 99.99 (9.99) \\
\hline & & & $20.27(0.11)$ & 99.99 (9.99) & $19.13(0.14)$ & $19.22(0.22)$ & $20.03(0.11)$ & 99.99 (9.99) \\
\hline & & & $19.90(0.37)$ & $19.09(0.19)$ & 99.99 (9.99) & 99.99 (9.99) & 99.99 (9.99) & $21.06(0.14)$ \\
\hline & & & $20.50(0.12)$ & $20.33(0.27)$ & $18.79(0.11)$ & $22.88(0.46)$ & $21.63(0.20)$ & $20.47(0.33)$ \\
\hline 795 & 46.919 & 66.360 & $20.07(0.09)$ & $21.18(0.20)$ & $20.83(0.17)$ & $21.26(0.24)$ & $20.14(0.08)$ & $20.03(0.08)$ \\
\hline & & & $19.94(0.08)$ & $20.08(0.08)$ & $20.13(0.08)$ & $20.11(0.07)$ & $20.32(0.10)$ & $20.56(0.16)$ \\
\hline & & & $19.79(0.06)$ & $19.66(0.07)$ & $20.07(0.10)$ & $20.19(0.11)$ & $20.01(0.13)$ & $19.78(0.07)$ \\
\hline & & & $19.65(0.06)$ & $19.96(0.09)$ & $19.83(0.08)$ & $19.65(0.06)$ & $19.72(0.06)$ & $19.99(0.16)$ \\
\hline 797 & 511.539 & 298.404 & $19.80(0.05)$ & $19.70(0.08)$ & $19.68(0.06)$ & $19.88(0.06)$ & $19.84(0.05)$ & $19.73(0.05)$ \\
\hline & & & $19.70(0.05)$ & $19.89(0.05)$ & $19.77(0.05)$ & $19.80(0.05)$ & $19.66(0.05)$ & $19.66(0.05)$ \\
\hline & & & $20.50(0.07)$ & $20.59(0.05)$ & $20.48(0.07)$ & $20.36(0.06)$ & $20.35(0.06)$ & $20.21(0.06)$ \\
\hline & & & $20.02(0.06)$ & $20.09(0.05)$ & $19.96(0.05)$ & $19.86(0.05)$ & $19.83(0.05)$ & $20.24(0.09)$ \\
\hline 799 & 864.499 & 45.122 & $19.71(0.04)$ & 99.99 (9.99) & $19.44(0.05)$ & $19.44(0.05)$ & $20.05(0.05)$ & $19.89(0.05)$ \\
\hline & & & $19.99(0.05)$ & $19.71(0.05)$ & $19.68(0.05)$ & $21.06(0.08)$ & $21.54(0.14)$ & $20.86(0.07)$ \\
\hline & & & $19.83(0.05)$ & $19.96(0.05)$ & $20.09(0.06)$ & $20.13(0.06)$ & $20.37(0.07)$ & $20.24(0.06)$ \\
\hline & & & $20.73(0.09)$ & $20.68(0.07)$ & $20.65(0.08)$ & $20.92(0.11)$ & $21.39(0.22)$ & $20.81(0.25)$ \\
\hline 802 & 604.547 & 212.523 & $19.63(0.08)$ & $19.72(0.09)$ & $20.18(0.15)$ & $19.46(0.12)$ & $19.37(0.10)$ & $20.04(0.13)$ \\
\hline & & & $20.36(0.26)$ & $21.86(1.97)$ & $20.12(0.20)$ & $19.39(0.12)$ & $20.83(0.12)$ & $19.68(0.10)$ \\
\hline & & & $20.99(0.31)$ & $20.67(0.26)$ & $20.39(0.22)$ & $20.02(0.15)$ & $20.44(0.22)$ & $21.09(0.37)$ \\
\hline & & & $21.65(0.26)$ & $20.85(0.37)$ & $21.74(0.59)$ & $21.02(0.24)$ & $21.04(0.26)$ & $19.80(0.16)$ \\
\hline 805 & 849.506 & 87.339 & $19.76(0.04)$ & $19.89(0.07)$ & $19.79(0.07)$ & $19.60(0.05)$ & $19.85(0.06)$ & $19.90(0.05)$ \\
\hline & & & $19.83(0.05)$ & $19.77(0.05)$ & $19.80(0.05)$ & $19.73(0.05)$ & $19.73(0.05)$ & $19.55(0.05)$ \\
\hline & & & $20.23(0.06)$ & $20.35(0.05)$ & $20.36(0.05)$ & $20.24(0.06)$ & $20.42(0.07)$ & $20.20(0.06)$ \\
\hline & & & $20.04(0.06)$ & $20.38(0.06)$ & $19.91(0.05)$ & $19.96(0.06)$ & $19.98(0.05)$ & $19.76(0.09)$ \\
\hline 808 & 783.843 & 412.300 & $20.26(0.05)$ & $20.23(0.07)$ & $20.34(0.07)$ & $20.37(0.07)$ & $20.27(0.05)$ & $20.33(0.05)$ \\
\hline & & & $20.43(0.08)$ & $20.55(0.09)$ & $20.43(0.05)$ & $20.11(0.06)$ & $20.20(0.06)$ & $20.38(0.05)$ \\
\hline & & & $19.60(0.05)$ & $19.57(0.04)$ & $19.58(0.04)$ & $19.60(0.04)$ & $19.67(0.05)$ & $19.74(0.05)$ \\
\hline & & & $19.87(0.05)$ & $19.84(0.05)$ & $19.97(0.05)$ & $20.14(0.05)$ & $20.42(0.06)$ & $19.86(0.11)$ \\
\hline
\end{tabular}


Table 8. continuation.

\begin{tabular}{|c|c|c|c|c|c|c|c|c|}
\hline \multirow[t]{4}{*}{$\overline{\mathrm{F} 2 \mathrm{ID}}$} & \multirow[t]{4}{*}{$\mathrm{x}$} & \multirow[t]{4}{*}{$\mathrm{y}$} & $2 \mathrm{Ks} 10(\sigma)$ & $2 \mathrm{Ks} 01(\sigma)$ & $2 \mathrm{Ks} 02(\sigma)$ & $2 \mathrm{Ks} 03(\sigma)$ & $2 \mathrm{Ks} 05(\sigma)$ & $2 \mathrm{Ks06}(\sigma)$ \\
\hline & & & $2 \mathrm{Ks} 07(\sigma)$ & $2 \mathrm{Ks} 08(\sigma)$ & $2 \mathrm{Ks} 09(\sigma)$ & $2 \mathrm{Ks} 11(\sigma)$ & $2 \mathrm{Ks} 12(\sigma)$ & $2 \mathrm{Ks} 13(\sigma)$ \\
\hline & & & $2 \mathrm{Ks} 14(\sigma)$ & $2 \mathrm{Ks} 15(\sigma)$ & $2 \mathrm{Ks} 16(\sigma)$ & $2 \mathrm{Ks} 17(\sigma)$ & $2 \mathrm{Ks} 18(\sigma)$ & $2 \mathrm{Ks} 19(\sigma)$ \\
\hline & & & $2 \mathrm{Ks} 20(\sigma)$ & $2 \mathrm{Ks} 21(\sigma)$ & $2 \mathrm{Ks} 22(\sigma)$ & $2 \mathrm{Ks} 23(\sigma)$ & $2 \mathrm{Ks} 24(\sigma)$ & $2 \mathrm{Ks} 04(\sigma)$ \\
\hline \multirow[t]{4}{*}{$\overline{8809}$} & 131.859 & 25.982 & $19.93(0.05)$ & $20.04(0.07)$ & $20.01(0.06)$ & $20.16(0.06)$ & $20.21(0.06)$ & $20.06(0.05)$ \\
\hline & & & $20.10(0.05)$ & $20.06(0.07)$ & $19.98(0.05)$ & $21.05(0.15)$ & $20.11(0.05)$ & $20.06(0.05)$ \\
\hline & & & $19.62(0.05)$ & $19.37(0.05)$ & $19.61(0.07)$ & $19.85(0.10)$ & $19.63(0.06)$ & $19.81(0.05)$ \\
\hline & & & $19.88(0.05)$ & $19.88(0.05)$ & $20.05(0.06)$ & $20.33(0.06)$ & $20.26(0.08)$ & $20.04(0.18)$ \\
\hline \multirow[t]{4}{*}{814} & 287.076 & 201.624 & $19.78(0.04)$ & $20.23(0.06)$ & $19.96(0.06)$ & $19.76(0.05)$ & $20.34(0.05)$ & $20.01(0.05)$ \\
\hline & & & $20.01(0.06)$ & $19.97(0.05)$ & $19.86(0.05)$ & $20.02(0.05)$ & $19.80(0.05)$ & $19.71(0.05)$ \\
\hline & & & $20.27(0.06)$ & $20.16(0.05)$ & $20.25(0.05)$ & $20.20(0.06)$ & $20.24(0.06)$ & $20.14(0.05)$ \\
\hline & & & $19.90(0.06)$ & $20.08(0.08)$ & $19.75(0.05)$ & $19.67(0.05)$ & $19.60(0.05)$ & $20.48(0.15)$ \\
\hline \multirow[t]{4}{*}{815} & 716.371 & 310.165 & $19.58(0.04)$ & $19.55(0.06)$ & $19.57(0.06)$ & $19.59(0.05)$ & $19.83(0.05)$ & $19.65(0.04)$ \\
\hline & & & $19.77(0.05)$ & $19.68(0.05)$ & $19.65(0.04)$ & $20.43(0.05)$ & $20.12(0.06)$ & $19.91(0.04)$ \\
\hline & & & $20.04(0.05)$ & $20.21(0.05)$ & $20.29(0.05)$ & $20.36(0.05)$ & $20.46(0.06)$ & $20.34(0.06)$ \\
\hline & & & $20.31(0.06)$ & $20.42(0.07)$ & $20.55(0.05)$ & $20.30(0.05)$ & $20.14(0.05)$ & $20.44(0.10)$ \\
\hline \multirow[t]{4}{*}{816} & 549.096 & 166.588 & $20.14(0.05)$ & $19.51(0.05)$ & $19.46(0.05)$ & $19.44(0.05)$ & $21.31(0.08)$ & $21.27(0.09)$ \\
\hline & & & $21.44(0.14)$ & $21.21(0.10)$ & $20.86(0.08)$ & $21.23(0.08)$ & $21.65(0.15)$ & $22.02(0.13)$ \\
\hline & & & $19.85(0.05)$ & $19.83(0.05)$ & $19.83(0.04)$ & $19.81(0.05)$ & $19.83(0.05)$ & $19.81(0.05)$ \\
\hline & & & $19.94(0.05)$ & $19.81(0.06)$ & $19.95(0.05)$ & $20.05(0.05)$ & $20.27(0.06)$ & $20.81(0.12)$ \\
\hline \multirow[t]{4}{*}{817} & 346.288 & 464.295 & $19.88(0.05)$ & $20.41(0.07)$ & $20.00(0.06)$ & $19.92(0.07)$ & $19.86(0.05)$ & $19.74(0.05)$ \\
\hline & & & $19.83(0.05)$ & $19.72(0.05)$ & $19.79(0.05)$ & $19.69(0.05)$ & $19.78(0.05)$ & $20.05(0.05)$ \\
\hline & & & $20.47(0.06)$ & $20.38(0.05)$ & $20.28(0.05)$ & $20.02(0.05)$ & $19.96(0.05)$ & $19.82(0.05)$ \\
\hline & & & $19.77(0.05)$ & $19.70(0.05)$ & $19.75(0.05)$ & $19.85(0.05)$ & $19.97(0.05)$ & $20.69(0.13)$ \\
\hline \multirow[t]{4}{*}{820} & 608.691 & 220.076 & $19.53(0.09)$ & $19.80(0.13)$ & $20.10(0.14)$ & $19.99(0.16)$ & $19.04(0.12)$ & $19.12(0.10)$ \\
\hline & & & $19.77(0.15)$ & $19.74(0.21)$ & $19.34(0.11)$ & $19.73(0.12)$ & $20.23(0.10)$ & $19.81(0.13)$ \\
\hline & & & $20.50(0.22)$ & $20.05(0.11)$ & $19.51(0.23)$ & $19.96(0.20)$ & $20.15(0.10)$ & $20.50(0.16)$ \\
\hline & & & $20.55(0.09)$ & $20.36(0.18)$ & $20.64(0.16)$ & 99.99 (9.99) & $21.29(0.24)$ & $19.97(0.17)$ \\
\hline \multirow[t]{4}{*}{828} & 528.831 & 805.376 & $20.18(0.05)$ & $20.21(0.07)$ & $20.63(0.09)$ & $19.99(0.06)$ & $20.14(0.05)$ & 20.63 \\
\hline & & & $20.31(0.07)$ & $20.32(0.06)$ & $20.43(0.05)$ & $19.67(0.04)$ & $19.71(0.05)$ & $19.93(0.05)$ \\
\hline & & & $20.14(0.06)$ & $20.11(0.05)$ & $19.99(0.05)$ & $20.06(0.05)$ & $19.84(0.05)$ & $19.85(0.04)$ \\
\hline & & & $19.67(0.05)$ & $19.59(0.05)$ & $19.78(0.05)$ & $19.48(0.05)$ & $19.47(0.05)$ & $20.13(0.06)$ \\
\hline \multirow[t]{4}{*}{832} & 51.849 & 373.813 & $19.81(0.05)$ & $20.09(0.06)$ & $20.06(0.06)$ & $20.18(0.06)$ & $19.93(0.05)$ & $19.57(0.05)$ \\
\hline & & & $19.77(0.05)$ & $19.51(0.05)$ & $19.72(0.05)$ & $19.87(0.05)$ & $19.82(0.05)$ & $19.70(0.05)$ \\
\hline & & & $20.61(0.07)$ & $20.52(0.06)$ & $20.52(0.07)$ & $20.47(0.09)$ & $20.38(0.07)$ & $20.27(0.06)$ \\
\hline & & & $20.15(0.06)$ & $20.21(0.08)$ & $20.09(0.05)$ & $19.90(0.05)$ & $19.80(0.05)$ & $20.06(0.07)$ \\
\hline \multirow[t]{4}{*}{839} & 328.852 & 173.045 & $20.35(0.05)$ & $20.67(0.07)$ & $20.71(0.09)$ & $21.08(0.12)$ & $20.19(0.05)$ & $20.48(0.06)$ \\
\hline & & & $20.30(0.06)$ & $20.65(0.07)$ & $20.34(0.05)$ & $19.82(0.04)$ & $19.98(0.06)$ & $20.20(0.05)$ \\
\hline & & & $20.18(0.05)$ & $20.08(0.05)$ & $19.98(0.05)$ & $19.83(0.05)$ & $19.73(0.04)$ & $19.76(0.05)$ \\
\hline & & & $19.60(0.05)$ & $19.58(0.05)$ & $19.65(0.04)$ & $19.71(0.05)$ & $19.85(0.05)$ & $19.91(0.08)$ \\
\hline \multirow[t]{4}{*}{841} & 350.130 & 761.766 & $20.13(0.06)$ & $20.41(0.08)$ & $20.00(0.06)$ & $20.04(0.07)$ & $19.79(0.05)$ & $19.86(0.05)$ \\
\hline & & & $19.76(0.06)$ & $19.85(0.06)$ & $19.92(0.06)$ & $19.85(0.05)$ & $20.11(0.07)$ & $20.38(0.07)$ \\
\hline & & & $19.67(0.05)$ & $19.73(0.05)$ & $19.77(0.05)$ & $19.78(0.06)$ & $19.78(0.06)$ & $19.90(0.06)$ \\
\hline & & & $19.97(0.06)$ & $20.11(0.07)$ & $20.22(0.06)$ & $20.24(0.06)$ & $20.15(0.07)$ & $19.93(0.09)$ \\
\hline \multirow[t]{4}{*}{843} & 386.502 & 661.370 & $19.64(0.04)$ & $20.02(0.05)$ & $19.85(0.05)$ & $19.58(0.05)$ & $19.91(0.05)$ & $19.99(0.05)$ \\
\hline & & & $20.05(0.05)$ & $20.00(0.05)$ & $19.90(0.05)$ & $20.05(0.05)$ & $20.00(0.06)$ & $20.02(0.05)$ \\
\hline & & & $19.56(0.05)$ & $19.71(0.05)$ & $19.87(0.05)$ & $19.98(0.05)$ & $20.05(0.05)$ & $20.26(0.05)$ \\
\hline & & & $20.18(0.06)$ & $20.37(0.07)$ & $20.40(0.05)$ & $20.49(0.06)$ & $20.26(0.06)$ & $20.22(0.11)$ \\
\hline \multirow[t]{4}{*}{852} & 233.105 & 436.758 & $20.18(0.05)$ & $20.36(0.07)$ & $20.43(0.07)$ & $20.89(0.12)$ & $20.60(0.06)$ & $21.24(0.28)$ \\
\hline & & & $20.51(0.09)$ & $21.53(0.30)$ & $20.19(0.06)$ & $20.48(0.05)$ & $20.64(0.08)$ & $20.89(0.08)$ \\
\hline & & & $19.73(0.05)$ & $19.69(0.05)$ & $19.61(0.05)$ & $19.55(0.05)$ & $19.59(0.05)$ & $19.71(0.05)$ \\
\hline & & & $19.85(0.06)$ & $19.76(0.06)$ & $19.90(0.05)$ & $20.12(0.05)$ & $20.46(0.06)$ & $20.24(0.13)$ \\
\hline
\end{tabular}


Table 8. continuation.

\begin{tabular}{|c|c|c|c|c|c|c|c|c|}
\hline \multirow[t]{4}{*}{ F2 ID } & \multirow[t]{4}{*}{$\mathrm{x}$} & \multirow[t]{4}{*}{$\mathrm{y}$} & $2 \mathrm{Ks} 10(\sigma)$ & $2 \mathrm{Ks} 01(\sigma)$ & $2 \mathrm{Ks} 02(\sigma)$ & $2 \mathrm{Ks} 03(\sigma)$ & $2 \mathrm{Ks} 05(\sigma)$ & $2 \mathrm{Ks} 06(\sigma)$ \\
\hline & & & $2 \mathrm{Ks} 07(\sigma)$ & $2 \mathrm{Ks} 08(\sigma)$ & $2 \mathrm{Ks} 09(\sigma)$ & $2 \mathrm{Ks} 11(\sigma)$ & $2 \mathrm{Ks} 12(\sigma)$ & $2 \mathrm{Ks} 13(\sigma)$ \\
\hline & & & $2 \mathrm{Ks} 14(\sigma)$ & $2 \mathrm{Ks} 15(\sigma)$ & $2 \mathrm{Ks} 16(\sigma)$ & $2 \mathrm{Ks} 17(\sigma)$ & $2 \mathrm{Ks} 18(\sigma)$ & $2 \mathrm{Ks} 19(\sigma)$ \\
\hline & & & $2 \mathrm{Ks} 20(\sigma)$ & $2 \mathrm{Ks} 21(\sigma)$ & $2 \mathrm{Ks} 22(\sigma)$ & $2 \mathrm{Ks} 23(\sigma)$ & $2 \mathrm{Ks} 24(\sigma)$ & $2 \mathrm{Ks} 04(\sigma)$ \\
\hline \multirow[t]{4}{*}{853} & \multirow[t]{4}{*}{302.687} & \multirow[t]{4}{*}{543.326} & $20.48(0.05)$ & $20.15(0.07)$ & $20.47(0.08)$ & $20.41(0.08)$ & $\bar{~} 19.80(0.05)$ & $20.00(0.05)$ \\
\hline & & & $20.16(0.07)$ & $20.26(0.06)$ & $20.32(0.06)$ & $19.61(0.04)$ & $19.76(0.05)$ & $20.01(0.04)$ \\
\hline & & & $20.28(0.05)$ & $20.18(0.05)$ & $20.03(0.05)$ & $19.84(0.05)$ & $19.85(0.05)$ & $19.77(0.05)$ \\
\hline & & & $19.74(0.05)$ & $19.73(0.05)$ & $19.73(0.05)$ & $19.98(0.05)$ & $19.91(0.05)$ & $19.66(0.07)$ \\
\hline \multirow[t]{4}{*}{856} & \multirow[t]{4}{*}{123.108} & \multirow[t]{4}{*}{645.115} & $19.59(0.06)$ & $20.16(0.06)$ & $20.05(0.07)$ & $19.87(0.07)$ & $20.03(0.07)$ & $19.76(0.05)$ \\
\hline & & & $19.81(0.06)$ & $19.64(0.05)$ & $19.67(0.06)$ & $19.85(0.06)$ & $19.86(0.07)$ & $19.76(0.06)$ \\
\hline & & & $20.17(0.06)$ & $20.23(0.06)$ & $20.24(0.06)$ & $20.18(0.08)$ & $20.21(0.08)$ & $20.15(0.06)$ \\
\hline & & & $20.05(0.06)$ & $20.17(0.06)$ & $19.93(0.05)$ & $19.90(0.06)$ & $19.75(0.06)$ & $20.20(0.14)$ \\
\hline \multirow[t]{4}{*}{857} & \multirow[t]{4}{*}{144.135} & \multirow[t]{4}{*}{662.058} & $20.38(0.05)$ & $20.46(0.07)$ & $20.46(0.08)$ & $20.38(0.08)$ & $19.97(0.05)$ & $20.10(0.05)$ \\
\hline & & & $20.35(0.06)$ & $20.34(0.06)$ & $20.29(0.06)$ & $19.58(0.05)$ & $19.63(0.05)$ & $19.74(0.05)$ \\
\hline & & & $20.38(0.07)$ & $20.38(0.05)$ & $20.27(0.06)$ & $20.13(0.05)$ & $19.99(0.06)$ & $19.90(0.05)$ \\
\hline & & & $19.83(0.06)$ & $19.78(0.05)$ & $19.78(0.05)$ & $19.79(0.05)$ & $19.67(0.05)$ & $19.74(0.07)$ \\
\hline \multirow[t]{4}{*}{858} & \multirow[t]{4}{*}{889.946} & \multirow[t]{4}{*}{524.230} & $20.44(0.08)$ & 99.99 (9.99) & $20.51(0.08)$ & $20.32(0.08)$ & $20.00(0.04)$ & $20.11(0.05)$ \\
\hline & & & $20.36(0.07)$ & $20.01(0.20)$ & $20.42(0.06)$ & $19.65(0.04)$ & $19.65(0.05)$ & $19.62(0.05)$ \\
\hline & & & $20.42(0.07)$ & $20.43(0.05)$ & $20.42(0.06)$ & $19.93(1.00)$ & $20.47(0.07)$ & $20.13(0.05)$ \\
\hline & & & 99.99 (9.99) & $19.97(0.07)$ & $19.74(0.05)$ & $19.68(0.05)$ & $19.60(0.05)$ & $19.76(0.07)$ \\
\hline \multirow[t]{4}{*}{860} & \multirow[t]{4}{*}{508.933} & \multirow[t]{4}{*}{137.441} & $20.46(0.05)$ & $20.28(0.06)$ & $20.33(0.08)$ & $20.26(0.06)$ & $20.43(0.06)$ & $20.81(0.07)$ \\
\hline & & & $20.62(0.07)$ & $20.45(0.13)$ & $20.61(0.07)$ & $19.53(0.06)$ & $19.92(0.05)$ & $19.97(0.05)$ \\
\hline & & & $20.41(0.08)$ & $20.35(0.05)$ & $20.33(0.05)$ & $20.09(0.06)$ & $19.99(0.05)$ & 19.77 \\
\hline & & & $19.63(0.05)$ & $19.67(0.06)$ & $19.52(0.04)$ & $19.54(0.05)$ & $19.49(0.05)$ & $20.04(0.09)$ \\
\hline 861 & 549.033 & 372.270 & $20.05(0.05)$ & $20.16(0.07)$ & $20.16(0.06)$ & $20.23(0.07)$ & $20.14(0.06)$ & $20.20(0.05)$ \\
\hline & & & $20.27(0.07)$ & $20.15(0.06)$ & $20.20(0.05)$ & $19.96(0.05)$ & $20.16(0.06)$ & $20.16(0.05)$ \\
\hline & & & $19.65(0.05)$ & $19.64(0.05)$ & $19.67(0.05)$ & $19.72(0.05)$ & $19.78(0.05)$ & $19.84(0.05)$ \\
\hline & & & $19.87(0.05)$ & $19.88(0.05)$ & $20.01(0.05)$ & $20.04(0.05)$ & $20.03(0.05)$ & $19.90(0.08)$ \\
\hline 864 & 717.957 & 378.409 & $20.00(0.05)$ & $20.08(0.07)$ & $20.47(0.10)$ & 20.49 (0.09) & $19.67(0.04)$ & $19.67(0.04)$ \\
\hline & & & $19.87(0.05)$ & $19.97(0.05)$ & $19.89(0.05)$ & $19.68(0.04)$ & $19.59(0.05)$ & $19.58(0.05)$ \\
\hline & & & $20.44(0.06)$ & $20.41(0.05)$ & $20.44(0.06)$ & $20.37(0.05)$ & $20.52(0.05)$ & $20.44(0.05)$ \\
\hline & & & $20.49(0.07)$ & $20.49(0.07)$ & $20.51(0.05)$ & $20.06(0.05)$ & $20.00(0.05)$ & $19.88(0.09)$ \\
\hline 869 & 261.072 & 301.476 & $19.78(0.04)$ & $20.68(0.07)$ & $20.71(0.08)$ & $20.22(0.07)$ & $20.23(0.05)$ & $19.87(0.05)$ \\
\hline & & & $19.92(0.05)$ & $19.73(0.06)$ & $19.67(0.05)$ & $18.10(0.09)$ & $19.85(0.10)$ & $19.87(0.05)$ \\
\hline & & & $19.94(0.16)$ & $20.25(0.05)$ & $20.40(0.05)$ & $20.22(0.05)$ & $20.10(0.05)$ & $19.88(0.05)$ \\
\hline & & & $19.67(0.05)$ & $19.77(0.06)$ & $19.71(0.05)$ & $19.59(0.07)$ & $19.46(0.06)$ & $20.14(0.08)$ \\
\hline 870 & 265.326 & 15.040 & $20.38(0.05)$ & $20.14(0.08)$ & $20.27(0.08)$ & $20.16(0.08)$ & $19.71(0.05)$ & $20.11(0.06)$ \\
\hline & & & $20.12(0.06)$ & $20.65(0.12)$ & $20.26(0.05)$ & $19.88(0.05)$ & $19.84(0.05)$ & $19.85(0.05)$ \\
\hline & & & $20.44(0.07)$ & $20.19(0.05)$ & $20.26(0.06)$ & $20.23(0.07)$ & $20.03(0.05)$ & $19.95(0.05)$ \\
\hline & & & $19.75(0.06)$ & $19.76(0.05)$ & $19.67(0.05)$ & $19.57(0.05)$ & $19.55(0.05)$ & $19.67(0.09)$ \\
\hline 872 & 543.791 & 359.843 & $19.87(0.05)$ & $19.57(0.08)$ & $19.71(0.06)$ & $19.90(0.06)$ & $19.77(0.05)$ & $19.77(0.05)$ \\
\hline & & & $19.81(0.05)$ & $19.74(0.05)$ & $19.77(0.05)$ & $19.90(0.05)$ & $19.82(0.05)$ & $19.71(0.05)$ \\
\hline & & & $20.44(0.06)$ & $20.46(0.06)$ & $20.37(0.07)$ & $20.43(0.10)$ & $20.46(0.08)$ & $20.34(0.05)$ \\
\hline & & & $20.19(0.06)$ & $20.35(0.07)$ & $20.21(0.05)$ & $20.01(0.05)$ & $19.90(0.05)$ & $20.07(0.09)$ \\
\hline 874 & 470.740 & 678.097 & $19.72(0.04)$ & $20.36(0.06)$ & $20.04(0.07)$ & $20.04(0.05)$ & $20.00(0.05)$ & $19.98(0.05)$ \\
\hline & & & $19.74(0.05)$ & $19.80(0.06)$ & $19.71(0.04)$ & $19.83(0.04)$ & $19.76(0.05)$ & $19.73(0.05)$ \\
\hline & & & $20.42(0.06)$ & $20.40(0.05)$ & $20.36(0.05)$ & $20.25(0.05)$ & $20.05(0.05)$ & $20.02(0.05)$ \\
\hline & & & $19.80(0.06)$ & $19.86(0.05)$ & $19.82(0.05)$ & $19.92(0.06)$ & $19.93(0.05)$ & $20.31(0.13)$ \\
\hline 876 & 599.405 & 44.980 & $20.41(0.05)$ & $19.94(0.05)$ & $20.18(0.06)$ & $20.17(0.08)$ & $19.92(0.05)$ & $20.28(0.06)$ \\
\hline & & & $20.24(0.07)$ & $20.56(0.07)$ & $20.44(0.06)$ & $20.14(0.05)$ & $20.09(0.06)$ & $20.37(0.05)$ \\
\hline & & & $19.62(0.05)$ & $19.58(0.05)$ & $19.64(0.05)$ & $19.61(0.05)$ & $19.75(0.05)$ & $19.72(0.05)$ \\
\hline & & & $20.11(0.06)$ & $20.01(0.06)$ & $20.20(0.05)$ & $20.40(0.06)$ & $20.37(0.06)$ & $19.82(0.06)$ \\
\hline
\end{tabular}


Table 8. continuation.

\begin{tabular}{|c|c|c|c|c|c|c|c|c|}
\hline \multirow[t]{4}{*}{$\bar{F}$ F ID } & \multirow[t]{4}{*}{$\mathrm{x}$} & \multirow[t]{4}{*}{$\mathrm{y}$} & $2 \mathrm{Ks} 10(\sigma)$ & $2 \mathrm{Ks} 01(\sigma)$ & $2 \mathrm{Ks} 02(\sigma)$ & $2 \mathrm{Ks} 03(\sigma)$ & $2 \mathrm{Ks} 05(\sigma)$ & $2 \mathrm{Ks} 06(\sigma)$ \\
\hline & & & $2 \mathrm{Ks} 07(\sigma)$ & $2 \mathrm{Ks} 08(\sigma)$ & $2 \mathrm{Ks} 09(\sigma)$ & $2 \mathrm{Ks} 11(\sigma)$ & $2 \mathrm{Ks} 12(\sigma)$ & $2 \mathrm{Ks} 13(\sigma)$ \\
\hline & & & $2 \mathrm{Ks} 14(\sigma)$ & $2 \mathrm{Ks} 15(\sigma)$ & $2 \mathrm{Ks} 16(\sigma)$ & $2 \mathrm{Ks} 17(\sigma)$ & $2 \mathrm{Ks} 18(\sigma)$ & $2 \mathrm{Ks} 19(\sigma)$ \\
\hline & & & $2 \mathrm{Ks} 20(\sigma)$ & $2 \mathrm{Ks} 21(\sigma)$ & $2 \mathrm{Ks} 22(\sigma)$ & $2 \mathrm{Ks} 23(\sigma)$ & $2 \mathrm{Ks} 24(\sigma)$ & $2 \mathrm{Ks} 04(\sigma)$ \\
\hline \multirow[t]{4}{*}{877} & 195.617 & 278.930 & $19.75(0.04)$ & $20.36(0.06)$ & $20.37(0.08)$ & $20.45(0.07)$ & $20.22(0.05)$ & $19.97(0.05)$ \\
\hline & & & $19.88(0.05)$ & $19.82(0.05)$ & $19.79(0.05)$ & $19.93(0.05)$ & $19.95(0.06)$ & $20.06(0.05)$ \\
\hline & & & $20.05(0.05)$ & $19.92(0.05)$ & $19.95(0.04)$ & $19.76(0.05)$ & $19.76(0.05)$ & $19.83(0.05)$ \\
\hline & & & $19.80(0.05)$ & $19.80(0.06)$ & $19.94(0.05)$ & $20.16(0.05)$ & $20.32(0.06)$ & $20.47(0.11)$ \\
\hline \multirow[t]{4}{*}{883} & 136.275 & 129.456 & $20.44(0.06)$ & $20.14(0.08)$ & $19.91(0.07)$ & $19.81(0.06)$ & $19.76(0.05)$ & $19.99(0.05)$ \\
\hline & & & $20.13(0.05)$ & $20.05(0.06)$ & $20.21(0.05)$ & $20.23(0.05)$ & $20.11(0.06)$ & $20.10(0.05)$ \\
\hline & & & $19.65(0.05)$ & $19.62(0.04)$ & $19.81(0.05)$ & $19.97(0.07)$ & $19.79(0.05)$ & $19.89(0.05)$ \\
\hline & & & $19.77(0.06)$ & $19.96(0.07)$ & $20.21(0.05)$ & $20.19(0.05)$ & $19.96(0.05)$ & $19.64(0.09)$ \\
\hline \multirow[t]{4}{*}{884} & 587.084 & 469.637 & $19.82(0.04)$ & $19.88(0.05)$ & $19.68(0.05)$ & $19.79(0.05)$ & $20.02(0.05)$ & $19.91(0.05)$ \\
\hline & & & $20.00(0.05)$ & $19.81(0.06)$ & 19.93 & $19.84(0.05)$ & $19.67(0.05)$ & $19.73(0.05)$ \\
\hline & & & $20.36(0.06)$ & $20.35(0.04)$ & $20.22(0.04)$ & $20.09(0.05)$ & $20.19(0.06)$ & $20.18(0.05)$ \\
\hline & & & $19.82(0.05)$ & $19.89(0.05)$ & $19.72(0.05)$ & $19.79(0.05)$ & $19.79(0.05)$ & $20.21(0.09)$ \\
\hline \multirow[t]{4}{*}{895} & 343.661 & 20.594 & $20.00(0.06)$ & $20.22(0.09)$ & $20.18(0.07)$ & $20.35(0.08)$ & $20.20(0.05)$ & $20.23(0.05)$ \\
\hline & & & $20.07(0.0$ & $20.04(0.06)$ & 20.17 (0. & $19.90(0.05)$ & $19.79(0$ & $19.98(0.06)$ \\
\hline & & & $19.78(0.05)$ & $19.69(0.05)$ & 19.92 & 19.91 & 19.75 & 19.66 \\
\hline & & & $19.84(0.05)$ & $19.82(0.05)$ & $20.02(0.05)$ & 20.11 & 20.33 & $20.10(0.09)$ \\
\hline \multirow[t]{4}{*}{896} & 148.425 & 12.985 & $20.05(0.05)$ & $20.14(0.12)$ & $20.15(0.06)$ & $20.21(0.07)$ & $20.11(0.06)$ & $20.19(0.05)$ \\
\hline & & & $20.15(0.06)$ & $20.17(0.07)$ & $20.16(0.06)$ & $20.61(0.12)$ & $19.99(0.06)$ & $20.13(0.05)$ \\
\hline & & & 19.96 & 19.59 & 19.75 & 19.87 & 19.66 & 19.73 \\
\hline & & & $19.80(0.05)$ & $19.85(0.05)$ & $19.72(0.06)$ & $19.98(0.06)$ & $19.99(0.05)$ & $20.14(0.30)$ \\
\hline \multirow[t]{4}{*}{897} & 496.569 & 370.754 & $20.34(0.05)$ & $20.16(0.06)$ & $20.47(0.09)$ & $20.86(0.10)$ & $20.21(0.05)$ & $20.44(0.07)$ \\
\hline & & & $20.55(0.08)$ & $20.72(0.09)$ & $20.56(0.07)$ & $19.69(0.04)$ & $19.82(0.05)$ & $20.06(0.05)$ \\
\hline & & & $20.48(0.06)$ & $20.37(0.05)$ & 20.04 & $19.96(0.05)$ & 19.93 & $19.84(0.04)$ \\
\hline & & & $19.72(0.05)$ & $19.68(0.05)$ & $19.73(0.05)$ & $19.75(0.05)$ & 19.78 & $19.78(0.08)$ \\
\hline \multirow[t]{4}{*}{899} & 874.834 & 660.431 & $20.36(0.18)$ & $99.99(9.99)$ & $20.46(0.18)$ & $19.72(0.16)$ & $19.40(0.15)$ & $19.86(0.23)$ \\
\hline & & & $22.68(1.98)$ & $18.91(0.14)$ & $19.98(0.16)$ & $19.57(0.10)$ & $20.93(0.10)$ & $19.24(0.09)$ \\
\hline & & & 99.99 (9.99) & $21.18(0.29)$ & $20.83(0.67)$ & $21.46(0.23)$ & 99.99 (9.99) & $21.55(0.40)$ \\
\hline & & & & & 20.91 & 99.99 & 99.99 & 20.19 \\
\hline \multirow[t]{4}{*}{900} & 108.720 & 859.735 & $20.14(0.05)$ & $19.89(0.05)$ & $20.23(0.08)$ & $20.36(0.07)$ & $19.69(0.05)$ & $19.82(0.05)$ \\
\hline & & & $19.83(0.05)$ & $19.86(0.06)$ & $19.90(0.05)$ & 99.99 (9.99) & 99.99 (9.99) & 99.99 (9.99) \\
\hline & & & 99.99 (9.99) & 99.99 (9.99) & 99.99 (9.99) & $20.42(0.06)$ & $20.43(0.09)$ & $20.55(0.06)$ \\
\hline & & & & 99.99 (9.99) & & 99.99 (9.99) & 99.99 (9.99) & $20.60(0.40)$ \\
\hline \multirow[t]{4}{*}{901} & 70.901 & 646.594 & & $20.24(0.06)$ & $20.17(0.06)$ & $20.31(0.06)$ & $19.80(0.04)$ & $19.87(0.05)$ \\
\hline & & & $20.06(0.05)$ & $20.09(0.07)$ & $20.38(0.07)$ & $19.64(0.05)$ & $19.74(0.05)$ & $19.82(0.05)$ \\
\hline & & & $20.32(0.05)$ & $20.46(0.05)$ & $20.32(0.05)$ & $20.22(0.06)$ & $20.07(0.06)$ & $19.97(0.05)$ \\
\hline & & & & & $19.79(0.05)$ & $19.67(0.05)$ & $19.63(0.05)$ & $19.49(0.12)$ \\
\hline \multirow[t]{4}{*}{904} & 613.064 & 539.520 & $20.27(0.04)$ & $19.94(0.06)$ & $20.44(0.09)$ & $20.92(0.09)$ & $19.87(0.05)$ & $19.99(0.05)$ \\
\hline & & & $19.94(0.06)$ & $20.38(0.06)$ & $20.21(0.05)$ & $19.83(0.05)$ & $19.95(0.05)$ & $20.18(0.05)$ \\
\hline & & & $20.11(0.06)$ & $19.97(0.05)$ & $19.86(0.05)$ & $19.82(0.05)$ & $19.83(0.04)$ & $19.74(0.05)$ \\
\hline & & & & $19.66(0.05)$ & $19.75(0.05)$ & $19.99(0.05)$ & $20.08(0.05)$ & $19.90(0.10)$ \\
\hline \multirow[t]{4}{*}{905} & 442.866 & 243.989 & $19.78(0.04)$ & $20.42(0.07)$ & $20.55(0.07)$ & $20.54(0.07)$ & $20.20(0.05)$ & $20.09(0.05)$ \\
\hline & & & $20.05(0.05)$ & $19.77(0.05)$ & $19.83(0.05)$ & $20.07(0.05)$ & $19.97(0.05)$ & $19.89(0.05)$ \\
\hline & & & $20.24(0.05)$ & $20.21(0.05)$ & $20.10(0.05)$ & $19.96(0.05)$ & $19.92(0.05)$ & $19.74(0.05)$ \\
\hline & & & $19.78(0.05)$ & $19.73(0.06)$ & $19.75(0.05)$ & $19.85(0.05)$ & $20.12(0.06)$ & $20.21(0.07)$ \\
\hline \multirow[t]{4}{*}{906} & 839.347 & 811.938 & $19.69(0.05)$ & $20.26(0.12)$ & $20.36(0.08)$ & $20.35(0.07)$ & $20.25(0.06)$ & $20.06(0.05)$ \\
\hline & & & $19.90(0.05)$ & $19.96(0.06)$ & $19.82(0.05)$ & $19.91(0.05)$ & $19.82(0.06)$ & $19.74(0.06)$ \\
\hline & & & $20.40(0.07)$ & $20.20(0.06)$ & $20.43(0.07)$ & $20.42(0.13)$ & $20.12(0.10)$ & $19.93(0.06)$ \\
\hline & & & $19.74(0.05)$ & $19.79(0.07)$ & $19.71(0.07)$ & $19.68(0.06)$ & $19.72(0.05)$ & $20.56(0.33)$ \\
\hline
\end{tabular}


Table 8. continuation.

\begin{tabular}{|c|c|c|c|c|c|c|c|c|}
\hline \multirow[t]{4}{*}{ F2 ID } & \multirow[t]{4}{*}{$\mathrm{x}$} & \multirow[t]{4}{*}{$\mathrm{y}$} & $2 \mathrm{Ks} 10(\sigma)$ & $2 \mathrm{Ks} 01(\sigma)$ & $2 \mathrm{Ks} 02(\sigma)$ & $2 \mathrm{Ks} 03(\sigma)$ & $2 \mathrm{Ks} 05(\sigma)$ & $\overline{2 \mathrm{Ks} 06(\sigma)}$ \\
\hline & & & $2 \mathrm{Ks} 07(\sigma)$ & $2 \mathrm{Ks} 08(\sigma)$ & $2 \mathrm{Ks} 09(\sigma)$ & $2 \mathrm{Ks} 11(\sigma)$ & $2 \mathrm{Ks} 12(\sigma)$ & $2 \mathrm{Ks} 13(\sigma)$ \\
\hline & & & $2 \mathrm{Ks} 14(\sigma)$ & $2 \mathrm{Ks} 15(\sigma)$ & $2 \mathrm{Ks} 16(\sigma)$ & $2 \mathrm{Ks} 17(\sigma)$ & $2 \mathrm{Ks} 18(\sigma)$ & $2 \mathrm{Ks} 19(\sigma)$ \\
\hline & & & $2 \mathrm{Ks} 20(\sigma)$ & $2 \mathrm{Ks} 21(\sigma)$ & $2 \mathrm{Ks} 22(\sigma)$ & $2 \mathrm{Ks} 23(\sigma)$ & $2 \mathrm{Ks} 24(\sigma)$ & $2 \mathrm{Ks} 04(\sigma)$ \\
\hline \multirow[t]{4}{*}{$\overline{9917}$} & $\overline{761.600}$ & 134.913 & $19.93(0.04)$ & $20.04(0.06)$ & $19.71(0.06)$ & $19.59(0.05)$ & $20.33(0.05)$ & $20.36(0.06)$ \\
\hline & & & $20.31(0.07)$ & $20.20(0.06)$ & $20.26(0.07)$ & $20.40(0.06)$ & $20.25(0.05)$ & $20.21(0.05)$ \\
\hline & & & $19.49(0.05)$ & $19.65(0.05)$ & $19.72(0.05)$ & $19.76(0.05)$ & $19.93(0.05)$ & $19.89(0.05)$ \\
\hline & & & $20.18(0.06)$ & $20.12(0.06)$ & $20.10(0.05)$ & $20.19(0.05)$ & $20.27(0.06)$ & $20.69(0.14)$ \\
\hline \multirow[t]{4}{*}{918} & 311.623 & 31.425 & $19.75(0.05)$ & $20.41(0.09)$ & $20.30(0.07)$ & $20.02(0.07)$ & $20.23(0.05)$ & $20.15(0.05)$ \\
\hline & & & $20.01(0.05)$ & $19.79(0.05)$ & $19.93(0.05)$ & $20.13(0.05)$ & $19.93(0.05)$ & $19.91(0.05)$ \\
\hline & & & $20.14(0.06)$ & $20.11(0.05)$ & $20.21(0.05)$ & $20.18(0.07)$ & $19.97(0.06)$ & $19.85(0.05)$ \\
\hline & & & $19.77(0.05)$ & $19.87(0.06)$ & $19.75(0.05)$ & $19.66(0.05)$ & $19.87(0.06)$ & $20.17(0.08)$ \\
\hline \multirow[t]{4}{*}{920} & 894.150 & 151.063 & 99.99 (9.99) & 99.99 (9.99) & $19.68(0.06)$ & $19.72(0.05)$ & $19.72(0.05)$ & $19.60(0.05)$ \\
\hline & & & 99.99 (9.99) & $99.99(9.99)$ & $20.68(0.37)$ & $20.39(0.05)$ & $20.59(0.09)$ & $20.64(0.06)$ \\
\hline & & & $20.19(0.07)$ & $20.16(0.05)$ & $20.49(0.86)$ & 99.99 (9.99) & $20.18(0.06)$ & $19.83(0.05)$ \\
\hline & & & 99.99 (9.99) & 99.99 (9.99) & 99.99 (9.99) & $19.82(0.06)$ & $20.01(0.09)$ & $20.03(0.12)$ \\
\hline \multirow[t]{4}{*}{921} & 249.834 & 263.876 & $20.86(0.10)$ & $21.42(0.25)$ & $20.71(0.15)$ & $20.83(0.14)$ & $20.83(0.10)$ & $18.29(0.14)$ \\
\hline & & & $20.91(0.11)$ & $18.15(0.17)$ & $21.05(0.13)$ & $18.02(0.10)$ & $19.65(0.07)$ & $20.77(0.25)$ \\
\hline & & & $19.40(0.16)$ & $20.27(0.33)$ & $21.20(0.19)$ & $20.78(0.13)$ & $20.78(0.15)$ & $19.52(0.09)$ \\
\hline & & & $19.65(0.14)$ & $20.76(0.15)$ & $18.82(0.10)$ & $20.88(0.25)$ & $20.03(0.07)$ & $19.63(0.24)$ \\
\hline \multirow[t]{4}{*}{924} & 251.805 & 266.782 & 99.99 (9.99) & $19.93(0.42)$ & $22.01(0.80)$ & $21.06(0.42)$ & 99.99 (9.99) & $19.08(0.25)$ \\
\hline & & & $21.62(0.39)$ & $18.32(0.16)$ & $21.01(0.44)$ & $19.18(0.23)$ & $20.29(0.14)$ & 99.99 (9.99) \\
\hline & & & 99.99 (9.99) & $19.34(0.13)$ & $20.54(0.51)$ & $22.55(2.69)$ & $99.99(9.99)$ & $21.24(0.33)$ \\
\hline & & & 99.99 (9.99) & $20.89(0.37)$ & $19.90(0.14)$ & $20.24(0.12)$ & $21.07(0.14)$ & $19.82(0.28)$ \\
\hline \multirow[t]{4}{*}{927} & 39.207 & 632.947 & $19.71(0.04)$ & $19.70(0.05)$ & $19.62(0.06)$ & $19.60(0.06)$ & $19.97(0.05)$ & $19.88(0.05)$ \\
\hline & & & $19.86(0.05)$ & $19.66(0.05)$ & $19.65(0.05)$ & $20.39(0.06)$ & $20.09(0.06)$ & $19.79(0.04)$ \\
\hline & & & $20.89(0.08)$ & $21.15(0.07)$ & $20.89(0.06)$ & $21.14(0.14)$ & $20.89(0.09)$ & $20.85(0.07)$ \\
\hline & & & $20.83(0.08)$ & $20.81(0.09)$ & $20.92(0.08)$ & $20.72(0.07)$ & $20.85(0.09)$ & $20.50(0.23)$ \\
\hline \multirow[t]{4}{*}{929} & 72.743 & 19.972 & $20.70(0.07)$ & $20.49(0.06)$ & $20.49(0.08)$ & $20.64(0.10)$ & $20.60(0.05)$ & $20.19(0.12)$ \\
\hline & & & $21.05(0.11)$ & $19.94(0.08)$ & $20.90(0.08)$ & $19.61(0.05)$ & $20.21(0.06)$ & $20.00(0.11)$ \\
\hline & & & $19.73(0.05)$ & $19.68(0.05)$ & $19.97(0.06)$ & $20.38(0.08)$ & $19.80(0.06)$ & $19.85(0.05)$ \\
\hline & & & $19.62(0.05)$ & $19.68(0.05)$ & $19.65(0.05)$ & $20.31(0.06)$ & $20.36(0.05)$ & $19.87(0.15)$ \\
\hline \multirow[t]{4}{*}{935} & 276.218 & 750.716 & $20.25(0.05)$ & $19.92(0.05)$ & $20.04(0.08)$ & $20.08(0.07)$ & $19.72(0.04)$ & $19.96(0.05)$ \\
\hline & & & $19.91(0.05)$ & $20.02(0.06)$ & $20.15(0.06)$ & $19.86(0.05)$ & $19.84(0.05)$ & $20.08(0.05)$ \\
\hline & & & $20.41(0.06)$ & $20.47(0.05)$ & $20.31(0.05)$ & $20.15(0.06)$ & $20.03(0.05)$ & $19.94(0.05)$ \\
\hline & & & $19.93(0.05)$ & $19.84(0.06)$ & $19.83(0.05)$ & $19.82(0.05)$ & $19.76(0.05)$ & $19.79(0.08)$ \\
\hline \multirow[t]{4}{*}{938} & 384.965 & 361.451 & $19.76(0.04)$ & $19.52(0.06)$ & $19.65(0.05)$ & $19.92(0.06)$ & $19.96(0.05)$ & $19.84(0.05)$ \\
\hline & & & $19.90(0.05)$ & $19.88(0.05)$ & $19.75(0.05)$ & $20.03(0.05)$ & $19.89(0.05)$ & $19.59(0.04)$ \\
\hline & & & $20.44(0.06)$ & $20.41(0.05)$ & $20.45(0.05)$ & $20.46(0.06)$ & $20.40(0.06)$ & $20.27(0.05)$ \\
\hline & & & $20.22(0.07)$ & $20.34(0.06)$ & $20.26(0.05)$ & $20.41(0.06)$ & $20.37(0.07)$ & $20.46(0.12)$ \\
\hline \multirow[t]{4}{*}{940} & 611.253 & 230.397 & $19.79(0.05)$ & $20.24(0.07)$ & $20.01(0.06)$ & $19.89(0.06)$ & $19.90(0.04)$ & $20.24(0.11)$ \\
\hline & & & $20.13(0.09)$ & $19.42(0.18)$ & $19.98(0.05)$ & $19.33(0.07)$ & $21.13(0.29)$ & $20.28(0.05)$ \\
\hline & & & $19.65(0.08)$ & $19.76(0.05)$ & $19.82(0.05)$ & $19.95(0.05)$ & $19.97(0.04)$ & $20.08(0.08)$ \\
\hline & & & $20.26(0.06)$ & $20.14(0.06)$ & $20.17(0.07)$ & $20.30(0.08)$ & $20.34(0.09)$ & $20.40(0.13)$ \\
\hline \multirow[t]{4}{*}{941} & 471.261 & 171.558 & $20.37(0.09)$ & $19.63(0.07)$ & $19.89(0.07)$ & $20.12(0.07)$ & $20.03(0.09)$ & $20.61(0.09)$ \\
\hline & & & $20.31(0.08)$ & $21.27(0.44)$ & $20.19(0.07)$ & $19.90(0.06)$ & $20.00(0.06)$ & $20.75(0.09)$ \\
\hline & & & $20.24(0.05)$ & $19.83(0.05)$ & $20.05(0.05)$ & $20.18(0.05)$ & $20.03(0.05)$ & $20.32(0.05)$ \\
\hline & & & $20.41(0.07)$ & $20.07(0.06)$ & $19.87(0.05)$ & $19.74(0.05)$ & $19.65(0.05)$ & $19.98(0.12)$ \\
\hline \multirow[t]{4}{*}{942} & 738.350 & 482.234 & $20.11(0.05)$ & $20.09(0.06)$ & $20.20(0.07)$ & $20.27(0.07)$ & $19.63(0.05)$ & $19.76(0.05)$ \\
\hline & & & $19.90(0.05)$ & $20.12(0.06)$ & $19.98(0.05)$ & $19.56(0.05)$ & $19.54(0.05)$ & $19.63(0.05)$ \\
\hline & & & $20.47(0.08)$ & $20.47(0.06)$ & $20.57(0.06)$ & $20.63(0.06)$ & $20.77(0.06)$ & $20.66(0.06)$ \\
\hline & & & $20.45(0.07)$ & $20.63(0.09)$ & $20.25(0.05)$ & $19.97(0.05)$ & $19.85(0.05)$ & $19.56(0.09)$ \\
\hline
\end{tabular}


Table 8. continuation.

\begin{tabular}{|c|c|c|c|c|c|c|c|c|}
\hline \multirow[t]{4}{*}{ F2 ID } & \multirow[t]{4}{*}{$\mathrm{x}$} & \multirow[t]{4}{*}{$\mathrm{y}$} & $2 \mathrm{Ks} 10(\sigma)$ & $2 \mathrm{Ks} 01(\sigma)$ & $2 \mathrm{Ks} 02(\sigma)$ & $2 \mathrm{Ks} 03(\sigma)$ & $2 \mathrm{Ks} 05(\sigma)$ & $\overline{2 \mathrm{Ks} 06(\sigma)}$ \\
\hline & & & $2 \mathrm{Ks} 07(\sigma)$ & $2 \mathrm{Ks} 08(\sigma)$ & $2 \mathrm{Ks} 09(\sigma)$ & $2 \mathrm{Ks} 11(\sigma)$ & $2 \mathrm{Ks} 12(\sigma)$ & $2 \mathrm{Ks} 13(\sigma)$ \\
\hline & & & $2 \mathrm{Ks} 14(\sigma)$ & $2 \mathrm{Ks} 15(\sigma)$ & $2 \mathrm{Ks} 16(\sigma)$ & $2 \mathrm{Ks} 17(\sigma)$ & $2 \mathrm{Ks} 18(\sigma)$ & $2 \mathrm{Ks} 19(\sigma)$ \\
\hline & & & $2 \mathrm{Ks} 20(\sigma)$ & $2 \mathrm{Ks} 21(\sigma)$ & $2 \mathrm{Ks} 22(\sigma)$ & $2 \mathrm{Ks} 23(\sigma)$ & $2 \mathrm{Ks} 24(\sigma)$ & $2 \mathrm{Ks} 04(\sigma)$ \\
\hline \multirow[t]{4}{*}{$\overline{944}$} & 624.171 & 204.963 & $19.98(0.10)$ & $19.89(0.12)$ & $\overline{999.99(9.99)}$ & $20.00(0.16)$ & $\overline{~ 19.34(0.12)}$ & $\bar{~} 19.54(0.16)$ \\
\hline & & & $19.87(0.18)$ & $19.52(0.22)$ & $19.81(0.11)$ & $19.36(0.08)$ & $20.31(0.09)$ & $19.19(0.10)$ \\
\hline & & & 99.99 (9.99) & $20.90(0.24)$ & $20.47(0.23)$ & $20.35(0.18)$ & $20.69(0.22)$ & $20.16(0.10)$ \\
\hline & & & $20.43(0.11)$ & $21.12(0.33)$ & $19.96(0.11)$ & $20.74(0.13)$ & $20.15(0.08)$ & $20.27(0.28)$ \\
\hline \multirow[t]{4}{*}{945} & 456.772 & 44.377 & $20.31(0.06)$ & $20.31(0.08)$ & $20.33(0.10)$ & $20.45(0.08)$ & $20.27(0.06)$ & $20.49(0.06)$ \\
\hline & & & $20.45(0.08)$ & $20.48(0.08)$ & $20.38(0.07)$ & $20.24(0.06)$ & $20.27(0.07)$ & $20.14(0.06)$ \\
\hline & & & $19.54(0.05)$ & $19.52(0.05)$ & $19.66(0.07)$ & $19.73(0.07)$ & $19.76(0.07)$ & $19.82(0.05)$ \\
\hline & & & $19.95(0.06)$ & $19.98(0.06)$ & $20.09(0.05)$ & $20.21(0.06)$ & $20.32(0.06)$ & $20.06(0.08)$ \\
\hline \multirow[t]{4}{*}{946} & 674.638 & 817.162 & $19.78(0.05)$ & $19.87(0.08)$ & $19.76(0.07)$ & $19.83(0.07)$ & $19.91(0.05)$ & $19.95(0.05)$ \\
\hline & & & $19.73(0.05)$ & $19.95(0.05)$ & 19.75 & $19.89(0.05)$ & $19.76(0.05)$ & $19.66(0.05)$ \\
\hline & & & $20.75(0.06)$ & 20.64 & 20.73 & 20.67 & 20.63 & $20.43(0.06)$ \\
\hline & & & $20.17(0.07)$ & $20.26(0.06)$ & $20.15(0.06)$ & $19.96(0.06)$ & $20.00(0.05)$ & $20.38(0.14)$ \\
\hline \multirow[t]{4}{*}{954} & 168.503 & 285.342 & $20.23(0.05)$ & $20.40(0.08)$ & $20.32(0.08)$ & $20.38(0.07)$ & $20.25(0.05)$ & $20.11(0.05)$ \\
\hline & & & $20.27(0.07)$ & $20.36(0.08)$ & 20.19 & $20.38(0.05)$ & $20.22(0.06)$ & $20.22(0.05)$ \\
\hline & & & $19.59(0.05)$ & $19.58(0.04)$ & 19.70 & 19.75 & 19.82 & 20.02 \\
\hline & & & $20.05(0.06)$ & 20.07 & 20.21 & 20.19 & 20.08 & 20.27 \\
\hline \multirow[t]{4}{*}{958} & 104.947 & -20.160 & 99.99 (9.99) & $20.92(0.15)$ & 99.99 (9.99) & 99.99 (9.99) & 99.99 (9.99) & 99.99 (9.99) \\
\hline & & & 99.99 (9.99) & 99.99 (9.99) & 99.99 (9.99) & $17.54(0.08)$ & $19.70(0.29)$ & $19.75(0.17)$ \\
\hline & & & $99.99(9$ & $20.13(0.09)$ & 20.75 & 99.9 & 20.13 & $20.1^{\prime}$ \\
\hline & & & $20.06(0.07)$ & 20.26 & 20.22 & 20.99 & 20.53 & $20.93(0.44)$ \\
\hline \multirow[t]{4}{*}{962} & 416.830 & 712.250 & $19.84(0.04)$ & $19.77(0.05)$ & $19.75(0.06)$ & $19.76(0.06)$ & $19.73(0.05)$ & $19.81(0.05)$ \\
\hline & & & $19.75(0.05)$ & $19.84(0.05)$ & $19.76(0.05)$ & $20.17(0.05)$ & $20.00(0.05)$ & $19.89(0.05)$ \\
\hline & & & $20.78(0.06)$ & $20.78(0.07)$ & $20.76(0.06)$ & $20.66(0.08)$ & $20.54(0.06)$ & $20.44(0.06)$ \\
\hline & & & $20.22(0.06)$ & $20.24(0.06)$ & $20.14(0.06)$ & 19.99 & 19.91 & $20.18(0.10)$ \\
\hline \multirow[t]{4}{*}{964} & 700.009 & 428.179 & $20.11(0.05)$ & $20.14(0.06)$ & $20.18(0.06)$ & $20.22(0.07)$ & $20.01(0.05)$ & $20.15(0.05)$ \\
\hline & & & $20.28(0.06)$ & $20.32(0.07)$ & $20.10(0.05)$ & $20.09(0.05)$ & $20.34(0.06)$ & $20.46(0.05)$ \\
\hline & & & & $19.76(0.04)$ & $19.72(0.05)$ & $19.77(0.04)$ & $19.83(0.05)$ & $19.86(0.05)$ \\
\hline & & & & & 20.03 & 20.12 & 20.40 & \\
\hline \multirow[t]{4}{*}{966} & 461.003 & 9.151 & $19.56(0.05)$ & $20.06(0.05)$ & $19.96(0.06)$ & $19.85(0.06)$ & $20.07(0.05)$ & $20.04(0.05)$ \\
\hline & & & $19.92(0.05)$ & $19.63(0.06)$ & $19.66(0.05)$ & $20.13(0.05)$ & $20.18(0.05)$ & $20.00(0.05)$ \\
\hline & & & $19.91(0.05)$ & $19.87(0.05)$ & $20.13(0.06)$ & $20.18(0.06)$ & $20.21(0.05)$ & $20.33(0.06)$ \\
\hline & & & & $20.42(0.10)$ & & $20.27(0.07)$ & $20.45(0.07)$ & $20.46(0.11)$ \\
\hline \multirow[t]{4}{*}{970} & 14.395 & 722.569 & $20.50(0.05)$ & & & & 19.95 & $20.10(0.05)$ \\
\hline & & & $20.20(0.06)$ & $20.57(0.08)$ & $20.36(0.07)$ & $19.83(0.06)$ & $19.97(0.05)$ & $20.22(0.07)$ \\
\hline & & & $20.11(0.06)$ & $20.02(0.05)$ & $19.70(0.05)$ & 99.99 (9.99) & $19.72(0.07)$ & $19.67(0.04)$ \\
\hline & & & & & $19.78(0.05)$ & & $20.30(0.07)$ & \\
\hline \multirow[t]{4}{*}{973} & 728.236 & 285.185 & $20.30(0.05)$ & $19.81(0.07)$ & $19.92(0.06)$ & $20.33(0.08)$ & $19.84(0.05)$ & $19.97(0.05)$ \\
\hline & & & $20.21(0.06)$ & $20.22(0.06)$ & $20.15(0.05)$ & $20.05(0.05)$ & $20.22(0.06)$ & $20.40(0.05)$ \\
\hline & & & $19.75(0.05)$ & $19.78(0.04)$ & $19.79(0.05)$ & $19.84(0.05)$ & $19.91(0.05)$ & $19.97(0.05)$ \\
\hline & & & & $20.06(0.06)$ & $20.20(0.05)$ & $20.41(0.06)$ & $20.53(0.06)$ & $19.81(0.11)$ \\
\hline \multirow[t]{4}{*}{975} & 219.651 & 848.037 & $19.74(0.04)$ & $19.62(0.05)$ & $19.59(0.06)$ & $19.58(0.06)$ & $20.06(0$. & $20.03(0.05)$ \\
\hline & & & $19.93(0.05)$ & $19.76(0.05)$ & $19.78(0.05)$ & $20.21(0.05)$ & $20.05(0.05)$ & $19.88(0.05)$ \\
\hline & & & $20.25(0.06)$ & $20.45(0.06)$ & $20.31(0.05)$ & $20.25(0.05)$ & $20.25(0.07)$ & $20.26(0.05)$ \\
\hline & & & $20.19(0.06)$ & $20.19(0.06)$ & $20.16(0.05)$ & $20.10(0.21)$ & 99.99 (9.99) & $20.23(0.14)$ \\
\hline \multirow[t]{4}{*}{976} & 833.153 & 409.515 & $20.28(0.05)$ & $19.73(0.05)$ & $20.00(0.07)$ & $20.12(0.07)$ & $19.70(0.04)$ & $19.87(0.05)$ \\
\hline & & & $19.61(0.05)$ & $20.26(0.07)$ & $19.98(0.05)$ & $19.82(0.05)$ & $19.61(0.05)$ & $19.63(0.04)$ \\
\hline & & & $20.52(0.06)$ & $20.44(0.05)$ & $20.29(0.05)$ & $20.61(0.05)$ & $20.56(0.05)$ & $20.64(0.06)$ \\
\hline & & & $20.32(0.07)$ & $20.11(0.06)$ & $20.27(0.05)$ & $20.18(0.05)$ & $20.11(0.05)$ & $19.49(0.06)$ \\
\hline
\end{tabular}


Table 8. continuation.

\begin{tabular}{|c|c|c|c|c|c|c|c|c|}
\hline \multirow[t]{4}{*}{ F2 ID } & \multirow[t]{4}{*}{$\mathrm{x}$} & \multirow[t]{4}{*}{$\mathrm{y}$} & $2 \mathrm{Ks} 10(\sigma)$ & $2 \mathrm{Ks} 01(\sigma)$ & $2 \mathrm{Ks} 02(\sigma)$ & $2 \mathrm{Ks} 03(\sigma)$ & $2 \mathrm{Ks} 05(\sigma)$ & $\overline{2 \mathrm{Ks} 06(\sigma)}$ \\
\hline & & & $2 \mathrm{Ks} 07(\sigma)$ & $2 \mathrm{Ks} 08(\sigma)$ & $2 \mathrm{Ks} 09(\sigma)$ & $2 \mathrm{Ks} 11(\sigma)$ & $2 \mathrm{Ks} 12(\sigma)$ & $2 \mathrm{Ks} 13(\sigma)$ \\
\hline & & & $2 \mathrm{Ks} 14(\sigma)$ & $2 \mathrm{Ks} 15(\sigma)$ & $2 \mathrm{Ks} 16(\sigma)$ & $2 \mathrm{Ks} 17(\sigma)$ & $2 \mathrm{Ks} 18(\sigma)$ & $2 \mathrm{Ks} 19(\sigma)$ \\
\hline & & & $2 \mathrm{Ks} 20(\sigma)$ & $2 \mathrm{Ks} 21(\sigma)$ & $2 \mathrm{Ks} 22(\sigma)$ & $2 \mathrm{Ks} 23(\sigma)$ & $2 \mathrm{Ks} 24(\sigma)$ & $2 \mathrm{Ks} 04(\sigma)$ \\
\hline \multirow[t]{4}{*}{$\overline{9981}$} & 1.907 & 76.444 & $20.17(0.05)$ & $20.14(0.10)$ & $20.22(0.66)$ & $20.40(0.07)$ & $99.99(9.99)$ & $20.43(0.13)$ \\
\hline & & & $20.21(0.06)$ & $20.30(0.06)$ & $20.21(0.06)$ & 99.99 (9.99) & 99.99 (9.99) & 99.99 (9.99) \\
\hline & & & 99.99 (9.99) & 99.99 (9.99) & $19.80(0.05)$ & 99.99 (9.99) & 99.99 (9.99) & $19.68(0.06)$ \\
\hline & & & $19.69(0.05)$ & $19.66(0.06)$ & $20.30(0.28)$ & 99.99 (9.99) & 99.99 (9.99) & $20.03(0.22)$ \\
\hline \multirow[t]{4}{*}{982} & 443.991 & 671.618 & $19.99(0.05)$ & $20.25(0.07)$ & $20.20(0.05)$ & $20.22(0.06)$ & $20.00(0.05)$ & $20.30(0.05)$ \\
\hline & & & $20.22(0.06)$ & $20.19(0.06)$ & $20.09(0.05)$ & $20.04(0.05)$ & $20.18(0.06)$ & $20.23(0.04)$ \\
\hline & & & $19.82(0.05)$ & $19.84(0.05)$ & $19.71(0.05)$ & $19.73(0.05)$ & $19.72(0.05)$ & $19.90(0.05)$ \\
\hline & & & $19.99(0.05)$ & $19.77(0.05)$ & $20.03(0.05)$ & $20.29(0.07)$ & $20.50(0.08)$ & $19.82(0.07)$ \\
\hline \multirow[t]{4}{*}{986} & 773.864 & 71.346 & $20.52(0.05)$ & $19.92(0.05)$ & $19.83(0.05)$ & $19.81(0.06)$ & $20.72(0.08)$ & $20.66(0.06)$ \\
\hline & & & $20.80(0.07)$ & $20.54(0.07)$ & $20.45(0.06)$ & $20.42(0.06)$ & $20.70(0.06)$ & $20.75(0.06)$ \\
\hline & & & $19.86(0.05)$ & $19.76(0.04)$ & $19.82(0.06)$ & $19.73(0.05)$ & $19.81(0.05)$ & $19.63(0.05)$ \\
\hline & & & $19.82(0.05)$ & $19.80(0.05)$ & $19.79(0.05)$ & $20.05(0.05)$ & $20.30(0.07)$ & $21.48(0.24)$ \\
\hline \multirow[t]{4}{*}{988} & 708.433 & 429.740 & $19.94(0.05)$ & $20.12(0.06)$ & $19.86(0.05)$ & $19.81(0.05)$ & $20.16(0.06)$ & $19.93(0.05)$ \\
\hline & & & $20.12(0.05)$ & $20.14(0.06)$ & $19.90(0.05)$ & $19.85(0.05)$ & $19.80(0.05)$ & $19.77(0.05)$ \\
\hline & & & $20.21(0.06)$ & $20.32(0.05)$ & $20.22(0.05)$ & $20.14(0.05)$ & $20.21(0.06)$ & $20.00(0.05)$ \\
\hline & & & $19.94(0.05)$ & $19.97(0.06)$ & $19.88(0.05)$ & $19.92(0.05)$ & $20.07(0.06)$ & $20.73(0.12)$ \\
\hline \multirow[t]{4}{*}{991} & 41.210 & 298.708 & $19.77(0.05)$ & $20.43(0.08)$ & $20.22(0.07)$ & $20.07(0.06)$ & $20.15(0.05)$ & $19.87(0.05)$ \\
\hline & & & $19.82(0.05)$ & $19.84(0.05)$ & $19.76(0.05)$ & $20.08(0.05)$ & $19.95(0.06)$ & $19.92(0.05)$ \\
\hline & & & $20.39(0.07)$ & $20.44(0.06)$ & $20.28(0.05)$ & $20.30(0.06)$ & $20.18(0.05)$ & $20.07(0.06)$ \\
\hline & & & $19.96(0.06)$ & $19.95(0.06)$ & $19.87(0.05)$ & $19.77(0.05)$ & $19.81(0.05)$ & $20.31(0.10)$ \\
\hline \multirow[t]{4}{*}{993} & 28.742 & 806.438 & $20.30(0.07)$ & $20.29(0.09)$ & $20.39(0.10)$ & $20.20(0.09)$ & $20.03(0.06)$ & $20.15(0.06)$ \\
\hline & & & $20.23(0.06)$ & $20.13(0.06)$ & $20.35(0.08)$ & $19.82(0.05)$ & $20.02(0.07)$ & $20.12(0.06)$ \\
\hline & & & $19.61(0.05)$ & $19.75(0.05)$ & $19.72(0.05)$ & 99.99 (9.99) & $20.01(0.08)$ & $19.97(0.06)$ \\
\hline & & & $20.13(0.06)$ & $20.29(0.07)$ & $20.23(0.05)$ & $20.40(0.08)$ & $20.26(0.08)$ & $20.41(0.29)$ \\
\hline \multirow[t]{4}{*}{995} & 80.395 & -10.595 & $99.99(9.99)$ & $99.99(9.99)$ & $99.99(9.99)$ & 99.99 (9.99) & $99.99(9.99)$ & 99.99 (9.99) \\
\hline & & & $99.99(9.99)$ & $99.99(9.99)$ & $99.99(9.99)$ & $20.10(0.06)$ & $20.45(0.07)$ & 99.99 (9.99) \\
\hline & & & $20.80(0.09)$ & $20.33(0.07)$ & $20.14(0.09)$ & 99.99 (9.99) & $19.74(0.07)$ & $19.88(0.06)$ \\
\hline & & & $20.35(0.07)$ & $20.11(0.07)$ & $19.72(0.05)$ & $19.44(0.07)$ & $19.56(0.07)$ & $20.83(0.50)$ \\
\hline \multirow[t]{4}{*}{998} & 675.854 & 236.591 & $19.54(0.04)$ & $19.93(0.05)$ & $19.74(0.06)$ & $19.74(0.07)$ & $20.07(0.05)$ & $19.97(0.05)$ \\
\hline & & & $19.95(0.05)$ & $19.85(0.05)$ & $19.72(0.05)$ & $20.36(0.06)$ & $20.29(0.06)$ & $20.24(0.05)$ \\
\hline & & & $19.83(0.05)$ & $19.94(0.05)$ & $20.09(0.04)$ & $20.14(0.05)$ & $20.30(0.05)$ & $20.27(0.05)$ \\
\hline & & & $20.25(0.07)$ & $20.16(0.07)$ & $20.04(0.05)$ & $19.98(0.05)$ & $20.15(0.06)$ & $20.30(0.14)$ \\
\hline \multirow[t]{4}{*}{1004} & 169.996 & 493.106 & $20.64(0.05)$ & $20.05(0.05)$ & $20.27(0.08)$ & $20.51(0.08)$ & $20.09(0.05)$ & $20.17(0.05)$ \\
\hline & & & $20.30(0.06)$ & $20.77(0.09)$ & $20.42(0.06)$ & $19.97(0.05)$ & $20.11(0.06)$ & $20.33(0.05)$ \\
\hline & & & $19.98(0.05)$ & $19.84(0.05)$ & $19.78(0.05)$ & $19.61(0.05)$ & $19.66(0.05)$ & $19.64(0.04)$ \\
\hline & & & $19.76(0.05)$ & $19.66(0.05)$ & $19.89(0.05)$ & $20.18(0.05)$ & $20.29(0.08)$ & $19.82(0.08)$ \\
\hline \multirow[t]{4}{*}{1012} & 631.397 & 109.589 & $20.12(0.05)$ & $20.46(0.09)$ & $20.50(0.06)$ & $20.49(0.06)$ & $20.18(0.05)$ & $20.21(0.05)$ \\
\hline & & & $20.31(0.07)$ & $20.26(0.06)$ & $20.17(0.05)$ & $20.25(0.05)$ & $20.36(0.06)$ & $20.37(0.05)$ \\
\hline & & & $19.74(0.05)$ & $19.69(0.04)$ & $19.75(0.05)$ & $19.80(0.04)$ & $19.91(0.05)$ & $19.74(0.05)$ \\
\hline & & & $20.11(0.07)$ & $19.98(0.06)$ & $20.02(0.05)$ & $20.29(0.06)$ & $20.39(0.07)$ & $19.99(0.11)$ \\
\hline \multirow[t]{4}{*}{1014} & 798.490 & 34.797 & $19.90(0.05)$ & $20.28(0.07)$ & $20.39(0.08)$ & $20.22(0.09)$ & $19.92(0.06)$ & $19.82(0.05)$ \\
\hline & & & $20.00(0.06)$ & $19.72(0.05)$ & $19.88(0.06)$ & $19.96(0.06)$ & $20.19(0.06)$ & $20.30(0.07)$ \\
\hline & & & $19.74(0.05)$ & $19.91(0.05)$ & $20.01(0.06)$ & $20.10(0.07)$ & $20.16(0.06)$ & $20.04(0.06)$ \\
\hline & & & $20.23(0.07)$ & $20.35(0.10)$ & $20.15(0.05)$ & $20.29(0.06)$ & $20.55(0.09)$ & $19.84(0.14)$ \\
\hline \multirow[t]{4}{*}{1017} & 501.398 & 309.847 & $20.81(0.05)$ & $21.09(0.12)$ & $21.02(0.12)$ & $20.84(0.11)$ & $20.93(0.07)$ & $20.74(0.06)$ \\
\hline & & & $20.96(0.10)$ & 99.99 (9.99) & $20.86(0.07)$ & $20.54(0.05)$ & $20.60(0.06)$ & $20.70(0.06)$ \\
\hline & & & $19.91(0.05)$ & $19.87(0.05)$ & $19.80(0.05)$ & $19.74(0.04)$ & $19.73(0.05)$ & $19.71(0.05)$ \\
\hline & & & $19.77(0.05)$ & $19.75(0.06)$ & $19.79(0.05)$ & $19.89(0.05)$ & $20.12(0.06)$ & $20.66(0.14)$ \\
\hline
\end{tabular}


Table 8. continuation.

\begin{tabular}{|c|c|c|c|c|c|c|c|c|}
\hline \multirow[t]{4}{*}{ F2 ID } & \multirow[t]{4}{*}{$\mathrm{x}$} & \multirow[t]{4}{*}{$y$} & $2 \mathrm{Ks} 10(\sigma)$ & $2 \mathrm{Ks} 01(\sigma)$ & $2 \mathrm{Ks} 02(\sigma)$ & $2 \mathrm{Ks} 03(\sigma)$ & $2 \mathrm{Ks} 05(\sigma)$ & $2 \mathrm{Ks} 06(\sigma)$ \\
\hline & & & $2 \mathrm{Ks} 07(\sigma)$ & $2 \mathrm{Ks} 08(\sigma)$ & $2 \mathrm{Ks} 09(\sigma)$ & $2 \mathrm{Ks} 11(\sigma)$ & $2 \mathrm{Ks} 12(\sigma)$ & $2 \mathrm{Ks} 13(\sigma)$ \\
\hline & & & $2 \mathrm{Ks} 14(\sigma)$ & $2 \mathrm{Ks} 15(\sigma)$ & $2 \mathrm{Ks} 16(\sigma)$ & $2 \mathrm{Ks} 17(\sigma)$ & $2 \mathrm{Ks} 18(\sigma)$ & $2 \mathrm{Ks} 19(\sigma)$ \\
\hline & & & $2 \mathrm{Ks} 20(\sigma)$ & $2 \mathrm{Ks} 21(\sigma)$ & $2 \mathrm{Ks} 22(\sigma)$ & $2 \mathrm{Ks} 23(\sigma)$ & $2 \mathrm{Ks} 24(\sigma)$ & $2 \mathrm{Ks} 04(\sigma)$ \\
\hline \multirow[t]{4}{*}{1019} & 145.017 & 297.283 & $20.36(0.05)$ & $20.10(0.06)$ & $20.50(0.09)$ & $20.26(0.07)$ & $20.51(0.05)$ & $20.32(0.05)$ \\
\hline & & & $20.54(0.07)$ & $20.33(0.06)$ & $20.40(0.06)$ & $20.44(0.06)$ & $20.22(0.06)$ & $20.08(0.05)$ \\
\hline & & & $19.65(0.05)$ & $19.52(0.04)$ & $19.64(0.05)$ & $19.69(0.05)$ & $19.73(0.05)$ & $20.02(0.05)$ \\
\hline & & & $20.19(0.05)$ & $20.20(0.06)$ & $20.28(0.05)$ & $20.41(0.06)$ & $20.37(0.07)$ & $20.46(0.12)$ \\
\hline \multirow[t]{4}{*}{1023} & 131.064 & -34.965 & $99.99(9.99)$ & $99.99(9.99)$ & $99.99(9.99)$ & 99.99 (9.99) & $99.99(9.99)$ & 99.99 (9.99) \\
\hline & & & 99.99 (9.99) & 99.99 (9.99) & 99.99 (9.99) & $18.75(0.31)$ & $19.24(0.16)$ & $18.29(0.15)$ \\
\hline & & & 99.99 (9.99) & 99.99 (9.99) & $21.44(1.05)$ & 99.99 (9.99) & 99.99 (9.99) & 99.99 (9.99) \\
\hline & & & $20.41(0.10)$ & $21.01(0.12)$ & $18.42(0.21)$ & $21.79(1.10)$ & $20.60(0.15)$ & $20.83(0.33)$ \\
\hline \multirow[t]{4}{*}{1027} & 387.202 & 150.105 & $20.20(0.05)$ & $20.15(0.05)$ & $20.08(0.07)$ & $20.06(0.06)$ & $20.32(0.05)$ & $20.34(0.05)$ \\
\hline & & & $20.27(0.06)$ & $20.25(0.06)$ & $20.19(0.05)$ & $20.22(0.05)$ & $20.14(0.05)$ & $20.13(0.05)$ \\
\hline & & & $19.72(0.05)$ & $19.63(0.04)$ & $19.82(0.05)$ & $19.81(0.05)$ & $19.87(0.05)$ & $20.03(0.05)$ \\
\hline & & & $20.02(0.05)$ & $20.11(0.06)$ & $20.01(0.05)$ & $20.12(0.06)$ & $20.08(0.06)$ & $20.04(0.09)$ \\
\hline \multirow[t]{4}{*}{1028} & 494.779 & 27.265 & $19.97(0.05)$ & $20.56(0.08)$ & $20.59(0.09)$ & $20.70(0.10)$ & $20.47(0.05)$ & $20.35(0.05)$ \\
\hline & & & $20.10(0.06)$ & $20.00(0.06)$ & $20.11(0.05)$ & $19.81(0.05)$ & $19.79(0.05)$ & $19.89(0.05)$ \\
\hline & & & $20.19(0.06)$ & $19.95(0.05)$ & $19.99(0.05)$ & $19.99(0.06)$ & $19.86(0.05)$ & $19.67(0.05)$ \\
\hline & & & $19.85(0.06)$ & $19.85(0.06)$ & $19.84(0.05)$ & $20.09(0.05)$ & $20.25(0.06)$ & $20.43(0.15)$ \\
\hline \multirow[t]{4}{*}{1032} & 786.150 & 72.583 & $19.95(0.04)$ & $20.44(0.07)$ & $20.37(0.09)$ & $20.18(0.07)$ & $19.99(0.05)$ & $19.95(0.05)$ \\
\hline & & & $19.98(0.05)$ & $20.04(0.06)$ & $19.87(0.04)$ & $19.92(0.05)$ & $20.04(0.05)$ & $20.15(0.05)$ \\
\hline & & & $19.81(0.05)$ & $19.84(0.05)$ & $19.83(0.05)$ & $19.88(0.05)$ & $20.09(0.06)$ & $20.02(0.05)$ \\
\hline & & & $20.57(0.08)$ & $20.35(0.07)$ & $20.54(0.05)$ & $20.64(0.07)$ & $20.75(0.07)$ & $20.38(0.10)$ \\
\hline \multirow[t]{4}{*}{1037} & 741.930 & 653.414 & $20.02(0.06)$ & $20.13(0.07)$ & $20.23(0.06)$ & $19.94(0.06)$ & $20.44(0.06)$ & $20.41(0.06)$ \\
\hline & & & $20.38(0.07)$ & $20.22(0.05)$ & $20.10(0.06)$ & $19.99(0.05)$ & $19.81(0.05)$ & $19.81(0.06)$ \\
\hline & & & $20.16(0.05)$ & $20.22(0.05)$ & $20.21(0.06)$ & $20.16(0.05)$ & $20.06(0.06)$ & $19.99(0.05)$ \\
\hline & & & $19.77(0.05)$ & $19.90(0.05)$ & $19.74(0.05)$ & $19.70(0.05)$ & $19.91(0.05)$ & $20.51(0.13)$ \\
\hline \multirow[t]{4}{*}{1039} & 692.432 & 94.454 & $20.76(0.06)$ & $20.37(0.08)$ & $20.46(0.09)$ & $20.42(0.10)$ & $20.05(0.05)$ & $20.48(0.06)$ \\
\hline & & & $20.42(0.06)$ & $20.75(0.08)$ & $20.52(0.06)$ & $20.02(0.05)$ & $20.18(0.06)$ & $20.38(0.06)$ \\
\hline & & & $20.03(0.05)$ & $20.00(0.05)$ & $19.90(0.05)$ & $19.86(0.05)$ & $19.85(0.05)$ & $19.69(0.05)$ \\
\hline & & & $19.84(0.06)$ & & $19.77(0.05)$ & $19.90(0.05)$ & $20.06(0.05)$ & $19.53(0.05)$ \\
\hline \multirow[t]{4}{*}{1044} & 766.510 & 748.804 & $20.39(0.04)$ & $19.71(0.07)$ & $19.67(0.05)$ & $19.78(0.06)$ & $19.83(0.05)$ & $20.07(0.05)$ \\
\hline & & & $20.01(0.05)$ & $20.33(0.05)$ & $20.26(0.05)$ & $20.05(0.05)$ & $20.03(0.05)$ & $20.21(0.05)$ \\
\hline & & & $20.13(0.06)$ & $19.99(0.05)$ & $19.85(0.04)$ & $19.90(0.09)$ & $19.73(0.05)$ & $19.88(0.05)$ \\
\hline & & & $19.82(0.05)$ & $19.83(0.06)$ & $19.92(0.05)$ & $20.07(0.05)$ & $20.27(0.06)$ & $19.89(0.07)$ \\
\hline \multirow[t]{4}{*}{1045} & 547.012 & 590.337 & $20.35(0.04)$ & $20.48(0.05)$ & $20.65(0.08)$ & $20.56(0.09)$ & $19.71(0.04)$ & $19.90(0.05)$ \\
\hline & & & $20.05(0.06)$ & $20.20(0.06)$ & $20.01(0.05)$ & $19.38(0.04)$ & $19.48(0.05)$ & $19.59(0.04)$ \\
\hline & & & $20.83(0.07)$ & $20.76(0.05)$ & $20.67(0.05)$ & $20.74(0.05)$ & $20.79(0.06)$ & $20.89(0.07)$ \\
\hline & & & $20.88(0.12)$ & $20.80(0.09)$ & $20.55(0.05)$ & $20.29(0.05)$ & $20.06(0.05)$ & $19.53(0.09)$ \\
\hline \multirow[t]{4}{*}{1059} & 245.611 & 319.988 & $19.97(0.05)$ & $21.16(0.10)$ & $21.21(0.14)$ & $21.33(0.17)$ & $19.75(0.05)$ & $19.86(0.05)$ \\
\hline & & & $19.84(0.05)$ & $19.75(0.05)$ & $19.84(0.04)$ & $20.87(0.08)$ & $20.59(0.08)$ & $20.04(0.05)$ \\
\hline & & & $19.94(0.05)$ & $20.04(0.05)$ & $20.03(0.05)$ & $20.03(0.05)$ & $20.18(0.05)$ & $20.35(0.05)$ \\
\hline & & & $20.42(0.07)$ & $20.40(0.06)$ & $20.57(0.07)$ & $21.20(0.11)$ & $20.98(0.08)$ & $19.70(0.09)$ \\
\hline \multirow[t]{4}{*}{1061} & 224.933 & 56.899 & $20.29(0.05)$ & $20.81(0.10)$ & $21.17(0.12)$ & $21.02(0.10)$ & $19.95(0.05)$ & $20.23(0.06)$ \\
\hline & & & $19.95(0.05)$ & $20.31(0.06)$ & $20.23(0.05)$ & $19.59(0.04)$ & $19.54(0.05)$ & $19.67(0.06)$ \\
\hline & & & $20.44(0.06)$ & $20.30(0.05)$ & $20.46(0.06)$ & $20.39(0.06)$ & $20.18(0.06)$ & $20.13(0.05)$ \\
\hline & & & $20.14(0.06)$ & $20.27(0.07)$ & $20.18(0.05)$ & $20.26(0.05)$ & $19.98(0.05)$ & $19.81(0.06)$ \\
\hline \multirow[t]{4}{*}{1065} & 137.791 & 178.163 & $20.30(0.05)$ & $20.20(0.07)$ & $20.44(0.08)$ & $20.21(0.06)$ & $20.20(0.05)$ & $20.44(0.06)$ \\
\hline & & & $20.46(0.06)$ & $20.47(0.07)$ & $20.43(0.05)$ & $20.21(0.05)$ & $20.43(0.07)$ & $20.65(0.06)$ \\
\hline & & & $19.92(0.06)$ & $19.82(0.05)$ & $19.82(0.05)$ & $19.74(0.06)$ & $19.66(0.05)$ & $19.70(0.05)$ \\
\hline & & & $19.76(0.05)$ & $19.72(0.06)$ & $19.91(0.05)$ & $20.06(0.05)$ & $20.23(0.05)$ & $19.65(0.07)$ \\
\hline
\end{tabular}


Table 8. continuation.

\begin{tabular}{|c|c|c|c|c|c|c|c|c|}
\hline \multirow[t]{4}{*}{$\bar{F}$ F2 ID } & \multirow[t]{4}{*}{$\mathrm{x}$} & \multirow[t]{4}{*}{$\mathrm{y}$} & $2 \mathrm{Ks} 10(\sigma)$ & $2 \mathrm{Ks} 01(\sigma)$ & $2 \mathrm{Ks} 02(\sigma)$ & $2 \mathrm{Ks} 03(\sigma)$ & $2 \mathrm{Ks} 05(\sigma)$ & $\overline{2 \mathrm{Ks} 06(\sigma)}$ \\
\hline & & & $2 \mathrm{Ks} 07(\sigma)$ & $2 \mathrm{Ks} 08(\sigma)$ & $2 \mathrm{Ks} 09(\sigma)$ & $2 \mathrm{Ks} 11(\sigma)$ & $2 \mathrm{Ks} 12(\sigma)$ & $2 \mathrm{Ks} 13(\sigma)$ \\
\hline & & & $2 \mathrm{Ks} 14(\sigma)$ & $2 \mathrm{Ks} 15(\sigma)$ & $2 \mathrm{Ks} 16(\sigma)$ & $2 \mathrm{Ks} 17(\sigma)$ & $2 \mathrm{Ks} 18(\sigma)$ & $2 \mathrm{Ks} 19(\sigma)$ \\
\hline & & & $2 \mathrm{Ks} 20(\sigma)$ & $2 \mathrm{Ks} 21(\sigma)$ & $2 \mathrm{Ks} 22(\sigma)$ & $2 \mathrm{Ks} 23(\sigma)$ & $2 \mathrm{Ks} 24(\sigma)$ & $2 \mathrm{Ks} 04(\sigma)$ \\
\hline \multirow[t]{4}{*}{$\overline{1074}$} & $\overline{851.034}$ & 88.744 & $20.21(0.05)$ & $19.99(0.07)$ & $\overline{20.17(0.08)}$ & $20.61(0.09)$ & $19.67(0.05)$ & 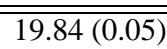 \\
\hline & & & $19.91(0.05)$ & $20.11(0.06)$ & $20.00(0.05)$ & $19.84(0.05)$ & $19.97(0.05)$ & $19.85(0.05)$ \\
\hline & & & $20.23(0.06)$ & $20.31(0.05)$ & $20.19(0.05)$ & $20.31(0.07)$ & $20.34(0.07)$ & $20.08(0.05)$ \\
\hline & & & $20.31(0.07)$ & $20.21(0.06)$ & $19.95(0.05)$ & $20.10(0.06)$ & $19.99(0.05)$ & $20.14(0.11)$ \\
\hline \multirow[t]{4}{*}{1081} & 314.049 & 818.506 & $20.59(0.05)$ & $20.00(0.05)$ & $20.20(0.08)$ & $20.22(0.06)$ & $19.84(0.05)$ & $20.30(0.06)$ \\
\hline & & & $20.12(0.05)$ & $20.38(0.08)$ & $20.24(0.05)$ & $19.92(0.05)$ & $19.97(0.06)$ & $20.04(0.05)$ \\
\hline & & & $20.39(0.06)$ & $20.37(0.05)$ & $20.21(0.05)$ & $20.03(0.05)$ & $19.98(0.05)$ & $20.08(0.05)$ \\
\hline & & & $19.92(0.05)$ & $19.78(0.05)$ & $19.85(0.05)$ & $19.76(0.05)$ & $19.69(0.05)$ & $19.62(0.11)$ \\
\hline \multirow[t]{4}{*}{1082} & 863.847 & 428.322 & $20.50(0.05)$ & 99.99 (9.99) & $20.84(0.12)$ & $20.96(0.14)$ & $20.06(0.05)$ & $20.22(0.06)$ \\
\hline & & & $20.31(0.06)$ & $20.70(0.08)$ & $20.59(0.06)$ & $20.01(0.05)$ & $20.11(0.06)$ & $20.26(0.05)$ \\
\hline & & & $20.16(0.05)$ & $20.01(0.05)$ & $19.87(0.05)$ & $19.88(0.06)$ & $19.84(0.05)$ & $19.75(0.05)$ \\
\hline & & & $19.80(0.05)$ & $19.75(0.06)$ & $19.81(0.05)$ & $20.02(0.05)$ & $20.19(0.06)$ & $19.85(0.09)$ \\
\hline \multirow[t]{4}{*}{1096} & 164.605 & 652.455 & $20.44(0.05)$ & $19.91(0.06)$ & $20.04(0.06)$ & $20.17(0.06)$ & $19.93(0.04)$ & $20.04(0.05)$ \\
\hline & & & $20.19(0.06)$ & $20.07(0.06)$ & $20.24(0.05)$ & $19.87(0.05)$ & $20.08(0.06)$ & $20.15(0.05)$ \\
\hline & & & $20.28(0.06)$ & $20.31(0.05)$ & $20.07(0.05)$ & $19.84(0.05)$ & $19.81(0.04)$ & $19.91(0.04)$ \\
\hline & & & $19.94(0.05)$ & $19.87(0.06)$ & $19.95(0.05)$ & $20.15(0.05)$ & $20.54(0.06)$ & $20.38(0.08)$ \\
\hline \multirow[t]{4}{*}{1098} & 683.389 & 648.228 & $19.59(0.04)$ & $19.79(0.08)$ & $19.68(0.05)$ & $19.54(0.05)$ & $20.18(0.05)$ & $19.93(0.05)$ \\
\hline & & & $19.94(0.06)$ & $19.96(0.06)$ & $19.73(0.05)$ & $20.43(0.05)$ & $20.29(0.06)$ & $20.06(0.04)$ \\
\hline & & & $19.98(0.05)$ & $20.04(0.04)$ & $20.10(0.05)$ & $20.29(0.05)$ & $20.31(0.05)$ & $20.46(0.05)$ \\
\hline & & & $20.51(0.07)$ & $20.49(0.07)$ & $20.59(0.05)$ & $20.51(0.07)$ & $20.41(0.06)$ & $20.25(0.12)$ \\
\hline \multirow[t]{4}{*}{1100} & 455.267 & 724.707 & $20.09(0.06)$ & $19.97(0.08)$ & $19.94(0.08)$ & $19.80(0.07)$ & $20.16(0.06)$ & $20.24(0.05)$ \\
\hline & & & $20.21(0.07)$ & $20.20(0.06)$ & $20.22(0.06)$ & $19.79(0.05)$ & $19.71(0.05)$ & $19.82(0.06)$ \\
\hline & & & $19.72(0.05)$ & $19.82(0.05)$ & $19.98(0.08)$ & $20.05(0.06)$ & $20.25(0.07)$ & $20.26(0.06)$ \\
\hline & & & $20.37(0.07)$ & $20.25(0.07)$ & $20.22(0.06)$ & $20.28(0.06)$ & $20.18(0.06)$ & $20.00(0.08)$ \\
\hline \multirow[t]{4}{*}{1104} & 616.080 & 212.496 & $20.13(0.15)$ & $19.80(0.13)$ & $20.12(0.16)$ & $19.62(0.26)$ & $18.92(0.13)$ & $99.99(9.99)$ \\
\hline & & & $19.81(0.14)$ & 99.99 (9.99) & $19.38(0.11)$ & $20.05(0.15)$ & $20.56(0.14)$ & $19.38(0.15)$ \\
\hline & & & 99.99 (9.99) & $20.70(0.16)$ & $20.28(0.34)$ & $20.15(0.16)$ & $20.61(0.18)$ & $20.84(0.25)$ \\
\hline & & & $20.67(0.10)$ & $20.28(0.15)$ & $99.99(9.99)$ & $20.48(0.38)$ & $20.50(0.30)$ & $20.53(0.66)$ \\
\hline \multirow[t]{4}{*}{1108} & 497.846 & 595.427 & $20.41(0.05)$ & $20.52(0.09)$ & $20.50(0.08)$ & $20.21(0.06)$ & $20.51(0.06)$ & $20.53(0.07)$ \\
\hline & & & $20.36(0.05)$ & $20.39(0.07)$ & $20.38(0.05)$ & $20.48(0.05)$ & $20.45(0.08)$ & $20.51(0.06)$ \\
\hline & & & $19.70(0.05)$ & $19.73(0.05)$ & $19.74(0.05)$ & $19.84(0.05)$ & $19.85(0.04)$ & $19.92(0.05)$ \\
\hline & & & $20.10(0.06)$ & $20.00(0.05)$ & $20.19(0.06)$ & $20.43(0.06)$ & $20.62(0.07)$ & $20.63(0.10)$ \\
\hline \multirow[t]{4}{*}{1110} & 9.058 & 379.270 & $20.65(0.06)$ & $20.46(0.09)$ & $20.60(0.12)$ & $20.45(0.08)$ & $20.25(0.08)$ & $20.15(0.06)$ \\
\hline & & & $20.50(0.06)$ & $20.87(0.09)$ & $20.55(0.06)$ & 99.99 (9.99) & $19.61(1.61)$ & 99.99 (9.99) \\
\hline & & & $20.53(0.06)$ & $20.41(0.05)$ & $20.33(0.06)$ & 99.99 (9.99) & $19.94(0.05)$ & $19.93(0.05)$ \\
\hline & & & $19.73(0.05)$ & $19.84(0.05)$ & $19.69(0.04)$ & 99.99 (9.99) & $19.67(0.05)$ & $19.79(0.15)$ \\
\hline \multirow[t]{4}{*}{1111} & 174.542 & 430.648 & $20.57(0.06)$ & $20.97(0.12)$ & $21.15(0.13)$ & $20.68(0.09)$ & $20.13(0.05)$ & $20.32(0.05)$ \\
\hline & & & $20.50(0.07)$ & $21.08(0.11)$ & $20.50(0.06)$ & $19.80(0.05)$ & $19.89(0.05)$ & $20.08(0.05)$ \\
\hline & & & $20.60(0.07)$ & $20.59(0.06)$ & $20.31(0.06)$ & $20.06(0.05)$ & $19.96(0.05)$ & $20.04(0.05)$ \\
\hline & & & $19.85(0.05)$ & $19.81(0.06)$ & $19.75(0.05)$ & $19.78(0.05)$ & $19.74(0.06)$ & $19.98(0.09)$ \\
\hline \multirow[t]{4}{*}{1112} & 620.794 & 311.416 & $19.61(0.04)$ & $20.19(0.06)$ & $19.91(0.07)$ & $19.78(0.06)$ & $20.20(0.05)$ & $20.02(0.05)$ \\
\hline & & & $20.04(0.05)$ & $19.86(0.06)$ & $19.75(0.04)$ & $20.27(0.05)$ & $20.18(0.06)$ & $20.05(0.05)$ \\
\hline & & & $20.11(0.06)$ & $20.21(0.05)$ & $20.34(0.05)$ & $20.32(0.05)$ & $20.41(0.05)$ & $20.11(0.05)$ \\
\hline & & & $20.28(0.06)$ & $20.18(0.06)$ & $20.17(0.05)$ & $20.12(0.05)$ & $20.00(0.05)$ & $20.35(0.10)$ \\
\hline \multirow[t]{4}{*}{1115} & 563.077 & 161.583 & $20.27(0.05)$ & $19.88(0.05)$ & $19.98(0.06)$ & $19.88(0.05)$ & $20.72(0.07)$ & $20.57(0.06)$ \\
\hline & & & $20.72(0.09)$ & $20.71(0.09)$ & $20.48(0.06)$ & $20.47(0.06)$ & $20.51(0.07)$ & $20.52(0.06)$ \\
\hline & & & $20.09(0.05)$ & $19.91(0.05)$ & $20.00(0.05)$ & $19.96(0.05)$ & $20.00(0.06)$ & $19.81(0.05)$ \\
\hline & & & $19.92(0.05)$ & $19.85(0.06)$ & $19.79(0.05)$ & $19.95(0.06)$ & $20.02(0.05)$ & $20.47(0.12)$ \\
\hline
\end{tabular}


Table 8. continuation.

\begin{tabular}{|c|c|c|c|c|c|c|c|c|}
\hline \multirow[t]{4}{*}{$\bar{F} 2$ ID } & \multirow[t]{4}{*}{$\mathrm{x}$} & \multirow[t]{4}{*}{$\mathrm{y}$} & $2 \mathrm{Ks} 10(\sigma)$ & $2 \mathrm{Ks} 01(\sigma)$ & $2 \mathrm{Ks} 02(\sigma)$ & $2 \mathrm{Ks} 03(\sigma)$ & $2 \mathrm{Ks} 05(\sigma)$ & $2 \mathrm{Ks} 06(\sigma)$ \\
\hline & & & $2 \mathrm{Ks} 07(\sigma)$ & $2 \mathrm{Ks} 08(\sigma)$ & $2 \mathrm{Ks} 09(\sigma)$ & $2 \mathrm{Ks} 11(\sigma)$ & $2 \mathrm{Ks} 12(\sigma)$ & $2 \mathrm{Ks} 13(\sigma)$ \\
\hline & & & $2 \mathrm{Ks} 14(\sigma)$ & $2 \mathrm{Ks} 15(\sigma)$ & $2 \mathrm{Ks} 16(\sigma)$ & $2 \mathrm{Ks} 17(\sigma)$ & $2 \mathrm{Ks} 18(\sigma)$ & $2 \mathrm{Ks} 19(\sigma)$ \\
\hline & & & $2 \mathrm{Ks} 20(\sigma)$ & $2 \mathrm{Ks} 21(\sigma)$ & $2 \mathrm{Ks} 22(\sigma)$ & $2 \mathrm{Ks} 23(\sigma)$ & $2 \mathrm{Ks} 24(\sigma)$ & $2 \mathrm{Ks} 04(\sigma)$ \\
\hline \multirow[t]{4}{*}{1121} & \multirow[t]{4}{*}{284.613} & \multirow[t]{4}{*}{499.730} & $19.99(0.05)$ & $20.56(0.10)$ & $20.67(0.08)$ & $21.07(0.10)$ & $19.93(0.05)$ & $20.03(0.05)$ \\
\hline & & & $19.92(0.06)$ & $19.84(0.06)$ & $19.93(0.05)$ & $20.77(0.06)$ & $20.58(0.07)$ & $20.43(0.06)$ \\
\hline & & & $19.70(0.05)$ & $19.69(0.05)$ & $20.00(0.05)$ & $19.98(0.05)$ & $20.04(0.05)$ & $20.09(0.05)$ \\
\hline & & & $20.38(0.06)$ & $20.30(0.08)$ & $20.32(0.05)$ & $20.58(0.06)$ & $20.67(0.07)$ & $20.08(0.07)$ \\
\hline \multirow[t]{4}{*}{1122} & \multirow[t]{4}{*}{452.259} & \multirow[t]{4}{*}{624.100} & $19.85(0.04)$ & $20.70(0.09)$ & $20.60(0.11)$ & $20.18(0.07)$ & $20.52(0.09)$ & $20.28(0.05)$ \\
\hline & & & $20.21(0.07)$ & $20.11(0.06)$ & $20.00(0.05)$ & $19.98(0.05)$ & $19.85(0.05)$ & $19.72(0.05)$ \\
\hline & & & $20.73(0.08)$ & $20.63(0.07)$ & $20.49(0.05)$ & $20.31(0.05)$ & $20.30(0.05)$ & $20.10(0.05)$ \\
\hline & & & $20.10(0.06)$ & $19.99(0.05)$ & $19.98(0.05)$ & $20.03(0.05)$ & $20.22(0.06)$ & $20.64(0.10)$ \\
\hline \multirow[t]{4}{*}{1124} & \multirow[t]{4}{*}{881.169} & \multirow[t]{4}{*}{122.298} & $20.00(0.04)$ & 99.99 (9.99) & $19.89(0.06)$ & $19.84(0.06)$ & $19.98(0.05)$ & $19.89(0.05)$ \\
\hline & & & $19.96(0.06)$ & $20.04(0.06)$ & $19.98(0.05)$ & $19.96(0.05)$ & $19.87(0.05)$ & $19.78(0.05)$ \\
\hline & & & $20.46(0.05)$ & $20.49(0.05)$ & $20.72(0.05)$ & $20.59(0.08)$ & $20.76(0.09)$ & $20.32(0.06)$ \\
\hline & & & $99.99(9.99)$ & $20.26(0.08)$ & $20.02(0.06)$ & $19.91(0.05)$ & $19.99(0.06)$ & $20.42(0.10)$ \\
\hline \multirow[t]{4}{*}{1125} & \multirow[t]{4}{*}{710.975} & \multirow[t]{4}{*}{410.796} & $20.17(0.05)$ & $20.24(0.06)$ & $20.10(0.06)$ & $19.84(0.06)$ & $20.32(0.05)$ & $20.10(0.05)$ \\
\hline & & & $20.16(0.06)$ & $20.48(0.07)$ & $20.22(0.05)$ & $20.57(0.06)$ & $20.56(0.08)$ & $20.27(0.05)$ \\
\hline & & & $19.67(0.05)$ & $19.77(0.04)$ & $19.75(0.04)$ & $19.92(0.05)$ & $20.04(0.05)$ & $20.13(0.05)$ \\
\hline & & & $20.41(0.07)$ & $20.20(0.05)$ & $20.33(0.05)$ & $20.71(0.07)$ & $20.53(0.07)$ & $20.69(0.20)$ \\
\hline \multirow[t]{4}{*}{1129} & \multirow[t]{4}{*}{541.726} & \multirow[t]{4}{*}{448.683} & $20.27(0.05)$ & $19.80(0.06)$ & $19.79(0.05)$ & $19.82(0.05)$ & $19.96(0.05)$ & $20.25(0.05)$ \\
\hline & & & $20.22(0.06)$ & $20.47(0.07)$ & $20.30(0.06)$ & $20.25(0.05)$ & $20.26(0.07)$ & $19.91(0.05)$ \\
\hline & & & $20.46(0.06)$ & $20.36(0.05)$ & $20.48(0.06)$ & $20.40(0.06)$ & $20.36(0.05)$ & $20.07(0.05)$ \\
\hline & & & $19.91(0.06)$ & $19.95(0.07)$ & $19.76(0.05)$ & $19.85(0.05)$ & $19.87(0.05)$ & $19.79(0.07)$ \\
\hline 1133 & 71.767 & 566.213 & $20.68(0.07)$ & $20.99(0.11)$ & $21.19(0.13)$ & $21.09(0.15)$ & $20.38(0.05)$ & $20.48(0.07)$ \\
\hline & & & $20.63(0.08)$ & $20.51(0.09)$ & $20.65(0.07)$ & $19.66(0.05)$ & $19.81(0.06)$ & $20.17(0.05)$ \\
\hline & & & $20.66(0.08)$ & $20.76(0.06)$ & $20.40(0.06)$ & $20.16(0.06)$ & $19.98(0.05)$ & $19.95(0.05)$ \\
\hline & & & $19.72(0.06)$ & $19.87(0.07)$ & $19.81(0.05)$ & $19.74(0.05)$ & $19.72(0.05)$ & $20.06(0.13)$ \\
\hline 1134 & 690.081 & 79.855 & $19.86(0.05)$ & $19.71(0.05)$ & $19.66(0.06)$ & $19.76(0.06)$ & $19.94(0.05)$ & $19.95(0.05)$ \\
\hline & & & $19.93(0.06)$ & $19.83(0.06)$ & $19.84(0.05)$ & $20.29(0.05)$ & $20.05(0.05)$ & $19.88(0.05)$ \\
\hline & & & $20.67(0.08)$ & $20.74(0.05)$ & $20.77(0.06)$ & $20.61(0.07)$ & $20.72(0.05)$ & $20.44(0.06)$ \\
\hline & & & $20.43(0.07)$ & $20.38(0.06)$ & $20.20(0.05)$ & $20.06(0.05)$ & $19.93(0.05)$ & $20.08(0.08)$ \\
\hline 1135 & 91.713 & 2.622 & $20.36(0.06)$ & $20.25(0.10)$ & $20.54(0.09)$ & $20.51(0.09)$ & $20.35(0.06)$ & $20.55(0.07)$ \\
\hline & & & $20.35(0.07)$ & $20.45(0.07)$ & 99.99 (9.99) & $20.91(0.14)$ & $20.16(0.06)$ & $20.44(0.08)$ \\
\hline & & & $19.58(0.05)$ & $19.39(0.06)$ & $19.54(0.07)$ & 99.99 (9.99) & $19.66(0.08)$ & $19.86(0.06)$ \\
\hline & & & $19.96(0.06)$ & $19.98(0.06)$ & $20.01(0.06)$ & $20.64(0.08)$ & $20.41(0.07)$ & $19.89(0.21)$ \\
\hline 1138 & 351.421 & 437.518 & $19.81(0.05)$ & $19.91(0.06)$ & $19.77(0.07)$ & $19.73(0.07)$ & $20.16(0.05)$ & $19.94(0.05)$ \\
\hline & & & $19.85(0.05)$ & $19.89(0.05)$ & $19.84(0.05)$ & $20.06(0.05)$ & $19.98(0.05)$ & $19.81(0.04)$ \\
\hline & & & $20.40(0.07)$ & $20.39(0.06)$ & $20.39(0.05)$ & $20.21(0.06)$ & $20.30(0.05)$ & $20.34(0.06)$ \\
\hline & & & $20.48(0.09)$ & $20.28(0.06)$ & $20.40(0.06)$ & $20.46(0.06)$ & $20.23(0.06)$ & $20.22(0.11)$ \\
\hline 1144 & 406.090 & 412.486 & $19.89(0.04)$ & $20.46(0.07)$ & $20.26(0.06)$ & $20.50(0.08)$ & $20.22(0.06)$ & $20.11(0.05)$ \\
\hline & & & $20.06(0.06)$ & $19.92(0.05)$ & $19.96(0.05)$ & $20.05(0.05)$ & $19.92(0.05)$ & $19.77(0.05)$ \\
\hline & & & $20.31(0.07)$ & $20.45(0.05)$ & $20.45(0.05)$ & $20.39(0.06)$ & $20.41(0.06)$ & $20.17(0.05)$ \\
\hline & & & $20.03(0.06)$ & $20.09(0.07)$ & $19.96(0.05)$ & $19.92(0.05)$ & $20.06(0.06)$ & $20.47(0.08)$ \\
\hline 1147 & 234.777 & 555.588 & $19.88(0.04)$ & $20.34(0.08)$ & $20.15(0.07)$ & $20.04(0.06)$ & $20.28(0.05)$ & $20.09(0.05)$ \\
\hline & & & $20.02(0.05)$ & $19.94(0.06)$ & $19.94(0.05)$ & $20.19(0.05)$ & $19.99(0.05)$ & $19.89(0.05)$ \\
\hline & & & $20.35(0.06)$ & $20.34(0.06)$ & $20.28(0.05)$ & $20.20(0.05)$ & $20.26(0.05)$ & $20.10(0.05)$ \\
\hline & & & $20.00(0.05)$ & $19.90(0.06)$ & $19.81(0.05)$ & $19.81(0.05)$ & $19.76(0.05)$ & $20.40(0.12)$ \\
\hline 1152 & 815.906 & 748.255 & $20.63(0.06)$ & $20.62(0.11)$ & $20.47(0.10)$ & $20.33(0.09)$ & $20.28(0.05)$ & $20.62(0.07)$ \\
\hline & & & $20.48(0.06)$ & $21.13(0.12)$ & $20.70(0.07)$ & $19.79(0.05)$ & $19.74(0.05)$ & $19.80(0.04)$ \\
\hline & & & $20.61(0.06)$ & $20.29(0.05)$ & $20.33(0.05)$ & $20.41(0.12)$ & $20.27(0.06)$ & $20.32(0.06)$ \\
\hline & & & $19.98(0.06)$ & $19.95(0.07)$ & $19.85(0.05)$ & $19.78(0.05)$ & $19.61(0.05)$ & $19.99(0.14)$ \\
\hline
\end{tabular}


Table 8. continuation.

\begin{tabular}{|c|c|c|c|c|c|c|c|c|}
\hline \multirow[t]{4}{*}{ F2 ID } & \multirow[t]{4}{*}{$\mathrm{x}$} & \multirow[t]{4}{*}{$\mathrm{y}$} & $2 \mathrm{Ks} 10(\sigma)$ & $2 \mathrm{Ks} 01(\sigma)$ & $2 \mathrm{Ks} 02(\sigma)$ & $2 \mathrm{Ks} 03(\sigma)$ & $2 \mathrm{Ks} 05(\sigma)$ & $2 \mathrm{Ks} 06(\sigma)$ \\
\hline & & & $2 \mathrm{Ks} 07(\sigma)$ & $2 \mathrm{Ks} 08(\sigma)$ & $2 \mathrm{Ks} 09(\sigma)$ & $2 \mathrm{Ks} 11(\sigma)$ & $2 \mathrm{Ks} 12(\sigma)$ & $2 \mathrm{Ks} 13(\sigma)$ \\
\hline & & & $2 \mathrm{Ks} 14(\sigma)$ & $2 \mathrm{Ks} 15(\sigma)$ & $2 \mathrm{Ks} 16(\sigma)$ & $2 \mathrm{Ks} 17(\sigma)$ & $2 \mathrm{Ks} 18(\sigma)$ & $2 \mathrm{Ks} 19(\sigma)$ \\
\hline & & & $2 \mathrm{Ks} 20(\sigma)$ & $2 \mathrm{Ks} 21(\sigma)$ & $2 \mathrm{Ks} 22(\sigma)$ & $2 \mathrm{Ks} 23(\sigma)$ & $2 \mathrm{Ks} 24(\sigma)$ & $2 \mathrm{Ks} 04(\sigma)$ \\
\hline \multirow[t]{4}{*}{1153} & 176.104 & 27.906 & 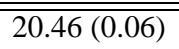 & $\overline{20.56(0.12)}$ & 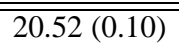 & $\overline{20.68(0.10)}$ & 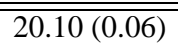 & $\overline{20.29(0.06)}$ \\
\hline & & & $20.27(0.07)$ & $20.24(0.08)$ & $20.38(0.06)$ & $19.64(0.05)$ & $19.60(0.05)$ & $19.68(0.06)$ \\
\hline & & & $20.47(0.07)$ & $20.19(0.06)$ & $20.36(0.05)$ & $20.45(0.07)$ & $20.23(0.07)$ & $20.20(0.06)$ \\
\hline & & & $20.18(0.06)$ & $20.31(0.07)$ & $20.00(0.05)$ & $19.95(0.06)$ & $19.68(0.06)$ & $19.67(0.13)$ \\
\hline \multirow[t]{4}{*}{1154} & 818.647 & 625.910 & $19.74(0.05)$ & $20.74(0.15)$ & $20.49(0.06)$ & $20.13(0.06)$ & $20.29(0.05)$ & $20.08(0.05)$ \\
\hline & & & $20.06(0.06)$ & $20.22(0.06)$ & $19.93(0.05)$ & $20.17(0.05)$ & $20.00(0.05)$ & $19.89(0.05)$ \\
\hline & & & $20.49(0.06)$ & $20.41(0.05)$ & $20.30(0.06)$ & $20.31(0.08)$ & $20.13(0.05)$ & $19.96(0.05)$ \\
\hline & & & $19.76(0.05)$ & $19.87(0.06)$ & $19.84(0.05)$ & $19.81(0.05)$ & $20.05(0.05)$ & $20.54(0.12)$ \\
\hline \multirow[t]{4}{*}{1159} & 590.311 & 348.722 & $19.91(0.05)$ & $20.44(0.06)$ & $20.11(0.06)$ & $20.32(0.07)$ & $20.34(0.05)$ & $19.87(0.06)$ \\
\hline & & & $20.19(0.07)$ & $19.98(0.11)$ & $19.96(0.05)$ & $20.14(0.06)$ & $19.92(0.05)$ & $19.78(0.06)$ \\
\hline & & & $20.33(0.07)$ & $20.36(0.07)$ & $20.39(0.06)$ & $20.48(0.05)$ & $20.40(0.06)$ & $20.14(0.06)$ \\
\hline & & & $20.05(0.06)$ & $20.14(0.06)$ & $19.89(0.05)$ & $19.79(0.05)$ & $19.83(0.05)$ & $20.26(0.11)$ \\
\hline \multirow[t]{4}{*}{1161} & 264.717 & 721.636 & $20.20(0.05)$ & $20.46(0.07)$ & $20.62(0.08)$ & $20.84(0.11)$ & $19.69(0.04)$ & $19.88(0.05)$ \\
\hline & & & $19.83(0.05)$ & $19.92(0.06)$ & $19.98(0.05)$ & $20.03(0.05)$ & $19.93(0.05)$ & $19.86(0.04)$ \\
\hline & & & $20.92(0.09)$ & $21.04(0.07)$ & $20.83(0.06)$ & $20.96(0.06)$ & $21.10(0.07)$ & $21.10(0.07)$ \\
\hline & & & $21.67(0.13)$ & $21.13(0.11)$ & $21.28(0.08)$ & $21.09(0.07)$ & $21.18(0.08)$ & $19.68(0.06)$ \\
\hline \multirow[t]{4}{*}{1162} & 599.665 & 143.639 & $20.17(0.05)$ & $20.41(0.07)$ & $20.20(0.06)$ & $20.22(0.07)$ & $20.46(0.05)$ & $20.57(0.06)$ \\
\hline & & & $20.43(0.07)$ & $20.57(0.08)$ & $20.42(0.06)$ & $20.50(0.05)$ & $20.54(0.07)$ & $20.52(0.06)$ \\
\hline & & & $19.75(0.05)$ & $19.74(0.05)$ & $19.76(0.04)$ & $19.83(0.0$ & $19.89(0$ & 19.88 \\
\hline & & & $20.14(0.06)$ & $20.01(0.06)$ & $20.20(0.05)$ & $20.31(0.06)$ & $20.57(0.07)$ & $20.01(0.14)$ \\
\hline \multirow[t]{4}{*}{1165} & 779.517 & 44.128 & $19.83(0.04)$ & $19.92(0.06)$ & $20.17(0.07)$ & $20.28(0.09)$ & $20.15(0.05)$ & $20.26(0.06)$ \\
\hline & & & $20.04(0.06)$ & $20.16(0.06)$ & $19.93(0.05)$ & $20.97(0.07)$ & $21.01(0.11)$ & $21.10(0.08)$ \\
\hline & & & $20.82(0.09)$ & $20.45(0.05)$ & $20.23(0.05)$ & $20.21(0.06)$ & $20.25(0.07)$ & $20.03(0.05)$ \\
\hline & & & $20.32(0.06)$ & $20.18(0.07)$ & $19.90(0.05)$ & $19.73(0.05)$ & $19.72(0.05)$ & $20.79(0.13)$ \\
\hline \multirow[t]{4}{*}{1167} & 895.388 & 660.961 & $99.99(9.99)$ & $99.99(9.99)$ & $19.48(0.10)$ & $19.75(1.18)$ & $19.39(0.08)$ & $19.67(0.33)$ \\
\hline & & & 99.99 (9.99) & 99.99 (9.99) & 99.99 (9.99) & $19.26(0.09)$ & $20.57(0.08)$ & $19.44(0.12)$ \\
\hline & & & $18.25(3.68)$ & $20.54(2.94)$ & 99.99 (9.99) & 99.99 (9.99) & $19.17(1.34)$ & $21.19(2.01)$ \\
\hline & & & 99.99 (9.99) & 99.99 (9.99) & 99.99 (9.99) & $21.05(0.07)$ & $20.48(0.42)$ & 20.02 \\
\hline \multirow[t]{4}{*}{1168} & 626.103 & 102.738 & $20.24(0.05)$ & $19.91(0.07)$ & $20.04(0.06)$ & $20.09(0.07)$ & $20.35(0.06)$ & $20.34(0.05)$ \\
\hline & & & $20.59(0.08)$ & $20.29(0.08)$ & $20.34(0.05)$ & $19.90(0.05)$ & $19.79(0.05)$ & $19.80(0.05)$ \\
\hline & & & $19.99(0.05)$ & $20.08(0.05)$ & $20.04(0.05)$ & $19.97(0.06)$ & $19.95(0.06)$ & $19.82(0.05)$ \\
\hline & & & & $20.04(0.05)$ & $20.14(0.05)$ & $20.40(0.06)$ & $20.17(0.06)$ & $19.93(0.08)$ \\
\hline \multirow[t]{4}{*}{1178} & 655.254 & 418.403 & $20.74(0.05)$ & $21.00(0.10)$ & $20.66(0.09)$ & $21.07(0.12)$ & $20.38(0.05)$ & $20.69(0.07)$ \\
\hline & & & $20.66(0.07)$ & $21.01(0.09)$ & $20.90(0.09)$ & $19.84(0.05)$ & $20.03(0.06)$ & $20.24(0.05)$ \\
\hline & & & $20.34(0.06)$ & $20.21(0.05)$ & $19.96(0.04)$ & $19.96(0.05)$ & $19.95(0.05)$ & $19.94(0.05)$ \\
\hline & & & $19.89(0.06)$ & $19.81(0.06)$ & $19.87(0.05)$ & $19.83(0.05)$ & $19.89(0.05)$ & $20.26(0.07)$ \\
\hline \multirow[t]{4}{*}{1181} & 604.368 & 485.248 & $20.35(0.05)$ & $20.13(0.07)$ & $20.41(0.08)$ & $20.64(0.08)$ & $19.91(0.05)$ & $20.21(0.06)$ \\
\hline & & & $20.22(0.07)$ & $20.60(0.08)$ & $20.31(0.06)$ & $20.06(0.05)$ & $20.30(0.07)$ & $20.48(0.05)$ \\
\hline & & & $19.93(0.05)$ & $19.90(0.04)$ & $19.80(0.05)$ & $19.82(0.05)$ & $19.84(0.05)$ & $19.96(0.05)$ \\
\hline & & & $20.03(0.06)$ & $20.00(0.05)$ & $20.08(0.05)$ & $20.41(0.05)$ & $20.47(0.06)$ & $19.95(0.09)$ \\
\hline \multirow[t]{4}{*}{1184} & 690.203 & 725.324 & $20.38(0.06)$ & $19.83(0.09)$ & $19.74(0.06)$ & $19.76(0.05)$ & $19.86(0.05)$ & $20.12(0.05)$ \\
\hline & & & $20.06(0.06)$ & $20.26(0.07)$ & $20.21(0.05)$ & $20.07(0.05)$ & $20.26(0.07)$ & $20.47(0.06)$ \\
\hline & & & $19.80(0.05)$ & $19.87(0.05)$ & $20.04(0.07)$ & $20.12(0.06)$ & $20.18(0.06)$ & $20.35(0.06)$ \\
\hline & & & $20.53(0.09)$ & $20.37(0.08)$ & $20.31(0.06)$ & $20.33(0.05)$ & $20.25(0.06)$ & $19.78(0.10)$ \\
\hline \multirow[t]{4}{*}{1185} & 555.668 & 148.297 & $19.82(0.04)$ & $20.33(0.07)$ & $20.08(0.08)$ & $20.11(0.07)$ & $20.30(0.05)$ & $20.10(0.05)$ \\
\hline & & & $19.99(0.05)$ & $19.88(0.06)$ & $19.86(0.05)$ & $20.31(0.06)$ & $19.96(0.06)$ & $19.80(0.05)$ \\
\hline & & & $20.51(0.06)$ & $20.62(0.06)$ & $20.61(0.05)$ & $20.65(0.06)$ & $20.55(0.07)$ & $20.38(0.07)$ \\
\hline & & & $20.24(0.07)$ & $20.31(0.07)$ & $20.11(0.05)$ & $19.97(0.05)$ & $19.84(0.05)$ & $20.71(0.26)$ \\
\hline
\end{tabular}


Table 8. continuation.

\begin{tabular}{|c|c|c|c|c|c|c|c|c|}
\hline \multirow[t]{4}{*}{ F2 ID } & \multirow[t]{4}{*}{$\mathrm{x}$} & \multirow[t]{4}{*}{$\mathrm{y}$} & $2 \mathrm{Ks} 10(\sigma)$ & $2 \mathrm{Ks} 01(\sigma)$ & $2 \mathrm{Ks} 02(\sigma)$ & $2 \mathrm{Ks} 03(\sigma)$ & $2 \mathrm{Ks} 05(\sigma)$ & $2 \mathrm{Ks} 06(\sigma)$ \\
\hline & & & $2 \mathrm{Ks} 07(\sigma)$ & $2 \mathrm{Ks} 08(\sigma)$ & $2 \mathrm{Ks} 09(\sigma)$ & $2 \mathrm{Ks} 11(\sigma)$ & $2 \mathrm{Ks} 12(\sigma)$ & $2 \mathrm{Ks} 13(\sigma)$ \\
\hline & & & $2 \mathrm{Ks} 14(\sigma)$ & $2 \mathrm{Ks} 15(\sigma)$ & $2 \mathrm{Ks} 16(\sigma)$ & $2 \mathrm{Ks} 17(\sigma)$ & $2 \mathrm{Ks} 18(\sigma)$ & $2 \mathrm{Ks} 19(\sigma)$ \\
\hline & & & $2 \mathrm{Ks} 20(\sigma)$ & $2 \mathrm{Ks} 21(\sigma)$ & $2 \mathrm{Ks} 22(\sigma)$ & $2 \mathrm{Ks} 23(\sigma)$ & $2 \mathrm{Ks} 24(\sigma)$ & $2 \mathrm{Ks} 04(\sigma)$ \\
\hline \multirow[t]{4}{*}{1190} & 521.926 & 783.060 & $\overline{20.27(0.06)}$ & 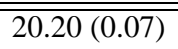 & 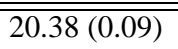 & $20.34(0.07)$ & 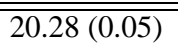 & 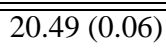 \\
\hline & & & $20.40(0.08)$ & $20.37(0.07)$ & $20.30(0.06)$ & $20.29(0.06)$ & $20.32(0.08)$ & $20.34(0.06)$ \\
\hline & & & $19.78(0.05)$ & $19.72(0.05)$ & $19.79(0.05)$ & $19.84(0.05)$ & $19.87(0.05)$ & $20.03(0.06)$ \\
\hline & & & $20.16(0.07)$ & $20.05(0.07)$ & $20.33(0.07)$ & $20.28(0.06)$ & $20.47(0.07)$ & $19.94(0.08)$ \\
\hline \multirow[t]{4}{*}{1194} & 149.128 & -28.142 & 99.99 (9.99) & 99.99 (9.99) & 99.99 (9.99) & $99.99(9.99)$ & 99.99 (9.99) & 99.99 (9.99) \\
\hline & & & 99.99 (9.99) & 99.99 (9.99) & 99.99 (9.99) & $17.87(0.09)$ & $19.48(0.27)$ & $21.21(0.23)$ \\
\hline & & & 99.99 (9.99) & $20.17(0.07)$ & $21.20(0.38)$ & 99.99 (9.99) & $20.60(0.08)$ & 99.99 (9.99) \\
\hline & & & $20.60(0.09)$ & $21.00(0.13)$ & $20.36(0.39)$ & $20.22(0.10)$ & $21.45(0.28)$ & $19.82(0.20)$ \\
\hline \multirow[t]{4}{*}{1195} & 240.108 & 329.556 & $20.11(0.05)$ & $20.12(0.06)$ & $20.45(0.08)$ & $20.53(0.08)$ & $19.98(0.05)$ & $20.20(0.05)$ \\
\hline & & & $20.23(0.06)$ & $20.23(0.06)$ & $20.19(0.05)$ & $20.37(0.05)$ & $20.56(0.07)$ & $20.43(0.05)$ \\
\hline & & & $19.67(0.05)$ & 19.86 & 19.83 & 19.88 & 19.85 & 19.96 \\
\hline & & & $20.02(0.05)$ & $20.02(0.06)$ & $20.20(0.05)$ & $20.23(0.05)$ & $20.31(0.07)$ & $19.94(0.08)$ \\
\hline \multirow[t]{4}{*}{1200} & 885.058 & 465.589 & $19.85(0.05)$ & 99.99 (9.99) & $20.00(0.06)$ & $19.91(0.06)$ & $20.31(0.05)$ & $20.13(0.06)$ \\
\hline & & & $20.15(0.0$ & $20.30(0.07)$ & $19.99(0$. & $20.09(0.05)$ & $20.00(0$. & $19.71(0.05)$ \\
\hline & & & $20.43(0.06)$ & $20.43(0.05)$ & 20.44 & 20.52 & 20.52 & 20.45 \\
\hline & & & 99.99 (9.99) & $20.26(0.07)$ & 20.02 & 19.85 & 19.85 & 20.48 \\
\hline \multirow[t]{4}{*}{1204} & 319.576 & 816.011 & $19.79(0.05)$ & $20.00(0.05)$ & $19.81(0.05)$ & $19.59(0.06)$ & $20.37(0.05)$ & $20.06(0.05)$ \\
\hline & & & $20.08(0.06)$ & $19.91(0.06)$ & $19.94(0.05)$ & $20.23(0.06)$ & $20.17(0.05)$ & $19.99(0.05)$ \\
\hline & & & $20.46(0$ & 20.41 & 20.40 & 20.33 & 20.19 & 20.24 \\
\hline & & & $20.16(0.06)$ & $20.22(0.06)$ & $20.26(0.06)$ & 20.14 & 20.09 & $20.32(0.20)$ \\
\hline \multirow[t]{4}{*}{1205} & 109.527 & 261.751 & $20.29(0.05)$ & $20.27(0.07)$ & $20.39(0.08)$ & $20.54(0.08)$ & $20.00(0.05)$ & $19.84(0.05)$ \\
\hline & & & $20.04(0.06)$ & $20.14(0.06)$ & $20.10(0.05)$ & $19.90(0.05)$ & $19.89(0.05)$ & $19.95(0.05)$ \\
\hline & & & $20.52(0.06)$ & $20.46(0.05)$ & 20.48 & 20.38 & 20.32 & $20.25(0.05)$ \\
\hline & & & 20.01 & $20.10(0.06)$ & 19.97 & 19.81 & 19.75 & 19.86 \\
\hline \multirow[t]{4}{*}{1211} & 266.011 & -3.585 & 99.99 (9.99) & $20.61(0.10)$ & 99.99 (9.99) & $21.06(0.17)$ & $20.62(0.06)$ & $20.51(0.06)$ \\
\hline & & & 99.99 (9.99) & 99.99 (9.99) & 99.99 (9.99) & $20.31(0.06)$ & $20.21(0.07)$ & $20.03(0.05)$ \\
\hline & & & $20.24(0.06)$ & $20.12(0.05)$ & $20.17(0.05)$ & 99.99 (9.99) & $19.94(0.05)$ & $19.81(0.05)$ \\
\hline & & & 19.76 & & 19.90 & & 20.23 & 20.40 \\
\hline \multirow[t]{4}{*}{1216} & 232.343 & 42.286 & $20.48(0.05)$ & $19.96(0.08)$ & $20.15(0.06)$ & $20.35(0.07)$ & $19.91(0.05)$ & $20.23(0.06)$ \\
\hline & & & $20.20(0.12)$ & $20.40(0.07)$ & $20.26(0.06)$ & $19.98(0.05)$ & $20.17(0.07)$ & $20.50(0.06)$ \\
\hline & & & $20.23(0.06)$ & $20.01(0.06)$ & $19.91(0.06)$ & $19.90(0.07)$ & $19.74(0.06)$ & $19.82(0.05)$ \\
\hline & & & & & & & & $19.94(0.10)$ \\
\hline \multirow[t]{4}{*}{1221} & 480.870 & -1.880 & & & & $20.25(0.08)$ & $20.21(0.05)$ & $20.31(0.06)$ \\
\hline & & & $19.47(0.10)$ & $20.09(0.67)$ & 99.99 (9.99) & $20.13(0.05)$ & $20.15(0.05)$ & $20.28(0.05)$ \\
\hline & & & $19.96(0.06)$ & $19.83(0.04)$ & $19.85(0.05)$ & 99.99 (9.99) & $19.93(0.05)$ & $20.00(0.05)$ \\
\hline & & & & & & $20.70(0.07)$ & 20.40 & $20.49(0.10)$ \\
\hline \multirow[t]{4}{*}{1224} & 611.563 & 36.604 & $19.93(0.05)$ & $19.65(0.05)$ & $19.50(0.05)$ & $19.42(0.05)$ & $20.67(0.06)$ & $20.29(0.05)$ \\
\hline & & & $20.33(0.07)$ & $20.19(0.07)$ & $20.08(0.05)$ & $20.92(0.06)$ & $20.71(0.07)$ & $20.71(0.06)$ \\
\hline & & & $19.80(0.05)$ & $19.95(0.05)$ & $20.07(0.05)$ & $20.09(0.05)$ & $20.24(0.05)$ & $20.24(0.05)$ \\
\hline & & & & $20.35(0.07)$ & $20.31(0.05)$ & $20.50(0.07)$ & $20.49(0.09)$ & $20.64(0.13)$ \\
\hline \multirow[t]{4}{*}{1233} & 78.433 & 616.813 & $20.04(0.05)$ & $21.08(0.11)$ & $20.50(0.10)$ & $20.58(0.12)$ & $20.25(0.12)$ & $19.94(0.09)$ \\
\hline & & & $19.90(0.05)$ & $20.25(0.13)$ & $20.38(0.10)$ & $20.20(0.05)$ & $20.42(0.06)$ & $21.63(0.42)$ \\
\hline & & & $20.75(0.12)$ & $20.60(0.09)$ & $20.05(0.18)$ & $19.88(0.05)$ & $19.90(0.05)$ & $20.05(0.05)$ \\
\hline & & & $20.44(0.07)$ & $19.88(0.05)$ & $20.05(0.05)$ & $20.23(0.06)$ & $20.38(0.05)$ & $20.27(0.14)$ \\
\hline \multirow[t]{4}{*}{1234} & 127.027 & 561.476 & $20.08(0.04)$ & $20.35(0.07)$ & $20.73(0.10)$ & $20.87(0.09)$ & $19.84(0.05)$ & $19.93(0.05)$ \\
\hline & & & $19.88(0.05)$ & $19.91(0.06)$ & $19.87(0.05)$ & $19.95(0.05)$ & $19.81(0.05)$ & $19.71(0.05)$ \\
\hline & & & $20.57(0.06)$ & $20.68(0.06)$ & $20.64(0.05)$ & $20.68(0.07)$ & $20.69(0.06)$ & $20.85(0.08)$ \\
\hline & & & $20.88(0.10)$ & $20.86(0.08)$ & $20.78(0.07)$ & $21.02(0.08)$ & $20.85(0.08)$ & $19.94(0.09)$ \\
\hline
\end{tabular}


Table 8. continuation.

\begin{tabular}{|c|c|c|c|c|c|c|c|c|}
\hline \multirow[t]{4}{*}{ F2 ID } & \multirow[t]{4}{*}{$\mathrm{x}$} & \multirow[t]{4}{*}{$\mathrm{y}$} & $2 \mathrm{Ks} 10(\sigma)$ & $2 \mathrm{Ks} 01(\sigma)$ & $2 \mathrm{Ks} 02(\sigma)$ & $2 \mathrm{Ks} 03(\sigma)$ & $2 \mathrm{Ks} 05(\sigma)$ & $2 \mathrm{Ks} 06(\sigma)$ \\
\hline & & & $2 \mathrm{Ks} 07(\sigma)$ & $2 \mathrm{Ks} 08(\sigma)$ & $2 \mathrm{Ks} 09(\sigma)$ & $2 \operatorname{Ks} 11(\sigma)$ & $2 \mathrm{Ks} 12(\sigma)$ & $2 \mathrm{Ks} 13(\sigma)$ \\
\hline & & & $2 \mathrm{Ks} 14(\sigma)$ & $2 \mathrm{Ks} 15(\sigma)$ & $2 \mathrm{Ks} 16(\sigma)$ & $2 \mathrm{Ks} 17(\sigma)$ & $2 \mathrm{Ks} 18(\sigma)$ & $2 \mathrm{Ks} 19(\sigma)$ \\
\hline & & & $2 \mathrm{Ks} 20(\sigma)$ & $2 \mathrm{Ks} 21(\sigma)$ & $2 \mathrm{Ks} 22(\sigma)$ & $2 \mathrm{Ks} 23(\sigma)$ & $2 \mathrm{Ks} 24(\sigma)$ & $2 \mathrm{Ks} 04(\sigma)$ \\
\hline \multirow[t]{4}{*}{1243} & 541.883 & 392.268 & $20.21(0.05)$ & $20.63(0.08)$ & $20.27(0.08)$ & $20.26(0.08)$ & 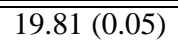 & $19.80(0.05)$ \\
\hline & & & $19.82(0.05)$ & $20.01(0.05)$ & $19.98(0.05)$ & $20.22(0.05)$ & $20.38(0.07)$ & $20.56(0.05)$ \\
\hline & & & $19.82(0.05)$ & $19.90(0.04)$ & $20.07(0.05)$ & $20.26(0.05)$ & $20.35(0.06)$ & $20.57(0.06)$ \\
\hline & & & $20.64(0.08)$ & $20.58(0.07)$ & $20.48(0.06)$ & $20.42(0.06)$ & $20.39(0.06)$ & $20.30(0.09)$ \\
\hline \multirow[t]{4}{*}{1251} & 592.318 & 113.564 & $19.93(0.04)$ & $20.58(0.09)$ & $20.74(0.10)$ & $20.80(0.09)$ & $20.40(0.05)$ & $20.10(0.06)$ \\
\hline & & & $20.13(0.06)$ & $19.66(0.05)$ & $20.01(0.05)$ & $20.59(0.09)$ & $19.93(0.05)$ & $20.36(0.05)$ \\
\hline & & & $19.85(0.05)$ & $19.84(0.05)$ & $20.04(0.05)$ & $20.19(0.05)$ & $20.29(0.06)$ & $20.30(0.06)$ \\
\hline & & & $20.29(0.08)$ & $20.82(0.06)$ & $20.66(0.06)$ & $20.19(0.07)$ & $20.37(0.07)$ & $21.15(0.44)$ \\
\hline \multirow[t]{4}{*}{1255} & 673.457 & 23.479 & $20.59(0.05)$ & $20.38(0.09)$ & $20.26(0.09)$ & $20.17(0.06)$ & $20.89(0.07)$ & $20.83(0.07)$ \\
\hline & & & $20.75(0.07)$ & $20.94(0.10)$ & $20.60(0.07)$ & $20.75(0.06)$ & $20.80(0.08)$ & $20.76(0.06)$ \\
\hline & & & $19.96(0.06)$ & $19.87(0.04)$ & $19.90(0.05)$ & $19.85(0.05)$ & $19.87(0.04)$ & $19.88(0.05)$ \\
\hline & & & $19.96(0.05)$ & $20.02(0.05)$ & $20.04(0.05)$ & $20.27(0.06)$ & $20.69(0.07)$ & $21.56(0.24)$ \\
\hline \multirow[t]{4}{*}{1258} & 43.960 & 221.394 & $20.36(0.07)$ & $20.47(0.14)$ & $20.92(0.12)$ & $20.86(0.13)$ & $20.29(0.06)$ & $20.25(0.07)$ \\
\hline & & & $20.52(0.08)$ & $20.49(0.09)$ & $20.45(0.07)$ & $20.49(0.08)$ & $20.59(0.09)$ & $20.56(0.08)$ \\
\hline & & & $19.82(0.06)$ & $19.65(0.05)$ & $19.84(0.05)$ & $19.86(0.09)$ & $19.92(0.06)$ & $19.99(0.06)$ \\
\hline & & & $20.24(0.07)$ & $20.14(0.06)$ & $20.33(0.06)$ & $20.11(0.06)$ & $20.21(0.07)$ & $19.97(0.15)$ \\
\hline \multirow[t]{4}{*}{1260} & 831.726 & 578.119 & $19.90(0.05)$ & $19.57(0.07)$ & $19.51(0.05)$ & $19.50(0.06)$ & $20.34(0.05)$ & $20.09(0.06)$ \\
\hline & & & $19.98(0.06)$ & $20.16(0.07)$ & $19.94(0.05)$ & $20.16(0.05)$ & $19.92(0.05)$ & $19.74(0.05)$ \\
\hline & & & $20.85(0.09)$ & $20.85(0.06)$ & $20.89(0.06)$ & $20.85(0.08)$ & $21.00(0$. & 21.13 \\
\hline & & & $20.82(0.10)$ & $21.06(0.09)$ & $21.00(0.08)$ & $20.82(0.08)$ & $20.24(0.06)$ & $20.75(0.11)$ \\
\hline \multirow[t]{4}{*}{1261} & 257.534 & 681.248 & $19.70(0.04)$ & $20.20(0.05)$ & $19.97(0.06)$ & $19.67(0.05)$ & $20.23(0.05)$ & $20.02(0.05)$ \\
\hline & & & $19.94(0.05)$ & $19.80(0.06)$ & $19.78(0.05)$ & $20.47(0.06)$ & $20.25(0.05)$ & $20.04(0.05)$ \\
\hline & & & $20.39(0.06)$ & $20.69(0.05)$ & $20.62(0.06)$ & $20.66(0.06)$ & $20.75(0.05)$ & $20.72(0.06)$ \\
\hline & & & $20.86(0.09)$ & $20.80(0.09)$ & $20.72(0.06)$ & $20.80(0.07)$ & $20.50(0.07)$ & $20.30(0.10)$ \\
\hline \multirow[t]{4}{*}{1262} & 171.765 & 513.055 & $20.64(0.06)$ & $20.37(0.07)$ & $20.69(0.11)$ & $20.72(0.09)$ & $20.26(0.05)$ & $20.33(0.06)$ \\
\hline & & & $20.44(0.07)$ & $21.12(0.10)$ & $20.54(0.05)$ & $20.41(0.06)$ & $20.42(0.06)$ & $20.70(0.06)$ \\
\hline & & & $19.86(0.05)$ & $19.82(0.04)$ & $19.81(0.04)$ & $19.83(0.05)$ & $19.92(0.05)$ & $20.12(0.05)$ \\
\hline & & & $20.30(0.06)$ & $20.14(0.06)$ & $20.34(0.05)$ & $20.57(0.06)$ & $20.90(0.09)$ & $19.88(0.07)$ \\
\hline \multirow[t]{4}{*}{1267} & 351.482 & 692.093 & $19.81(0.04)$ & $19.93(0.07)$ & $20.01(0.07)$ & $20.09(0.08)$ & $19.90(0.05)$ & $19.90(0.05)$ \\
\hline & & & $19.91(0.05)$ & $19.86(0.06)$ & $19.74(0.05)$ & $20.30(0.05)$ & $20.04(0.05)$ & $19.93(0.04)$ \\
\hline & & & $20.89(0.08)$ & $20.98(0.07)$ & $20.80(0.06)$ & $20.76(0.06)$ & $20.82(0.07)$ & $20.90(0.07)$ \\
\hline & & & $20.92(0.13)$ & $20.81(0.09)$ & $20.89(0.06)$ & $20.65(0.07)$ & $20.38(0.06)$ & $20.95(0.15)$ \\
\hline \multirow[t]{4}{*}{1271} & 103.029 & 229.035 & $20.14(0.05)$ & $20.15(0.05)$ & $20.06(0.07)$ & $19.93(0.06)$ & $19.90(0.04)$ & $19.79(0.05)$ \\
\hline & & & $19.93(0.05)$ & $19.86(0.05)$ & $20.04(0.05)$ & $20.15(0.05)$ & $20.40(0.07)$ & $20.61(0.05)$ \\
\hline & & & $20.14(0.06)$ & $20.12(0.05)$ & $20.16(0.05)$ & $20.24(0.08)$ & $20.31(0.05)$ & $20.31(0.06)$ \\
\hline & & & $19.90(0.10)$ & $20.61(0.07)$ & $20.55(0.06)$ & $20.45(0.07)$ & $19.87(0.10)$ & $19.88(0.07)$ \\
\hline \multirow[t]{4}{*}{1272} & 752.651 & 190.181 & $20.46(0.05)$ & $20.36(0.09)$ & $20.56(0.12)$ & $20.96(0.15)$ & $19.93(0.05)$ & $20.14(0.06)$ \\
\hline & & & $20.43(0.07)$ & $20.57(0.08)$ & $20.30(0.06)$ & $20.16(0.05)$ & $20.42(0.06)$ & $20.49(0.05)$ \\
\hline & & & $19.88(0.05)$ & $19.88(0.05)$ & $19.89(0.05)$ & $19.87(0.05)$ & $19.99(0.05)$ & $20.03(0.05)$ \\
\hline & & & $20.22(0.06)$ & $20.14(0.06)$ & $20.29(0.05)$ & $20.63(0.06)$ & $20.75(0.09)$ & $19.84(0.06)$ \\
\hline \multirow[t]{4}{*}{1282} & 863.396 & 656.010 & $20.45(0.05)$ & 99.99 (9.99) & $20.01(0.06)$ & $20.04(0.09)$ & $19.97(0.05)$ & $20.08(0.09)$ \\
\hline & & & $20.27(0.05)$ & 99.99 (9.99) & $20.52(0.05)$ & $19.32(0.07)$ & 99.99 (9.99) & $20.48(0.06)$ \\
\hline & & & $20.14(0.22)$ & $19.84(0.06)$ & $19.78(0.05)$ & $19.92(0.08)$ & $19.96(0.05)$ & $20.19(0.14)$ \\
\hline & & & $20.15(0.06)$ & $20.30(0.06)$ & $20.35(0.06)$ & $21.59(0.12)$ & $21.30(0.12)$ & $19.97(0.10)$ \\
\hline \multirow[t]{4}{*}{1283} & 13.011 & 491.855 & $19.86(0.05)$ & $20.00(0.06)$ & $19.91(0.06)$ & $20.02(0.06)$ & $20.11(0.05)$ & $19.89(0.05)$ \\
\hline & & & $20.06(0.06)$ & $20.06(0.06)$ & $19.95(0.05)$ & $20.16(1.64)$ & $20.25(0.08)$ & $20.15(0.06)$ \\
\hline & & & $20.87(0.09)$ & $20.92(0.07)$ & $20.79(0.07)$ & 99.99 (9.99) & $20.58(0.08)$ & $20.74(0.07)$ \\
\hline & & & $20.46(0.08)$ & $20.42(0.07)$ & $20.34(0.06)$ & $20.36(0.26)$ & $20.11(0.06)$ & $20.59(0.26)$ \\
\hline
\end{tabular}


Table 8. continuation.

\begin{tabular}{|c|c|c|c|c|c|c|c|c|}
\hline \multirow[t]{4}{*}{$\bar{F} 2$ ID } & \multirow[t]{4}{*}{$\mathrm{x}$} & \multirow[t]{4}{*}{$\mathrm{y}$} & $2 \mathrm{Ks} 10(\sigma)$ & $2 \mathrm{Ks} 01(\sigma)$ & $2 \mathrm{Ks} 02(\sigma)$ & $2 \mathrm{Ks} 03(\sigma)$ & $2 \mathrm{Ks} 05(\sigma)$ & $2 \mathrm{Ks} 06(\sigma)$ \\
\hline & & & $2 \mathrm{Ks} 07(\sigma)$ & $2 \mathrm{Ks} 08(\sigma)$ & $2 \mathrm{Ks} 09(\sigma)$ & $2 \mathrm{Ks} 11(\sigma)$ & $2 \mathrm{Ks} 12(\sigma)$ & $2 \mathrm{Ks} 13(\sigma)$ \\
\hline & & & $2 \mathrm{Ks} 14(\sigma)$ & $2 \mathrm{Ks} 15(\sigma)$ & $2 \mathrm{Ks} 16(\sigma)$ & $2 \mathrm{Ks} 17(\sigma)$ & $2 \mathrm{Ks} 18(\sigma)$ & $2 \mathrm{Ks} 19(\sigma)$ \\
\hline & & & $2 \mathrm{Ks} 20(\sigma)$ & $2 \mathrm{Ks} 21(\sigma)$ & $2 \mathrm{Ks} 22(\sigma)$ & $2 \mathrm{Ks} 23(\sigma)$ & $2 \mathrm{Ks} 24(\sigma)$ & $2 \mathrm{Ks} 04(\sigma)$ \\
\hline \multirow[t]{4}{*}{1287} & 420.344 & 569.240 & 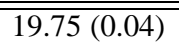 & 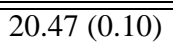 & $20.45(0.09)$ & $20.45(0.07)$ & $\overline{20.27(0.05)}$ & 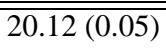 \\
\hline & & & $20.16(0.06)$ & $19.99(0.05)$ & $19.91(0.05)$ & $20.41(0.05)$ & $20.28(0.07)$ & $19.87(0.05)$ \\
\hline & & & $20.48(0.07)$ & $20.48(0.05)$ & $20.25(0.05)$ & $20.12(0.06)$ & $20.19(0.05)$ & $20.27(0.05)$ \\
\hline & & & $20.33(0.06)$ & $20.23(0.07)$ & $20.40(0.06)$ & $20.43(0.06)$ & $20.09(0.05)$ & $20.06(0.09)$ \\
\hline \multirow[t]{4}{*}{1294} & 171.705 & 600.459 & $20.15(0.05)$ & $20.29(0.07)$ & $20.44(0.09)$ & $20.18(0.06)$ & $20.29(0.05)$ & $20.38(0.06)$ \\
\hline & & & $20.47(0.06)$ & $20.39(0.06)$ & $20.45(0.06)$ & $20.46(0.06)$ & $20.15(0.06)$ & $19.82(0.05)$ \\
\hline & & & $20.35(0.06)$ & $20.30(0.05)$ & $20.20(0.05)$ & $20.15(0.05)$ & $20.21(0.05)$ & $20.34(0.05)$ \\
\hline & & & $20.13(0.06)$ & $19.96(0.05)$ & $19.83(0.04)$ & $19.79(0.05)$ & $19.63(0.05)$ & $20.67(0.11)$ \\
\hline \multirow[t]{4}{*}{1295} & 659.530 & 568.547 & $20.52(0.05)$ & $19.70(0.05)$ & $19.85(0.06)$ & $19.92(0.05)$ & $19.72(0.05)$ & $20.10(0.05)$ \\
\hline & & & $20.00(0.06)$ & $20.59(0.07)$ & $20.46(0.06)$ & $20.59(0.06)$ & $20.60(0.07)$ & $20.54(0.05)$ \\
\hline & & & $19.91(0.05)$ & $19.94(0.05)$ & $20.02(0.05)$ & $20.16(0.05)$ & $20.27(0.05)$ & $20.49(0.07)$ \\
\hline & & & $20.54(0.07)$ & $20.48(0.07)$ & $20.49(0.06)$ & $20.47(0.06)$ & $20.35(0.06)$ & $19.94(0.14)$ \\
\hline \multirow[t]{4}{*}{1300} & 721.038 & 644.433 & $20.09(0.06)$ & $20.36(0.09)$ & $20.50(0.09)$ & $20.16(0.08)$ & $20.39(0.06)$ & $20.40(0.06)$ \\
\hline & & & $20.43(0.08)$ & $20.42(0.06)$ & $20.19(0.06)$ & $20.28(0.06)$ & $20.40(0.06)$ & $20.33(0.05)$ \\
\hline & & & $19.85(0.05)$ & $19.82(0.05)$ & $19.85(0.06)$ & $19.89(0.05)$ & $19.93(0.05)$ & $20.00(0.05)$ \\
\hline & & & $20.21(0.06)$ & $20.09(0.06)$ & $20.12(0.05)$ & $20.20(0.05)$ & $20.26(0.06)$ & $20.15(0.11)$ \\
\hline \multirow[t]{4}{*}{1311} & 144.601 & 757.731 & $19.79(0.05)$ & $20.08(0.07)$ & $19.97(0.07)$ & $19.67(0.05)$ & $20.15(0.07)$ & $20.72(0.12)$ \\
\hline & & & $20.13(0.05)$ & $20.87(0.10)$ & $19.91(0.06)$ & $20.40(0.07)$ & $20.49(0.08)$ & $21.04(0.20)$ \\
\hline & & & $20.18(0.07)$ & $20.09(0.05)$ & $20.22(0.06)$ & $20.31(0.07)$ & $20.26(0.07)$ & $20.23(0.08)$ \\
\hline & & & $20.64(0.08)$ & $20.66(0.09)$ & $20.44(0.12)$ & $20.83(0.11)$ & 20.47 (0.09) & $20.13(0.15)$ \\
\hline \multirow[t]{4}{*}{1318} & 131.437 & 385.234 & $19.99(0.05)$ & $20.65(0.09)$ & $20.88(0.11)$ & $21.02(0.12)$ & $20.52(0.06)$ & $20.04(0.05)$ \\
\hline & & & $20.18(0.06)$ & $20.06(0.06)$ & $20.03(0.05)$ & $20.01(0.05)$ & $19.98(0.06)$ & $20.38(0.05)$ \\
\hline & & & $20.12(0.05)$ & $20.01(0.05)$ & $19.95(0.05)$ & $19.96(0.06)$ & $20.01(0.05)$ & $20.14(0.05)$ \\
\hline & & & $20.29(0.08)$ & $20.12(0.0$ & $20.32(0.05)$ & $20.45(0.0$ & $20.46(0.07)$ & $20.56(0.11)$ \\
\hline \multirow[t]{4}{*}{1323} & 894.573 & 566.546 & 99.99 (9.99) & 99.99 (9.99) & $20.68(0.10)$ & $20.82(0.11)$ & $20.68(0.05)$ & $20.79(0.08)$ \\
\hline & & & 99.99 (9.99) & 99.99 (9.99) & 99.99 (9.99) & $19.98(0.05)$ & $19.88(0.05)$ & $20.11(0.05)$ \\
\hline & & & $20.08(0.22)$ & $20.64(0.08)$ & $19.70(0.11)$ & 99.99 (9.99) & $20.66(0.09)$ & $21.11(0.10)$ \\
\hline & & & 99.99 (9.99) & 99.99 (9.99) & 99.99 (9.99) & $20.04(0.05)$ & $19.68(0.05)$ & $20.45(0.12)$ \\
\hline \multirow[t]{4}{*}{1325} & 539.054 & 700.875 & $20.31(0.05)$ & $19.72(0.05)$ & $20.01(0.07)$ & $20.24(0.06)$ & 19.76 & $20.05(0.05)$ \\
\hline & & & $19.92(0.05)$ & $20.28(0.07)$ & $20.04(0.05)$ & $20.07(0.05)$ & $19.96(0.06)$ & $19.94(0.05)$ \\
\hline & & & $20.97(0.09)$ & $20.92(0.06)$ & $20.87(0.05)$ & $20.76(0.07)$ & $20.64(0.06)$ & $20.51(0.06)$ \\
\hline & & & $20.28(0.06)$ & $20.17(0.06)$ & $20.14(0.05)$ & $20.07(0.06)$ & $19.96(0.05)$ & $19.83(0.10)$ \\
\hline \multirow[t]{4}{*}{1334} & 564.474 & 110.939 & $19.87(0.05)$ & $19.78(0.05)$ & $19.92(0.06)$ & $19.81(0.05)$ & 99.99 (9.99) & $20.30(0.06)$ \\
\hline & & & $19.77(0.07)$ & $19.90(0.07)$ & $19.81(0.05)$ & 99.99 (9.99) & $20.92(0.08)$ & $20.58(0.09)$ \\
\hline & & & $21.33(0.10)$ & $21.34(0.11)$ & $21.04(0.07)$ & $20.85(0.06)$ & $20.95(0.07)$ & $20.89(0.07)$ \\
\hline & & & $20.88(0.09)$ & 99.99 (9.99) & $20.81(0.08)$ & $20.45(0.06)$ & $20.12(0.06)$ & $20.69(0.19)$ \\
\hline \multirow[t]{4}{*}{1337} & 630.755 & 532.113 & $20.46(0.07)$ & $20.97(0.14)$ & $20.88(0.11)$ & $20.46(0.07)$ & $20.05(0.05)$ & $20.23(0.05)$ \\
\hline & & & $20.28(0.08)$ & $20.54(0.07)$ & $20.35(0.07)$ & $19.78(0.05)$ & $19.71(0.05)$ & $19.65(0.06)$ \\
\hline & & & $20.15(0.06)$ & $20.30(0.06)$ & $20.32(0.07)$ & $20.42(0.09)$ & $20.50(0.06)$ & $20.44(0.07)$ \\
\hline & & & $20.42(0.06)$ & $20.54(0.11)$ & $20.47(0.06)$ & $20.41(0.06)$ & $20.46(0.07)$ & $19.87(0.10)$ \\
\hline \multirow[t]{4}{*}{1343} & 50.503 & 354.209 & $20.13(0.05)$ & $21.15(0.11)$ & $20.85(0.10)$ & $20.94(0.09)$ & $20.67(0.06)$ & $20.39(0.06)$ \\
\hline & & & $20.67(0.09)$ & $20.55(0.07)$ & $20.45(0.06)$ & $20.30(0.05)$ & $20.07(0.06)$ & $19.95(0.05)$ \\
\hline & & & $20.59(0.07)$ & $20.40(0.06)$ & $20.43(0.06)$ & $20.39(0.08)$ & $20.16(0.06)$ & $20.04(0.05)$ \\
\hline & & & $19.86(0.05)$ & $19.93(0.07)$ & $19.86(0.05)$ & $19.79(0.05)$ & $19.97(0.05)$ & $20.42(0.15)$ \\
\hline \multirow[t]{4}{*}{1345} & 28.113 & 159.253 & $20.21(0.05)$ & $19.88(0.06)$ & $20.34(0.07)$ & $19.64(0.08)$ & $20.12(0.05)$ & $17.87(0.14)$ \\
\hline & & & $20.47(0.06)$ & $18.82(0.29)$ & $20.39(0.06)$ & 99.99 (9.99) & $20.43(0.10)$ & $20.52(0.38)$ \\
\hline & & & 99.99 (9.99) & 99.99 (9.99) & $21.10(0.21)$ & 99.99 (9.99) & $21.07(0.11)$ & $22.32(0.32)$ \\
\hline & & & $21.74(0.28)$ & $20.86(0.11)$ & $18.57(0.08)$ & $21.53(0.16)$ & 99.99 (9.99) & $19.75(0.16)$ \\
\hline
\end{tabular}


Table 8. continuation.

\begin{tabular}{|c|c|c|c|c|c|c|c|c|}
\hline \multirow[t]{4}{*}{$\bar{F}$ F2 ID } & \multirow[t]{4}{*}{$\mathrm{x}$} & \multirow[t]{4}{*}{$\mathrm{y}$} & $2 \mathrm{Ks} 10(\sigma)$ & $2 \mathrm{Ks} 01(\sigma)$ & $2 \mathrm{Ks} 02(\sigma)$ & $2 \mathrm{Ks} 03(\sigma)$ & $2 \mathrm{Ks} 05(\sigma)$ & $\overline{2 \mathrm{Ks} 06(\sigma)}$ \\
\hline & & & $2 \mathrm{Ks} 07(\sigma)$ & $2 \mathrm{Ks} 08(\sigma)$ & $2 \mathrm{Ks} 09(\sigma)$ & $2 \mathrm{Ks} 11(\sigma)$ & $2 \mathrm{Ks} 12(\sigma)$ & $2 \mathrm{Ks} 13(\sigma)$ \\
\hline & & & $2 \mathrm{Ks} 14(\sigma)$ & $2 \mathrm{Ks} 15(\sigma)$ & $2 \mathrm{Ks} 16(\sigma)$ & $2 \mathrm{Ks} 17(\sigma)$ & $2 \mathrm{Ks} 18(\sigma)$ & $2 \mathrm{Ks} 19(\sigma)$ \\
\hline & & & $2 \mathrm{Ks} 20(\sigma)$ & $2 \mathrm{Ks} 21(\sigma)$ & $2 \mathrm{Ks} 22(\sigma)$ & $2 \mathrm{Ks} 23(\sigma)$ & $2 \mathrm{Ks} 24(\sigma)$ & $2 \mathrm{Ks} 04(\sigma)$ \\
\hline \multirow[t]{4}{*}{$\overline{1349}$} & 872.937 & 654.931 & $20.03(0.13)$ & $99.99(9.99)$ & $19.84(0.10)$ & $19.56(0.11)$ & $20.01(0.16)$ & 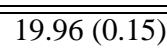 \\
\hline & & & $19.79(0.09)$ & $18.71(0.17)$ & $19.86(0.07)$ & $19.20(0.08)$ & $20.51(0.08)$ & $19.80(0.13)$ \\
\hline & & & $20.34(0.27)$ & $20.21(0.11)$ & $20.07(0.35)$ & $20.21(0.12)$ & $20.55(0.18)$ & $20.00(0.09)$ \\
\hline & & & $20.48(0.08)$ & $21.25(0.16)$ & $20.14(0.10)$ & $21.89(0.12)$ & $21.19(0.09)$ & $20.23(0.20)$ \\
\hline \multirow[t]{4}{*}{1352} & 278.642 & 272.301 & $21.18(0.18)$ & $21.36(0.29)$ & $21.82(0.29)$ & $21.09(0.25)$ & $21.11(0.24)$ & $99.99(9.99)$ \\
\hline & & & $21.25(0.30)$ & $19.20(0.40)$ & $21.00(0.14)$ & $19.71(0.24)$ & $20.17(0.19)$ & $22.75(2.91)$ \\
\hline & & & $19.50(0.19)$ & $19.61(0.25)$ & $21.29(0.16)$ & $21.36(0.27)$ & $21.32(0.33)$ & $20.08(0.16)$ \\
\hline & & & $19.55(0.13)$ & $20.99(0.32)$ & $20.44(0.36)$ & $19.66(0.07)$ & $20.51(0.12)$ & $19.28(0.16)$ \\
\hline \multirow[t]{4}{*}{1356} & 297.561 & 737.609 & $20.78(0.07)$ & $21.05(0.11)$ & $20.69(0.09)$ & $20.13(0.07)$ & $21.07(0.09)$ & $21.18(0.07)$ \\
\hline & & & $21.48(0.13)$ & $21.15(0.12)$ & $20.96(0.08)$ & $20.52(0.06)$ & $20.66(0.09)$ & $20.71(0.06)$ \\
\hline & & & $19.92(0.05)$ & $19.86(0.05)$ & $19.82(0.05)$ & $19.82(0.05)$ & $19.83(0.05)$ & $19.93(0.05)$ \\
\hline & & & $20.12(0.07)$ & $20.08(0.06)$ & $20.24(0.06)$ & $20.53(0.07)$ & $20.63(0.08)$ & $20.73(0.14)$ \\
\hline \multirow[t]{4}{*}{1362} & 887.622 & 654.633 & $19.82(0.09)$ & 99.99 (9.99) & $19.94(0.14)$ & $19.90(0.10)$ & $19.17(0.11)$ & $19.67(0.11)$ \\
\hline & & & $20.73(0.20)$ & $19.73(0.33)$ & $19.77(0.13)$ & $20.01(0.11)$ & $21.01(0.10)$ & $19.16(0.12)$ \\
\hline & & & $20.21(0.16)$ & $20.29(0.13)$ & $19.38(0.24)$ & $22.77(1.98)$ & $21.48(0.44)$ & $20.62(0.16)$ \\
\hline & & & 99.99 (9.99) & 99.99 (9.99) & $21.83(0.13)$ & $22.68(0.23)$ & $22.93(0.38)$ & $21.14(0.22)$ \\
\hline \multirow[t]{4}{*}{1364} & 154.398 & 108.098 & $20.39(0.05)$ & $20.04(0.06)$ & $20.28(0.06)$ & $20.50(0.06)$ & $19.96(0.05)$ & $20.12(0.05)$ \\
\hline & & & $20.00(0.05)$ & $20.40(0.06)$ & $20.19(0.05)$ & $20.28(0.05)$ & $20.40(0.06)$ & $20.74(0.05)$ \\
\hline & & & $20.08(0.05)$ & $19.84(0.05)$ & $19.99(0.06)$ & $19.87(0.05)$ & $19.88(0.04)$ & $20.04(0.05)$ \\
\hline & & & $20.20(0.06)$ & $20.10(0.06)$ & $20.35(0.05)$ & $20.41(0.06)$ & $20.42(0.06)$ & $20.34(0.07)$ \\
\hline \multirow[t]{4}{*}{1367} & 376.052 & -10.798 & $99.99(9.99)$ & $19.84(0.06)$ & $99.99(9.99)$ & 99.99 (9.99) & $20.40(1.01)$ & $99.99(9.99)$ \\
\hline & & & 99.99 (9.99) & 99.99 (9.99) & 99.99 (9.99) & $20.36(0.05)$ & $20.17(0.06)$ & $20.22(0.05)$ \\
\hline & & & $20.50(0.06)$ & $20.47(0.05)$ & $20.52(0.06)$ & 99.99 (9.99) & $20.27(0.05)$ & $20.09(0.05)$ \\
\hline & & & $19.95(0.06)$ & $20.07(0.06)$ & $19.90(0.05)$ & $19.86(0.05)$ & $19.79(0.05)$ & $20.76(0.13)$ \\
\hline \multirow[t]{4}{*}{1374} & 100.095 & 253.502 & $20.55(0.06)$ & $20.10(0.06)$ & $20.39(0.08)$ & $20.48(0.06)$ & $20.12(0.05)$ & $20.28(0.05)$ \\
\hline & & & $20.37(0.06)$ & $20.46(0.07)$ & $20.54(0.06)$ & $20.19(0.05)$ & $20.33(0.06)$ & $20.49(0.06)$ \\
\hline & & & $19.90(0.05)$ & $19.86(0.05)$ & $19.90(0.05)$ & $20.03(0.06)$ & $20.00(0.05)$ & $20.15(0.05)$ \\
\hline & & & $20.32(0.06)$ & $20.21(0.06)$ & $20.51(0.06)$ & $20.66(0.07)$ & $20.45(0.07)$ & $19.78(0.07)$ \\
\hline \multirow[t]{4}{*}{1376} & 294.451 & 571.822 & $19.78(0.05)$ & $20.22(0.05)$ & $19.94(0.06)$ & $19.80(0.05)$ & $20.53(0.07)$ & $20.25(0.05)$ \\
\hline & & & $20.13(0.05)$ & $19.94(0.06)$ & $20.02(0.05)$ & $20.23(0.05)$ & $20.00(0.05)$ & $19.82(0.05)$ \\
\hline & & & $20.42(0.07)$ & $20.40(0.05)$ & $20.37(0.05)$ & $20.32(0.05)$ & $20.39(0.06)$ & $20.34(0.05)$ \\
\hline & & & $20.24(0.06)$ & $20.41(0.05)$ & $20.29(0.05)$ & $20.13(0.06)$ & $19.78(0.05)$ & $20.47(0.12)$ \\
\hline \multirow[t]{4}{*}{1377} & 381.153 & 313.570 & $20.01(0.06)$ & $20.39(0.07)$ & $20.71(0.10)$ & $20.64(0.08)$ & $20.55(0.07)$ & $20.41(0.05)$ \\
\hline & & & $20.42(0.07)$ & $20.24(0.07)$ & $20.12(0.06)$ & $20.04(0.05)$ & $19.90(0.06)$ & $19.88(0.06)$ \\
\hline & & & $20.65(0.08)$ & $20.55(0.06)$ & $20.50(0.07)$ & $20.38(0.07)$ & $20.35(0.06)$ & $20.02(0.05)$ \\
\hline & & & $19.97(0.06)$ & $19.92(0.06)$ & $19.89(0.06)$ & $20.03(0.05)$ & $20.09(0.06)$ & $20.23(0.09)$ \\
\hline \multirow[t]{4}{*}{1378} & 135.920 & 532.695 & $20.46(0.05)$ & $19.98(0.05)$ & $20.12(0.07)$ & $20.44(0.08)$ & $20.03(0.05)$ & $20.07(0.05)$ \\
\hline & & & $20.27(0.06)$ & $20.42(0.08)$ & $20.28(0.05)$ & $20.13(0.05)$ & $20.18(0.06)$ & $20.31(0.05)$ \\
\hline & & & $20.12(0.05)$ & $20.09(0.05)$ & $19.96(0.05)$ & $19.88(0.06)$ & $19.93(0.05)$ & $20.02(0.05)$ \\
\hline & & & $20.27(0.06)$ & $20.27(0.07)$ & $20.37(0.05)$ & $20.44(0.06)$ & $20.49(0.06)$ & $20.23(0.08)$ \\
\hline \multirow[t]{4}{*}{1380} & 691.007 & 27.103 & $19.90(0.04)$ & $20.50(0.07)$ & $20.29(0.06)$ & $20.02(0.07)$ & $20.46(0.05)$ & $20.36(0.05)$ \\
\hline & & & $20.25(0.06)$ & $20.19(0.07)$ & $19.98(0.05)$ & $20.13(0.05)$ & $20.13(0.06)$ & $20.06(0.05)$ \\
\hline & & & $19.86(0.05)$ & $20.08(0.05)$ & $20.13(0.05)$ & $20.39(0.05)$ & $20.25(0.05)$ & $20.12(0.06)$ \\
\hline & & & $20.39(0.07)$ & $20.57(0.07)$ & $20.34(0.06)$ & $20.59(0.07)$ & $20.39(0.06)$ & $20.61(0.12)$ \\
\hline \multirow[t]{4}{*}{1382} & 110.288 & 722.837 & $19.91(0.06)$ & $20.18(0.09)$ & $19.93(0.07)$ & $19.80(0.05)$ & $20.45(0.07)$ & $20.37(0.07)$ \\
\hline & & & $20.48(0.07)$ & $19.98(0.06)$ & $19.94(0.06)$ & $20.33(0.06)$ & $20.40(0.08)$ & $20.48(0.07)$ \\
\hline & & & $19.92(0.05)$ & $20.02(0.06)$ & $20.07(0.07)$ & $20.17(0.08)$ & $20.25(0.06)$ & $20.33(0.06)$ \\
\hline & & & $20.43(0.08)$ & $20.68(0.09)$ & $20.50(0.07)$ & $20.65(0.08)$ & $20.61(0.07)$ & $20.34(0.17)$ \\
\hline
\end{tabular}


Table 8. continuation.

\begin{tabular}{|c|c|c|c|c|c|c|c|c|}
\hline \multirow[t]{4}{*}{$\overline{\mathrm{F} 2 \mathrm{ID}}$} & \multirow[t]{4}{*}{$\mathrm{x}$} & \multirow[t]{4}{*}{$\mathrm{y}$} & $2 \mathrm{Ks} 10(\sigma)$ & $2 \mathrm{Ks} 01(\sigma)$ & $2 \mathrm{Ks} 02(\sigma)$ & $2 \mathrm{Ks} 03(\sigma)$ & $2 \mathrm{Ks} 05(\sigma)$ & $2 \mathrm{Ks06}(\sigma)$ \\
\hline & & & $2 \mathrm{Ks} 07(\sigma)$ & $2 \mathrm{Ks} 08(\sigma)$ & $2 \mathrm{Ks} 09(\sigma)$ & $2 \mathrm{Ks} 11(\sigma)$ & $2 \mathrm{Ks} 12(\sigma)$ & $2 \mathrm{Ks} 13(\sigma)$ \\
\hline & & & $2 \mathrm{Ks} 14(\sigma)$ & $2 \mathrm{Ks} 15(\sigma)$ & $2 \mathrm{Ks} 16(\sigma)$ & $2 \mathrm{Ks} 17(\sigma)$ & $2 \mathrm{Ks} 18(\sigma)$ & $2 \mathrm{Ks} 19(\sigma)$ \\
\hline & & & $2 \mathrm{Ks} 20(\sigma)$ & $2 \mathrm{Ks} 21(\sigma)$ & $2 \mathrm{Ks} 22(\sigma)$ & $2 \mathrm{Ks} 23(\sigma)$ & $2 \mathrm{Ks} 24(\sigma)$ & $2 \mathrm{Ks} 04(\sigma)$ \\
\hline \multirow[t]{4}{*}{1385} & \multirow[t]{4}{*}{870.608} & \multirow[t]{4}{*}{38.459} & $20.45(0.05)$ & $99.99(9.99)$ & $20.85(0.15)$ & $20.85(0.10)$ & $21.75(0.18)$ & $21.46(0.15)$ \\
\hline & & & $20.26(0.09)$ & $21.92(0.25)$ & $20.16(0.05)$ & $20.45(0.06)$ & $20.10(0.06)$ & $20.68(0.07)$ \\
\hline & & & $20.27(0.06)$ & $20.40(0.05)$ & $20.44(0.06)$ & 99.99 (9.99) & 99.99 (9.99) & 99.99 (9.99) \\
\hline & & & $20.68(0.08)$ & 99.99 (9.99) & $21.25(0.11)$ & $19.65(0.05)$ & $19.49(0.05)$ & 99.99 (9.99) \\
\hline \multirow[t]{4}{*}{1387} & \multirow[t]{4}{*}{83.990} & \multirow[t]{4}{*}{774.122} & $19.93(0.05)$ & $20.24(0.06)$ & $20.02(0.06)$ & $19.81(0.05)$ & $20.31(0.06)$ & $20.22(0.05)$ \\
\hline & & & $20.18(0.06)$ & $19.98(0.06)$ & $19.99(0.05)$ & $20.04(0.05)$ & $19.91(0.05)$ & $19.80(0.05)$ \\
\hline & & & $20.60(0.07)$ & $20.68(0.06)$ & $20.46(0.05)$ & $20.49(0.08)$ & $20.51(0.07)$ & $20.58(0.06)$ \\
\hline & & & $20.47(0.06)$ & $20.71(0.08)$ & $20.52(0.06)$ & $20.25(0.06)$ & $20.09(0.07)$ & $21.02(0.54)$ \\
\hline \multirow[t]{4}{*}{1389} & \multirow[t]{4}{*}{807.249} & \multirow[t]{4}{*}{555.021} & $20.16(0.05)$ & $20.62(0.08)$ & $20.21(0.06)$ & $19.99(0.06)$ & $20.00(0.05)$ & $19.98(0.05)$ \\
\hline & & & $19.83(0.06)$ & $20.59(0.07)$ & $19.99(0.05)$ & $20.08(0.05)$ & $20.17(0.06)$ & $20.38(0.05)$ \\
\hline & & & $19.83(0.05)$ & $20.03(0.05)$ & $20.07(0.05)$ & $20.11(0.05)$ & $20.26(0.05)$ & $20.28(0.05)$ \\
\hline & & & $20.42(0.07)$ & $20.37(0.07)$ & $20.41(0.05)$ & $20.21(0.05)$ & $20.52(0.06)$ & $21.04(0.14)$ \\
\hline \multirow[t]{4}{*}{1390} & \multirow[t]{4}{*}{756.298} & \multirow[t]{4}{*}{-9.293} & 99.99 (9.99) & $20.52(0.08)$ & $99.99(9.99)$ & $99.99(9.99)$ & $20.09(0.07)$ & 99.99 (9.99) \\
\hline & & & 99.99 (9.99) & 99.99 (9.99) & 99.99 (9.99) & $20.10(0.06)$ & $20.14(0.06)$ & $19.85(0.05)$ \\
\hline & & & $20.36(0.06)$ & $20.46(0.06)$ & $20.46(0.05)$ & 99.99 (9.99) & $20.49(0.06)$ & $20.13(0.06)$ \\
\hline & & & $20.41(0.08)$ & $20.33(0.07)$ & $19.99(0.05)$ & $19.92(0.06)$ & $19.85(0.05)$ & $20.57(0.13)$ \\
\hline \multirow[t]{4}{*}{1401} & \multirow[t]{4}{*}{450.796} & \multirow[t]{4}{*}{258.307} & $19.88(0.05)$ & $19.88(0.06)$ & $19.74(0.06)$ & $19.91(0.08)$ & $20.18(0.05)$ & $20.03(0.05)$ \\
\hline & & & $19.98(0.05)$ & $20.05(0.06)$ & $19.85(0.05)$ & $20.47(0.07)$ & $20.19(0.07)$ & $19.99(0.05)$ \\
\hline & & & $20.68(0.06)$ & $20.69(0.06)$ & $20.75(0.05)$ & $20.54(0.07)$ & $20.62(0.07)$ & $20.71(0.08)$ \\
\hline & & & $20.60(0.07)$ & $20.48(0.10)$ & $20.45(0.06)$ & $20.18(0.07)$ & $19.92(0.06)$ & $20.77(0.14)$ \\
\hline 1403 & 570.336 & 356.383 & $20.48(0.05)$ & $20.43(0.07)$ & $20.41(0.08)$ & $20.47(0.08)$ & $20.63(0.05)$ & $20.04(0.09)$ \\
\hline & & & $20.59(0.07)$ & $21.50(0.23)$ & $20.58(0.08)$ & $20.27(0.06)$ & $20.49(0.07)$ & $20.36(0.06)$ \\
\hline & & & $19.71(0.05)$ & $19.80(0.05)$ & $19.82(0.05)$ & $19.97(0.05)$ & $20.09(0.05)$ & $20.22(0.05)$ \\
\hline & & & $20.40(0.07)$ & $20.35(0.07)$ & $20.39(0.05)$ & $20.90(0.08)$ & $20.66(0.07)$ & $20.27(0.10)$ \\
\hline 1413 & 660.172 & 73.683 & $20.39(0.05)$ & $20.94(0.08)$ & $21.09(0.12)$ & $21.21(0.10)$ & $20.37(0.05)$ & $20.57(0.06)$ \\
\hline & & & $20.59(0.08)$ & $20.78(0.08)$ & $20.50(0.06)$ & $20.17(0.05)$ & $20.52(0.07)$ & $20.56(0.05)$ \\
\hline & & & $19.90(0.05)$ & $19.85(0.05)$ & $19.89(0.04)$ & $19.89(0.05)$ & $20.03(0.05)$ & $20.02(0.05)$ \\
\hline & & & $20.33(0.07)$ & $20.14(0.05)$ & $20.19(0.05)$ & $20.56(0.07)$ & $20.89(0.08)$ & $19.95(0.09)$ \\
\hline 1416 & 193.155 & 727.949 & $19.97(0.04)$ & $19.85(0.05)$ & $19.98(0.07)$ & $19.98(0.07)$ & $20.22(0.05)$ & $20.12(0.05)$ \\
\hline & & & $20.19(0.05)$ & $20.05(0.06)$ & $19.93(0.05)$ & $20.26(0.05)$ & $20.25(0.06)$ & $20.04(0.05)$ \\
\hline & & & $20.25(0.06)$ & $20.39(0.05)$ & $20.44(0.05)$ & $20.47(0.06)$ & $20.48(0.07)$ & $20.46(0.06)$ \\
\hline & & & $20.41(0.07)$ & $20.47(0.07)$ & $20.45(0.06)$ & $20.44(0.06)$ & $20.36(0.06)$ & $20.47(0.13)$ \\
\hline 1428 & 310.168 & 448.889 & $20.19(0.05)$ & $20.61(0.08)$ & $20.71(0.07)$ & $20.90(0.12)$ & $20.50(0.06)$ & $20.36(0.05)$ \\
\hline & & & $20.28(0.06)$ & $20.46(0.07)$ & $20.30(0.05)$ & $20.56(0.06)$ & $20.50(0.07)$ & $20.45(0.05)$ \\
\hline & & & $19.80(0.05)$ & $19.83(0.05)$ & $19.85(0.05)$ & $19.95(0.06)$ & $20.04(0.05)$ & $20.18(0.06)$ \\
\hline & & & $20.26(0.07)$ & $20.16(0.06)$ & $20.30(0.06)$ & $20.45(0.06)$ & $20.38(0.06)$ & $20.33(0.09)$ \\
\hline 1447 & 781.259 & 221.809 & $19.89(0.05)$ & $20.27(0.08)$ & $19.89(0.06)$ & $19.82(0.06)$ & $20.53(0.05)$ & $20.21(0.05)$ \\
\hline & & & $20.29(0.05)$ & $20.27(0.06)$ & $20.09(0.05)$ & $20.34(0.05)$ & $20.08(0.05)$ & $19.82(0.05)$ \\
\hline & & & $20.61(0.06)$ & $20.67(0.06)$ & $20.55(0.05)$ & $20.69(0.06)$ & $20.77(0.05)$ & $20.61(0.06)$ \\
\hline & & & $20.34(0.07)$ & $20.39(0.08)$ & $20.16(0.05)$ & $19.92(0.05)$ & $19.82(0.05)$ & $20.74(0.15)$ \\
\hline 1450 & 342.254 & 598.411 & $20.32(0.05)$ & $20.72(0.08)$ & $20.79(0.11)$ & $20.74(0.09)$ & $19.77(0.05)$ & $19.93(0.05)$ \\
\hline & & & $19.99(0.05)$ & $20.21(0.06)$ & $20.11(0.05)$ & $20.20(0.05)$ & $19.92(0.05)$ & $19.78(0.04)$ \\
\hline & & & $20.26(0.06)$ & $20.39(0.05)$ & $20.50(0.05)$ & $20.54(0.06)$ & $20.73(0.06)$ & $20.72(0.06)$ \\
\hline & & & $20.98(0.09)$ & $20.94(0.09)$ & $20.83(0.07)$ & $21.42(0.12)$ & $21.10(0.07)$ & $19.74(0.09)$ \\
\hline 1452 & 891.524 & 655.324 & $20.18(2.91)$ & 99.99 (9.99) & $20.55(0.20)$ & $21.21(0.51)$ & $19.75(0.18)$ & $19.93(0.24)$ \\
\hline & & & $20.44(2.76)$ & $18.81(3.19)$ & $20.69(0.27)$ & $19.93(0.13)$ & $21.41(0.19)$ & $20.92(0.50)$ \\
\hline & & & $21.32(0.50)$ & $20.54(0.18)$ & $19.46(0.09)$ & 99.99 (9.99) & $21.68(0.56)$ & $20.92(0.26)$ \\
\hline & & & 99.99 (9.99) & 99.99 (9.99) & 99.99 (9.99) & $22.74(0.35)$ & $22.08(0.24)$ & $21.57(0.42)$ \\
\hline
\end{tabular}


Table 8. continuation.

\begin{tabular}{|c|c|c|c|c|c|c|c|c|}
\hline \multirow[t]{4}{*}{$\bar{F}$ F2 ID } & \multirow[t]{4}{*}{$\mathrm{x}$} & \multirow[t]{4}{*}{$\mathrm{y}$} & $2 \mathrm{Ks} 10(\sigma)$ & $2 \mathrm{Ks} 01(\sigma)$ & $2 \mathrm{Ks} 02(\sigma)$ & $2 \mathrm{Ks} 03(\sigma)$ & $2 \mathrm{Ks} 05(\sigma)$ & $\overline{2 \mathrm{Ks} 06(\sigma)}$ \\
\hline & & & $2 \mathrm{Ks} 07(\sigma)$ & $2 \mathrm{Ks} 08(\sigma)$ & $2 \mathrm{Ks} 09(\sigma)$ & $2 \mathrm{Ks} 11(\sigma)$ & $2 \mathrm{Ks} 12(\sigma)$ & $2 \mathrm{Ks} 13(\sigma)$ \\
\hline & & & $2 \mathrm{Ks} 14(\sigma)$ & $2 \mathrm{Ks} 15(\sigma)$ & $2 \mathrm{Ks} 16(\sigma)$ & $2 \mathrm{Ks} 17(\sigma)$ & $2 \mathrm{Ks} 18(\sigma)$ & $2 \mathrm{Ks} 19(\sigma)$ \\
\hline & & & $2 \mathrm{Ks} 20(\sigma)$ & $2 \mathrm{Ks} 21(\sigma)$ & $2 \mathrm{Ks} 22(\sigma)$ & $2 \mathrm{Ks} 23(\sigma)$ & $2 \mathrm{Ks} 24(\sigma)$ & $2 \mathrm{Ks} 04(\sigma)$ \\
\hline \multirow[t]{4}{*}{ 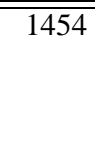 } & $\overline{661.897}$ & 796.132 & $19.87(0.05)$ & $20.48(0.07)$ & $20.45(0.09)$ & $20.24(0.07)$ & $20.36(0.05)$ & 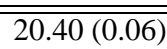 \\
\hline & & & $20.36(0.07)$ & $20.02(0.05)$ & $20.02(0.05)$ & $20.33(0.05)$ & $20.00(0.05)$ & $19.67(0.05)$ \\
\hline & & & $20.43(0.06)$ & $20.39(0.05)$ & $20.29(0.06)$ & $20.42(0.07)$ & $20.40(0.06)$ & $20.41(0.06)$ \\
\hline & & & $20.33(0.07)$ & $20.45(0.07)$ & $20.11(0.05)$ & $20.07(0.07)$ & $19.84(0.08)$ & $21.39(0.59)$ \\
\hline \multirow[t]{4}{*}{1456} & 16.619 & 61.724 & $20.18(0.05)$ & $20.99(0.14)$ & $20.56(0.10)$ & $20.74(0.08)$ & $20.51(0.05)$ & $21.11(0.11)$ \\
\hline & & & $20.40(0.07)$ & $20.57(0.10)$ & $20.31(0.06)$ & $20.19(0.05)$ & $19.92(0.06)$ & $20.21(0.07)$ \\
\hline & & & $20.36(0.06)$ & $19.94(0.06)$ & $19.99(0.07)$ & 99.99 (9.99) & $19.78(0.09)$ & $19.86(0.06)$ \\
\hline & & & $20.22(0.06)$ & $20.08(0.09)$ & $20.09(0.07)$ & $19.53(0.08)$ & $19.49(0.08)$ & 99.99 (9.99) \\
\hline \multirow[t]{4}{*}{1457} & 179.029 & 357.745 & $19.96(0.05)$ & $20.39(0.08)$ & $20.23(0.06)$ & $20.15(0.06)$ & $20.72(0.07)$ & $20.36(0.06)$ \\
\hline & & & $20.41(0.07)$ & $20.24(0.06)$ & $20.18(0.05)$ & $20.15(0.05)$ & $19.98(0.06)$ & $19.84(0.05)$ \\
\hline & & & $20.85(0.10)$ & $20.53(0.06)$ & $20.59(0.05)$ & $20.48(0.07)$ & $20.55(0.06)$ & $20.51(0.06)$ \\
\hline & & & $20.18(0.06)$ & $20.21(0.06)$ & $19.99(0.05)$ & $19.94(0.05)$ & $19.91(0.05)$ & $20.83(0.18)$ \\
\hline \multirow[t]{4}{*}{1465} & 830.918 & 266.779 & $20.68(0.06)$ & $19.78(0.06)$ & $19.84(0.06)$ & $19.92(0.05)$ & $19.99(0.05)$ & $19.95(0.05)$ \\
\hline & & & $20.31(0.06)$ & $20.36(0.06)$ & $20.48(0.06)$ & $20.14(0.05)$ & $20.52(0.06)$ & $20.48(0.08)$ \\
\hline & & & $20.09(0.05)$ & $20.02(0.05)$ & $20.02(0.06)$ & $20.15(0.06)$ & $20.18(0.06)$ & $20.13(0.06)$ \\
\hline & & & $20.24(0.06)$ & $20.37(0.07)$ & $20.39(0.05)$ & $20.63(0.06)$ & $20.49(0.06)$ & $20.29(0.10)$ \\
\hline \multirow[t]{4}{*}{1476} & 706.694 & 203.573 & $19.92(0.05)$ & $20.56(0.07)$ & $20.60(0.09)$ & $20.50(0.09)$ & $20.37(0.05)$ & $20.16(0.05)$ \\
\hline & & & $20.22(0.06)$ & $20.19(0.06)$ & $20.10(0.05)$ & $20.10(0.05)$ & $20.07(0.06)$ & $19.93(0.05)$ \\
\hline & & & $20.27(0.06)$ & $20.44(0.06)$ & $20.43(0.05)$ & $20.42(0.05)$ & $20.37(0.05)$ & $20.11(0.05)$ \\
\hline & & & $20.01(0.06)$ & $20.14(0.06)$ & $20.01(0.05)$ & $20.11(0.06)$ & $20.20(0.06)$ & $20.50(0.13)$ \\
\hline \multirow[t]{4}{*}{1478} & 868.002 & 667.055 & $22.12(0.85)$ & $99.99(9.99)$ & $20.35(0.14)$ & $21.35(0.78)$ & $20.15(0.13)$ & $19.94(0.16)$ \\
\hline & & & $20.61(0.17)$ & $19.27(0.16)$ & $21.08(0.22)$ & $19.53(0.06)$ & $21.32(0.12)$ & $20.13(0.20)$ \\
\hline & & & $20.36(0.17)$ & 99.99 (9.99) & 99.99 (9.99) & 99.99 (9.99) & $22.18(0.61)$ & $21.12(0.35)$ \\
\hline & & & 99.99 (9.99) & $23.09(0.34)$ & $21.30(0.15)$ & $22.55(0.23)$ & $22.37(0.31)$ & $21.25(0.27)$ \\
\hline \multirow[t]{4}{*}{1481} & 488.082 & 193.035 & $20.30(0.07)$ & $20.41(0.11)$ & $20.45(0.08)$ & $20.47(0.08)$ & $20.35(0.09)$ & $20.43(0.09)$ \\
\hline & & & $20.38(0.06)$ & $20.97(0.37)$ & $20.30(0.08)$ & $19.80(0.07)$ & $20.15(0.05)$ & $20.31(0.08)$ \\
\hline & & & $20.30(0.06)$ & $20.35(0.08)$ & $20.32(0.09)$ & $20.45(0.05)$ & $20.51(0.05)$ & $20.43(0.07)$ \\
\hline & & & $20.23(0.07)$ & $20.55(0.09)$ & $19.98(0.05)$ & $19.91(0.05)$ & $19.79(0.05)$ & $20.22(0.13)$ \\
\hline \multirow[t]{4}{*}{1486} & 861.583 & 68.406 & $20.63(0.06)$ & 99.99 (9.99) & $20.15(0.08)$ & $20.10(0.07)$ & $20.75(0.06)$ & $21.01(0.08)$ \\
\hline & & & $20.74(0.08)$ & $21.00(0.10)$ & $20.79(0.07)$ & $20.47(0.05)$ & $20.73(0.07)$ & $20.53(0.06)$ \\
\hline & & & $19.95(0.06)$ & $19.95(0.05)$ & $19.91(0.07)$ & $19.91(0.06)$ & $20.02(0.06)$ & $19.82(0.05)$ \\
\hline & & & $20.03(0.06)$ & $20.06(0.06)$ & $20.00(0.05)$ & $20.30(0.07)$ & $20.39(0.09)$ & $20.65(0.23)$ \\
\hline \multirow[t]{4}{*}{1494} & 70.381 & 424.654 & $20.00(0.05)$ & $20.47(0.05)$ & $20.26(0.07)$ & $20.20(0.08)$ & $20.42(0.07)$ & $20.99(0.09)$ \\
\hline & & & $20.29(0.06)$ & $20.68(0.09)$ & $20.13(0.06)$ & $20.32(0.05)$ & $20.04(0.06)$ & $19.90(0.06)$ \\
\hline & & & $20.42(0.06)$ & $20.50(0.06)$ & $20.43(0.06)$ & $20.49(0.08)$ & $20.33(0.06)$ & $20.27(0.06)$ \\
\hline & & & $19.97(0.06)$ & $20.05(0.07)$ & $19.98(0.05)$ & $19.99(0.05)$ & $19.95(0.05)$ & $20.63(0.12)$ \\
\hline \multirow[t]{4}{*}{1499} & 665.151 & 396.558 & $20.28(0.06)$ & $20.28(0.07)$ & $20.52(0.08)$ & $20.59(0.07)$ & $20.07(0.05)$ & $19.85(0.05)$ \\
\hline & & & $20.05(0.06)$ & $20.21(0.05)$ & $20.05(0.05)$ & $20.54(0.06)$ & $20.65(0.08)$ & $20.65(0.08)$ \\
\hline & & & $20.28(0.06)$ & $20.41(0.07)$ & $20.39(0.07)$ & $20.50(0.08)$ & $20.61(0.07)$ & $20.44(0.06)$ \\
\hline & & & $20.26(0.06)$ & $20.34(0.09)$ & $20.10(0.05)$ & $20.00(0.05)$ & $20.05(0.05)$ & $20.41(0.15)$ \\
\hline \multirow[t]{4}{*}{1502} & 851.204 & 443.255 & $19.98(0.04)$ & $21.23(0.13)$ & $21.13(0.14)$ & $21.06(0.11)$ & $20.55(0.06)$ & $20.11(0.05)$ \\
\hline & & & $20.22(0.05)$ & $20.39(0.07)$ & $20.02(0.05)$ & $20.11(0.05)$ & $20.12(0.06)$ & $20.19(0.05)$ \\
\hline & & & $20.43(0.06)$ & $20.23(0.05)$ & $20.16(0.05)$ & $20.12(0.05)$ & $20.12(0.05)$ & $20.12(0.05)$ \\
\hline & & & $20.29(0.07)$ & $20.17(0.07)$ & $20.44(0.05)$ & $20.78(0.08)$ & $21.01(0.09)$ & $20.98(0.21)$ \\
\hline \multirow[t]{4}{*}{1505} & 290.979 & 788.720 & $20.42(0.06)$ & $20.56(0.06)$ & $20.54(0.08)$ & $20.22(0.07)$ & $20.59(0.05)$ & $20.63(0.05)$ \\
\hline & & & $20.57(0.07)$ & $20.47(0.08)$ & $20.45(0.05)$ & $20.40(0.05)$ & $20.25(0.05)$ & $20.19(0.05)$ \\
\hline & & & $19.85(0.05)$ & $19.83(0.05)$ & $19.89(0.05)$ & $19.92(0.05)$ & $19.94(0.05)$ & $20.23(0.05)$ \\
\hline & & & $20.29(0.06)$ & $20.24(0.06)$ & $20.33(0.05)$ & $20.38(0.06)$ & $20.22(0.06)$ & $20.20(0.08)$ \\
\hline
\end{tabular}


Table 8. continuation.

\begin{tabular}{|c|c|c|c|c|c|c|c|c|}
\hline \multirow[t]{4}{*}{ F2 ID } & \multirow[t]{4}{*}{$\mathrm{x}$} & \multirow[t]{4}{*}{$y$} & $2 \mathrm{Ks} 10(\sigma)$ & $2 \mathrm{Ks} 01(\sigma)$ & $2 \mathrm{Ks} 02(\sigma)$ & $2 \mathrm{Ks} 03(\sigma)$ & $2 \mathrm{Ks} 05(\sigma)$ & $2 \mathrm{Ks} 06(\sigma)$ \\
\hline & & & $2 \mathrm{Ks} 07(\sigma)$ & $2 \mathrm{Ks} 08(\sigma)$ & $2 \mathrm{Ks} 09(\sigma)$ & $2 \mathrm{Ks} 11(\sigma)$ & $2 \mathrm{Ks} 12(\sigma)$ & $2 \mathrm{Ks} 13(\sigma)$ \\
\hline & & & $2 \mathrm{Ks} 14(\sigma)$ & $2 \mathrm{Ks} 15(\sigma)$ & $2 \mathrm{Ks} 16(\sigma)$ & $2 \mathrm{Ks} 17(\sigma)$ & $2 \mathrm{Ks} 18(\sigma)$ & $2 \mathrm{Ks} 19(\sigma)$ \\
\hline & & & $2 \mathrm{Ks} 20(\sigma)$ & $2 \mathrm{Ks} 21(\sigma)$ & $2 \mathrm{Ks} 22(\sigma)$ & $2 \mathrm{Ks} 23(\sigma)$ & $2 \mathrm{Ks} 24(\sigma)$ & $2 \mathrm{Ks} 04(\sigma)$ \\
\hline \multirow[t]{4}{*}{1508} & 529.299 & 819.837 & $20.32(0.05)$ & $19.91(0.06)$ & $19.96(0.06)$ & $19.97(0.08)$ & $19.90(0.05)$ & 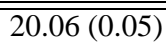 \\
\hline & & & $20.03(0.06)$ & $20.20(0.07)$ & $20.02(0.05)$ & $20.53(0.05)$ & $20.52(0.07)$ & $20.58(0.05)$ \\
\hline & & & $20.04(0.05)$ & $20.00(0.05)$ & $20.07(0.05)$ & $20.07(0.06)$ & $20.07(0.05)$ & $20.32(0.05)$ \\
\hline & & & $20.41(0.06)$ & $20.45(0.06)$ & $20.47(0.05)$ & $20.40(0.05)$ & $20.52(0.07)$ & $20.09(0.07)$ \\
\hline \multirow[t]{4}{*}{1512} & 869.103 & 267.056 & $20.00(0.05)$ & $99.99(9.99)$ & $20.92(0.13)$ & $20.66(0.10)$ & $20.63(0.06)$ & $20.10(0.05)$ \\
\hline & & & $20.19(0.06)$ & $20.35(0.06)$ & $19.98(0.05)$ & $20.03(0.05)$ & $20.20(0.06)$ & $20.33(0.05)$ \\
\hline & & & $19.93(0.05)$ & $20.02(0.05)$ & $20.13(0.05)$ & $20.33(0.07)$ & $20.56(0.07)$ & $20.64(0.05)$ \\
\hline & & & $20.75(0.07)$ & $20.67(0.09)$ & $20.66(0.07)$ & $20.51(0.06)$ & $20.66(0.07)$ & $20.35(0.09)$ \\
\hline \multirow[t]{4}{*}{1518} & 342.464 & 322.107 & $20.79(0.06)$ & $20.76(0.09)$ & $20.64(0.12)$ & $20.64(0.09)$ & $20.91(0.07)$ & $20.76(0.07)$ \\
\hline & & & $20.97(0.10)$ & $21.01(0.10)$ & $20.71(0.07)$ & $20.32(0.05)$ & $20.35(0.06)$ & $20.28(0.05)$ \\
\hline & & & $19.75(0.05)$ & $19.85(0.05)$ & $20.02(0.05)$ & $20.02(0.05)$ & $20.13(0.05)$ & $20.43(0.06)$ \\
\hline & & & $20.34(0.06)$ & $20.41(0.08)$ & $20.55(0.05)$ & $20.64(0.06)$ & $20.28(0.06)$ & $20.61(0.13)$ \\
\hline \multirow[t]{4}{*}{1522} & 373.323 & 462.304 & $20.43(0.05)$ & $20.20(0.06)$ & $20.57(0.11)$ & $20.89(0.10)$ & $19.95(0.05)$ & $20.13(0.05)$ \\
\hline & & & $20.15(0.06)$ & $20.64(0.10)$ & $20.15(0.05)$ & $19.78(0.05)$ & $19.80(0.05)$ & $20.03(0.05)$ \\
\hline & & & $21.05(0.09)$ & $21.18(0.09)$ & $20.94(0.05)$ & $20.75(0.07)$ & $20.68(0.06)$ & $20.56(0.07)$ \\
\hline & & & $20.40(0.07)$ & $20.34(0.08)$ & $20.20(0.05)$ & $20.23(0.05)$ & $20.27(0.06)$ & $19.99(0.07)$ \\
\hline \multirow[t]{4}{*}{1528} & 424.559 & 645.769 & $20.12(0.06)$ & $20.68(0.09)$ & $20.71(0.10)$ & $20.62(0.10)$ & $20.21(0.07)$ & $20.07(0.05)$ \\
\hline & & & $19.96(0.05)$ & $19.86(0.06)$ & $20.03(0.06)$ & $20.21(0.06)$ & $20.31(0.07)$ & $20.54(0.07)$ \\
\hline & & & $20.01(0.06)$ & $20.09(0.05)$ & $20.25(0.08)$ & $20.34(0.08)$ & $20.46(0.11)$ & $20.54(0.06)$ \\
\hline & & & $20.45(0.08)$ & $20.79(0.12)$ & $20.67(0.07)$ & $20.68(0.08)$ & $20.72(0.09)$ & $20.48(0.15)$ \\
\hline \multirow[t]{4}{*}{1536} & 414.598 & 114.209 & $20.32(0.06)$ & $20.67(0.08)$ & $20.71(0.10)$ & $20.51(0.09)$ & $20.64(0.06)$ & $20.58(0.06)$ \\
\hline & & & $20.52(0.07)$ & $20.30(0.06)$ & $20.34(0.06)$ & $20.54(0.05)$ & $20.63(0.08)$ & $20.52(0.06)$ \\
\hline & & & $19.80(0.05)$ & $19.80(0.05)$ & $19.90(0.06)$ & $19.98(0.05)$ & $20.07(0.05)$ & $20.10(0.05)$ \\
\hline & & & $20.37(0.06)$ & $20.27(0.07)$ & $20.48(0.06)$ & $20.59(0.06)$ & $20.65(0.07)$ & $20.20(0.09)$ \\
\hline \multirow[t]{4}{*}{1542} & 791.883 & 392.269 & $20.23(0.05)$ & $19.90(0.06)$ & $19.81(0.06)$ & $19.82(0.05)$ & $19.97(0.05)$ & $20.08(0.06)$ \\
\hline & & & $19.93(0.06)$ & $20.26(0.06)$ & $20.12(0.06)$ & $20.30(0.05)$ & $20.30(0.07)$ & $20.43(0.05)$ \\
\hline & & & $20.04(0.05)$ & $20.06(0.05)$ & $20.16(0.05)$ & $20.33(0.05)$ & $20.47(0.05)$ & $20.60(0.06)$ \\
\hline & & & $20.88(0.10)$ & $20.79(0.11)$ & $20.74(0.07)$ & $20.74(0.07)$ & $20.74(0.08)$ & $20.26(0.11)$ \\
\hline \multirow[t]{4}{*}{1547} & 249.170 & 142.121 & $20.35(0.05)$ & $20.62(0.08)$ & $20.62(0.12)$ & $20.48(0.07)$ & $20.53(0.05)$ & $20.79(0.06)$ \\
\hline & & & $20.71(0.08)$ & $20.49(0.07)$ & $20.37(0.06)$ & $20.62(0.06)$ & $20.66(0.08)$ & $20.59(0.05)$ \\
\hline & & & $19.75(0.05)$ & $19.71(0.04)$ & $19.95(0.05)$ & $20.08(0.06)$ & $20.10(0.05)$ & $20.30(0.05)$ \\
\hline & & & $20.44(0.07)$ & $20.40(0.06)$ & $20.56(0.06)$ & $20.72(0.06)$ & $20.69(0.07)$ & $20.21(0.08)$ \\
\hline \multirow[t]{4}{*}{1552} & 619.005 & 163.799 & $20.16(0.05)$ & $20.66(0.09)$ & $20.57(0.08)$ & $20.54(0.08)$ & $20.16(0.05)$ & $20.09(0.05)$ \\
\hline & & & $20.07(0.06)$ & $20.14(0.06)$ & $20.05(0.05)$ & $20.17(0.05)$ & $20.26(0.06)$ & $20.45(0.05)$ \\
\hline & & & $19.95(0.05)$ & $20.03(0.05)$ & $20.09(0.05)$ & $20.21(0.05)$ & $20.32(0.05)$ & $20.23(0.06)$ \\
\hline & & & $20.46(0.07)$ & $20.62(0.08)$ & $20.64(0.05)$ & $20.55(0.06)$ & $20.67(0.08)$ & $20.64(0.18)$ \\
\hline \multirow[t]{4}{*}{1553} & 826.799 & 327.667 & $20.42(0.05)$ & $20.58(0.07)$ & $20.17(0.06)$ & $20.10(0.06)$ & $20.70(0.06)$ & $20.64(0.08)$ \\
\hline & & & $20.62(0.08)$ & $20.90(0.08)$ & $20.45(0.06)$ & $20.51(0.06)$ & $20.50(0.07)$ & $20.62(0.05)$ \\
\hline & & & $20.02(0.05)$ & $19.95(0.05)$ & $19.92(0.05)$ & $19.88(0.05)$ & $20.02(0.05)$ & $19.94(0.05)$ \\
\hline & & & $20.09(0.05)$ & $20.19(0.06)$ & $20.17(0.05)$ & $20.32(0.06)$ & $20.49(0.07)$ & $20.70(0.11)$ \\
\hline \multirow[t]{4}{*}{1556} & 694.829 & 817.735 & $20.00(0.05)$ & $20.81(0.09)$ & $20.83(0.09)$ & $20.83(0.08)$ & $20.99(0.07)$ & $20.79(0.07)$ \\
\hline & & & $20.36(0.05)$ & $20.41(0.06)$ & $20.28(0.06)$ & $20.11(0.05)$ & $19.93(0.05)$ & $19.87(0.05)$ \\
\hline & & & $21.07(0.09)$ & $20.88(0.07)$ & $20.63(0.05)$ & $20.46(0.08)$ & $20.28(0.06)$ & $20.20(0.05)$ \\
\hline & & & $20.06(0.06)$ & $19.95(0.06)$ & $20.05(0.05)$ & $20.13(0.06)$ & $20.21(0.05)$ & $20.82(0.15)$ \\
\hline \multirow[t]{4}{*}{1559} & 640.348 & 70.001 & $19.97(0.05)$ & $20.50(0.08)$ & $20.62(0.08)$ & $20.86(0.10)$ & $20.35(0.05)$ & $20.49(0.05)$ \\
\hline & & & $20.56(0.06)$ & $20.25(0.06)$ & $20.17(0.05)$ & $20.38(0.06)$ & $20.14(0.06)$ & $19.90(0.05)$ \\
\hline & & & $20.58(0.07)$ & $20.64(0.06)$ & $20.64(0.06)$ & $20.48(0.05)$ & $20.43(0.06)$ & $20.19(0.06)$ \\
\hline & & & $20.33(0.06)$ & $20.24(0.07)$ & $19.93(0.05)$ & $19.98(0.05)$ & $19.88(0.05)$ & $20.52(0.16)$ \\
\hline
\end{tabular}


Table 8. continuation.

\begin{tabular}{|c|c|c|c|c|c|c|c|c|}
\hline \multirow[t]{4}{*}{$\bar{F}$ F2 ID } & \multirow[t]{4}{*}{$\mathrm{x}$} & \multirow[t]{4}{*}{$\mathrm{y}$} & $2 \mathrm{Ks} 10(\sigma)$ & $2 \mathrm{Ks} 01(\sigma)$ & $2 \mathrm{Ks} 02(\sigma)$ & $2 \mathrm{Ks} 03(\sigma)$ & $2 \mathrm{Ks} 05(\sigma)$ & $\overline{2 \mathrm{Ks} 06(\sigma)}$ \\
\hline & & & $2 \mathrm{Ks} 07(\sigma)$ & $2 \mathrm{Ks} 08(\sigma)$ & $2 \mathrm{Ks} 09(\sigma)$ & $2 \mathrm{Ks} 11(\sigma)$ & $2 \mathrm{Ks} 12(\sigma)$ & $2 \mathrm{Ks} 13(\sigma)$ \\
\hline & & & $2 \mathrm{Ks} 14(\sigma)$ & $2 \mathrm{Ks} 15(\sigma)$ & $2 \mathrm{Ks} 16(\sigma)$ & $2 \mathrm{Ks} 17(\sigma)$ & $2 \mathrm{Ks} 18(\sigma)$ & $2 \mathrm{Ks} 19(\sigma)$ \\
\hline & & & $2 \mathrm{Ks} 20(\sigma)$ & $2 \mathrm{Ks} 21(\sigma)$ & $2 \mathrm{Ks} 22(\sigma)$ & $2 \mathrm{Ks} 23(\sigma)$ & $2 \mathrm{Ks} 24(\sigma)$ & $2 \mathrm{Ks} 04(\sigma)$ \\
\hline \multirow[t]{4}{*}{$\overline{1560}$} & 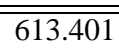 & 357.201 & $20.12(0.05)$ & $20.72(0.09)$ & $\overline{20.54(0.07)}$ & $20.57(0.09)$ & $20.19(0.05)$ & 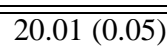 \\
\hline & & & $20.04(0.06)$ & $20.17(0.06)$ & $19.94(0.05)$ & $20.15(0.05)$ & $20.34(0.07)$ & $20.51(0.05)$ \\
\hline & & & $20.00(0.05)$ & $20.01(0.05)$ & $20.05(0.04)$ & $20.27(0.06)$ & $20.41(0.05)$ & $20.70(0.06)$ \\
\hline & & & $20.93(0.09)$ & $20.79(0.10)$ & $20.96(0.06)$ & $20.91(0.08)$ & $21.10(0.10)$ & $20.94(0.15)$ \\
\hline \multirow[t]{4}{*}{1579} & 359.919 & 637.705 & $19.86(0.05)$ & $20.66(0.11)$ & $20.28(0.08)$ & $20.40(0.08)$ & $20.54(0.06)$ & $20.32(0.05)$ \\
\hline & & & $20.36(0.06)$ & $20.27(0.06)$ & $20.03(0.05)$ & $20.25(0.06)$ & $20.15(0.06)$ & $19.81(0.05)$ \\
\hline & & & $20.82(0.07)$ & $20.54(0.06)$ & $20.43(0.05)$ & $20.47(0.05)$ & $20.33(0.05)$ & $20.53(0.05)$ \\
\hline & & & $20.68(0.09)$ & $20.52(0.08)$ & $20.43(0.05)$ & $20.30(0.06)$ & $20.02(0.05)$ & $20.68(0.14)$ \\
\hline \multirow[t]{4}{*}{1588} & 887.266 & 609.134 & $20.11(0.05)$ & 99.99 (9.99) & $19.98(0.06)$ & $20.26(0.07)$ & $20.70(0.05)$ & $20.49(0.06)$ \\
\hline & & & $20.32(0.06)$ & $20.59(0.08)$ & $20.31(0.06)$ & $21.47(0.10)$ & $20.75(0.07)$ & $20.87(0.05)$ \\
\hline & & & $19.83(0.05)$ & $19.85(0.05)$ & $19.91(0.05)$ & $19.95(0.07)$ & $20.09(0.05)$ & $20.25(0.05)$ \\
\hline & & & 99.99 (9.99) & $20.35(0.06)$ & $20.41(0.05)$ & $20.75(0.07)$ & $20.75(0.08)$ & $20.97(0.23)$ \\
\hline \multirow[t]{4}{*}{1600} & 93.163 & 244.983 & $20.53(0.06)$ & $20.13(0.08)$ & $20.39(0.08)$ & $20.64(0.07)$ & $20.40(0.06)$ & $20.59(0.07)$ \\
\hline & & & $20.59(0.07)$ & $20.77(0.10)$ & $20.54(0.06)$ & $20.87(0.06)$ & $20.59(0.07)$ & $20.50(0.05)$ \\
\hline & & & $19.84(0.05)$ & $19.74(0.05)$ & $19.91(0.06)$ & $20.08(0.06)$ & $20.03(0.05)$ & $20.34(0.05)$ \\
\hline & & & $20.62(0.08)$ & $20.39(0.07)$ & $20.62(0.06)$ & $20.71(0.06)$ & $20.46(0.07)$ & $20.29(0.13)$ \\
\hline \multirow[t]{4}{*}{1602} & 628.858 & 196.393 & $20.36(0.05)$ & $20.36(0.07)$ & $20.40(0.08)$ & $20.07(0.05)$ & $20.04(0.06)$ & $20.40(0.26)$ \\
\hline & & & $20.22(0.06)$ & $19.62(0.18)$ & $20.31(0.06)$ & $19.71(0.18)$ & $20.92(0.20)$ & $20.34(0.06)$ \\
\hline & & & $20.12(0.13)$ & $20.41(0.06)$ & $20.36(0.07)$ & $20.47(0.07)$ & $20.48(0.07)$ & $19.68(0.10)$ \\
\hline & & & $20.46(0.07)$ & $20.36(0.07)$ & $19.79(0.11)$ & $20.19(0.07)$ & $19.83(0.05)$ & $20.03(0.09)$ \\
\hline \multirow[t]{4}{*}{1605} & 203.969 & 493.424 & $20.93(0.05)$ & $21.02(0.13)$ & $20.96(0.13)$ & $21.46(0.15)$ & $20.58(0.06)$ & $20.66(0.06)$ \\
\hline & & & $20.87(0.09)$ & $21.12(0.11)$ & $20.83(0.06)$ & $20.15(0.05)$ & $20.19(0.06)$ & $20.52(0.05)$ \\
\hline & & & $20.46(0.06)$ & $20.31(0.05)$ & $20.20(0.05)$ & $20.02(0.05)$ & $19.99(0.05)$ & $19.93(0.05)$ \\
\hline & & & $19.88(0.05)$ & $20.00(0.06)$ & $19.93(0.05)$ & $20.15(0.05)$ & $20.22(0.07)$ & $20.34(0.12)$ \\
\hline \multirow[t]{4}{*}{1612} & 606.099 & 225.538 & $20.33(0.05)$ & $20.40(0.06)$ & $20.58(0.06)$ & $20.28(0.08)$ & $20.29(0.06)$ & $18.39(0.07)$ \\
\hline & & & $20.40(0.07)$ & $20.06(0.37)$ & $20.33(0.05)$ & $19.14(0.10)$ & $20.01(0.07)$ & $19.46(0.14)$ \\
\hline & & & $19.65(0.11)$ & $19.82(0.13)$ & $20.43(0.06)$ & $20.41(0.06)$ & $20.47(0.06)$ & $20.35(0.19)$ \\
\hline & & & $20.33(0.10)$ & $20.25(0.08)$ & $19.77(0.08)$ & $21.88(0.28)$ & $20.30(0.11)$ & $19.77(0.13)$ \\
\hline \multirow[t]{4}{*}{1613} & 848.538 & 389.540 & $20.01(0.04)$ & $19.98(0.08)$ & $19.90(0.07)$ & $19.92(0.06)$ & $20.41(0.05)$ & $20.23(0.06)$ \\
\hline & & & $20.20(0.08)$ & $20.35(0.07)$ & $20.04(0.05)$ & $20.41(0.05)$ & $20.23(0.06)$ & $19.85(0.05)$ \\
\hline & & & $20.79(0.09)$ & $20.70(0.05)$ & $20.52(0.05)$ & $20.47(0.06)$ & $20.60(0.06)$ & $20.51(0.05)$ \\
\hline & & & $20.54(0.08)$ & $20.40(0.07)$ & $20.50(0.06)$ & $20.27(0.05)$ & $20.07(0.05)$ & $20.88(0.19)$ \\
\hline \multirow[t]{4}{*}{1616} & 101.122 & 391.106 & $20.73(0.08)$ & $20.53(0.10)$ & $20.30(0.10)$ & $20.21(0.09)$ & $20.00(0.08)$ & $20.02(0.05)$ \\
\hline & & & $20.23(0.07)$ & $20.31(0.07)$ & $20.50(0.07)$ & $20.42(0.06)$ & $20.31(0.07)$ & $20.64(0.10)$ \\
\hline & & & $20.55(0.07)$ & $20.43(0.07)$ & $20.58(0.09)$ & $20.50(0.09)$ & $20.56(0.09)$ & $20.39(0.06)$ \\
\hline & & & $20.11(0.06)$ & $20.27(0.06)$ & $20.12(0.06)$ & $19.96(0.05)$ & $19.99(0.05)$ & $19.95(0.10)$ \\
\hline \multirow[t]{4}{*}{1617} & 571.782 & 6.244 & $19.93(0.05)$ & $20.05(0.05)$ & $20.20(0.08)$ & $20.19(0.07)$ & $20.19(0.05)$ & $20.27(0.05)$ \\
\hline & & & $20.10(0.06)$ & $20.02(0.06)$ & $19.98(0.05)$ & $20.56(0.05)$ & $20.27(0.05)$ & $20.08(0.05)$ \\
\hline & & & $20.41(0.06)$ & $20.56(0.06)$ & $20.61(0.06)$ & $20.53(0.09)$ & $20.53(0.05)$ & $20.36(0.05)$ \\
\hline & & & $20.36(0.07)$ & $20.31(0.07)$ & $20.31(0.05)$ & $20.46(0.06)$ & $20.31(0.06)$ & $20.54(0.12)$ \\
\hline \multirow[t]{4}{*}{1622} & 541.392 & 443.704 & $20.20(0.05)$ & $20.59(0.07)$ & $20.56(0.08)$ & $20.56(0.08)$ & $20.08(0.05)$ & $20.01(0.05)$ \\
\hline & & & $19.98(0.05)$ & $20.10(0.06)$ & $20.04(0.06)$ & $20.24(0.05)$ & $20.29(0.06)$ & $20.42(0.05)$ \\
\hline & & & $19.99(0.05)$ & $20.09(0.05)$ & $20.10(0.05)$ & $20.23(0.05)$ & $20.38(0.05)$ & $20.77(0.06)$ \\
\hline & & & $20.62(0.07)$ & $20.60(0.07)$ & $20.89(0.07)$ & $21.03(0.07)$ & $20.79(0.07)$ & $20.42(0.09)$ \\
\hline \multirow[t]{4}{*}{1623} & 787.079 & 288.645 & $19.92(0.04)$ & $20.45(0.07)$ & $20.34(0.08)$ & $20.40(0.06)$ & $20.41(0.05)$ & $20.36(0.06)$ \\
\hline & & & $20.39(0.06)$ & $20.44(0.07)$ & $20.14(0.05)$ & $20.24(0.05)$ & $20.07(0.05)$ & $19.78(0.04)$ \\
\hline & & & $20.66(0.07)$ & $20.83(0.06)$ & $20.70(0.05)$ & $20.66(0.05)$ & $20.80(0.05)$ & $20.52(0.05)$ \\
\hline & & & $20.66(0.08)$ & $20.82(0.08)$ & $20.61(0.06)$ & $20.39(0.06)$ & $20.21(0.05)$ & $20.25(0.07)$ \\
\hline
\end{tabular}


Table 8. continuation.

\begin{tabular}{|c|c|c|c|c|c|c|c|c|}
\hline \multirow[t]{4}{*}{$\overline{\mathrm{F} 2 \mathrm{ID}}$} & \multirow[t]{4}{*}{$\mathrm{x}$} & \multirow[t]{4}{*}{$\mathrm{y}$} & $2 \mathrm{Ks} 10(\sigma)$ & $2 \mathrm{Ks} 01(\sigma)$ & $2 \mathrm{Ks} 02(\sigma)$ & $2 \mathrm{Ks} 03(\sigma)$ & $2 \mathrm{Ks} 05(\sigma)$ & $2 \mathrm{Ks06}(\sigma)$ \\
\hline & & & $2 \mathrm{Ks} 07(\sigma)$ & $2 \mathrm{Ks} 08(\sigma)$ & $2 \mathrm{Ks} 09(\sigma)$ & $2 \mathrm{Ks} 11(\sigma)$ & $2 \mathrm{Ks} 12(\sigma)$ & $2 \mathrm{Ks} 13(\sigma)$ \\
\hline & & & $2 \mathrm{Ks} 14(\sigma)$ & $2 \mathrm{Ks} 15(\sigma)$ & $2 \mathrm{Ks} 16(\sigma)$ & $2 \mathrm{Ks} 17(\sigma)$ & $2 \mathrm{Ks} 18(\sigma)$ & $2 \mathrm{Ks} 19(\sigma)$ \\
\hline & & & $2 \mathrm{Ks} 20(\sigma)$ & $2 \mathrm{Ks} 21(\sigma)$ & $2 \mathrm{Ks} 22(\sigma)$ & $2 \mathrm{Ks} 23(\sigma)$ & $2 \mathrm{Ks} 24(\sigma)$ & $2 \mathrm{Ks} 04(\sigma)$ \\
\hline \multirow[t]{4}{*}{1629} & 872.381 & 666.590 & $20.39(0.11)$ & $99.99(9.99)$ & $20.17(0.18)$ & $20.02(0.18)$ & $19.80(0.15)$ & $20.01(0.16)$ \\
\hline & & & $20.17(0.11)$ & $20.21(0.54)$ & $20.29(0.09)$ & $20.09(0.14)$ & $20.88(0.11)$ & $20.72(0.23)$ \\
\hline & & & $19.48(0.09)$ & $21.94(0.98)$ & $19.41(0.14)$ & $20.70(0.21)$ & $21.57(0.40)$ & $20.44(0.15)$ \\
\hline & & & $19.08(0.14)$ & $21.88(0.30)$ & $21.81(0.21)$ & $23.32(0.55)$ & $21.33(0.11)$ & $19.85(0.19)$ \\
\hline \multirow[t]{4}{*}{1631} & 679.773 & 105.185 & $20.61(0.06)$ & $20.09(0.06)$ & $20.23(0.07)$ & $20.64(0.09)$ & $20.03(0.05)$ & $20.16(0.05)$ \\
\hline & & & $20.31(0.06)$ & $20.42(0.07)$ & $20.51(0.06)$ & $20.44(0.05)$ & $20.60(0.08)$ & $20.52(0.05)$ \\
\hline & & & $20.01(0.05)$ & $19.92(0.05)$ & $20.08(0.05)$ & $20.17(0.05)$ & $20.28(0.06)$ & $20.30(0.05)$ \\
\hline & & & $20.65(0.07)$ & $20.55(0.07)$ & $20.60(0.06)$ & $20.43(0.05)$ & $20.55(0.06)$ & $20.07(0.07)$ \\
\hline \multirow[t]{4}{*}{1645} & 49.166 & 103.181 & $20.88(0.07)$ & $20.28(0.07)$ & $20.81(0.09)$ & $20.96(0.09)$ & $20.30(0.05)$ & $20.62(0.08)$ \\
\hline & & & $20.52(0.08)$ & $21.23(0.15)$ & $20.78(0.09)$ & $20.43(0.06)$ & $20.50(0.07)$ & $20.77(0.06)$ \\
\hline & & & $20.01(0.05)$ & $19.79(0.05)$ & $19.89(0.06)$ & $20.04(0.10)$ & $19.78(0.07)$ & $19.94(0.05)$ \\
\hline & & & $20.14(0.07)$ & $20.02(0.06)$ & $20.28(0.05)$ & $20.57(0.08)$ & $20.83(0.09)$ & $20.46(0.35)$ \\
\hline \multirow[t]{4}{*}{1683} & 677.682 & 104.014 & $20.01(0.05)$ & $21.22(0.11)$ & $21.44(0.15)$ & $20.88(0.10)$ & $20.40(0.05)$ & $20.24(0.05)$ \\
\hline & & & $20.09(0.05)$ & $20.00(0.06)$ & $20.01(0.05)$ & $20.12(0.05)$ & $20.33(0.07)$ & $20.62(0.06)$ \\
\hline & & & $20.37(0.06)$ & $20.29(0.05)$ & $20.23(0.05)$ & $20.23(0.05)$ & $20.27(0.05)$ & $20.23(0.05)$ \\
\hline & & & $20.42(0.07)$ & $20.47(0.07)$ & $20.57(0.06)$ & $20.95(0.08)$ & $20.68(0.08)$ & $20.53(0.14)$ \\
\hline \multirow[t]{4}{*}{1685} & 370.284 & 103.559 & $20.06(0.04)$ & $19.77(0.05)$ & $19.80(0.06)$ & $19.72(0.05)$ & $20.47(0.05)$ & $20.47(0.05)$ \\
\hline & & & $20.45(0.07)$ & $20.23(0.07)$ & $20.22(0.05)$ & $20.36(0.05)$ & $20.07(0.05)$ & $19.96(0.05)$ \\
\hline & & & $20.25(0.06)$ & $20.36(0.05)$ & $20.48(0.05)$ & $20.63(0.06)$ & $20.66(0.06)$ & $20.73(0.06)$ \\
\hline & & & $20.61(0.07)$ & $20.50(0.08)$ & $20.68(0.06)$ & $20.76(0.07)$ & $20.60(0.08)$ & $20.70(0.09)$ \\
\hline \multirow[t]{4}{*}{1688} & 592.385 & 217.938 & $20.08(0.04)$ & $20.67(0.07)$ & $21.19(0.17)$ & $20.76(0.09)$ & $20.72(0.05)$ & $20.29(0.09)$ \\
\hline & & & $20.68(0.08)$ & $20.60(0.26)$ & $20.24(0.05)$ & $19.12(0.07)$ & $19.87(0.07)$ & $19.97(0.04)$ \\
\hline & & & $22.06(0.82)$ & $20.93(0.09)$ & $20.84(0.06)$ & $20.86(0.06)$ & $20.70(0.06)$ & $20.46(0.09)$ \\
\hline & & & $20.43(0.07)$ & $20.37(0.06)$ & $20.30(0.09)$ & $20.40(0.06)$ & $20.49(0.09)$ & $20.90(0.37)$ \\
\hline \multirow[t]{4}{*}{1690} & 702.089 & 302.619 & $20.30(0.05)$ & $20.34(0.07)$ & $20.61(0.09)$ & $20.24(0.07)$ & $20.12(0.05)$ & $19.90(0.05)$ \\
\hline & & & $20.08(0.05)$ & $20.02(0.05)$ & $20.08(0.05)$ & $20.13(0.05)$ & $20.36(0.06)$ & $20.30(0.05)$ \\
\hline & & & $20.18(0.06)$ & $20.29(0.05)$ & $20.48(0.05)$ & $20.52(0.06)$ & $20.53(0.06)$ & $20.53(0.06)$ \\
\hline & & & $20.45(0.09)$ & $20.50(0.08)$ & $20.46(0.05)$ & $20.23(0.05)$ & $20.42(0.06)$ & $20.45(0.12)$ \\
\hline \multirow[t]{4}{*}{1692} & 239.523 & 661.270 & $20.21(0.05)$ & $20.95(0.08)$ & $20.85(0.10)$ & $20.52(0.07)$ & $20.26(0.05)$ & $20.10(0.05)$ \\
\hline & & & $20.07(0.06)$ & $20.14(0.06)$ & $20.11(0.05)$ & $20.15(0.05)$ & $20.19(0.05)$ & $20.47(0.06)$ \\
\hline & & & $20.28(0.06)$ & $20.21(0.05)$ & $20.17(0.05)$ & $20.11(0.05)$ & $20.17(0.05)$ & $20.31(0.05)$ \\
\hline & & & $20.32(0.07)$ & $20.59(0.07)$ & $20.64(0.06)$ & $20.68(0.06)$ & $20.58(0.07)$ & $20.47(0.15)$ \\
\hline \multirow[t]{4}{*}{1697} & 887.821 & 314.828 & $20.34(0.08)$ & $99.99(9.99)$ & $20.75(0.10)$ & $20.50(0.08)$ & $20.80(0.09)$ & $20.41(0.07)$ \\
\hline & & & $20.53(0.09)$ & $20.62(0.09)$ & $20.78(0.09)$ & $20.38(0.06)$ & $20.12(0.06)$ & $19.97(0.05)$ \\
\hline & & & $20.25(0.07)$ & $20.48(0.07)$ & $20.58(0.06)$ & $20.52(0.07)$ & $20.50(0.05)$ & $20.22(0.06)$ \\
\hline & & & $99.99(9.99)$ & $20.28(0.08)$ & $20.00(0.05)$ & $19.91(0.05)$ & $19.99(0.06)$ & $20.70(0.19)$ \\
\hline \multirow[t]{4}{*}{1699} & 119.719 & 434.180 & $23.89(0.62)$ & $99.99(9.99)$ & $99.99(9.99)$ & 99.99 (9.99) & $99.99(9.99)$ & 99.99 (9.99) \\
\hline & & & 99.99 (9.99) & $19.76(0.25)$ & 99.99 (9.99) & $19.73(0.09)$ & $20.59(0.09)$ & 99.99 (9.99) \\
\hline & & & $23.06(1.03)$ & $24.63(2.00)$ & $24.78(1.14)$ & $24.43(1.19)$ & $24.21(0.83)$ & 99.99 (9.99) \\
\hline & & & 99.99 (9.99) & 99.99 (9.99) & 99.99 (9.99) & $24.41(2.37)$ & $23.03(0.56)$ & $20.77(0.21)$ \\
\hline \multirow[t]{4}{*}{1700} & 131.430 & 614.878 & $20.60(0.05)$ & $20.21(0.05)$ & $20.22(0.06)$ & $20.03(0.06)$ & $20.78(0.06)$ & $20.78(0.07)$ \\
\hline & & & $20.72(0.08)$ & $21.11(0.10)$ & $20.79(0.08)$ & $20.89(0.06)$ & $20.81(0.09)$ & $20.57(0.05)$ \\
\hline & & & $19.91(0.05)$ & $19.91(0.05)$ & $19.95(0.05)$ & $20.02(0.05)$ & $20.07(0.05)$ & $20.38(0.05)$ \\
\hline & & & $20.57(0.07)$ & $20.33(0.05)$ & $20.62(0.06)$ & $20.69(0.07)$ & $20.54(0.08)$ & $21.04(0.15)$ \\
\hline \multirow[t]{4}{*}{1701} & 50.788 & 204.785 & $20.46(0.07)$ & $20.14(0.08)$ & $20.24(0.08)$ & $20.43(0.09)$ & $20.71(0.07)$ & $20.99(0.16)$ \\
\hline & & & $20.61(0.08)$ & $20.79(0.08)$ & $20.51(0.07)$ & $20.48(0.15)$ & $20.85(0.13)$ & $20.41(0.06)$ \\
\hline & & & $20.29(0.07)$ & $20.14(0.07)$ & $20.43(0.07)$ & $20.43(0.09)$ & $20.42(0.07)$ & $20.16(0.06)$ \\
\hline & & & $19.98(0.06)$ & $20.17(0.07)$ & $20.02(0.05)$ & $19.90(0.06)$ & $20.09(0.06)$ & $20.11(0.18)$ \\
\hline
\end{tabular}


Table 8. continuation.

\begin{tabular}{|c|c|c|c|c|c|c|c|c|}
\hline \multirow[t]{4}{*}{$\bar{F}$ F2 ID } & \multirow[t]{4}{*}{$\mathrm{x}$} & \multirow[t]{4}{*}{$\mathrm{y}$} & $2 \mathrm{Ks} 10(\sigma)$ & $2 \mathrm{Ks} 01(\sigma)$ & $2 \mathrm{Ks} 02(\sigma)$ & $2 \mathrm{Ks} 03(\sigma)$ & $2 \mathrm{Ks} 05(\sigma)$ & $\overline{2 \mathrm{Ks} 06(\sigma)}$ \\
\hline & & & $2 \mathrm{Ks} 07(\sigma)$ & $2 \mathrm{Ks} 08(\sigma)$ & $2 \mathrm{Ks} 09(\sigma)$ & $2 \mathrm{Ks} 11(\sigma)$ & $2 \mathrm{Ks} 12(\sigma)$ & $2 \mathrm{Ks} 13(\sigma)$ \\
\hline & & & $2 \mathrm{Ks} 14(\sigma)$ & $2 \mathrm{Ks} 15(\sigma)$ & $2 \mathrm{Ks} 16(\sigma)$ & $2 \mathrm{Ks} 17(\sigma)$ & $2 \mathrm{Ks} 18(\sigma)$ & $2 \mathrm{Ks} 19(\sigma)$ \\
\hline & & & $2 \mathrm{Ks} 20(\sigma)$ & $2 \mathrm{Ks} 21(\sigma)$ & $2 \mathrm{Ks} 22(\sigma)$ & $2 \mathrm{Ks} 23(\sigma)$ & $2 \mathrm{Ks} 24(\sigma)$ & $2 \mathrm{Ks} 04(\sigma)$ \\
\hline \multirow[t]{4}{*}{$\overline{1703}$} & 186.144 & 267.475 & $20.11(0.05)$ & $20.72(0.09)$ & $\overline{20.74(0.09)}$ & $20.89(0.12)$ & $20.39(0.05)$ & 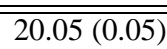 \\
\hline & & & $20.17(0.06)$ & $20.01(0.06)$ & $20.03(0.06)$ & $20.31(0.05)$ & $20.12(0.06)$ & $20.16(0.04)$ \\
\hline & & & $20.78(0.07)$ & $20.70(0.07)$ & $20.67(0.05)$ & $20.51(0.05)$ & $20.35(0.05)$ & $20.29(0.05)$ \\
\hline & & & $20.07(0.06)$ & $20.08(0.07)$ & $20.15(0.05)$ & $20.20(0.05)$ & $20.15(0.06)$ & $20.78(0.10)$ \\
\hline \multirow[t]{4}{*}{1709} & 377.356 & 658.374 & $20.61(0.06)$ & $20.31(0.06)$ & $20.29(0.07)$ & $20.18(0.07)$ & $20.05(0.05)$ & $20.30(0.06)$ \\
\hline & & & $20.43(0.07)$ & $20.60(0.08)$ & $20.47(0.06)$ & $19.88(0.05)$ & $19.92(0.05)$ & $20.09(0.05)$ \\
\hline & & & $21.00(0.10)$ & $21.05(0.09)$ & $21.11(0.07)$ & $21.23(0.08)$ & $21.02(0.07)$ & $20.88(0.07)$ \\
\hline & & & $20.43(0.07)$ & $20.63(0.07)$ & $20.22(0.05)$ & $20.14(0.06)$ & $19.97(0.06)$ & $19.69(0.06)$ \\
\hline \multirow[t]{4}{*}{1710} & 711.522 & 550.778 & $20.23(0.05)$ & $20.73(0.08)$ & $20.81(0.09)$ & $20.44(0.06)$ & $20.22(0.05)$ & $20.01(0.05)$ \\
\hline & & & $20.22(0.06)$ & $20.24(0.06)$ & $20.13(0.05)$ & $20.26(0.05)$ & $20.38(0.07)$ & $20.48(0.05)$ \\
\hline & & & $19.96(0.05)$ & $20.08(0.05)$ & $20.09(0.05)$ & $20.21(0.05)$ & $20.27(0.05)$ & $20.41(0.05)$ \\
\hline & & & $21.02(0.11)$ & $20.48(0.09)$ & $20.60(0.05)$ & $20.56(0.06)$ & $20.62(0.06)$ & $21.25(0.21)$ \\
\hline \multirow[t]{4}{*}{1728} & 184.289 & 636.129 & $21.11(0.07)$ & $20.65(0.06)$ & $20.64(0.08)$ & $20.67(0.11)$ & $20.56(0.05)$ & $21.00(0.07)$ \\
\hline & & & $20.96(0.08)$ & $21.49(0.16)$ & $21.06(0.08)$ & $20.84(0.08)$ & $21.24(0.11)$ & $21.04(0.06)$ \\
\hline & & & $19.96(0.05)$ & $19.95(0.05)$ & $19.85(0.05)$ & $19.94(0.05)$ & $20.05(0.05)$ & $20.32(0.05)$ \\
\hline & & & $20.42(0.06)$ & $20.36(0.07)$ & $20.67(0.06)$ & $20.84(0.07)$ & $20.80(0.08)$ & $20.12(0.07)$ \\
\hline \multirow[t]{4}{*}{1729} & 534.104 & 395.756 & $19.94(0.04)$ & $20.59(0.05)$ & $20.47(0.09)$ & $20.30(0.07)$ & $20.27(0.05)$ & $20.18(0.05)$ \\
\hline & & & $20.09(0.05)$ & $20.08(0.06)$ & $19.95(0.05)$ & $20.30(0.05)$ & $20.25(0.07)$ & $20.15(0.05)$ \\
\hline & & & $21.00(0.06)$ & $21.11(0.06)$ & $21.04(0.05)$ & $21.27(0.08)$ & $21.10(0.07)$ & $20.75(0.06)$ \\
\hline & & & $20.70(0.09)$ & $20.58(0.08)$ & $20.38(0.06)$ & $20.24(0.05)$ & $20.18(0.05)$ & $20.94(0.13)$ \\
\hline \multirow[t]{4}{*}{1730} & 541.524 & 536.047 & $19.93(0.05)$ & $20.07(0.05)$ & $20.26(0.06)$ & $20.50(0.09)$ & $20.55(0.06)$ & $20.16(0.05)$ \\
\hline & & & $20.26(0.05)$ & $20.20(0.06)$ & $19.99(0.05)$ & $21.06(0.08)$ & $21.50(0.13)$ & $21.21(0.08)$ \\
\hline & & & $20.77(0.08)$ & $20.49(0.06)$ & $20.43(0.06)$ & $20.39(0.07)$ & $20.38(0.06)$ & $20.35(0.05)$ \\
\hline & & & $20.23(0.06)$ & $20.34(0.07)$ & $20.34(0.05)$ & $20.65(0.08)$ & $20.55(0.07)$ & $21.04(0.23)$ \\
\hline \multirow[t]{4}{*}{1731} & 341.025 & 304.310 & $20.22(0.05)$ & $20.62(0.07)$ & $21.04(0.12)$ & $20.72(0.08)$ & $20.45(0.06)$ & $20.43(0.06)$ \\
\hline & & & $20.52(0.08)$ & $20.82(0.09)$ & $20.41(0.06)$ & $20.57(0.06)$ & $20.25(0.06)$ & $20.09(0.04)$ \\
\hline & & & $20.44(0.06)$ & $20.46(0.05)$ & $20.38(0.05)$ & $20.32(0.05)$ & $20.34(0.05)$ & $20.25(0.05)$ \\
\hline & & & $20.02(0.06)$ & $20.04(0.06)$ & $20.02(0.05)$ & $20.15(0.06)$ & $20.30(0.06)$ & $20.60(0.12)$ \\
\hline \multirow[t]{4}{*}{1732} & 802.774 & 165.105 & $20.25(0.05)$ & $19.76(0.06)$ & $19.92(0.06)$ & $20.04(0.06)$ & $20.20(0.05)$ & $20.39(0.06)$ \\
\hline & & & $20.56(0.07)$ & $20.57(0.07)$ & $20.38(0.06)$ & $20.63(0.06)$ & $20.67(0.08)$ & $20.52(0.05)$ \\
\hline & & & $20.46(0.06)$ & $20.52(0.05)$ & $20.45(0.06)$ & $20.39(0.06)$ & $20.45(0.06)$ & $20.24(0.05)$ \\
\hline & & & $20.18(0.06)$ & $20.41(0.07)$ & $20.22(0.05)$ & $20.05(0.05)$ & $19.88(0.05)$ & $20.03(0.06)$ \\
\hline \multirow[t]{4}{*}{1735} & 830.730 & 41.451 & $20.59(0.07)$ & $20.76(0.11)$ & $20.59(0.08)$ & $20.35(0.08)$ & $20.16(0.06)$ & $20.11(0.05)$ \\
\hline & & & $20.21(0.06)$ & $20.23(0.07)$ & $20.33(0.07)$ & $20.51(0.09)$ & $20.65(0.08)$ & $20.60(0.08)$ \\
\hline & & & $20.30(0.07)$ & $20.43(0.08)$ & $20.48(0.09)$ & $20.47(0.08)$ & $20.49(0.07)$ & $20.08(0.06)$ \\
\hline & & & $20.15(0.06)$ & $20.54(0.08)$ & $20.22(0.06)$ & $19.96(0.06)$ & $20.02(0.07)$ & $20.62(0.17)$ \\
\hline \multirow[t]{4}{*}{1736} & 40.346 & 627.771 & $20.72(0.06)$ & $20.33(0.09)$ & $20.60(0.08)$ & $20.76(0.12)$ & $20.11(0.04)$ & $20.15(0.05)$ \\
\hline & & & $20.43(0.07)$ & $20.59(0.08)$ & $20.36(0.06)$ & $20.20(0.05)$ & $20.31(0.06)$ & $20.33(0.05)$ \\
\hline & & & $20.83(0.08)$ & $20.65(0.05)$ & $20.22(0.05)$ & $20.24(0.06)$ & $20.15(0.07)$ & $20.07(0.06)$ \\
\hline & & & $20.11(0.06)$ & $20.07(0.07)$ & $20.13(0.05)$ & $20.20(0.06)$ & $20.42(0.07)$ & $20.12(0.18)$ \\
\hline \multirow[t]{4}{*}{1749} & 832.748 & 178.959 & $20.64(0.05)$ & $20.34(0.07)$ & $20.37(0.10)$ & $20.04(0.06)$ & $20.16(0.05)$ & $20.58(0.06)$ \\
\hline & & & $20.60(0.08)$ & $21.15(0.09)$ & $20.75(0.06)$ & $20.49(0.05)$ & $20.26(0.06)$ & $20.16(0.05)$ \\
\hline & & & $20.44(0.07)$ & $20.35(0.05)$ & $20.25(0.06)$ & $20.16(0.05)$ & $20.21(0.05)$ & $19.98(0.05)$ \\
\hline & & & $20.23(0.06)$ & $20.20(0.07)$ & $20.16(0.05)$ & $20.33(0.05)$ & $20.49(0.07)$ & $20.09(0.10)$ \\
\hline \multirow[t]{4}{*}{1776} & 337.907 & -7.156 & 99.99 (9.99) & $20.71(0.11)$ & $99.99(9.99)$ & 99.99 (9.99) & $20.53(0.07)$ & 99.99 (9.99) \\
\hline & & & $99.99(9.99)$ & 99.99 (9.99) & $99.99(9.99)$ & $20.23(0.07)$ & $21.31(0.11)$ & $20.98(0.11)$ \\
\hline & & & $19.85(0.05)$ & $19.97(0.07)$ & $19.98(0.07)$ & 99.99 (9.99) & $20.41(0.07)$ & $20.51(0.06)$ \\
\hline & & & $20.96(0.10)$ & $20.72(0.09)$ & $20.91(0.09)$ & $21.59(0.18)$ & $22.10(0.31)$ & $20.41(0.09)$ \\
\hline
\end{tabular}


Table 8. continuation.

\begin{tabular}{|c|c|c|c|c|c|c|c|c|}
\hline \multirow[t]{4}{*}{$\overline{\mathrm{F} 2 \mathrm{ID}}$} & \multirow[t]{4}{*}{$\mathrm{x}$} & \multirow[t]{4}{*}{$\mathrm{y}$} & $2 \mathrm{Ks} 10(\sigma)$ & $2 \mathrm{Ks} 01(\sigma)$ & $2 \mathrm{Ks} 02(\sigma)$ & $2 \mathrm{Ks} 03(\sigma)$ & $2 \mathrm{Ks} 05(\sigma)$ & $2 \mathrm{Ks} 06(\sigma)$ \\
\hline & & & $2 \mathrm{Ks} 07(\sigma)$ & $2 \mathrm{Ks} 08(\sigma)$ & $2 \mathrm{Ks} 09(\sigma)$ & $2 \mathrm{Ks} 11(\sigma)$ & $2 \mathrm{Ks} 12(\sigma)$ & $2 \mathrm{Ks} 13(\sigma)$ \\
\hline & & & $2 \mathrm{Ks} 14(\sigma)$ & $2 \mathrm{Ks} 15(\sigma)$ & $2 \mathrm{Ks} 16(\sigma)$ & $2 \mathrm{Ks} 17(\sigma)$ & $2 \mathrm{Ks} 18(\sigma)$ & $2 \mathrm{Ks} 19(\sigma)$ \\
\hline & & & $2 \mathrm{Ks} 20(\sigma)$ & $2 \mathrm{Ks} 21(\sigma)$ & $2 \mathrm{Ks} 22(\sigma)$ & $2 \mathrm{Ks} 23(\sigma)$ & $2 \mathrm{Ks} 24(\sigma)$ & $2 \mathrm{Ks} 04(\sigma)$ \\
\hline \multirow[t]{4}{*}{1784} & 711.917 & 559.903 & $20.47(0.06)$ & 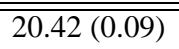 & $20.36(0.08)$ & 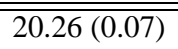 & $19.94(0.05)$ & 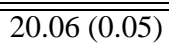 \\
\hline & & & $20.03(0.06)$ & $20.30(0.06)$ & $20.14(0.05)$ & $20.43(0.05)$ & $20.58(0.08)$ & $20.77(0.06)$ \\
\hline & & & $20.10(0.05)$ & $20.24(0.05)$ & $20.29(0.05)$ & $20.46(0.06)$ & 20.57 (0.07) & $20.67(0.07)$ \\
\hline & & & $20.56(0.08)$ & $20.68(0.10)$ & $20.54(0.06)$ & $20.63(0.06)$ & $20.38(0.06)$ & $20.31(0.13)$ \\
\hline \multirow[t]{4}{*}{1794} & 491.605 & 75.748 & $19.92(0.05)$ & $19.81(0.05)$ & $19.87(0.05)$ & $20.09(0.07)$ & $20.20(0.06)$ & $20.14(0.05)$ \\
\hline & & & $20.19(0.07)$ & $20.14(0.06)$ & $20.03(0.06)$ & $20.76(0.06)$ & $20.29(0.06)$ & $20.03(0.04)$ \\
\hline & & & $20.80(0.07)$ & $20.84(0.07)$ & $20.83(0.05)$ & $20.79(0.07)$ & $20.99(0.06)$ & $20.77(0.07)$ \\
\hline & & & $20.92(0.09)$ & $20.86(0.09)$ & $20.71(0.05)$ & $20.57(0.06)$ & $20.24(0.05)$ & $22.20(0.66)$ \\
\hline \multirow[t]{4}{*}{1795} & 405.954 & 409.103 & $20.31(0.05)$ & $20.68(0.10)$ & $20.86(0.09)$ & $20.73(0.09)$ & $20.68(0.05)$ & $20.84(0.06)$ \\
\hline & & & $20.70(0.09)$ & $20.49(0.07)$ & $20.57(0.05)$ & $20.57(0.05)$ & $20.38(0.06)$ & $20.35(0.05)$ \\
\hline & & & $19.95(0.05)$ & $19.85(0.05)$ & $20.04(0.04)$ & $20.20(0.05)$ & $20.29(0.05)$ & $20.47(0.06)$ \\
\hline & & & $20.73(0.09)$ & $20.95(0.11)$ & $21.06(0.07)$ & $20.97(0.07)$ & $20.85(0.07)$ & $20.08(0.09)$ \\
\hline \multirow[t]{4}{*}{1797} & 481.968 & -33.851 & 99.99 (9.99) & 99.99 (9.99) & 99.99 (9.99) & 99.99 (9.99) & 99.99 (9.99) & 99.99 (9.99) \\
\hline & & & 99.99 (9.99) & $99.99(9.99)$ & 99.99 (9.99) & $20.19(0.05)$ & $20.52(0.05)$ & $20.75(0.06)$ \\
\hline & & & $21.45(0.49)$ & $19.91(C$ & $20.20(0.06)$ & 99.99 (9.99) & 99.99 (9.99) & 99.99 \\
\hline & & & $20.46(0.08)$ & $20.73(0.08)$ & $20.70(0.07)$ & $21.06(0.09)$ & $20.89(0.09)$ & 20.32 \\
\hline \multirow[t]{4}{*}{1799} & 575.492 & 194.880 & $20.59(0.06)$ & $20.00(0.07)$ & $20.11(0.06)$ & $20.17(0.07)$ & $20.21(0.05)$ & $20.30(0.05)$ \\
\hline & & & $20.45(0.07)$ & $20.47(0.07)$ & $20.52(0.06)$ & $20.23(0.05)$ & $20.40(0.06)$ & $20.36(0.05)$ \\
\hline & & & $19.95(0.05)$ & $20.04(0$. & 20.18 (C & 20.36( & 20.47 & 20.63 \\
\hline & & & $20.82(0.08)$ & $20.66(0.09)$ & $20.64(0.07)$ & $20.41(0.06)$ & $20.33(0.06)$ & $20.42(0.12)$ \\
\hline \multirow[t]{4}{*}{1803} & 786.644 & 606.898 & $20.52(0.05)$ & $21.34(0.15)$ & $20.63(0.09)$ & $20.71(0.08)$ & $20.58(0.05)$ & $20.78(0.06)$ \\
\hline & & & $20.76(0.09)$ & $21.15(0.11)$ & $20.69(0.08)$ & $20.87(0.10)$ & $20.83(0.07)$ & $21.00(0.06)$ \\
\hline & & & $20.12(0.05)$ & $20.08(0.05)$ & $19.94(0.06)$ & $19.99(0.05)$ & $20.03(0.05)$ & $20.08(0.05)$ \\
\hline & & & $20.24(0.06)$ & $20.03(0.05)$ & $20.29(0.05)$ & $20.37(0.06)$ & 20.64 & $20.28(0.11)$ \\
\hline \multirow[t]{4}{*}{1804} & 605.698 & 879.856 & $20.36(0.22)$ & $19.70(0.07)$ & $21.17(0.14)$ & $20.89(0.10)$ & $19.55(1.56)$ & $21.54(0.34)$ \\
\hline & & & $19.79(0.13)$ & $22.28(0.38)$ & $21.09(0.09)$ & 99.99 (9.99) & 99.99 (9.99) & 99.99 (9.99) \\
\hline & & & 99.99 (9.99) & 99.99 (9.99) & 99.99 (9.99) & 99.99 (9.99) & 99.99 (9.99) & 99.99 (9.99) \\
\hline & & & 99.99 (9.99) & 99.99 & 99.99 & 99.99 & 99.99 & 99.99 (9.99) \\
\hline \multirow[t]{4}{*}{1805} & 359.442 & 396.001 & $20.22(0.05)$ & $20.65(0.07)$ & $21.10(0.13)$ & $20.96(0.13)$ & $20.57(0.05)$ & $20.37(0.05)$ \\
\hline & & & $20.59(0.07)$ & $20.71(0.08)$ & $20.36(0.05)$ & $20.23(0.05)$ & $20.09(0.06)$ & $20.19(0.05)$ \\
\hline & & & $20.41(0.06)$ & $20.20(0.05)$ & $20.16(0.05)$ & $20.06(0.06)$ & $20.05(0.05)$ & $20.08(0.05)$ \\
\hline & & & $20.34(0.07)$ & & $20.42(0.05)$ & & & $20.66(0.10)$ \\
\hline \multirow[t]{4}{*}{1809} & 849.471 & 740.460 & $20.46(0.05)$ & & & $20.20(0.07)$ & $20.15(0.05)$ & $20.37(0.06)$ \\
\hline & & & $20.34(0.06)$ & $20.75(0.08)$ & $20.46(0.07)$ & $20.52(0.05)$ & $20.70(0.08)$ & $20.53(0.05)$ \\
\hline & & & $20.01(0.05)$ & $19.90(0.05)$ & $19.99(0.05)$ & $20.15(0.10)$ & $20.21(0.06)$ & $20.33(0.06)$ \\
\hline & & & & & $20.61(0.07)$ & $20.45(0.06)$ & $20.62(0.07)$ & $19.94(0.15)$ \\
\hline \multirow[t]{4}{*}{1817} & 679.902 & 51.165 & $20.47(0.06)$ & $20.33(0.07)$ & $20.40(0.08)$ & $20.56(0.08)$ & $20.63(0.06)$ & $20.55(0.06)$ \\
\hline & & & $20.69(0.09)$ & $20.79(0.10)$ & $20.64(0.07)$ & $20.26(0.06)$ & $20.34(0.06)$ & $20.25(0.07)$ \\
\hline & & & $20.07(0.06)$ & $20.14(0.05)$ & $20.15(0.06)$ & $20.11(0.05)$ & $20.13(0.05)$ & $20.01(0.06)$ \\
\hline & & & 99.99 (9.99) & $20.52(0.07)$ & $20.60(0.08)$ & 99.99 (9.99) & 99.99 (9.99) & 99.99 (9.99) \\
\hline \multirow[t]{4}{*}{1820} & 780.108 & 653.670 & $20.24(0.05)$ & $20.12(0.06)$ & $20.20(0.06)$ & $20.41(0.07)$ & $20.16(0.05)$ & $20.32(0.05)$ \\
\hline & & & $20.25(0.06)$ & $20.46(0.07)$ & $20.32(0.05)$ & $20.51(0.06)$ & $20.40(0.07)$ & $20.43(0.05)$ \\
\hline & & & $20.00(0.05)$ & $20.00(0.05)$ & $20.18(0.05)$ & $20.44(0.07)$ & $20.48(0.05)$ & $20.50(0.05)$ \\
\hline & & & $20.65(0.07)$ & $20.53(0.09)$ & $20.63(0.06)$ & $20.50(0.06)$ & $20.70(0.08)$ & $20.23(0.09)$ \\
\hline \multirow[t]{4}{*}{1825} & 273.977 & 402.602 & $20.35(0.06)$ & $20.90(0.10)$ & $21.16(0.18)$ & $21.27(0.13)$ & $20.75(0.07)$ & $20.55(0.07)$ \\
\hline & & & $20.66(0.09)$ & $20.64(0.09)$ & $20.68(0.07)$ & $20.25(0.06)$ & $20.18(0.07)$ & $20.15(0.06)$ \\
\hline & & & $20.53(0.08)$ & $20.58(0.06)$ & $20.43(0.07)$ & $20.29(0.08)$ & $20.29(0.09)$ & $20.05(0.05)$ \\
\hline & & & $19.97(0.06)$ & $20.05(0.06)$ & $20.05(0.06)$ & $20.15(0.05)$ & $20.37(0.07)$ & $20.77(0.30)$ \\
\hline
\end{tabular}


Table 8. continuation.

\begin{tabular}{|c|c|c|c|c|c|c|c|c|}
\hline \multirow[t]{4}{*}{ F2 ID } & \multirow[t]{4}{*}{$\mathrm{x}$} & \multirow[t]{4}{*}{$\mathrm{y}$} & $2 \mathrm{Ks} 10(\sigma)$ & $2 \mathrm{Ks} 01(\sigma)$ & $2 \mathrm{Ks} 02(\sigma)$ & $2 \mathrm{Ks} 03(\sigma)$ & $2 \mathrm{Ks} 05(\sigma)$ & $2 \mathrm{Ks} 06(\sigma)$ \\
\hline & & & $2 \mathrm{Ks} 07(\sigma)$ & $2 \mathrm{Ks} 08(\sigma)$ & $2 \mathrm{Ks} 09(\sigma)$ & $2 \mathrm{Ks} 11(\sigma)$ & $2 \mathrm{Ks} 12(\sigma)$ & $2 \mathrm{Ks} 13(\sigma)$ \\
\hline & & & $2 \mathrm{Ks} 14(\sigma)$ & $2 \mathrm{Ks} 15(\sigma)$ & $2 \mathrm{Ks} 16(\sigma)$ & $2 \mathrm{Ks} 17(\sigma)$ & $2 \mathrm{Ks} 18(\sigma)$ & $2 \mathrm{Ks} 19(\sigma)$ \\
\hline & & & $2 \mathrm{Ks} 20(\sigma)$ & $2 \mathrm{Ks} 21(\sigma)$ & $2 \mathrm{Ks} 22(\sigma)$ & $2 \mathrm{Ks} 23(\sigma)$ & $2 \mathrm{Ks} 24(\sigma)$ & $2 \mathrm{Ks} 04(\sigma)$ \\
\hline \multirow[t]{4}{*}{1834} & 113.048 & 406.136 & $20.53(0.05)$ & $20.16(0.07)$ & $20.03(0.06)$ & $20.11(0.07)$ & $20.03(0.05)$ & $19.98(0.05)$ \\
\hline & & & $20.26(0.05)$ & $20.42(0.07)$ & $20.47(0.06)$ & $20.49(0.06)$ & $20.27(0.07)$ & $20.40(0.05)$ \\
\hline & & & $20.21(0.06)$ & $20.26(0.05)$ & $20.28(0.05)$ & $20.42(0.06)$ & $20.44(0.06)$ & $20.48(0.06)$ \\
\hline & & & $20.54(0.07)$ & $20.46(0.07)$ & $20.41(0.06)$ & $20.48(0.06)$ & $20.56(0.07)$ & $20.00(0.08)$ \\
\hline \multirow[t]{4}{*}{1837} & 359.384 & 756.684 & $20.66(0.05)$ & $21.03(0.12)$ & $21.04(0.15)$ & $21.55(0.21)$ & $20.63(0.06)$ & $21.08(0.07)$ \\
\hline & & & $21.06(0.10)$ & $20.96(0.09)$ & $20.75(0.08)$ & $20.79(0.06)$ & $20.71(0.07)$ & $20.73(0.05)$ \\
\hline & & & $19.96(0.05)$ & $19.96(0.05)$ & $19.98(0.05)$ & $20.03(0.04)$ & $20.07(0.05)$ & $20.50(0.05)$ \\
\hline & & & 20.49 (0.07) & $20.33(0.07)$ & 20.77 (0.06) & $20.87(0.08)$ & $20.85(0.07)$ & $20.41(0.14)$ \\
\hline \multirow[t]{4}{*}{1841} & 278.849 & 8.182 & $20.72(0.05)$ & $20.64(0.10)$ & $21.00(0.11)$ & $20.76(0.11)$ & $20.53(0.05)$ & $20.56(0.07)$ \\
\hline & & & $20.61(0.09)$ & $20.38(0.10)$ & $20.58(0.06)$ & $20.59(0.10)$ & $20.56(0.09)$ & $20.84(0.06)$ \\
\hline & & & $19.99(0.06)$ & $19.81(0.05)$ & $19.99(0.05)$ & $20.10(0.08)$ & $20.02(0.05)$ & $20.09(0.06)$ \\
\hline & & & $20.17(0.07)$ & $20.26(0.07)$ & $20.25(0.06)$ & $20.27(0.07)$ & $20.34(0.07)$ & $20.17(0.11)$ \\
\hline \multirow[t]{4}{*}{1852} & 219.645 & 704.799 & $21.31(0.08)$ & $21.73(0.13)$ & $20.70(0.10)$ & $20.60(0.09)$ & $21.24(0.08)$ & $21.23(0.09)$ \\
\hline & & & $21.22(0.09)$ & $21.39(0.13)$ & $21.31(0.12)$ & 20.77 (0.07) & $20.77(0.10)$ & $20.95(0.06)$ \\
\hline & & & $20.16(0.05)$ & $20.01(0.05)$ & $20.01(0.05)$ & $19.98(0.05)$ & $20.06(0.05)$ & $20.13(0.05)$ \\
\hline & & & $20.20(0.06)$ & $20.30(0.07)$ & $20.38(0.06)$ & $20.48(0.06)$ & $20.73(0.08)$ & $20.83(0.15)$ \\
\hline \multirow[t]{4}{*}{1863} & 395.169 & 623.677 & $20.50(0.05)$ & $20.46(0.09)$ & $20.76(0.08)$ & $20.81(0.10)$ & $20.87(0.07)$ & $20.69(0.06)$ \\
\hline & & & $20.86(0.08)$ & $20.89(0.08)$ & $20.64(0.06)$ & $20.36(0.05)$ & $20.19(0.06)$ & $20.05(0.05)$ \\
\hline & & & $20.62(0.07)$ & $20.54(0.06)$ & $20.45(0.05)$ & $20.25(0.05)$ & $20.14(0.05)$ & $20.08(0.05)$ \\
\hline & & & $20.06(0.06)$ & $19.86(0.06)$ & $20.15(0.05)$ & $20.22(0.05)$ & $20.49(0.07)$ & $20.55(0.16)$ \\
\hline \multirow[t]{4}{*}{1868} & 135.708 & 343.444 & $20.62(0.07)$ & $20.63(0.08)$ & $20.33(0.08)$ & $20.32(0.07)$ & $20.53(0.06)$ & $20.86(0.07)$ \\
\hline & & & $20.73(0.06)$ & $20.63(0.06)$ & $20.70(0.07)$ & $20.23(0.05)$ & $20.04(0.06)$ & $20.21(0.05)$ \\
\hline & & & $20.08(0.05)$ & $20.06(0.05)$ & $20.12(0.06)$ & $20.10(0.05)$ & $20.15(0.06)$ & $20.27(0.06)$ \\
\hline & & & $20.27(0.06)$ & $20.53(0.08)$ & $20.54(0.06)$ & $20.44(0.06)$ & $20.30(0.06)$ & $20.03(0.08)$ \\
\hline \multirow[t]{4}{*}{1870} & 843.018 & 828.104 & $20.17(0.05)$ & $20.60(0.13)$ & $20.19(0.07)$ & $20.28(0.07)$ & $20.26(0.05)$ & $20.36(0.05)$ \\
\hline & & & $20.02(0.06)$ & $20.51(0.07)$ & $20.12(0.05)$ & $20.34(0.05)$ & $20.47(0.06)$ & $20.65(0.06)$ \\
\hline & & & $20.26(0.06)$ & $20.08(0.05)$ & $20.22(0.06)$ & $20.40(0.14)$ & $20.23(0.09)$ & 20.45 (0.09) \\
\hline & & & $20.67(0.08)$ & $20.55(0.06)$ & $20.90(0.07)$ & $20.72(0.08)$ & $21.07(0.09)$ & $21.50(0.58)$ \\
\hline \multirow[t]{4}{*}{1871} & 870.020 & 231.107 & $20.70(0.06)$ & 99.99 (9.99) & $20.03(0.06)$ & $19.99(0.06)$ & $20.04(0.05)$ & $20.29(0.06)$ \\
\hline & & & $20.35(0.06)$ & $20.69(0.08)$ & $20.57(0.06)$ & $20.45(0.06)$ & $20.60(0.07)$ & $20.34(0.05)$ \\
\hline & & & $19.97(0.05)$ & $20.13(0.05)$ & $20.30(0.05)$ & $20.47(0.07)$ & $20.59(0.07)$ & $20.56(0.06)$ \\
\hline & & & $20.54(0.08)$ & $20.55(0.05)$ & $20.58(0.06)$ & $20.32(0.06)$ & $20.47(0.08)$ & $20.17(0.10)$ \\
\hline \multirow[t]{4}{*}{1880} & 847.714 & 649.539 & $20.52(0.06)$ & $20.22(0.08)$ & $20.01(0.07)$ & $20.19(0.07)$ & $20.08(0.05)$ & $20.48(0.06)$ \\
\hline & & & $20.27(0.07)$ & $20.45(0.07)$ & $20.51(0.06)$ & $20.42(0.06)$ & $20.75(0.07)$ & $20.72(0.06)$ \\
\hline & & & $20.05(0.05)$ & $20.04(0.05)$ & $20.14(0.07)$ & $20.32(0.09)$ & $20.43(0.07)$ & $20.64(0.07)$ \\
\hline & & & $20.84(0.10)$ & $20.60(0.08)$ & $20.68(0.07)$ & $20.71(0.07)$ & $20.45(0.06)$ & $20.10(0.12)$ \\
\hline \multirow[t]{4}{*}{1881} & 245.502 & 278.014 & $20.90(0.13)$ & $21.47(0.32)$ & $21.91(0.30)$ & $21.45(0.46)$ & $21.17(0.14)$ & $18.14(0.15)$ \\
\hline & & & $20.80(0.12)$ & 99.99 (9.99) & $21.15(0.23)$ & $19.54(0.21)$ & $20.09(0.18)$ & $18.73(0.14)$ \\
\hline & & & $19.84(0.28)$ & $22.41(2.07)$ & $21.70(0.12)$ & $21.20(0.18)$ & $21.05(0.18)$ & $20.69(0.26)$ \\
\hline & & & $22.13(1.08)$ & $21.51(0.41)$ & $19.36(0.10)$ & 99.99 (9.99) & $21.92(0.30)$ & $19.49(0.18)$ \\
\hline \multirow[t]{4}{*}{1882} & 512.132 & 412.280 & $20.23(0.05)$ & $20.73(0.09)$ & $20.39(0.07)$ & $20.43(0.08)$ & $20.42(0.05)$ & $20.09(0.05)$ \\
\hline & & & 20.09 (0.06) & $20.11(0.06)$ & $20.15(0.06)$ & $20.22(0.05)$ & $20.34(0.06)$ & $20.59(0.06)$ \\
\hline & & & $20.05(0.05)$ & $20.21(0.06)$ & $20.23(0.07)$ & $20.38(0.07)$ & $20.61(0.07)$ & $20.62(0.06)$ \\
\hline & & & $20.78(0.08)$ & $20.61(0.07)$ & $20.46(0.06)$ & $20.45(0.06)$ & $20.43(0.06)$ & $20.34(0.10)$ \\
\hline \multirow[t]{4}{*}{1884} & 599.866 & 473.719 & $20.32(0.05)$ & $20.15(0.07)$ & $20.54(0.09)$ & $20.73(0.10)$ & $19.97(0.05)$ & $20.03(0.05)$ \\
\hline & & & $20.13(0.07)$ & $20.48(0.07)$ & $20.24(0.05)$ & $20.32(0.05)$ & $20.27(0.06)$ & $20.21(0.05)$ \\
\hline & & & $21.25(0.09)$ & $21.30(0.07)$ & $21.21(0.07)$ & $21.44(0.10)$ & $21.47(0.14)$ & $21.32(0.10)$ \\
\hline & & & $21.18(0.10)$ & $21.20(0.13)$ & $20.64(0.06)$ & $20.55(0.06)$ & $20.43(0.06)$ & $20.53(0.11)$ \\
\hline
\end{tabular}


Table 8. continuation.

\begin{tabular}{|c|c|c|c|c|c|c|c|c|}
\hline \multirow[t]{4}{*}{ F2 ID } & \multirow[t]{4}{*}{$\mathrm{x}$} & \multirow[t]{4}{*}{$y$} & $2 \mathrm{Ks} 10(\sigma)$ & $2 \mathrm{Ks} 01(\sigma)$ & $2 \mathrm{Ks} 02(\sigma)$ & $2 \mathrm{Ks} 03(\sigma)$ & $2 \mathrm{Ks} 05(\sigma)$ & $2 \mathrm{Ks} 06(\sigma)$ \\
\hline & & & $2 \mathrm{Ks} 07(\sigma)$ & $2 \mathrm{Ks} 08(\sigma)$ & $2 \mathrm{Ks} 09(\sigma)$ & $2 \mathrm{Ks} 11(\sigma)$ & $2 \mathrm{Ks} 12(\sigma)$ & $2 \mathrm{Ks} 13(\sigma)$ \\
\hline & & & $2 \mathrm{Ks} 14(\sigma)$ & $2 \mathrm{Ks} 15(\sigma)$ & $2 \mathrm{Ks} 16(\sigma)$ & $2 \mathrm{Ks} 17(\sigma)$ & $2 \mathrm{Ks} 18(\sigma)$ & $2 \mathrm{Ks} 19(\sigma)$ \\
\hline & & & $2 \mathrm{Ks} 20(\sigma)$ & $2 \mathrm{Ks} 21(\sigma)$ & $2 \mathrm{Ks} 22(\sigma)$ & $2 \mathrm{Ks} 23(\sigma)$ & $2 \mathrm{Ks} 24(\sigma)$ & $2 \mathrm{Ks} 04(\sigma)$ \\
\hline \multirow[t]{4}{*}{1909} & 506.830 & 444.480 & $20.18(0.05)$ & 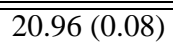 & 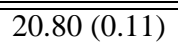 & $\bar{~} 21.13(0.09)$ & "20.64(0.06) & 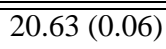 \\
\hline & & & $20.46(0.07)$ & $20.58(0.07)$ & $20.39(0.06)$ & $20.12(0.05)$ & $19.97(0.05)$ & $20.17(0.06)$ \\
\hline & & & $20.43(0.06)$ & $20.30(0.05)$ & $20.28(0.06)$ & $20.16(0.07)$ & $20.20(0.06)$ & $20.21(0.05)$ \\
\hline & & & $20.19(0.06)$ & $20.17(0.07)$ & $20.37(0.06)$ & $20.63(0.06)$ & $20.78(0.08)$ & $20.78(0.11)$ \\
\hline \multirow[t]{4}{*}{1914} & 554.137 & 80.880 & $20.16(0.05)$ & $20.78(0.13)$ & $20.88(0.10)$ & $20.83(0.11)$ & $20.55(0.05)$ & $20.65(0.06)$ \\
\hline & & & $20.57(0.09)$ & $20.57(0.07)$ & $20.37(0.06)$ & $20.54(0.06)$ & $20.27(0.05)$ & $20.12(0.05)$ \\
\hline & & & $20.65(0.07)$ & $20.48(0.05)$ & $20.51(0.07)$ & $20.49(0.06)$ & $20.41(0.05)$ & $20.20(0.06)$ \\
\hline & & & $20.12(0.07)$ & $20.12(0.06)$ & $20.11(0.05)$ & $20.10(0.06)$ & $20.15(0.06)$ & $20.78(0.15)$ \\
\hline \multirow[t]{4}{*}{1915} & 598.682 & 641.232 & $20.69(0.06)$ & $20.29(0.08)$ & $20.14(0.06)$ & $20.18(0.07)$ & $20.02(0.05)$ & $20.19(0.05)$ \\
\hline & & & $20.13(0.06)$ & $20.62(0.07)$ & $20.44(0.06)$ & $20.48(0.06)$ & $20.81(0.08)$ & $20.70(0.06)$ \\
\hline & & & $20.16(0.05)$ & $20.17(0.04)$ & $20.21(0.05)$ & $20.37(0.05)$ & $20.43(0.06)$ & $20.71(0.07)$ \\
\hline & & & $20.84(0.10)$ & $20.72(0.11)$ & $20.86(0.06)$ & $21.08(0.07)$ & $20.98(0.08)$ & $20.48(0.10)$ \\
\hline \multirow[t]{4}{*}{1917} & 583.137 & 658.359 & $20.58(0.05)$ & $20.40(0.08)$ & $20.40(0.09)$ & $20.55(0.11)$ & $20.62(0.06)$ & $20.89(0.06)$ \\
\hline & & & $20.49(0.07)$ & $21.43(0.16)$ & $20.56(0.06)$ & $21.17(0.09)$ & $21.01(0.09)$ & $20.89(0.06)$ \\
\hline & & & $20.02(0.05)$ & $20.04(0.05)$ & $20.00(0.05)$ & $20.10(0.05)$ & $20.13(0.06)$ & $20.21(0.05)$ \\
\hline & & & $20.50(0.06)$ & $20.35(0.06)$ & $20.75(0.06)$ & $20.51(0.06)$ & $20.78(0.08)$ & $20.89(0.16)$ \\
\hline \multirow[t]{4}{*}{1922} & 54.686 & 283.433 & $20.28(0.05)$ & $20.47(0.07)$ & $20.43(0.09)$ & $20.40(0.08)$ & $20.42(0.05)$ & $20.01(0.05)$ \\
\hline & & & $20.29(0.05)$ & $20.66(0.08)$ & $20.26(0.06)$ & $20.29(0.05)$ & $20.28(0.06)$ & $20.56(0.06)$ \\
\hline & & & $20.19(0.06)$ & $20.08(0.05)$ & $20.14(0.05)$ & $20.28(0.08)$ & $20.22(0.05)$ & $20.60(0.06)$ \\
\hline & & & $20.54(0.08)$ & $20.59(0.08)$ & $20.79(0.06)$ & $20.88(0.06)$ & $20.81(0.08)$ & $20.62(0.12)$ \\
\hline \multirow[t]{4}{*}{1936} & 421.521 & 282.357 & $20.90(0.06)$ & $19.75(0.07)$ & $19.71(0.05)$ & $19.78(0.07)$ & $21.07(0.06)$ & $20.85(0.06)$ \\
\hline & & & $20.97(0.09)$ & $20.97(0.08)$ & $21.09(0.08)$ & $19.80(0.05)$ & $19.99(0.05)$ & $20.23(0.05)$ \\
\hline & & & $20.89(0.08)$ & $21.07(0.08)$ & $21.43(0.11)$ & $21.46(0.11)$ & $21.26(0.09)$ & $21.30(0.10)$ \\
\hline & & & $20.95(0.09)$ & $21.49(0.16)$ & $20.87(0.06)$ & $20.64(0.0$ & $20.35(0.06)$ & $20.61(0.11)$ \\
\hline \multirow[t]{4}{*}{1952} & 683.717 & 606.454 & $20.67(0.05)$ & $20.23(0.06)$ & $20.28(0.07)$ & $20.36(0.09)$ & $20.66(0.06)$ & $20.70(0.06)$ \\
\hline & & & $20.78(0.09)$ & $20.81(0.09)$ & $20.66(0.06)$ & $20.44(0.05)$ & $20.21(0.05)$ & $20.13(0.04)$ \\
\hline & & & $20.52(0.06)$ & $20.32(0.05)$ & $20.21(0.05)$ & $20.18(0.06)$ & $20.22(0.05)$ & $20.26(0.05)$ \\
\hline & & & $20.28(0.07)$ & $20.24(0.07)$ & $20.67(0.06)$ & $20.81(0.07)$ & $20.72(0.07)$ & $20.33(0.13)$ \\
\hline \multirow[t]{4}{*}{1954} & 430.979 & 26.005 & $20.09(0.06)$ & $20.79(0.10)$ & $20.84(0.11)$ & $20.92(0.09)$ & $20.48(0.05)$ & $20.31(0.05)$ \\
\hline & & & $20.13(0.05)$ & $20.24(0.06)$ & $20.08(0.05)$ & $20.08(0.05)$ & $20.42(0.06)$ & $20.80(0.07)$ \\
\hline & & & $20.12(0.05)$ & $20.20(0.05)$ & $20.56(0.06)$ & $20.60(0.06)$ & $20.65(0.06)$ & $20.52(0.07)$ \\
\hline & & & $20.59(0.08)$ & $20.67(0.10)$ & $20.42(0.06)$ & $20.47(0.06)$ & $20.55(0.07)$ & $20.40(0.15)$ \\
\hline \multirow[t]{4}{*}{1955} & 293.224 & 671.359 & $20.57(0.05)$ & $21.06(0.09)$ & $21.05(0.10)$ & $20.76(0.0$ & 20.10 & $20.37(0.05)$ \\
\hline & & & $20.36(0.06)$ & $20.50(0.08)$ & $20.42(0.06)$ & $19.94(0.05)$ & $19.98(0.06)$ & $19.95(0.05)$ \\
\hline & & & $21.00(0.08)$ & $21.05(0.07)$ & $21.04(0.08)$ & $21.09(0.06)$ & $21.09(0.06)$ & $21.19(0.09)$ \\
\hline & & & $21.03(0.10)$ & $21.23(0.10)$ & $20.81(0.06)$ & $20.54(0.06)$ & $20.41(0.06)$ & $19.99(0.06)$ \\
\hline \multirow[t]{4}{*}{1956} & 740.211 & 45.215 & $20.09(0.05)$ & $20.77(0.09)$ & $20.92(0.12)$ & $21.04(0.10)$ & $20.84(0.06)$ & $20.58(0.07)$ \\
\hline & & & $20.45(0.08)$ & $20.40(0.07)$ & $20.18(0.05)$ & $20.15(0.05)$ & $20.18(0.05)$ & $20.25(0.05)$ \\
\hline & & & $20.59(0.07)$ & $20.33(0.05)$ & $20.28(0.06)$ & $20.26(0.05)$ & $20.37(0.05)$ & $20.30(0.06)$ \\
\hline & & & $20.54(0.08)$ & $20.36(0.06)$ & $20.62(0.06)$ & $20.86(0.08)$ & $21.30(0.13)$ & $20.82(0.18)$ \\
\hline \multirow[t]{4}{*}{1979} & 697.548 & 522.166 & 99.99 (9.99) & 99.99 (9.99) & 99.99 (9.99) & $23.13(0.62)$ & $99.99(9.99)$ & $19.52(0.07)$ \\
\hline & & & 99.99 (9.99) & 99.99 (9.99) & 99.99 (9.99) & $21.09(0.16)$ & $22.67(0.51)$ & $20.71(0.19)$ \\
\hline & & & 99.99 (9.99) & $21.43(0.11)$ & 99.99 (9.99) & $24.24(1.32)$ & 99.99 (9.99) & $22.55(0.27)$ \\
\hline & & & $21.94(0.27)$ & 99.99 (9.99) & $21.29(0.12)$ & 99.99 (9.99) & $25.51(3.41)$ & $21.86(0.70)$ \\
\hline \multirow[t]{4}{*}{1983} & 77.576 & 340.094 & $20.29(0.05)$ & $20.44(0.09)$ & $20.41(0.08)$ & $20.43(0.06)$ & $20.51(0.05)$ & $20.33(0.06)$ \\
\hline & & & $20.37(0.07)$ & $20.48(0.07)$ & $20.36(0.06)$ & $20.99(0.07)$ & $20.85(0.10)$ & $20.67(0.06)$ \\
\hline & & & $20.02(0.05)$ & $19.99(0.05)$ & $20.10(0.05)$ & $20.20(0.05)$ & $20.30(0.06)$ & $20.51(0.06)$ \\
\hline & & & $20.60(0.08)$ & $20.65(0.07)$ & $20.72(0.07)$ & $20.56(0.07)$ & $20.61(0.07)$ & $19.99(0.07)$ \\
\hline
\end{tabular}


Table 8. continuation.

\begin{tabular}{|c|c|c|c|c|c|c|c|c|}
\hline \multirow[t]{4}{*}{ F2 ID } & \multirow[t]{4}{*}{$\mathrm{x}$} & \multirow[t]{4}{*}{$\mathrm{y}$} & $2 \mathrm{Ks} 10(\sigma)$ & 2Ks01 $(\sigma)$ & $2 \mathrm{Ks} 02(\sigma)$ & $2 \mathrm{Ks} 03(\sigma)$ & $2 \mathrm{Ks} 05(\sigma)$ & $2 \mathrm{Ks} 06(\sigma)$ \\
\hline & & & $2 \mathrm{Ks} 07(\sigma)$ & $2 \mathrm{Ks} 08(\sigma)$ & $2 \mathrm{Ks} 09(\sigma)$ & $2 \mathrm{Ks} 11(\sigma)$ & $2 \mathrm{Ks} 12(\sigma)$ & $2 \mathrm{Ks} 13(\sigma)$ \\
\hline & & & $2 \mathrm{Ks} 14(\sigma)$ & $2 \mathrm{Ks} 15(\sigma)$ & $2 \mathrm{Ks} 16(\sigma)$ & $2 \mathrm{Ks} 17(\sigma)$ & $2 \mathrm{Ks} 18(\sigma)$ & $2 \mathrm{Ks} 19(\sigma)$ \\
\hline & & & $2 \mathrm{Ks} 20(\sigma)$ & $2 \mathrm{Ks} 21(\sigma)$ & $2 \mathrm{Ks} 22(\sigma)$ & $2 \mathrm{Ks} 23(\sigma)$ & $2 \mathrm{Ks} 24(\sigma)$ & $2 \mathrm{Ks} 04(\sigma)$ \\
\hline \multirow[t]{4}{*}{1997} & 724.672 & 477.335 & $20.14(0.05)$ & 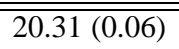 & 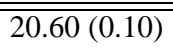 & $20.44(0.08)$ & 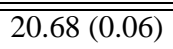 & $20.46(0.05)$ \\
\hline & & & $20.38(0.07)$ & $20.25(0.06)$ & $20.25(0.06)$ & 21.57 (0.09) & $21.23(0.10)$ & $21.02(0.08)$ \\
\hline & & & $20.05(0.06)$ & $20.13(0.05)$ & $20.19(0.05)$ & $20.30(0.06)$ & $20.39(0.05)$ & $20.47(0.05)$ \\
\hline & & & $20.51(0.07)$ & $20.67(0.08)$ & $20.81(0.07)$ & $21.05(0.09)$ & $20.89(0.08)$ & $21.49(0.33)$ \\
\hline \multirow[t]{4}{*}{1999} & 671.946 & 537.017 & $20.38(0.08)$ & $20.65(0.10)$ & $20.66(0.09)$ & $20.69(0.13)$ & $20.16(0.07)$ & $20.01(0.05)$ \\
\hline & & & $19.95(0.07)$ & $20.22(0.07)$ & $20.11(0.07)$ & $20.41(0.06)$ & $20.69(0.09)$ & $20.55(0.08)$ \\
\hline & & & $20.29(0.06)$ & $20.60(0.07)$ & $20.73(0.12)$ & $20.74(0.09)$ & $20.71(0.09)$ & $20.60(0.07)$ \\
\hline & & & $20.58(0.07)$ & $20.51(0.10)$ & $20.47(0.08)$ & $20.57(0.07)$ & $20.42(0.06)$ & $20.26(0.11)$ \\
\hline \multirow[t]{4}{*}{2002} & 108.682 & 335.946 & $20.38(0.05)$ & $21.25(0.09)$ & $21.00(0.16)$ & $20.55(0.09)$ & $20.14(0.06)$ & $19.93(0.05)$ \\
\hline & & & $20.11(0.06)$ & $20.16(0.06)$ & $20.32(0.06)$ & $20.58(0.06)$ & $20.57(0.07)$ & $20.81(0.07)$ \\
\hline & & & $20.24(0.06)$ & $20.29(0.05)$ & $20.43(0.07)$ & $20.61(0.07)$ & $20.51(0.07)$ & $20.55(0.07)$ \\
\hline & & & $20.61(0.09)$ & 20.57 (0.09) & $20.52(0.07)$ & $20.42(0.06)$ & $20.15(0.06)$ & $20.50(0.15)$ \\
\hline \multirow[t]{4}{*}{2006} & 810.785 & 406.280 & $20.08(0.05)$ & $20.91(0.12)$ & $20.98(0.12)$ & $20.89(0.12)$ & $20.65(0.06)$ & $20.22(0.06)$ \\
\hline & & & $20.20(0.07)$ & $20.32(0.06)$ & $20.13(0.05)$ & $20.28(0.05)$ & $20.30(0.06)$ & $20.48(0.05)$ \\
\hline & & & $20.10(0.06)$ & $20.18(0.05)$ & $20.15(0.05)$ & $20.30(0.05)$ & $20.43(0.05)$ & $20.38(0.05)$ \\
\hline & & & $20.66(0.09)$ & $20.73(0.07)$ & $20.81(0.05)$ & $20.86(0.07)$ & $20.63(0.07)$ & $20.66(0.15)$ \\
\hline \multirow[t]{4}{*}{2010} & 275.337 & 130.635 & $20.43(0.06)$ & $20.31(0.06)$ & $20.69(0.06)$ & $20.55(0.08)$ & $20.59(0.05)$ & $20.62(0.06)$ \\
\hline & & & $20.63(0.07)$ & $20.59(0.07)$ & $20.62(0.06)$ & $20.42(0.05)$ & $20.19(0.06)$ & $20.31(0.05)$ \\
\hline & & & $20.45(0.06)$ & $20.41(0.05)$ & $20.31(0.05)$ & $20.21(C$ & 20.07 & $.05)$ \\
\hline & & & $20.07(0.06)$ & $20.09(0.06)$ & $20.24(0.06)$ & $20.54(0.06)$ & $20.59(0.07)$ & $20.62(0.12)$ \\
\hline \multirow[t]{4}{*}{2011} & 165.858 & 722.627 & $20.52(0.05)$ & $20.15(0.05)$ & $20.21(0.07)$ & $20.32(0.07)$ & $20.16(0.05)$ & $20.40(0.05)$ \\
\hline & & & $20.33(0.06)$ & $20.63(0.07)$ & $20.46(0.06)$ & $20.56(0.06)$ & $20.66(0.08)$ & $20.73(0.05)$ \\
\hline & & & $20.00(0.05)$ & $20.18(0.05)$ & $20.16(0.05)$ & $20.26(0.05)$ & $20.34(0.06)$ & $20.44(0.06)$ \\
\hline & & & $20.67(0.08)$ & $20.63(0.11)$ & $20.73(0.06)$ & $20.77(0.06)$ & $20.71(0.08)$ & $20.01(0.10)$ \\
\hline \multirow[t]{4}{*}{2016} & 152.743 & 576.538 & $20.45(0.05)$ & $20.71(0.08)$ & $20.47(0.07)$ & $20.47(0.08)$ & $20.23(0.05)$ & $20.23(0.05)$ \\
\hline & & & $20.30(0.06)$ & $20.50(0.09)$ & $20.29(0.06)$ & $20.57(0.06)$ & $20.63(0.07)$ & $20.72(0.05)$ \\
\hline & & & $20.34(0.06)$ & $20.19(0.05)$ & $20.05(0.05)$ & $20.07(0.05)$ & $20.13(0.06)$ & $20.34(0.06)$ \\
\hline & & & $20.62(0.08)$ & $20.43(0.06)$ & $20.90(0.07)$ & 21.10 & $21.14(0.1$ & 21.07 \\
\hline \multirow[t]{4}{*}{2023} & 729.523 & -31.503 & 99.99 (9.99) & 99.99 (9.99) & 99.99 (9.99) & 99.99 (9.99) & 99.99 (9.99) & 99.99 (9.99) \\
\hline & & & 99.99 (9.99) & 99.99 (9.99) & 99.99 (9.99) & $21.41(0.09)$ & $21.31(0.11)$ & $21.31(0.13)$ \\
\hline & & & $20.94(0.09)$ & $21.16(0.09)$ & $21.46(0.14)$ & 99.99 (9.99) & $21.87(0.21)$ & 99.99 (9.99) \\
\hline & & & & $22.48(0.33)$ & 99.99 (9.99) & $19.97(0.05)$ & $19.94(0.05)$ & 99.99 (9.99) \\
\hline \multirow[t]{4}{*}{2028} & 207.790 & 519.523 & $20.32(0.05)$ & $20.59(0.07)$ & $20.90(0.16)$ & $20.90(0.09)$ & $20.70(0.06)$ & $20.54(0.06)$ \\
\hline & & & $20.68(0.09)$ & $20.73(0.10)$ & $20.46(0.06)$ & $20.19(0.05)$ & $20.18(0.05)$ & $20.12(0.05)$ \\
\hline & & & $20.64(0.07)$ & $20.54(0.05)$ & $20.52(0.05)$ & $20.39(0.05)$ & $20.41(0.06)$ & $20.26(0.05)$ \\
\hline & & & $20.23(0.06)$ & $20.15(0.07)$ & $20.17(0.05)$ & $20.33(0.05)$ & $20.36(0.07)$ & $20.85(0.16)$ \\
\hline \multirow[t]{4}{*}{2042} & 462.821 & 421.725 & $20.76(0.06)$ & $19.97(0.10)$ & $20.17(0.07)$ & $20.37(0.08)$ & $20.17(0.06)$ & $20.37(0.06)$ \\
\hline & & & $20.37(0.06)$ & $20.53(0.07)$ & $20.65(0.06)$ & $20.51(0.07)$ & $20.57(0.08)$ & $20.56(0.06)$ \\
\hline & & & $19.98(0.05)$ & $20.09(0.05)$ & $20.16(0.05)$ & $20.30(0.06)$ & $20.40(0.05)$ & $20.52(0.06)$ \\
\hline & & & $20.79(0.09)$ & $20.56(0.08)$ & $20.53(0.06)$ & $20.41(0.06)$ & $20.29(0.07)$ & $19.91(0.08)$ \\
\hline \multirow[t]{4}{*}{2043} & 375.293 & 718.491 & $20.88(0.05)$ & $20.46(0.09)$ & $20.69(0.08)$ & $20.87(0.09)$ & $20.28(0.05)$ & $20.72(0.07)$ \\
\hline & & & $20.60(0.07)$ & $20.90(0.10)$ & $20.80(0.07)$ & $20.49(0.05)$ & $20.56(0.08)$ & $20.68(0.05)$ \\
\hline & & & $20.07(0.05)$ & $20.20(0.05)$ & $20.08(0.05)$ & $20.07(0.05)$ & $20.11(0.05)$ & $20.30(0.05)$ \\
\hline & & & $20.45(0.07)$ & $20.35(0.07)$ & $20.59(0.06)$ & $20.70(0.07)$ & $21.00(0.11)$ & $20.12(0.08)$ \\
\hline \multirow[t]{4}{*}{2047} & 404.297 & 72.172 & $20.41(0.05)$ & $20.68(0.08)$ & $20.72(0.10)$ & $20.77(0.08)$ & $20.57(0.06)$ & $20.58(0.06)$ \\
\hline & & & $20.35(0.07)$ & $20.22(0.06)$ & $20.31(0.06)$ & $20.23(0.05)$ & $20.25(0.06)$ & $20.73(0.05)$ \\
\hline & & & $20.25(0.06)$ & $20.10(0.05)$ & $20.14(0.05)$ & $20.21(0.05)$ & $20.25(0.05)$ & $20.31(0.05)$ \\
\hline & & & $20.59(0.08)$ & $20.56(0.09)$ & $20.89(0.07)$ & $20.74(0.08)$ & $20.86(0.08)$ & $20.79(0.15)$ \\
\hline
\end{tabular}


Table 8. continuation.

\begin{tabular}{|c|c|c|c|c|c|c|c|c|}
\hline \multirow[t]{4}{*}{$\overline{\mathrm{F} 2 \mathrm{ID}}$} & \multirow[t]{4}{*}{$\mathrm{x}$} & \multirow[t]{4}{*}{$\mathrm{y}$} & $2 \mathrm{Ks} 10(\sigma)$ & $2 \mathrm{Ks} 01(\sigma)$ & $2 \mathrm{Ks} 02(\sigma)$ & $2 \mathrm{Ks} 03(\sigma)$ & $2 \mathrm{Ks} 05(\sigma)$ & $2 \mathrm{Ks06}(\sigma)$ \\
\hline & & & $2 \mathrm{Ks} 07(\sigma)$ & $2 \mathrm{Ks} 08(\sigma)$ & $2 \mathrm{Ks} 09(\sigma)$ & $2 \mathrm{Ks} 11(\sigma)$ & $2 \mathrm{Ks} 12(\sigma)$ & $2 \mathrm{Ks} 13(\sigma)$ \\
\hline & & & $2 \mathrm{Ks} 14(\sigma)$ & $2 \mathrm{Ks} 15(\sigma)$ & $2 \mathrm{Ks} 16(\sigma)$ & $2 \mathrm{Ks} 17(\sigma)$ & $2 \mathrm{Ks} 18(\sigma)$ & $2 \mathrm{Ks} 19(\sigma)$ \\
\hline & & & $2 \mathrm{Ks} 20(\sigma)$ & $2 \mathrm{Ks} 21(\sigma)$ & $2 \mathrm{Ks} 22(\sigma)$ & $2 \mathrm{Ks} 23(\sigma)$ & $2 \mathrm{Ks} 24(\sigma)$ & $2 \mathrm{Ks} 04(\sigma)$ \\
\hline \multirow[t]{4}{*}{2053} & \multirow[t]{4}{*}{200.940} & \multirow[t]{4}{*}{$\overline{249.853}$} & $20.31(0.05)$ & $20.77(0.10)$ & $20.74(0.09)$ & $20.74(0.07)$ & $20.52(0.05)$ & $20.29(0.05)$ \\
\hline & & & $20.43(0.06)$ & $20.39(0.07)$ & $20.39(0.05)$ & $20.50(0.05)$ & $20.48(0.06)$ & $20.61(0.05)$ \\
\hline & & & $20.14(0.05)$ & $20.10(0.05)$ & $20.13(0.05)$ & $20.20(0.05)$ & $20.22(0.05)$ & $20.49(0.05)$ \\
\hline & & & $20.65(0.09)$ & $20.58(0.06)$ & $20.83(0.07)$ & $20.72(0.06)$ & $20.67(0.07)$ & $20.49(0.10)$ \\
\hline \multirow[t]{4}{*}{2089} & \multirow[t]{4}{*}{756.694} & \multirow[t]{4}{*}{173.340} & $20.49(0.05)$ & $20.28(0.08)$ & $20.34(0.08)$ & $20.99(0.12)$ & $20.57(0.06)$ & $20.47(0.06)$ \\
\hline & & & $20.71(0.08)$ & $20.68(0.07)$ & $20.64(0.06)$ & $20.68(0.07)$ & $20.57(0.07)$ & $20.30(0.05)$ \\
\hline & & & $20.49(0.07)$ & $20.62(0.06)$ & $20.63(0.06)$ & $20.50(0.05)$ & $20.50(0.05)$ & $20.10(0.06)$ \\
\hline & & & $20.09(0.06)$ & $20.15(0.06)$ & $20.04(0.06)$ & $19.89(0.05)$ & $20.07(0.05)$ & $20.34(0.13)$ \\
\hline \multirow[t]{4}{*}{2097} & \multirow[t]{4}{*}{189.588} & \multirow[t]{4}{*}{266.447} & $20.95(0.05)$ & $20.17(0.06)$ & $20.20(0.07)$ & $20.94(0.11)$ & $20.46(0.06)$ & $20.94(0.07)$ \\
\hline & & & $20.83(0.07)$ & $21.79(0.21)$ & $21.03(0.09)$ & $20.74(0.05)$ & $20.78(0.08)$ & $20.66(0.05)$ \\
\hline & & & $19.88(0.05)$ & $19.87(0.05)$ & $19.99(0.05)$ & $20.10(0.05)$ & $20.19(0.05)$ & $20.48(0.05)$ \\
\hline & & & $20.83(0.09)$ & $20.64(0.07)$ & $20.83(0.06)$ & $20.66(0.06)$ & $20.73(0.07)$ & $20.15(0.10)$ \\
\hline \multirow[t]{4}{*}{2100} & \multirow[t]{4}{*}{556.260} & \multirow[t]{4}{*}{252.061} & $20.46(0.06)$ & $20.36(0.08)$ & $20.30(0.09)$ & $20.18(0.08)$ & $20.94(0.06)$ & $20.82(0.06)$ \\
\hline & & & $20.82(0.09)$ & $21.11(0.09)$ & $20.79(0.09)$ & $20.61(0.06)$ & $20.60(0.09)$ & $20.58(0.05)$ \\
\hline & & & $19.89(0.05)$ & $20.03(0.05)$ & $20.12(0.04)$ & $20.23(0.06)$ & $20.27(0.05)$ & $20.55(0.06)$ \\
\hline & & & $20.83(0.10)$ & $20.58(0.07)$ & $20.73(0.05)$ & $20.82(0.07)$ & $20.96(0.09)$ & $20.82(0.15)$ \\
\hline \multirow[t]{4}{*}{2104} & \multirow[t]{4}{*}{213.147} & \multirow[t]{4}{*}{364.457} & $20.24(0.05)$ & $20.67(0.12)$ & $20.69(0.09)$ & $20.76(0.09)$ & $20.90(0.06)$ & $20.66(0.06)$ \\
\hline & & & $20.68(0.08)$ & $20.52(0.09)$ & $20.41(0.05)$ & $20.12(0.05)$ & $20.15(0.06)$ & $20.47(0.05)$ \\
\hline & & & $20.09(0.05)$ & $20.12(0.05)$ & $20.23(0.05)$ & $20.32(0.05)$ & $20.35(0.05)$ & $20.71(0.07)$ \\
\hline & & & $20.68(0.09)$ & $20.84(0.09)$ & $20.79(0.07)$ & $20.63(0.07)$ & $20.72(0.08)$ & $21.16(0.20)$ \\
\hline 2109 & 827.857 & 18.619 & $20.38(0.06)$ & $20.28(0.07)$ & $20.27(0.09)$ & $20.24(0.07)$ & $20.49(0.05)$ & $20.65(0.06)$ \\
\hline & & & $20.68(0.08)$ & $20.48(0.08)$ & $20.55(0.06)$ & $20.25(0.06)$ & $20.64(0.07)$ & $20.07(0.06)$ \\
\hline & & & $20.29(0.06)$ & $20.45(0.06)$ & $20.51(0.07)$ & $20.64(0.07)$ & $20.71(0.06)$ & $20.23(0.06)$ \\
\hline & & & $20.08(0.06)$ & $20.47(0.06)$ & $20.15(0.05)$ & $20.23(0.06)$ & $20.23(0.07)$ & $20.13(0.18)$ \\
\hline 2111 & 440.356 & 523.504 & $20.35(0.05)$ & $20.82(0.11)$ & $21.03(0.12)$ & $21.34(0.13)$ & $20.50(0.05)$ & $20.34(0.06)$ \\
\hline & & & $20.49(0.08)$ & $20.43(0.07)$ & $20.44(0.06)$ & $20.57(0.08)$ & $20.42(0.07)$ & $20.31(0.05)$ \\
\hline & & & $20.70(0.08)$ & $20.68(0.06)$ & $20.77(0.05)$ & $20.58(0.08)$ & $20.61(0.06)$ & $20.38(0.06)$ \\
\hline & & & $20.06(0.06)$ & $20.21(0.06)$ & $20.13(0.05)$ & $20.02(0.05)$ & $19.97(0.05)$ & $20.52(0.16)$ \\
\hline 2112 & 296.300 & 208.554 & $20.35(0.05)$ & $20.74(0.08)$ & $20.73(0.09)$ & $20.78(0.09)$ & $20.31(0.05)$ & $20.15(0.05)$ \\
\hline & & & $20.29(0.06)$ & $20.20(0.07)$ & $20.22(0.05)$ & $20.31(0.05)$ & $20.54(0.07)$ & $20.74(0.06)$ \\
\hline & & & $20.22(0.06)$ & $20.23(0.05)$ & $20.36(0.06)$ & $20.44(0.05)$ & $20.46(0.05)$ & $20.57(0.06)$ \\
\hline & & & $20.52(0.07)$ & $20.66(0.08)$ & $20.66(0.07)$ & $20.73(0.07)$ & $20.62(0.08)$ & $20.79(0.13)$ \\
\hline 2115 & 468.654 & 668.942 & $20.27(0.05)$ & $20.81(0.11)$ & $20.86(0.14)$ & $20.41(0.07)$ & $20.57(0.05)$ & $20.32(0.05)$ \\
\hline & & & $20.37(0.07)$ & $20.43(0.07)$ & $20.20(0.05)$ & $20.20(0.05)$ & $20.52(0.06)$ & $20.80(0.06)$ \\
\hline & & & $20.22(0.06)$ & $20.25(0.05)$ & $20.19(0.05)$ & $20.23(0.05)$ & $20.42(0.05)$ & $20.62(0.05)$ \\
\hline & & & $20.87(0.10)$ & $20.83(0.09)$ & $20.89(0.07)$ & $20.92(0.08)$ & $20.82(0.08)$ & $21.14(0.11)$ \\
\hline 2116 & 698.278 & 147.312 & $20.59(0.05)$ & $20.21(0.08)$ & $20.83(0.08)$ & $20.75(0.09)$ & $20.68(0.06)$ & $20.92(0.07)$ \\
\hline & & & $20.80(0.07)$ & $20.63(0.08)$ & $20.65(0.06)$ & $20.26(0.05)$ & $20.18(0.06)$ & $20.10(0.05)$ \\
\hline & & & $20.46(0.05)$ & $20.41(0.05)$ & $20.30(0.05)$ & $20.22(0.05)$ & $20.26(0.06)$ & $20.16(0.05)$ \\
\hline & & & $20.27(0.06)$ & $20.15(0.06)$ & $20.50(0.05)$ & $20.60(0.06)$ & $20.57(0.07)$ & $21.20(0.24)$ \\
\hline 2127 & 520.114 & 847.988 & $20.71(0.06)$ & $20.67(0.13)$ & $21.17(0.10)$ & $20.60(0.18)$ & $20.31(0.08)$ & $19.96(0.07)$ \\
\hline & & & $20.77(0.07)$ & $20.61(0.09)$ & $20.18(0.06)$ & $20.85(0.07)$ & $20.39(0.09)$ & $20.07(0.09)$ \\
\hline & & & $21.47(0.10)$ & $20.83(0.12)$ & $20.93(0.16)$ & $20.41(0.11)$ & $20.25(0.06)$ & $20.33(0.05)$ \\
\hline & & & $20.25(0.06)$ & $20.28(0.08)$ & $20.15(0.05)$ & $20.04(0.12)$ & $99.99(9.99)$ & $20.35(0.11)$ \\
\hline 2128 & 651.229 & 824.181 & $20.57(0.05)$ & $20.11(0.10)$ & $20.03(0.06)$ & $19.98(0.08)$ & $20.06(0.06)$ & $20.33(0.05)$ \\
\hline & & & $20.54(0.08)$ & $20.58(0.07)$ & $20.52(0.06)$ & $20.43(0.05)$ & $20.57(0.09)$ & $20.60(0.06)$ \\
\hline & & & $20.66(0.05)$ & $20.67(0.06)$ & $20.75(0.06)$ & $20.73(0.09)$ & $20.72(0.07)$ & $20.68(0.06)$ \\
\hline & & & $20.33(0.08)$ & $20.46(0.08)$ & $20.14(0.05)$ & $20.07(0.05)$ & $20.07(0.06)$ & $20.03(0.10)$ \\
\hline
\end{tabular}


Table 8. continuation.

\begin{tabular}{|c|c|c|c|c|c|c|c|c|}
\hline \multirow[t]{4}{*}{ F2 ID } & \multirow[t]{4}{*}{$\mathrm{x}$} & \multirow[t]{4}{*}{$\mathrm{y}$} & $2 \mathrm{Ks} 10(\sigma)$ & $\overline{2 \mathrm{Ks} 01(\sigma)}$ & $2 \mathrm{Ks} 02(\sigma)$ & $2 \mathrm{Ks} 03(\sigma)$ & $2 \mathrm{Ks} 05(\sigma)$ & $2 \mathrm{Ks} 06(\sigma)$ \\
\hline & & & $2 \mathrm{Ks} 07(\sigma)$ & $2 \mathrm{Ks} 08(\sigma)$ & $2 \mathrm{Ks} 09(\sigma)$ & $2 \mathrm{Ks} 11(\sigma)$ & $2 \mathrm{Ks} 12(\sigma)$ & $2 \mathrm{Ks} 13(\sigma)$ \\
\hline & & & $2 \mathrm{Ks} 14(\sigma)$ & $2 \mathrm{Ks} 15(\sigma)$ & $2 \mathrm{Ks} 16(\sigma)$ & $2 \mathrm{Ks} 17(\sigma)$ & $2 \mathrm{Ks} 18(\sigma)$ & $2 \mathrm{Ks} 19(\sigma)$ \\
\hline & & & $2 \mathrm{Ks} 20(\sigma)$ & $2 \mathrm{Ks} 21(\sigma)$ & $2 \mathrm{Ks} 22(\sigma)$ & $2 \mathrm{Ks} 23(\sigma)$ & $2 \mathrm{Ks} 24(\sigma)$ & $2 \mathrm{Ks} 04(\sigma)$ \\
\hline \multirow[t]{4}{*}{2130} & \multirow[t]{4}{*}{161.405} & \multirow[t]{4}{*}{456.508} & $20.70(0.05)$ & $20.61(0.08)$ & $20.70(0.08)$ & $20.99(0.11)$ & $20.87(0.05)$ & $20.52(0.06)$ \\
\hline & & & $20.70(0.08)$ & $21.23(0.12)$ & $20.75(0.05)$ & $20.71(0.06)$ & $20.56(0.06)$ & $20.23(0.05)$ \\
\hline & & & $20.63(0.07)$ & $20.52(0.05)$ & $20.56(0.05)$ & $20.31(0.06)$ & $20.31(0.05)$ & $20.22(0.05)$ \\
\hline & & & $20.12(0.06)$ & $20.00(0.06)$ & $20.08(0.04)$ & $20.17(0.05)$ & $20.17(0.05)$ & $20.70(0.15)$ \\
\hline \multirow[t]{4}{*}{2131} & \multirow[t]{4}{*}{569.326} & \multirow[t]{4}{*}{701.240} & $21.16(0.07)$ & $20.55(0.06)$ & $20.12(0.07)$ & $20.07(0.06)$ & $21.38(0.06)$ & $21.42(0.10)$ \\
\hline & & & $21.29(0.11)$ & $21.56(0.17)$ & $21.12(0.07)$ & $20.79(0.06)$ & $20.88(0.10)$ & $21.11(0.07)$ \\
\hline & & & $20.36(0.06)$ & $20.25(0.05)$ & $20.17(0.05)$ & $20.06(0.06)$ & $20.10(0.05)$ & $20.20(0.05)$ \\
\hline & & & $20.28(0.06)$ & $20.08(0.06)$ & $20.26(0.05)$ & $20.34(0.05)$ & $20.44(0.05)$ & $21.01(0.21)$ \\
\hline \multirow[t]{4}{*}{2135} & \multirow[t]{4}{*}{486.286} & \multirow[t]{4}{*}{169.442} & $20.77(0.10)$ & $20.43(0.09)$ & $20.35(0.09)$ & $20.56(0.11)$ & $20.13(0.11)$ & $20.56(0.15)$ \\
\hline & & & $20.71(0.10)$ & $19.93(0.14)$ & $20.76(0.08)$ & $20.20(0.09)$ & $20.63(0.06)$ & $22.47(0.32)$ \\
\hline & & & $20.11(0.07)$ & $20.14(0.05)$ & $19.95(0.06)$ & $20.12(0.07)$ & $20.37(0.06)$ & $21.63(0.21)$ \\
\hline & & & $21.01(0.11)$ & $20.93(0.10)$ & $21.06(0.10)$ & $20.89(0.07)$ & $20.58(0.07)$ & $20.41(0.11)$ \\
\hline \multirow[t]{4}{*}{2139} & \multirow[t]{4}{*}{605.706} & \multirow[t]{4}{*}{164.195} & $20.29(0.05)$ & $20.44(0.08)$ & $20.98(0.13)$ & $20.53(0.07)$ & $20.34(0.05)$ & $20.27(0.05)$ \\
\hline & & & $20.27(0.06)$ & $20.13(0.06)$ & $20.23(0.05)$ & $20.32(0.05)$ & $20.64(0.07)$ & $20.73(0.06)$ \\
\hline & & & $20.23(0.05)$ & $20.27(0.05)$ & $20.29(0.05)$ & $20.40(0.05)$ & $20.44(0.05)$ & $20.48(0.05)$ \\
\hline & & & $20.73(0.08)$ & $20.69(0.10)$ & $20.80(0.06)$ & $20.70(0.06)$ & $20.69(0.07)$ & $21.07(0.16)$ \\
\hline \multirow[t]{4}{*}{2143} & \multirow[t]{4}{*}{643.003} & \multirow[t]{4}{*}{586.010} & $20.32(0.05)$ & $21.01(0.08)$ & $20.87(0.11)$ & $20.75(0.12)$ & $20.34(0.05)$ & $20.54(0.06)$ \\
\hline & & & $20.36(0.06)$ & $20.92(0.10)$ & $20.53(0.06)$ & $20.71(0.06)$ & $20.43(0.07)$ & $20.16(0.05)$ \\
\hline & & & $20.53(0.06)$ & $20.46(0.05)$ & $20.52(0.05)$ & $20.61(0.06)$ & $20.63(0.06)$ & $20.66(0.06)$ \\
\hline & & & $20.48(0.07)$ & $20.51(0.07)$ & $20.18(0.05)$ & $20.01(0.05)$ & $20.07(0.06)$ & $20.99(0.14)$ \\
\hline 2144 & 739.896 & 55.852 & $20.54(0.06)$ & $20.65(0.09)$ & $20.61(0.10)$ & $20.18(0.07)$ & $20.05(0.05)$ & $20.02(0.05)$ \\
\hline & & & $20.18(0.05)$ & $20.09(0.06)$ & $20.21(0.05)$ & $20.59(0.06)$ & $20.90(0.09)$ & $20.55(0.05)$ \\
\hline & & & $20.39(0.06)$ & $20.54(0.06)$ & $20.64(0.06)$ & $20.56(0.05)$ & $20.75(0.05)$ & $20.48(0.06)$ \\
\hline & & & $20.56(0.09)$ & $20.49(0.07)$ & $20.64(0.06)$ & $20.43(0.06)$ & $20.31(0.06)$ & $20.37(0.12)$ \\
\hline 2145 & 296.250 & 99.696 & $21.00(0.06)$ & $20.46(0.08)$ & $20.75(0.11)$ & $20.87(0.09)$ & $21.21(0.08)$ & $21.15(0.07)$ \\
\hline & & & $21.39(0.11)$ & $21.25(0.12)$ & $21.28(0.09)$ & $20.78(0.06)$ & $20.50(0.07)$ & $20.23(0.05)$ \\
\hline & & & $20.62(0.07)$ & $20.53(0.06)$ & $20.49(0.06)$ & $20.48(0.06)$ & $20.21(0.05)$ & $20.13(0.05)$ \\
\hline & & & $19.99(0.06)$ & $20.07(0.06)$ & $20.02(0.05)$ & $20.12(0.05)$ & $20.54(0.06)$ & $20.78(0.14)$ \\
\hline 2147 & 716.631 & 540.415 & $20.22(0.05)$ & $20.24(0.07)$ & $20.36(0.07)$ & $20.72(0.11)$ & $20.69(0.05)$ & $20.53(0.06)$ \\
\hline & & & $20.56(0.06)$ & $20.74(0.08)$ & $20.53(0.06)$ & $20.21(0.05)$ & $20.14(0.05)$ & $20.36(0.05)$ \\
\hline & & & $20.37(0.06)$ & $20.32(0.05)$ & $20.24(0.05)$ & $20.36(0.05)$ & $20.37(0.05)$ & $20.46(0.06)$ \\
\hline & & & $20.70(0.07)$ & $20.63(0.11)$ & $20.71(0.06)$ & $20.96(0.08)$ & $20.81(0.08)$ & $20.90(0.24)$ \\
\hline 2149 & 421.425 & 429.891 & $20.89(0.07)$ & $20.38(0.07)$ & $20.96(0.11)$ & $21.26(0.14)$ & $20.46(0.05)$ & $20.70(0.07)$ \\
\hline & & & $20.81(0.10)$ & $20.74(0.09)$ & $20.83(0.07)$ & $20.60(0.08)$ & $20.81(0.11)$ & $20.73(0.06)$ \\
\hline & & & $19.87(0.05)$ & $19.99(0.05)$ & $20.05(0.05)$ & $20.17(0.06)$ & $20.31(0.05)$ & $20.56(0.07)$ \\
\hline & & & $20.69(0.08)$ & $20.86(0.09)$ & $21.04(0.06)$ & $21.21(0.10)$ & $21.17(0.13)$ & $20.21(0.08)$ \\
\hline 2151 & 406.827 & 782.052 & $20.13(0.05)$ & $20.72(0.08)$ & $20.40(0.07)$ & $20.25(0.06)$ & $20.48(0.05)$ & $20.57(0.06)$ \\
\hline & & & $20.43(0.07)$ & $20.27(0.06)$ & $20.25(0.05)$ & $20.35(0.05)$ & $20.16(0.06)$ & $19.92(0.05)$ \\
\hline & & & $20.96(0.08)$ & $20.98(0.06)$ & $20.98(0.07)$ & $20.90(0.06)$ & $20.98(0.06)$ & $20.86(0.07)$ \\
\hline & & & $20.98(0.09)$ & $20.68(0.07)$ & $20.69(0.06)$ & $20.41(0.06)$ & $20.31(0.06)$ & $20.98(0.15)$ \\
\hline 2157 & 97.973 & 635.122 & $20.24(0.09)$ & $20.93(0.11)$ & $20.69(0.11)$ & $20.87(0.14)$ & $19.03(0.12)$ & $21.07(0.32)$ \\
\hline & & & $20.28(0.09)$ & $19.42(0.08)$ & $20.70(0.11)$ & $20.09(0.06)$ & $20.53(0.08)$ & $20.15(0.09)$ \\
\hline & & & $20.50(0.09)$ & $20.21(0.08)$ & $19.32(0.15)$ & $20.44(0.14)$ & $20.52(0.14)$ & $20.50(0.07)$ \\
\hline & & & $20.54(0.09)$ & $20.83(0.16)$ & $20.43(0.08)$ & $20.88(0.07)$ & $20.64(0.06)$ & $20.10(0.13)$ \\
\hline 2159 & 234.546 & 122.594 & $20.26(0.05)$ & $20.59(0.07)$ & $20.63(0.09)$ & $20.77(0.09)$ & $20.85(0.06)$ & $20.62(0.07)$ \\
\hline & & & $20.57(0.07)$ & $20.73(0.09)$ & $20.57(0.06)$ & $20.24(0.05)$ & $20.14(0.06)$ & $20.43(0.05)$ \\
\hline & & & $20.24(0.07)$ & $20.16(0.05)$ & $20.25(0.07)$ & $20.21(0.05)$ & $20.24(0.05)$ & $20.24(0.06)$ \\
\hline & & & $20.57(0.08)$ & $20.61(0.07)$ & $20.68(0.06)$ & $20.67(0.07)$ & $20.49(0.07)$ & $20.55(0.15)$ \\
\hline
\end{tabular}


Table 8. continuation.

\begin{tabular}{|c|c|c|c|c|c|c|c|c|}
\hline \multirow[t]{4}{*}{$\overline{\mathrm{F} 2 \mathrm{ID}}$} & \multirow[t]{4}{*}{$\mathrm{x}$} & \multirow[t]{4}{*}{$\mathrm{y}$} & $2 \mathrm{Ks} 10(\sigma)$ & $2 \mathrm{Ks} 01(\sigma)$ & $2 \mathrm{Ks} 02(\sigma)$ & $2 \mathrm{Ks} 03(\sigma)$ & $2 \mathrm{Ks} 05(\sigma)$ & $2 \mathrm{Ks06}(\sigma)$ \\
\hline & & & $2 \mathrm{Ks} 07(\sigma)$ & $2 \mathrm{Ks} 08(\sigma)$ & $2 \mathrm{Ks} 09(\sigma)$ & $2 \mathrm{Ks} 11(\sigma)$ & $2 \mathrm{Ks} 12(\sigma)$ & $2 \mathrm{Ks} 13(\sigma)$ \\
\hline & & & $2 \mathrm{Ks} 14(\sigma)$ & $2 \mathrm{Ks} 15(\sigma)$ & $2 \mathrm{Ks} 16(\sigma)$ & $2 \mathrm{Ks} 17(\sigma)$ & $2 \mathrm{Ks} 18(\sigma)$ & $2 \mathrm{Ks} 19(\sigma)$ \\
\hline & & & $2 \mathrm{Ks} 20(\sigma)$ & $2 \mathrm{Ks} 21(\sigma)$ & $2 \mathrm{Ks} 22(\sigma)$ & $2 \mathrm{Ks} 23(\sigma)$ & $2 \mathrm{Ks} 24(\sigma)$ & $2 \mathrm{Ks} 04(\sigma)$ \\
\hline \multirow[t]{4}{*}{2160} & $\overline{622.434}$ & 620.733 & $20.03(0.06)$ & $20.37(0.09)$ & $20.81(0.09)$ & $20.77(0.09)$ & $20.55(0.06)$ & $20.53(0.07)$ \\
\hline & & & $20.47(0.07)$ & $20.26(0.06)$ & $20.27(0.07)$ & $20.07(0.06)$ & $20.15(0.06)$ & $20.47(0.06)$ \\
\hline & & & $20.18(0.06)$ & $20.17(0.05)$ & $20.30(0.07)$ & $20.50(0.07)$ & $20.54(0.05)$ & $20.64(0.07)$ \\
\hline & & & $20.57(0.09)$ & $21.12(0.11)$ & $20.71(0.08)$ & $20.68(0.08)$ & $20.66(0.07)$ & $20.73(0.15)$ \\
\hline \multirow[t]{4}{*}{2175} & 99.192 & 452.047 & $21.15(0.09)$ & $20.30(0.07)$ & $20.32(0.10)$ & $20.56(0.18)$ & $19.85(0.30)$ & 99.99 (9.99) \\
\hline & & & $20.86(0.09)$ & 99.99 (9.99) & $20.79(0.19)$ & $21.35(0.12)$ & $20.72(0.14)$ & 99.99 (9.99) \\
\hline & & & $20.59(0.15)$ & 99.99 (9.99) & $20.34(0.08)$ & $20.17(0.07)$ & $20.07(0.08)$ & $20.38(0.07)$ \\
\hline & & & $20.89(0.09)$ & $20.23(0.06)$ & $19.99(0.06)$ & $20.92(0.10)$ & $20.62(0.09)$ & $19.99(0.12)$ \\
\hline \multirow[t]{4}{*}{2183} & 106.757 & 436.531 & $20.69(0.10)$ & $20.77(0.17)$ & $20.92(0.16)$ & $20.67(0.13)$ & $20.54(0.19)$ & $20.49(0.77)$ \\
\hline & & & $20.74(0.10)$ & $19.90(0.27)$ & $20.12(0.12)$ & $20.36(0.12)$ & $20.46(0.11)$ & $20.16(0.29)$ \\
\hline & & & $20.09(0.09)$ & $20.81(0.27)$ & $20.98(0.15)$ & $21.11(0.13)$ & $20.83(0.12)$ & $19.98(0.05)$ \\
\hline & & & $20.15(0.07)$ & $20.90(0.14)$ & $20.89(0.08)$ & $20.55(0.11)$ & $20.83(0.10)$ & $20.35(0.21)$ \\
\hline \multirow[t]{4}{*}{2185} & 611.151 & 223.807 & $21.66(0.39)$ & $22.02(0.95)$ & 99.99 (9.99) & 99.99 (9.99) & $19.99(0.30)$ & $20.91(0.40)$ \\
\hline & & & 99.99 (9.99) & $20.93(0.70)$ & $21.27(0.34)$ & $19.20(0.12)$ & $21.25(0.28)$ & $19.70(0.20)$ \\
\hline & & & $21.51(0.60)$ & $19.85(0.12)$ & $99.99(9.99)$ & $20.90(0.23)$ & $21.94(0.52)$ & $20.40(0.13)$ \\
\hline & & & $20.97(0.14)$ & $21.55(0.46)$ & $20.69(0.18)$ & $21.42(0.19)$ & $20.99(0.15)$ & $20.59(0.24)$ \\
\hline \multirow[t]{4}{*}{2193} & 260.297 & 405.949 & $20.69(0.05)$ & $20.88(0.08)$ & $20.50(0.07)$ & $20.87(0.10)$ & $20.47(0.05)$ & $20.49(0.06)$ \\
\hline & & & $20.62(0.07)$ & $20.48(0.06)$ & $20.46(0.06)$ & $20.94(0.09)$ & $20.70(0.09)$ & $20.86(0.08)$ \\
\hline & & & $20.19(0.05)$ & $20.20(0.06)$ & $20.22(0.06)$ & $20.16(0.06)$ & $20.23(0.06)$ & $20.22(0.06)$ \\
\hline & & & $20.28(0.07)$ & $20.27(0.08)$ & $20.29(0.05)$ & $20.27(0.06)$ & $20.42(0.06)$ & $20.08(0.13)$ \\
\hline \multirow[t]{4}{*}{2194} & 768.241 & 550.935 & $20.37(0.05)$ & $20.45(0.07)$ & $20.67(0.09)$ & $20.55(0.08)$ & $20.22(0.05)$ & $20.37(0.06)$ \\
\hline & & & $20.55(0.07)$ & $20.62(0.09)$ & $20.51(0.06)$ & $20.73(0.07)$ & $20.78(0.08)$ & $20.77(0.05)$ \\
\hline & & & $19.93(0.05)$ & $20.08(0.05)$ & $20.14(0.05)$ & $20.32(0.06)$ & $20.47(0.05)$ & $20.61(0.05)$ \\
\hline & & & $20.68(0.08)$ & $20.67(0.07)$ & $20.99(0.07)$ & $20.82(0.09)$ & $20.92(0.10)$ & $20.17(0.15)$ \\
\hline \multirow[t]{4}{*}{2200} & 799.343 & 510.013 & $20.60(0.05)$ & $20.40(0.09)$ & $20.91(0.13)$ & $20.87(0.10)$ & $20.68(0.06)$ & $20.66(0.07)$ \\
\hline & & & $20.60(0.07)$ & $21.02(0.11)$ & $20.75(0.06)$ & $20.57(0.06)$ & $20.37(0.06)$ & $20.32(0.04)$ \\
\hline & & & $20.44(0.06)$ & $20.26(0.05)$ & $20.15(0.05)$ & $20.23(0.05)$ & $20.23(0.05)$ & $20.37(0.05)$ \\
\hline & & & $20.47(0.07)$ & $20.52(0.07)$ & $20.76(0.06)$ & $20.90(0.09)$ & $20.91(0.08)$ & $20.74(0.12)$ \\
\hline \multirow[t]{4}{*}{2206} & 511.707 & 825.415 & $20.37(0.06)$ & $20.29(0.08)$ & $20.42(0.08)$ & $20.25(0.08)$ & $20.26(0.05)$ & 20.82 \\
\hline & & & $20.43(0.08)$ & $21.84(0.20)$ & $20.27(0.06)$ & $21.07(0.06)$ & $20.89(0.07)$ & $20.31(0.08)$ \\
\hline & & & $20.49(0.06)$ & $20.28(0.06)$ & $20.25(0.05)$ & $20.13(0.06)$ & $20.18(0.05)$ & $20.52(0.05)$ \\
\hline & & & $20.68(0.08)$ & $20.16(0.07)$ & $20.48(0.06)$ & $20.64(0.05)$ & $20.27(0.06)$ & $20.43(0.14)$ \\
\hline \multirow[t]{4}{*}{2222} & 414.360 & 218.193 & $20.33(0.05)$ & $20.95(0.10)$ & $21.04(0.12)$ & $21.72(0.30)$ & $20.41(0.05)$ & $20.21(0.05)$ \\
\hline & & & $20.33(0.06)$ & $20.23(0.07)$ & $20.04(0.05)$ & $20.60(0.06)$ & $20.60(0.07)$ & $20.96(0.07)$ \\
\hline & & & $20.28(0.05)$ & $20.23(0.05)$ & $20.29(0.05)$ & $20.48(0.05)$ & $20.55(0.05)$ & $20.83(0.07)$ \\
\hline & & & $21.26(0.14)$ & $21.16(0.15)$ & $21.23(0.11)$ & $21.97(0.19)$ & $21.36(0.10)$ & $20.76(0.16)$ \\
\hline \multirow[t]{4}{*}{2228} & 194.227 & 762.506 & $20.90(0.06)$ & $20.22(0.07)$ & $20.23(0.06)$ & $20.49(0.07)$ & $20.84(0.06)$ & $20.98(0.07)$ \\
\hline & & & $21.00(0.08)$ & $21.23(0.12)$ & $21.06(0.07)$ & $20.30(0.05)$ & $20.19(0.06)$ & $20.10(0.05)$ \\
\hline & & & $20.63(0.07)$ & $20.52(0.05)$ & $20.36(0.06)$ & $20.22(0.06)$ & $20.19(0.05)$ & $20.34(0.05)$ \\
\hline & & & $20.35(0.07)$ & $20.28(0.08)$ & $20.42(0.06)$ & $20.66(0.06)$ & $20.84(0.08)$ & $20.23(0.09)$ \\
\hline \multirow[t]{4}{*}{2231} & 332.270 & 207.499 & $20.91(0.06)$ & $20.29(0.07)$ & $20.48(0.08)$ & $20.39(0.07)$ & $20.32(0.05)$ & $20.50(0.06)$ \\
\hline & & & $20.71(0.08)$ & $20.68(0.08)$ & $20.83(0.08)$ & $20.48(0.06)$ & $20.65(0.07)$ & $20.87(0.06)$ \\
\hline & & & $20.01(0.06)$ & $20.03(0.05)$ & $20.14(0.05)$ & $20.21(0.05)$ & $20.31(0.06)$ & $20.42(0.06)$ \\
\hline & & & $20.70(0.09)$ & $20.61(0.06)$ & $20.92(0.08)$ & $20.78(0.07)$ & $20.74(0.08)$ & $19.95(0.12)$ \\
\hline \multirow[t]{4}{*}{2233} & 538.403 & 244.348 & $20.36(0.05)$ & $20.83(0.13)$ & $20.72(0.08)$ & $20.48(0.07)$ & $20.31(0.06)$ & $20.12(0.05)$ \\
\hline & & & $20.18(0.07)$ & $20.25(0.06)$ & $20.17(0.05)$ & $20.39(0.06)$ & $20.63(0.08)$ & $20.88(0.08)$ \\
\hline & & & $20.15(0.06)$ & $20.20(0.06)$ & $20.45(0.06)$ & $20.69(0.06)$ & $20.77(0.07)$ & $20.87(0.08)$ \\
\hline & & & $21.08(0.11)$ & $21.49(0.15)$ & $21.20(0.10)$ & $21.04(0.09)$ & $21.04(0.11)$ & $20.82(0.22)$ \\
\hline
\end{tabular}


Table 8. continuation.

\begin{tabular}{|c|c|c|c|c|c|c|c|c|}
\hline \multirow[t]{4}{*}{ F2 ID } & \multirow[t]{4}{*}{$\mathrm{x}$} & \multirow[t]{4}{*}{$\mathrm{y}$} & $2 \mathrm{Ks} 10(\sigma)$ & $2 \mathrm{Ks} 01(\sigma)$ & $2 \mathrm{Ks} 02(\sigma)$ & $2 \mathrm{Ks} 03(\sigma)$ & $2 \mathrm{Ks} 05(\sigma)$ & $2 \mathrm{Ks} 06(\sigma)$ \\
\hline & & & $2 \mathrm{Ks} 07(\sigma)$ & $2 \mathrm{Ks} 08(\sigma)$ & $2 \mathrm{Ks} 09(\sigma)$ & $2 \mathrm{Ks} 11(\sigma)$ & $2 \mathrm{Ks} 12(\sigma)$ & $2 \mathrm{Ks} 13(\sigma)$ \\
\hline & & & $2 \mathrm{Ks} 14(\sigma)$ & $2 \mathrm{Ks} 15(\sigma)$ & $2 \mathrm{Ks} 16(\sigma)$ & $2 \mathrm{Ks} 17(\sigma)$ & $2 \mathrm{Ks} 18(\sigma)$ & $2 \mathrm{Ks} 19(\sigma)$ \\
\hline & & & $2 \mathrm{Ks} 20(\sigma)$ & $2 \mathrm{Ks} 21(\sigma)$ & $2 \mathrm{Ks} 22(\sigma)$ & $2 \mathrm{Ks} 23(\sigma)$ & $2 \mathrm{Ks} 24(\sigma)$ & $2 \mathrm{Ks} 04(\sigma)$ \\
\hline \multirow[t]{4}{*}{2240} & 176.015 & 88.968 & 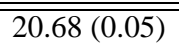 & 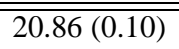 & $21.19(0.13)$ & 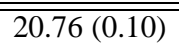 & 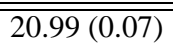 & 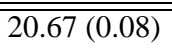 \\
\hline & & & $20.88(0.06)$ & $20.42(0.09)$ & $20.82(0.07)$ & $20.47(0.06)$ & $20.32(0.07)$ & $20.34(0.07)$ \\
\hline & & & $20.62(0.07)$ & $20.36(0.07)$ & $20.25(0.07)$ & $20.20(0.08)$ & $20.00(0.06)$ & $20.08(0.05)$ \\
\hline & & & $20.11(0.06)$ & $20.15(0.06)$ & $20.30(0.05)$ & $20.44(0.06)$ & $20.55(0.07)$ & $20.49(0.09)$ \\
\hline \multirow[t]{4}{*}{2247} & 289.713 & 806.131 & $20.00(0.04)$ & $20.35(0.09)$ & $20.18(0.07)$ & $20.07(0.06)$ & $20.63(0.05)$ & $20.65(0.06)$ \\
\hline & & & $20.42(0.06)$ & $20.36(0.05)$ & $20.19(0.05)$ & $20.78(0.07)$ & $20.66(0.07)$ & $20.27(0.05)$ \\
\hline & & & $20.71(0.07)$ & $20.72(0.06)$ & $20.82(0.06)$ & $20.74(0.06)$ & $20.70(0.07)$ & $20.79(0.06)$ \\
\hline & & & $20.71(0.08)$ & $20.83(0.08)$ & $20.77(0.06)$ & $20.61(0.07)$ & $20.38(0.06)$ & $20.83(0.17)$ \\
\hline \multirow[t]{4}{*}{2251} & 64.773 & 16.630 & $21.18(0.09)$ & $20.30(0.13)$ & $20.35(0.08)$ & $20.83(0.10)$ & $20.98(0.07)$ & $20.66(0.07)$ \\
\hline & & & $21.57(0.19)$ & $21.49(0.22)$ & $21.35(0.10)$ & $20.54(0.07)$ & $20.73(0.08)$ & $20.34(0.07)$ \\
\hline & & & $20.66(0.09)$ & $20.38(0.08)$ & $20.45(0.09)$ & $20.66(0.11)$ & $20.39(0.06)$ & $20.26(0.06)$ \\
\hline & & & $20.01(0.06)$ & $20.09(0.08)$ & $20.05(0.06)$ & $20.19(0.07)$ & $20.25(0.07)$ & $21.34(0.71)$ \\
\hline \multirow[t]{4}{*}{2255} & 225.671 & 511.834 & $20.51(0.06)$ & $20.60(0.07)$ & $21.00(0.10)$ & $21.10(0.11)$ & $20.49(0.05)$ & $20.65(0.07)$ \\
\hline & & & $20.47(0.07)$ & $20.82(0.08)$ & $20.73(0.07)$ & $20.70(0.06)$ & $20.60(0.07)$ & $20.86(0.07)$ \\
\hline & & & $20.06(0.06)$ & $20.14(0.05)$ & $20.20(0.05)$ & $20.17(0.05)$ & $20.40(0.06)$ & $20.50(0.06)$ \\
\hline & & & $20.53(0.08)$ & $20.56(0.06)$ & $20.56(0.07)$ & $20.55(0.07)$ & $20.53(0.07)$ & $20.03(0.08)$ \\
\hline \multirow[t]{4}{*}{2272} & 213.546 & 261.986 & $20.40(0.05)$ & $20.23(0.06)$ & $20.75(0.11)$ & $21.06(0.08)$ & $20.76(0.05)$ & $20.58(0.05)$ \\
\hline & & & $20.61(0.08)$ & $20.63(0.08)$ & $20.51(0.06)$ & $20.62(0.06)$ & $20.64(0.06)$ & $20.57(0.05)$ \\
\hline & & & $20.69(0.08)$ & $20.43(0.06)$ & $20.52(0.05)$ & 20.54 & 20.65 & 20.40 \\
\hline & & & $20.24(0.06)$ & $20.28(0.05)$ & $20.09(0.05)$ & $20.09(0.05)$ & $20.11(0.06)$ & $20.18(0.07)$ \\
\hline \multirow[t]{4}{*}{2273} & 397.683 & 301.931 & $20.55(0.05)$ & $20.68(0.08)$ & $20.36(0.08)$ & $20.44(0.08)$ & $20.07(0.05)$ & $20.12(0.05)$ \\
\hline & & & $20.37(0.07)$ & $20.32(0.07)$ & $20.41(0.06)$ & $20.73(0.06)$ & $20.76(0.07)$ & $20.80(0.06)$ \\
\hline & & & $20.17(0.06)$ & $20.30(0.05)$ & $20.50(0.05)$ & $20.52(0.06)$ & $20.63(0.06)$ & $20.77(0.07)$ \\
\hline & & & $20.66(0.07)$ & $20.71(0.10)$ & $20.79(0.07)$ & $20.65(0.07)$ & $20.49(0.07)$ & $20.34(0.12)$ \\
\hline \multirow[t]{4}{*}{2285} & 125.038 & 494.064 & $20.26(0.05)$ & $20.64(0.09)$ & $20.97(0.11)$ & $21.72(0.20)$ & $20.79(0.07)$ & $20.40(0.05)$ \\
\hline & & & $20.81(0.10)$ & $20.44(0.07)$ & $20.52(0.06)$ & $20.21(0.05)$ & $20.46(0.07)$ & $20.57(0.05)$ \\
\hline & & & $20.33(0.06)$ & $20.16(0.05)$ & $20.30(0.05)$ & $20.36(0.06)$ & $20.46(0.07)$ & $20.59(0.06)$ \\
\hline & & & $20.73(0.10)$ & $21.00(0.12)$ & $20.92(0.07)$ & $20.87(0.08)$ & 20.88 & 20.83 \\
\hline \multirow[t]{4}{*}{2287} & 489.746 & 552.798 & $20.82(0.06)$ & $20.17(0.07)$ & $20.64(0.10)$ & $20.62(0.07)$ & $20.17(0.05)$ & $20.59(0.06)$ \\
\hline & & & $20.92(0.14)$ & $20.98(0.11)$ & $20.87(0.08)$ & $20.75(0.06)$ & $20.64(0.07)$ & $20.62(0.05)$ \\
\hline & & & $20.07(0.05)$ & $20.26(0.05)$ & $20.29(0.04)$ & $20.39(0.07)$ & $20.52(0.05)$ & $20.67(0.07)$ \\
\hline & & & $20.65(0.08)$ & $21.06(0.12)$ & $20.62(0.05)$ & $20.61(0.07)$ & $20.48(0.06)$ & $20.21(0.13)$ \\
\hline \multirow[t]{4}{*}{2291} & 330.198 & 797.906 & $20.14(0.05)$ & $20.63(0.06)$ & $20.83(0.09)$ & $21.01(0.11)$ & $20.68(0.06)$ & $20.65(0.06)$ \\
\hline & & & $20.71(0.07)$ & $20.51(0.07)$ & $20.37(0.05)$ & $20.29(0.05)$ & $20.27(0.06)$ & $20.61(0.05)$ \\
\hline & & & $20.34(0.05)$ & $20.30(0.05)$ & $20.30(0.05)$ & $20.28(0.05)$ & $20.21(0.06)$ & $20.38(0.05)$ \\
\hline & & & $20.66(0.07)$ & $20.64(0.06)$ & $20.71(0.06)$ & $20.79(0.06)$ & $20.90(0.08)$ & $20.80(0.11)$ \\
\hline \multirow[t]{4}{*}{2305} & 53.673 & 209.730 & $20.48(0.06)$ & $20.62(0.10)$ & $20.81(0.11)$ & $20.85(0.09)$ & $20.86(0.06)$ & $21.09(0.09)$ \\
\hline & & & $20.79(0.08)$ & $20.75(0.09)$ & $20.68(0.07)$ & $20.35(0.06)$ & $20.23(0.07)$ & $20.21(0.05)$ \\
\hline & & & $20.71(0.06)$ & $20.32(0.05)$ & $20.35(0.05)$ & $20.34(0.09)$ & $20.13(0.06)$ & $20.37(0.06)$ \\
\hline & & & $20.39(0.06)$ & $20.24(0.06)$ & $20.37(0.06)$ & $20.61(0.06)$ & $20.55(0.07)$ & $20.76(0.18)$ \\
\hline \multirow[t]{4}{*}{2307} & 319.996 & 617.377 & $20.76(0.06)$ & $20.47(0.09)$ & $20.41(0.08)$ & $20.12(0.07)$ & $20.70(0.06)$ & $20.59(0.06)$ \\
\hline & & & $21.14(0.08)$ & $20.67(0.09)$ & $20.64(0.07)$ & $20.17(0.05)$ & $20.19(0.06)$ & $20.39(0.06)$ \\
\hline & & & $20.25(0.06)$ & $20.29(0.05)$ & $20.34(0.06)$ & $20.38(0.05)$ & $20.44(0.06)$ & $20.66(0.07)$ \\
\hline & & & $20.52(0.07)$ & $20.78(0.07)$ & $20.72(0.07)$ & $20.58(0.06)$ & $20.59(0.07)$ & $20.15(0.09)$ \\
\hline \multirow[t]{4}{*}{2311} & 337.757 & 176.217 & $20.56(0.05)$ & $20.89(0.12)$ & $20.89(0.11)$ & $21.30(0.14)$ & $20.83(0.05)$ & $20.80(0.06)$ \\
\hline & & & 20.77 (0.09) & $21.16(0.12)$ & $20.80(0.06)$ & $20.64(0.06)$ & $20.60(0.08)$ & $20.27(0.05)$ \\
\hline & & & $20.70(0.08)$ & $20.50(0.06)$ & $20.50(0.06)$ & $20.56(0.05)$ & $20.48(0.05)$ & $20.42(0.05)$ \\
\hline & & & $20.40(0.07)$ & $20.17(0.08)$ & $20.04(0.05)$ & $20.23(0.06)$ & $20.09(0.06)$ & $20.92(0.14)$ \\
\hline
\end{tabular}


Table 8. continuation.

\begin{tabular}{|c|c|c|c|c|c|c|c|c|}
\hline \multirow[t]{4}{*}{$\overline{\mathrm{F} 2 \mathrm{ID}}$} & \multirow[t]{4}{*}{$\mathrm{x}$} & \multirow[t]{4}{*}{$\mathrm{y}$} & $2 \mathrm{Ks} 10(\sigma)$ & $2 \mathrm{Ks} 01(\sigma)$ & $2 \mathrm{Ks} 02(\sigma)$ & $2 \mathrm{Ks} 03(\sigma)$ & $2 \mathrm{Ks} 05(\sigma)$ & $2 \mathrm{Ks06}(\sigma)$ \\
\hline & & & $2 \mathrm{Ks} 07(\sigma)$ & $2 \mathrm{Ks} 08(\sigma)$ & $2 \mathrm{Ks} 09(\sigma)$ & $2 \mathrm{Ks} 11(\sigma)$ & $2 \mathrm{Ks} 12(\sigma)$ & $2 \mathrm{Ks} 13(\sigma)$ \\
\hline & & & $2 \mathrm{Ks} 14(\sigma)$ & $2 \mathrm{Ks} 15(\sigma)$ & $2 \mathrm{Ks} 16(\sigma)$ & $2 \mathrm{Ks} 17(\sigma)$ & $2 \mathrm{Ks} 18(\sigma)$ & $2 \mathrm{Ks} 19(\sigma)$ \\
\hline & & & $2 \mathrm{Ks} 20(\sigma)$ & $2 \mathrm{Ks} 21(\sigma)$ & $2 \mathrm{Ks} 22(\sigma)$ & $2 \mathrm{Ks} 23(\sigma)$ & $2 \mathrm{Ks} 24(\sigma)$ & $2 \mathrm{Ks} 04(\sigma)$ \\
\hline \multirow[t]{4}{*}{2312} & $\overline{403.463}$ & 508.011 & $20.30(0.05)$ & $20.41(0.07)$ & $20.98(0.12)$ & $20.96(0.11)$ & $20.71(0.06)$ & $20.61(0.07)$ \\
\hline & & & $20.73(0.08)$ & $20.72(0.08)$ & $20.65(0.07)$ & $20.15(0.05)$ & $20.27(0.06)$ & $20.45(0.06)$ \\
\hline & & & $20.15(0.05)$ & $20.24(0.05)$ & $20.29(0.05)$ & $20.33(0.05)$ & $20.47(0.04)$ & $20.61(0.06)$ \\
\hline & & & $20.68(0.09)$ & $20.81(0.08)$ & $20.71(0.07)$ & $20.77(0.07)$ & $20.73(0.08)$ & $20.81(0.16)$ \\
\hline \multirow[t]{4}{*}{2314} & 158.728 & 199.856 & $20.28(0.05)$ & $20.77(0.09)$ & $21.36(0.13)$ & $21.07(0.15)$ & $20.87(0.06)$ & $20.78(0.06)$ \\
\hline & & & $20.63(0.07)$ & $20.73(0.10)$ & $20.40(0.05)$ & $20.67(0.08)$ & $20.53(0.08)$ & $20.58(0.06)$ \\
\hline & & & $20.29(0.06)$ & $20.21(0.05)$ & $20.23(0.06)$ & $20.31(0.07)$ & $20.24(0.05)$ & $20.34(0.05)$ \\
\hline & & & $20.41(0.08)$ & $20.37(0.07)$ & $20.46(0.06)$ & $20.76(0.07)$ & $20.50(0.08)$ & $20.77(0.16)$ \\
\hline \multirow[t]{4}{*}{2318} & 84.369 & 34.552 & $20.50(0.07)$ & $20.31(0.18)$ & $20.93(0.10)$ & $20.40(0.12)$ & $20.98(0.10)$ & $21.38(0.17)$ \\
\hline & & & $21.62(0.16)$ & $21.11(0.29)$ & $21.45(0.09)$ & $20.84(0.11)$ & $21.33(0.10)$ & $20.93(0.14)$ \\
\hline & & & $21.06(0.07)$ & $20.96(0.17)$ & $19.91(0.07)$ & $20.33(0.08)$ & $20.32(0.11)$ & $20.17(0.06)$ \\
\hline & & & $20.25(0.07)$ & $20.20(0.07)$ & $20.12(0.05)$ & $20.39(0.06)$ & $20.62(0.09)$ & $21.52(0.72)$ \\
\hline \multirow[t]{4}{*}{2320} & 473.832 & 347.226 & $20.54(0.05)$ & $20.65(0.09)$ & $20.73(0.08)$ & $20.85(0.10)$ & $20.90(0.06)$ & $21.08(0.13)$ \\
\hline & & & $21.03(0.11)$ & $21.04(0.16)$ & $20.79(0.06)$ & $20.21(0.06)$ & $20.39(0.06)$ & $20.67(0.06)$ \\
\hline & & & $20.05(0.05)$ & $20.11(0.05)$ & $20.26(0.05)$ & $20.33(0.06)$ & $20.47(0.06)$ & $21.04(0.07)$ \\
\hline & & & $21.06(0.11)$ & $20.77(0.10)$ & $20.60(0.05)$ & $20.59(0.06)$ & $20.68(0.07)$ & $20.93(0.14)$ \\
\hline \multirow[t]{4}{*}{2323} & 165.667 & 250.222 & $20.80(0.06)$ & $20.02(0.05)$ & $20.02(0.07)$ & $20.14(0.06)$ & $20.41(0.05)$ & $20.51(0.06)$ \\
\hline & & & $20.68(0.07)$ & $20.76(0.08)$ & $20.76(0.07)$ & $20.76(0.07)$ & $20.83(0.08)$ & $20.58(0.06)$ \\
\hline & & & $20.68(0.07)$ & $20.77(0.06)$ & $20.94(0.05)$ & $20.78(0.06)$ & $20.71(0.07)$ & $20.61(0.06)$ \\
\hline & & & $20.18(0.07)$ & $20.27(0.08)$ & $20.16(0.06)$ & $20.01(0.05)$ & $20.05(0.05)$ & $19.93(0.09)$ \\
\hline \multirow[t]{4}{*}{2324} & 429.095 & 18.648 & $20.24(0.05)$ & $20.59(0.09)$ & $20.91(0.10)$ & $21.04(0.09)$ & $20.38(0.05)$ & $20.49(0.06)$ \\
\hline & & & $20.46(0.07)$ & $20.41(0.08)$ & $20.31(0.06)$ & $22.03(0.17)$ & $21.39(0.14)$ & $21.29(0.08)$ \\
\hline & & & $20.11(0.06)$ & $20.14(0.05)$ & $20.38(0.05)$ & $20.45(0.06)$ & $20.54(0.06)$ & $20.57(0.06)$ \\
\hline & & & $20.79(0.09)$ & $20.56(0.09)$ & $21.19(0.10)$ & $20.91(0.08)$ & $21.05(0.11)$ & $20.60(0.09)$ \\
\hline \multirow[t]{4}{*}{2333} & 508.225 & 852.286 & $20.29(0.07)$ & $20.74(0.09)$ & $21.02(0.15)$ & $20.75(0.08)$ & $20.15(0.05)$ & $20.46(0.07)$ \\
\hline & & & $20.11(0.07)$ & $20.77(0.08)$ & $20.20(0.06)$ & $22.03(0.34)$ & $22.60(2.96)$ & $21.30(0.74)$ \\
\hline & & & $21.34(0.14)$ & $20.94(0.12)$ & $20.61(0.09)$ & $20.68(0.10)$ & $20.72(0.06)$ & $20.79(0.06)$ \\
\hline & & & $20.15(0.52)$ & $19.92(2.63)$ & $20.32(0.06)$ & 99.99 (9.99) & 99.99 (9.99) & $20.40(0.11)$ \\
\hline \multirow[t]{4}{*}{2335} & 699.863 & 145.770 & $20.59(0.06)$ & $20.32(0.09)$ & $19.94(0.06)$ & $20.09(0.07)$ & $20.26(0.05)$ & 20.18 \\
\hline & & & $20.31(0.06)$ & $20.70(0.08)$ & $20.38(0.06)$ & $20.76(0.07)$ & $20.88(0.08)$ & $20.88(0.07)$ \\
\hline & & & $20.24(0.05)$ & $20.40(0.05)$ & $20.66(0.05)$ & $20.69(0.07)$ & $20.72(0.06)$ & $20.78(0.07)$ \\
\hline & & & $20.99(0.11)$ & $21.15(0.12)$ & $20.53(0.06)$ & $20.76(0.06)$ & $20.80(0.09)$ & $20.14(0.07)$ \\
\hline \multirow[t]{4}{*}{2350} & 466.810 & 195.625 & $20.86(0.07)$ & $19.87(0.06)$ & $19.97(0.06)$ & $20.03(0.06)$ & $21.50(0.10)$ & $20.85(0.13)$ \\
\hline & & & $21.35(0.15)$ & $20.27(0.18)$ & $21.76(0.14)$ & $20.41(0.10)$ & $21.35(0.13)$ & $21.48(0.11)$ \\
\hline & & & $21.78(0.24)$ & $21.39(0.11)$ & $21.09(0.07)$ & $20.74(0.06)$ & $20.59(0.07)$ & $20.65(0.09)$ \\
\hline & & & $20.25(0.06)$ & $20.41(0.06)$ & $20.16(0.05)$ & $20.40(0.06)$ & $20.30(0.06)$ & $21.58(0.50)$ \\
\hline \multirow[t]{4}{*}{2355} & 117.225 & 453.458 & $21.44(0.18)$ & $20.09(0.15)$ & $20.39(0.22)$ & $19.20(0.14)$ & $19.60(0.24)$ & 99.99 (9.99) \\
\hline & & & $21.64(0.17)$ & 99.99 (9.99) & $19.96(0.16)$ & 20.19 (0.09) & $21.68(0.30)$ & $20.51(0.18)$ \\
\hline & & & $20.15(0.09)$ & $20.84(0.25)$ & $99.99(9.99)$ & $20.85(0.15)$ & $20.12(0.22)$ & $21.42(0.13)$ \\
\hline & & & 99.99 (9.99) & $21.74(0.31)$ & $20.80(0.11)$ & 99.99 (9.99) & 99.99 (9.99) & $20.38(0.20)$ \\
\hline \multirow[t]{4}{*}{2357} & 612.716 & 491.961 & $20.31(0.05)$ & $20.76(0.09)$ & $21.09(0.13)$ & $21.75(0.19)$ & $20.07(0.05)$ & $20.16(0.06)$ \\
\hline & & & $20.30(0.06)$ & $20.50(0.08)$ & $20.26(0.05)$ & $20.92(0.08)$ & $20.45(0.07)$ & $20.30(0.05)$ \\
\hline & & & $20.72(0.07)$ & $20.76(0.06)$ & $20.91(0.05)$ & $21.06(0.08)$ & $21.10(0.06)$ & $21.19(0.08)$ \\
\hline & & & $21.59(0.18)$ & $21.47(0.13)$ & $21.70(0.16)$ & $21.88(0.13)$ & $21.50(0.13)$ & $20.44(0.10)$ \\
\hline \multirow[t]{4}{*}{2371} & 409.452 & 459.005 & $20.95(0.07)$ & $20.71(0.10)$ & $20.52(0.10)$ & $20.46(0.07)$ & $20.46(0.06)$ & $20.45(0.07)$ \\
\hline & & & $20.54(0.07)$ & $20.93(0.11)$ & $20.68(0.07)$ & $20.96(0.08)$ & $21.07(0.13)$ & $21.06(0.07)$ \\
\hline & & & $20.26(0.06)$ & $20.28(0.05)$ & $20.15(0.05)$ & $20.21(0.06)$ & $20.32(0.06)$ & $20.63(0.06)$ \\
\hline & & & $20.95(0.10)$ & $20.85(0.09)$ & $21.06(0.09)$ & $21.40(0.13)$ & $21.49(0.14)$ & $20.79(0.15)$ \\
\hline
\end{tabular}


Table 8. continuation.

\begin{tabular}{|c|c|c|c|c|c|c|c|c|}
\hline \multirow[t]{4}{*}{$\bar{F}$ F2 ID } & \multirow[t]{4}{*}{$\mathrm{x}$} & \multirow[t]{4}{*}{$\mathrm{y}$} & $2 \mathrm{Ks} 10(\sigma)$ & $2 \mathrm{Ks} 01(\sigma)$ & $2 \mathrm{Ks} 02(\sigma)$ & $2 \mathrm{Ks} 03(\sigma)$ & $2 \mathrm{Ks} 05(\sigma)$ & $\overline{2 \mathrm{Ks} 06(\sigma)}$ \\
\hline & & & $2 \mathrm{Ks} 07(\sigma)$ & $2 \mathrm{Ks} 08(\sigma)$ & $2 \mathrm{Ks} 09(\sigma)$ & $2 \mathrm{Ks} 11(\sigma)$ & $2 \mathrm{Ks} 12(\sigma)$ & $2 \mathrm{Ks} 13(\sigma)$ \\
\hline & & & $2 \mathrm{Ks} 14(\sigma)$ & $2 \mathrm{Ks} 15(\sigma)$ & $2 \mathrm{Ks} 16(\sigma)$ & $2 \mathrm{Ks} 17(\sigma)$ & $2 \mathrm{Ks} 18(\sigma)$ & $2 \mathrm{Ks} 19(\sigma)$ \\
\hline & & & $2 \mathrm{Ks} 20(\sigma)$ & $2 \mathrm{Ks} 21(\sigma)$ & $2 \mathrm{Ks} 22(\sigma)$ & $2 \mathrm{Ks} 23(\sigma)$ & $2 \mathrm{Ks} 24(\sigma)$ & $2 \mathrm{Ks} 04(\sigma)$ \\
\hline \multirow[t]{4}{*}{2372} & 145.677 & 37.058 & $20.71(0.07)$ & $20.72(0.11)$ & $20.43(0.09)$ & $20.20(0.07)$ & $20.36(0.06)$ & 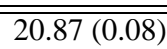 \\
\hline & & & $20.53(0.08)$ & $20.85(0.10)$ & $20.69(0.08)$ & $21.37(0.11)$ & $20.78(0.08)$ & $20.81(0.06)$ \\
\hline & & & $20.74(0.09)$ & $20.53(0.06)$ & $20.67(0.06)$ & $20.75(0.13)$ & $20.51(0.06)$ & $20.50(0.06)$ \\
\hline & & & $20.31(0.07)$ & $20.36(0.07)$ & $20.17(0.05)$ & $20.07(0.06)$ & $20.10(0.06)$ & $20.42(0.22)$ \\
\hline \multirow[t]{4}{*}{2380} & 708.827 & 734.763 & $20.65(0.06)$ & $20.02(0.07)$ & $20.13(0.06)$ & $20.33(0.07)$ & $20.26(0.05)$ & $20.55(0.06)$ \\
\hline & & & $20.67(0.09)$ & $21.10(0.10)$ & $20.73(0.06)$ & $20.67(0.06)$ & $20.72(0.08)$ & $20.56(0.05)$ \\
\hline & & & $20.06(0.05)$ & $20.15(0.05)$ & $20.32(0.05)$ & $20.51(0.08)$ & $20.50(0.05)$ & $20.81(0.06)$ \\
\hline & & & $21.00(0.09)$ & $20.71(0.09)$ & $20.86(0.06)$ & $20.79(0.06)$ & $20.64(0.08)$ & $19.99(0.08)$ \\
\hline \multirow[t]{4}{*}{2395} & 574.495 & 153.833 & $20.61(0.05)$ & $20.58(0.10)$ & $20.78(0.10)$ & $20.69(0.09)$ & $20.69(0.06)$ & $20.70(0.07)$ \\
\hline & & & $20.96(0.08)$ & $21.15(0.09)$ & $20.66(0.05)$ & $20.90(0.10)$ & $20.60(0.09)$ & $20.25(0.05)$ \\
\hline & & & $20.60(0.07)$ & $20.47(0.05)$ & $20.52(0.05)$ & $20.52(0.05)$ & $20.58(0.06)$ & $20.40(0.06)$ \\
\hline & & & $20.28(0.08)$ & $20.35(0.07)$ & $20.09(0.05)$ & $20.00(0.06)$ & $20.05(0.06)$ & $20.87(0.17)$ \\
\hline \multirow[t]{4}{*}{2413} & 9.806 & 687.280 & $20.60(0.05)$ & $20.41(0.08)$ & $20.54(0.08)$ & $20.69(0.09)$ & $20.82(0.07)$ & $20.66(0.06)$ \\
\hline & & & $20.76(0.09)$ & $21.05(0.13)$ & $20.66(0.07)$ & 99.99 (9.99) & $20.62(0.82)$ & 99.99 (9.99) \\
\hline & & & $20.76(0.09)$ & $20.70(0.05)$ & $20.58(0.07)$ & 99.99 (9.99) & $20.35(0.06)$ & $20.35(0.05)$ \\
\hline & & & $20.18(0.06)$ & $20.23(0.07)$ & $20.18(0.05)$ & 99.99 (9.99) & $20.39(0.06)$ & $21.26(0.28)$ \\
\hline \multirow[t]{4}{*}{2414} & 669.380 & 466.236 & $20.65(0.05)$ & $20.03(0.06)$ & $20.32(0.08)$ & $20.44(0.07)$ & $20.07(0.05)$ & $20.24(0.06)$ \\
\hline & & & $20.39(0.06)$ & $20.77(0.08)$ & $20.57(0.06)$ & $20.66(0.06)$ & $20.68(0.08)$ & $20.60(0.05)$ \\
\hline & & & $20.24(0.05)$ & $20.38(0.05)$ & $20.43(0.05)$ & $20.59(0.06)$ & $20.71(0.06)$ & $20.77(0.06)$ \\
\hline & & & $20.72(0.08)$ & $20.57(0.09)$ & $20.63(0.06)$ & $20.77(0.06)$ & $20.79(0.07)$ & $20.16(0.10)$ \\
\hline \multirow[t]{4}{*}{2421} & 383.677 & 511.695 & $20.77(0.06)$ & $20.31(0.07)$ & $20.21(0.06)$ & $20.14(0.07)$ & $20.28(0.05)$ & $20.47(0.05)$ \\
\hline & & & $20.77(0.09)$ & $20.73(0.10)$ & $20.85(0.08)$ & $20.60(0.06)$ & $20.59(0.07)$ & $20.58(0.05)$ \\
\hline & & & $20.76(0.08)$ & $20.64(0.05)$ & $20.70(0.06)$ & $20.75(0.06)$ & $20.82(0.08)$ & $20.58(0.07)$ \\
\hline & & & $20.51(0.07)$ & $20.43(0.09)$ & $20.23(0.05)$ & $20.26(0.05)$ & $20.27(0.06)$ & $20.28(0.10)$ \\
\hline \multirow[t]{4}{*}{2423} & 19.841 & 169.698 & $20.72(0.06)$ & $20.72(0.08)$ & $20.95(0.08)$ & $20.65(0.11)$ & $20.74(0.07)$ & $17.90(0.23)$ \\
\hline & & & $20.84(0.08)$ & $18.42(0.26)$ & $20.79(0.07)$ & $18.44(0.10)$ & $19.87(0.12)$ & $20.29(0.23)$ \\
\hline & & & $18.75(0.15)$ & $20.41(0.23)$ & $20.39(0.08)$ & 99.99 (9.99) & $20.43(0.08)$ & $20.97(0.11)$ \\
\hline & & & $20.22(0.09)$ & $20.92(0.11)$ & $18.90(0.11)$ & $21.06(0.12)$ & $20.13(0.08)$ & $19.95(0.27)$ \\
\hline \multirow[t]{4}{*}{2424} & 637.782 & 571.600 & $21.04(0.07)$ & $20.23(0.06)$ & $20.63(0.10)$ & $21.30(0.12)$ & $20.89(0.06)$ & $21.11(0.08)$ \\
\hline & & & $21.13(0.10)$ & $21.62(0.15)$ & $21.13(0.08)$ & $20.78(0.06)$ & $20.58(0.06)$ & $20.25(0.05)$ \\
\hline & & & $20.87(0.09)$ & $20.86(0.06)$ & $20.63(0.06)$ & $20.60(0.05)$ & $20.51(0.06)$ & $20.36(0.05)$ \\
\hline & & & $20.30(0.06)$ & $20.14(0.06)$ & $20.21(0.05)$ & $20.25(0.06)$ & $20.29(0.05)$ & $20.42(0.17)$ \\
\hline \multirow[t]{4}{*}{2427} & 319.817 & 513.646 & $20.79(0.06)$ & $20.28(0.08)$ & $20.69(0.08)$ & $20.91(0.12)$ & $21.08(0.08)$ & $21.20(0.09)$ \\
\hline & & & $20.90(0.09)$ & $21.14(0.13)$ & $20.84(0.07)$ & $20.78(0.07)$ & $20.76(0.09)$ & $20.29(0.05)$ \\
\hline & & & $20.75(0.07)$ & $20.81(0.07)$ & $20.71(0.06)$ & $20.64(0.07)$ & $20.59(0.05)$ & $20.55(0.06)$ \\
\hline & & & $20.40(0.07)$ & $20.12(0.06)$ & $20.13(0.05)$ & $20.23(0.06)$ & $20.39(0.07)$ & $20.50(0.11)$ \\
\hline \multirow[t]{4}{*}{2428} & 698.016 & 398.013 & $20.83(0.07)$ & $20.19(0.07)$ & $20.47(0.07)$ & $20.79(0.08)$ & $20.35(0.06)$ & $20.65(0.07)$ \\
\hline & & & $20.69(0.08)$ & $20.77(0.08)$ & $20.86(0.07)$ & $20.60(0.07)$ & $20.75(0.07)$ & $20.99(0.08)$ \\
\hline & & & $20.06(0.06)$ & $20.19(0.05)$ & $20.32(0.05)$ & $20.46(0.05)$ & $20.53(0.06)$ & $20.62(0.06)$ \\
\hline & & & $20.73(0.08)$ & $20.85(0.08)$ & $20.93(0.07)$ & $20.70(0.08)$ & $20.83(0.11)$ & $19.90(0.09)$ \\
\hline \multirow[t]{4}{*}{2432} & 806.787 & 834.417 & $20.48(0.06)$ & $20.03(0.06)$ & $20.50(0.10)$ & $19.90(0.06)$ & $20.28(0.05)$ & $20.51(0.06)$ \\
\hline & & & $20.20(0.05)$ & $20.11(0.06)$ & $20.43(0.05)$ & $20.70(0.06)$ & $20.57(0.08)$ & $21.03(0.06)$ \\
\hline & & & $20.78(0.07)$ & $20.62(0.05)$ & $20.80(0.05)$ & 99.99 (9.99) & $21.03(0.06)$ & 99.99 (9.99) \\
\hline & & & $20.93(0.09)$ & $20.59(0.06)$ & 99.99 (9.99) & $20.35(0.06)$ & $20.57(0.11)$ & 99.99 (9.99) \\
\hline \multirow[t]{4}{*}{2436} & 643.429 & 672.785 & $20.58(0.05)$ & $21.06(0.09)$ & $20.66(0.09)$ & $20.63(0.09)$ & $20.18(0.05)$ & $20.26(0.05)$ \\
\hline & & & $20.34(0.06)$ & $20.40(0.07)$ & $20.39(0.05)$ & $20.41(0.05)$ & $20.73(0.07)$ & $21.04(0.06)$ \\
\hline & & & $20.32(0.06)$ & $20.20(0.05)$ & $20.42(0.06)$ & $20.67(0.05)$ & $20.57(0.06)$ & $20.87(0.07)$ \\
\hline & & & $21.10(0.12)$ & $21.01(0.09)$ & $20.88(0.06)$ & $20.51(0.07)$ & $20.70(0.08)$ & $22.35(0.73)$ \\
\hline
\end{tabular}


Table 8. continuation.

\begin{tabular}{|c|c|c|c|c|c|c|c|c|}
\hline \multirow[t]{4}{*}{$\overline{\mathrm{F} 2 \mathrm{ID}}$} & \multirow[t]{4}{*}{$\mathrm{x}$} & \multirow[t]{4}{*}{$\mathrm{y}$} & $2 \mathrm{Ks} 10(\sigma)$ & $2 \mathrm{Ks} 01(\sigma)$ & $2 \mathrm{Ks} 02(\sigma)$ & $2 \mathrm{Ks} 03(\sigma)$ & $2 \mathrm{Ks} 05(\sigma)$ & $2 \mathrm{Ks06}(\sigma)$ \\
\hline & & & $2 \mathrm{Ks} 07(\sigma)$ & $2 \mathrm{Ks} 08(\sigma)$ & $2 \mathrm{Ks} 09(\sigma)$ & $2 \mathrm{Ks} 11(\sigma)$ & $2 \mathrm{Ks} 12(\sigma)$ & $2 \mathrm{Ks} 13(\sigma)$ \\
\hline & & & $2 \mathrm{Ks} 14(\sigma)$ & $2 \mathrm{Ks} 15(\sigma)$ & $2 \mathrm{Ks} 16(\sigma)$ & $2 \mathrm{Ks} 17(\sigma)$ & $2 \mathrm{Ks} 18(\sigma)$ & $2 \mathrm{Ks} 19(\sigma)$ \\
\hline & & & $2 \mathrm{Ks} 20(\sigma)$ & $2 \mathrm{Ks} 21(\sigma)$ & $2 \mathrm{Ks} 22(\sigma)$ & $2 \mathrm{Ks} 23(\sigma)$ & $2 \mathrm{Ks} 24(\sigma)$ & $2 \mathrm{Ks} 04(\sigma)$ \\
\hline \multirow[t]{4}{*}{2438} & 860.170 & 34.060 & $20.70(0.05)$ & $99.99(9.99)$ & $20.57(0.07)$ & $20.43(0.08)$ & $20.36(0.06)$ & 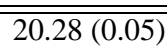 \\
\hline & & & $20.41(0.07)$ & $20.57(0.08)$ & $20.45(0.05)$ & $20.48(0.06)$ & $20.90(0.08)$ & $21.19(0.11)$ \\
\hline & & & $20.26(0.06)$ & $20.27(0.05)$ & $20.26(0.06)$ & $20.46(0.08)$ & $20.64(0.08)$ & $20.43(0.06)$ \\
\hline & & & $20.84(0.08)$ & $20.75(0.09)$ & $20.99(0.07)$ & $20.87(0.08)$ & $20.74(0.09)$ & $21.17(0.30)$ \\
\hline \multirow[t]{4}{*}{2447} & 232.207 & 4.549 & $20.71(0.06)$ & $21.08(0.10)$ & $21.22(0.13)$ & $20.97(0.11)$ & $21.10(0.09)$ & $21.43(0.08)$ \\
\hline & & & $21.18(0.08)$ & $21.22(0.13)$ & $21.00(0.10)$ & $20.54(0.06)$ & $20.50(0.07)$ & $20.36(0.05)$ \\
\hline & & & $20.54(0.07)$ & $20.31(0.06)$ & $20.30(0.05)$ & $20.25(0.08)$ & $20.11(0.05)$ & $20.21(0.06)$ \\
\hline & & & $20.48(0.07)$ & $20.36(0.07)$ & $20.58(0.06)$ & $21.26(0.13)$ & $20.96(0.10)$ & $21.07(0.24)$ \\
\hline \multirow[t]{4}{*}{2451} & 501.540 & 825.126 & $20.64(0.05)$ & $20.58(0.09)$ & $20.20(0.07)$ & $20.17(0.07)$ & $20.18(0.05)$ & $20.44(0.05)$ \\
\hline & & & $20.30(0.06)$ & $20.60(0.07)$ & $20.46(0.06)$ & $20.48(0.06)$ & $99.99(9.99)$ & $21.06(0.07)$ \\
\hline & & & $20.27(0.05)$ & $20.34(0.05)$ & $20.47(0.06)$ & $20.65(0.06)$ & $20.63(0.05)$ & $20.88(0.08)$ \\
\hline & & & $21.06(0.11)$ & $21.01(0.12)$ & $21.19(0.10)$ & $21.91(0.17)$ & $20.97(0.09)$ & $20.94(0.22)$ \\
\hline \multirow[t]{4}{*}{2466} & 377.006 & 64.798 & $20.54(0.05)$ & $20.75(0.08)$ & $20.77(0.10)$ & $20.85(0.09)$ & $20.40(0.05)$ & $20.29(0.05)$ \\
\hline & & & $20.19(0.06)$ & $20.51(0.07)$ & $20.22(0.05)$ & $21.24(0.11)$ & $21.21(0.12)$ & $20.93(0.06)$ \\
\hline & & & $20.57(0.07)$ & $20.66(0.06)$ & $20.80(0.07)$ & $20.75(0.05)$ & $20.58(0.05)$ & $20.71(0.07)$ \\
\hline & & & $20.75(0.09)$ & $20.44(0.09)$ & $20.54(0.05)$ & $20.45(0.06)$ & $20.16(0.05)$ & $20.84(0.20)$ \\
\hline \multirow[t]{4}{*}{2467} & 133.825 & 116.259 & $21.04(0.07)$ & $20.14(0.07)$ & $20.19(0.07)$ & $20.74(0.10)$ & $20.30(0.05)$ & $20.65(0.07)$ \\
\hline & & & $20.82(0.09)$ & $21.43(0.17)$ & $20.99(0.07)$ & $20.89(0.07)$ & $20.86(0.10)$ & $20.95(0.08)$ \\
\hline & & & $20.25(0.06)$ & $20.03(0.06)$ & $20.20(0.06)$ & $20.40(0.09)$ & $20.24(0.06)$ & $20.57(0.07)$ \\
\hline & & & $20.68(0.09)$ & $20.58(0.08)$ & $20.81(0.06)$ & $20.55(0.07)$ & $20.74(0.07)$ & $20.10(0.13)$ \\
\hline \multirow[t]{4}{*}{2471} & 600.781 & 221.610 & $21.21(0.14)$ & $21.66(0.24)$ & $22.49(0.60)$ & $21.30(0.17)$ & $21.29(0.19)$ & $20.26(0.25)$ \\
\hline & & & $21.51(0.22)$ & $18.89(0.17)$ & $21.29(0.13)$ & $19.15(0.11)$ & $20.25(0.12)$ & $19.61(0.19)$ \\
\hline & & & $19.99(0.10)$ & $20.34(0.16)$ & $21.38(0.23)$ & $21.67(0.19)$ & $21.35(0.19)$ & $22.27(0.79)$ \\
\hline & & & $21.21(0.18)$ & $21.14(0.22)$ & $20.13(0.13)$ & $22.19(0.61)$ & $21.65(0.35)$ & $20.76(0.34)$ \\
\hline \multirow[t]{4}{*}{2482} & 488.647 & 486.885 & $20.63(0.05)$ & $20.88(0.08)$ & $21.19(0.11)$ & $22.45(0.29)$ & $20.95(0.07)$ & $20.87(0.07)$ \\
\hline & & & $21.23(0.11)$ & $21.63(0.12)$ & $20.88(0.07)$ & $20.61(0.06)$ & $20.37(0.06)$ & $20.24(0.05)$ \\
\hline & & & $21.03(0.10)$ & $20.95(0.06)$ & $20.56(0.05)$ & $20.25(0.05)$ & $20.32(0.05)$ & $20.21(0.05)$ \\
\hline & & & $20.23(0.06)$ & $20.21(0.06)$ & $20.21(0.05)$ & $20.55(0.06)$ & $20.87(0.07)$ & $21.25(0.22)$ \\
\hline \multirow[t]{4}{*}{2487} & 647.817 & 43.051 & $20.44(0.05)$ & $20.66(0.08)$ & $20.82(0.09)$ & $20.48(0.10)$ & $20.27(0.05)$ & $20.29(0.05)$ \\
\hline & & & $20.21(0.06)$ & $20.42(0.07)$ & $20.21(0.05)$ & $20.84(0.06)$ & $21.30(0.11)$ & $20.99(0.05)$ \\
\hline & & & $20.21(0.05)$ & $20.40(0.05)$ & $20.49(0.05)$ & $20.65(0.05)$ & $20.79(0.06)$ & $20.70(0.06)$ \\
\hline & & & $20.79(0.10)$ & $20.75(0.09)$ & $20.76(0.07)$ & $20.79(0.08)$ & $20.78(0.09)$ & $20.71(0.14)$ \\
\hline \multirow[t]{4}{*}{2488} & 565.277 & 368.955 & $20.99(0.07)$ & $21.43(0.17)$ & $21.53(0.11)$ & $22.07(0.24)$ & $20.41(0.05)$ & $20.67(0.07)$ \\
\hline & & & $20.70(0.08)$ & $21.13(0.12)$ & $20.85(0.06)$ & $19.97(0.05)$ & $20.03(0.05)$ & $19.98(0.05)$ \\
\hline & & & $21.16(0.11)$ & $21.10(0.07)$ & $21.21(0.05)$ & $21.17(0.06)$ & $21.05(0.07)$ & $21.25(0.09)$ \\
\hline & & & $21.06(0.12)$ & $21.43(0.12)$ & $21.00(0.06)$ & $20.67(0.08)$ & $20.76(0.07)$ & $20.08(0.08)$ \\
\hline \multirow[t]{4}{*}{2489} & 847.263 & 472.621 & $20.62(0.06)$ & $20.05(0.07)$ & $19.98(0.06)$ & $20.10(0.06)$ & $20.10(0.05)$ & $20.22(0.05)$ \\
\hline & & & $20.43(0.07)$ & $20.54(0.07)$ & $20.63(0.06)$ & $21.04(0.07)$ & $20.79(0.08)$ & $21.03(0.06)$ \\
\hline & & & $20.50(0.07)$ & $20.53(0.05)$ & $20.70(0.06)$ & $20.87(0.07)$ & $20.94(0.06)$ & $20.92(0.07)$ \\
\hline & & & $20.94(0.10)$ & $21.12(0.15)$ & $20.85(0.07)$ & $20.63(0.06)$ & $20.51(0.06)$ & $20.08(0.08)$ \\
\hline \multirow[t]{4}{*}{2497} & 24.327 & 491.417 & $20.89(0.05)$ & $20.15(0.06)$ & $20.04(0.07)$ & $20.03(0.05)$ & $20.21(0.05)$ & $20.20(0.05)$ \\
\hline & & & $20.67(0.07)$ & $20.81(0.11)$ & $20.68(0.06)$ & $20.70(0.06)$ & $20.59(0.08)$ & $20.61(0.05)$ \\
\hline & & & $20.55(0.06)$ & $20.59(0.06)$ & $20.53(0.06)$ & 99.99 (9.99) & $20.69(0.07)$ & $20.70(0.06)$ \\
\hline & & & $20.65(0.08)$ & $20.66(0.10)$ & $20.79(0.06)$ & $20.81(0.08)$ & $20.51(0.09)$ & $20.21(0.13)$ \\
\hline \multirow[t]{4}{*}{2502} & 23.400 & 631.599 & $20.65(0.05)$ & $20.62(0.09)$ & $20.56(0.07)$ & $20.89(0.10)$ & $20.51(0.05)$ & $20.43(0.05)$ \\
\hline & & & $20.80(0.07)$ & $20.96(0.11)$ & $20.66(0.06)$ & $21.10(0.07)$ & $21.14(0.14)$ & $21.08(0.07)$ \\
\hline & & & $20.05(0.05)$ & $20.09(0.05)$ & $20.18(0.06)$ & 99.99 (9.99) & $20.36(0.06)$ & $20.46(0.06)$ \\
\hline & & & $20.73(0.08)$ & $20.52(0.07)$ & $20.62(0.05)$ & $20.91(0.08)$ & $20.91(0.10)$ & $20.17(0.24)$ \\
\hline
\end{tabular}


Table 8. continuation.

\begin{tabular}{|c|c|c|c|c|c|c|c|c|}
\hline \multirow[t]{4}{*}{$\bar{F}$ F2 ID } & \multirow[t]{4}{*}{$\mathrm{x}$} & \multirow[t]{4}{*}{$\mathrm{y}$} & $2 \mathrm{Ks} 10(\sigma)$ & $2 \mathrm{Ks} 01(\sigma)$ & $2 \mathrm{Ks} 02(\sigma)$ & $2 \mathrm{Ks} 03(\sigma)$ & $2 \mathrm{Ks} 05(\sigma)$ & $\overline{2 \mathrm{Ks} 06(\sigma)}$ \\
\hline & & & $2 \mathrm{Ks} 07(\sigma)$ & $2 \mathrm{Ks} 08(\sigma)$ & $2 \mathrm{Ks} 09(\sigma)$ & $2 \mathrm{Ks} 11(\sigma)$ & $2 \mathrm{Ks} 12(\sigma)$ & $2 \mathrm{Ks} 13(\sigma)$ \\
\hline & & & $2 \mathrm{Ks} 14(\sigma)$ & $2 \mathrm{Ks} 15(\sigma)$ & $2 \mathrm{Ks} 16(\sigma)$ & $2 \mathrm{Ks} 17(\sigma)$ & $2 \mathrm{Ks} 18(\sigma)$ & $2 \mathrm{Ks} 19(\sigma)$ \\
\hline & & & $2 \mathrm{Ks} 20(\sigma)$ & $2 \mathrm{Ks} 21(\sigma)$ & $2 \mathrm{Ks} 22(\sigma)$ & $2 \mathrm{Ks} 23(\sigma)$ & $2 \mathrm{Ks} 24(\sigma)$ & $2 \mathrm{Ks} 04(\sigma)$ \\
\hline \multirow[t]{4}{*}{$\overline{2506}$} & $\overline{85.337}$ & 339.939 & $20.61(0.06)$ & $20.46(0.10)$ & $\overline{20.76(0.11)}$ & $21.28(0.11)$ & $20.71(0.05)$ & 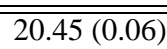 \\
\hline & & & $20.74(0.08)$ & $20.83(0.09)$ & $20.68(0.07)$ & $20.85(0.07)$ & $20.57(0.07)$ & $20.36(0.05)$ \\
\hline & & & $20.89(0.08)$ & $20.62(0.07)$ & $20.64(0.06)$ & $20.61(0.09)$ & $20.47(0.06)$ & $20.37(0.05)$ \\
\hline & & & $20.26(0.06)$ & $20.29(0.05)$ & $20.23(0.05)$ & $20.31(0.06)$ & $20.54(0.06)$ & $20.89(0.12)$ \\
\hline \multirow[t]{4}{*}{2510} & 71.269 & 18.369 & $22.38(0.18)$ & 99.99 (9.99) & $21.67(0.16)$ & $21.25(0.18)$ & $22.24(0.17)$ & 99.99 (9.99) \\
\hline & & & $21.93(0.19)$ & 99.99 (9.99) & $21.89(0.14)$ & $21.64(0.19)$ & $21.13(0.10)$ & $22.45(0.38)$ \\
\hline & & & $22.75(0.30)$ & $21.09(0.10)$ & $21.28(0.17)$ & $20.30(0.07)$ & $20.42(0.11)$ & $20.53(0.06)$ \\
\hline & & & $20.73(0.08)$ & $20.70(0.11)$ & $20.76(0.07)$ & $20.08(0.05)$ & $20.13(0.05)$ & 99.99 (9.99) \\
\hline \multirow[t]{4}{*}{2515} & 542.975 & 765.505 & $20.98(0.05)$ & $19.99(0.06)$ & $20.09(0.06)$ & $20.08(0.06)$ & $20.43(0.05)$ & $20.68(0.06)$ \\
\hline & & & $20.51(0.06)$ & $21.16(0.09)$ & $20.78(0.06)$ & $20.70(0.06)$ & $20.63(0.07)$ & $20.43(0.06)$ \\
\hline & & & $20.81(0.07)$ & $20.86(0.06)$ & $20.74(0.05)$ & $20.86(0.09)$ & $20.71(0.07)$ & $20.60(0.05)$ \\
\hline & & & $20.52(0.07)$ & $20.53(0.07)$ & $20.27(0.05)$ & $20.19(0.05)$ & $20.11(0.06)$ & $20.03(0.10)$ \\
\hline \multirow[t]{4}{*}{2517} & 430.972 & 423.967 & $20.33(0.05)$ & $20.67(0.10)$ & $21.03(0.10)$ & $21.04(0.11)$ & $20.14(0.05)$ & $20.33(0.06)$ \\
\hline & & & $20.30(0.06)$ & $20.32(0.08)$ & $20.26(0.05)$ & $20.84(0.08)$ & $20.53(0.07)$ & $20.26(0.06)$ \\
\hline & & & $20.67(0.07)$ & $20.84(0.07)$ & $20.77(0.06)$ & $20.89(0.07)$ & $20.99(0.05)$ & $21.39(0.09)$ \\
\hline & & & $21.39(0.13)$ & $21.32(0.10)$ & $21.25(0.10)$ & $21.54(0.12)$ & $21.78(0.21)$ & $20.53(0.09)$ \\
\hline \multirow[t]{4}{*}{2519} & 870.694 & 395.502 & $20.18(0.04)$ & 99.99 (9.99) & $20.54(0.09)$ & $20.51(0.08)$ & $20.57(0.06)$ & $20.29(0.05)$ \\
\hline & & & $20.38(0.07)$ & $20.84(0.07)$ & $20.27(0.05)$ & $21.90(0.15)$ & $21.95(0.30)$ & $21.26(0.06)$ \\
\hline & & & $20.43(0.06)$ & $20.49(0.05)$ & $20.53(0.05)$ & $20.73(0.10)$ & $20.91(0.06)$ & $20.80(0.07)$ \\
\hline & & & $21.56(0.19)$ & $21.01(0.09)$ & $21.37(0.09)$ & $21.42(0.13)$ & $21.69(0.16)$ & $21.29(0.28)$ \\
\hline \multirow[t]{4}{*}{2522} & 740.714 & 381.765 & $20.43(0.05)$ & $20.77(0.08)$ & $21.16(0.14)$ & $20.76(0.11)$ & $20.90(0.06)$ & $20.71(0.07)$ \\
\hline & & & $20.93(0.12)$ & $20.98(0.09)$ & $20.73(0.08)$ & $20.22(0.05)$ & $20.31(0.06)$ & $20.37(0.05)$ \\
\hline & & & $20.56(0.06)$ & $20.41(0.05)$ & $20.33(0.05)$ & $20.42(0.07)$ & $20.51(0.05)$ & $20.46(0.06)$ \\
\hline & & & $20.62(0.09)$ & $20.55(0.08)$ & $20.86(0.07)$ & $20.80(0.07)$ & $20.79(0.09)$ & $21.08(0.26)$ \\
\hline \multirow[t]{4}{*}{2530} & 112.241 & 590.792 & $21.18(0.07)$ & $20.73(0.09)$ & $20.69(0.12)$ & $20.44(0.08)$ & $20.64(0.05)$ & $20.97(0.08)$ \\
\hline & & & $21.29(0.10)$ & $21.66(0.15)$ & $21.30(0.10)$ & $20.54(0.05)$ & $20.56(0.07)$ & $20.35(0.05)$ \\
\hline & & & $20.62(0.07)$ & $20.57(0.05)$ & $20.38(0.05)$ & $20.21(0.05)$ & $20.26(0.05)$ & $20.36(0.05)$ \\
\hline & & & $20.75(0.10)$ & $20.38(0.06)$ & $20.87(0.08)$ & $21.01(0.08)$ & $20.91(0.08)$ & $20.32(0.12)$ \\
\hline \multirow[t]{4}{*}{2535} & 482.608 & 510.925 & $21.68(0.19)$ & $20.50(0.15)$ & $21.80(0.40)$ & $20.81(0.25)$ & $20.30(0.14)$ & $19.48(0.08)$ \\
\hline & & & $21.15(0.13)$ & $19.01(0.08)$ & $21.66(0.23)$ & $20.77(0.12)$ & $21.21(0.15)$ & $20.66(0.21)$ \\
\hline & & & $20.81(0.10)$ & $21.17(0.14)$ & $21.23(0.18)$ & $22.75(0.50)$ & $22.01(0.24)$ & $21.24(0.13)$ \\
\hline & & & $20.88(0.11)$ & $21.34(0.16)$ & $20.81(0.15)$ & $21.48(0.18)$ & $22.99(0.73)$ & $21.18(0.29)$ \\
\hline \multirow[t]{4}{*}{2549} & 447.949 & 58.256 & $20.02(0.05)$ & $20.57(0.09)$ & $20.62(0.08)$ & $20.54(0.11)$ & $20.66(0.06)$ & $20.63(0.06)$ \\
\hline & & & $20.56(0.06)$ & $20.35(0.07)$ & $20.21(0.05)$ & $20.70(0.06)$ & $20.56(0.07)$ & $20.29(0.05)$ \\
\hline & & & $20.55(0.06)$ & $20.75(0.06)$ & $20.79(0.06)$ & $20.86(0.07)$ & $20.86(0.07)$ & $20.82(0.06)$ \\
\hline & & & $20.88(0.11)$ & $20.66(0.11)$ & $20.72(0.07)$ & $20.79(0.07)$ & $20.74(0.09)$ & $21.38(0.27)$ \\
\hline \multirow[t]{4}{*}{2550} & 97.797 & 624.016 & $21.56(0.16)$ & $21.26(0.23)$ & $21.12(0.13)$ & $20.32(0.13)$ & $19.54(0.08)$ & $99.99(9.99)$ \\
\hline & & & $21.65(0.22)$ & 99.99 (9.99) & $21.15(0.17)$ & $20.43(0.06)$ & $21.72(0.17)$ & $21.28(0.26)$ \\
\hline & & & 99.99 (9.99) & $21.81(0.37)$ & 99.99 (9.99) & $99.99(9.99)$ & $99.99(9.99)$ & $22.54(0.65)$ \\
\hline & & & 99.99 (9.99) & $25.50(7.57)$ & $21.98(0.37)$ & 99.99 (9.99) & 99.99 (9.99) & $20.02(0.42)$ \\
\hline \multirow[t]{4}{*}{2554} & 629.672 & 131.178 & $20.76(0.05)$ & $20.65(0.09)$ & $20.50(0.07)$ & $20.07(0.06)$ & $20.70(0.05)$ & $21.25(0.08)$ \\
\hline & & & $20.81(0.08)$ & $21.28(0.11)$ & $20.99(0.08)$ & $19.88(0.05)$ & $20.01(0.05)$ & $20.27(0.05)$ \\
\hline & & & $21.39(0.12)$ & $21.29(0.09)$ & $21.19(0.09)$ & $21.07(0.07)$ & $21.23(0.08)$ & $21.19(0.08)$ \\
\hline & & & $21.08(0.09)$ & $21.49(0.17)$ & $21.05(0.07)$ & $21.03(0.08)$ & $20.47(0.06)$ & $20.30(0.09)$ \\
\hline \multirow[t]{4}{*}{2567} & 503.912 & 145.854 & $20.68(0.06)$ & $20.30(0.07)$ & $20.35(0.09)$ & $20.54(0.09)$ & $20.20(0.05)$ & $20.34(0.06)$ \\
\hline & & & $20.47(0.08)$ & $20.53(0.08)$ & $20.78(0.07)$ & $20.03(0.07)$ & $21.17(0.11)$ & $20.87(0.07)$ \\
\hline & & & $20.38(0.07)$ & $20.55(0.07)$ & $20.79(0.07)$ & $20.89(0.09)$ & $20.85(0.08)$ & $20.74(0.08)$ \\
\hline & & & $20.83(0.10)$ & $20.62(0.12)$ & $20.63(0.07)$ & $20.75(0.07)$ & $20.36(0.06)$ & $19.94(0.08)$ \\
\hline
\end{tabular}


Table 8. continuation.

\begin{tabular}{|c|c|c|c|c|c|c|c|c|}
\hline \multirow[t]{4}{*}{$\overline{\mathrm{F} 2 \mathrm{ID}}$} & \multirow[t]{4}{*}{$\mathrm{x}$} & \multirow[t]{4}{*}{$\mathrm{y}$} & $2 \mathrm{Ks} 10(\sigma)$ & $2 \mathrm{Ks} 01(\sigma)$ & $2 \mathrm{Ks} 02(\sigma)$ & $2 \mathrm{Ks} 03(\sigma)$ & $2 \mathrm{Ks} 05(\sigma)$ & $2 \mathrm{Ks06}(\sigma)$ \\
\hline & & & $2 \mathrm{Ks} 07(\sigma)$ & $2 \mathrm{Ks} 08(\sigma)$ & $2 \mathrm{Ks} 09(\sigma)$ & $2 \mathrm{Ks} 11(\sigma)$ & $2 \mathrm{Ks} 12(\sigma)$ & $2 \mathrm{Ks} 13(\sigma)$ \\
\hline & & & $2 \mathrm{Ks} 14(\sigma)$ & $2 \mathrm{Ks} 15(\sigma)$ & $2 \mathrm{Ks} 16(\sigma)$ & $2 \mathrm{Ks} 17(\sigma)$ & $2 \mathrm{Ks} 18(\sigma)$ & $2 \mathrm{Ks} 19(\sigma)$ \\
\hline & & & $2 \mathrm{Ks} 20(\sigma)$ & $2 \mathrm{Ks} 21(\sigma)$ & $2 \mathrm{Ks} 22(\sigma)$ & $2 \mathrm{Ks} 23(\sigma)$ & $2 \mathrm{Ks} 24(\sigma)$ & $2 \mathrm{Ks} 04(\sigma)$ \\
\hline \multirow[t]{4}{*}{2590} & 8817.535 & 94.909 & $21.01(0.08)$ & $20.68(0.12)$ & $20.78(0.10)$ & $20.83(0.07)$ & $20.42(0.07)$ & $20.25(0.05)$ \\
\hline & & & $20.71(0.09)$ & $20.82(0.11)$ & $20.59(0.07)$ & $20.65(0.07)$ & $20.61(0.07)$ & $20.71(0.07)$ \\
\hline & & & $21.22(0.09)$ & $20.93(0.06)$ & $20.67(0.10)$ & $20.60(0.09)$ & $20.66(0.10)$ & $20.63(0.09)$ \\
\hline & & & $20.34(0.07)$ & $20.35(0.08)$ & $20.35(0.06)$ & $20.00(0.06)$ & $20.34(0.06)$ & $20.55(0.15)$ \\
\hline \multirow[t]{4}{*}{2591} & 674.688 & 425.903 & $20.57(0.06)$ & $21.02(0.09)$ & $20.70(0.08)$ & $20.98(0.08)$ & $20.13(0.05)$ & $20.21(0.05)$ \\
\hline & & & $20.41(0.07)$ & $20.53(0.07)$ & $20.51(0.06)$ & $20.83(0.07)$ & $20.67(0.07)$ & $20.71(0.06)$ \\
\hline & & & $20.64(0.06)$ & $20.69(0.05)$ & $20.77(0.06)$ & $20.89(0.08)$ & $20.78(0.06)$ & $20.52(0.06)$ \\
\hline & & & $20.34(0.05)$ & $20.49(0.08)$ & $20.30(0.05)$ & $20.33(0.06)$ & $20.69(0.07)$ & $20.75(0.11)$ \\
\hline \multirow[t]{4}{*}{2593} & 723.163 & 474.360 & $20.90(0.05)$ & $20.67(0.07)$ & $20.26(0.07)$ & $20.31(0.09)$ & $20.78(0.06)$ & $20.65(0.05)$ \\
\hline & & & $20.96(0.09)$ & $21.39(0.11)$ & $20.86(0.07)$ & $20.37(0.05)$ & $20.37(0.07)$ & $20.37(0.05)$ \\
\hline & & & $20.49(0.07)$ & $20.34(0.05)$ & $20.25(0.05)$ & $20.38(0.05)$ & $20.53(0.05)$ & $20.66(0.06)$ \\
\hline & & & $20.73(0.08)$ & $21.04(0.10)$ & $20.95(0.06)$ & $20.59(0.07)$ & $20.83(0.06)$ & $20.32(0.09)$ \\
\hline \multirow[t]{4}{*}{2603} & 25.643 & 161.351 & $21.05(0.10)$ & $20.72(0.09)$ & $20.87(0.12)$ & $20.92(0.16)$ & $20.89(0.10)$ & $18.59(0.21)$ \\
\hline & & & $20.91(0.10)$ & $18.09(0.26)$ & $20.83(0.08)$ & $18.41(0.09)$ & $23.14(1.47)$ & $20.71(0.39)$ \\
\hline & & & $20.03(0.47)$ & $19.36(0.35)$ & $20.86(0.09)$ & 99.99 (9.99) & $20.86(0.13)$ & $21.17(0.15)$ \\
\hline & & & $20.31(0.10)$ & $20.85(0.13)$ & $19.56(0.19)$ & $21.45(0.18)$ & $21.38(0.20)$ & $19.73(0.25)$ \\
\hline \multirow[t]{4}{*}{2623} & 828.670 & 837.169 & $20.44(0.05)$ & $20.17(0.06)$ & $20.84(0.13)$ & $20.57(0.09)$ & $20.40(0.06)$ & $20.76(0.07)$ \\
\hline & & & $20.64(0.07)$ & $20.62(0.07)$ & $20.47(0.06)$ & $20.84(0.06)$ & $20.68(0.07)$ & $20.59(0.05)$ \\
\hline & & & $20.45(0.06)$ & $20.77(0.06)$ & $20.75(0.05)$ & 99.99 (9.99) & $21.04(0.11)$ & $21.19(0.11)$ \\
\hline & & & $20.49(0.07)$ & $20.63(0.09)$ & $21.02(0.06)$ & $19.85(0.05)$ & 99.99 (9.99) & 99.99 (9.99) \\
\hline \multirow[t]{4}{*}{2645} & 79.945 & 365.099 & $20.76(0.06)$ & $20.31(0.06)$ & $20.27(0.07)$ & $20.61(0.07)$ & $20.33(0.05)$ & $20.39(0.05)$ \\
\hline & & & $20.73(0.07)$ & $20.90(0.13)$ & $20.71(0.07)$ & $20.83(0.06)$ & $20.70(0.08)$ & $20.84(0.06)$ \\
\hline & & & $20.22(0.05)$ & $20.26(0.05)$ & $20.40(0.06)$ & $20.55(0.07)$ & $20.63(0.05)$ & $20.81(0.06)$ \\
\hline & & & $20.81(0.09)$ & $20.89(0.09)$ & $20.93(0.07)$ & $20.91(0.07)$ & $20.64(0.07)$ & $20.05(0.08)$ \\
\hline \multirow[t]{4}{*}{2654} & 642.679 & 572.352 & $20.69(0.05)$ & $20.07(0.06)$ & $20.24(0.06)$ & $20.23(0.06)$ & $20.18(0.05)$ & $20.40(0.06)$ \\
\hline & & & $20.47(0.07)$ & $20.99(0.10)$ & $20.63(0.06)$ & $20.73(0.06)$ & $20.77(0.07)$ & $20.66(0.05)$ \\
\hline & & & $20.41(0.06)$ & $20.46(0.05)$ & $20.62(0.06)$ & $20.72(0.07)$ & $20.74(0.06)$ & $20.97(0.06)$ \\
\hline & & & $20.91(0.09)$ & $20.75(0.08)$ & $20.82(0.07)$ & $20.93(0.07)$ & $20.79(0.08)$ & $20.00(0.11)$ \\
\hline \multirow[t]{4}{*}{2665} & 159.258 & 341.052 & $21.03(0.06)$ & $20.28(0.06)$ & $20.38(0.09)$ & $20.77(0.10)$ & $20.99(0.06)$ & 20.80 \\
\hline & & & $21.08(0.11)$ & $21.06(0.09)$ & $20.90(0.07)$ & $20.82(0.06)$ & $20.57(0.07)$ & $20.42(0.05)$ \\
\hline & & & $20.88(0.07)$ & $20.63(0.06)$ & $20.41(0.05)$ & $20.41(0.05)$ & $20.30(0.05)$ & $20.39(0.05)$ \\
\hline & & & $20.27(0.06)$ & $20.24(0.06)$ & $20.34(0.05)$ & $20.64(0.06)$ & $21.06(0.08)$ & $20.47(0.10)$ \\
\hline \multirow[t]{4}{*}{2667} & 641.325 & 564.165 & $20.57(0.05)$ & $20.44(0.07)$ & $20.67(0.09)$ & $20.98(0.13)$ & $20.90(0.06)$ & $20.86(0.07)$ \\
\hline & & & $20.85(0.08)$ & $21.27(0.12)$ & $20.72(0.06)$ & $20.43(0.05)$ & $20.26(0.06)$ & $20.46(0.05)$ \\
\hline & & & $20.40(0.06)$ & $20.41(0.06)$ & $20.32(0.05)$ & $20.27(0.05)$ & $20.39(0.05)$ & $20.49(0.06)$ \\
\hline & & & $20.67(0.07)$ & $20.74(0.08)$ & $21.11(0.09)$ & $21.04(0.08)$ & $21.06(0.08)$ & $20.88(0.14)$ \\
\hline \multirow[t]{4}{*}{2669} & 241.936 & 58.066 & $20.68(0.06)$ & $20.37(0.09)$ & $20.78(0.11)$ & $20.91(0.12)$ & $20.60(0.05)$ & $20.91(0.06)$ \\
\hline & & & $20.81(0.08)$ & $21.02(0.12)$ & $20.70(0.07)$ & $20.94(0.09)$ & $20.92(0.09)$ & $20.71(0.05)$ \\
\hline & & & $20.45(0.07)$ & $20.38(0.05)$ & $20.63(0.06)$ & $20.68(0.07)$ & $20.46(0.06)$ & $20.55(0.06)$ \\
\hline & & & $20.44(0.07)$ & $20.55(0.07)$ & $20.25(0.05)$ & $20.17(0.06)$ & $20.10(0.06)$ & $20.16(0.09)$ \\
\hline \multirow[t]{4}{*}{2680} & 34.000 & 437.282 & $22.15(0.17)$ & $21.37(0.33)$ & $21.85(0.32)$ & $21.43(0.94)$ & $20.31(0.22)$ & $19.32(0.12)$ \\
\hline & & & $22.54(0.29)$ & $19.64(0.12)$ & $21.80(0.47)$ & 99.99 (9.99) & $22.94(0.71)$ & $20.86(0.34)$ \\
\hline & & & $22.79(0.38)$ & 99.99 (9.99) & $20.38(0.19)$ & 99.99 (9.99) & 99.99 (9.99) & $21.94(0.21)$ \\
\hline & & & 99.99 (9.99) & 99.99 (9.99) & $22.52(0.47)$ & $99.99(9.99)$ & $99.99(9.99)$ & 99.99 (9.99) \\
\hline \multirow[t]{4}{*}{2689} & 151.908 & 256.661 & $20.38(0.05)$ & $21.04(0.10)$ & $21.03(0.12)$ & $21.32(0.13)$ & $21.11(0.07)$ & $21.03(0.07)$ \\
\hline & & & $21.10(0.11)$ & $20.80(0.08)$ & $20.68(0.06)$ & $20.30(0.05)$ & $20.38(0.06)$ & $20.48(0.05)$ \\
\hline & & & $20.28(0.06)$ & $20.21(0.05)$ & $20.47(0.06)$ & $20.50(0.05)$ & $20.66(0.05)$ & $20.81(0.07)$ \\
\hline & & & $20.89(0.09)$ & $21.06(0.10)$ & $21.00(0.07)$ & $21.10(0.07)$ & $21.10(0.11)$ & $21.21(0.18)$ \\
\hline
\end{tabular}


Table 8. continuation.

\begin{tabular}{|c|c|c|c|c|c|c|c|c|}
\hline \multirow[t]{4}{*}{$\bar{F}$ F2 ID } & \multirow[t]{4}{*}{$\mathrm{x}$} & \multirow[t]{4}{*}{$\mathrm{y}$} & $2 \mathrm{Ks} 10(\sigma)$ & $2 \mathrm{Ks} 01(\sigma)$ & $2 \mathrm{Ks} 02(\sigma)$ & $2 \mathrm{Ks} 03(\sigma)$ & $2 \mathrm{Ks} 05(\sigma)$ & $\overline{2 \mathrm{Ks} 06(\sigma)}$ \\
\hline & & & $2 \mathrm{Ks} 07(\sigma)$ & $2 \mathrm{Ks} 08(\sigma)$ & $2 \mathrm{Ks} 09(\sigma)$ & $2 \mathrm{Ks} 11(\sigma)$ & $2 \mathrm{Ks} 12(\sigma)$ & $2 \mathrm{Ks} 13(\sigma)$ \\
\hline & & & $2 \mathrm{Ks} 14(\sigma)$ & $2 \mathrm{Ks} 15(\sigma)$ & $2 \mathrm{Ks} 16(\sigma)$ & $2 \mathrm{Ks} 17(\sigma)$ & $2 \mathrm{Ks} 18(\sigma)$ & $2 \mathrm{Ks} 19(\sigma)$ \\
\hline & & & $2 \mathrm{Ks} 20(\sigma)$ & $2 \mathrm{Ks} 21(\sigma)$ & $2 \mathrm{Ks} 22(\sigma)$ & $2 \mathrm{Ks} 23(\sigma)$ & $2 \mathrm{Ks} 24(\sigma)$ & $2 \mathrm{Ks} 04(\sigma)$ \\
\hline \multirow[t]{4}{*}{2691} & 308.731 & 775.795 & $20.47(0.05)$ & $21.13(0.09)$ & $20.83(0.11)$ & $20.90(0.09)$ & $20.99(0.07)$ & $\overline{21.10(0.07)}$ \\
\hline & & & $21.14(0.10)$ & $20.75(0.09)$ & $20.78(0.06)$ & $20.73(0.06)$ & $20.37(0.05)$ & $20.14(0.05)$ \\
\hline & & & $20.86(0.08)$ & $20.90(0.06)$ & $20.80(0.05)$ & $20.75(0.07)$ & $20.63(0.06)$ & $20.86(0.06)$ \\
\hline & & & $20.78(0.08)$ & $20.80(0.12)$ & $20.58(0.05)$ & $20.21(0.05)$ & $20.06(0.06)$ & $20.84(0.14)$ \\
\hline \multirow[t]{4}{*}{2695} & 595.973 & 699.644 & $20.45(0.05)$ & $20.67(0.09)$ & $20.92(0.13)$ & $21.11(0.09)$ & $21.15(0.06)$ & $21.09(0.07)$ \\
\hline & & & $20.95(0.10)$ & $21.01(0.08)$ & $20.84(0.07)$ & $20.21(0.05)$ & $20.12(0.05)$ & $20.34(0.05)$ \\
\hline & & & $20.83(0.09)$ & $20.55(0.05)$ & $20.47(0.06)$ & $20.46(0.07)$ & $20.52(0.06)$ & $20.69(0.06)$ \\
\hline & & & $20.71(0.08)$ & $20.65(0.07)$ & $20.82(0.08)$ & $20.99(0.08)$ & $20.92(0.09)$ & $20.85(0.13)$ \\
\hline \multirow[t]{4}{*}{2696} & 155.024 & 109.834 & $20.70(0.05)$ & $20.23(0.07)$ & $20.31(0.06)$ & $20.31(0.06)$ & $20.67(0.06)$ & $21.02(0.07)$ \\
\hline & & & $20.74(0.09)$ & $20.66(0.07)$ & $20.79(0.07)$ & $20.85(0.06)$ & $20.72(0.07)$ & $20.49(0.05)$ \\
\hline & & & $20.86(0.08)$ & $20.51(0.06)$ & $20.50(0.08)$ & $20.66(0.06)$ & $20.32(0.06)$ & $20.40(0.05)$ \\
\hline & & & $20.39(0.06)$ & $20.55(0.08)$ & $20.64(0.05)$ & $21.01(0.08)$ & $20.84(0.07)$ & $19.90(0.06)$ \\
\hline \multirow[t]{4}{*}{2712} & 46.154 & 364.506 & $20.97(0.07)$ & $21.35(0.15)$ & $21.37(0.23)$ & $21.48(0.15)$ & $20.40(0.05)$ & $20.26(0.06)$ \\
\hline & & & $20.61(0.09)$ & $20.92(0.09)$ & $20.82(0.08)$ & $21.33(0.08)$ & $21.61(0.15)$ & $21.08(0.07)$ \\
\hline & & & $21.06(0.08)$ & $20.77(0.06)$ & $20.72(0.07)$ & $20.72(0.07)$ & $20.72(0.06)$ & $20.56(0.06)$ \\
\hline & & & $20.39(0.07)$ & $20.34(0.06)$ & $20.14(0.05)$ & $20.26(0.05)$ & $20.38(0.06)$ & $21.11(0.24)$ \\
\hline \multirow[t]{4}{*}{2719} & 182.164 & 425.840 & $20.88(0.05)$ & $20.32(0.07)$ & $20.34(0.07)$ & $20.91(0.09)$ & $20.97(0.06)$ & $20.97(0.07)$ \\
\hline & & & $21.01(0.10)$ & $21.34(0.15)$ & $20.82(0.07)$ & $21.18(0.10)$ & $20.87(0.08)$ & $20.25(0.05)$ \\
\hline & & & $21.29(0.12)$ & $21.07(0.07)$ & $20.88(0.07)$ & $20.49(0.06)$ & $20.39(0.05)$ & $20.33(0.06)$ \\
\hline & & & $20.32(0.07)$ & $20.23(0.07)$ & $20.39(0.05)$ & $20.57(0.06)$ & $20.76(0.07)$ & $20.38(0.15)$ \\
\hline \multirow[t]{4}{*}{2722} & 892.692 & 803.489 & $99.99(9.99)$ & $99.99(9.99)$ & $21.07(0.15)$ & $20.99(0.14)$ & $22.10(0.09)$ & $22.19(0.19)$ \\
\hline & & & $22.14(1.05)$ & 99.99 (9.99) & 99.99 (9.99) & $20.46(0.05)$ & $20.51(0.07)$ & $20.80(0.06)$ \\
\hline & & & $20.41(0.06)$ & $20.29(0.05)$ & $20.41(0.06)$ & 99.99 (9.99) & $20.51(0.09)$ & $20.79(0.06)$ \\
\hline & & & 99.99 (9.99) & 99.99 (9.99) & $19.41(3.13)$ & $21.36(0.15)$ & $21.34(0.13)$ & $21.51(0.52)$ \\
\hline \multirow[t]{4}{*}{2738} & 515.265 & 863.676 & $20.86(0.05)$ & $20.75(0.09)$ & $20.64(0.09)$ & $21.22(0.17)$ & $20.47(0.06)$ & $21.01(0.06)$ \\
\hline & & & $20.89(0.09)$ & $21.09(0.09)$ & $20.91(0.07)$ & 99.99 (9.99) & 99.99 (9.99) & 99.99 (9.99) \\
\hline & & & 99.99 (9.99) & 99.99 (9.99) & 99.99 (9.99) & $19.93(0.07)$ & 99.99 (9.99) & $20.16(0.05)$ \\
\hline & & & $99.99(9.99)$ & 99.99 (9.99) & 99.99 (9.99) & $99.99(9.99)$ & $99.99(9.99)$ & $20.24(0.19)$ \\
\hline \multirow[t]{4}{*}{2745} & 56.166 & 169.856 & $21.74(0.15)$ & $21.56(0.16)$ & $22.07(0.42)$ & $20.79(0.14)$ & $20.61(0.10)$ & 99.99 (9.99) \\
\hline & & & 23.77 (1.04) & $17.46(0.13)$ & $21.28(0.22)$ & $18.41(0.13)$ & $20.57(0.13)$ & $20.24(0.47)$ \\
\hline & & & $19.62(0.35)$ & $21.36(1.94)$ & $20.77(0.08)$ & $22.12(0.16)$ & $21.99(0.15)$ & $20.92(0.12)$ \\
\hline & & & $20.73(0.15)$ & $21.71(0.29)$ & 99.99 (9.99) & $20.91(0.21)$ & 99.99 (9.99) & $19.71(0.24)$ \\
\hline \multirow[t]{4}{*}{2760} & 169.819 & 228.192 & $20.66(0.06)$ & $20.87(0.09)$ & $20.78(0.10)$ & $20.97(0.10)$ & $20.88(0.08)$ & $20.45(0.10)$ \\
\hline & & & $21.39(0.15)$ & $21.35(0.17)$ & $20.96(0.08)$ & $21.03(0.08)$ & $20.48(0.07)$ & $20.25(0.09)$ \\
\hline & & & $20.94(0.09)$ & $20.74(0.08)$ & $20.78(0.11)$ & $20.81(0.09)$ & $20.67(0.06)$ & $20.53(0.06)$ \\
\hline & & & $20.44(0.09)$ & $20.40(0.08)$ & $20.24(0.05)$ & $20.40(0.05)$ & $20.21(0.06)$ & $21.02(0.19)$ \\
\hline \multirow[t]{4}{*}{2763} & 878.699 & 393.997 & $20.59(0.05)$ & $99.99(9.99)$ & $20.05(0.06)$ & $20.26(0.06)$ & $20.23(0.05)$ & $20.26(0.06)$ \\
\hline & & & $20.42(0.08)$ & $20.89(0.11)$ & $20.65(0.06)$ & $20.78(0.06)$ & $20.69(0.08)$ & $20.61(0.05)$ \\
\hline & & & $20.56(0.07)$ & $20.79(0.06)$ & $20.79(0.06)$ & $20.91(0.07)$ & $20.90(0.07)$ & $20.68(0.06)$ \\
\hline & & & $20.20(1.24)$ & $20.93(0.09)$ & $20.66(0.06)$ & $20.60(0.07)$ & $20.44(0.07)$ & $20.19(0.08)$ \\
\hline \multirow[t]{4}{*}{2766} & 118.033 & 820.844 & $20.55(0.05)$ & $20.68(0.07)$ & $20.82(0.10)$ & $20.33(0.06)$ & $20.55(0.05)$ & $20.52(0.06)$ \\
\hline & & & $20.54(0.06)$ & $20.45(0.06)$ & $20.43(0.05)$ & $20.45(0.05)$ & $20.91(0.08)$ & $20.74(0.06)$ \\
\hline & & & $20.23(0.06)$ & $20.39(0.06)$ & $20.46(0.06)$ & $20.71(0.07)$ & $20.83(0.08)$ & $20.99(0.08)$ \\
\hline & & & $21.19(0.13)$ & $21.09(0.12)$ & $21.25(0.09)$ & $20.93(0.10)$ & $21.13(0.11)$ & $21.64(0.46)$ \\
\hline \multirow[t]{4}{*}{2800} & 296.371 & 123.840 & $20.79(0.06)$ & $21.11(0.11)$ & $21.09(0.10)$ & $21.03(0.10)$ & $20.46(0.05)$ & $20.52(0.06)$ \\
\hline & & & $20.72(0.08)$ & $20.74(0.08)$ & $20.89(0.07)$ & $20.58(0.06)$ & $20.70(0.07)$ & $20.75(0.07)$ \\
\hline & & & $20.69(0.08)$ & $20.63(0.07)$ & $20.80(0.06)$ & $20.96(0.08)$ & $20.77(0.06)$ & $20.62(0.07)$ \\
\hline & & & $20.25(0.06)$ & $20.31(0.07)$ & $20.32(0.05)$ & $20.26(0.05)$ & $20.42(0.06)$ & $20.67(0.17)$ \\
\hline
\end{tabular}


Table 8. continuation.

\begin{tabular}{|c|c|c|c|c|c|c|c|c|}
\hline \multirow[t]{4}{*}{ F2 ID } & \multirow[t]{4}{*}{$\mathrm{x}$} & \multirow[t]{4}{*}{$\mathrm{y}$} & $2 \mathrm{Ks} 10(\sigma)$ & $2 \mathrm{Ks} 01(\sigma)$ & $2 \mathrm{Ks} 02(\sigma)$ & $2 \mathrm{Ks} 03(\sigma)$ & $2 \mathrm{Ks} 05(\sigma)$ & $2 \mathrm{Ks06}(\sigma)$ \\
\hline & & & $2 \mathrm{Ks} 07(\sigma)$ & $2 \mathrm{Ks} 08(\sigma)$ & $2 \mathrm{Ks} 09(\sigma)$ & $2 \mathrm{Ks} 11(\sigma)$ & $2 \mathrm{Ks} 12(\sigma)$ & $2 \mathrm{Ks} 13(\sigma)$ \\
\hline & & & $2 \mathrm{Ks} 14(\sigma)$ & $2 \mathrm{Ks} 15(\sigma)$ & $2 \mathrm{Ks} 16(\sigma)$ & $2 \mathrm{Ks} 17(\sigma)$ & $2 \mathrm{Ks} 18(\sigma)$ & $2 \mathrm{Ks} 19(\sigma)$ \\
\hline & & & $2 \mathrm{Ks} 20(\sigma)$ & $2 \mathrm{Ks} 21(\sigma)$ & $2 \mathrm{Ks} 22(\sigma)$ & $2 \mathrm{Ks} 23(\sigma)$ & $2 \mathrm{Ks} 24(\sigma)$ & $2 \mathrm{Ks} 04(\sigma)$ \\
\hline \multirow[t]{4}{*}{2818} & 696.376 & 337.355 & $20.66(0.06)$ & $20.73(0.10)$ & $21.33(0.14)$ & $22.56(0.43)$ & $21.23(0.08)$ & $21.46(0.09)$ \\
\hline & & & $21.15(0.09)$ & $21.36(0.12)$ & $20.94(0.08)$ & $20.44(0.06)$ & $20.41(0.06)$ & $20.65(0.06)$ \\
\hline & & & $20.46(0.06)$ & $20.36(0.05)$ & $20.33(0.05)$ & $20.47(0.05)$ & $20.56(0.05)$ & $20.66(0.06)$ \\
\hline & & & $21.58(0.20)$ & $20.97(0.11)$ & $21.24(0.08)$ & $21.34(0.10)$ & $21.22(0.11)$ & $21.19(0.24)$ \\
\hline \multirow[t]{4}{*}{2820} & 827.596 & 836.270 & $21.22(0.06)$ & $99.99(9.99)$ & $20.65(0.09)$ & $21.05(0.13)$ & $21.45(0.11)$ & $21.36(0.11)$ \\
\hline & & & $20.89(0.09)$ & $21.53(0.12)$ & $21.42(0.10)$ & $20.68(0.05)$ & $20.92(0.09)$ & $20.57(0.05)$ \\
\hline & & & $21.10(0.09)$ & $20.42(0.06)$ & $20.73(0.05)$ & $20.05(0.06)$ & $20.28(0.06)$ & $20.33(0.06)$ \\
\hline & & & $20.80(0.08)$ & $20.42(0.08)$ & $20.16(0.05)$ & $22.45(0.27)$ & $18.83(3.19)$ & $20.17(0.17)$ \\
\hline \multirow[t]{4}{*}{2821} & 809.613 & 297.404 & $20.64(0.05)$ & $20.47(0.07)$ & $20.61(0.08)$ & $21.04(0.12)$ & $20.96(0.07)$ & $20.68(0.07)$ \\
\hline & & & $20.84(0.09)$ & $20.91(0.08)$ & $20.76(0.07)$ & $20.50(0.05)$ & $20.48(0.07)$ & $20.10(0.05)$ \\
\hline & & & $21.25(0.10)$ & $21.31(0.07)$ & $21.13(0.06)$ & $20.98(0.06)$ & $20.83(0.05)$ & $20.51(0.06)$ \\
\hline & & & $20.31(0.07)$ & $20.24(0.07)$ & $20.40(0.05)$ & $20.43(0.06)$ & $20.39(0.06)$ & $20.22(0.12)$ \\
\hline \multirow[t]{4}{*}{2827} & 567.302 & 271.132 & $20.92(0.11)$ & $20.98(0.17)$ & $20.83(0.13)$ & $21.01(0.14)$ & $20.36(0.09)$ & $20.74(0.10)$ \\
\hline & & & $20.90(0.14)$ & $21.53(0.17)$ & $20.84(0.11)$ & $20.22(0.08)$ & $20.55(0.10)$ & $20.52(0.09)$ \\
\hline & & & $20.52(0.09)$ & $20.84(0.11)$ & $21.05(0.13)$ & $20.97(0.15)$ & $21.22(0.13)$ & $20.92(0.13)$ \\
\hline & & & $20.86(0.10)$ & $21.32(0.20)$ & $20.86(0.10)$ & $19.86(0.08)$ & $19.94(0.08)$ & $21.24(0.64)$ \\
\hline \multirow[t]{4}{*}{2836} & 574.932 & 643.524 & $21.21(0.07)$ & $20.88(0.08)$ & $20.77(0.09)$ & $20.93(0.12)$ & $20.43(0.05)$ & $20.66(0.06)$ \\
\hline & & & $20.66(0.08)$ & $21.00(0.10)$ & $20.93(0.07)$ & $20.84(0.07)$ & $20.54(0.07)$ & $20.31(0.05)$ \\
\hline & & & $20.74(0.08)$ & $20.52(0.06)$ & $20.42(0.06)$ & $20.38(0.07)$ & $20.33(0.06)$ & $20.48(0.06)$ \\
\hline & & & $20.74(0.08)$ & $20.51(0.08)$ & $20.83(0.07)$ & $21.17(0.07)$ & $21.49(0.13)$ & $20.56(0.10)$ \\
\hline \multirow[t]{4}{*}{2840} & 849.378 & 27.277 & $20.53(0.05)$ & $20.92(0.11)$ & $20.67(0.10)$ & $20.72(0.10)$ & $20.47(0.05)$ & $20.19(0.05)$ \\
\hline & & & $20.35(0.06)$ & $20.55(0.07)$ & $20.30(0.05)$ & $20.36(0.05)$ & $20.59(0.07)$ & $20.77(0.06)$ \\
\hline & & & $20.46(0.07)$ & $20.63(0.05)$ & $20.82(0.06)$ & $21.26(0.06)$ & $21.12(0.06)$ & $20.77(0.07)$ \\
\hline & & & $21.10(0.14)$ & $21.39(0.14)$ & $20.74(0.07)$ & $20.91(0.11)$ & $20.87(0.11)$ & 99.99 (9.99) \\
\hline \multirow[t]{4}{*}{2847} & 629.045 & 575.200 & $21.02(0.07)$ & $20.96(0.09)$ & $21.49(0.14)$ & $21.46(0.13)$ & $21.34(0.09)$ & $21.17(0.09)$ \\
\hline & & & $21.16(0.10)$ & $21.37(0.12)$ & $21.18(0.10)$ & $20.63(0.06)$ & $20.45(0.06)$ & $20.32(0.06)$ \\
\hline & & & $20.70(0.08)$ & $20.58(0.07)$ & $20.62(0.07)$ & $20.58(0.07)$ & $20.67(0.06)$ & $20.57(0.06)$ \\
\hline & & & $20.40(0.06)$ & $20.57(0.07)$ & $20.25(0.06)$ & $20.27(0.06)$ & $20.18(0.06)$ & $20.91(0.22)$ \\
\hline \multirow[t]{4}{*}{2861} & 220.957 & 500.059 & $20.65(0.05)$ & $20.48(0.07)$ & $20.77(0.08)$ & $21.18(0.15)$ & $20.67(0.06)$ & 20.70 \\
\hline & & & $20.81(0.09)$ & $20.69(0.08)$ & $20.65(0.06)$ & $20.86(0.07)$ & $20.79(0.07)$ & $20.41(0.05)$ \\
\hline & & & $21.00(0.11)$ & $21.06(0.07)$ & $20.86(0.07)$ & $20.86(0.07)$ & $20.73(0.06)$ & $20.65(0.06)$ \\
\hline & & & $20.25(0.06)$ & $20.45(0.08)$ & $20.31(0.06)$ & $20.28(0.06)$ & $20.21(0.06)$ & $20.30(0.15)$ \\
\hline \multirow[t]{4}{*}{2872} & 70.099 & 269.646 & $20.72(0.06)$ & $20.14(0.06)$ & $20.30(0.08)$ & $20.44(0.08)$ & $20.44(0.05)$ & $20.43(0.06)$ \\
\hline & & & $20.64(0.07)$ & $20.73(0.07)$ & $20.90(0.08)$ & $20.67(0.05)$ & $20.61(0.07)$ & $20.51(0.06)$ \\
\hline & & & $21.06(0.09)$ & $20.89(0.07)$ & $21.04(0.09)$ & $20.94(0.07)$ & $20.75(0.07)$ & $21.00(0.10)$ \\
\hline & & & $20.49(0.08)$ & $21.00(0.09)$ & $20.52(0.07)$ & $20.37(0.06)$ & $20.37(0.06)$ & $20.07(0.10)$ \\
\hline \multirow[t]{4}{*}{2877} & 768.979 & 122.517 & $20.60(0.05)$ & $20.63(0.07)$ & $20.43(0.08)$ & $20.16(0.07)$ & $20.39(0.05)$ & $20.26(0.05)$ \\
\hline & & & $20.24(0.06)$ & $20.52(0.07)$ & $20.35(0.05)$ & $21.07(0.08)$ & $21.26(0.11)$ & $21.22(0.07)$ \\
\hline & & & $20.39(0.06)$ & $20.59(0.05)$ & $20.75(0.05)$ & $20.91(0.09)$ & $21.18(0.09)$ & $21.09(0.08)$ \\
\hline & & & $21.19(0.13)$ & $21.12(0.12)$ & $20.95(0.07)$ & $21.14(0.08)$ & $20.59(0.07)$ & $20.58(0.11)$ \\
\hline \multirow[t]{4}{*}{2878} & 41.405 & 736.545 & $21.13(0.09)$ & $21.58(0.17)$ & $21.48(0.18)$ & $21.26(0.15)$ & $20.72(0.08)$ & $20.99(0.09)$ \\
\hline & & & $21.39(0.12)$ & $20.90(0.10)$ & $21.66(0.16)$ & $20.62(0.06)$ & $21.27(0.15)$ & $21.38(0.11)$ \\
\hline & & & $21.24(0.12)$ & $21.68(0.14)$ & $21.68(0.26)$ & $20.22(0.09)$ & $20.34(0.12)$ & $20.61(0.07)$ \\
\hline & & & $21.24(0.12)$ & $20.81(0.07)$ & $20.56(0.07)$ & $19.87(0.06)$ & $19.98(0.06)$ & 99.99 (9.99) \\
\hline \multirow[t]{4}{*}{2894} & 642.332 & 39.686 & $20.52(0.06)$ & $20.30(0.07)$ & $20.45(0.07)$ & $20.70(0.10)$ & $20.57(0.06)$ & $20.86(0.07)$ \\
\hline & & & $20.54(0.06)$ & $20.75(0.08)$ & $20.57(0.07)$ & $20.62(0.06)$ & $20.68(0.08)$ & $20.48(0.06)$ \\
\hline & & & $20.81(0.09)$ & $20.81(0.07)$ & $20.88(0.05)$ & $20.93(0.07)$ & $20.90(0.07)$ & $20.89(0.07)$ \\
\hline & & & $20.69(0.09)$ & $20.75(0.08)$ & $20.48(0.06)$ & $20.22(0.05)$ & $20.14(0.06)$ & $20.24(0.07)$ \\
\hline
\end{tabular}


Table 8. continuation.

\begin{tabular}{|c|c|c|c|c|c|c|c|c|}
\hline \multirow[t]{4}{*}{$\bar{F}$ F2 ID } & \multirow[t]{4}{*}{$\mathrm{x}$} & \multirow[t]{4}{*}{$\mathrm{y}$} & $2 \mathrm{Ks} 10(\sigma)$ & $2 \mathrm{Ks} 01(\sigma)$ & $2 \mathrm{Ks} 02(\sigma)$ & $2 \mathrm{Ks} 03(\sigma)$ & $2 \mathrm{Ks} 05(\sigma)$ & $\overline{2 \mathrm{Ks} 06(\sigma)}$ \\
\hline & & & $2 \mathrm{Ks} 07(\sigma)$ & $2 \mathrm{Ks} 08(\sigma)$ & $2 \mathrm{Ks} 09(\sigma)$ & $2 \mathrm{Ks} 11(\sigma)$ & $2 \mathrm{Ks} 12(\sigma)$ & $2 \mathrm{Ks} 13(\sigma)$ \\
\hline & & & $2 \mathrm{Ks} 14(\sigma)$ & $2 \mathrm{Ks} 15(\sigma)$ & $2 \mathrm{Ks} 16(\sigma)$ & $2 \mathrm{Ks} 17(\sigma)$ & $2 \mathrm{Ks} 18(\sigma)$ & $2 \mathrm{Ks} 19(\sigma)$ \\
\hline & & & $2 \mathrm{Ks} 20(\sigma)$ & $2 \mathrm{Ks} 21(\sigma)$ & $2 \mathrm{Ks} 22(\sigma)$ & $2 \mathrm{Ks} 23(\sigma)$ & $2 \mathrm{Ks} 24(\sigma)$ & $2 \mathrm{Ks} 04(\sigma)$ \\
\hline \multirow[t]{4}{*}{2916} & $\overline{6649.996}$ & 832.180 & $20.95(0.07)$ & $20.65(0.09)$ & $20.42(0.07)$ & $20.39(0.10)$ & $20.77(0.07)$ & 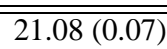 \\
\hline & & & $21.27(0.11)$ & $21.09(0.10)$ & $21.42(0.12)$ & $20.39(0.05)$ & $20.36(0.06)$ & $20.34(0.06)$ \\
\hline & & & $20.44(0.06)$ & $20.41(0.06)$ & $20.46(0.07)$ & $20.51(0.10)$ & $20.44(0.07)$ & $20.78(0.06)$ \\
\hline & & & $20.79(0.09)$ & $20.66(0.08)$ & $21.14(0.10)$ & $20.95(0.08)$ & $20.77(0.07)$ & $20.31(0.15)$ \\
\hline \multirow[t]{4}{*}{2938} & 783.337 & 462.572 & $21.22(0.07)$ & $21.52(0.18)$ & $21.05(0.11)$ & $20.97(0.11)$ & $20.33(0.05)$ & $20.32(0.06)$ \\
\hline & & & $20.61(0.07)$ & $21.02(0.09)$ & $20.83(0.07)$ & $21.09(0.07)$ & $21.21(0.13)$ & $20.48(0.05)$ \\
\hline & & & $20.85(0.09)$ & $20.76(0.05)$ & $20.50(0.05)$ & $20.57(0.06)$ & $20.48(0.05)$ & $20.58(0.06)$ \\
\hline & & & $20.61(0.08)$ & $20.44(0.06)$ & $20.69(0.06)$ & $21.15(0.11)$ & $20.95(0.09)$ & $20.24(0.09)$ \\
\hline \multirow[t]{4}{*}{2942} & 403.046 & 806.695 & $20.83(0.05)$ & $20.29(0.07)$ & $20.59(0.10)$ & $20.55(0.08)$ & $20.85(0.05)$ & $21.05(0.07)$ \\
\hline & & & $20.96(0.07)$ & $20.88(0.09)$ & $20.70(0.07)$ & $20.89(0.07)$ & $20.58(0.07)$ & $20.26(0.05)$ \\
\hline & & & $21.09(0.10)$ & $20.93(0.07)$ & $20.83(0.06)$ & $20.71(0.06)$ & $20.54(0.06)$ & $20.50(0.06)$ \\
\hline & & & $20.43(0.07)$ & $20.33(0.05)$ & $20.40(0.05)$ & $20.45(0.06)$ & $20.68(0.08)$ & $20.65(0.11)$ \\
\hline \multirow[t]{4}{*}{2960} & 867.599 & 815.115 & $21.21(0.08)$ & 99.99 (9.99) & $20.58(0.11)$ & $20.76(0.13)$ & $20.75(0.06)$ & $20.87(0.06)$ \\
\hline & & & $21.14(0.13)$ & $21.55(0.13)$ & $21.23(0.07)$ & $20.74(0.06)$ & $20.89(0.09)$ & $20.96(0.05)$ \\
\hline & & & $20.08(0.05)$ & $20.07(0.05)$ & $20.23(0.05)$ & $20.50(0.14)$ & $20.38(0.08)$ & $20.60(0.07)$ \\
\hline & & & $20.76(0.08)$ & $20.91(0.09)$ & $20.88(0.07)$ & $20.84(0.08)$ & $21.11(0.11)$ & $20.73(0.40)$ \\
\hline \multirow[t]{4}{*}{2964} & 315.637 & 137.195 & $21.12(0.07)$ & $21.16(0.14)$ & $21.09(0.13)$ & $21.47(0.19)$ & $21.38(0.09)$ & $20.88(0.07)$ \\
\hline & & & $21.34(0.11)$ & $20.83(0.09)$ & $21.26(0.11)$ & $20.43(0.06)$ & $20.64(0.08)$ & $20.89(0.06)$ \\
\hline & & & $20.74(0.09)$ & $20.60(0.06)$ & $20.67(0.05)$ & $20.60(0.06)$ & $20.58(0.07)$ & $20.58(0.06)$ \\
\hline & & & $20.34(0.08)$ & $20.39(0.07)$ & $20.31(0.05)$ & $20.20(0.05)$ & $20.35(0.06)$ & $20.53(0.11)$ \\
\hline \multirow[t]{4}{*}{2966} & 292.007 & 641.779 & $20.75(0.05)$ & $20.60(0.09)$ & $20.65(0.08)$ & $20.81(0.08)$ & $20.39(0.05)$ & $20.52(0.06)$ \\
\hline & & & $20.38(0.06)$ & $20.74(0.09)$ & $20.65(0.07)$ & $20.47(0.05)$ & $20.35(0.06)$ & $20.29(0.05)$ \\
\hline & & & $21.31(0.10)$ & $21.66(0.11)$ & $21.54(0.08)$ & $21.41(0.09)$ & $21.25(0.08)$ & $21.32(0.09)$ \\
\hline & & & $20.94(0.09)$ & $21.30(0.12)$ & $20.93(0.06)$ & $20.72(0.08)$ & $20.57(0.07)$ & $20.81(0.16)$ \\
\hline \multirow[t]{4}{*}{2977} & 534.652 & 425.818 & $20.89(0.06)$ & $20.89(0.10)$ & $20.45(0.09)$ & $20.46(0.09)$ & $21.01(0.06)$ & $21.35(0.08)$ \\
\hline & & & $21.36(0.13)$ & $21.40(0.11)$ & $21.24(0.10)$ & $20.57(0.06)$ & $20.43(0.07)$ & $20.42(0.05)$ \\
\hline & & & $20.54(0.07)$ & $20.50(0.05)$ & $20.40(0.05)$ & $20.43(0.06)$ & $20.50(0.06)$ & $20.70(0.06)$ \\
\hline & & & $20.61(0.06)$ & $20.71(0.08)$ & $20.79(0.07)$ & $20.66(0.06)$ & $20.73(0.07)$ & $20.85(0.17)$ \\
\hline \multirow[t]{4}{*}{2981} & 145.374 & 182.201 & $20.82(0.06)$ & $20.81(0.09)$ & $20.43(0.06)$ & $20.54(0.09)$ & $20.27(0.05)$ & $20.41(0.06)$ \\
\hline & & & $20.30(0.06)$ & $20.76(0.08)$ & $20.44(0.06)$ & $20.81(0.06)$ & $20.81(0.09)$ & $20.95(0.06)$ \\
\hline & & & $20.64(0.07)$ & $20.61(0.06)$ & $20.84(0.06)$ & $20.86(0.07)$ & $20.67(0.06)$ & $20.83(0.07)$ \\
\hline & & & $20.72(0.08)$ & $20.71(0.08)$ & $20.79(0.07)$ & $20.78(0.08)$ & $20.42(0.06)$ & $20.86(0.15)$ \\
\hline \multirow[t]{4}{*}{2988} & 501.439 & 630.638 & $20.85(0.06)$ & $20.93(0.13)$ & $21.06(0.13)$ & $20.54(0.08)$ & $20.46(0.05)$ & $20.85(0.07)$ \\
\hline & & & 20.89 (0.09) & $21.15(0.11)$ & $21.01(0.08)$ & $20.89(0.07)$ & $20.90(0.09)$ & $20.53(0.05)$ \\
\hline & & & $20.89(0.08)$ & $20.78(0.06)$ & $20.59(0.06)$ & $20.57(0.06)$ & $20.42(0.05)$ & $20.46(0.06)$ \\
\hline & & & $20.53(0.07)$ & $20.45(0.07)$ & $20.57(0.06)$ & $20.78(0.07)$ & $20.77(0.07)$ & $20.64(0.13)$ \\
\hline \multirow[t]{4}{*}{2998} & 307.871 & 427.580 & $20.80(0.05)$ & $20.38(0.06)$ & $20.28(0.06)$ & $20.42(0.08)$ & $20.21(0.05)$ & $20.26(0.05)$ \\
\hline & & & $20.37(0.06)$ & $20.72(0.07)$ & $20.74(0.07)$ & $20.93(0.06)$ & $20.52(0.07)$ & $20.72(0.05)$ \\
\hline & & & $20.82(0.07)$ & $20.93(0.06)$ & $20.95(0.06)$ & $20.99(0.06)$ & $20.98(0.06)$ & $20.83(0.06)$ \\
\hline & & & $21.09(0.11)$ & $20.73(0.08)$ & $20.81(0.06)$ & $20.72(0.06)$ & $20.54(0.07)$ & $20.17(0.08)$ \\
\hline \multirow[t]{4}{*}{3017} & 806.094 & 833.476 & $21.42(0.08)$ & $23.02(0.49)$ & $20.94(0.16)$ & $23.38(0.83)$ & $21.21(0.07)$ & $21.16(0.08)$ \\
\hline & & & $21.34(0.10)$ & 99.99 (9.99) & $21.32(0.08)$ & $20.79(0.06)$ & $21.18(0.12)$ & $21.12(0.06)$ \\
\hline & & & $21.20(0.09)$ & $20.98(0.06)$ & $20.73(0.07)$ & $20.04(0.05)$ & $20.31(0.05)$ & $20.01(0.06)$ \\
\hline & & & $20.54(0.07)$ & $20.94(0.11)$ & $20.23(0.05)$ & $23.27(0.57)$ & $23.74(1.02)$ & $20.41(0.15)$ \\
\hline \multirow[t]{4}{*}{3021} & 807.149 & 169.294 & $20.51(0.05)$ & $20.47(0.08)$ & $20.54(0.08)$ & $21.09(0.14)$ & $20.78(0.06)$ & $20.80(0.07)$ \\
\hline & & & $20.89(0.07)$ & $20.76(0.08)$ & $20.61(0.07)$ & $20.57(0.06)$ & $20.60(0.07)$ & $20.41(0.05)$ \\
\hline & & & $21.00(0.08)$ & $20.99(0.06)$ & $20.92(0.06)$ & $20.83(0.09)$ & $20.97(0.06)$ & $20.78(0.07)$ \\
\hline & & & $20.76(0.09)$ & $20.74(0.09)$ & $20.51(0.06)$ & $20.31(0.06)$ & $20.13(0.05)$ & $20.84(0.14)$ \\
\hline
\end{tabular}


Table 8. continuation.

\begin{tabular}{|c|c|c|c|c|c|c|c|c|}
\hline \multirow[t]{4}{*}{ F2 ID } & \multirow[t]{4}{*}{$\mathrm{x}$} & \multirow[t]{4}{*}{$\mathrm{y}$} & $2 \mathrm{Ks} 10(\sigma)$ & $2 \mathrm{Ks} 01(\sigma)$ & $2 \mathrm{Ks} 02(\sigma)$ & $2 \mathrm{Ks} 03(\sigma)$ & $2 \mathrm{Ks} 05(\sigma)$ & $2 \mathrm{Ks} 06(\sigma)$ \\
\hline & & & $2 \mathrm{Ks} 07(\sigma)$ & $2 \mathrm{Ks} 08(\sigma)$ & $2 \mathrm{Ks} 09(\sigma)$ & $2 \mathrm{Ks} 11(\sigma)$ & $2 \mathrm{Ks} 12(\sigma)$ & $2 \mathrm{Ks} 13(\sigma)$ \\
\hline & & & $2 \mathrm{Ks} 14(\sigma)$ & $2 \mathrm{Ks} 15(\sigma)$ & $2 \mathrm{Ks} 16(\sigma)$ & $2 \mathrm{Ks} 17(\sigma)$ & $2 \mathrm{Ks} 18(\sigma)$ & $2 \mathrm{Ks} 19(\sigma)$ \\
\hline & & & $2 \mathrm{Ks} 20(\sigma)$ & $2 \mathrm{Ks} 21(\sigma)$ & $2 \mathrm{Ks} 22(\sigma)$ & $2 \mathrm{Ks} 23(\sigma)$ & $2 \mathrm{Ks} 24(\sigma)$ & $2 \mathrm{Ks} 04(\sigma)$ \\
\hline \multirow[t]{4}{*}{3042} & \multirow[t]{4}{*}{382.359} & \multirow[t]{4}{*}{678.613} & $20.47(0.05)$ & $20.78(0.08)$ & $21.12(0.14)$ & $20.48(0.08)$ & $20.22(0.05)$ & $20.33(0.06)$ \\
\hline & & & $20.20(0.06)$ & $20.55(0.06)$ & $20.20(0.06)$ & $20.87(0.06)$ & $20.97(0.11)$ & $20.95(0.06)$ \\
\hline & & & $21.22(0.10)$ & $21.35(0.08)$ & $21.44(0.07)$ & $21.44(0.11)$ & $21.36(0.09)$ & $21.59(0.11)$ \\
\hline & & & $21.60(0.18)$ & $21.39(0.11)$ & $21.26(0.10)$ & $21.09(0.09)$ & $20.74(0.08)$ & $20.97(0.28)$ \\
\hline \multirow[t]{4}{*}{3048} & \multirow[t]{4}{*}{787.507} & \multirow[t]{4}{*}{356.560} & $20.54(0.05)$ & $20.53(0.07)$ & $20.82(0.10)$ & $20.75(0.09)$ & $20.73(0.06)$ & $20.35(0.05)$ \\
\hline & & & $20.65(0.07)$ & $20.34(0.06)$ & $20.29(0.06)$ & $20.94(0.09)$ & $20.86(0.09)$ & $20.80(0.07)$ \\
\hline & & & $21.11(0.09)$ & $20.95(0.07)$ & $21.02(0.06)$ & $20.93(0.08)$ & $20.91(0.05)$ & $20.56(0.06)$ \\
\hline & & & $20.35(0.07)$ & $20.51(0.09)$ & $20.38(0.06)$ & $20.45(0.07)$ & $20.71(0.09)$ & $20.64(0.15)$ \\
\hline \multirow[t]{4}{*}{3058} & \multirow[t]{4}{*}{897.064} & \multirow[t]{4}{*}{203.126} & 99.99 (9.99) & 99.99 (9.99) & $20.91(0.15)$ & $18.74(0.07)$ & $21.17(0.09)$ & $21.65(4.25)$ \\
\hline & & & 99.99 (9.99) & 99.99 (9.99) & 99.99 (9.99) & $21.03(0.08)$ & $21.28(0.08)$ & $20.74(0.05)$ \\
\hline & & & 99.99 (9.99) & 99.99 (9.99) & $99.99(9.99)$ & 99.99 (9.99) & 99.99 (9.99) & $21.10(0.30)$ \\
\hline & & & $99.99(9.99)$ & 99.99 (9.99) & 99.99 (9.99) & $20.99(0.08)$ & 99.99 (9.99) & $21.25(0.18)$ \\
\hline \multirow[t]{4}{*}{3059} & \multirow[t]{4}{*}{50.935} & \multirow[t]{4}{*}{645.011} & $21.05(0.06)$ & $21.00(0.09)$ & $21.25(0.16)$ & $21.41(0.16)$ & $20.45(0.06)$ & $20.48(0.07)$ \\
\hline & & & $20.80(0.10)$ & $20.97(0.10)$ & $20.94(0.08)$ & $21.43(0.11)$ & $20.94(0.11)$ & $20.48(0.05)$ \\
\hline & & & $21.16(0.09)$ & $20.84(0.07)$ & $20.45(0.05)$ & $20.46(0.05)$ & $20.40(0.06)$ & $20.43(0.06)$ \\
\hline & & & $20.65(0.08)$ & $20.75(0.08)$ & $20.64(0.06)$ & $20.83(0.08)$ & $20.88(0.09)$ & $20.71(0.20)$ \\
\hline \multirow[t]{4}{*}{3074} & \multirow[t]{4}{*}{473.478} & \multirow[t]{4}{*}{183.960} & $20.31(0.08)$ & $20.27(0.12)$ & $20.78(0.11)$ & $20.35(0.07)$ & $20.22(0.13)$ & $20.20(0.08)$ \\
\hline & & & $20.85(0.08)$ & 99.99 (9.99) & $20.35(0.07)$ & $99.99(9.99)$ & $20.89(0.08)$ & 99.99 (9.99) \\
\hline & & & $21.44(0.16)$ & $21.02(0.09)$ & $20.38(0.09)$ & $20.44(0.06)$ & $20.65(0.07)$ & $21.16(0.11)$ \\
\hline & & & $21.19(0.11)$ & $21.02(0.11)$ & $21.06(0.11)$ & 99.99 (9.99) & $21.16(0.09)$ & 99.99 (9.99) \\
\hline 3077 & 215.977 & 359.867 & $20.72(0.06)$ & $20.70(0.09)$ & $21.03(0.11)$ & $21.20(0.17)$ & $20.81(0.06)$ & $20.50(0.05)$ \\
\hline & & & $20.45(0.07)$ & $20.63(0.09)$ & $20.78(0.06)$ & $21.05(0.08)$ & $20.85(0.09)$ & $20.81(0.07)$ \\
\hline & & & $21.04(0.10)$ & $20.84(0.07)$ & $20.82(0.06)$ & $20.80(0.06)$ & $20.68(0.06)$ & $20.50(0.05)$ \\
\hline & & & $20.47(0.07)$ & $20.44(0.07)$ & $20.53(0.07)$ & $20.32(0.06)$ & $20.21(0.06)$ & 99.99 (9.99) \\
\hline 3094 & 755.853 & 791.767 & $21.00(0.06)$ & $21.02(0.15)$ & $20.41(0.09)$ & $20.31(0.07)$ & $20.38(0.05)$ & $20.76(0.06)$ \\
\hline & & & $20.69(0.09)$ & $21.45(0.12)$ & $20.95(0.07)$ & $21.12(0.06)$ & $21.11(0.09)$ & $21.08(0.06)$ \\
\hline & & & $20.64(0.06)$ & $20.60(0.05)$ & $20.67(0.05)$ & $20.67(0.09)$ & $20.39(0.06)$ & $20.58(0.05)$ \\
\hline & & & $20.52(0.06)$ & $20.51(0.07)$ & $20.49(0.06)$ & $20.25(0.06)$ & $20.17(0.06)$ & $20.51(0.09)$ \\
\hline 3102 & 119.432 & 457.504 & $20.70(0.12)$ & $20.89(0.45)$ & $21.16(0.19)$ & $20.38(0.18)$ & $19.44(0.18)$ & 99.99 (9.99) \\
\hline & & & $21.25(0.16)$ & $18.96(0.16)$ & $20.31(0.20)$ & $20.18(0.08)$ & $20.93(0.12)$ & $19.37(0.16)$ \\
\hline & & & $20.53(0.16)$ & $20.05(0.14)$ & $20.55(0.14)$ & $22.45(0.60)$ & $21.49(0.20)$ & $21.21(0.10)$ \\
\hline & & & $20.68(0.10)$ & $21.23(0.17)$ & $21.07(0.10)$ & $22.41(0.30)$ & $21.89(0.18)$ & $20.53(0.19)$ \\
\hline 3116 & 398.267 & 539.442 & $21.05(0.06)$ & $21.00(0.09)$ & $20.66(0.09)$ & $20.48(0.08)$ & $20.60(0.08)$ & $20.59(0.06)$ \\
\hline & & & $20.92(0.11)$ & $21.21(0.11)$ & $20.97(0.07)$ & $20.91(0.06)$ & $20.78(0.08)$ & $20.48(0.05)$ \\
\hline & & & $21.35(0.11)$ & $21.30(0.08)$ & $20.96(0.06)$ & $20.77(0.07)$ & $20.56(0.06)$ & $20.44(0.06)$ \\
\hline & & & $20.37(0.06)$ & $20.27(0.07)$ & $20.25(0.05)$ & $20.55(0.07)$ & $20.81(0.08)$ & $20.48(0.11)$ \\
\hline 3118 & 331.157 & 843.819 & $20.44(0.05)$ & $20.90(0.09)$ & $21.30(0.14)$ & $20.99(0.12)$ & $21.17(0.07)$ & $21.04(0.08)$ \\
\hline & & & $20.89(0.07)$ & $20.77(0.09)$ & $20.84(0.06)$ & $20.84(0.07)$ & $20.38(0.06)$ & $20.67(0.05)$ \\
\hline & & & $20.54(0.06)$ & $20.46(0.05)$ & $20.39(0.05)$ & $20.52(0.05)$ & $20.60(0.06)$ & $20.88(0.08)$ \\
\hline & & & $21.11(0.10)$ & $21.11(0.10)$ & $21.17(0.08)$ & $21.59(0.12)$ & 99.99 (9.99) & $21.34(0.16)$ \\
\hline 3121 & 206.707 & 260.865 & $20.93(0.06)$ & $20.60(0.07)$ & $20.92(0.11)$ & $21.27(0.10)$ & $21.15(0.09)$ & $21.12(0.07)$ \\
\hline & & & $21.46(0.14)$ & $21.31(0.12)$ & $21.04(0.08)$ & $20.77(0.06)$ & $20.65(0.09)$ & $20.62(0.05)$ \\
\hline & & & $20.75(0.07)$ & $20.46(0.06)$ & $20.39(0.06)$ & $20.35(0.05)$ & $20.36(0.05)$ & $20.57(0.07)$ \\
\hline & & & $20.93(0.10)$ & $20.68(0.11)$ & $21.15(0.08)$ & $21.42(0.10)$ & $21.16(0.09)$ & $20.50(0.19)$ \\
\hline 3152 & 139.489 & 250.324 & $20.87(0.07)$ & $20.78(0.11)$ & $20.78(0.10)$ & $20.92(0.11)$ & $20.99(0.07)$ & $21.04(0.08)$ \\
\hline & & & $21.00(0.09)$ & $21.55(0.15)$ & $21.00(0.08)$ & $20.95(0.08)$ & $20.67(0.07)$ & $20.64(0.06)$ \\
\hline & & & $20.66(0.07)$ & $20.39(0.05)$ & $20.44(0.06)$ & $20.25(0.06)$ & $20.34(0.06)$ & $20.57(0.06)$ \\
\hline & & & $20.79(0.11)$ & $20.62(0.08)$ & $21.06(0.08)$ & $21.45(0.12)$ & $21.22(0.12)$ & $20.48(0.12)$ \\
\hline
\end{tabular}


Table 8. continuation.

\begin{tabular}{|c|c|c|c|c|c|c|c|c|}
\hline \multirow[t]{4}{*}{ F2 ID } & \multirow[t]{4}{*}{$\mathrm{x}$} & \multirow[t]{4}{*}{$\mathrm{y}$} & $2 \mathrm{Ks} 10(\sigma)$ & 2Ks01 $(\sigma)$ & $2 \mathrm{Ks} 02(\sigma)$ & $2 \mathrm{Ks} 03(\sigma)$ & $2 \mathrm{Ks} 05(\sigma)$ & $2 \mathrm{Ks} 06(\sigma)$ \\
\hline & & & $2 \mathrm{Ks} 07(\sigma)$ & $2 \mathrm{Ks} 08(\sigma)$ & $2 \mathrm{Ks} 09(\sigma)$ & $2 \mathrm{Ks} 11(\sigma)$ & $2 \mathrm{Ks} 12(\sigma)$ & $2 \mathrm{Ks} 13(\sigma)$ \\
\hline & & & $2 \mathrm{Ks} 14(\sigma)$ & $2 \mathrm{Ks} 15(\sigma)$ & $2 \mathrm{Ks} 16(\sigma)$ & $2 \mathrm{Ks} 17(\sigma)$ & $2 \mathrm{Ks} 18(\sigma)$ & $2 \mathrm{Ks} 19(\sigma)$ \\
\hline & & & $2 \mathrm{Ks} 20(\sigma)$ & $2 \mathrm{Ks} 21(\sigma)$ & $2 \mathrm{Ks} 22(\sigma)$ & $2 \mathrm{Ks} 23(\sigma)$ & $2 \mathrm{Ks} 24(\sigma)$ & $2 \mathrm{Ks} 04(\sigma)$ \\
\hline \multirow[t]{4}{*}{3159} & 215.796 & 325.273 & 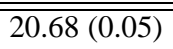 & $20.97(0.07)$ & $21.28(0.15)$ & $20.95(0.09)$ & $21.02(0.06)$ & $21.21(0.08)$ \\
\hline & & & $20.72(0.09)$ & $20.39(0.07)$ & $21.11(0.07)$ & $20.80(0.06)$ & $20.49(0.06)$ & $20.45(0.05)$ \\
\hline & & & $21.16(0.09)$ & $20.88(0.08)$ & $20.80(0.06)$ & $20.58(0.05)$ & $20.54(0.05)$ & $20.37(0.05)$ \\
\hline & & & $20.34(0.06)$ & $20.38(0.06)$ & $20.69(0.07)$ & $21.45(0.10)$ & 99.99 (9.99) & $21.12(0.22)$ \\
\hline \multirow[t]{4}{*}{3167} & 397.800 & 834.029 & $20.96(0.07)$ & $20.69(0.07)$ & $21.27(0.13)$ & $21.10(0.13)$ & $20.47(0.05)$ & $20.91(0.06)$ \\
\hline & & & $20.80(0.09)$ & $20.92(0.07)$ & $20.89(0.08)$ & $20.59(0.05)$ & $20.97(0.09)$ & $21.01(0.07)$ \\
\hline & & & $20.91(0.09)$ & $21.02(0.07)$ & $20.89(0.07)$ & $20.63(0.06)$ & $20.40(0.06)$ & $20.51(0.06)$ \\
\hline & & & $20.35(0.06)$ & $20.43(0.06)$ & $20.61(0.06)$ & $20.92(0.08)$ & $21.69(0.41)$ & $20.44(0.11)$ \\
\hline \multirow[t]{4}{*}{3169} & 545.314 & 695.390 & $20.95(0.06)$ & $21.31(0.10)$ & $20.96(0.08)$ & $20.79(0.09)$ & $20.84(0.06)$ & $20.98(0.08)$ \\
\hline & & & $21.21(0.10)$ & $20.91(0.08)$ & $21.08(0.08)$ & $20.63(0.06)$ & $20.54(0.07)$ & $20.41(0.05)$ \\
\hline & & & $20.49(0.06)$ & $20.52(0.06)$ & $20.38(0.05)$ & $20.52(0.06)$ & $20.59(0.05)$ & $20.72(0.06)$ \\
\hline & & & $20.70(0.09)$ & $20.89(0.08)$ & $21.11(0.08)$ & $20.83(0.07)$ & $20.80(0.07)$ & $20.22(0.09)$ \\
\hline \multirow[t]{4}{*}{3173} & 122.506 & 226.249 & $20.88(0.05)$ & $20.69(0.10)$ & $20.40(0.07)$ & $20.60(0.08)$ & $20.38(0.05)$ & $20.50(0.06)$ \\
\hline & & & $20.74(0.09)$ & $21.40(0.12)$ & $21.05(0.08)$ & $21.08(0.07)$ & $20.93(0.09)$ & $20.93(0.06)$ \\
\hline & & & $20.83(0.07)$ & $20.79(0.06)$ & $20.88(0.08)$ & $20.78(0.06)$ & $20.67(0.07)$ & $20.87(0.07)$ \\
\hline & & & $20.78(0.09)$ & $21.01(0.10)$ & $20.52(0.06)$ & $20.34(0.06)$ & $20.24(0.06)$ & $20.18(0.09)$ \\
\hline \multirow[t]{4}{*}{3201} & 671.573 & 133.334 & $20.39(0.06)$ & $20.59(0.10)$ & $20.30(0.08)$ & $20.18(0.06)$ & $20.86(0.07)$ & $20.86(0.08)$ \\
\hline & & & $20.86(0.08)$ & $21.21(0.11)$ & $20.69(0.07)$ & $20.71(0.09)$ & $20.37(0.06)$ & $20.40(0.05)$ \\
\hline & & & $20.49(0.08)$ & $20.74(0.06)$ & $20.86(0.06)$ & $21.12(C$ & 21.30 & 21.21 \\
\hline & & & $21.16(0.12)$ & $21.44(0.20)$ & $20.99(0.08)$ & $21.00(0.10)$ & $20.89(0.10)$ & $21.12(0.17)$ \\
\hline \multirow[t]{4}{*}{3223} & 574.027 & 634.704 & $20.57(0.05)$ & $21.36(0.10)$ & $21.94(0.27)$ & $21.88(0.29)$ & $21.37(0.09)$ & $20.90(0.07)$ \\
\hline & & & $20.99(0.09)$ & $20.92(0.10)$ & $20.92(0.09)$ & $20.37(0.05)$ & $20.44(0.06)$ & $20.66(0.06)$ \\
\hline & & & $20.48(0.07)$ & $20.54(0.06)$ & $20.41(0.07)$ & $20.49(0.09)$ & $20.50(0.0$ & $20.68(0.06)$ \\
\hline & & & $20.78(0.08)$ & $20.88(0.12)$ & $20.78(0.06)$ & $21.22(0.10)$ & $21.03(0.09)$ & $21.30(0.19)$ \\
\hline \multirow[t]{4}{*}{3231} & 541.879 & 386.689 & $20.67(0.05)$ & $20.83(0.09)$ & $20.88(0.12)$ & $20.78(0.09)$ & $20.31(0.05)$ & $20.35(0.05)$ \\
\hline & & & $20.32(0.06)$ & $20.51(0.07)$ & $20.40(0.05)$ & $20.83(0.06)$ & $20.78(0.07)$ & $20.74(0.06)$ \\
\hline & & & $20.89(0.10)$ & $20.94(0.07)$ & $20.91(0.05)$ & $21.01(0.09)$ & $20.84(0.05)$ & $20.92(0.07)$ \\
\hline & & & $21.29(0.12)$ & $21.19(0.11)$ & $20.87(0.07)$ & $20.76(0$. & 20.64 & 20.87 \\
\hline \multirow[t]{4}{*}{3238} & 516.011 & 222.659 & $20.90(0.06)$ & $20.42(0.07)$ & $20.61(0.09)$ & $21.20(0.12)$ & $20.84(0.06)$ & $21.09(0.08)$ \\
\hline & & & $20.93(0.09)$ & $21.37(0.12)$ & $20.85(0.07)$ & $20.90(0.06)$ & $20.69(0.09)$ & $20.48(0.05)$ \\
\hline & & & $21.43(0.11)$ & $20.88(0.05)$ & $20.73(0.05)$ & $20.59(0.07)$ & $20.50(0.07)$ & $20.35(0.05)$ \\
\hline & & & $20.49(0.07)$ & $20.38(0.07)$ & $20.56(0.05)$ & $20.74(0.07)$ & $20.77(0.06)$ & $20.58(0.12)$ \\
\hline \multirow[t]{4}{*}{3255} & 832.131 & 409.754 & $20.86(0.07)$ & $21.89(0.23)$ & $21.38(0.18)$ & 99.99 (9.99) & $20.83(0.07)$ & $20.62(0.07)$ \\
\hline & & & 99.99 (9.99) & $21.00(0.12)$ & $21.05(0.07)$ & $20.48(0.06)$ & $21.08(0.09)$ & $20.77(0.06)$ \\
\hline & & & $20.73(0.07)$ & $20.94(0.06)$ & $21.11(0.07)$ & $20.77(0.06)$ & $20.84(0.06)$ & $20.45(0.05)$ \\
\hline & & & $20.75(0.09)$ & $20.99(0.10)$ & $20.41(0.06)$ & $20.32(0.06)$ & $20.40(0.06)$ & 99.99 (9.99) \\
\hline \multirow[t]{4}{*}{3277} & 483.024 & 174.466 & $20.44(0.11)$ & $20.45(0.19)$ & $20.93(0.14)$ & $20.28(0.10)$ & $20.03(0.15)$ & $19.99(0.07)$ \\
\hline & & & $20.56(0.11)$ & $20.16(0.18)$ & $20.38(0.12)$ & $19.94(0.09)$ & $21.26(0.16)$ & $20.49(0.13)$ \\
\hline & & & $21.34(0.11)$ & $21.41(0.11)$ & $21.18(0.15)$ & $21.73(0.17)$ & $21.18(0.11)$ & $20.90(0.09)$ \\
\hline & & & $21.38(0.12)$ & $21.51(0.16)$ & $20.95(0.08)$ & 99.99 (9.99) & 99.99 (9.99) & $19.98(0.34)$ \\
\hline \multirow[t]{4}{*}{3312} & 262.010 & 322.619 & $20.38(0.05)$ & $20.91(0.10)$ & $21.30(0.13)$ & $21.36(0.22)$ & $20.65(0.06)$ & $20.47(0.06)$ \\
\hline & & & $20.40(0.07)$ & $20.53(0.08)$ & $20.34(0.05)$ & $20.75(0.06)$ & $20.93(0.08)$ & $21.01(0.05)$ \\
\hline & & & $20.96(0.08)$ & $20.90(0.06)$ & $20.86(0.06)$ & $20.74(0.06)$ & $20.86(0.05)$ & $20.93(0.07)$ \\
\hline & & & $20.76(0.07)$ & $20.78(0.11)$ & $20.95(0.06)$ & $20.50(0.06)$ & $20.44(0.06)$ & $20.57(0.14)$ \\
\hline \multirow[t]{4}{*}{3327} & 549.657 & 606.106 & $20.94(0.06)$ & $20.87(0.07)$ & $20.90(0.12)$ & $20.90(0.10)$ & $20.59(0.05)$ & $20.77(0.07)$ \\
\hline & & & $20.60(0.08)$ & $21.55(0.15)$ & $21.05(0.09)$ & $21.03(0.07)$ & $20.59(0.07)$ & $20.56(0.05)$ \\
\hline & & & $20.66(0.07)$ & $20.46(0.05)$ & $20.48(0.05)$ & $20.64(0.06)$ & $20.68(0.06)$ & $20.99(0.07)$ \\
\hline & & & $21.20(0.14)$ & $21.33(0.11)$ & $21.40(0.10)$ & $21.36(0.10)$ & $21.46(0.14)$ & $20.73(0.19)$ \\
\hline
\end{tabular}


Table 8. continuation.

\begin{tabular}{|c|c|c|c|c|c|c|c|c|}
\hline \multirow[t]{4}{*}{ F2 ID } & \multirow[t]{4}{*}{$\mathrm{x}$} & \multirow[t]{4}{*}{$\mathrm{y}$} & $2 \mathrm{Ks} 10(\sigma)$ & $2 \mathrm{Ks} 01(\sigma)$ & $2 \mathrm{Ks} 02(\sigma)$ & $2 \mathrm{Ks} 03(\sigma)$ & $2 \mathrm{Ks} 05(\sigma)$ & $2 \mathrm{Ks06}(\sigma)$ \\
\hline & & & $2 \mathrm{Ks} 07(\sigma)$ & $2 \mathrm{Ks} 08(\sigma)$ & $2 \mathrm{Ks} 09(\sigma)$ & $2 \mathrm{Ks} 11(\sigma)$ & $2 \mathrm{Ks} 12(\sigma)$ & $2 \mathrm{Ks} 13(\sigma)$ \\
\hline & & & $2 \mathrm{Ks} 14(\sigma)$ & $2 \mathrm{Ks} 15(\sigma)$ & $2 \mathrm{Ks} 16(\sigma)$ & $2 \mathrm{Ks} 17(\sigma)$ & $2 \mathrm{Ks} 18(\sigma)$ & $2 \mathrm{Ks} 19(\sigma)$ \\
\hline & & & $2 \mathrm{Ks} 20(\sigma)$ & $2 \mathrm{Ks} 21(\sigma)$ & $2 \mathrm{Ks} 22(\sigma)$ & $2 \mathrm{Ks} 23(\sigma)$ & $2 \mathrm{Ks} 24(\sigma)$ & $2 \mathrm{Ks} 04(\sigma)$ \\
\hline \multirow[t]{4}{*}{3364} & $\overline{994.439}$ & 172.691 & $20.84(0.06)$ & $20.54(0.08)$ & $20.52(0.09)$ & $20.85(0.11)$ & $21.40(0.11)$ & 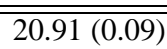 \\
\hline & & & $21.00(0.10)$ & $21.32(0.13)$ & $21.26(0.09)$ & $20.44(0.06)$ & $20.35(0.07)$ & $20.59(0.06)$ \\
\hline & & & $20.87(0.08)$ & $20.42(0.06)$ & $20.51(0.06)$ & $20.63(0.09)$ & $20.58(0.07)$ & $20.83(0.07)$ \\
\hline & & & $21.07(0.11)$ & $21.01(0.11)$ & $21.11(0.08)$ & $21.46(0.11)$ & $21.42(0.14)$ & $20.81(0.19)$ \\
\hline \multirow[t]{4}{*}{3366} & 530.536 & 409.101 & $21.12(0.11)$ & $21.22(0.15)$ & $21.18(0.17)$ & $21.15(0.09)$ & $20.53(0.07)$ & $20.64(0.06)$ \\
\hline & & & $20.64(0.07)$ & $21.42(0.13)$ & $21.05(0.12)$ & $20.45(0.06)$ & $20.35(0.07)$ & $20.70(0.10)$ \\
\hline & & & $20.55(0.07)$ & $20.91(0.07)$ & $21.16(0.11)$ & $21.19(0.12)$ & $21.14(0.13)$ & $20.95(0.08)$ \\
\hline & & & $21.05(0.10)$ & $20.93(0.12)$ & $20.89(0.09)$ & $20.57(0.06)$ & $20.51(0.07)$ & $21.10(0.34)$ \\
\hline \multirow[t]{4}{*}{3378} & 240.495 & 539.909 & $20.44(0.05)$ & $21.27(0.12)$ & $21.17(0.09)$ & $20.98(0.14)$ & $20.78(0.07)$ & $20.32(0.05)$ \\
\hline & & & $20.37(0.09)$ & $20.54(0.08)$ & $20.43(0.05)$ & $20.68(0.07)$ & $20.83(0.09)$ & $20.99(0.05)$ \\
\hline & & & $20.78(0.07)$ & $20.93(0.07)$ & $21.12(0.07)$ & $20.97(0.06)$ & $20.85(0.06)$ & $20.90(0.06)$ \\
\hline & & & $20.85(0.07)$ & $20.59(0.07)$ & $20.67(0.06)$ & $20.77(0.06)$ & $20.33(0.06)$ & $21.65(0.29)$ \\
\hline \multirow[t]{4}{*}{3390} & 421.316 & 212.971 & $21.15(0.06)$ & $21.18(0.13)$ & $20.86(0.13)$ & $21.40(0.14)$ & $20.50(0.05)$ & $20.63(0.07)$ \\
\hline & & & $20.95(0.09)$ & $21.08(0.11)$ & $21.04(0.08)$ & $21.16(0.07)$ & $20.78(0.08)$ & $20.51(0.06)$ \\
\hline & & & $20.51(0.07)$ & $20.43(0.05)$ & $20.39(0.07)$ & $20.40(0.07)$ & $20.49(0.06)$ & $20.80(0.07)$ \\
\hline & & & $21.09(0.11)$ & $20.82(0.09)$ & $21.23(0.08)$ & $21.33(0.10)$ & $21.20(0.13)$ & $20.78(0.13)$ \\
\hline \multirow[t]{4}{*}{3402} & 700.282 & 491.063 & $21.14(0.16)$ & $21.49(0.23)$ & $21.52(0.20)$ & $21.86(0.31)$ & $21.32(0.25)$ & $20.48(0.18)$ \\
\hline & & & 99.99 (9.99) & $18.70(0.09)$ & $21.45(0.18)$ & $20.24(0.09)$ & $20.92(0.13)$ & $20.44(0.11)$ \\
\hline & & & $20.92(0.14)$ & $21.74(0.18)$ & $21.60(0.15)$ & $21.76(0.20)$ & $21.75(0.17)$ & $20.80(0.10)$ \\
\hline & & & $20.80(0.08)$ & $21.38(0.18)$ & $20.55(0.09)$ & $21.76(0.16)$ & $21.23(0.12)$ & $20.67(0.15)$ \\
\hline \multirow[t]{4}{*}{3435} & 242.104 & 343.433 & $20.53(0.06)$ & $20.78(0.07)$ & $21.54(0.20)$ & $21.87(0.20)$ & $21.22(0.08)$ & $21.12(0.09)$ \\
\hline & & & $21.16(0.12)$ & $21.19(0.10)$ & $20.76(0.07)$ & $20.62(0.07)$ & $20.49(0.08)$ & $20.90(0.06)$ \\
\hline & & & $20.42(0.07)$ & $20.45(0.06)$ & $20.56(0.05)$ & $20.62(0.05)$ & $20.67(0.06)$ & $21.01(0.09)$ \\
\hline & & & $21.23(0.12)$ & $21.45(0.19)$ & $21.18(0.08)$ & $21.45(0.10)$ & $21.19(0.10)$ & $21.78(0.51)$ \\
\hline \multirow[t]{4}{*}{3438} & 881.416 & 650.027 & $23.25(1.30)$ & $99.99(9.99)$ & $22.51(0.99)$ & $20.65(0.27)$ & $21.84(0.21)$ & $19.96(0.16)$ \\
\hline & & & $21.36(0.52)$ & $19.68(0.43)$ & $21.83(0.49)$ & 99.99 (9.99) & $20.88(0.09)$ & 99.99 (9.99) \\
\hline & & & 99.99 (9.99) & $21.78(0.38)$ & 99.99 (9.99) & 99.99 (9.99) & $21.53(0.40)$ & $20.33(0.13)$ \\
\hline & & & 99.99 (9.99) & $21.61(0.14)$ & $20.35(0.09)$ & $21.90(0.12)$ & $20.36(0.07)$ & $21.01(0.26)$ \\
\hline \multirow[t]{4}{*}{3451} & 102.962 & 631.117 & $20.94(0.14)$ & $21.24(0.14)$ & $21.26(0.18)$ & $21.01(0.14)$ & $19.73(0.16)$ & 19.91 \\
\hline & & & $20.93(0.13)$ & $19.62(0.10)$ & $20.93(0.12)$ & $20.58(0.10)$ & $20.82(0.10)$ & $20.05(0.12)$ \\
\hline & & & $20.78(0.13)$ & $20.58(0.13)$ & $20.40(0.29)$ & $22.28(0.26)$ & $21.11(0.15)$ & $20.55(0.11)$ \\
\hline & & & $20.86(0.11)$ & $20.99(0.11)$ & $20.50(0.10)$ & $21.16(0.10)$ & $21.39(0.11)$ & $20.64(0.23)$ \\
\hline \multirow[t]{4}{*}{3460} & 661.932 & 529.525 & $20.97(0.06)$ & $20.96(0.14)$ & $21.52(0.14)$ & $21.90(0.23)$ & $20.46(0.05)$ & $20.68(0.06)$ \\
\hline & & & $20.66(0.09)$ & $20.99(0.09)$ & $20.75(0.07)$ & $21.48(0.10)$ & $20.99(0.11)$ & $20.63(0.05)$ \\
\hline & & & $21.02(0.10)$ & $20.68(0.05)$ & $20.48(0.05)$ & $20.51(0.05)$ & $20.66(0.06)$ & $20.76(0.06)$ \\
\hline & & & $20.77(0.09)$ & $20.86(0.10)$ & $21.25(0.09)$ & $21.47(0.08)$ & $21.37(0.11)$ & $21.14(0.18)$ \\
\hline \multirow[t]{4}{*}{3470} & 169.439 & 28.400 & $22.65(0.22)$ & 99.99 (9.99) & $23.25(1.09)$ & 99.99 (9.99) & $99.99(9.99)$ & $99.99(9.99)$ \\
\hline & & & $25.46(3.99)$ & 99.99 (9.99) & $23.59(0.71)$ & $23.43(0.49)$ & $21.69(0.18)$ & $22.39(0.21)$ \\
\hline & & & $99.99(9.99)$ & $22.85(0.44)$ & $22.00(0.20)$ & $20.36(0.05)$ & $21.02(0.10)$ & $21.80(0.21)$ \\
\hline & & & $21.38(0.13)$ & $21.25(0.10)$ & $21.17(0.11)$ & $20.85(0.07)$ & $20.80(0.08)$ & 99.99 (9.99) \\
\hline \multirow[t]{4}{*}{3474} & 556.286 & 827.190 & $21.00(0.07)$ & $21.49(0.16)$ & $21.33(0.19)$ & $21.01(0.12)$ & $20.35(0.05)$ & $20.52(0.05)$ \\
\hline & & & $20.53(0.07)$ & $20.80(0.08)$ & $20.74(0.07)$ & $21.38(0.09)$ & $21.61(0.16)$ & $21.27(0.07)$ \\
\hline & & & $21.09(0.10)$ & $21.26(0.09)$ & $20.94(0.07)$ & $21.00(0.09)$ & $20.83(0.07)$ & $21.09(0.10)$ \\
\hline & & & $20.86(0.10)$ & $21.02(0.09)$ & $20.72(0.06)$ & $20.53(0.06)$ & $20.62(0.07)$ & $21.24(0.25)$ \\
\hline \multirow[t]{4}{*}{3491} & 434.079 & 220.827 & $21.26(0.09)$ & $20.60(0.06)$ & $20.75(0.08)$ & $22.09(0.26)$ & $21.27(0.08)$ & $21.73(0.12)$ \\
\hline & & & $21.79(0.20)$ & $22.24(0.31)$ & $21.39(0.12)$ & $20.91(0.07)$ & $20.94(0.09)$ & $20.41(0.05)$ \\
\hline & & & $20.87(0.07)$ & $20.88(0.06)$ & $20.77(0.05)$ & $20.68(0.05)$ & $20.70(0.05)$ & $20.56(0.06)$ \\
\hline & & & $21.00(0.10)$ & $20.55(0.09)$ & $20.55(0.06)$ & $20.40(0.06)$ & $20.94(0.08)$ & $20.75(0.23)$ \\
\hline
\end{tabular}


Table 8. continuation.

\begin{tabular}{|c|c|c|c|c|c|c|c|c|}
\hline \multirow[t]{4}{*}{$\bar{F}$ F2 ID } & \multirow[t]{4}{*}{$\mathrm{x}$} & \multirow[t]{4}{*}{$\mathrm{y}$} & $2 \mathrm{Ks} 10(\sigma)$ & $2 \mathrm{Ks} 01(\sigma)$ & $2 \mathrm{Ks} 02(\sigma)$ & $2 \mathrm{Ks} 03(\sigma)$ & $2 \mathrm{Ks} 05(\sigma)$ & $\overline{2 \mathrm{Ks} 06(\sigma)}$ \\
\hline & & & $2 \mathrm{Ks} 07(\sigma)$ & $2 \mathrm{Ks} 08(\sigma)$ & $2 \mathrm{Ks} 09(\sigma)$ & $2 \mathrm{Ks} 11(\sigma)$ & $2 \mathrm{Ks} 12(\sigma)$ & $2 \mathrm{Ks} 13(\sigma)$ \\
\hline & & & $2 \mathrm{Ks} 14(\sigma)$ & $2 \mathrm{Ks} 15(\sigma)$ & $2 \mathrm{Ks} 16(\sigma)$ & $2 \mathrm{Ks} 17(\sigma)$ & $2 \mathrm{Ks} 18(\sigma)$ & $2 \mathrm{Ks} 19(\sigma)$ \\
\hline & & & $2 \mathrm{Ks} 20(\sigma)$ & $2 \mathrm{Ks} 21(\sigma)$ & $2 \mathrm{Ks} 22(\sigma)$ & $2 \mathrm{Ks} 23(\sigma)$ & $2 \mathrm{Ks} 24(\sigma)$ & $2 \mathrm{Ks} 04(\sigma)$ \\
\hline \multirow[t]{4}{*}{3509} & 4881.177 & 518.413 & $20.80(0.11)$ & $20.95(0.22)$ & $20.82(0.15)$ & $21.05(0.23)$ & $20.04(0.13)$ & $\overline{20.32(0.13)}$ \\
\hline & & & $20.78(0.10)$ & $20.27(0.30)$ & $20.95(0.17)$ & $20.30(0.06)$ & $21.18(0.11)$ & $20.43(0.22)$ \\
\hline & & & $23.04(0.77)$ & $21.77(0.12)$ & $21.94(0.24)$ & $21.93(0.25)$ & $22.98(0.49)$ & 99.99 (9.99) \\
\hline & & & $21.40(0.14)$ & 99.99 (9.99) & $22.11(0.23)$ & 99.99 (9.99) & 99.99 (9.99) & $21.15(0.25)$ \\
\hline \multirow[t]{4}{*}{3519} & 726.396 & 584.699 & $20.45(0.04)$ & $20.68(0.09)$ & $21.04(0.10)$ & $21.16(0.12)$ & $21.07(0.06)$ & $20.69(0.06)$ \\
\hline & & & $20.70(0.07)$ & $20.76(0.08)$ & $20.64(0.06)$ & $21.22(0.08)$ & $21.19(0.13)$ & $20.97(0.06)$ \\
\hline & & & $21.18(0.09)$ & $20.97(0.07)$ & $21.17(0.06)$ & $20.97(0.05)$ & $21.00(0.07)$ & $20.86(0.07)$ \\
\hline & & & $20.64(0.08)$ & $20.65(0.07)$ & $20.51(0.05)$ & $20.84(0.07)$ & $20.98(0.09)$ & $21.12(0.13)$ \\
\hline \multirow[t]{4}{*}{3524} & 327.911 & 236.230 & $21.10(0.07)$ & $20.65(0.08)$ & $20.58(0.11)$ & $20.91(0.11)$ & $20.80(0.06)$ & $21.00(0.07)$ \\
\hline & & & $21.36(0.11)$ & $21.65(0.18)$ & $21.27(0.08)$ & $21.02(0.08)$ & $20.89(0.09)$ & $20.62(0.05)$ \\
\hline & & & $20.93(0.09)$ & $20.94(0.07)$ & $20.84(0.06)$ & $20.72(0.07)$ & $20.53(0.05)$ & $20.50(0.06)$ \\
\hline & & & $20.46(0.08)$ & $20.28(0.07)$ & $20.67(0.06)$ & $20.83(0.07)$ & $21.10(0.10)$ & $20.42(0.13)$ \\
\hline \multirow[t]{4}{*}{3544} & 215.668 & 618.383 & $20.55(0.05)$ & $20.97(0.10)$ & $21.44(0.14)$ & $21.24(0.15)$ & $20.84(0.06)$ & $20.51(0.06)$ \\
\hline & & & $20.55(0.07)$ & $20.52(0.06)$ & $20.52(0.05)$ & $21.27(0.09)$ & $21.19(0.09)$ & $21.20(0.05)$ \\
\hline & & & $21.17(0.11)$ & $21.36(0.08)$ & $21.07(0.08)$ & $21.34(0.07)$ & $21.33(0.07)$ & $20.98(0.08)$ \\
\hline & & & $20.47(0.07)$ & $20.96(0.07)$ & $20.50(0.05)$ & $20.49(0.06)$ & $20.67(0.06)$ & $20.93(0.19)$ \\
\hline \multirow[t]{4}{*}{3550} & 77.913 & 621.555 & $20.61(0.09)$ & $21.20(0.14)$ & $21.40(0.15)$ & $21.64(0.14)$ & 99.99 (9.99) & $20.01(0.14)$ \\
\hline & & & $20.93(0.10)$ & $22.75(1.31)$ & $21.53(0.13)$ & $20.17(0.06)$ & $21.11(0.09)$ & $20.91(0.24)$ \\
\hline & & & $21.10(0.14)$ & $20.86(0.13)$ & $19.45(0.16)$ & $20.49(0.07)$ & $20.94(0.08)$ & $20.65(0.09)$ \\
\hline & & & $21.07(0.09)$ & $21.37(0.14)$ & $20.86(0.09)$ & $21.66(0.14)$ & $21.37(0.11)$ & $20.71(0.27)$ \\
\hline \multirow[t]{4}{*}{3577} & 30.904 & 415.011 & $21.21(0.07)$ & $21.28(0.10)$ & $21.24(0.13)$ & $21.39(0.17)$ & $21.32(0.11)$ & $99.99(9.99)$ \\
\hline & & & $21.18(0.10)$ & $20.04(0.13)$ & $21.50(0.12)$ & $20.06(0.07)$ & $20.41(0.08)$ & 99.99 (9.99) \\
\hline & & & $20.32(0.06)$ & $20.74(0.17)$ & $21.64(0.13)$ & 99.99 (9.99) & $21.60(0.08)$ & $21.07(0.08)$ \\
\hline & & & $20.69(0.09)$ & $21.94(0.26)$ & $21.49(0.14)$ & $20.62(0.06)$ & $20.75(0.07)$ & $20.32(0.09)$ \\
\hline \multirow[t]{4}{*}{3610} & 222.338 & 70.817 & $20.57(0.05)$ & $20.72(0.41)$ & $21.19(0.11)$ & $21.58(0.16)$ & $20.94(0.07)$ & $21.03(0.06)$ \\
\hline & & & $20.88(0.09)$ & $20.93(0.11)$ & $20.61(0.07)$ & $20.75(0.06)$ & $20.61(0.07)$ & $21.08(0.07)$ \\
\hline & & & $20.44(0.07)$ & $20.38(0.05)$ & $20.63(0.09)$ & $20.84(0.08)$ & $20.89(0.07)$ & 20.99 (0.09) \\
\hline & & & $21.39(0.14)$ & $21.25(0.12)$ & $21.20(0.08)$ & $20.99(0.08)$ & $20.90(0.09)$ & $21.03(0.14)$ \\
\hline \multirow[t]{4}{*}{3622} & 607.527 & 129.783 & $21.00(0.06)$ & $20.58(0.10)$ & $20.52(0.10)$ & $20.40(0.10)$ & $20.57(0.05)$ & $21.11(0.08)$ \\
\hline & & & $20.95(0.09)$ & $21.61(0.14)$ & $21.21(0.11)$ & $20.90(0.08)$ & $20.86(0.07)$ & $20.52(0.05)$ \\
\hline & & & $21.07(0.09)$ & $21.07(0.07)$ & $21.00(0.07)$ & $20.74(0.08)$ & $20.78(0.06)$ & $20.60(0.06)$ \\
\hline & & & $20.61(0.07)$ & $20.59(0.07)$ & $20.69(0.06)$ & $20.90(0.08)$ & $21.25(0.13)$ & $20.28(0.09)$ \\
\hline \multirow[t]{4}{*}{3642} & 308.610 & 837.671 & $20.66(0.06)$ & $21.03(0.11)$ & $20.60(0.10)$ & $20.50(0.09)$ & $21.15(0.07)$ & $21.06(0.08)$ \\
\hline & & & $20.84(0.08)$ & $20.83(0.10)$ & $20.52(0.05)$ & $21.24(0.09)$ & $20.90(0.10)$ & $20.54(0.06)$ \\
\hline & & & $20.54(0.07)$ & $20.52(0.06)$ & $20.66(0.08)$ & $20.84(0.07)$ & $20.87(0.09)$ & $21.04(0.07)$ \\
\hline & & & $21.11(0.09)$ & $21.49(0.15)$ & $21.28(0.11)$ & $20.95(0.08)$ & 99.99 (9.99) & $20.58(0.17)$ \\
\hline \multirow[t]{4}{*}{3643} & 777.644 & 587.259 & $21.46(0.11)$ & $21.29(0.08)$ & $20.78(0.12)$ & $21.03(0.10)$ & $20.68(0.12)$ & $20.81(0.11)$ \\
\hline & & & $21.34(0.11)$ & $19.87(0.20)$ & $20.88(0.12)$ & $21.99(0.24)$ & $20.69(0.08)$ & 99.99 (9.99) \\
\hline & & & $20.82(0.11)$ & $20.45(0.05)$ & $20.51(0.21)$ & $21.18(0.11)$ & $20.35(0.06)$ & $21.51(0.10)$ \\
\hline & & & $21.72(0.18)$ & $20.87(0.12)$ & $21.29(0.16)$ & $21.02(0.09)$ & $21.12(0.09)$ & $20.51(0.20)$ \\
\hline \multirow[t]{4}{*}{3668} & 577.917 & 197.946 & $21.15(0.06)$ & $21.45(0.13)$ & $21.52(0.13)$ & $21.09(0.11)$ & $20.32(0.05)$ & $20.70(0.06)$ \\
\hline & & & $20.90(0.09)$ & $21.81(0.20)$ & $20.94(0.07)$ & $21.27(0.09)$ & $21.34(0.14)$ & $20.99(0.06)$ \\
\hline & & & $21.06(0.08)$ & $21.27(0.08)$ & $21.23(0.07)$ & $21.10(0.06)$ & $20.90(0.06)$ & $20.75(0.07)$ \\
\hline & & & $20.64(0.08)$ & $20.51(0.07)$ & $20.52(0.06)$ & $20.39(0.06)$ & $20.66(0.07)$ & $20.41(0.11)$ \\
\hline \multirow[t]{4}{*}{3679} & 589.101 & 506.609 & $21.23(0.07)$ & $20.86(0.07)$ & $21.39(0.16)$ & $22.14(0.28)$ & $20.39(0.05)$ & $20.48(0.05)$ \\
\hline & & & $20.45(0.07)$ & $21.23(0.09)$ & $21.12(0.09)$ & $20.55(0.06)$ & $20.66(0.08)$ & $20.75(0.06)$ \\
\hline & & & $20.77(0.08)$ & $20.89(0.06)$ & $21.09(0.06)$ & $21.12(0.08)$ & $21.24(0.07)$ & $21.00(0.08)$ \\
\hline & & & $20.79(0.09)$ & $21.05(0.10)$ & $21.02(0.07)$ & $20.61(0.07)$ & $20.90(0.08)$ & $21.77(0.35)$ \\
\hline
\end{tabular}


Table 8. continuation.

\begin{tabular}{|c|c|c|c|c|c|c|c|c|}
\hline \multirow[t]{4}{*}{$\overline{\mathrm{F} 2 \mathrm{ID}}$} & \multirow[t]{4}{*}{$\mathrm{x}$} & \multirow[t]{4}{*}{$\mathrm{y}$} & $2 \mathrm{Ks} 10(\sigma)$ & $2 \mathrm{Ks} 01(\sigma)$ & $2 \mathrm{Ks} 02(\sigma)$ & $2 \mathrm{Ks} 03(\sigma)$ & $2 \mathrm{Ks} 05(\sigma)$ & $2 \mathrm{Ks06}(\sigma)$ \\
\hline & & & $2 \mathrm{Ks} 07(\sigma)$ & $2 \mathrm{Ks} 08(\sigma)$ & $2 \mathrm{Ks} 09(\sigma)$ & $2 \mathrm{Ks} 11(\sigma)$ & $2 \mathrm{Ks} 12(\sigma)$ & $2 \mathrm{Ks} 13(\sigma)$ \\
\hline & & & $2 \mathrm{Ks} 14(\sigma)$ & $2 \mathrm{Ks} 15(\sigma)$ & $2 \mathrm{Ks} 16(\sigma)$ & $2 \mathrm{Ks} 17(\sigma)$ & $2 \mathrm{Ks} 18(\sigma)$ & $2 \mathrm{Ks} 19(\sigma)$ \\
\hline & & & $2 \mathrm{Ks} 20(\sigma)$ & $2 \mathrm{Ks} 21(\sigma)$ & $2 \mathrm{Ks} 22(\sigma)$ & $2 \mathrm{Ks} 23(\sigma)$ & $2 \mathrm{Ks} 24(\sigma)$ & $2 \mathrm{Ks} 04(\sigma)$ \\
\hline \multirow[t]{4}{*}{3682} & 646.109 & 97.475 & $21.44(0.06)$ & $20.61(0.08)$ & $21.11(0.13)$ & $21.12(0.10)$ & $21.31(0.08)$ & $21.15(0.08)$ \\
\hline & & & $21.50(0.15)$ & $21.73(0.16)$ & $21.54(0.11)$ & $20.73(0.06)$ & $20.57(0.07)$ & $20.34(0.05)$ \\
\hline & & & $21.04(0.09)$ & $21.00(0.07)$ & $21.04(0.06)$ & $21.17(0.07)$ & $21.07(0.06)$ & $20.75(0.07)$ \\
\hline & & & $20.69(0.09)$ & $20.53(0.08)$ & $20.40(0.06)$ & $20.38(0.07)$ & $20.45(0.06)$ & $20.96(0.15)$ \\
\hline \multirow[t]{4}{*}{3712} & 424.787 & 437.690 & $21.28(0.06)$ & $21.02(0.10)$ & $21.45(0.14)$ & $21.26(0.14)$ & $20.60(0.05)$ & $20.64(0.06)$ \\
\hline & & & $21.02(0.10)$ & $21.35(0.11)$ & $21.13(0.08)$ & $20.93(0.06)$ & $20.74(0.08)$ & $20.49(0.05)$ \\
\hline & & & $20.91(0.08)$ & $20.69(0.06)$ & $20.65(0.05)$ & $20.66(0.07)$ & $20.68(0.06)$ & $20.81(0.07)$ \\
\hline & & & $21.08(0.12)$ & $20.93(0.07)$ & $21.16(0.08)$ & $21.47(0.10)$ & $21.24(0.08)$ & $21.46(0.31)$ \\
\hline \multirow[t]{4}{*}{3722} & 179.207 & 837.567 & $20.66(0.05)$ & $21.20(0.12)$ & $21.05(0.15)$ & $20.87(0.13)$ & $20.54(0.05)$ & $20.60(0.06)$ \\
\hline & & & $20.51(0.07)$ & $20.59(0.08)$ & $20.40(0.06)$ & $20.94(0.06)$ & $20.98(0.10)$ & $20.86(0.06)$ \\
\hline & & & $21.60(0.15)$ & $21.19(0.08)$ & $21.34(0.07)$ & $21.12(0.06)$ & $21.15(0.06)$ & $20.85(0.06)$ \\
\hline & & & $20.95(0.11)$ & $20.91(0.11)$ & $20.83(0.06)$ & $20.46(0.06)$ & $20.95(2.31)$ & $22.39(0.86)$ \\
\hline \multirow[t]{4}{*}{3761} & 165.993 & 669.807 & $20.88(0.06)$ & $20.26(0.07)$ & $20.27(0.06)$ & $20.14(0.06)$ & $20.50(0.05)$ & $20.59(0.06)$ \\
\hline & & & $20.62(0.07)$ & $20.90(0.09)$ & $20.93(0.07)$ & $21.27(0.08)$ & $21.50(0.14)$ & $21.03(0.06)$ \\
\hline & & & $21.12(0.07)$ & $21.29(0.08)$ & $21.06(0.06)$ & $21.23(0.07)$ & $21.13(0.06)$ & $21.16(0.07)$ \\
\hline & & & $21.22(0.16)$ & $20.91(0.10)$ & $21.02(0.07)$ & $21.18(0.09)$ & $20.54(0.06)$ & $20.37(0.09)$ \\
\hline \multirow[t]{4}{*}{3770} & 510.257 & 325.189 & $21.03(0.08)$ & $20.94(0.11)$ & $21.63(0.21)$ & $21.80(0.21)$ & $20.60(0.07)$ & $20.50(0.06)$ \\
\hline & & & $20.72(0.10)$ & $21.16(0.16)$ & $21.10(0.11)$ & $20.95(0.10)$ & $20.61(0.08)$ & $21.00(0.08)$ \\
\hline & & & $20.82(0.09)$ & $20.87(0.06)$ & $21.30(0.12)$ & $21.04(0.06)$ & $20.99(0.06)$ & $20.89(0.10)$ \\
\hline & & & $20.48(0.08)$ & $20.79(0.13)$ & $20.59(0.08)$ & $20.39(0.07)$ & $20.51(0.07)$ & $21.13(0.23)$ \\
\hline \multirow[t]{4}{*}{3792} & 126.725 & 511.100 & $21.11(0.06)$ & $21.19(0.12)$ & $21.62(0.17)$ & $21.76(0.17)$ & $20.63(0.06)$ & $20.57(0.06)$ \\
\hline & & & $20.82(0.09)$ & $21.08(0.11)$ & $20.98(0.07)$ & $21.33(0.09)$ & $21.17(0.12)$ & $21.15(0.07)$ \\
\hline & & & $21.16(0.10)$ & $21.32(0.10)$ & $20.99(0.06)$ & $20.85(0.07)$ & $20.73(0.06)$ & $20.54(0.06)$ \\
\hline & & & $20.56(0.09)$ & $20.44(0.06)$ & $20.43(0.06)$ & $20.60(0.06)$ & $21.00(0.08)$ & $21.41(0.26)$ \\
\hline \multirow[t]{4}{*}{3806} & 35.897 & 416.674 & $20.89(0.08)$ & $21.22(0.11)$ & $21.31(0.14)$ & $21.92(0.20)$ & $21.29(0.08)$ & 19.71 \\
\hline & & & $21.18(0.09)$ & $20.15(0.16)$ & $21.09(0.08)$ & $20.29(0.07)$ & $20.70(0.07)$ & $21.13(0.18)$ \\
\hline & & & $20.58(0.11)$ & $20.79(0.13)$ & $20.97(0.07)$ & $20.28(0.13)$ & $21.22(0.12)$ & $20.64(0.08)$ \\
\hline & & & $20.70(0.08)$ & $20.91(0.16)$ & $20.53(0.07)$ & $21.07(0.10)$ & $21.13(0.11)$ & $20.84(0.18)$ \\
\hline \multirow[t]{4}{*}{3846} & 167.066 & 449.521 & $20.99(0.06)$ & $21.28(0.09)$ & $21.77(0.21)$ & $21.47(0.13)$ & $20.42(0.06)$ & 20.37 \\
\hline & & & $20.78(0.07)$ & $20.83(0.09)$ & $20.75(0.07)$ & $21.30(0.09)$ & $21.11(0.12)$ & $20.91(0.05)$ \\
\hline & & & $21.16(0.10)$ & $21.37(0.10)$ & $21.22(0.08)$ & $21.11(0.06)$ & $21.19(0.09)$ & $20.98(0.07)$ \\
\hline & & & $20.48(0.08)$ & $20.63(0.08)$ & $20.53(0.06)$ & $20.54(0.07)$ & $20.77(0.08)$ & $20.95(0.20)$ \\
\hline \multirow[t]{4}{*}{3848} & 615.164 & 452.077 & $20.44(0.05)$ & $21.24(0.08)$ & $21.46(0.15)$ & $21.19(0.13)$ & $21.38(0.08)$ & $21.04(0.07)$ \\
\hline & & & $20.80(0.08)$ & $20.49(0.07)$ & $20.53(0.05)$ & $20.57(0.05)$ & $20.98(0.09)$ & $20.93(0.08)$ \\
\hline & & & $20.80(0.09)$ & $21.04(0.06)$ & $21.17(0.07)$ & $21.26(0.09)$ & $21.31(0.08)$ & $21.13(0.09)$ \\
\hline & & & $21.00(0.10)$ & $21.19(0.09)$ & $21.15(0.09)$ & $21.07(0.08)$ & $20.59(0.07)$ & $21.27(0.24)$ \\
\hline \multirow[t]{4}{*}{3864} & 695.073 & 132.244 & $20.93(0.07)$ & $20.37(0.09)$ & $20.46(0.06)$ & $20.97(0.11)$ & $21.71(0.10)$ & $21.54(0.11)$ \\
\hline & & & $21.71(0.17)$ & $21.73(0.20)$ & $21.52(0.10)$ & $20.84(0.10)$ & $20.49(0.07)$ & $20.82(0.06)$ \\
\hline & & & $20.41(0.06)$ & $20.52(0.06)$ & $20.61(0.07)$ & $20.70(0.06)$ & $20.99(0.05)$ & $21.07(0.09)$ \\
\hline & & & $21.47(0.17)$ & $21.73(0.16)$ & $21.30(0.13)$ & $21.41(0.13)$ & $21.16(0.13)$ & $20.63(0.23)$ \\
\hline \multirow[t]{4}{*}{3876} & 81.905 & 614.520 & $21.29(0.13)$ & $21.61(0.16)$ & $21.01(0.11)$ & $21.36(0.18)$ & $19.16(0.09)$ & $21.36(0.34)$ \\
\hline & & & $22.11(0.24)$ & $20.42(0.14)$ & $21.55(0.18)$ & $22.86(0.52)$ & $21.42(0.13)$ & $21.31(0.16)$ \\
\hline & & & $21.87(0.33)$ & $22.54(0.45)$ & 99.99 (9.99) & $23.64(0.66)$ & $22.36(0.32)$ & $21.28(0.20)$ \\
\hline & & & $22.40(0.34)$ & $23.58(0.94)$ & $23.16(0.54)$ & $23.29(0.64)$ & $99.99(9.99)$ & $21.78(0.31)$ \\
\hline \multirow[t]{4}{*}{3888} & 797.245 & 635.009 & $21.31(0.05)$ & $21.18(0.12)$ & $20.92(0.12)$ & $20.88(0.11)$ & $20.78(0.05)$ & $21.07(0.07)$ \\
\hline & & & $21.41(0.11)$ & $21.54(0.15)$ & $21.17(0.08)$ & $20.96(0.07)$ & $20.66(0.08)$ & $20.47(0.05)$ \\
\hline & & & $20.86(0.09)$ & $20.67(0.05)$ & $20.56(0.05)$ & $20.66(0.07)$ & $20.78(0.06)$ & $21.07(0.08)$ \\
\hline & & & $21.39(0.14)$ & $21.59(0.17)$ & $21.41(0.10)$ & $21.24(0.10)$ & $20.99(0.09)$ & $20.52(0.10)$ \\
\hline
\end{tabular}


Table 8. continuation.

\begin{tabular}{|c|c|c|c|c|c|c|c|c|}
\hline \multirow[t]{4}{*}{$\bar{F} 2$ ID } & \multirow[t]{4}{*}{$\mathrm{X}$} & \multirow[t]{4}{*}{$\mathrm{y}$} & $2 \mathrm{Ks} 10(\sigma)$ & $2 \mathrm{Ks} 01(\sigma)$ & $2 \mathrm{Ks} 02(\sigma)$ & $2 \mathrm{Ks} 03(\sigma)$ & $2 \mathrm{Ks} 05(\sigma)$ & $2 \mathrm{Ks} 06(\sigma)$ \\
\hline & & & $2 \mathrm{Ks} 07(\sigma)$ & $2 \mathrm{Ks} 08(\sigma)$ & $2 \mathrm{Ks} 09(\sigma)$ & $2 \mathrm{Ks} 11(\sigma)$ & $2 \mathrm{Ks} 12(\sigma)$ & $2 \mathrm{Ks} 13(\sigma)$ \\
\hline & & & $2 \mathrm{Ks} 14(\sigma)$ & $2 \mathrm{Ks} 15(\sigma)$ & $2 \mathrm{Ks} 16(\sigma)$ & $2 \mathrm{Ks} 17(\sigma)$ & $2 \mathrm{Ks} 18(\sigma)$ & $2 \mathrm{Ks} 19(\sigma)$ \\
\hline & & & $2 \mathrm{Ks} 20(\sigma)$ & $2 \mathrm{Ks} 21(\sigma)$ & $2 \mathrm{Ks} 22(\sigma)$ & $2 \mathrm{Ks} 23(\sigma)$ & $2 \mathrm{Ks} 24(\sigma)$ & $2 \mathrm{Ks} 04(\sigma)$ \\
\hline \multirow[t]{4}{*}{3889} & 125.703 & 2.655 & $21.02(0.09)$ & $22.25(0.30)$ & $22.71(0.49)$ & $21.43(0.19)$ & $21.65(0.15)$ & $20.53(0.13)$ \\
\hline & & & $21.42(0.15)$ & $20.80(0.13)$ & $22.13(5.07)$ & $17.76(0.10)$ & $99.99(9.99)$ & $21.63(0.22)$ \\
\hline & & & $21.40(2.14)$ & $21.00(0.11)$ & $21.76(0.09)$ & 99.99 (9.99) & $21.28(0.13)$ & 99.99 (9.99) \\
\hline & & & $20.74(0.11)$ & $21.67(0.33)$ & $20.92(0.70)$ & $20.60(0.16)$ & $21.46(0.38)$ & $20.33(0.37)$ \\
\hline \multirow[t]{4}{*}{3912} & 248.918 & 713.633 & $21.12(0.06)$ & $20.31(0.06)$ & $20.43(0.09)$ & $20.76(0.12)$ & $20.83(0.06)$ & $21.06(0.07)$ \\
\hline & & & $21.26(0.13)$ & $21.62(0.15)$ & $21.25(0.07)$ & $20.80(0.06)$ & $20.63(0.07)$ & $20.48(0.05)$ \\
\hline & & & $20.60(0.06)$ & $20.76(0.06)$ & $20.90(0.06)$ & $20.96(0.06)$ & $20.90(0.06)$ & $21.22(0.08)$ \\
\hline & & & $21.46(0.14)$ & $21.27(0.10)$ & $21.12(0.07)$ & $21.59(0.14)$ & $21.36(0.14)$ & $20.59(0.11)$ \\
\hline \multirow[t]{4}{*}{3914} & 256.811 & 133.548 & $20.87(0.06)$ & $20.87(0.10)$ & $21.22(0.13)$ & $21.66(0.21)$ & $20.56(0.06)$ & $20.59(0.06)$ \\
\hline & & & $20.82(0.08)$ & $20.87(0.09)$ & $20.84(0.08)$ & $20.65(0.07)$ & $20.43(0.07)$ & $20.56(0.06)$ \\
\hline & & & $21.17(0.10)$ & $21.37(0.09)$ & $21.29(0.08)$ & $21.18(0.08)$ & $21.31(0.09)$ & $21.06(0.09)$ \\
\hline & & & $21.01(0.10)$ & $21.05(0.10)$ & $21.21(0.10)$ & $21.04(0.09)$ & $20.74(0.07)$ & $20.43(0.12)$ \\
\hline \multirow[t]{4}{*}{3916} & 58.056 & 179.244 & $21.42(0.10)$ & $21.68(0.24)$ & $21.58(0.20)$ & $21.16(0.13)$ & $21.06(0.12)$ & 99.99 (9.99) \\
\hline & & & $21.65(0.16)$ & 99.99 (9.99) & $21.24(0.15)$ & $18.91(0.13)$ & $20.37(0.13)$ & $21.00(0.17)$ \\
\hline & & & $19.48(0.19)$ & 99.99 (9.99) & $22.12(0.57)$ & $20.95(0.15)$ & $20.95(0.13)$ & $20.73(0.12)$ \\
\hline & & & $20.88(0.12)$ & $21.45(0.16)$ & $19.42(0.13)$ & $21.71(0.16)$ & $21.55(0.27)$ & $19.99(0.26)$ \\
\hline \multirow[t]{4}{*}{3928} & 790.322 & 76.998 & $20.60(0.05)$ & $20.97(0.11)$ & $20.74(0.11)$ & $20.65(0.09)$ & $20.98(0.07)$ & $21.06(0.07)$ \\
\hline & & & $21.25(0.10)$ & $21.25(0.14)$ & $20.91(0.07)$ & $21.23(0.08)$ & $21.00(0.09)$ & $21.15(0.06)$ \\
\hline & & & $20.96(0.11)$ & $21.13(0.06)$ & $21.07(0.08)$ & $21.13(0.08)$ & $21.08(0.07)$ & $20.65(0.05)$ \\
\hline & & & $20.75(0.08)$ & $20.73(0.07)$ & $20.56(0.06)$ & $20.40(0.06)$ & $20.78(0.08)$ & $20.51(0.12)$ \\
\hline \multirow[t]{4}{*}{3941} & 365.734 & 210.995 & $20.70(0.05)$ & $20.90(0.09)$ & $21.26(0.15)$ & $21.69(0.20)$ & $21.60(0.13)$ & 20.95 \\
\hline & & & $20.88(0.09)$ & $20.85(0.11)$ & $20.90(0.07)$ & $21.14(0.08)$ & $21.21(0.11)$ & $21.20(0.07)$ \\
\hline & & & $21.69(0.18)$ & $21.18(0.08)$ & $21.24(0.07)$ & $21.02(0.06)$ & $21.03(0.07)$ & $20.65(0.07)$ \\
\hline & & & $20.43(0.07)$ & $20.51(0.06)$ & $20.44(0.05)$ & $20.73(0.08)$ & $21.01(0.10)$ & $20.93(0.20)$ \\
\hline \multirow[t]{4}{*}{4004} & 359.017 & 404.042 & $20.71(0.05)$ & $20.88(0.10)$ & $20.63(0.08)$ & $21.49(0.14)$ & $21.56(0.09)$ & 21.06 \\
\hline & & & $21.21(0.11)$ & $21.11(0.10)$ & $20.83(0.06)$ & $21.09(0.06)$ & $21.20(0.11)$ & $20.75(0.06)$ \\
\hline & & & $21.07(0.10)$ & $20.81(0.06)$ & $20.73(0.05)$ & $20.54(0.06)$ & $20.61(0.05)$ & $20.83(0.06)$ \\
\hline & & & $21.10(0.11)$ & $20.98(0.12)$ & $21.29(0.08)$ & $21.44(0.11)$ & $21.39(0.11)$ & $21.11(0.18)$ \\
\hline \multirow[t]{4}{*}{4017} & 428.467 & 358.329 & $20.44(0.05)$ & $20.71(0.09)$ & $20.93(0.08)$ & $21.53(0.17)$ & $20.98(0.06)$ & $20.82(0.07)$ \\
\hline & & & $21.07(0.12)$ & $20.92(0.10)$ & $20.59(0.06)$ & $21.16(0.07)$ & $21.47(0.15)$ & 21.30 \\
\hline & & & $21.23(0.09)$ & $21.31(0.09)$ & $21.21(0.05)$ & $21.13(0.07)$ & $21.12(0.06)$ & $21.03(0.07)$ \\
\hline & & & $21.28(0.16)$ & $21.20(0.14)$ & $21.16(0.08)$ & $20.74(0.06)$ & $20.68(0.07)$ & $21.25(0.26)$ \\
\hline \multirow[t]{4}{*}{4021} & 700.288 & 494.977 & $20.79(0.10)$ & $20.69(0.10)$ & $21.16(0.15)$ & $20.99(0.13)$ & $20.16(0.15)$ & $20.57(0.18)$ \\
\hline & & & $20.62(0.11)$ & $20.57(0.51)$ & $20.49(0.11)$ & $20.00(0.09)$ & $20.71(0.10)$ & $21.48(0.38)$ \\
\hline & & & $21.96(0.26)$ & $22.50(0.35)$ & $20.84(0.27)$ & $21.42(0.14)$ & $20.82(0.11)$ & $21.45(0.12)$ \\
\hline & & & $21.55(0.16)$ & $21.26(0.16)$ & $21.26(0.11)$ & $22.06(0.23)$ & $21.25(0.11)$ & $20.50(0.14)$ \\
\hline \multirow[t]{4}{*}{4034} & 727.690 & 229.530 & $21.03(0.07)$ & $21.07(0.14)$ & $21.19(0.11)$ & $21.20(0.17)$ & $20.46(0.06)$ & $20.60(0.07)$ \\
\hline & & & $20.62(0.10)$ & $21.02(0.10)$ & $20.85(0.07)$ & $21.04(0.09)$ & $21.02(0.09)$ & $20.67(0.07)$ \\
\hline & & & $21.37(0.10)$ & $21.37(0.08)$ & $21.18(0.06)$ & $21.13(0.08)$ & $21.04(0.06)$ & $20.78(0.07)$ \\
\hline & & & $20.89(0.09)$ & $20.51(0.06)$ & $20.64(0.06)$ & $20.74(0.08)$ & $20.86(0.07)$ & $20.48(0.12)$ \\
\hline \multirow[t]{4}{*}{4035} & 590.112 & 30.280 & $21.12(0.06)$ & $20.93(0.08)$ & $21.63(0.17)$ & $21.21(0.16)$ & $20.68(0.06)$ & $20.64(0.06)$ \\
\hline & & & $20.57(0.06)$ & $21.30(0.14)$ & $20.76(0.06)$ & $21.24(0.07)$ & $20.98(0.10)$ & $20.68(0.05)$ \\
\hline & & & $20.56(0.07)$ & $20.61(0.05)$ & $20.83(0.05)$ & $20.94(0.07)$ & $21.08(0.06)$ & $21.20(0.09)$ \\
\hline & & & $20.97(0.11)$ & $21.45(0.16)$ & $21.18(0.09)$ & $21.10(0.09)$ & $20.79(0.08)$ & $21.18(0.20)$ \\
\hline \multirow[t]{4}{*}{4053} & 507.566 & 223.257 & $21.08(0.06)$ & $20.44(0.07)$ & $20.80(0.12)$ & $21.00(0.13)$ & $21.25(0.08)$ & $21.03(0.07)$ \\
\hline & & & $21.10(0.09)$ & $21.36(0.15)$ & $21.18(0.06)$ & $21.08(0.07)$ & $20.93(0.11)$ & $20.61(0.06)$ \\
\hline & & & $21.06(0.08)$ & $20.97(0.07)$ & $20.85(0.07)$ & $20.68(0.07)$ & $20.69(0.06)$ & $20.54(0.06)$ \\
\hline & & & $20.69(0.09)$ & $20.68(0.06)$ & $20.64(0.06)$ & $20.99(0.08)$ & $21.06(0.10)$ & $20.62(0.19)$ \\
\hline
\end{tabular}


Table 8. continuation.

\begin{tabular}{|c|c|c|c|c|c|c|c|c|}
\hline \multirow[t]{4}{*}{ F2 ID } & \multirow[t]{4}{*}{$\mathrm{x}$} & \multirow[t]{4}{*}{$y$} & $2 \mathrm{Ks} 10(\sigma)$ & $2 \mathrm{Ks} 01(\sigma)$ & $2 \mathrm{Ks} 02(\sigma)$ & $2 \mathrm{Ks} 03(\sigma)$ & $2 \mathrm{Ks} 05(\sigma)$ & $2 \mathrm{Ks} 06(\sigma)$ \\
\hline & & & $2 \mathrm{Ks} 07(\sigma)$ & $2 \mathrm{Ks} 08(\sigma)$ & $2 \mathrm{Ks} 09(\sigma)$ & $2 \mathrm{Ks} 11(\sigma)$ & $2 \mathrm{Ks} 12(\sigma)$ & $2 \mathrm{Ks} 13(\sigma)$ \\
\hline & & & $2 \mathrm{Ks} 14(\sigma)$ & $2 \mathrm{Ks} 15(\sigma)$ & $2 \mathrm{Ks} 16(\sigma)$ & $2 \mathrm{Ks} 17(\sigma)$ & $2 \mathrm{Ks} 18(\sigma)$ & $2 \mathrm{Ks} 19(\sigma)$ \\
\hline & & & $2 \mathrm{Ks} 20(\sigma)$ & $2 \mathrm{Ks} 21(\sigma)$ & $2 \mathrm{Ks} 22(\sigma)$ & $2 \mathrm{Ks} 23(\sigma)$ & $2 \mathrm{Ks} 24(\sigma)$ & $2 \mathrm{Ks} 04(\sigma)$ \\
\hline \multirow[t]{4}{*}{4054} & 17.627 & 422.046 & $21.29(0.14)$ & $20.93(0.20)$ & $21.04(0.19)$ & $20.03(0.08)$ & $20.71(0.18)$ & 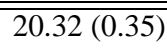 \\
\hline & & & $22.29(0.31)$ & $20.85(0.37)$ & $21.11(0.16)$ & $21.03(0.10)$ & $21.48(0.15)$ & $20.25(0.11)$ \\
\hline & & & $22.22(0.32)$ & $21.18(0.26)$ & 99.99 (9.99) & 99.99 (9.99) & $22.89(0.36)$ & $22.28(0.28)$ \\
\hline & & & $21.99(0.24)$ & $24.93(5.16)$ & $21.55(0.15)$ & 99.99 (9.99) & 99.99 (9.99) & $22.09(0.72)$ \\
\hline \multirow[t]{4}{*}{4097} & 526.847 & 688.469 & $20.82(0.06)$ & $20.76(0.07)$ & $20.78(0.09)$ & $20.98(0.12)$ & $20.98(0.06)$ & $20.58(0.06)$ \\
\hline & & & $20.70(0.09)$ & $20.38(0.06)$ & $20.62(0.06)$ & $21.03(0.06)$ & $20.97(0.08)$ & $21.06(0.06)$ \\
\hline & & & $21.08(0.09)$ & $21.23(0.09)$ & $21.24(0.07)$ & $21.33(0.07)$ & $21.22(0.07)$ & $21.07(0.08)$ \\
\hline & & & $20.73(0.08)$ & $20.97(0.10)$ & $20.65(0.07)$ & $20.62(0.06)$ & $20.59(0.07)$ & $21.51(0.36)$ \\
\hline \multirow[t]{4}{*}{4103} & 468.065 & 505.340 & $20.73(0.10)$ & $20.83(0.28)$ & $21.63(0.24)$ & $20.56(0.12)$ & $20.28(0.17)$ & $20.48(0.26)$ \\
\hline & & & $21.33(0.18)$ & $20.81(0.79)$ & $21.22(0.15)$ & $19.96(0.08)$ & $21.17(0.16)$ & $20.95(0.38)$ \\
\hline & & & $21.20(0.12)$ & $21.39(0.11)$ & $21.28(0.17)$ & $21.79(0.23)$ & $21.35(0.23)$ & $21.37(0.20)$ \\
\hline & & & $22.11(0.52)$ & $22.40(0.49)$ & $21.25(0.14)$ & $22.44(0.28)$ & 99.99 (9.99) & $20.68(0.25)$ \\
\hline \multirow[t]{4}{*}{4141} & 713.961 & 504.932 & $21.25(0.09)$ & $21.23(0.16)$ & $21.15(0.15)$ & $20.99(0.14)$ & $20.15(0.15)$ & $19.95(0.09)$ \\
\hline & & & $21.16(0.13)$ & $20.08(0.28)$ & $20.63(0.12)$ & $20.59(0.09)$ & 99.99 (9.99) & $20.97(0.22)$ \\
\hline & & & $21.89(0.30)$ & $21.75(0.20)$ & $22.08(1.25)$ & $22.44(0.54)$ & $21.64(0.19)$ & $22.00(0.23)$ \\
\hline & & & 99.99 (9.99) & $22.05(0.27)$ & $21.70(0.22)$ & 99.99 (9.99) & 99.99 (9.99) & $20.72(0.17)$ \\
\hline \multirow[t]{4}{*}{4173} & 764.284 & 587.884 & $21.15(0.13)$ & $21.21(0.14)$ & $21.09(0.18)$ & $20.64(0.16)$ & $20.28(0.14)$ & 99.99 (9.99) \\
\hline & & & $22.43(0.38)$ & 99.99 (9.99) & $24.10(2.05)$ & $20.81(0.13)$ & $20.66(0.08)$ & $21.24(0.14)$ \\
\hline & & & $20.80(0.14)$ & $22.02(0.19)$ & $20.15(0.21)$ & $21.37(0.16)$ & $22.63(0.23)$ & $21.30(0.10)$ \\
\hline & & & $21.09(0.14)$ & $21.43(0.13)$ & $20.68(0.08)$ & $19.81(0.13)$ & $20.03(0.13)$ & $20.85(0.96)$ \\
\hline \multirow[t]{4}{*}{4182} & 728.664 & 434.580 & $21.07(0.06)$ & $21.45(0.13)$ & $21.77(0.20)$ & $21.97(0.20)$ & $20.56(0.06)$ & $20.89(0.07)$ \\
\hline & & & $21.56(0.13)$ & $21.75(0.20)$ & $21.36(0.12)$ & $20.78(0.06)$ & $20.70(0.08)$ & $20.68(0.05)$ \\
\hline & & & $20.53(0.06)$ & $20.68(0.07)$ & $20.69(0.05)$ & $21.05(0.06)$ & $21.17(0.06)$ & $21.24(0.09)$ \\
\hline & & & $21.16(0.14)$ & $21.39(0.15)$ & $21.30(0.09)$ & $21.43(0.11)$ & $21.12(0.09)$ & $20.79(0.13)$ \\
\hline \multirow[t]{4}{*}{4196} & 162.064 & 292.952 & $20.98(0.06)$ & $20.98(0.11)$ & $21.01(0.10)$ & $21.71(0.27)$ & $21.62(0.10)$ & $21.18(0.08)$ \\
\hline & & & $20.73(0.07)$ & $20.68(0.08)$ & $20.67(0.06)$ & $21.65(0.11)$ & $21.40(0.13)$ & $21.09(0.07)$ \\
\hline & & & $21.88(0.15)$ & $21.37(0.08)$ & $20.93(0.06)$ & $20.73(0.06)$ & $20.65(0.05)$ & $20.79(0.06)$ \\
\hline & & & $20.50(0.07)$ & $20.70(0.09)$ & $21.19(0.07)$ & $23.74(0.83)$ & 99.99 (9.99) & $20.97(0.09)$ \\
\hline \multirow[t]{4}{*}{4233} & 694.949 & 499.693 & $20.76(0.10)$ & $20.95(0.15)$ & $20.74(0.13)$ & $20.47(0.09)$ & $20.02(0.13)$ & $99.99(9.99)$ \\
\hline & & & $21.02(0.15)$ & $20.28(0.30)$ & $20.74(0.10)$ & $20.11(0.11)$ & $21.43(0.12)$ & $99.99(9.99)$ \\
\hline & & & $21.38(0.14)$ & $21.22(0.12)$ & $20.67(0.32)$ & $21.80(0.10)$ & $20.74(0.11)$ & $20.91(0.10)$ \\
\hline & & & $20.56(0.09)$ & $21.33(0.16)$ & $21.49(0.16)$ & $23.18(0.61)$ & $21.46(0.12)$ & $20.57(0.14)$ \\
\hline \multirow[t]{4}{*}{4272} & 126.101 & 446.682 & $21.46(0.15)$ & $21.60(0.30)$ & $23.19(0.82)$ & $21.93(0.37)$ & $21.93(0.45)$ & 99.99 (9.99) \\
\hline & & & $21.64(0.19)$ & 99.99 (9.99) & $21.87(0.24)$ & $20.26(0.10)$ & $21.27(0.17)$ & $22.52(1.30)$ \\
\hline & & & $20.76(0.14)$ & $20.75(0.15)$ & $21.78(0.22)$ & $21.65(0.20)$ & $22.11(0.21)$ & $20.70(0.10)$ \\
\hline & & & $21.01(0.12)$ & $21.52(0.14)$ & $20.43(0.07)$ & $20.97(0.09)$ & $21.65(0.16)$ & $20.41(0.17)$ \\
\hline \multirow[t]{4}{*}{4277} & 520.737 & 226.877 & $20.60(0.06)$ & $20.38(0.06)$ & $20.55(0.09)$ & $20.52(0.08)$ & $20.98(0.06)$ & $20.64(0.07)$ \\
\hline & & & $20.83(0.10)$ & $20.83(0.07)$ & $20.75(0.06)$ & $21.43(0.11)$ & $21.54(0.14)$ & $20.99(0.06)$ \\
\hline & & & $20.99(0.09)$ & $21.30(0.09)$ & $21.64(0.13)$ & $21.45(0.09)$ & $21.56(0.10)$ & $21.29(0.09)$ \\
\hline & & & $22.44(0.34)$ & $21.68(0.13)$ & $21.70(0.12)$ & $21.74(0.13)$ & $21.90(0.17)$ & $21.35(0.22)$ \\
\hline \multirow[t]{4}{*}{4286} & 265.024 & 257.490 & $21.45(0.11)$ & $21.61(0.15)$ & $21.62(0.21)$ & $20.99(0.11)$ & $21.41(0.15)$ & 99.99 (9.99) \\
\hline & & & $21.72(0.16)$ & $19.24(0.23)$ & $21.15(0.14)$ & $18.55(0.10)$ & 99.99 (9.99) & $21.32(0.13)$ \\
\hline & & & 99.99 (9.99) & $21.91(0.92)$ & $21.50(0.13)$ & $21.49(0.09)$ & $21.40(0.14)$ & $20.22(0.17)$ \\
\hline & & & $20.59(0.12)$ & $21.86(0.20)$ & 99.99 (9.99) & $21.06(0.30)$ & 99.99 (9.99) & $19.51(0.18)$ \\
\hline \multirow[t]{4}{*}{4291} & 124.064 & 247.886 & $21.23(0.06)$ & $21.16(0.11)$ & $21.12(0.19)$ & $21.11(0.10)$ & $21.14(0.06)$ & $21.26(0.08)$ \\
\hline & & & $21.62(0.13)$ & $21.93(0.20)$ & $21.34(0.09)$ & $20.82(0.06)$ & $20.53(0.07)$ & $20.80(0.07)$ \\
\hline & & & $20.61(0.06)$ & $20.49(0.06)$ & $20.72(0.05)$ & $20.81(0.08)$ & $20.89(0.06)$ & $21.01(0.06)$ \\
\hline & & & $21.47(0.16)$ & $21.26(0.18)$ & $21.04(0.07)$ & $21.10(0.07)$ & $21.21(0.10)$ & $20.56(0.07)$ \\
\hline
\end{tabular}


Table 8. continuation.

\begin{tabular}{|c|c|c|c|c|c|c|c|c|}
\hline \multirow[t]{4}{*}{$\bar{F}$ F2 ID } & \multirow[t]{4}{*}{$\mathrm{x}$} & \multirow[t]{4}{*}{$\mathrm{y}$} & $2 \mathrm{Ks} 10(\sigma)$ & $2 \mathrm{Ks} 01(\sigma)$ & $2 \mathrm{Ks} 02(\sigma)$ & $2 \mathrm{Ks} 03(\sigma)$ & $2 \mathrm{Ks} 05(\sigma)$ & $\overline{2 \mathrm{Ks} 06(\sigma)}$ \\
\hline & & & $2 \mathrm{Ks} 07(\sigma)$ & $2 \mathrm{Ks} 08(\sigma)$ & $2 \mathrm{Ks} 09(\sigma)$ & $2 \mathrm{Ks} 11(\sigma)$ & $2 \mathrm{Ks} 12(\sigma)$ & $2 \mathrm{Ks} 13(\sigma)$ \\
\hline & & & $2 \mathrm{Ks} 14(\sigma)$ & $2 \mathrm{Ks} 15(\sigma)$ & $2 \mathrm{Ks} 16(\sigma)$ & $2 \mathrm{Ks} 17(\sigma)$ & $2 \mathrm{Ks} 18(\sigma)$ & $2 \mathrm{Ks} 19(\sigma)$ \\
\hline & & & $2 \mathrm{Ks} 20(\sigma)$ & $2 \mathrm{Ks} 21(\sigma)$ & $2 \mathrm{Ks} 22(\sigma)$ & $2 \mathrm{Ks} 23(\sigma)$ & $2 \mathrm{Ks} 24(\sigma)$ & $2 \mathrm{Ks} 04(\sigma)$ \\
\hline \multirow[t]{4}{*}{$\overline{4298}$} & $\overline{367.091}$ & $\overline{~ 62.301}$ & $21.21(0.08)$ & $21.41(0.12)$ & $21.18(0.12)$ & $20.85(0.12)$ & $21.09(0.08)$ & $21.37(0.11)$ \\
\hline & & & $21.11(0.09)$ & $21.69(0.22)$ & $21.37(0.11)$ & $21.17(0.08)$ & $20.80(0.09)$ & $20.84(0.05)$ \\
\hline & & & $20.61(0.08)$ & $20.59(0.05)$ & $20.68(0.06)$ & $20.70(0.07)$ & $20.67(0.06)$ & $20.93(0.07)$ \\
\hline & & & $21.25(0.14)$ & $21.26(0.15)$ & $21.21(0.10)$ & $21.33(0.09)$ & $21.04(0.08)$ & $20.59(0.15)$ \\
\hline \multirow[t]{4}{*}{4371} & 117.656 & 759.908 & $21.43(0.12)$ & $21.46(0.23)$ & $20.61(0.13)$ & $20.16(0.10)$ & 99.99 (9.99) & $21.55(0.19)$ \\
\hline & & & $21.59(0.14)$ & $21.85(0.30)$ & $21.24(0.13)$ & $20.98(0.11)$ & $21.25(0.15)$ & 99.99 (9.99) \\
\hline & & & $20.66(0.08)$ & $22.18(0.23)$ & $20.44(0.26)$ & $21.41(0.18)$ & $20.56(0.06)$ & $21.46(0.15)$ \\
\hline & & & $20.97(0.10)$ & $21.16(0.13)$ & $21.12(0.09)$ & $21.92(0.13)$ & $21.83(0.20)$ & $20.97(0.28)$ \\
\hline \multirow[t]{4}{*}{4394} & 173.995 & 551.437 & $20.99(0.07)$ & $21.58(0.14)$ & $22.09(0.21)$ & $21.57(0.14)$ & $20.80(0.05)$ & $21.24(0.09)$ \\
\hline & & & $21.17(0.11)$ & $21.49(0.15)$ & $21.19(0.10)$ & $20.73(0.06)$ & $20.58(0.06)$ & $20.74(0.05)$ \\
\hline & & & $20.99(0.08)$ & $20.86(0.07)$ & $20.80(0.06)$ & $20.86(0.07)$ & $21.16(0.10)$ & $21.31(0.11)$ \\
\hline & & & $21.73(0.16)$ & $21.76(0.24)$ & $21.45(0.11)$ & $21.72(0.13)$ & $21.14(0.09)$ & $20.84(0.12)$ \\
\hline \multirow[t]{4}{*}{4442} & 846.485 & 11.054 & $21.10(0.06)$ & $21.36(0.14)$ & $21.60(0.19)$ & $21.11(0.14)$ & $21.28(0.08)$ & $20.67(0.07)$ \\
\hline & & & $20.96(0.11)$ & $20.86(0.09)$ & $21.40(0.10)$ & $20.68(0.08)$ & $21.18(0.12)$ & $21.27(0.08)$ \\
\hline & & & $20.82(0.09)$ & $21.02(0.06)$ & $21.25(0.07)$ & $20.90(0.07)$ & $20.82(0.07)$ & $20.52(0.06)$ \\
\hline & & & $21.55(0.16)$ & $20.77(0.08)$ & $20.53(0.07)$ & $21.01(0.10)$ & $20.59(0.07)$ & $20.61(0.22)$ \\
\hline \multirow[t]{4}{*}{4487} & 371.452 & 94.176 & $20.46(0.05)$ & $20.76(0.12)$ & $21.58(0.14)$ & $21.88(0.18)$ & $21.40(0.09)$ & $20.91(0.07)$ \\
\hline & & & $20.84(0.10)$ & $20.83(0.09)$ & $20.49(0.07)$ & $20.97(0.08)$ & $21.04(0.09)$ & $21.34(0.08)$ \\
\hline & & & $21.21(0.13)$ & $21.12(0.08)$ & $21.23(0.06)$ & $21.12(0.05)$ & $21.08(0.07)$ & $21.34(0.08)$ \\
\hline & & & $21.28(0.12)$ & $21.09(0.13)$ & $20.87(0.06)$ & $20.91(0.08)$ & $20.53(0.07)$ & $22.23(0.61)$ \\
\hline \multirow[t]{4}{*}{4492} & 708.879 & 510.591 & $21.20(0.12)$ & $20.68(0.13)$ & $20.81(0.17)$ & $20.43(0.09)$ & $19.92(0.13)$ & $22.90(2.18)$ \\
\hline & & & $21.06(0.11)$ & 99.99 (9.99) & $20.23(0.11)$ & $20.84(0.14)$ & $21.04(0.11)$ & $21.05(0.32)$ \\
\hline & & & $22.87(0.70)$ & $21.25(0.13)$ & $20.72(0.22)$ & $21.51(0.12)$ & $21.23(0.09)$ & $21.87(0.15)$ \\
\hline & & & $23.70(1.68)$ & $21.52(0.31)$ & $22.45(0.31)$ & 99.99 (9.99) & 99.99 (9.99) & $19.66(0.40)$ \\
\hline \multirow[t]{4}{*}{4534} & 711.397 & 491.549 & $21.56(0.10)$ & $22.00(0.26)$ & $22.37(0.29)$ & $22.10(0.20)$ & $22.34(0.28)$ & $20.97(0.27)$ \\
\hline & & & $22.00(0.17)$ & 99.99 (9.99) & $21.60(0.11)$ & $19.75(0.06)$ & $20.63(0.09)$ & $21.33(0.18)$ \\
\hline & & & $21.12(0.19)$ & $21.38(0.09)$ & $21.56(0.12)$ & $21.92(0.11)$ & $21.56(0.08)$ & $20.77(0.08)$ \\
\hline & & & $21.21(0.14)$ & $21.51(0.18)$ & $21.25(0.10)$ & $21.16(0.10)$ & $21.10(0.11)$ & $20.88(0.17)$ \\
\hline \multirow[t]{4}{*}{4589} & 622.493 & 194.710 & $21.65(0.12)$ & $21.92(0.30)$ & $21.69(0.19)$ & $21.92(0.29)$ & $21.78(0.13)$ & $20.50(0.28)$ \\
\hline & & & $21.47(0.14)$ & $20.09(0.36)$ & $21.83(0.20)$ & $19.35(0.14)$ & $20.18(0.11)$ & $20.92(0.29)$ \\
\hline & & & $20.01(0.17)$ & $21.03(0.31)$ & $21.63(0.12)$ & $21.89(0.15)$ & $21.85(0.17)$ & $20.24(0.16)$ \\
\hline & & & $20.65(0.13)$ & $22.44(0.49)$ & $20.22(0.11)$ & $20.99(0.09)$ & $20.29(0.15)$ & $19.98(0.22)$ \\
\hline \multirow[t]{4}{*}{4608} & 780.725 & 572.034 & $22.47(0.18)$ & $99.99(9.99)$ & $22.30(0.36)$ & 99.99 (9.99) & $23.45(0.58)$ & $20.27(0.09)$ \\
\hline & & & 99.99 (9.99) & $19.03(0.10)$ & $22.61(0.17)$ & $20.78(0.10)$ & $21.65(0.24)$ & $21.57(0.22)$ \\
\hline & & & $21.10(0.17)$ & $21.87(0.15)$ & $23.96(0.63)$ & $23.10(0.39)$ & $23.27(0.40)$ & $21.79(0.18)$ \\
\hline & & & $21.71(0.21)$ & 99.99 (9.99) & $22.09(0.25)$ & $22.07(0.21)$ & $21.27(0.13)$ & $21.54(0.54)$ \\
\hline \multirow[t]{4}{*}{4638} & 86.897 & 40.083 & $21.26(0.12)$ & $21.37(0.13)$ & $22.27(0.38)$ & $20.49(0.11)$ & $21.34(0.12)$ & $99.99(9.99)$ \\
\hline & & & $21.99(0.25)$ & $21.38(0.33)$ & $21.94(0.22)$ & $20.58(0.08)$ & $21.06(0.11)$ & 99.99 (9.99) \\
\hline & & & $22.29(0.21)$ & $21.05(0.20)$ & $20.77(0.17)$ & $21.44(0.18)$ & $21.05(0.16)$ & $21.24(0.11)$ \\
\hline & & & $20.75(0.10)$ & $21.60(0.14)$ & $21.43(0.11)$ & $20.70(0.06)$ & $20.61(0.08)$ & $21.96(0.79)$ \\
\hline \multirow[t]{4}{*}{4661} & 52.470 & 160.751 & $22.39(0.23)$ & $21.85(0.16)$ & $21.31(0.19)$ & $21.79(0.30)$ & $21.90(0.31)$ & $19.20(0.31)$ \\
\hline & & & $22.71(0.46)$ & 99.99 (9.99) & $21.74(0.15)$ & $18.76(0.13)$ & $20.17(0.10)$ & 99.99 (9.99) \\
\hline & & & $19.72(0.39)$ & $21.76(2.20)$ & $21.56(0.23)$ & $21.82(0.10)$ & $21.76(0.16)$ & $20.83(0.16)$ \\
\hline & & & $21.47(0.35)$ & $23.85(1.29)$ & $22.20(3.26)$ & $21.02(0.15)$ & 99.99 (9.99) & $20.08(0.24)$ \\
\hline \multirow[t]{4}{*}{4678} & 703.973 & 513.781 & $21.40(0.12)$ & $21.40(0.23)$ & $20.90(0.16)$ & $20.84(0.14)$ & $20.24(0.14)$ & 99.99 (9.99) \\
\hline & & & $21.43(0.16)$ & 99.99 (9.99) & $21.38(0.17)$ & $20.29(0.08)$ & $22.07(0.28)$ & $20.75(0.21)$ \\
\hline & & & 99.99 (9.99) & $22.64(0.33)$ & 99.99 (9.99) & $23.23(0.79)$ & $22.35(0.15)$ & $22.90(0.43)$ \\
\hline & & & $99.99(9.99)$ & $99.99(9.99)$ & $22.83(0.46)$ & $99.99(9.99)$ & $99.99(9.99)$ & $20.17(0.38)$ \\
\hline
\end{tabular}


Table 8. continuation.

\begin{tabular}{|c|c|c|c|c|c|c|c|c|}
\hline \multirow[t]{4}{*}{$\overline{\mathrm{F} 2 \mathrm{ID}}$} & \multirow[t]{4}{*}{$\mathrm{x}$} & \multirow[t]{4}{*}{$\mathrm{y}$} & $2 \mathrm{Ks} 10(\sigma)$ & $2 \mathrm{Ks} 01(\sigma)$ & $2 \mathrm{Ks} 02(\sigma)$ & $2 \mathrm{Ks} 03(\sigma)$ & $2 \mathrm{Ks} 05(\sigma)$ & $2 \mathrm{Ks06}(\sigma)$ \\
\hline & & & $2 \mathrm{Ks} 07(\sigma)$ & $2 \mathrm{Ks} 08(\sigma)$ & $2 \mathrm{Ks} 09(\sigma)$ & $2 \mathrm{Ks} 11(\sigma)$ & $2 \mathrm{Ks} 12(\sigma)$ & $2 \mathrm{Ks} 13(\sigma)$ \\
\hline & & & $2 \mathrm{Ks} 14(\sigma)$ & $2 \mathrm{Ks} 15(\sigma)$ & $2 \mathrm{Ks} 16(\sigma)$ & $2 \mathrm{Ks} 17(\sigma)$ & $2 \mathrm{Ks} 18(\sigma)$ & $2 \mathrm{Ks} 19(\sigma)$ \\
\hline & & & $2 \mathrm{Ks} 20(\sigma)$ & $2 \mathrm{Ks} 21(\sigma)$ & $2 \mathrm{Ks} 22(\sigma)$ & $2 \mathrm{Ks} 23(\sigma)$ & $2 \mathrm{Ks} 24(\sigma)$ & $2 \mathrm{Ks} 04(\sigma)$ \\
\hline \multirow[t]{4}{*}{ 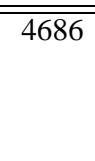 } & $\overline{668.814}$ & 80.933 & $21.28(0.10)$ & $20.45(0.12)$ & $20.69(0.07)$ & $21.44(0.20)$ & $20.93(0.08)$ & $21.13(0.08)$ \\
\hline & & & $21.57(0.16)$ & $22.58(0.32)$ & $21.41(0.10)$ & $21.05(0.09)$ & $21.30(0.13)$ & $20.90(0.06)$ \\
\hline & & & $21.71(0.17)$ & $21.18(0.07)$ & $21.54(0.11)$ & $21.68(0.16)$ & $21.27(0.07)$ & $21.38(0.09)$ \\
\hline & & & $20.70(0.08)$ & $21.02(0.14)$ & $20.73(0.07)$ & $20.51(0.07)$ & $20.49(0.07)$ & $20.39(0.30)$ \\
\hline \multirow[t]{4}{*}{4707} & 409.992 & 310.652 & $20.82(0.06)$ & $20.94(0.12)$ & $20.93(0.11)$ & $21.42(0.11)$ & $21.50(0.09)$ & $21.21(0.08)$ \\
\hline & & & $21.46(0.12)$ & $21.39(0.12)$ & $21.44(0.11)$ & $21.15(0.09)$ & $21.10(0.10)$ & $21.39(0.07)$ \\
\hline & & & $21.15(0.09)$ & $21.32(0.08)$ & $21.12(0.06)$ & $21.07(0.06)$ & $20.82(0.05)$ & $20.81(0.07)$ \\
\hline & & & $20.61(0.10)$ & $20.67(0.08)$ & $20.63(0.05)$ & $20.72(0.06)$ & $20.94(0.07)$ & $21.07(0.15)$ \\
\hline \multirow[t]{4}{*}{4762} & 715.893 & 492.961 & $23.09(0.37)$ & $23.78(1.32)$ & $24.00(2.25)$ & 99.99 (9.99) & $24.78(2.73)$ & $20.02(0.12)$ \\
\hline & & & $23.74(1.13)$ & $19.04(0.14)$ & $23.88(0.74)$ & $20.76(0.16)$ & $21.63(0.22)$ & $23.12(0.63)$ \\
\hline & & & $21.54(0.20)$ & $24.12(1.41)$ & 99.99 (9.99) & 99.99 (9.99) & $25.49(3.23)$ & $22.02(0.23)$ \\
\hline & & & $22.67(0.44)$ & $23.32(0.91)$ & $22.19(0.19)$ & $22.34(0.31)$ & $22.06(0.20)$ & $21.37(0.21)$ \\
\hline \multirow[t]{4}{*}{4812} & 756.235 & 588.293 & $21.27(0.09)$ & $21.15(0.15)$ & $20.89(0.14)$ & $21.00(0.13)$ & $20.45(0.13)$ & $20.51(0.08)$ \\
\hline & & & $21.29(0.14)$ & $20.02(0.20)$ & $21.12(0.13)$ & $20.49(0.08)$ & $21.86(0.22)$ & $21.54(0.16)$ \\
\hline & & & $21.17(0.15)$ & $20.86(0.08)$ & $20.41(0.21)$ & $21.42(0.21)$ & $21.41(0.17)$ & $21.53(0.21)$ \\
\hline & & & $21.70(0.17)$ & $22.01(0.34)$ & $21.20(0.13)$ & $22.59(0.28)$ & $21.82(0.16)$ & $21.10(0.19)$ \\
\hline \multirow[t]{4}{*}{4818} & 886.928 & 345.232 & $21.56(0.08)$ & $99.99(9.99)$ & $22.07(0.39)$ & $21.44(0.16)$ & $20.78(0.05)$ & $21.45(0.18)$ \\
\hline & & & $21.65(0.16)$ & $22.01(0.36)$ & $21.45(0.09)$ & $20.61(0.07)$ & $20.66(0.07)$ & $20.60(0.07)$ \\
\hline & & & $20.75(0.06)$ & $20.86(0.07)$ & $21.00(0.09)$ & $21.11(0.08)$ & $21.54(0.07)$ & $21.35(0.09)$ \\
\hline & & & 99.99 (9.99) & $21.21(0.08)$ & $21.24(0.09)$ & $21.52(0.14)$ & $20.90(0.08)$ & $21.00(0.17)$ \\
\hline \multirow[t]{4}{*}{4822} & 50.178 & 191.103 & $21.92(0.14)$ & $22.08(0.35)$ & $22.08(0.23)$ & $22.48(0.50)$ & $21.95(0.14)$ & $20.09(0.66)$ \\
\hline & & & $22.60(0.34)$ & 99.99 (9.99) & $23.26(0.48)$ & 99.99 (9.99) & $20.33(0.14)$ & $22.25(0.17)$ \\
\hline & & & $19.66(0.32)$ & $20.88(0.83)$ & $21.48(0.19)$ & $21.97(0.14)$ & $21.55(0.10)$ & $21.04(0.17)$ \\
\hline & & & $22.55(0.47)$ & $21.41(0.13)$ & $19.46(0.24)$ & $20.10(0.07)$ & $21.68(0.22)$ & $20.03(0.19)$ \\
\hline \multirow[t]{4}{*}{4844} & 790.515 & 407.132 & $20.70(0.07)$ & $21.29(0.14)$ & $21.82(0.25)$ & $22.11(0.20)$ & $20.69(0.05)$ & $20.90(0.08)$ \\
\hline & & & $21.04(0.11)$ & $20.86(0.08)$ & $20.78(0.06)$ & $21.76(0.14)$ & $21.61(0.19)$ & $21.60(0.09)$ \\
\hline & & & $20.71(0.07)$ & $20.92(0.06)$ & $21.00(0.07)$ & $21.26(0.09)$ & $21.34(0.07)$ & $21.25(0.11)$ \\
\hline & & & $21.23(0.12)$ & $21.67(0.16)$ & $21.46(0.10)$ & $21.65(0.11)$ & $23.94(1.30)$ & $20.83(0.17)$ \\
\hline \multirow[t]{4}{*}{4869} & 817.172 & 410.033 & $21.28(0.09)$ & $21.48(0.13)$ & $20.86(0.10)$ & $21.05(0.14)$ & $21.11(0.09)$ & 21.29 \\
\hline & & & $21.26(0.11)$ & $21.75(0.12)$ & $21.63(0.15)$ & $20.66(0.07)$ & $20.82(0.09)$ & $21.41(0.14)$ \\
\hline & & & $20.96(0.10)$ & $21.00(0.08)$ & $21.50(0.12)$ & $21.54(0.11)$ & $21.37(0.09)$ & $21.32(0.09)$ \\
\hline & & & $21.13(0.14)$ & $20.99(0.11)$ & $20.71(0.07)$ & $20.64(0.07)$ & $20.71(0.08)$ & $20.52(0.10)$ \\
\hline \multirow[t]{4}{*}{4950} & 351.475 & 586.850 & $21.63(0.09)$ & $20.82(0.10)$ & $20.82(0.09)$ & $20.81(0.09)$ & $20.90(0.06)$ & $21.27(0.08)$ \\
\hline & & & $21.22(0.10)$ & $21.55(0.15)$ & $21.79(0.13)$ & $20.99(0.07)$ & $20.96(0.10)$ & $21.42(0.07)$ \\
\hline & & & $21.72(0.14)$ & $21.38(0.08)$ & $21.30(0.07)$ & $20.91(0.06)$ & $20.82(0.05)$ & $20.81(0.06)$ \\
\hline & & & $20.82(0.09)$ & $20.80(0.11)$ & $21.30(0.08)$ & $21.76(0.14)$ & $21.67(0.15)$ & $22.25(0.34)$ \\
\hline \multirow[t]{4}{*}{5022} & 826.351 & 18.085 & $20.96(0.07)$ & $22.05(0.17)$ & $99.99(9.99)$ & $22.57(0.39)$ & $21.47(0.08)$ & $21.39(0.10)$ \\
\hline & & & $21.36(0.12)$ & 99.99 (9.99) & $21.47(0.11)$ & 99.99 (9.99) & $20.97(0.09)$ & $20.92(0.10)$ \\
\hline & & & $22.09(0.22)$ & $21.45(0.09)$ & $21.40(0.13)$ & $21.11(0.10)$ & $21.27(0.07)$ & $21.33(0.11)$ \\
\hline & & & 99.99 (9.99) & $21.51(0.13)$ & $20.77(0.07)$ & $20.40(0.06)$ & $20.37(0.08)$ & 99.99 (9.99) \\
\hline \multirow[t]{4}{*}{5045} & 823.415 & 300.274 & $20.93(0.05)$ & $21.86(0.21)$ & $21.64(0.17)$ & $21.15(0.12)$ & $21.51(0.11)$ & $21.51(0.10)$ \\
\hline & & & $22.34(0.24)$ & $21.80(0.21)$ & $21.33(0.14)$ & $21.83(0.11)$ & $21.43(0.12)$ & $21.18(0.09)$ \\
\hline & & & $21.09(0.09)$ & $20.94(0.05)$ & $20.64(0.06)$ & $20.72(0.06)$ & $20.90(0.08)$ & $21.04(0.07)$ \\
\hline & & & $22.25(0.29)$ & $21.28(0.11)$ & $21.53(0.14)$ & $21.63(0.12)$ & $21.48(0.11)$ & $21.48(0.24)$ \\
\hline \multirow[t]{4}{*}{5060} & 33.263 & 194.560 & $21.25(0.10)$ & $21.71(0.23)$ & $21.74(0.14)$ & $21.40(0.12)$ & $21.55(0.09)$ & $21.03(0.36)$ \\
\hline & & & $21.77(0.18)$ & $20.39(0.86)$ & $21.35(0.10)$ & $19.43(0.32)$ & $20.69(0.14)$ & $21.25(0.11)$ \\
\hline & & & $99.99(9.99)$ & $21.01(0.38)$ & $21.16(0.11)$ & 99.99 (9.99) & $21.20(0.09)$ & $21.14(0.14)$ \\
\hline & & & $20.77(0.13)$ & $21.00(0.11)$ & $99.99(9.99)$ & $20.28(0.07)$ & $20.42(0.12)$ & $20.73(0.24)$ \\
\hline
\end{tabular}


Table 8. continuation.

\begin{tabular}{|c|c|c|c|c|c|c|c|c|}
\hline \multirow[t]{4}{*}{$\bar{F}$ F2 ID } & \multirow[t]{4}{*}{$\mathrm{x}$} & \multirow[t]{4}{*}{$\mathrm{y}$} & $2 \mathrm{Ks} 10(\sigma)$ & $2 \mathrm{Ks} 01(\sigma)$ & $2 \mathrm{Ks} 02(\sigma)$ & $2 \mathrm{Ks} 03(\sigma)$ & $2 \mathrm{Ks} 05(\sigma)$ & $\overline{2 \mathrm{Ks} 06(\sigma)}$ \\
\hline & & & $2 \mathrm{Ks} 07(\sigma)$ & $2 \mathrm{Ks} 08(\sigma)$ & $2 \mathrm{Ks} 09(\sigma)$ & $2 \mathrm{Ks} 11(\sigma)$ & $2 \mathrm{Ks} 12(\sigma)$ & $2 \mathrm{Ks} 13(\sigma)$ \\
\hline & & & $2 \mathrm{Ks} 14(\sigma)$ & $2 \mathrm{Ks} 15(\sigma)$ & $2 \mathrm{Ks} 16(\sigma)$ & $2 \mathrm{Ks} 17(\sigma)$ & $2 \mathrm{Ks} 18(\sigma)$ & $2 \mathrm{Ks} 19(\sigma)$ \\
\hline & & & $2 \mathrm{Ks} 20(\sigma)$ & $2 \mathrm{Ks} 21(\sigma)$ & $2 \mathrm{Ks} 22(\sigma)$ & $2 \mathrm{Ks} 23(\sigma)$ & $2 \mathrm{Ks} 24(\sigma)$ & $2 \mathrm{Ks} 04(\sigma)$ \\
\hline \multirow[t]{4}{*}{ 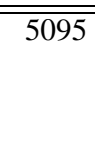 } & $\overline{470.747}$ & 191.450 & $21.70(0.19)$ & $22.01(0.64)$ & $21.74(0.20)$ & $21.51(0.24)$ & $21.23(0.14)$ & 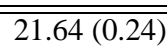 \\
\hline & & & $21.53(0.19)$ & $20.18(0.21)$ & $21.32(0.18)$ & $20.34(0.08)$ & $21.65(0.18)$ & $20.80(0.09)$ \\
\hline & & & $21.51(0.15)$ & $20.77(0.07)$ & $20.78(0.08)$ & $20.88(0.08)$ & $21.35(0.13)$ & $21.52(0.09)$ \\
\hline & & & $21.47(0.15)$ & $21.45(0.10)$ & $21.02(0.09)$ & $21.17(0.07)$ & $21.34(0.10)$ & $21.39(0.33)$ \\
\hline \multirow[t]{4}{*}{5161} & 108.786 & 382.722 & $22.74(0.22)$ & $21.04(0.13)$ & $20.71(0.08)$ & $20.87(0.12)$ & $22.94(0.22)$ & $22.46(0.31)$ \\
\hline & & & $23.80(0.78)$ & $22.87(0.48)$ & $22.42(0.21)$ & $20.71(0.06)$ & $20.70(0.08)$ & $20.87(0.06)$ \\
\hline & & & $22.31(0.27)$ & $22.00(0.16)$ & $21.83(0.11)$ & $21.98(0.19)$ & $21.65(0.10)$ & $21.95(0.17)$ \\
\hline & & & $21.75(0.21)$ & $21.57(0.11)$ & $21.16(0.09)$ & $21.06(0.10)$ & $20.76(0.06)$ & $22.20(0.77)$ \\
\hline \multirow[t]{4}{*}{5189} & 256.480 & 86.847 & $21.69(0.07)$ & $21.27(0.18)$ & $21.25(0.12)$ & $21.71(0.20)$ & $23.85(0.75)$ & $22.59(0.25)$ \\
\hline & & & $24.43(2.43)$ & $99.99(9.99)$ & $23.07(0.49)$ & $99.99(9.99)$ & $21.35(0.12)$ & $20.96(0.07)$ \\
\hline & & & $22.38(0.36)$ & $21.68(0.11)$ & $21.01(0.08)$ & $20.68(0.06)$ & $20.68(0.06)$ & $20.86(0.06)$ \\
\hline & & & $21.40(0.13)$ & $21.08(0.12)$ & $21.04(0.08)$ & $21.19(0.10)$ & $22.49(0.35)$ & $21.47(0.32)$ \\
\hline \multirow[t]{4}{*}{5251} & 31.060 & 440.962 & $21.70(0.11)$ & 99.99 (9.99) & 23.07 (1.97) & 99.99 (9.99) & $20.07(0.13)$ & $19.71(0.16)$ \\
\hline & & & $22.44(0.45)$ & $19.51(0.14)$ & 99.99 (9.99) & 99.99 (9.99) & $21.89(0.26)$ & $20.68(0.22)$ \\
\hline & & & $21.66(0.11)$ & $21.55(0.36)$ & $20.81(0.29)$ & 99.99 (9.99) & $23.30(0.29)$ & 99.99 (9.99) \\
\hline & & & $23.31(0.82)$ & 99.99 (9.99) & $21.79(0.20)$ & 99.99 (9.99) & $22.63(0.28)$ & $21.82(0.35)$ \\
\hline \multirow[t]{4}{*}{5259} & 744.804 & 365.482 & $20.67(0.05)$ & $20.85(0.10)$ & $20.50(0.08)$ & $20.45(0.07)$ & $21.29(0.08)$ & $21.09(0.10)$ \\
\hline & & & $21.03(0.10)$ & $20.77(0.07)$ & $21.03(0.07)$ & $21.60(0.10)$ & $21.39(0.13)$ & $21.43(0.08)$ \\
\hline & & & $21.12(0.08)$ & $21.11(0.06)$ & $21.19(0.06)$ & $21.29(0.08)$ & $21.60(0.09)$ & $21.95(0.15)$ \\
\hline & & & $22.77(0.43)$ & $22.06(0.21)$ & $21.90(0.11)$ & $22.00(0.16)$ & $21.89(0.18)$ & $21.16(0.23)$ \\
\hline \multirow[t]{4}{*}{5277} & 475.311 & 531.572 & $21.66(0.25)$ & $99.99(9.99)$ & $21.41(0.35)$ & $21.53(0.48)$ & $20.38(0.17)$ & $99.99(9.99)$ \\
\hline & & & $20.89(0.11)$ & $20.63(0.46)$ & $21.18(0.21)$ & $20.19(0.09)$ & $23.14(1.02)$ & 99.99 (9.99) \\
\hline & & & $22.26(0.30)$ & $21.11(0.16)$ & $21.64(0.20)$ & $21.55(0.13)$ & $21.48(0.21)$ & $21.64(0.15)$ \\
\hline & & & $21.58(0.18)$ & $21.90(0.30)$ & $21.50(0.15)$ & 99.99 (9.99) & 99.99 (9.99) & $21.20(0.35)$ \\
\hline \multirow[t]{4}{*}{5378} & 837.762 & 732.250 & $20.98(0.06)$ & $20.85(0.10)$ & $20.75(0.11)$ & $20.70(0.10)$ & $22.35(0.13)$ & $22.19(0.17)$ \\
\hline & & & $22.20(0.22)$ & $21.67(0.15)$ & $21.29(0.09)$ & $21.23(0.07)$ & $21.37(0.13)$ & $21.68(0.09)$ \\
\hline & & & $22.72(0.29)$ & $21.58(0.10)$ & $21.37(0.07)$ & $21.29(0.15)$ & $21.14(0.09)$ & $21.06(0.08)$ \\
\hline & & & $20.97(0.08)$ & $20.76(0.07)$ & $20.90(0.09)$ & $20.65(0.06)$ & $20.64(0.08)$ & $99.99(9.99)$ \\
\hline \multirow[t]{4}{*}{5454} & 467.379 & 172.238 & $21.40(0.10)$ & $21.66(0.23)$ & $21.59(0.18)$ & $21.21(0.13)$ & $21.32(0.16)$ & $20.78(0.11)$ \\
\hline & & & $21.79(0.20)$ & 99.99 (9.99) & $21.63(0.17)$ & $20.19(0.07)$ & $22.12(0.29)$ & $21.21(0.09)$ \\
\hline & & & $21.44(0.12)$ & $21.33(0.08)$ & $21.21(0.09)$ & $21.48(0.17)$ & $21.22(0.09)$ & $21.55(0.14)$ \\
\hline & & & $21.28(0.11)$ & $21.38(0.16)$ & $20.82(0.06)$ & $21.48(0.10)$ & $21.45(0.10)$ & $20.92(0.20)$ \\
\hline \multirow[t]{4}{*}{5595} & 575.631 & 243.740 & $21.38(0.09)$ & $21.10(0.13)$ & $21.30(0.14)$ & $22.38(0.33)$ & $20.82(0.06)$ & $20.99(0.08)$ \\
\hline & & & $21.21(0.10)$ & $21.71(0.16)$ & $21.20(0.07)$ & $20.78(0.07)$ & $20.72(0.06)$ & $21.06(0.06)$ \\
\hline & & & $20.99(0.09)$ & $21.33(0.08)$ & $21.47(0.08)$ & $21.42(0.07)$ & $21.76(0.09)$ & $21.68(0.10)$ \\
\hline & & & $21.59(0.17)$ & $21.37(0.14)$ & $21.08(0.09)$ & $20.88(0.08)$ & $20.93(0.09)$ & $21.54(0.30)$ \\
\hline \multirow[t]{4}{*}{5615} & 690.317 & 88.923 & $21.35(0.07)$ & $21.51(0.12)$ & $21.50(0.18)$ & $22.04(0.21)$ & $21.04(0.07)$ & $21.02(0.08)$ \\
\hline & & & $21.28(0.12)$ & $21.50(0.15)$ & $21.43(0.15)$ & $20.64(0.07)$ & $20.58(0.07)$ & $20.76(0.06)$ \\
\hline & & & $21.17(0.09)$ & $21.29(0.08)$ & $21.33(0.06)$ & $21.28(0.09)$ & $21.26(0.06)$ & $21.16(0.10)$ \\
\hline & & & $21.62(0.21)$ & $21.07(0.13)$ & $21.21(0.10)$ & $21.64(0.14)$ & $20.88(0.10)$ & $20.72(0.20)$ \\
\hline \multirow[t]{4}{*}{5616} & 625.235 & 227.382 & $21.31(0.09)$ & $21.36(0.11)$ & $21.83(0.21)$ & $21.82(0.17)$ & $21.54(0.07)$ & $20.62(0.06)$ \\
\hline & & & $21.44(0.11)$ & $21.08(0.19)$ & $21.40(0.10)$ & $19.57(0.16)$ & $20.81(0.15)$ & $21.32(0.09)$ \\
\hline & & & $20.46(0.15)$ & $20.98(0.08)$ & $21.44(0.08)$ & $21.32(0.10)$ & $21.34(0.10)$ & $21.04(0.10)$ \\
\hline & & & $20.89(0.08)$ & $21.42(0.17)$ & $20.97(0.09)$ & $20.79(0.08)$ & $21.02(0.12)$ & 99.99 (9.99) \\
\hline \multirow[t]{4}{*}{5635} & 811.716 & 705.519 & $20.64(0.06)$ & $22.00(0.32)$ & $22.07(0.28)$ & $21.49(0.18)$ & $21.51(0.07)$ & $21.34(0.09)$ \\
\hline & & & $21.37(0.13)$ & $21.32(0.10)$ & $21.06(0.08)$ & $21.42(0.09)$ & $21.49(0.11)$ & $20.98(0.07)$ \\
\hline & & & $21.11(0.10)$ & $21.05(0.07)$ & $21.38(0.08)$ & $21.56(0.10)$ & $21.53(0.13)$ & $21.70(0.14)$ \\
\hline & & & $21.25(0.12)$ & $21.62(0.23)$ & $21.22(0.09)$ & $20.87(0.08)$ & $20.73(0.07)$ & $20.79(0.12)$ \\
\hline
\end{tabular}


Table 8. continuation.

\begin{tabular}{|c|c|c|c|c|c|c|c|c|}
\hline \multirow[t]{4}{*}{$\overline{\mathrm{F} 2 \mathrm{ID}}$} & \multirow[t]{4}{*}{$\mathrm{x}$} & \multirow[t]{4}{*}{$\mathrm{y}$} & $2 \mathrm{Ks} 10(\sigma)$ & $2 \mathrm{Ks} 01(\sigma)$ & $2 \mathrm{Ks} 02(\sigma)$ & $2 \mathrm{Ks} 03(\sigma)$ & $2 \mathrm{Ks} 05(\sigma)$ & $2 \mathrm{Ks06}(\sigma)$ \\
\hline & & & $2 \mathrm{Ks} 07(\sigma)$ & $2 \mathrm{Ks} 08(\sigma)$ & $2 \mathrm{Ks} 09(\sigma)$ & $2 \mathrm{Ks} 11(\sigma)$ & $2 \mathrm{Ks} 12(\sigma)$ & $2 \mathrm{Ks} 13(\sigma)$ \\
\hline & & & $2 \mathrm{Ks} 14(\sigma)$ & $2 \mathrm{Ks} 15(\sigma)$ & $2 \mathrm{Ks} 16(\sigma)$ & $2 \mathrm{Ks} 17(\sigma)$ & $2 \mathrm{Ks} 18(\sigma)$ & $2 \mathrm{Ks} 19(\sigma)$ \\
\hline & & & $2 \mathrm{Ks} 20(\sigma)$ & $2 \mathrm{Ks} 21(\sigma)$ & $2 \mathrm{Ks} 22(\sigma)$ & $2 \mathrm{Ks} 23(\sigma)$ & $2 \mathrm{Ks} 24(\sigma)$ & $2 \mathrm{Ks} 04(\sigma)$ \\
\hline \multirow[t]{4}{*}{$\overline{56642}$} & $\overline{779.553}$ & $\overline{594.228}$ & $22.02(0.28)$ & $22.25(0.34)$ & $22.17(0.22)$ & $22.62(0.46)$ & $21.43(0.29)$ & $\overline{20.82(0.11)}$ \\
\hline & & & $21.90(0.19)$ & $19.22(0.11)$ & $21.73(0.23)$ & $21.06(0.15)$ & $22.83(0.55)$ & $21.60(0.25)$ \\
\hline & & & $20.96(0.10)$ & $21.98(0.24)$ & $20.94(0.31)$ & $24.22(1.07)$ & $22.49(0.34)$ & $21.84(0.15)$ \\
\hline & & & $20.95(0.09)$ & $22.69(0.43)$ & $21.74(0.14)$ & 99.99 (9.99) & $22.06(0.19)$ & $21.42(0.32)$ \\
\hline \multirow[t]{4}{*}{5656} & 776.083 & 583.138 & $21.31(0.14)$ & $21.16(0.17)$ & $21.60(0.29)$ & $21.09(0.15)$ & $20.40(0.15)$ & $20.49(0.11)$ \\
\hline & & & $21.62(0.18)$ & $20.78(0.50)$ & $21.23(0.21)$ & $20.58(0.08)$ & $21.42(0.16)$ & $21.97(0.20)$ \\
\hline & & & $20.82(0.17)$ & $22.05(0.24)$ & $21.14(0.32)$ & $22.22(0.20)$ & $22.55(0.24)$ & $21.74(0.11)$ \\
\hline & & & $99.99(9.99)$ & 99.99 (9.99) & $21.48(0.15)$ & 99.99 (9.99) & $21.90(0.21)$ & $20.57(0.21)$ \\
\hline \multirow[t]{4}{*}{5769} & 36.150 & 435.687 & $21.46(0.09)$ & $22.27(0.33)$ & $21.62(0.19)$ & $20.99(0.21)$ & $21.45(0.32)$ & 99.99 (9.99) \\
\hline & & & $22.48(0.36)$ & 99.99 (9.99) & $21.69(0.23)$ & $20.76(0.07)$ & $21.68(0.20)$ & $23.19(3.06)$ \\
\hline & & & $21.37(0.10)$ & $20.34(0.08)$ & 99.99 (9.99) & 99.99 (9.99) & $21.48(0.14)$ & $21.38(0.13)$ \\
\hline & & & $22.22(0.34)$ & $22.03(0.15)$ & $21.47(0.15)$ & 99.99 (9.99) & $21.88(0.17)$ & $21.36(0.28)$ \\
\hline \multirow[t]{4}{*}{5770} & 179.661 & 481.922 & $21.31(0.08)$ & $21.00(0.09)$ & $21.64(0.17)$ & $21.79(0.19)$ & $20.99(0.06)$ & $21.01(0.08)$ \\
\hline & & & $21.38(0.10)$ & $21.61(0.17)$ & $21.54(0.10)$ & $21.25(0.09)$ & $21.11(0.08)$ & $21.43(0.07)$ \\
\hline & & & $21.72(0.17)$ & $21.34(0.07)$ & $20.94(0.06)$ & $20.75(0.10)$ & $20.80(0.07)$ & $21.03(0.07)$ \\
\hline & & & $21.52(0.18)$ & $21.11(0.09)$ & $21.53(0.09)$ & $21.91(0.15)$ & $21.35(0.11)$ & $20.72(0.09)$ \\
\hline \multirow[t]{4}{*}{5897} & 198.408 & 281.172 & $20.96(0.06)$ & $21.36(0.10)$ & $21.21(0.18)$ & $20.83(0.09)$ & $21.49(0.08)$ & $21.10(0.08)$ \\
\hline & & & $21.37(0.12)$ & $21.53(0.14)$ & $20.75(0.06)$ & $21.46(0.10)$ & $20.97(0.09)$ & $21.01(0.06)$ \\
\hline & & & $21.07(0.09)$ & $21.24(0.08)$ & $21.57(0.08)$ & $21.68(0.09)$ & $21.65(0.11)$ & $21.35(0.10)$ \\
\hline & & & $21.49(0.16)$ & $21.25(0.09)$ & $21.67(0.11)$ & $20.95(0.07)$ & $20.98(0.08)$ & $22.37(0.48)$ \\
\hline \multirow[t]{4}{*}{5898} & 72.087 & 743.065 & $21.40(0.07)$ & $21.29(0.11)$ & $21.25(0.15)$ & $21.79(0.22)$ & 99.99 (9.99) & $22.17(0.18)$ \\
\hline & & & $22.81(0.53)$ & $22.43(0.38)$ & $22.04(0.16)$ & $20.89(0.07)$ & $20.92(0.09)$ & $20.78(0.05)$ \\
\hline & & & $21.61(0.17)$ & $21.05(0.06)$ & $21.22(0.09)$ & $21.77(0.14)$ & $21.76(0.17)$ & $22.00(0.16)$ \\
\hline & & & $21.59(0.17)$ & $22.32(0.29)$ & $22.35(0.16)$ & 99.99 (9.99) & $99.99(9.99)$ & $21.00(0.16)$ \\
\hline \multirow[t]{4}{*}{5970} & 31.470 & 419.341 & $21.70(0.21)$ & $21.59(0.26)$ & $21.71(0.20)$ & $21.58(0.38)$ & $20.51(0.19)$ & $21.01(0.41)$ \\
\hline & & & $21.89(0.22)$ & $21.30(0.61)$ & $21.61(0.25)$ & $20.66(0.07)$ & $22.03(0.22)$ & $20.07(0.18)$ \\
\hline & & & $22.08(0.23)$ & $21.34(0.25)$ & 99.99 (9.99) & 99.99 (9.99) & $22.17(0.23)$ & $23.04(0.61)$ \\
\hline & & & $23.54(1.03)$ & $22.02(0.24)$ & $26.55(9.98)$ & $22.06(0.20)$ & $21.59(0.14)$ & 99.99 (9.99) \\
\hline \multirow[t]{4}{*}{6079} & 765.569 & 567.395 & $21.96(0.19)$ & $22.53(0.37)$ & $24.58(2.84)$ & $22.76(0.65)$ & $21.94(0.13)$ & 20.43 \\
\hline & & & $22.08(0.27)$ & $19.72(0.18)$ & $22.22(0.23)$ & $20.50(0.08)$ & $21.11(0.14)$ & $21.59(0.16)$ \\
\hline & & & $20.92(0.16)$ & $22.15(0.22)$ & $22.83(0.28)$ & $22.23(0.18)$ & $22.30(0.18)$ & $21.13(0.10)$ \\
\hline & & & $21.04(0.10)$ & $21.62(0.13)$ & $20.93(0.11)$ & $21.66(0.15)$ & $21.02(0.08)$ & $20.95(0.18)$ \\
\hline \multirow[t]{4}{*}{6190} & 773.927 & 576.531 & $21.13(0.14)$ & $20.84(0.13)$ & $20.93(0.11)$ & $20.60(0.08)$ & $20.38(0.15)$ & $23.27(1.39)$ \\
\hline & & & $21.38(0.16)$ & $22.68(2.56)$ & $20.91(0.11)$ & $21.24(0.15)$ & $22.22(0.29)$ & $22.00(0.25)$ \\
\hline & & & $21.38(0.27)$ & $21.43(0.12)$ & $20.88(0.42)$ & $22.09(0.12)$ & $21.26(0.20)$ & $21.76(0.22)$ \\
\hline & & & $22.42(0.32)$ & $22.19(0.27)$ & $22.00(0.19)$ & $22.54(0.32)$ & $21.97(0.20)$ & $21.22(0.30)$ \\
\hline \multirow[t]{4}{*}{6476} & 92.030 & 446.583 & $21.61(0.07)$ & $22.23(0.30)$ & $21.99(0.24)$ & $21.91(0.27)$ & $21.94(0.12)$ & $18.79(0.15)$ \\
\hline & & & $21.46(0.13)$ & $19.36(0.19)$ & $21.94(0.13)$ & $20.42(0.10)$ & $21.38(0.16)$ & $22.15(0.92)$ \\
\hline & & & $20.68(0.15)$ & $21.28(0.39)$ & $21.81(0.12)$ & $21.54(0.11)$ & $21.45(0.09)$ & $20.89(0.17)$ \\
\hline & & & $20.98(0.14)$ & $22.34(0.44)$ & $20.82(0.10)$ & 99.99 (9.99) & $23.03(0.46)$ & $20.76(0.11)$ \\
\hline \multirow[t]{4}{*}{6484} & 792.447 & 481.648 & $21.67(0.08)$ & $20.80(0.09)$ & $21.57(0.18)$ & $21.51(0.17)$ & $21.21(0.08)$ & $21.21(0.10)$ \\
\hline & & & $21.63(0.18)$ & $21.09(0.10)$ & $21.71(0.14)$ & $22.10(0.14)$ & $23.27(0.66)$ & $21.64(0.09)$ \\
\hline & & & $21.96(0.19)$ & $21.54(0.09)$ & $21.37(0.07)$ & $21.40(0.10)$ & $21.37(0.07)$ & $22.51(0.27)$ \\
\hline & & & $22.15(0.23)$ & $21.37(0.19)$ & $21.24(0.08)$ & $20.58(0.06)$ & $20.87(0.08)$ & $21.92(0.26)$ \\
\hline \multirow[t]{4}{*}{6504} & 68.988 & 34.727 & $21.41(0.11)$ & $21.01(0.16)$ & $21.55(0.19)$ & $21.69(0.27)$ & $21.67(0.12)$ & $20.91(0.17)$ \\
\hline & & & $21.54(0.18)$ & $20.85(0.11)$ & $21.81(0.21)$ & $20.69(0.06)$ & $21.20(0.10)$ & $21.43(0.16)$ \\
\hline & & & $21.54(0.09)$ & $20.96(0.14)$ & $21.38(0.23)$ & $21.69(0.11)$ & $21.31(0.09)$ & $21.54(0.11)$ \\
\hline & & & $21.59(0.21)$ & $21.63(0.23)$ & $21.51(0.10)$ & $99.99(9.99)$ & $22.01(0.18)$ & $20.76(0.33)$ \\
\hline
\end{tabular}


Table 8. continuation.

\begin{tabular}{|c|c|c|c|c|c|c|c|c|}
\hline \multirow[t]{4}{*}{$\bar{F}$ F2 ID } & \multirow[t]{4}{*}{$\mathrm{x}$} & \multirow[t]{4}{*}{$\mathrm{y}$} & $2 \mathrm{Ks} 10(\sigma)$ & $2 \mathrm{Ks} 01(\sigma)$ & $2 \mathrm{Ks} 02(\sigma)$ & $2 \mathrm{Ks} 03(\sigma)$ & $2 \mathrm{Ks} 05(\sigma)$ & $\overline{2 \mathrm{Ks} 06(\sigma)}$ \\
\hline & & & $2 \mathrm{Ks} 07(\sigma)$ & $2 \mathrm{Ks} 08(\sigma)$ & $2 \mathrm{Ks} 09(\sigma)$ & $2 \mathrm{Ks} 11(\sigma)$ & $2 \mathrm{Ks} 12(\sigma)$ & $2 \mathrm{Ks} 13(\sigma)$ \\
\hline & & & $2 \mathrm{Ks} 14(\sigma)$ & $2 \mathrm{Ks} 15(\sigma)$ & $2 \mathrm{Ks} 16(\sigma)$ & $2 \mathrm{Ks} 17(\sigma)$ & $2 \mathrm{Ks} 18(\sigma)$ & $2 \mathrm{Ks} 19(\sigma)$ \\
\hline & & & $2 \mathrm{Ks} 20(\sigma)$ & $2 \mathrm{Ks} 21(\sigma)$ & $2 \mathrm{Ks} 22(\sigma)$ & $2 \mathrm{Ks} 23(\sigma)$ & $2 \mathrm{Ks} 24(\sigma)$ & $2 \mathrm{Ks} 04(\sigma)$ \\
\hline \multirow[t]{4}{*}{$\overline{66534}$} & $\overline{4662.763}$ & 507.712 & $222.31(0.25)$ & $21.14(0.31)$ & $\overline{22.02(0.35)}$ & $20.60(0.14)$ & $20.39(0.17)$ & 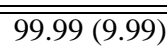 \\
\hline & & & $21.43(0.10)$ & $21.17(0.71)$ & $22.07(0.37)$ & $20.57(0.10)$ & $22.05(0.32)$ & $21.93(0.49)$ \\
\hline & & & $21.88(0.21)$ & $23.97(1.43)$ & $21.60(0.21)$ & $22.14(0.23)$ & $23.66(0.87)$ & $21.78(0.26)$ \\
\hline & & & 99.99 (9.99) & 99.99 (9.99) & $21.64(0.20)$ & $22.83(0.42)$ & $23.30(0.73)$ & $21.05(0.23)$ \\
\hline \multirow[t]{4}{*}{6606} & 880.138 & 827.235 & $22.61(0.35)$ & $99.99(9.99)$ & $21.76(0.26)$ & $99.99(9.99)$ & $22.65(0.30)$ & $21.98(0.15)$ \\
\hline & & & $22.32(0.40)$ & $21.89(0.22)$ & $22.53(0.33)$ & $21.63(0.14)$ & $21.86(0.21)$ & $22.02(0.18)$ \\
\hline & & & $22.47(0.27)$ & $21.74(0.12)$ & $21.71(0.17)$ & $21.04(0.07)$ & 99.99 (9.99) & $21.54(0.14)$ \\
\hline & & & $19.40(0.11)$ & $21.43(0.17)$ & $21.30(0.13)$ & $23.02(0.57)$ & $99.99(9.99)$ & $20.68(0.13)$ \\
\hline \multirow[t]{4}{*}{6837} & 121.917 & 845.695 & $21.02(0.05)$ & $21.86(0.17)$ & $23.32(0.81)$ & $21.06(0.13)$ & $21.75(0.11)$ & $21.95(0.15)$ \\
\hline & & & $21.89(0.14)$ & $22.04(0.23)$ & $21.33(0.08)$ & $21.96(0.15)$ & $21.61(0.12)$ & $21.18(0.07)$ \\
\hline & & & $21.01(0.10)$ & $21.07(0.07)$ & $21.09(0.06)$ & $21.53(0.09)$ & $21.65(0.10)$ & $21.47(0.11)$ \\
\hline & & & $21.89(0.24)$ & $21.73(0.14)$ & $21.73(0.16)$ & $22.60(0.31)$ & $99.99(9.99)$ & $21.22(0.35)$ \\
\hline \multirow[t]{4}{*}{6887} & 775.775 & 569.576 & $22.61(0.20)$ & $25.28(3.97)$ & $23.83(1.17)$ & 99.99 (9.99) & $25.35(2.37)$ & $21.06(0.11)$ \\
\hline & & & $23.68(0.86)$ & $21.05(0.50)$ & $23.25(0.40)$ & $20.53(0.08)$ & $21.85(0.21)$ & $21.93(0.21)$ \\
\hline & & & $21.46(0.20)$ & $22.01(0.23)$ & $22.94(0.36)$ & $23.90(0.80)$ & $22.33(0.23)$ & $21.29(0.12)$ \\
\hline & & & $22.09(0.23)$ & $22.82(0.62)$ & $21.96(0.18)$ & $22.50(0.27)$ & $21.88(0.20)$ & $20.78(0.18)$ \\
\hline \multirow[t]{4}{*}{7177} & 699.070 & 826.615 & $21.20(0.07)$ & $20.72(0.09)$ & $23.23(0.53)$ & $21.14(0.15)$ & $21.12(0.07)$ & $21.87(0.13)$ \\
\hline & & & $21.54(0.13)$ & $20.72(0.08)$ & $21.46(0.10)$ & $21.64(0.09)$ & $21.87(0.19)$ & $21.58(0.08)$ \\
\hline & & & $22.63(0.38)$ & $21.88(0.13)$ & $22.06(0.19)$ & $99.99(9.99)$ & $23.20(0.39)$ & $22.47(0.17)$ \\
\hline & & & 99.99 (9.99) & $21.58(0.20)$ & $22.52(0.20)$ & $21.12(0.08)$ & $21.65(0.18)$ & 99.99 (9.99) \\
\hline \multirow[t]{4}{*}{7294} & 691.671 & 48.960 & $21.56(0.07)$ & $21.78(0.14)$ & $21.57(0.19)$ & $21.98(0.22)$ & $21.56(0.06)$ & $21.61(0.09)$ \\
\hline & & & $20.88(0.10)$ & $21.89(0.20)$ & $21.53(0.13)$ & $21.49(0.11)$ & $21.70(0.16)$ & $21.27(0.08)$ \\
\hline & & & $21.61(0.12)$ & $21.00(0.08)$ & $21.53(0.08)$ & $21.54(0.12)$ & $21.56(0.09)$ & $21.13(0.07)$ \\
\hline & & & $22.68(0.44)$ & $21.37(0.16)$ & $21.13(0.10)$ & $20.72(0.07)$ & $21.74(0.16)$ & $21.59(0.31)$ \\
\hline \multirow[t]{4}{*}{7306} & 259.637 & 295.473 & $21.79(0.19)$ & $22.84(0.47)$ & $22.35(0.32)$ & $21.82(0.27)$ & $22.02(0.13)$ & $99.99(9.99)$ \\
\hline & & & $21.96(0.30)$ & 99.99 (9.99) & $21.88(0.22)$ & $20.01(0.38)$ & $19.97(0.13)$ & 99.99 (9.99) \\
\hline & & & 99.99 (9.99) & 99.99 (9.99) & $22.10(0.15)$ & $22.11(0.15)$ & $21.45(0.17)$ & $21.51(0.61)$ \\
\hline & & & $20.78(0.23)$ & $21.37(0.18)$ & $99.99(9.99)$ & $20.95(0.17)$ & $20.98(0.14)$ & $19.33(0.21)$ \\
\hline \multirow[t]{4}{*}{7353} & 128.704 & 774.632 & $21.59(0.14)$ & $22.07(0.20)$ & $21.13(0.19)$ & $22.38(0.57)$ & $19.91(0.15)$ & $21.43(0.11)$ \\
\hline & & & $21.87(0.20)$ & $20.46(0.10)$ & $21.70(0.17)$ & $21.11(0.09)$ & $22.28(0.32)$ & $21.60(0.16)$ \\
\hline & & & 99.99 (9.99) & $21.96(0.20)$ & $20.58(0.39)$ & 99.99 (9.99) & $22.31(0.16)$ & $22.08(0.15)$ \\
\hline & & & 99.99 (9.99) & $22.42(0.45)$ & $21.84(0.15)$ & $23.21(0.50)$ & $24.31(1.39)$ & $21.34(0.32)$ \\
\hline \multirow[t]{4}{*}{7536} & 506.814 & 110.240 & $21.83(0.13)$ & $21.78(0.16)$ & $99.99(9.99)$ & $21.42(0.13)$ & $21.21(0.10)$ & $21.24(0.09)$ \\
\hline & & & $21.89(0.22)$ & $22.32(0.68)$ & $21.91(0.13)$ & $20.49(0.08)$ & $22.83(0.31)$ & $21.35(0.12)$ \\
\hline & & & $22.80(0.54)$ & $23.46(0.52)$ & $22.20(0.17)$ & $22.80(0.24)$ & $23.48(0.36)$ & $22.75(0.41)$ \\
\hline & & & 99.99 (9.99) & 99.99 (9.99) & $22.20(0.17)$ & 99.99 (9.99) & 99.99 (9.99) & $23.48(1.81)$ \\
\hline \multirow[t]{4}{*}{7587} & 238.437 & 274.781 & $21.72(0.10)$ & $23.53(0.78)$ & $22.42(0.30)$ & $23.22(0.72)$ & $22.10(0.12)$ & $99.99(9.99)$ \\
\hline & & & $22.16(0.22)$ & 99.99 (9.99) & $22.57(0.22)$ & $18.27(0.09)$ & $19.78(0.32)$ & $21.74(0.06)$ \\
\hline & & & $20.51(0.36)$ & $22.42(0.21)$ & $22.19(0.15)$ & $21.94(0.13)$ & $21.73(0.10)$ & $21.93(0.14)$ \\
\hline & & & $21.83(0.21)$ & $22.03(0.18)$ & $21.73(0.15)$ & $20.56(0.11)$ & $21.34(0.16)$ & $21.44(0.34)$ \\
\hline \multirow[t]{4}{*}{7602} & 731.358 & 506.045 & $22.39(0.18)$ & $22.41(0.34)$ & $22.73(0.66)$ & $21.66(0.27)$ & $22.08(0.18)$ & 99.99 (9.99) \\
\hline & & & $23.07(0.58)$ & 99.99 (9.99) & $22.49(0.25)$ & $20.39(0.07)$ & 99.99 (9.99) & $22.03(0.13)$ \\
\hline & & & $24.07(1.27)$ & $22.64(0.22)$ & $22.41(0.19)$ & $23.45(0.49)$ & $22.61(0.23)$ & 99.99 (9.99) \\
\hline & & & $23.75(0.78)$ & 99.99 (9.99) & $22.76(0.19)$ & $99.99(9.99)$ & 99.99 (9.99) & $20.61(0.48)$ \\
\hline \multirow[t]{4}{*}{7623} & 453.342 & 675.695 & $21.70(0.09)$ & $21.53(0.10)$ & $21.57(0.19)$ & 99.99 (9.99) & $22.08(0.11)$ & $21.14(0.07)$ \\
\hline & & & $22.10(0.18)$ & $21.07(0.10)$ & $21.37(0.09)$ & $22.09(0.15)$ & $22.48(0.33)$ & $22.29(0.13)$ \\
\hline & & & $21.56(0.16)$ & $21.87(0.10)$ & $21.89(0.10)$ & $21.85(0.17)$ & $21.77(0.11)$ & $20.99(0.07)$ \\
\hline & & & $21.56(0.14)$ & $21.42(0.14)$ & $21.56(0.11)$ & $20.72(0.07)$ & $21.12(0.09)$ & $21.09(0.19)$ \\
\hline
\end{tabular}


Table 8. continuation.

\begin{tabular}{|c|c|c|c|c|c|c|c|c|}
\hline \multirow[t]{4}{*}{$\overline{\mathrm{F} 2 \mathrm{ID}}$} & \multirow[t]{4}{*}{$\mathrm{x}$} & \multirow[t]{4}{*}{$\mathrm{y}$} & $2 \mathrm{Ks} 10(\sigma)$ & $2 \mathrm{Ks} 01(\sigma)$ & $2 \mathrm{Ks} 02(\sigma)$ & $2 \mathrm{Ks} 03(\sigma)$ & $2 \mathrm{Ks} 05(\sigma)$ & $2 \mathrm{Ks06}(\sigma)$ \\
\hline & & & $2 \mathrm{Ks} 07(\sigma)$ & $2 \mathrm{Ks} 08(\sigma)$ & $2 \mathrm{Ks} 09(\sigma)$ & $2 \mathrm{Ks} 11(\sigma)$ & $2 \mathrm{Ks} 12(\sigma)$ & $2 \mathrm{Ks} 13(\sigma)$ \\
\hline & & & $2 \mathrm{Ks} 14(\sigma)$ & $2 \mathrm{Ks} 15(\sigma)$ & $2 \mathrm{Ks} 16(\sigma)$ & $2 \mathrm{Ks} 17(\sigma)$ & $2 \mathrm{Ks} 18(\sigma)$ & $2 \mathrm{Ks} 19(\sigma)$ \\
\hline & & & $2 \mathrm{Ks} 20(\sigma)$ & $2 \mathrm{Ks} 21(\sigma)$ & $2 \mathrm{Ks} 22(\sigma)$ & $2 \mathrm{Ks} 23(\sigma)$ & $2 \mathrm{Ks} 24(\sigma)$ & $2 \mathrm{Ks} 04(\sigma)$ \\
\hline \multirow[t]{4}{*}{7644} & $\overline{456.916}$ & 504.840 & $21.86(0.13)$ & $21.48(0.15)$ & $22.50(0.38)$ & $21.88(0.12)$ & $21.64(0.12)$ & 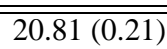 \\
\hline & & & $21.91(0.23)$ & $20.07(0.23)$ & $21.80(0.19)$ & $20.31(0.09)$ & $20.96(0.12)$ & $21.69(0.08)$ \\
\hline & & & $21.10(0.11)$ & $21.46(0.14)$ & $21.68(0.09)$ & $21.64(0.14)$ & $21.61(0.10)$ & $21.18(0.09)$ \\
\hline & & & $21.72(0.22)$ & $21.39(0.15)$ & $20.74(0.13)$ & $21.72(0.12)$ & $21.90(0.17)$ & $21.34(0.24)$ \\
\hline \multirow[t]{4}{*}{7775} & 492.606 & 525.767 & $23.22(0.34)$ & $25.00(2.67)$ & $24.49(1.86)$ & $99.99(9.99)$ & $22.74(0.18)$ & $99.99(9.99)$ \\
\hline & & & $22.96(0.78)$ & 99.99 (9.99) & $24.18(1.43)$ & $20.94(0.15)$ & $20.40(0.10)$ & $23.96(0.70)$ \\
\hline & & & $23.23(0.95)$ & $23.36(0.59)$ & 24.77 (1.66) & $22.88(0.34)$ & $23.61(0.72)$ & $22.25(0.20)$ \\
\hline & & & $23.42(1.01)$ & 99.99 (9.99) & $22.75(0.66)$ & $22.33(0.20)$ & $23.07(0.50)$ & $22.70(1.77)$ \\
\hline \multirow[t]{4}{*}{7785} & 486.544 & 526.428 & $22.88(0.25)$ & $24.48(3.00)$ & $25.45(6.73)$ & $23.13(0.56)$ & $99.99(9.99)$ & $22.19(1.25)$ \\
\hline & & & $24.51(2.98)$ & 99.99 (9.99) & 99.99 (9.99) & 99.99 (9.99) & $21.65(0.20)$ & $20.12(0.10)$ \\
\hline & & & 99.99 (9.99) & 99.99 (9.99) & $23.50(0.35)$ & 99.99 (9.99) & $22.70(0.14)$ & $23.76(0.95)$ \\
\hline & & & $23.90(1.77)$ & $23.36(1.22)$ & $22.78(0.41)$ & 25.15 (3.64) & $21.88(0.18)$ & $22.26(0.72)$ \\
\hline \multirow[t]{4}{*}{7830} & 452.040 & 516.979 & $21.73(0.07)$ & $22.03(0.34)$ & $22.06(0.24)$ & $22.38(0.35)$ & $21.69(0.11)$ & $20.93(0.24)$ \\
\hline & & & $22.09(0.21)$ & $19.88(0.20)$ & $22.05(0.15)$ & $20.11(0.10)$ & $20.21(0.12)$ & $21.68(0.08)$ \\
\hline & & & $21.16(0.17)$ & $21.64(0.14)$ & $21.93(0.13)$ & $21.71(0.08)$ & $21.81(0.09)$ & $21.15(0.13)$ \\
\hline & & & $21.03(0.10)$ & $21.33(0.11)$ & $21.32(0.13)$ & $20.96(0.11)$ & $21.58(0.12)$ & $20.93(0.20)$ \\
\hline \multirow[t]{4}{*}{7887} & 889.962 & 684.094 & $21.90(0.13)$ & $99.99(9.99)$ & $21.90(0.29)$ & $21.48(0.21)$ & $22.61(0.23)$ & $21.98(0.47)$ \\
\hline & & & $22.16(0.29)$ & 99.99 (9.99) & $22.86(0.36)$ & $20.85(0.35)$ & $20.76(0.11)$ & $21.80(0.12)$ \\
\hline & & & $21.31(0.47)$ & $21.84(0.35)$ & $22.64(0.14)$ & 99.99 (9.99) & $22.47(0.19)$ & $20.87(0.13)$ \\
\hline & & & 99.99 (9.99) & $21.39(0.26)$ & $21.41(0.11)$ & $20.93(0.07)$ & $21.49(0.13)$ & $20.99(0.35)$ \\
\hline \multirow[t]{4}{*}{8024} & 82.994 & 643.078 & $22.01(0.13)$ & $22.76(0.48)$ & $23.30(0.73)$ & $22.75(0.72)$ & $22.62(0.19)$ & $22.82(0.46)$ \\
\hline & & & $22.89(0.37)$ & $20.51(0.18)$ & $23.28(0.62)$ & $20.30(0.08)$ & $21.16(0.12)$ & $22.34(0.18)$ \\
\hline & & & $20.96(0.14)$ & $22.22(0.21)$ & $22.47(0.15)$ & $22.62(0.22)$ & $22.40(0.19)$ & $21.68(0.14)$ \\
\hline & & & $21.63(0.16)$ & 99.99 (9.99) & $21.93(0.12)$ & $21.59(0.11)$ & $22.97(0.39)$ & $21.72(0.38)$ \\
\hline \multirow[t]{4}{*}{8048} & 78.459 & 642.120 & $21.91(0.11)$ & $22.18(0.22)$ & $22.95(0.52)$ & $23.02(0.78)$ & $22.40(0.12)$ & $21.65(0.20)$ \\
\hline & & & $22.09(0.19)$ & $22.91(0.80)$ & $22.01(0.15)$ & $20.08(0.07)$ & $20.88(0.12)$ & $21.47(0.08)$ \\
\hline & & & $21.31(0.16)$ & $21.93(0.15)$ & $21.34(0.08)$ & $21.81(0.12)$ & $21.79(0.09)$ & $21.63(0.14)$ \\
\hline & & & $22.26(0.33)$ & $21.78(0.19)$ & $21.73(0.11)$ & $21.56(0.13)$ & $21.46(0.12)$ & $21.24(0.25)$ \\
\hline \multirow[t]{4}{*}{8259} & 161.000 & 806.380 & $21.97(0.11)$ & $22.71(0.41)$ & 99.99 (9.99) & $24.03(1.74)$ & 21.35 & 22.85 \\
\hline & & & $99.99(9.99)$ & $21.31(0.13)$ & $22.69(0.32)$ & $22.06(0.20)$ & $21.64(0.18)$ & $22.07(0.11)$ \\
\hline & & & $22.89(0.51)$ & $22.26(0.14)$ & 99.99 (9.99) & $21.36(0.10)$ & $21.15(0.07)$ & $22.12(0.15)$ \\
\hline & & & $21.76(0.20)$ & $21.06(0.11)$ & $22.24(0.16)$ & $21.07(0.10)$ & $20.98(0.09)$ & 99.99 (9.99) \\
\hline \multirow[t]{4}{*}{8315} & 518.321 & 828.124 & $22.03(0.13)$ & $21.75(0.15)$ & $21.88(0.15)$ & $22.49(0.66)$ & $21.01(0.10)$ & $20.58(0.08)$ \\
\hline & & & $22.21(0.22)$ & $22.12(0.22)$ & $21.59(0.18)$ & $21.77(0.12)$ & $22.46(0.28)$ & $21.11(0.15)$ \\
\hline & & & $21.96(0.14)$ & $21.78(0.24)$ & $99.99(9.99)$ & $21.41(0.18)$ & $23.30(0.62)$ & $21.65(0.10)$ \\
\hline & & & $21.66(0.15)$ & $21.70(0.16)$ & $21.46(0.10)$ & $22.35(0.20)$ & $21.63(0.18)$ & $23.31(1.32)$ \\
\hline \multirow[t]{4}{*}{8361} & 523.173 & 831.813 & $21.94(0.11)$ & $22.01(0.23)$ & $21.69(0.19)$ & $21.59(0.30)$ & $20.90(0.11)$ & $20.78(0.10)$ \\
\hline & & & $21.85(0.11)$ & $21.59(0.16)$ & $21.15(0.09)$ & $21.16(0.09)$ & $21.51(0.11)$ & $21.73(0.16)$ \\
\hline & & & $21.89(0.16)$ & $21.71(0.26)$ & $21.87(0.38)$ & $22.21(0.24)$ & $22.49(0.46)$ & $22.64(0.28)$ \\
\hline & & & $21.80(0.15)$ & $22.71(0.43)$ & $23.39(0.43)$ & $22.42(0.23)$ & $21.51(0.20)$ & $22.08(0.53)$ \\
\hline \multirow[t]{4}{*}{8531} & 700.970 & 523.287 & $99.99(9.99)$ & $99.99(9.99)$ & $22.61(0.30)$ & $99.99(9.99)$ & 99.99 (9.99) & $99.99(9.99)$ \\
\hline & & & $99.99(9.99)$ & 99.99 (9.99) & 99.99 (9.99) & $20.57(0.11)$ & $22.22(0.31)$ & $21.06(0.24)$ \\
\hline & & & $22.90(0.57)$ & $22.02(0.21)$ & 99.99 (9.99) & $23.42(0.45)$ & $22.79(0.29)$ & $23.55(0.51)$ \\
\hline & & & $21.91(0.25)$ & 99.99 (9.99) & $22.18(0.21)$ & 99.99 (9.99) & $23.34(0.52)$ & $21.07(0.30)$ \\
\hline \multirow[t]{4}{*}{8718} & 595.039 & 198.886 & $21.76(0.09)$ & $22.17(0.22)$ & $23.34(0.44)$ & $21.87(0.22)$ & $21.97(0.13)$ & 99.99 (9.99) \\
\hline & & & $21.98(0.21)$ & 99.99 (9.99) & $22.21(0.18)$ & $20.62(0.25)$ & $21.72(0.41)$ & $21.91(0.34)$ \\
\hline & & & $99.99(9.99)$ & 99.99 (9.99) & $21.91(0.17)$ & $21.75(0.14)$ & $22.01(0.13)$ & $22.12(0.62)$ \\
\hline & & & $20.71(0.09)$ & $22.04(0.26)$ & $20.73(0.19)$ & $20.76(0.15)$ & $21.05(0.17)$ & $20.63(0.28)$ \\
\hline
\end{tabular}


Table 8. continuation.

\begin{tabular}{|c|c|c|c|c|c|c|c|c|}
\hline \multirow[t]{4}{*}{$\bar{F}$ F2 ID } & \multirow[t]{4}{*}{$\mathrm{x}$} & \multirow[t]{4}{*}{$\mathrm{y}$} & $2 \mathrm{Ks} 10(\sigma)$ & $2 \mathrm{Ks} 01(\sigma)$ & $2 \mathrm{Ks} 02(\sigma)$ & $2 \mathrm{Ks} 03(\sigma)$ & $2 \mathrm{Ks} 05(\sigma)$ & $\overline{2 \mathrm{Ks} 06(\sigma)}$ \\
\hline & & & $2 \mathrm{Ks} 07(\sigma)$ & $2 \mathrm{Ks} 08(\sigma)$ & $2 \mathrm{Ks} 09(\sigma)$ & $2 \mathrm{Ks} 11(\sigma)$ & $2 \mathrm{Ks} 12(\sigma)$ & $2 \mathrm{Ks} 13(\sigma)$ \\
\hline & & & $2 \mathrm{Ks} 14(\sigma)$ & $2 \mathrm{Ks} 15(\sigma)$ & $2 \mathrm{Ks} 16(\sigma)$ & $2 \mathrm{Ks} 17(\sigma)$ & $2 \mathrm{Ks} 18(\sigma)$ & $2 \mathrm{Ks} 19(\sigma)$ \\
\hline & & & $2 \mathrm{Ks} 20(\sigma)$ & $2 \mathrm{Ks} 21(\sigma)$ & $2 \mathrm{Ks} 22(\sigma)$ & $2 \mathrm{Ks} 23(\sigma)$ & $2 \mathrm{Ks} 24(\sigma)$ & $2 \mathrm{Ks} 04(\sigma)$ \\
\hline \multirow[t]{4}{*}{$\overline{87455}$} & $\overline{802.736}$ & 847.321 & $22.39(0.15)$ & $99.99(9.99)$ & $999.99(9.99)$ & $21.36(0.13)$ & $22.62(0.18)$ & 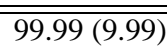 \\
\hline & & & $22.19(0.27)$ & $22.07(0.18)$ & $23.28(0.48)$ & $22.11(0.14)$ & $22.48(0.38)$ & $22.14(0.12)$ \\
\hline & & & $22.66(0.40)$ & $22.66(0.33)$ & $22.57(0.32)$ & $21.30(0.10)$ & $21.40(0.08)$ & $21.68(0.13)$ \\
\hline & & & $22.37(0.30)$ & 99.99 (9.99) & $22.02(0.16)$ & $21.02(0.22)$ & 99.99 (9.99) & $20.49(0.08)$ \\
\hline \multirow[t]{4}{*}{8837} & 204.386 & 427.624 & $23.13(0.25)$ & $24.00(1.56)$ & $99.99(9.99)$ & $23.86(1.77)$ & $23.74(0.94)$ & $20.61(0.09)$ \\
\hline & & & 99.99 (9.99) & 99.99 (9.99) & $23.94(0.59)$ & $99.99(9.99)$ & $23.85(1.25)$ & 23.77 (1.03) \\
\hline & & & $21.76(0.15)$ & $22.40(0.20)$ & $21.93(0.26)$ & $23.06(0.36)$ & $23.02(0.52)$ & $22.38(0.21)$ \\
\hline & & & $23.10(0.62)$ & $22.09(0.24)$ & $22.88(0.42)$ & $23.34(0.49)$ & $23.05(0.43)$ & 99.99 (9.99) \\
\hline \multirow[t]{4}{*}{8869} & 500.430 & 179.994 & $22.26(0.14)$ & $22.29(0.38)$ & $22.67(0.51)$ & $23.09(0.42)$ & $22.55(0.20)$ & $21.87(0.17)$ \\
\hline & & & $22.26(0.21)$ & $19.74(0.12)$ & $22.56(0.24)$ & $20.86(0.19)$ & $21.25(0.13)$ & $21.84(0.19)$ \\
\hline & & & $21.31(0.16)$ & $21.54(0.13)$ & $22.42(0.20)$ & $22.09(0.14)$ & $21.99(0.16)$ & $20.92(0.10)$ \\
\hline & & & $23.36(0.85)$ & $22.32(0.24)$ & $21.57(0.12)$ & $21.59(0.09)$ & $22.01(0.19)$ & $21.17(0.16)$ \\
\hline \multirow[t]{4}{*}{8955} & 8.396 & 158.185 & $23.15(0.28)$ & 99.99 (9.99) & $23.58(0.99)$ & $23.34(1.47)$ & $24.69(7.65)$ & $20.26(0.11)$ \\
\hline & & & 99.99 (9.99) & $21.48(0.24)$ & $22.88(0.23)$ & 99.99 (9.99) & $20.65(0.35)$ & 99.99 (9.99) \\
\hline & & & $23.32(0.80)$ & $22.22(0.22)$ & $23.29(0.66)$ & 99.99 (9.99) & $22.61(0.16)$ & $22.94(0.41)$ \\
\hline & & & 99.99 (9.99) & $22.94(0.60)$ & $22.75(0.37)$ & $99.99(9.99)$ & 99.99 (9.99) & 99.99 (9.99) \\
\hline \multirow[t]{4}{*}{9018} & 496.676 & 824.354 & $23.06(0.27)$ & $25.41(4.41)$ & $24.15(2.38)$ & 99.99 (9.99) & 99.99 (9.99) & $22.64(0.29)$ \\
\hline & & & $22.83(0.47)$ & $23.14(0.62)$ & 99.99 (9.99) & $20.83(0.07)$ & $23.26(0.73)$ & $22.80(0.20)$ \\
\hline & & & $99.99(9.99)$ & $23.00(0.35)$ & $22.86(0.25)$ & $23.23(0.35)$ & $99.99(9.99)$ & $22.38(0.20)$ \\
\hline & & & $22.96(0.55)$ & $22.41(0.35)$ & 99.99 (9.99) & $22.80(0.31)$ & $22.51(0.30)$ & 99.99 (9.99) \\
\hline \multirow[t]{4}{*}{9213} & 498.600 & 186.034 & $21.94(0.12)$ & $22.03(0.16)$ & $22.69(0.33)$ & $22.15(0.22)$ & $22.25(0.15)$ & $21.63(0.31)$ \\
\hline & & & $21.96(0.16)$ & 99.99 (9.99) & $22.43(0.22)$ & $20.83(0.13)$ & $21.00(0.09)$ & $21.40(0.22)$ \\
\hline & & & $21.85(0.17)$ & $21.33(0.09)$ & $22.12(0.14)$ & $22.29(0.13)$ & $21.82(0.12)$ & $21.50(0.09)$ \\
\hline & & & $21.64(0.18)$ & $21.89(0.23)$ & $21.29(0.09)$ & $21.80(0.15)$ & $22.16(0.23)$ & $20.63(0.13)$ \\
\hline \multirow[t]{4}{*}{9299} & 579.266 & 356.438 & $25.46(2.81)$ & $22.99(0.45)$ & $22.56(0.32)$ & 99.99 (9.99) & $99.99(9.99)$ & $23.13(0.45)$ \\
\hline & & & $23.51(0.86)$ & 99.99 (9.99) & $24.13(1.25)$ & $20.85(0.09)$ & $22.89(0.54)$ & $22.89(0.27)$ \\
\hline & & & 99.99 (9.99) & 99.99 (9.99) & 99.99 (9.99) & $22.98(0.33)$ & $23.30(0.45)$ & $22.43(0.22)$ \\
\hline & & & $22.50(0.37)$ & $22.20(0.23)$ & $22.75(0.31)$ & $99.99(9.99)$ & 99.99 (9.99) & $21.70(0.42)$ \\
\hline \multirow[t]{4}{*}{9383} & 782.842 & 577.054 & $22.42(0.23)$ & $22.80(0.73)$ & $22.23(0.40)$ & $22.38(0.41)$ & $22.59(0.39)$ & $20.86(0.11)$ \\
\hline & & & $22.41(0.30)$ & 99.99 (9.99) & $22.75(0.33)$ & $20.78(0.10)$ & $21.93(0.22)$ & $21.32(0.17)$ \\
\hline & & & $21.62(0.27)$ & $22.37(0.20)$ & $22.54(0.18)$ & $22.47(0.19)$ & $22.66(0.32)$ & $21.46(0.13)$ \\
\hline & & & $21.60(0.21)$ & $22.18(0.33)$ & $21.99(0.15)$ & $22.18(0.25)$ & $22.33(0.28)$ & $21.25(0.20)$ \\
\hline \multirow[t]{4}{*}{9628} & 131.268 & 734.555 & $23.37(0.40)$ & $22.63(0.42)$ & $99.99(9.99)$ & $23.81(1.22)$ & $22.85(0.14)$ & $22.61(0.30)$ \\
\hline & & & $22.78(0.30)$ & $22.30(0.32)$ & $22.86(0.19)$ & $20.51(0.07)$ & $22.88(0.46)$ & $22.67(0.20)$ \\
\hline & & & $22.77(0.35)$ & $22.46(0.26)$ & $24.24(1.10)$ & $23.17(0.29)$ & $23.02(0.33)$ & $22.75(0.29)$ \\
\hline & & & $24.11(1.57)$ & $25.03(4.48)$ & $22.69(0.27)$ & $22.76(0.35)$ & $23.01(0.58)$ & $22.92(1.32)$ \\
\hline \multirow[t]{4}{*}{9668} & 354.482 & -9.773 & $99.99(9.99)$ & $22.21(0.21)$ & $99.99(9.99)$ & 99.99 (9.99) & $22.81(0.36)$ & $99.99(9.99)$ \\
\hline & & & 99.99 (9.99) & 99.99 (9.99) & 99.99 (9.99) & $20.82(0.07)$ & $22.22(0.22)$ & $22.22(0.11)$ \\
\hline & & & $22.06(0.28)$ & $22.64(0.29)$ & $22.30(0.12)$ & 99.99 (9.99) & $22.44(0.19)$ & $22.07(0.20)$ \\
\hline & & & $24.15(2.07)$ & $22.89(0.63)$ & $22.54(0.31)$ & $25.51(3.69)$ & $24.30(1.29)$ & 99.99 (9.99) \\
\hline \multirow[t]{4}{*}{9844} & 101.202 & 471.023 & $22.31(0.14)$ & $22.08(0.14)$ & $24.24(1.53)$ & $99.99(9.99)$ & $22.47(0.17)$ & $21.54(0.45)$ \\
\hline & & & $22.36(0.28)$ & $20.67(0.46)$ & $22.68(0.35)$ & $20.80(0.12)$ & $21.78(0.28)$ & $22.75(0.32)$ \\
\hline & & & $21.06(0.19)$ & $21.12(0.28)$ & $22.40(0.26)$ & $22.25(0.15)$ & $21.86(0.11)$ & $21.35(0.15)$ \\
\hline & & & $21.65(0.18)$ & $22.13(0.31)$ & $21.21(0.07)$ & $21.77(0.12)$ & $22.47(0.27)$ & $21.73(0.43)$ \\
\hline \multirow[t]{4}{*}{9979} & 46.537 & 423.611 & $22.73(0.19)$ & 99.99 (9.99) & $99.99(9.99)$ & $24.70(9.85)$ & $23.62(0.33)$ & $21.05(0.09)$ \\
\hline & & & 99.99 (9.99) & $99.99(9.99)$ & $23.80(1.05)$ & $21.27(0.12)$ & $23.53(0.84)$ & $23.83(0.61)$ \\
\hline & & & $23.63(1.08)$ & $24.61(1.09)$ & $22.56(0.25)$ & $22.38(0.21)$ & $22.53(0.28)$ & $23.07(0.43)$ \\
\hline & & & $22.64(0.44)$ & $24.34(2.99)$ & 99.99 (9.99) & $23.23(0.51)$ & $99.99(9.99)$ & 99.99 (9.99) \\
\hline
\end{tabular}


Table 8. continuation.

\begin{tabular}{|c|c|c|c|c|c|c|c|c|}
\hline \multirow[t]{4}{*}{$\bar{F}$ F2 ID } & \multirow[t]{4}{*}{$\mathrm{x}$} & \multirow[t]{4}{*}{$\mathrm{y}$} & $2 \mathrm{Ks} 10(\sigma)$ & $2 \mathrm{Ks} 01(\sigma)$ & $2 \mathrm{Ks} 02(\sigma)$ & $2 \mathrm{Ks} 03(\sigma)$ & $2 \mathrm{Ks} 05(\sigma)$ & $2 \mathrm{Ks} 06(\sigma)$ \\
\hline & & & $2 \mathrm{Ks} 07(\sigma)$ & $2 \mathrm{Ks} 08(\sigma)$ & $2 \mathrm{Ks} 09(\sigma)$ & $2 \mathrm{Ks} 11(\sigma)$ & $2 \mathrm{Ks} 12(\sigma)$ & $2 \mathrm{Ks} 13(\sigma)$ \\
\hline & & & $2 \mathrm{Ks} 14(\sigma)$ & $2 \mathrm{Ks} 15(\sigma)$ & $2 \mathrm{Ks} 16(\sigma)$ & $2 \mathrm{Ks} 17(\sigma)$ & $2 \mathrm{Ks} 18(\sigma)$ & $2 \mathrm{Ks} 19(\sigma)$ \\
\hline & & & $2 \mathrm{Ks} 20(\sigma)$ & $2 \mathrm{Ks} 21(\sigma)$ & $2 \mathrm{Ks} 22(\sigma)$ & $2 \mathrm{Ks} 23(\sigma)$ & $2 \mathrm{Ks} 24(\sigma)$ & $2 \mathrm{Ks} 04(\sigma)$ \\
\hline \multirow[t]{4}{*}{10203} & $\overline{520.379}$ & 821.164 & $22.58(0.23)$ & $22.55(0.37)$ & $22.95(0.53)$ & $24.95(2.56)$ & $22.37(0.18)$ & $21.33(0.16)$ \\
\hline & & & $22.91(0.40)$ & $23.86(1.38)$ & $23.08(0.48)$ & $21.26(0.08)$ & $21.74(0.18)$ & $21.26(0.27)$ \\
\hline & & & $21.76(0.13)$ & $22.99(0.52)$ & $22.36(0.18)$ & $22.71(0.31)$ & $22.54(0.34)$ & $21.44(0.08)$ \\
\hline & & & $22.49(0.33)$ & $22.68(0.43)$ & $22.06(0.21)$ & $21.89(0.17)$ & $22.28(0.33)$ & $22.86(0.76)$ \\
\hline \multirow[t]{4}{*}{10761} & 227.931 & 439.216 & $22.29(0.11)$ & $22.29(0.25)$ & $22.14(0.28)$ & $23.04(1.94)$ & $21.26(0.15)$ & $20.60(0.11)$ \\
\hline & & & $22.76(0.41)$ & $20.97(0.15)$ & $22.88(0.31)$ & $21.93(0.13)$ & $22.18(0.23)$ & $21.39(0.18)$ \\
\hline & & & $22.66(0.40)$ & $22.39(0.23)$ & $21.54(0.19)$ & $22.66(0.24)$ & $22.09(0.14)$ & $22.33(0.23)$ \\
\hline & & & $25.09(2.91)$ & $22.41(0.37)$ & $22.64(0.29)$ & 24.03 (1.06) & $22.48(0.32)$ & $21.44(0.19)$ \\
\hline \multirow[t]{4}{*}{10854} & 49.091 & 429.543 & $22.53(0.21)$ & $22.53(0.36)$ & 23.44 (1.47) & $23.23(0.77)$ & $22.31(0.32)$ & $22.50(0.43)$ \\
\hline & & & $22.72(0.27)$ & $22.06(0.30)$ & $25.67(5.94)$ & $20.90(0.09)$ & $99.99(9.99)$ & $22.54(0.23)$ \\
\hline & & & 99.99 (9.99) & $22.67(0.19)$ & $22.93(0.30)$ & $23.28(0.35)$ & 99.99 (9.99) & $24.25(1.25)$ \\
\hline & & & $99.99(9.99)$ & 99.99 (9.99) & 99.99 (9.99) & $23.41(0.68)$ & $99.99(9.99)$ & 99.99 (9.99) \\
\hline \multirow[t]{4}{*}{11088} & 456.068 & 541.129 & $23.09(0.37)$ & $23.03(0.53)$ & $23.85(1.50)$ & $24.65(2.47)$ & 99.99 (9.99) & $22.54(0.20)$ \\
\hline & & & $24.05(1.41)$ & 99.99 (9.99) & $99.99(9.99)$ & $20.80(0.11)$ & $21.94(0.22)$ & $22.72(0.20)$ \\
\hline & & & $23.57(0.75)$ & $23.79(0.90)$ & $25.11(2.81)$ & 99.99 (9.99) & $24.68(2.06)$ & $22.90(0.33)$ \\
\hline & & & $24.58(3.33)$ & $23.44(0.71)$ & $22.77(0.33)$ & $24.29(1.37)$ & $23.28(0.71)$ & $99.99(9.99)$ \\
\hline \multirow[t]{4}{*}{11139} & 189.627 & 236.601 & $22.23(0.14)$ & $22.13(0.27)$ & 99.99 (9.99) & $23.80(1.27)$ & $22.57(0.22)$ & $21.12(0.09)$ \\
\hline & & & $22.93(0.48)$ & $22.90(0.86)$ & $22.54(0.22)$ & $21.76(0.11)$ & $22.55(0.35)$ & $22.05(0.19)$ \\
\hline & & & $21.55(0.11)$ & $21.98(0.16)$ & $22.18(0.16)$ & $22.52(0.18)$ & $22.33(0.21)$ & $22.46(0.18)$ \\
\hline & & & $22.22(0.32)$ & $22.52(0.4$ & $21.30(0.10)$ & 22.46 & $22.91(0.47)$ & $22.12(0.49)$ \\
\hline \multirow[t]{4}{*}{11139} & 189.627 & 236.601 & $22.23(0.14)$ & $22.13(0.27)$ & $99.99(9.99)$ & $23.80(1.27)$ & $22.57(0.22)$ & $21.12(0.09)$ \\
\hline & & & $22.93(0.48)$ & $22.90(0.86)$ & $22.54(0.22)$ & $21.76(0.11)$ & $22.55(0.35)$ & $22.05(0.19)$ \\
\hline & & & $21.55(0.11)$ & $21.98(0.16)$ & $22.18(0.16)$ & $22.52(0.18)$ & $22.33(0.21)$ & $22.46(0.18)$ \\
\hline & & & $22.22(0.32)$ & $22.52(0.48)$ & $21.30(0.10)$ & $22.46(0.28)$ & $22.91(0.47)$ & $22.12(0.49)$ \\
\hline \multirow[t]{4}{*}{11513} & 74.208 & 191.714 & $22.41(0.17)$ & $23.11(0.47)$ & $22.81(0.64)$ & $22.34(0.30)$ & 23.11 & $22.74(0.42)$ \\
\hline & & & $23.17(0.61)$ & $22.41(0.33)$ & $22.49(0.19)$ & $21.03(0.08)$ & 22.62 & $22.61(0.25)$ \\
\hline & & & $21.29(0.12)$ & $22.62(0.32)$ & $22.41(0.22)$ & $22.27(0.12)$ & $21.98(0.15)$ & $22.79(0.34)$ \\
\hline & & & $22.68(0.41)$ & $22.98(0.59)$ & $22.67(0.25)$ & $22.02(0.18)$ & $22.79(0.40)$ & 22.00 \\
\hline \multirow[t]{4}{*}{11635} & 166.246 & 208.354 & $22.26(0.16)$ & 23.26 & 25.15 & 99.99 & 23.07 & 21.36 \\
\hline & & & $23.75(1.00)$ & $21.49(0.30)$ & $99.99(9.99)$ & $21.23(0.08)$ & $22.82(0.51)$ & $22.21(0.28)$ \\
\hline & & & $22.74(0.36)$ & $22.59(0.21)$ & $22.13(0.17)$ & $22.18(0.15)$ & $22.45(0.19)$ & $23.02(0.39)$ \\
\hline & & & $22.87(0.47)$ & 99.99 (9.99) & $21.73(0.13)$ & 99.99 (9.99) & $22.37(0.32)$ & $22.28(0.60)$ \\
\hline \multirow[t]{4}{*}{11698} & 536.324 & 845.998 & $22.74(0.23)$ & $22.31(0.19)$ & $22.49(0.32)$ & $23.04(0.78)$ & $22.75(0.26)$ & $22.18(0.23)$ \\
\hline & & & $22.73(0.35)$ & $24.16(2.61)$ & $22.75(0.27)$ & $21.39(0.08)$ & $21.23(0.12)$ & $23.47(0.85)$ \\
\hline & & & $22.43(0.22)$ & $23.07(0.50)$ & $22.56(0.30)$ & $22.75(0.27)$ & $22.16(0.19)$ & 22.51 \\
\hline & & & $22.46(0.32)$ & $21.56(0.17)$ & $21.95(0.14)$ & $22.20(0.19)$ & 99.99 (9.99) & $22.66(0.69)$ \\
\hline \multirow[t]{4}{*}{12003} & 453.077 & 522.497 & $22.82(0.16)$ & $23.26(0.63)$ & $22.87(0.41)$ & $25.12(3.37)$ & $22.34(0.13)$ & 99.99 (9.99) \\
\hline & & & $22.39(0.28)$ & 99.99 (9.99) & $24.21(0.92)$ & $21.12(0.28)$ & $21.01(0.18)$ & $22.12(0.43)$ \\
\hline & & & $21.04(0.14)$ & $21.92(0.18)$ & $22.18(0.12)$ & $22.12(0.18)$ & $22.40(0.12)$ & $21.36(0.14)$ \\
\hline & & & $22.54(0.56)$ & $22.98(0.53)$ & $21.53(0.16)$ & $22.21(0.29)$ & $21.19(0.15)$ & $21.22(0.36)$ \\
\hline \multirow[t]{4}{*}{12041} & 807.046 & 508.901 & $22.27(0.18)$ & $22.35(0.23)$ & $23.35(0.72)$ & $22.57(0.41)$ & $22.13(0.15)$ & $22.79(0.38)$ \\
\hline & & & $22.79(0.60)$ & $22.83(0.31)$ & $22.81(0.28)$ & $23.17(0.47)$ & $22.29(0.34)$ & $22.17(0.16)$ \\
\hline & & & $23.01(0.55)$ & $22.31(0.13)$ & $21.62(0.06)$ & $22.21(0.23)$ & $22.09(0.14)$ & $22.96(0.37)$ \\
\hline & & & $23.28(0.89)$ & $21.94(0.25)$ & $22.13(0.19)$ & $22.33(0.23)$ & $22.58(0.36)$ & $21.99(0.47)$ \\
\hline \multirow[t]{4}{*}{12101} & 576.615 & 311.540 & $22.24(0.12)$ & $24.93(1.49)$ & $22.50(0.33)$ & $21.86(0.27)$ & $23.14(0.36)$ & $22.51(0.29)$ \\
\hline & & & $22.43(0.30)$ & $23.32(0.63)$ & $22.02(0.20)$ & $21.17(0.07)$ & $22.59(0.31)$ & $22.27(0.13)$ \\
\hline & & & $22.38(0.25)$ & $22.57(0.17)$ & $22.32(0.20)$ & $22.70(0.23)$ & $22.57(0.20)$ & $22.42(0.20)$ \\
\hline & & & $23.51(1.27)$ & $22.15(0.33)$ & $22.50(0.20)$ & $22.01(0.16)$ & $23.56(0.67)$ & $22.32(0.54)$ \\
\hline
\end{tabular}


Table 8. continuation.

\begin{tabular}{|c|c|c|c|c|c|c|c|c|}
\hline \multirow[t]{4}{*}{ F2 ID } & $\mathrm{x}$ & \multirow[t]{4}{*}{$\mathrm{y}$} & $2 \mathrm{Ks} 10(\sigma)$ & $2 \mathrm{Ks} 01(\sigma)$ & $2 \mathrm{Ks} 02(\sigma)$ & $2 \mathrm{Ks} 03(\sigma)$ & $2 \mathrm{Ks} 05(\sigma)$ & $2 \mathrm{Ks} 06(\sigma)$ \\
\hline & & & $2 \mathrm{Ks} 07(\sigma)$ & $2 \mathrm{Ks} 08(\sigma)$ & $2 \mathrm{Ks} 09(\sigma)$ & $2 \mathrm{Ks} 11(\sigma)$ & $2 \mathrm{Ks} 12(\sigma)$ & $2 \mathrm{Ks} 13(\sigma)$ \\
\hline & & & $2 \mathrm{Ks} 14(\sigma)$ & $2 \mathrm{Ks} 15(\sigma)$ & $2 \mathrm{Ks} 16(\sigma)$ & $2 \mathrm{Ks} 17(\sigma)$ & $2 \mathrm{Ks} 18(\sigma)$ & $2 \mathrm{Ks} 19(\sigma)$ \\
\hline & & & $2 \mathrm{Ks} 20(\sigma)$ & $2 \mathrm{Ks} 21(\sigma)$ & $2 \mathrm{Ks} 22(\sigma)$ & $2 \mathrm{Ks} 23(\sigma)$ & $2 \mathrm{Ks} 24(\sigma)$ & $2 \mathrm{Ks} 04(\sigma)$ \\
\hline \multirow[t]{4}{*}{12529} & 145.762 & 779.651 & 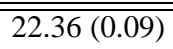 & 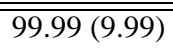 & $222.48(0.35)$ & $21.58(0.14)$ & $222.58(0.16)$ & 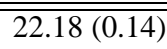 \\
\hline & & & $24.18(1.08)$ & $26.21(6.85)$ & $23.54(0.45)$ & $20.71(0.09)$ & $22.63(0.43)$ & $22.45(0.17)$ \\
\hline & & & $22.42(0.23)$ & $22.61(0.24)$ & $22.41(0.23)$ & $22.72(0.31)$ & $22.18(0.22)$ & $22.36(0.23)$ \\
\hline & & & $22.40(0.29)$ & $23.25(0.73)$ & $22.75(0.27)$ & $23.07(0.48)$ & $22.59(0.39)$ & $23.23(1.46)$ \\
\hline \multirow[t]{4}{*}{12881} & 649.102 & 266.481 & $23.14(0.35)$ & $23.27(0.48)$ & $23.82(1.24)$ & $24.37(1.73)$ & $23.01(0.20)$ & $21.08(0.09)$ \\
\hline & & & $25.83(7.51)$ & $22.45(0.26)$ & 99.99 (9.99) & $22.50(0.26)$ & $22.60(0.47)$ & $22.65(0.18)$ \\
\hline & & & $22.59(0.34)$ & $22.57(0.21)$ & $22.68(0.18)$ & $22.39(0.22)$ & $23.22(0.41)$ & $23.11(0.48)$ \\
\hline & & & $22.05(0.21)$ & $22.34(0.29)$ & $22.10(0.16)$ & 99.99 (9.99) & 99.99 (9.99) & $22.14(0.60)$ \\
\hline \multirow[t]{4}{*}{13140} & 273.655 & 820.992 & $22.84(0.22)$ & $24.48(2.01)$ & 99.99 (9.99) & $21.55(0.09)$ & $24.13(0.54)$ & 99.99 (9.99) \\
\hline & & & $23.08(0.67)$ & $22.54(0.72)$ & $23.45(0.63)$ & 99.99 (9 & 99.99 (9.99) & $23.66(0.54)$ \\
\hline & & & $23.37(0.76)$ & $23.38(0.40)$ & $24.09(0.79)$ & $24.14(0.84)$ & $23.13(0.45)$ & $23.17(0.50)$ \\
\hline & & & 99.99 (9.99) & $23.48(0.61)$ & $22.77(0.32)$ & $22.16(0.23)$ & $22.81(0.45)$ & $23.52(2.44)$ \\
\hline \multirow[t]{4}{*}{13468} & 253.199 & 381.898 & $22.50(0.14)$ & $21.92(0.27)$ & $24.21(1.15)$ & $22.70(0.46)$ & $23.56(0.37)$ & $22.71(0.30)$ \\
\hline & & & $23.37(0.70)$ & $22.31(0.33)$ & $24.26(1.14)$ & 99.99 (9.99) & $22.85(0.54)$ & $22.55(0.22)$ \\
\hline & & & $22.42(0.32)$ & $22.83(0.19)$ & $22.42(0.15)$ & $21.41(0.08)$ & $22.11(0.13)$ & $22.89(0.34)$ \\
\hline & & & $23.22(0.65)$ & $21.93(0.25)$ & $23.34(0.41)$ & $22.21(0.22)$ & $23.22(0.53)$ & $23.27(1.28)$ \\
\hline \multirow[t]{4}{*}{13620} & 728.246 & 491.702 & $23.50(0.47)$ & $23.21(0.59)$ & $22.35(0.35)$ & $23.17(0.76)$ & $22.72(0.24)$ & $23.25(0.53)$ \\
\hline & & & $23.07(0.35)$ & $21.62(0.20)$ & $23.12(0.46)$ & $20.37(0.14)$ & $24.57(2.71)$ & $22.47(0.13)$ \\
\hline & & & 99.99 (9.99) & $22.77(0.31)$ & $23.29(0.41)$ & $23.06(0.33)$ & $25.00(1.70)$ & 99.99 (9.99) \\
\hline & & & $22.93(0.59)$ & $22.83(0.46)$ & $23.56(0.89)$ & $23.73(0.88)$ & $22.67(0.34)$ & $22.77(0.66)$ \\
\hline \multirow[t]{4}{*}{14598} & 274.310 & 313.415 & $23.69(0.48)$ & 99.99 (9.99) & $24.18(1.74)$ & 99.99 (9.99) & $24.43(1.08)$ & $24.30(1.29)$ \\
\hline & & & $24.96(1.85)$ & $24.46(1.48)$ & 99.99 (9.99) & $22.52(0.22)$ & 99.99 (9.99) & $24.17(0.86)$ \\
\hline & & & $99.99(9.99)$ & $24.88(2.32)$ & $22.86(0.19)$ & $23.47(0.50)$ & $23.30(0.43)$ & $23.05(0.44)$ \\
\hline & & & 24.17 (1.48) & $23.11(0.59)$ & $23.09(0.39)$ & $21.63(0.11)$ & $23.56(0.74)$ & $22.29(0.56)$ \\
\hline \multirow[t]{4}{*}{14638} & 841.787 & 474.766 & $22.90(0.32)$ & $22.60(0.52)$ & $23.07(0.67)$ & $99.99(9.99)$ & $23.77(0.54)$ & $23.36(0.56)$ \\
\hline & & & 99.99 (9.99) & 99.99 (9.99) & $24.27(1.44)$ & $24.39(1.12)$ & $22.90(0.43)$ & $23.73(0.58)$ \\
\hline & & & $23.67(0.96)$ & $24.55(1.66)$ & $22.76(0.12)$ & $24.14(1.11)$ & $22.78(0.17)$ & $22.83(0.32)$ \\
\hline & & & $21.31(0.11)$ & 99.99 (9.99) & $23.21(0.49)$ & $21.92(0.13)$ & $22.82(0.37)$ & 99.99 (9.99) \\
\hline
\end{tabular}

